\title{
The genus Bilabrella Lindl. (Orchidaceae, Habenariinae): materials to the taxonomic revision
}

\author{
Marta Kras*, Natalia Olędrzyńska \& Dariusz L. Szlachetko
}

Department of Plant Taxonomy and Nature Conservation, Faculty of Biology, University of Gdańsk, Wita Stwosza 59, 80-308 Gdańsk, Poland

* corresponding author (e-mail: bgmk@univ.gda.pl)

\begin{abstract}
The aim of the paper is to present materials to the taxonomic revision of the African orchid genus Bilabrella Lindl., based on herbarium materials, specimens conserved in FAA as well as literature. It was compiled using classical taxonomic methods. The research involved determining differences between Bilabrella and other genera of Habenariinae in Africa. Its morphological structure was analysed and key features for the generic taxonomy defined. An infrageneric classification is proposed. A full description of each species and distribution data are provided. The ecological requirements and phenology of all species have been assembled and systematized.
\end{abstract}

Key words: taxonomy, revision, morphology, Orchidaceae, Habenaria, Bilabrella, Africa

\section{Introduction}

The African continent with lots of different habitats is home for many orchid species. Genus Bilabrella described by Lindley in 1834, is one of the most interesting taxa with paleotropical distribution. Most of Bilabrella species are noted from east and south parts of Africa, which are dominated by savanas and grassy ecosystems.

The terrestrial species from Bilabrella are recognizable by their flower features e.g. dorsal sepals with appendix, bilobed petals and elongated outgrowths of the stigma, rostellum and anther.

Like any other taxon with unclear systematic position, Bilabrella is a very interesting subject of study. So far, no revision of the genus has been published. Its high infrageneric variability, as well as the structure of perianth segments and the presence of species complex that are morphologically very similar, cause problems in determining the systematic position within the genus.

The aim of the present paper is to provide background for taxonomic revision of Bilabrella. Our work was based on the available herbarium material and specimens conserved in FAA as well as literature. The first stage of the research involved determining differences between Bilabrella and other genera of Habenariinae from Africa. Its morphological structure was analysed. The key for generic taxonomy was defined. Based on the data obtained, an infrageneric classification was proposed. Keys for determination together with detailed descriptions of all species should make them recognizable in the field. Moreover, any available information regarding the distribution of the species examined, as well as their ecological requirements and phenology were assembled and systematized.

\section{Material and methods}

This work was conducted using classical taxonomic methods - it is a standard research procedure in the case of taxa that have been poorly recognized and studied. In the course of research, there were used herbarium specimens collected in Africa from the following Herbaria: AMES, B, BM, BR, BRLU, C, E, F, HBG, $\mathrm{K}$, LISU, MA, MO, P, UGDA, W, WAG and WU, as well as specimens conserved in FAA. Duplicates of the specimens held in LBV and LISU were examined in other institutions.

Flowers of the examined plants were rehydrated, with their taxonomically important fragments drawn, and then measured and described. Based on the authors' 
own studies, features most important to the taxonomy of Bilabrella are:

- shape, dimensions and relative size of individual outgrowths of the gynostemium (stigmaphores, rostellophores, antherophores),

- presence or absence of glandular hairs on the stigmaphores,

- ratio of the central rostellum lobe to the connective,

- form and dimensions of the auriculae,

- form, relative dimensions and glandularity of two inner lobes of petals,

- form and dimensions of the labellum and spur.

Differences in the aforementioned characters were clear-cut, so they would be of greater diagnostic importance. In order to measure generative organs, flowers from the central part of inflorescences were used.

The features useful in identifying Bilabrella species are as follows:

- form and size of dorsal and lateral sepals,

- relative lengths of the ovary and peduncle and of the peduncle and floral bract,

- glandularity of the above-mentioned floral elements,

- leaf arrangement on the stem,

- leaf form and size,

- ratio of lamina length and width,

- inflorescence length and thickness,

- number of flowers.

Each specimen was photographed - in some cases, photocopies of the herbarium sheet were made and attached to the documentation. After database compilation, the data were compared with the original material - types, diagnoses and/or illustrations, if available.

In total, over 2000 specimens were examined, belonging to 93 species of Bilabrella. The Herbarium acronyms used conformed to those given in the Index Herbariorum (Holmgren et al. 1990). The type specimens of 90 species were located and made available. Further 6 type specimens were located but proved to be inaccessible. Lack of information made it impossible to find the place of deposition of 3 type specimens. The original diagnoses of all species were collected. The information thus acquired was augmented by a list of literature that described or quoted individual species, as well as by an illustrative material, if such existed.

Information on the ecology, distribution and phenology of individual species was obtained from herbarium labels or extracted from the literature as much as possible. The data were incomplete, however, and precluded the provision of a full description of the ecological requirements of the species examined.

We propose to divide the genus Bilabrella into several morphologically consistent groups in order to simplify determination of the species. In each group, species are listed according to the increasing grade of similarity to one another, what permits easy comparison between them.

We published a general information on the genus some years ago (Kras \& Szlachetko 2013). Below, we would like to briefly repeat the most important of items of information.

\section{History of the research on Bilabrella Lindl.}

Within the order Orchidales, a dozen or so of genera were described that comprised a considerable number of species. Until fairly recently, one of the larger taxa at the generic level was Habenaria Willd., which contained about 2100-2450 species' names (according to The International Plant Name Index 2018 and TropicosMissouri Botanical Garden database 2018). The genus was proposed by a German botanist, Willdenow, in the fourth edition of "Species Plantarum", which were established in 1805. The author included a description of two new species: Habenaria brachyceratitis Willd. and Habenaria macroceratitis Willd. Willdenow did not indicate the type for the genus, which was a standard procedure at that time. This quite brief description of the new genus caused serious taxonomic repercussions. Almost all terrestrial orchids with white or greenish and, usually, inconspicuous flowers, originating from tropical or subtropical areas started to be included in Habenaria. The confusion in the taxonomy of this group became even greater when genera from the temperate zone of the northern hemisphere such as Platanthera Rich., Coeloglossum Lindl., Piperia Rydb., Orchis L., Gymnadenia R.Br. and Dactylorhiza Neck., were included in Habenaria.

The genus Bilabrella was described by John Lindley in 1834. with only one species, Bilabrella falcicornis (Edwards's Bot. Reg. 20: sub. t. 1701. 1834) recognized. In the generic description, Lindley indicated the following diagnostic characters: fleshy sepals; lateral sepals of medium size; leathery and relatively large petals; 3-lobed, fleshy labellum; the anther with elongated, free and directed forwards or upwards antherophores; the rostellum evenly linear-lanceolate, with fleshy, stalked rostellophores; petiolate leaves with narrow blade; elongate inflorescence. None of these characters, however, could clearly differentiate Bilabrella from the broadly defined genus Habenaria. A few years later, Lindley himself included his genus to Habenaria.

In his monograph entitled "Beitrage zu einer Monographie der Gattung Habenaria Willd. (Allgemeiner Teil.)" from 1891, Kraenzlin selected $H$. macroceratitis Willd. as a type species for the genus. This, however, did not lead to defining unequivocal boundaries for the genus itself, nor for the section within it.

As a consequence, Rendle (1895), when describing a new species - Habenaria altior in "Flora of Eastern 
Tropical Africa" classified it within the section Bilabrellae, and similar species - Habenaria ndiana - in the section Replicatae. Rendle did not indicate differences between these sections nor did he justify his view on this issue.

In 1901 "Orchidacearum Genera et Species", another work by Kraenzlin was published and offered only a revision of Habenaria. Among other things, he mentioned 28 species there, which he included in the section Replicatae. He stated that differences between Replicatae and Bilabrella were so slight that these two should be amalgamated. Kraenzlin defined the section Replicatae by the following characters: 3-lobed labellum, 2-lobed petals and stigma with long stigmaphores.

In 1913, with no intention of revising the whole genus, Bolus published "Orchids of Southern Africa" where Habenaria species were listed in alphabetical order.

Also in 1913, "Flora Capensis" was published, with a chapter on orchids written by Rolfe. The author placed Habenaria under the number XXIV, giving descriptions of 37 species. Following Bolus, he did not recognize sections. Based on the Kraenzlin's revision, in Bilabrella, there should be 9 species described by Rolfe.

One of the best known researchers of African orchids was Summerhayes. He described a great number of Habenaria species. In early stages of his research on the genus, he accepted the existence of separate sections: Bilabrellae and Replicatae. This clearly resulted from species descriptions in a series of his articles (1931, 1932, 1942, 1960, 1962, 1966, 1968a, 1968b), in wich Habenaria limnophila Summerh. (1931) and Habenaria pauper (1932) were placed within sections Replicatae and Bilabrellae, respectively. With time, Summerhayes acquired more experience in his study of Habenaria and accepted Kraenzlin's proposal, uniting the two sections into one. Each new taxon description by Summerhayes showed great care for details. In 1968, two floras of western and eastern parts of tropical Africa were published by this author ("Flora of Tropical East Africa", "Flora of Tropical West Africa" ed. 2.), where the section Bilabrellae was not recognized. The section Replicatae was characterized by: anterior petal lobe longer than posterior lobe; both were narrow though, not always linear; spur almost straight or twisted; bract shorter than the combined length of ovary and pedicel. In "Flora of Tropical East Africa" (1968b), Summerhayes pinpointed a phenomenon which had not been described before by other researchers of this plant group. He noticed a relatively frequent presence of the so-called abnormal forms or atypically formed flowers. In his opinion, two species, Habenaria dactylostigma Kraenzl. and Habenaria inaequiloba Schltr., represented properly these forms. In Summerhayes' opinion, Habenaria simense, described by Henrich
Gustav Reichenbach in 1849 (Linnaea 22: 859) represented an abnormal form of Habenaria antennifera, which had been described by Richard 9 years earlier (Ann. Sci. Nat., Bot. 2(14): 268). Summerhayes placed information concerning this issue, among others, on the herbarium sheets of plants he had been studying.

Williamson (1977) published quite brief descriptions of selected 17 species of the section Replicatae and 5 unidentified species. Geerinck (1984) decided to omit the issue of sections under dispute. He gave descriptions and analytical figures for some of the 15 species from Central Africa, which, theoretically, should have belonged to the Replicatae section. In-depth research on Habenaria Willd. was conducted by la Croix and Cribb (1995). They noted 131 species of this genus from Mozambique, Malawi, Zimbabwe and Botswana. For each species, they provided information about the type specimen, precise quotation as well as the list of synonyms, a brief morphological description, site location and ecological data. These authors included 34 species in the Replicatae section. The results of analogous research conducted in Cameroon were published by Szlachetko and Olszewski in 1998 (1998a). A list of 25 species (where 8 of them were new to science) of the Replicatae section were given. In 2004, a volume was published by Szlachetko, Sawicka and Kras-Łapińska on Orchidaceae of Gabon. The authors did not include a Bilabrellae section in Habenaria. Plants with lanceolate or linear leaves, small resupinate flowers, a reflexed dorsal sepal, lateral sepals ending in an apiculus, petals divided into two basal lobes and gynostemium with outgrowths were all placed in the section Replicatae. The two species that occurred in Gabon were Habenaria genuflexa Rendle and Habenaria schimperiana Hochst. ex A. Rich.

In 2003, as a result of the research conducted on herbarium material, as well as data from literature, Szlachetko and Kras-Łapińska stated that there were no grounds for distinguishing Bilabrellae and Replicatae sections. At the same time, they restored the genus Bilabrella, with 60 species from the section Replicatae. In the course of further investigation, the authors changed the genus classification of other 11 species (Szlachetko $\&$ Kras 2006f). While analysing the materials collected for the present paper, other 22 taxa were included into Bilabrella (Kras \& Szlachetko 2009). Four species new to science were described (Kras \& Szlachetko 2008). Now the genus contains 93 species.

Taxonomic research on Bilabrella encountered many problems. First of all, many of the species presently included in this genus were described in the $19^{\text {th }}$ century and only on the basis of fragments of their inflorescences or single flowers. The condition in which they are found today often makes meaningful microscopic examination difficult or even impossible. An extreme 
case is that type material of Habenaria anaphysema Rchb. f., is available only as a drawing on a herbarium sheet. The type material for many species described by Schlechter (e.g. Bilabrella myodes 6, 1915) was burnt in Berlin during World War II. The location or existence of isotypes for many of Schlechter's taxa, e.g. Habenaria barbarae Schltr., are not known. In many cases, species descriptions published by their authors are brief and enigmatic making them not very useful (e.g. the diagnosis of Habenaria incarnata consists of one sentence only).

Another problem is a series of transitional forms between some species of the genus. The differentiation between the type materials is usually unproblematic, but the labelling of other materials may raise certain doubts. This seems to result from the rapidly developing speciation within not only Bilabrella but, more widely, in the majority of the Habenariinae, from the ease of hybrid creation and/or a high infrageneric variability.

Studies of ecological requirements of Bilabrella might provide the answer to the question as to whether the infrageneric variability observed is a result of adaptation to a different habitat or is genetic in nature. As a matter of fact, light exposure may considerably affect the size of a plant or its elements. Unfortunately, species of this genus have not been the subject to any ecological studies so far.

\section{Characteristics of the genus Bilabrella Lindl.}

\subsection{Systematic position}

In 1995, Szlachetko placed 13 genera in the subtribe Habenariinae: Ala Szlach., Arnottia A. Rich., Bonatea Willd., Centrostigma Schltr., Cynorkis Thouars, Diplomeris D. Don, Habenaria Willd., Kryptostoma (Summerh.) Geerinck, Megalorchis H. Perrier, Physoceras Schltr., Platycoryne Rchb. f., Roeperocharis Rchb. f., ?Tsaiorchis Tang. \& Wang. As a result of the research conducted by Szlachetko and his team since 2000, further genera have been extracted from Habenaria sensu latissimo, but they still belong to the Habenariinae which currently contains 42 genera.

Order: Orchidales Bromhaed

Family: Orchidaceae Juss.

Subfamily: Orchidoideae

Tribe: Orchideae

Subtribe: Habenariinae Benth.

Ala Szlach.

Alinorchis Szlach.

Arachnaria Szlach.

Arnottia A. Rich.

Ate Lindl.
Bertauxia Szlach.

Bicornella Lindl.

Bilabrella Lindl.

Bonatea Willd.

Centrostigma Schltr.

Ceratopetalorchis Szlach., Górn. \& Tuk.

Cynorkis Thouars

Diplomeris D. Don

Fimbrorchis Szlach.

Habenaria Willd.

Habenella Small

Kraenzlinorchis Szlach.

Kryptostoma (Summerh.) Geerinck

Kusibabella Szlach.

Lowiorchis Szlach.

Macrura Szlach. \& Sawicka

Medusorchis Szlach.

Megalorchis H. Perr.

Mirandorchis Szlach. \& Kras-Lap.

Monadeniorchis Szlach. \& Kras

Montolivaea Rchb. f.

Ochyrorchis Szlach.

Physoceras Schltr.

Plantaginorchis Szlach.

Platycoryne Rchb. f.

Platycorynoides Szlach.

Podandriella Szlach.

Pseudocoeloglossum Szlach.

Pseudohemipilia Szlach.

Renzorchis Szlach. \& Olszewski

Roeperocharis Rchb. f.

Schlechterorchis Szlach.

Senghasiella Szlach.

Smithanthe Szlach. \& Marg.

Trachypetalum Szlach. \& Sawicka

Tsaiorchis Tang \& F. T. Wang.

Veyretella Szlach. \& Olszewski

\subsection{Phylogeny and similarities}

The monophyly of Habenariinae was tested several times during last 20 years. Although the sampling of subtribe representatives was relatively poor, paraphyletic character of Habenariinae s.l. was confirmed by Bateman et al. (2003) and also Inda et al. (2010, 2012).

Based on data from two molecular markers (nuclear ITS region and plastid pseudogene matK), Batista et al. (2013) confirmed that Habenariinae s.l. (including Cynorkis and Stenoglottis) was a sister group to the Orchidinae subtribe. Although the monophyly of currently recognized sections within Habenaria was not supported, the authors indicated that there were a number of smaller, well-defined groups within the genus: 


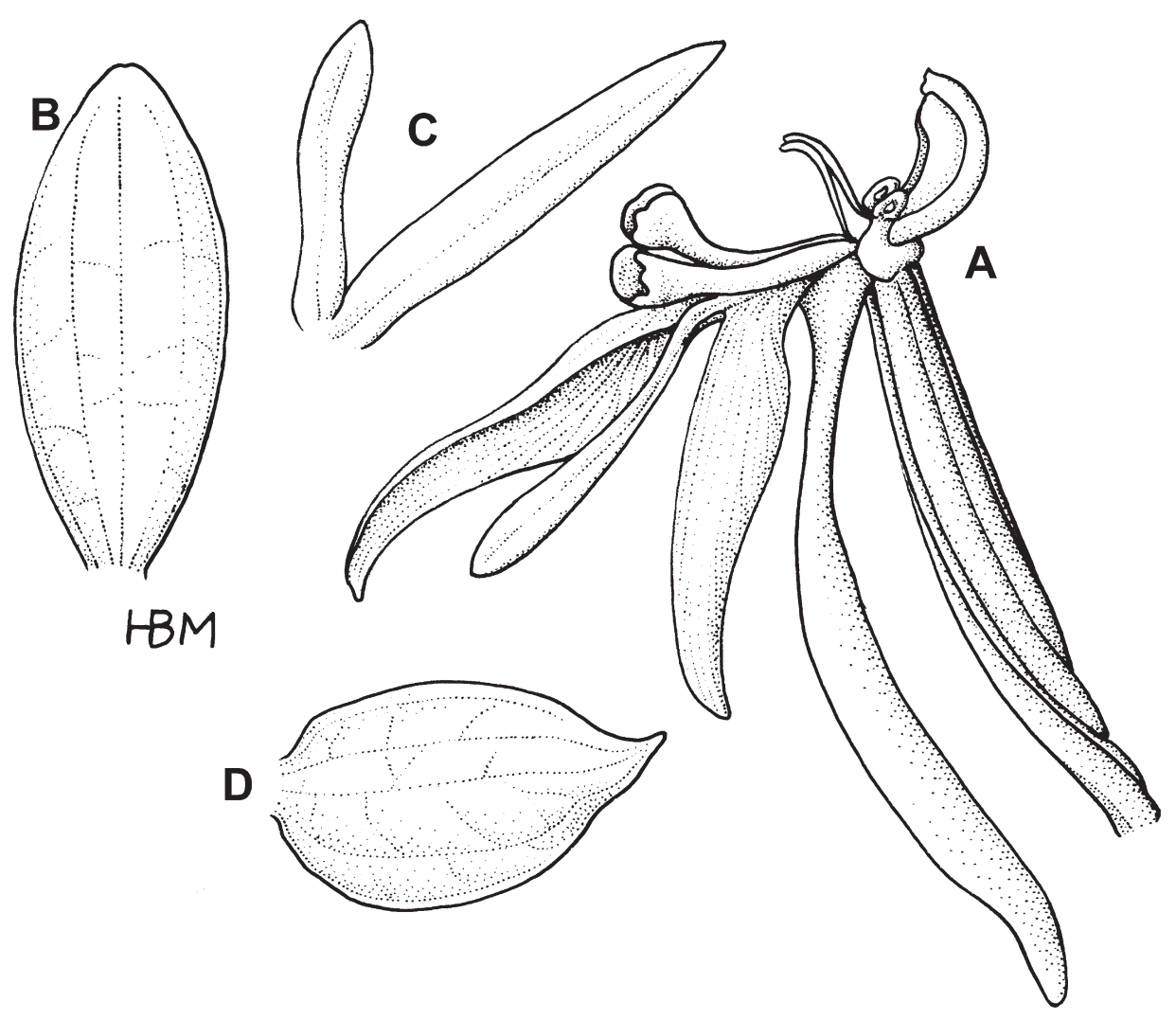

Fig. 1. Mirandorchis calvilabris (Summerh.) Szlach. \& Kras-Lap.

Explanations: A - habit; B - gynostemium, lip, spur and ovary; C - dorsal sepal; D - divided petal; E - lateral sepal (drawn by H. B. Margońska)

- clade consisting of Neotropical species;

- clade of African representatives (sect. Dolichostachyae, Podandria, Diphyllae, Ceratopetalae and section Bilabrellae), closely related to the previous one; - clade of Habenaria (sect. Chlorinae and Multipartitae) and genus Bonatea, which is a sister to the rest.

Moreover, the authors suggested an African orgin of the Neotropical clade as well as a relatively recent radiation of Habenaria in the New World.

Phylogenetic studies (Jin et al. 2014), where additionaly another plastid gene - rbcl - was investigated, showed that Habenaria consisted of two separated, well supported clades:

- one of species from tropical and subtropical Asia,

- and the other - of representatives from New and Old World Tropics (where also species from alpine regions were classified).

Since the paper of Jin et al. (2014) was published (where a large number of Asian taxa were sequenced), futher molecular studies on Habenariinae should focus on increasing sampling from African regions.

Bilabrella Lindl. seems to be related to a few other genera which have also been excluded from Habenaria sensu lattisimo.

Mirandorchis is a genus that appears to be morphologically similar to Bilabrella in habit. Mirandorchis was described by Szlachetko and Kras-Łapińska in 2003 (Fig. 1). Differences between the two genera may be observed when comparing the morphology of lateral sepals. In Mirandorchis, their apiculi are not prominent. Additionally, Mirandorchis resembles Bonatea Willd. in gynostemium structure. The pollen sacs are large and joined to a relatively narrow connective which protrudes over them. The central rostellum lobe is also large and subulate, while the stigmaphores are massive and wide in comparison with Bilabrella.

Many more differences can be found when comparing reproductive structures of Bilabrella and Renzorchis (Szlachetko \& Olszewski 1998b). Although, the two genera are very similar to each other in habit and structure of their floral parts (Fig. 2), representatives of the latter genus are characterized by a special structure of their gynostemium - the viscidia are roughly half as long as the lateral rostellum lobes, a feature not found in any other members of the Habenariinae.

In 2003, Szlachetko, Górniak and Tukałło raised the section Ceratopetalae Kraenzl. to generic level, under the name: Ceratopetalorchis (Fig. 3). The generative structure is almost identical to that found in Bilabrella. The form of the perianth elements, however, is different. Representatives of Ceratopetalorchis are characterized by a labellum with lanceolate, frequently divided, lateral 

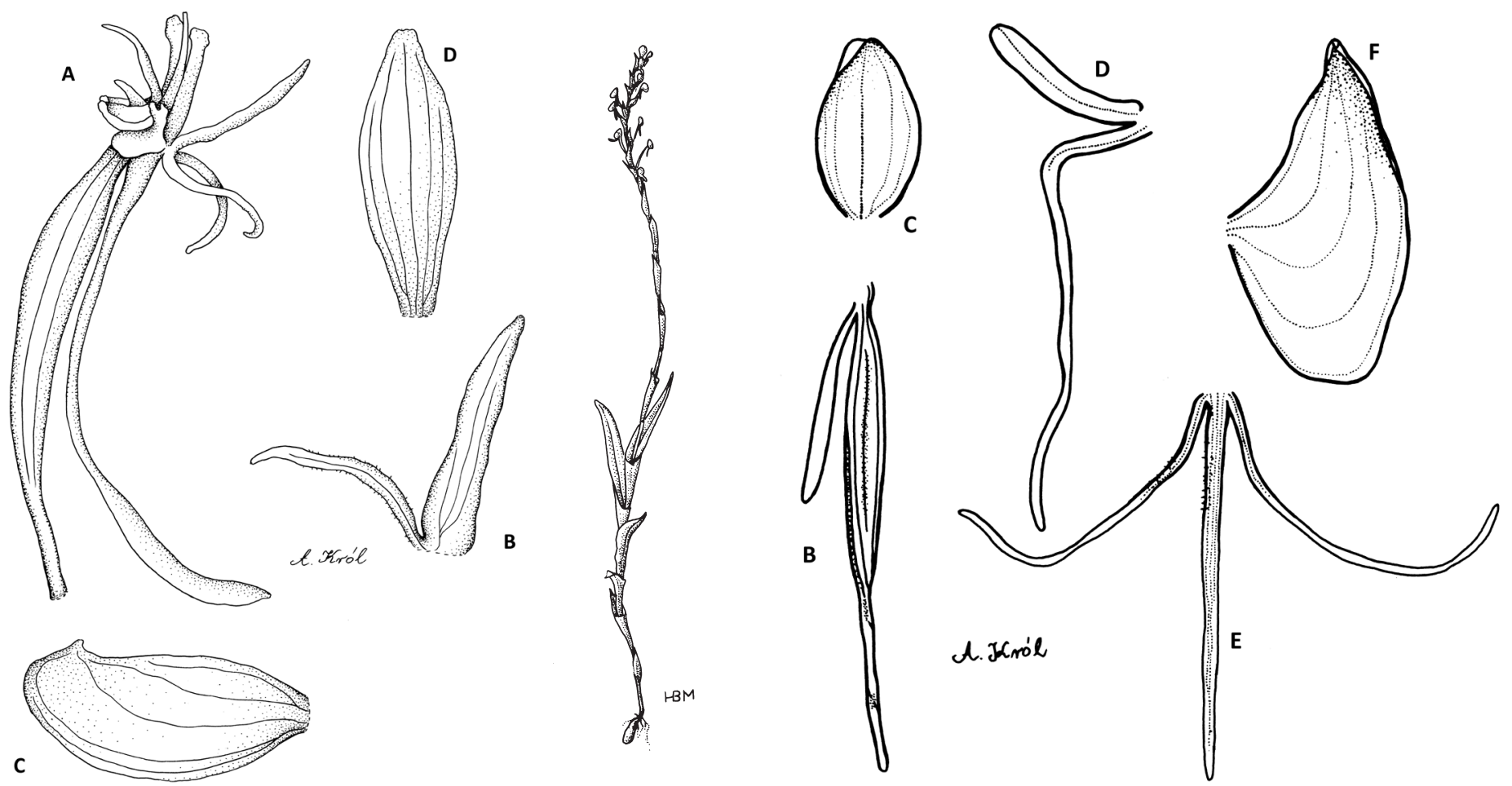

Fig. 2. Renzorchis pseudoplatycoryne Szlach. \& Olszewski

Explanations: A - habit; B - spur, ovary, and pedicel; C - dorsal sepal; D - divided petal; E - lateral sepal; F - lip (drawn by A. Król)

lobes; very long posterior lobes of petals which are intertwined above the helmet formed by the dorsal sepal and by anterior lobes of petals, giving these flowers a unique appearance. Vegetatively, Ceratopetalorchis differs from Bilabrella in its leaves, which are always lanceolate in shape and borne along the entire stem. Another genus similar to Bilabrella is Platycorynoides described by Szlachetko in 2005 (2005a) (Fig. 4). Similarities between representatives of these taxa may be noticed in the structure of their vegetative and floral

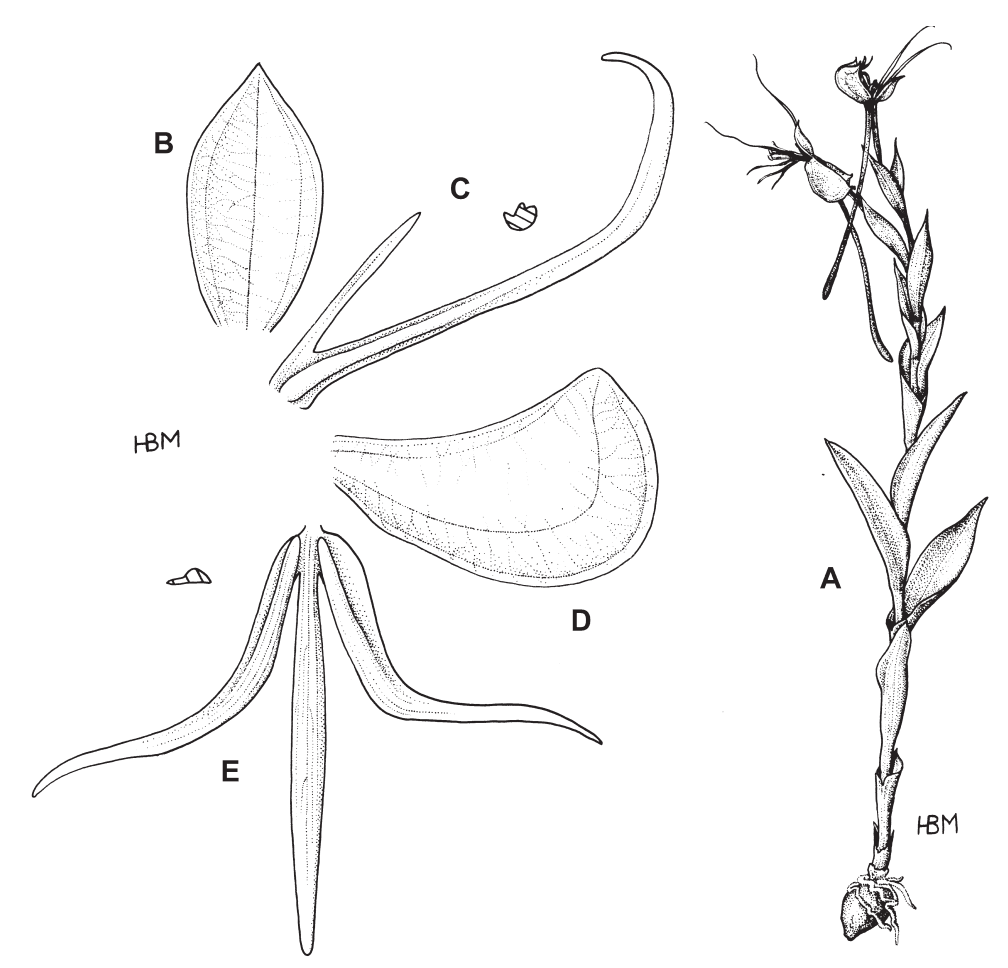

Fig. 3. Ceratopetalorchis sanfordiana (Szlach. \& Olszewski) Szlach., Górniak \& Tukałło Explanations: A - habit; B - dorsal sepal; C - petal; D - lateral sepal; E - lip (drawn by H. B. Margońska) 


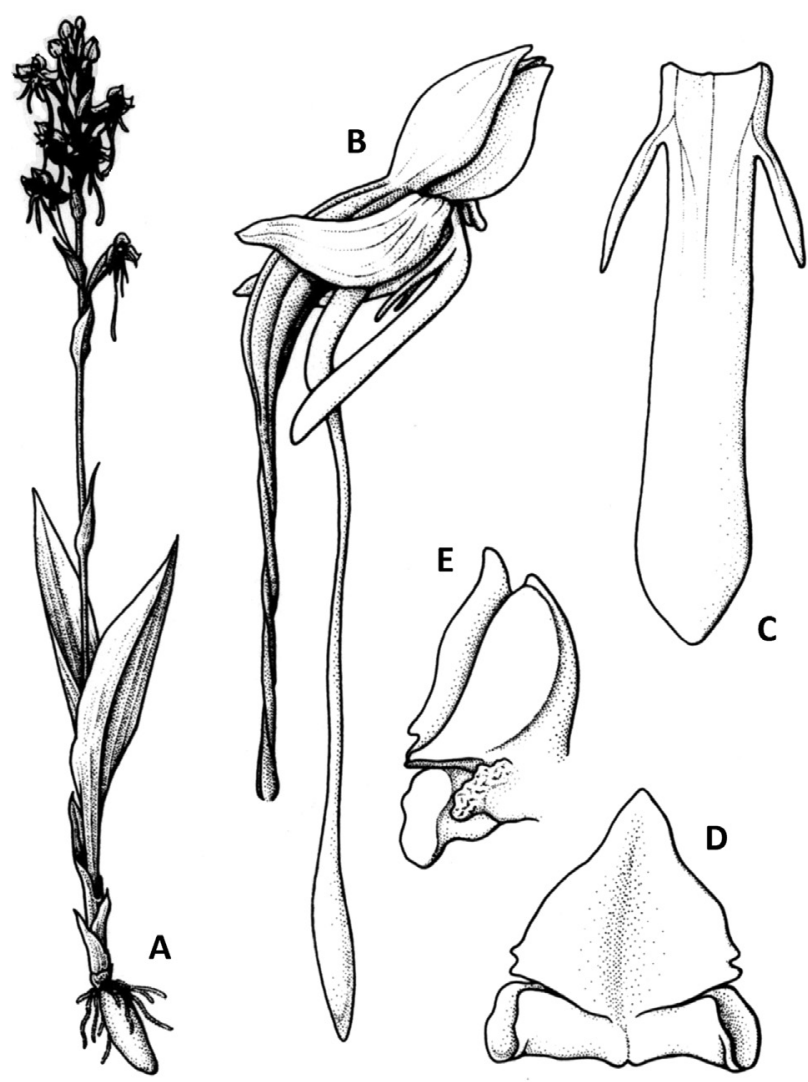

Fig. 4. Platycorynoides hircina (Rchb. f.) Szlach.

Explanations: A - habit; B - flower; C - lip; D - gynostemium, side view; E - gynostemium, front view (drawn by H. B. Margońska)

parts. Their generative structures, on the other hand, are totally different. The labellum in Platycorynoides is also 3-lobed, but its lateral lobes are short and linear. The gynostemium is very similar to that of Platycoryne Rchb. f. (short, massive, oval-conical) and differs greatly from that found in Bilabrella species.

\subsection{Morphological structure}

Bilabrella Lindl. differs from other Habenariinae by its unique set of features. The most important diagnostic characters include: strongly asymmetric and reflexed lateral sepals, pointed with an apiculus; petals divided into two lobes almost to the base; a deeply 3-lobed lip of usually filamentous or linear segments and the gynostemium structure.

The tuber is single, ovoid to ellipsoid, with a diameter ranging from $1 \mathrm{~cm}$ [e.g. Bilabrella hebes (la Croix \& P. J. Cribb) Szlach. \& Kras-Lap.] to $2.5 \mathrm{~cm}$ [Bilabrella falcicornis Lindl.]. Its length ranges from 1.2 $\mathrm{cm}$ to $2.2 \mathrm{~cm}$ in Bilabrella hochstetteriana (Kraenzl. \& Schltr.) Szlach. \& Kras (Lindley gives a length of $5 \mathrm{~cm}$ for this species). Roots are thin and rather short $-10 \mathrm{~cm}$ long and about $1 \mathrm{~mm}$ in diameter.

The tuber produces a single upright stem (Fig. 5), always glabrous and terminating in an inflorescence. The height varies from $12 \mathrm{~cm}$ in Bilabrella subaequalis

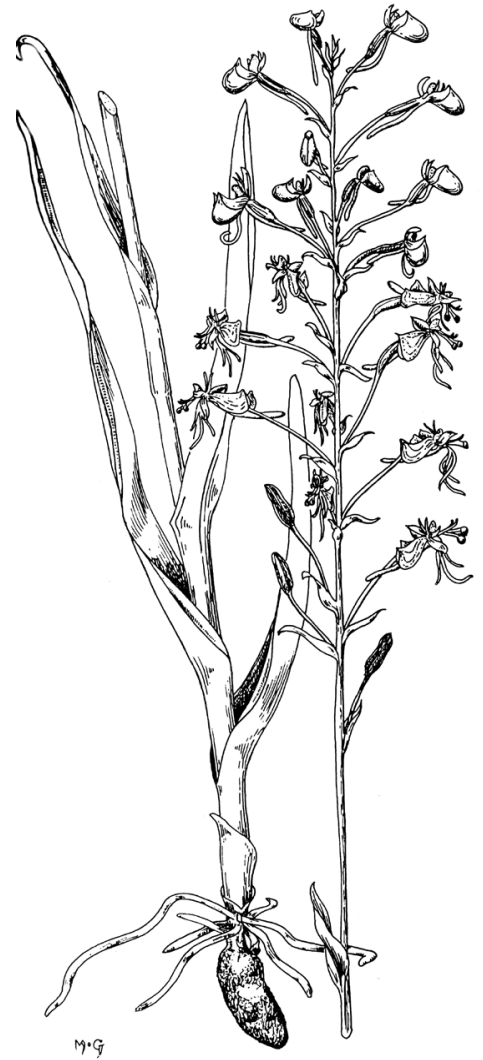

Fig. 5. Bilabrella Lindl. habit exemplified by Bilabrella macrotidion (Summerh.) Szlach. \& Kras-Lap. (ex La Croix \& Cribb in Pope, 1995)

(Summerh.) Szlach. \& Kras-Lap. (Summerhayes 1962) to about $100 \mathrm{~cm}$ in Bilabrella schimperiana (Hochst. ex A. Rich.) Szlach. \& Kras-Lap. Exceptionally, it can even reach $200 \mathrm{~cm}$, as in a specimen of Bilabrella sochensis (Rchb. f.) Szlach. \& Kras-Lap. reported by La Croix \& Cribb (1995).

Leaves are usually confined to the lower $1 / 3-1 / 4$ section of the stem. They gradually decrease in size upwards, providing a smooth transition upper leaves, and then the bracts. Leaves may be widely spaced or overlapping at the base, appressed or strongly patent. The lower 3 to 6 leaves tend to be linear or lanceolate, acute, sessile, sheathed and nonglandular. Upper leaves are of the same form or wider than the lower ones and may be glabrous, as in Bilabrella chirensis (Rchb. f.) Szlach. \& Kras-Lap., or glandular as in Bilabrella kyimbilae (Schltr.) Szlach. \& Kras-Lap. Some of them have characteristic reddish veins, as e.g. in Bilabrella hochstetteriana (Kraenzl. \& Schltr.) Szlach. \& Kras.

The inflorescence varies in length from $4 \mathrm{~cm}$ in Bilabrella cribbiana (Szlach. \& Olszewski) Szlach. \& Kras-Lap. (Szlachetko \& Olszewski 1998a) up to $30 \mathrm{~cm}$ or more as in Bilabrella disparilis (Summerh.) Szlach. \& Kras-Lap. $(27.1 \mathrm{~cm})$ or Bilabrella schimperiana (Hochst. ex A. Rich.) Szlach. \& Kras-Lap. $(31.5 \mathrm{~cm})$. It may be dense [Bilabrella dives (Rchb. f.) 


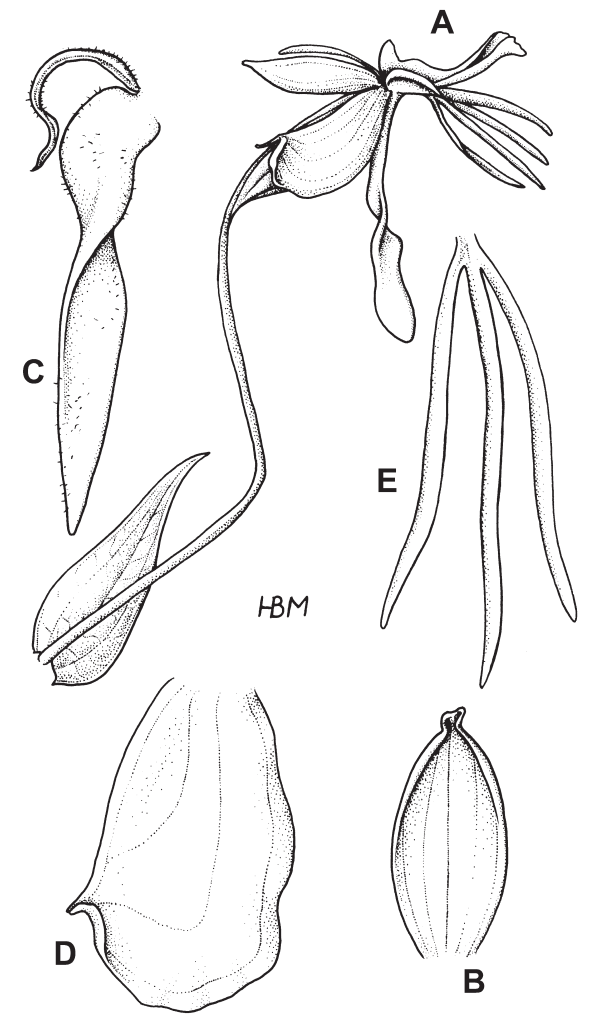

Fig. 6. Flower structure of the genus Bilabrella Lindl. exemplified by Bilabrella schimperiana (Hochst. ex A. Rich.) Szlach. \& Kras-Lap. Explanations: A - flower; B - dorsal sepal; C - petal; D - lateral sepal; E lip (drawn by H. B. Margońska)

Szlach. \& Kras] or lax [Bilabrella scimperiana (Hochst. ex A. Rich.) Szlach. \& Kras-Lap.]. The number of flowers usually ranges from 10 [Bilabrella macrostele (Summerh.) Szlach. \& Kras-Lap.] to 25, with some species such as Bilabrella weberana (Schltr.) Szlach. \& Kras-Lap. having 33 flowers and Bilabrella marxiana (Schltr.) Szlach. \& Kras as many as 50.

The flowers are usually green, white or yellowish, small, rather inconspicuous, always resupinate through $180^{\circ}$. On the surface of the ovary and peduncle, there are six clearly marked ribs that correspond to the accretion sites and carpel bundles. These ribs are sometimes corrugated. The ovary in Bilabrella galpinii (Bolus) Szlach. $\&$ Kras-Lap. is glandular. The petiole varies in length from $5 \mathrm{~mm}$ in Bilabrella anaphysema (Rchb. f.) Szlach. \& Kras-Lap. to $17 \mathrm{~mm}$ in Bilabrella huillensis (Rchb. f.) Szlach. \& Kras-Lap. Each flower is subtended by a bract which may tightly enfold the petiole or loosely surround it. The bract may reach half the length or enclose it completely. In most species, bracts are eglandular; however, they can be glandular throughout [Bilabrella marxiana (Schltr.) Szlach. \& Kras], or just up to half their length [Bilabrella tubifolia (La Croix \& P. J. Cribb) Szlach. \& Kras-Lap.] (Fig. 6A).

The dorsal sepal is usually folded or sunk about the central nerve, creating a shell-like appearance (Fig. 6B).
It may cover a part of the gynostemium or even take up a position so that it lies on the upper surface of the ovary. Unlike many Habenariinae, it never forms a helmet (a so-called galea) with posterior lobes of petals; they only lie in close proximity to one another. The mean dimensions of the dorsal sepal are $5 \mathrm{~mm}$ by $2 \mathrm{~mm}$. The smallest dorsal sepal was recorded in Bilabrella pedicellaris (Rchb. f.) Szlach. \& Kras (4 mm $\times 1.2 \mathrm{~mm})$, and the largest in Bilabrella kyimbilae (Schltr.) Szlach. \& Kras-Lap. $(6 \mathrm{~mm} \times 3 \mathrm{~mm})$.

Lateral sepals are much larger than dorsal ones; they are strongly asymmetric, apiculate, reflexed and appressed to the ovary. Their shape varies little across all species of the genus. Their margins are often involute. Sepal length ranges from 5 to $8.6 \mathrm{~mm}$ and width - from 3 to $6 \mathrm{~mm}$. The apiculus may be up to $0.5 \mathrm{~mm}$ long. Lateral sepals are always 5-nerved (Fig. 6D) and glabrous, except for Bilabrella macrostele (Summerh.) Szlach. \& Kras-Lap. where they are glandular on their outer surface (like the dorsal sepal).

Petals are divided into two lobes, posterior and anterior one, which differ in size or shape (Fig. 6C). They may fork at the base or be partly connate (up to $0.5 \mathrm{~mm}$ ) as in Bilabrella humilior (Rchb. f.) Szlach. \& Kras-Lap.

The posterior petal lobe, along with the dorsal sepal, may cover the gynostemium or bend upwards, or deviate to the back for half its length. It is linear, mostly measuring ca. $4 \mathrm{~mm}$ by $0.3 \mathrm{~mm}$ and is frequently 3 -nerved. In Bilabrella myodes (Summerh.) Szlach \& Kras-Lap. the posterior lobe is much larger $(15 \mathrm{~mm}$ by $1.1 \mathrm{~mm})$. In many species, it is glabrous but in some glandular, ciliate or ciliolate all over the surface (e.g. Bilabrella anaphysema (Rchb. f.) Szlach. \& Kras-Lap.) or at the base only (e.g. Bilabrella macrostele (Summerh.) Szlach. \& Kras-Lap.).

The anterior petal lobe deviates to the back and down or lies along the gynostemium outgrowths. It is usually much larger than the posterior lobe, linear or lanceolate, often very fleshy. It is usually between 6.5 and $15 \mathrm{~mm}$ long and from 1 to $2 \mathrm{~mm}$ wide. Like the posterior lobe, it may be glabrous, glandular all over, or only in the lower half.

Of course, there are exceptions. Thus, Bilabrella kilimanjari (Rchb. f.) Szlach. \& Kras-Lap. possesses petals which are more or less lobular shaped, the posterior lobe measuring $5.5 \mathrm{~mm} \times 1.5 \mathrm{~mm}$, and the anterior one $-4 \mathrm{~mm} \times 2 \mathrm{~mm}$.

The lip always consists of three lobes (Fig. 6E). They may be free to the base [Bilabrella cataphysema (Rchb. f.) Szlach. \& Kras-Lap.], or connate at the base for $0.5 \mathrm{~mm}$ [Bilabrella humbertii (Szlach. \& Olszewski) Szlach. \& Kras-Lap.] or united for $2.5 \mathrm{~mm}$ [Bilabrella pedicellaris (Rchb. f.) Szlach. \& Kras]. All the labellum parts are arranged parallel to one another and hang free, 

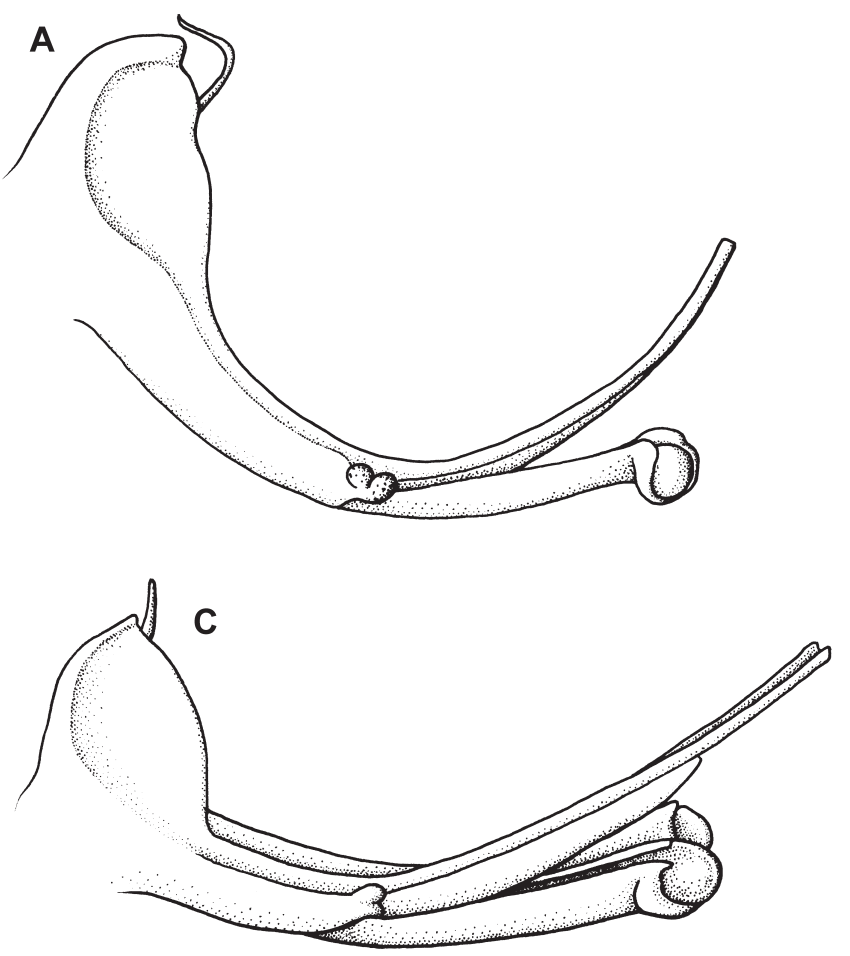

Fig. 7. Generative structure of the genus Bilabrella Lindl.

Explanations: A, C - side view; B, D - front view (drawn by D. L. Szlachetko)
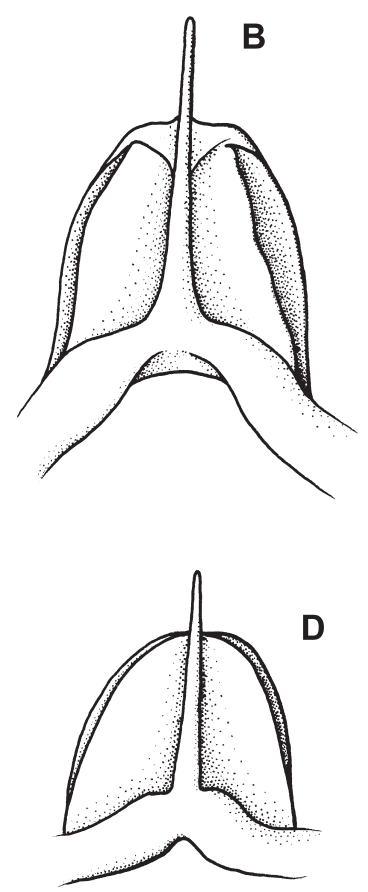

upturned at the tip, with lateral lobes curved outwards. All lobes are either similar in shape, linear, or the central one is linear and the lateral ones lanceolate as in Bilabrella incarnata (Lyall ex Lindl.) Szlach. \& KrasLap. All lobes are three-nerved and usually glabrous. In Bilabrella arianae (Geerinck) Szlach. \& Kras, the whole labellum is covered in papillae of various lengths, while in B. williamsonii - it is finely glandular. In Bilabrella marxiana (Schltr.) Szlach. \& Kras, the central lobe is fleshy and folded along the central nerve.

All Bilabrella Lindl. flowers possess a spur whose shape and size are diagnostically important at species level. It may be short and adjacent to the ovary [Bilabrella chirensis (Rchb. f.) Szlach. \& Kras-Lap.], or long and curved [Bilabrella burttii (Summerh.) Szlach. \& Kras-Lap.], or hang down freely. The bract may enfold it tightly, along with the pedicel and ovary. Usually, the spur is cylindrical, often swollen or ampullaceous at the top [Bilabrella cataphysema (Rchb. f.) Szlach. \& Kras-Lap.] and may be twisted.

The gynostemium varies from delicate to relatively massive (Fig. 7). The anther is straight, ovoid to ellipsoid. Generative features that distinguish Bilabrella from other Habenariinae and give them a unique appearance are their antherophores, stigmaphores, and rostellophores. Of these, the stigmaphores are usually longer than the others. All of them tend to be very thin, delicate, and may be glabrous, or partly glandular as in Bilabrella hebes (la Croix \& P. J. Cribb) Szlach. \& Kras-
Lap. In Bilabrella involuta (Bolus) Szlach. \& Kras, they are up to $5 \mathrm{~mm}$ long and $0.3-0.5 \mathrm{~mm}$ wide. The rostellum median lobe is acute at the apex and may extend out over the connective as in Bilabrella schimperiana (Hochst. ex A. Rich.) Szlach. \& Kras-Lap., or be fully covered by pollen sacs [Bilabrella myodes (Summerh.) Szlach. \& Kras-Lap.].

The connective is relatively narrow. Pollinia are from obovoid to ellipsoid, usually shorter than very thin caudicules. Auriculae are small [Bilabrella burtii (Summerh.) Szlach. \& Kras-Lap. $-0.4 \times 0.1 \mathrm{~mm}]$, or relatively large [Bilabrella tetrapetaloides (Schltr.) Szlach. \& Kras $-0.5 \times 0.9 \mathrm{~mm}]$, digitate and sculptured. They may be divided along their entire length [Bilabrella incarnata (Lyall ex Lindl.) Szlach. \& Kras-Lap.] or just at the tip [Bilabrella tubifolia (la Croix \& P.J. Cribb) Szlach. \& Kras-Lap.]. Undivided auriculae can be observed in e.g. Bilabrella falcata (G. Will.) Szlach. \& Kras-Lap. and Bilabrella robusta (Welw.) Szlach. \& Kras-Lap.

An exceptional generative structure for the genus is found in Bilabrella dives (Rchb. f.) Szlach. \& Kras, Bilabrella macrostele (Summerh.) Szlach. \& Kras-Lap. and Bilabrella orangana (Rchb. f.) Szlach. \& Kras, where antherophores, stigmaphores and rostellophores are relatively short.

The fruit is usually ellipsoid, more or less curved and, generally, tinted brown. 


\subsection{Geographical distribution}

Bilabrella comprises species found in Sub-Saharan Africa, Madagascar and neighbouring islands (Fig. 8). West-East, they extend right across the continent from the Atlantic to the Indian Oceans. To the north, they reach Senegal and southern Chad and in the south, extend right down to Cape Province. They have not been recorded in the Namib Desert, while Bilabrella retinervis (Summerh.) Szlach. \& Kras-Lap. is the only species of the genus to have been found in the Kalahari Desert.

Out of 93 species in the genus, 16 have a wide distribution that covers a considerable part of the continent (three or more regions in Africa's geobotanical division by Brummit 2001). For example, Bilabrella ichneumonea (Sw.) Szlach. \& Kras-Lap., one of the species with the widest range of occurrence, is known in 5 geobotanical regions (21 states).

The area that is richest in Bilabrella species is Tropical East Africa, where as many as 36 species have been found $(39 \%$ of the total number of taxa at this rank). Next come Tropical West-Central Africa and Tropical Southern Africa (both with 35 species). The plant cover in these areas is mainly savanna, a habitat preferred by most Bilabrella species.

The state richest in endemics is probably Tanzania, although only one of the seven recorded there was initially so defined. There are probably 7 endemics in the Republic of South Africa but confirmation requires further research. In the Democratic Republic of Congo, such endemics as: Bilabrella bertauxiana (Szlach. \& Olszewski) Szlach. \& Kras-Lap., Bilabrella humbertii (Szlach. \& Olszewski) Szlach. \& Kras-Lap., Bilabrella renziana (Szlach. \& Olszewski) Szlach. \& Kras-Lap., Bilabrella stanislawii Szlach. \& Kras and Bilabrella tangheana (Geerinck \& Schaijes) Szlach. \& Kras are found. These species were described in 1990s and are

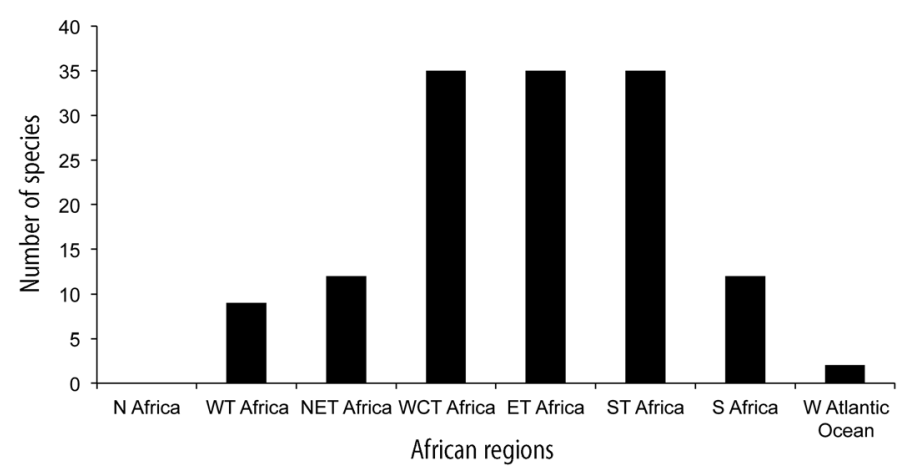

Fig. 8. Occurrence of Bilabrella Lindl. species in Africa's regions Explanations: N Africa - North Africa; WT Africa - West Tropical Africa; NET Africa - North-East Tropical Africa; WCT Africa - West-Central Tropical Africa; ET Africa - East Tropical Africa; ST Africa - Southern Tropical Africa; S Africa - South Africa; W Atlantic Ocean - West Indian Ocean

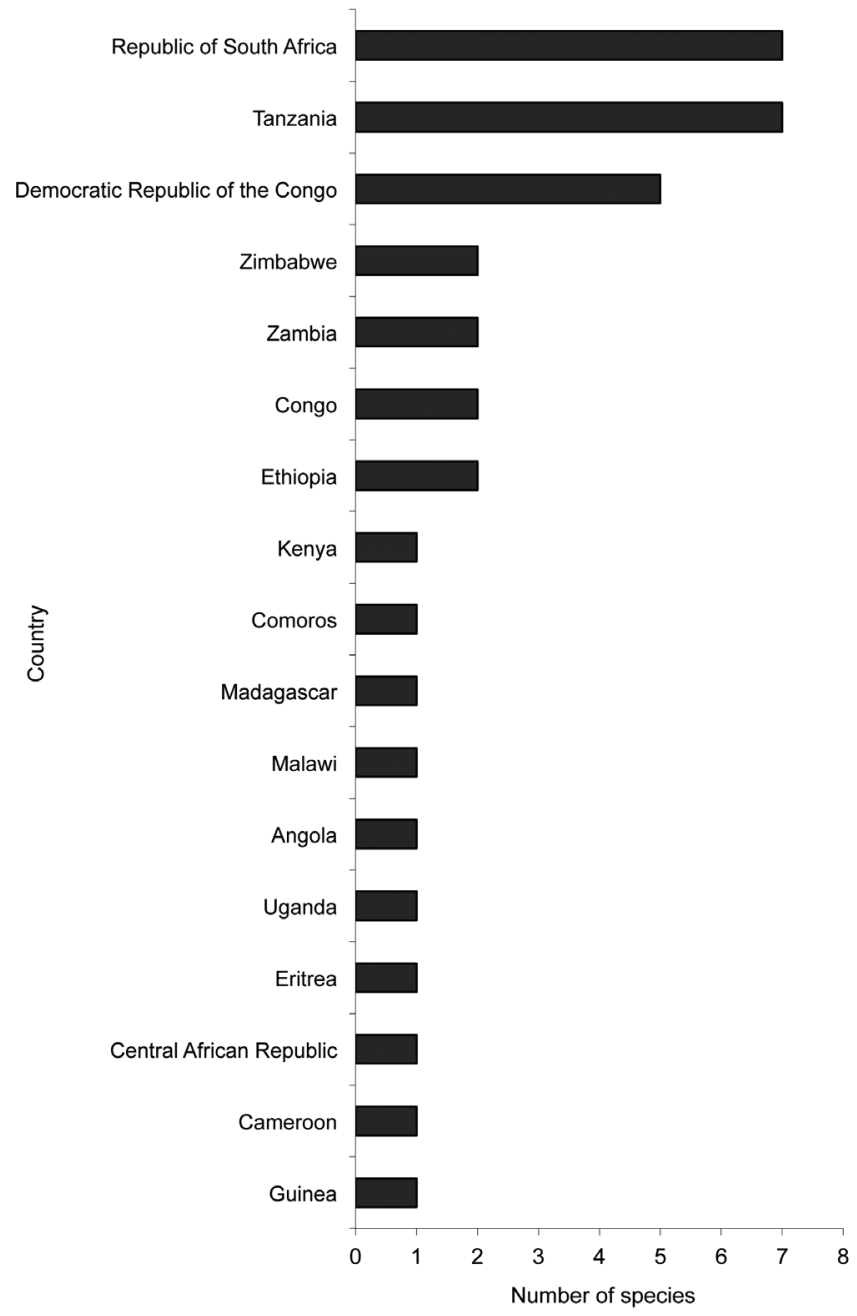

Fig. 9. Number of endemics recorded in African countries

known only from the type specimens. Two endemics were collected in each of the following countries: Ethiopia, Congo, Zambia and Zimbabwe and one in Angola, Cameroon, Central African Republic, Comoros, Eritrea, Guinea, Kenya, Madagascar, Malawi and Uganda (Fig. 9).

Africa is an upland continent, with valleys traversing its numerous plateaux. About $70 \%$ of the land area lies from 300 to 2000 metres above sea level, and the mean height of the continent is $660 \mathrm{~m}$ (Podbielkowski 1987). The lowest land is coastal or borders rivers, where it is covered in dense wet forest and mangrove swamp. These habitats are not favoured by Bilabrella, so few species grow below $500 \mathrm{~m}$. Only 13 species in total have been recorded including Bilabrella plectomaniaca (Rchb. f. \& S.Moore) Szlach. \& Kras-Lap. at a height between 30 and $420 \mathrm{~m}$ in South Africa and Bilabrella tetrapetala (Rchb. f.) Szlach. \& Kras has bee found at $100 \mathrm{~m}$.

The majority of species grow at the height between 500 to $2000 \mathrm{~m}$. At the optimum height of ca. $1000 \mathrm{~m}, 53$ species occur, which is way beyond the average height of the continent. The high-mountain species include e.g. Bilabrella altior (Rendle) Szlach. \& Kras-Lap., found 


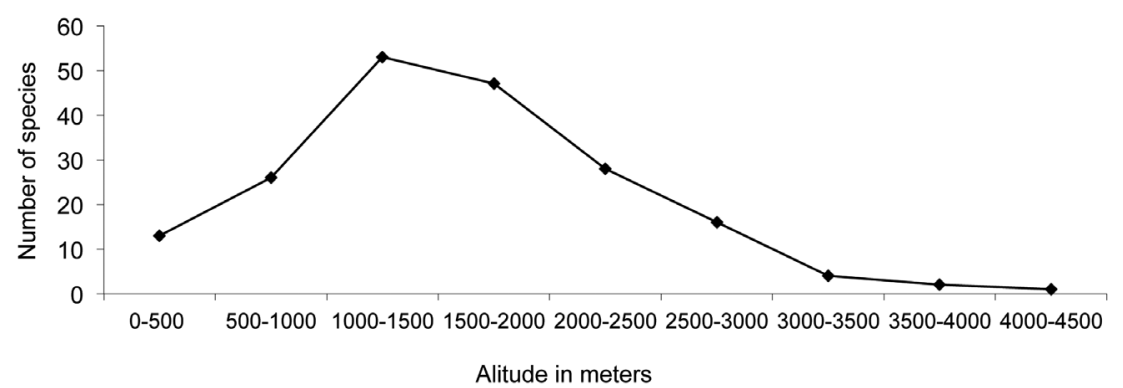

Fig. 10. Altitudinal distribution of Bilabrella Lindl.

on the slopes of Kilimanjaro in Tanzania at $3300 \mathrm{~m}$, and Bilabrella ichneumonea (Sw.) Szlach. \& Kras-Lap. collected at $3800 \mathrm{~m}$ in Angola (Fig. 10).

A sizable group of Bilabrella spp. tolerate a wide altitudinal range, e.g. Bilabrella welwitschii (Rchb. f.) Szlach. \& Kras-Lap. grows from sea level up to $2700 \mathrm{~m}$. Specimens of Bilabrella anaphysema (Rchb. f.) Szlach. \& Kras-Lap. were found at sea level in Ghana (Gold Coast), as well as at $4200 \mathrm{~m}$ in Zambia.

The analysis of the material collected suggests that 34 Bilabrella species are confined to a very narrow altitudinal band, e.g. Bilabrella strangulans (Summerh.) Szlach. \& Kras-Lap. grows in open forests between 1200 and $1300 \mathrm{~m}$. No information has been collected on the altitudinal distribution of 15 Bilabrella species.

\subsection{Ecology}

Information on the ecological requirements of Bilabrella is usually sketchy. It is gleaned from collectors' notes on herbarium labels or from incidental observations in the field. The genus has not been subjected to detailed ecological research so far.

Bilabrella is a terrestrial orchid that prefers lateritic soils associated with savannas, loams and red soils. Bilabrella schimperiana (Hochst. ex A. Rich.) Szlach. \& Kras-Lap. grows on hydromorphic, organically rich loams. A small group of the species are lithophytes appearing on thin layers of soil that develops between rocks, e.g. Bilabrella tortilis (P. J. Cribb) Szlach. \& Kras-Lap. They are also to be seen on granitie substrates and volcanic soils.

Based on data obtained from herbarium labels, it may be concluded that the majority of Bilabrella species are heliophytes. Thus, most species were recorded in open areas - savannas [Bilabrella urundiensis (Summerh.) Szlach. \& Kras], meadows and marshes of various kinds [Bilabrella thomsonii (Rchb. f.) Szlach. \& KrasLap.]. Relatively often, they were also found in dambos; Brachystegia spp. bushwood (Miombo forests), Uapaca spp. bushwood [Bilabrella tubifolia (la Croix \& P. J. Cribb) Szlach. \& Kras-Lap.] or of Acacia spp. [Bilabrella vollesenii (Thomas \& P. J. Cribb) Szlach.
\& Kras-Lap.]. They are rarely seen on forest fringes or steppes [Bilabrella macrostele (Summerh.) Szlach. \& Kras-Lap.]. Some species occur quite commonly in Eucalyptus plantations such as Bilabrella galpinii (Bolus) Szlach. \& Kras-Lap., or under pines [Bilabrella sochensis (Rchb. f.) Szlach. \& Kras-Lap.].

Some species, e.g. Bilabrella ichneumonea (Sw.) Szlach. \& Kras-Lap., are hydrophytes and grow in still, shallow water.

Based on the somewhat selective information, it is difficult to infer the phenology of these plants. Flowering usually extends over a few months. Specimens of one species were found in flower at different times of the year - flowering may be initiated by a drop in temperature for a few days or rain. Flowering specimens of some species were only recorded within a single month; e.g. specimens of Bilabrella furcipetala (Schltr.) Szlach. \& Kras were collected only in April.

Flowers develop gradually, starting from the base of the inflorescence, so that a single specimen sometimes has developing fruit open at the base, flowers attractive to pollinators - in the middle and unopened buds - at the top of the inflorescence. After seeding, the aboveground parts of the plant die. The underground tubers remain dormant for a few months and then, in the rainy season, produce stems again (Stewart \& Campbell 1996). Therefore, the life cycle of these plants resembles that of other species of the subtribes Habenariinae and Orchidinae.

Bilabrella species are insect pollinated. There have been no direct observations of the pollination of these plants. However, based on the inflorescence arrangement, flower structure and colour, it may be inferred that pollen is mainly transferred by night butterflies. The fragrance of flowers is most noticeable during the evening and night when these insects are active. An unpleasant, sweet and nauseating smell has been observed in Bilabrella chirensis (Rchb. f.) Szlach. \& Kras-Lap. and Bilabrella schimperiana (Hochst. ex A. Rich) Szlach. \& Kras-Lap. Sometimes, flowers have very strong smell as in Bilabrella myodes (Summerh.) Szlach. \& Kras-Lap. 
Adaptation to night butterflies pollination is evident from the structure of the individual parts of the flower: the long pointed spur, often widening at the top, forms a receptacle from which nectar may be collected; the twolobed petals; the three-lobed lip and long gynostemium outgrowths. The lip consists of narrow elements in all species. It has lost its original function as a landing site which it fulfils in the majority of other orchids as night butterflies do not alight on the flower, while seeking sustenance. It seems that specialization in the manner in which the pollen mass is deposited on an insects' body was a principal factor governing the evolution of the species. The long spur ensures that the pollinator, reaching for the nectar inside it with its proboscis, has to push its head, thorax or front legs on to the gynostemium. The lateral outgrowths, equipped with pollinia, touch the insect and makes it possible for the pollen to be transferred to its head or front legs. Hence, the pollinators of Bilabrella are primarily likely to be butterflies which are active at dusk or during the night (mostly from the Sphingidae and Acherotidae) (van der Cingel 2001).

\section{Key for the determination}

1. Gynostemium short and massive 2

1. Gynostemium slender, elongate 20

2. Gynostemium stalked 1. B. macrostele

2. Gynostemium sessile

... 3

3. Lip and petals densely covered by hairs of various length

2. B. arianae

3. Lip glabrous or minutely papillate. Petals glabrous or ciliate or papillate 4 4. Petals divided above the basal third or quarter ...... 5 4. Petals divided to the base ....................................... 7 5. Lip lateral lobes 3 times shorter than the middle one 3. B. robusta

5. Lip lateral lobes at least half as long as the middle one ...

6. Anterior petal lobe lanceolate, apiculate, posterior lobe oblong-lanceolate, acute to acuminate. Lip middle lobe wider than laterals

4. B. diselloides 6. Anterior petal lobe more or less deltoid, acute, posterior lobe linear to narrowly oblong, obtuse. Lip middle lobe as wide as laterals

5. B. dives

7. Auriculae bilobed 8

7. Auriculae entire 6. $B$. tortilis

8. Spur geniculate near the middle 7. $B$.

8. Spur not geniculate 7. B. isoantha

9. Spur more or less twisted 10

9. Spur not twisted 13

10. Spur twisted near the middle

8. B. ndiana

10. Spur twisted near the apex 11

11. Posterior petal lobe linear, subobtuse to subacute, ciliate
11. Posterior petal lobe filiform, obtuse, glabrous ...... 12 12. Leaves 8-11.5 $\times 0.3-0.6 \mathrm{~cm}$, anterior petal lobe 6.9$9.5 \times 0.3-0.6 \mathrm{~mm}$, filiform, acute, glabrous

10. B. compta

12. Leaves $11-23 \times 1.2-3 \mathrm{~cm}$, anterior petal lobe $7-13 \times$ $1.3 \mathrm{~mm}$, narrowly lanceolate-linear, falcate, acuminate to caudate, papillose-puberulate ........ 11. B. marxiana 13. Spur subequal in length to the lip middle lobe ..... 14 13. Spur prominently longer than lip middle lobe ..... 15 14. Petals glabrous; anterior lobe 7-10 × 1-2 mm, oblong-lanceolate, obtuse; posterior lobe $6-8 \times 1 \mathrm{~mm}$; stigmaphores and antherophores slender, upcurved, cylindrical-clavate

12. B. stolzii

14. Petals ciliolate; anterior lobe $3-4 \times 1.2 \mathrm{~mm}$, oblongoblanceolate, acute; posterior lobe $3-4 \times 0.3 \mathrm{~mm}$; stigmaphores and antherophores short and massive

13. B. tisseranti

15. Anterior petal lobe glabrous .............................. 16

15. Anterior petal lobe ciliolate or papillose ..............18

16. Posterior petal lobe glabrous ............. 14. B. woodii

16. Posterior petal lobe ciliolate ................................ 17

17. Leaves $13-15 \times 0.7 \mathrm{~cm}$, inflorescence $6-11 \mathrm{~cm}$ long, lip middle lobe $12 \mathrm{~mm}$ long, lateral lobes $10-11 \mathrm{~mm}$ long

15. B. stenophylla

17. Leaves $12-25 \times 2-5 \mathrm{~cm}$, inflorescence $15-40 \mathrm{~cm}$ long, lip middle lobe $10-17 \mathrm{~mm}$ long, lateral lobes $6-12 \mathrm{~mm}$ long

16. B. sochensis

18. Lip papillose ............................ 19. B. williamsonii

18. Lip glabrous ........................................................ 19

19. Petals minutely ciliolate, anterior lobe $4 \times 1.1 \mathrm{~mm}$, ligulate, rounded at the apex, posterior lobe $1.1 \times$ $0.8 \mathrm{~mm}$ wide, ovate to deltoid, obtuse, spur mouth widened

17. B. orangana 19. Petals ciliate, anterior lobe $13 \times 1-1.8 \mathrm{~mm}$, lanceolate, acute to acuminate, posterior lobe up to $4 \times 0.8 \mathrm{~mm}$, reflexed, linear-lanceolate, obtuse, often reduced in some plants, ciliate, spur mouth narrow

18. B. antennifera

20. Auriculae entire, not split into two lobules, sometimes irregularly sculpted .......................................... 21

20. Auriculae more or less split into two parts .......... 23

21. Posterior petal lobes much wider than anterior one

20. B. inaequiloba

21. Posterior petal lobes usually much narrower than

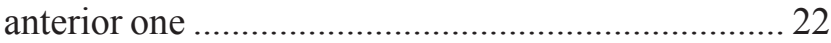

22. Anterior petal lobes at least 3 times wider than

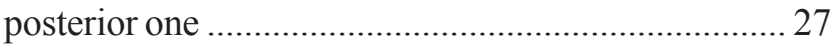

22. Anterior petal lobes less than twice wider than anterior one 45

23. Lip lobed near the middle, both lateral lobes crossed below the middle lobe 50. B. decaptera

23. Lip divided near the base .................................. 24 24. Both petal lobes lanceolate, oblong-lanceolate to oblanceolate, both lobes similar in form, usually anterior lobe smaller than posterior ones 
24. Both petal lobes dissimilar in form, anterior lobes usually larger than posterior ones ... 25

25. Anterior petal lobes bilobulate 56

25. Anterior petal lobes entire 26

26. Petals bilobulate above basal $2-3 \mathrm{~mm}$ 57

26. Petals bilobed to the base or so 58

27. Spur distinctly shorter than pedicel and ovary ... 28

27. Spur as long as or longer than pedicel and ovary ... 38

28. Spur prominently twisted near or below the middle

29

28. Spur not twisted

31

29. Spur slightly swollen in the apical third or so

21. B. incarnata

29. Spur ampullaceous in the apical third .. 30

30. Spur forming a complete loop .... 22. B. anaphysema

30. Spur without any loop 23. B. confusa

31. Spur cylindrical 24. B. pauper

31. Spur filiform, apically slightly swollen .. 32

32. Spur geniculate near the middle .. 25. B. urundiensis

32. Spur not geniculate 33

33. Lip 3-lobed above prominent basal undivided part

33. Lip 3-lobed to the base 35

34. Petals bilobed to the base, glabrous, anterior lobe $4 \times 0.3 \mathrm{~mm}$, lip middle lobe $7 \mathrm{~mm}$ long

26. B. pedicellaris

34. Petals bilobed to the base, ciliolate, anterior lobe 5.5-13 $\times 1-1.5 \mathrm{~mm}$, lip middle lobe 7-15 mm long ......

27. B. ichneumonea

35. Anterior petal lobes linear-lanceolate, widest at the base. . 36 35. Anterior petal lobes oblong-elliptic, oblong-lanceolate or ligulate, widest near the basal third or so ...... 37 36. Petals glabrous 28. B. cornutella

36. Petals densely ciliate 29. B. lisowskii

37. Petals posterior lobe papillose, spur $16-20 \mathrm{~mm}$ long 30. B. hochstetteriana 37. Petals posterior lobe ciliolate, spur $11.5-16.5 \mathrm{~mm}$ long..... 31. B. replicata 38. Spur only slightly swollen near the apex, if any .... 39 38. Spur distinctly swollen in the apical part ............ 42 39. Anterior petal lobe 9-14 mm long ... 32. B. huillensis 39. Anterior petal lobes usually less than $9 \mathrm{~mm}$ long ... 40

40. Stigmaphores papillose 33. B. falcata

40. Stigmaphores glabrous ...... 41

41. Anterior petal lobe glabrous, posterior one ciliolate all over 34. B. caffra 41. Anterior petal lobe ciliolate, posterior one ciliolate along margins only .... 35. B. falcicornis 42. Spur twisted 43

42. Spur not twisted 44

43. Lateral sepals obliquely triangular-ovate, widest at the apex, anterior petal lobes twice wider than posterior one
43. Lateral sepals obliquely oblong-ovate, widest just above the middle, anterior petal lobes ca 4-5 times wider than posterior one

37. B. humilior 44. Anterior petal lobes furculate apically, stigmaphores glabrous 38. B. nyikana 44. Anterior petal lobes entire, stigmaphores ciliolate .. 39. B. culcifera 45. Stigmaphores pubescent ......... 40. B. hirsutitrunci 45. Stigmaphores glabrous or papillate .................... 46 46. Spur ampullaceous in the apical third or so .......47 46. Spur not ampullaceous ...................................... 48 47. Spur twisted and geniculate near the middle

41. B. schimperiana

47. Spur parallel to the ovary, not twisted

42. B. cataphysema

48. Spur with acute apex 49

48. Spur blunt at the apex ...................................... 50

49. Leaves $10-28 \times 0.6-2.5 \mathrm{~cm}$, petals glabrous

43. B. weberana

49. Leaves 4-6 $60.9-1.2 \mathrm{~cm}$, petals papillose

44. B. linguiformis

50. Petals papillose ................................................... 51

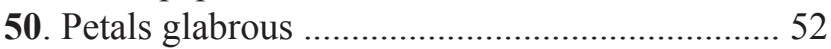

51. Both petals lobes similar in size and form

45. B. subaequalis

51. Anterior petal lobes much longer than posterior one

46. B. hebes

52. Dorsal sepal obscurely 3-lobed just above the middle, lip 3-lobed to the base ....... 47. B. orthocaulis 52. Dorsal sepal entire, lip 3-lobed above undivided basal claw 53 53. Leaves up to $9 \times 2-5 \mathrm{~cm}$, inflorescence up to $30 \mathrm{~cm}$ long, gynostemium stalked 48. B. ugandensis 53. Leaves $5-17 \times 0.5-1.7 \mathrm{~cm}$, inflorescence $1-15 \mathrm{~cm}$ long, gynostemium sessile 49. B. galpinii 54. Spur twisted. Petals divided distinctly above the base

51. B. peltates

54. Spur not twisted. Petals divided to the base ....... 55 55. The upper part of posterior petal lobe clearly toothed, auriculae obscurely bilobed ..... 52. B. martialis 55. The upper part of posterior petal lobe entire, glabrous, auriculae inconspicuous ....... 53. B. kilimanjari 56. Both anterior petal lobules oblong triangular, acute, glabrous

54. B. disparilis 56. Anterior petal lobe rounded, with laterally placed acute apex, ciliolate in the lower part

55. B. usambarae

57. Leaves up to 10 times longer than wide. Petals more or less 3-lobulate

56. B. tetrapetaloides 57. Leaves ca. 15-20 times longer than wide. Petals bilobed 57. B. dalzielii 58. Posterior petal lobe wider than anterior one ...... 59 58. Posterior petal lobe narrower than or subequal to anterior one 60 59. Petals bilobed nearly to the base, anterior lobe 
6-6.5 $\times 1 \mathrm{~mm}$, papillate-puberulate, posterior lobe 5-5.5 $\times 2-2.5 \mathrm{~mm}$, ciliolate 58. B. angustissima

59. Petals bilobed distinctly above the base, glabrous, anterior lobe $4 \times 0.5 \mathrm{~mm}$, posterior lobe $3 \times 2.1 \mathrm{~mm} \ldots$

59. B. kraenzliniana

60. Spur twisted 61

60. Spur not twisted .76

61 . Spur prominently swollen below the middle, swollen part at least twice wider than the basal one ......... 62

61. Spur only slightly swollen towards apex ........... 68

62. Spur geniculate near the middle .......................... 63

62. Spur not geniculate

63. Spur distinctly longer than lip middle lobe

60. B. altior

63. Spur subequal tot he lip middle lobe

64

64. Petals densely pubescent and ciliate, anterior lobe 9-11 $\times 1.2-2 \mathrm{~mm}$, posterior lobe $5.5-6 \times 0.2-0.3 \mathrm{~mm} \ldots$

61. B. kyimbilae

64. Petals puberulous or papillose with ciliolate margins, anterior lobe $8 \times 2-3 \mathrm{~mm}$, posterior lobe $6-7 \times 0.3$ $0.5 \mathrm{~mm}$

62. B. thomsonii

65. Lip lateral lobes oblong-lanceolate, subobtuse, different than the middle one

63. B. welwitschii 65. Lip lateral lobes linear, similar tot he middle one .. 66 66. Anterior petal lobe glabrous, posterior one ciliolate

64. B. leucoceras

66. Both petal lobes more or less ciliolate 67

67. Leaves up to 1(1.5) $\mathrm{cm}$ wide, anterior petal lobe 7-9 $\times 2 \mathrm{~mm}$, posterior lobe $3-5 \times 0.3 \mathrm{~mm}$

65. B. kubangensis

67. Leaves $1-3 \mathrm{~cm}$ wide, anterior petal lobe $9-18 \times 1.5$ $2.5 \mathrm{~mm}$, posterior lobe $5.5-7 \mathrm{~mm} \times 0.3 \mathrm{~mm}$

66. B. myodes

68. Spur usually shorter than pedicellate ovary ....... 69

68. Spur distinctly longer than pedicel and ovary ... 72

69. Stigmaphotes ciliate

67. B. humbertii

69. Stigmaphores glabrous 70

70. Lip 3-lobed nearly to the base

68. B. burttii

70. Lip 3-lobed above distinct claw, 1.5-2 mm long ... 71

71. Both petal lobes completely papillose

69. B. maitlandii

71. Anterior petal lobe glabrous, posterior one ciliolate 70. B. involuta

72. Both petal lobes similar in width, filiform

\section{B. kornasiorum}

72. Both petal lobes of different width, only posterior one filiform ... .73

73. Leaves $0.2-0.8 \mathrm{~cm}$ wide .74

73. Leaves $1-3 \mathrm{~cm}$ wide 75

74. Spur ca twice longer than pedicellate ovary 72. B. tubifolia

74. Spur as long as pedicellate ovary

73. B. strangulans

75. Floral bracts $12 \mathrm{~mm}$ long, ciliolate along margins, minutely glandular outside, stigmaphores $3 \mathrm{~mm}$ long, relatively massive, cylindrical, ciliate

74. B. bertauxiana

75. Floral bracts $10-25 \mathrm{~mm}$ long, glabrous, stigmaphores $4 \mathrm{~mm}$ long, slender cylindrical-clavate, minutely papillate 75. B. retinervis 76. Posterior petal lobe linear to ligulate, but never

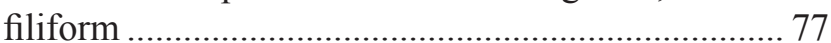
76. Posterior petal lobe filiform ................................. 79 77. Spur shorter than pedicellate ovary, both petal lobes papillose 76. B. furcipetala 77. Spur as long as or longer than pedicellate ovary, petals not as above ................................................. 78 78. Floral bracts rather densely ciliolate along margins and outwards, spur filiform, equal in thickness nearly to apex, acute

77. B. renziana

78. Floral bracts glabrous, spur narrowly cylindrical, apically inflated, subacute

78. B. rehmannii 79. Spur prominently shorter than pedicel and ovary .. 80 79. Spur usually as long as pedicel and ovary, occasionally somewhat longer or shorter ............................... 84 80. Spur cylindrical-filiform, not swollen, pendent ... 81 80. Spur cylindrical, somewhat swollen in the apical half, sigmoid 83 81. Anterior petal lobe linear, ca $1 \mathrm{~mm}$ wide, ciliolate ..

79. B. linderi

81. Anterior petal lobe ovate-lanceolate to narrowly oblanceolate, $1.5-2.5 \mathrm{~mm}$ wide, glabrous 82 82. Anterior petal lobe ovate-lanceolate, auriculae stalked, deeply bilobed, lobes elongate ....

80. B. macrotidion

82. Anterior petal lobe narrowly oblanceolate, auriculae sessile, massive, very unequally bilobed

81. B. bosseriana

83. Petals glabrous; anterior lobe $13.5 \times 1.1 \mathrm{~mm}$, linearlanceolate, posterior lobe $6 \times 0.5 \mathrm{~mm}$, linear

82. B. luegiana

83. Anterior petal lobe $7.5-9 \times 3.5-4 \mathrm{~mm}$, obliquely ovate-lanceolate to semi-ovate, margins minutely crenate, minutely papillose-pubescent above, mainly towards the base, posterior lobe $6 \times 0.4-0.5 \mathrm{~mm}$, linear, papillose-pubescent above with ciliolate margins

83. B. riparia

84. Spur clavately swollen towards apex ................ 85

84. Spur filiform, almost not swollen ....................... 89

85. Auriculae very large, massive ............................ 86

85. Auriculae small .................................................. 87

86. Pedicellate ovary up to $27 \mathrm{~mm}$ long, lip middle lobe 15-17 mm long, spur up to $22 \mathrm{~mm}$... 84. B. tangheana 86. Pedicellate ovary up to $13.5 \mathrm{~mm}$ long, lip middle lobe $9 \mathrm{~mm}$ long, spur $14 \mathrm{~mm}$ long ... 85. B. stanislawii 87. Lateral sepals oblong-obovate to elliptic-obovate, with short, erect apiculus placed near the middle of upper margin ....

86. B. cribbiana

87. Laterl sepals obliquely oblong-obovate, apiculus subapically placed 
88. Spur parallel to the ovary, pendent, anterior petal lobe glabrous, posterior lobe ciliolate .... 87. B. haareri 88. Spur horizontal or upcurved, petals densely pubescent 88. B. petraea 89. Ovary ciliate 89. B. incurva

89. Ovary glabrous 90

90. Spur 45-70 mm long, ca twice longer than pedicel and ovary 90. B. plectomaniaca 90. Spur much shorter, nearly as long as pedicellate ovary ...... 91 91. Anterior petal lobe glabrous 91. B. pseudokaessneriana

91. Antrior petal lobe papillose and/or ciliate 92 92. Lip 3-lobed just above the base .... 92. B. limnophila 92. Lip 3-lobed above the basal undivided part up to $1.5 \mathrm{~mm}$ long 93. B. chirensis

\section{Descriptions of subgenera, sections, subsections and species}

\section{Bilabrella Lind1.}

Bot. Reg. 20: t. 1701. 1835, emend. Szlach. \& KrasLap., Richardiana 3(3): 136-139. 2003; Lectotype (Szlachetko \& Kras-Lapińska 2003): Bilabrella falcicornis Lindl., Bot. Reg. 20: t. 1701. 1835. - Szlachetko \& Kras, Richardiana 6(4): 196-198. 2006. - Szlachetko \& Kowalkowska, Contrib. Orchid. Guinea, 25: 54. 2007 - Szlachetko, Orchid. Ivory Coast: 41. 2008. Szlachetko et al., Orchid. of Central-West Africa 1: 210. 2010.

= Habenaria Willd. sect. Replicatae Kraenzl., Bot. Jahrb. Syst. 16: 74, 85. 1893; Lectotype: (designated by Summerhayes [1968b: 119],

Habenaria kilimanjari Rchb. f., Otia Bot. Hamburg.: 119. 1881.

Key to subgenera

1. Gynostemium short and massive subgen. Brevicolumna

1. Gynostemium slender, elongate ... subgen. Bilabrella

\subsection{Subgenus Brevicolumna Szlach. \& Kras, subgen. nov.}

Type species: Bilabrella macrostele (Summerh.) Szlach. \& Kras-Lap. [झ Habenaria macrostele Summerh.].

Gynostemium short and relatively massive. Antherophores, stigmaphores and rostellophores usually as long as the fertile part of the anther. Caudicles more or less as long as pollen mass.

This group embraces 19 known species, with probably some still undescribed, and can be divided into 4 sections based on the gynostemium and flower perianths morphology.

\section{Key to sections}

1. Gynostemium stalked sect. Brevicolumna (B. macrostele)

1. Gynostemium sessile ... 2

2. Lip and petals densely covered by hairs of various length sect. Papillabiae (B. arianae)

2. Lip glabrous or minutely papillate. Petals glabrous or ciliate or papillate 3

3. Petals divided above the basal third or quarter sect. Diselloidae (23)

3. Petals divided to the base .... sect. Antenniferae (28)

\subsubsection{Section Brevicolumna}

Gynostemium stalked.

A monotypic section.

1. Bilabrella macrostele (Summerh.) Szlach. \& KrasLap., Richardiana 3(3): 141. 2003.

Type (as holotype): Zimbabwe, Norlindh \& Weimarck 4989 (lectotype, designated by la Croix \& Cribb [1995: 99], K!; isolectotypes, BM!, BR!, LD not seen).

Habenaria macrostele Summerh., Bot. Not. 1937: 184. 1937; Summerhayes, Mem. N. Y. Bot. Gard. 9(1): 80. 1954. - Goodier \& Phipps, Kirkia 1: 52. 1961. - Moriarty, Wild. Fls. Malawi: t. 26, 4. 1975. - Grosvenor, Excelsa 6: 83. 1976. - Geerinck, Fl. Afr. Centr., Orchid. 1: 110-111. 1984. - la Croix et al., Orchid. Malawi: 74. 1991. - la Croix \& Cribb, Fl. Zambes., Orchid. 1: 99. 1995. - Szlachetko \& Olszewski, Fl. Cam., Orchid. 34(1): 168. 1998.

Habenaria huillensis sensu Geerinck \& Coutrez, Not. Taxon. Orchid. Afr. Centr., Habenaria: 9. 1977.

Habenaria tournayana Geerinck, Bull. Jard. Bot. Belg. 52(1-2): 148. 1982.

Type: Burundi, Reekmans 8842 (holotype, BR!; isotypes, LG not seen, WAG!). - Geerinck, Fl. Afr. Centr., Orchid. 1: 116. 1984, syn. nov.

Tubers 2, 1-2.5 $\mathrm{cm}$ long, $0.7-1.2 \mathrm{~cm}$ in diameter, ovoid or ellipsoid. Stem $30-90 \mathrm{~cm}$ tall, erect, stout to slender, glabrous. Leaves 7-15, 4-18 cm long, 0.8$1.7 \mathrm{~cm}$ wide, narrowly lanceolate, acute, erect or semispreading, decreasing in size up the stem. Inflorescence 10-24 cm long, ca. 25-40-flowered, rather lax. Flowers green, with white centre, smelling very unpleasant. Floral bracts $12-19 \mathrm{~mm}$ long, densely glandular on margins and outwards. Pedicel and ovary $15-25 \mathrm{~mm}$ long, glabrous. Dorsal sepal 4-6 mm long, 0.5-2.1 mm wide, oblong-obovate or elliptic, cochleate, with obtuse slightly thickened apiculus, glabrous. Petals bilobed to the base, thickened; anterior lobe 6-9.2 mm long, 1-1.8 $\mathrm{mm}$ wide, linear-lanceolate, falcate, acute, pendent, ciliate near the base; posterior lobe 4-6 $\mathrm{mm}$ long, $0.5-1 \mathrm{~mm}$ wide, linear, falcate, subobtuse, erect, ciliate. Lateral sepals 5.5-7 mm long, 2.7-3.2 mm wide, falcate obovate 


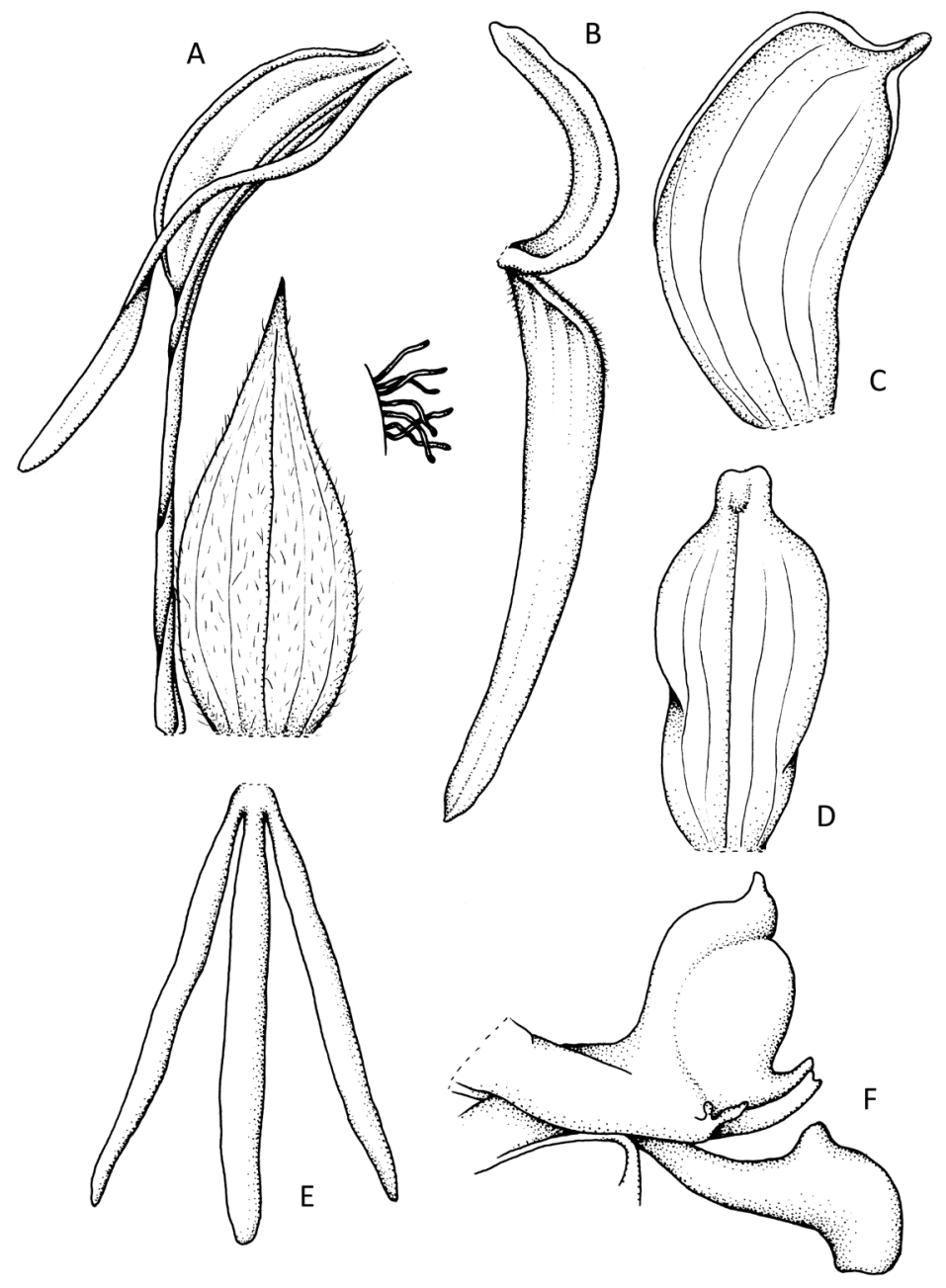

Fig. 11. Bilabrella macrostele (Summerh.) Szlach. \& Kras-Lap. Explanations: A - ovary, pedicel, spur, floral bract; B - petal; C - lateral sepal; D - dorsal sepal; E - lip; F - gynostemium, side view (drawn by H. B. Margońska from lectotype of Habenaria macrostele Summerh. - Norlindh \& Weimarck 4989, K)

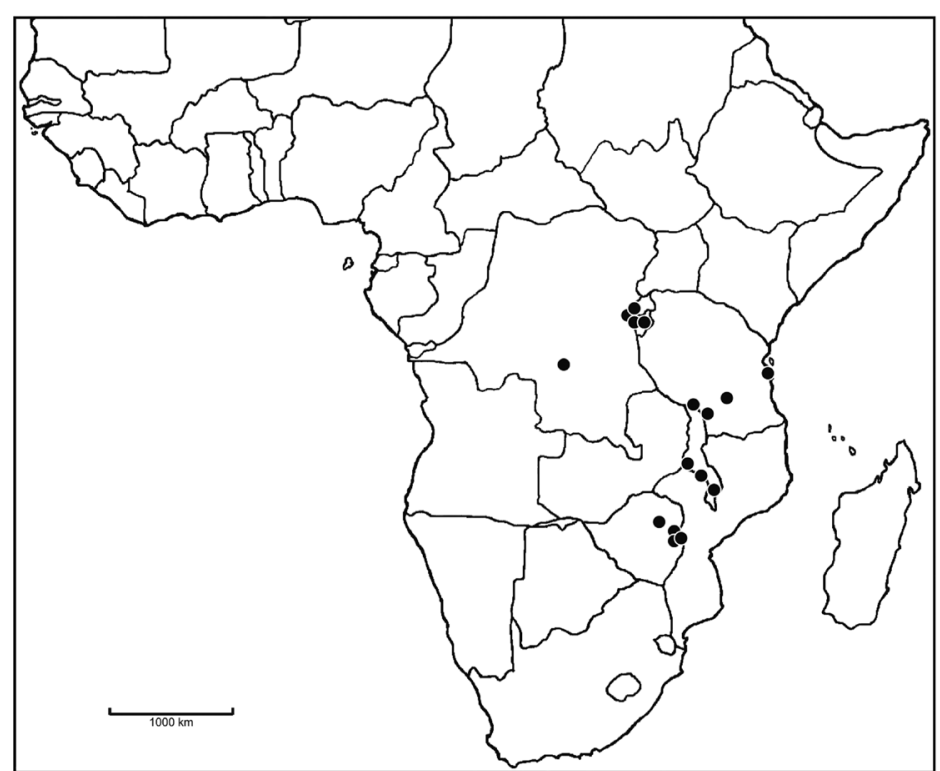

Fig. 13. Distribution of Bilabrella macrostele (Summerh.) Szlach. \& Kras-Lap.

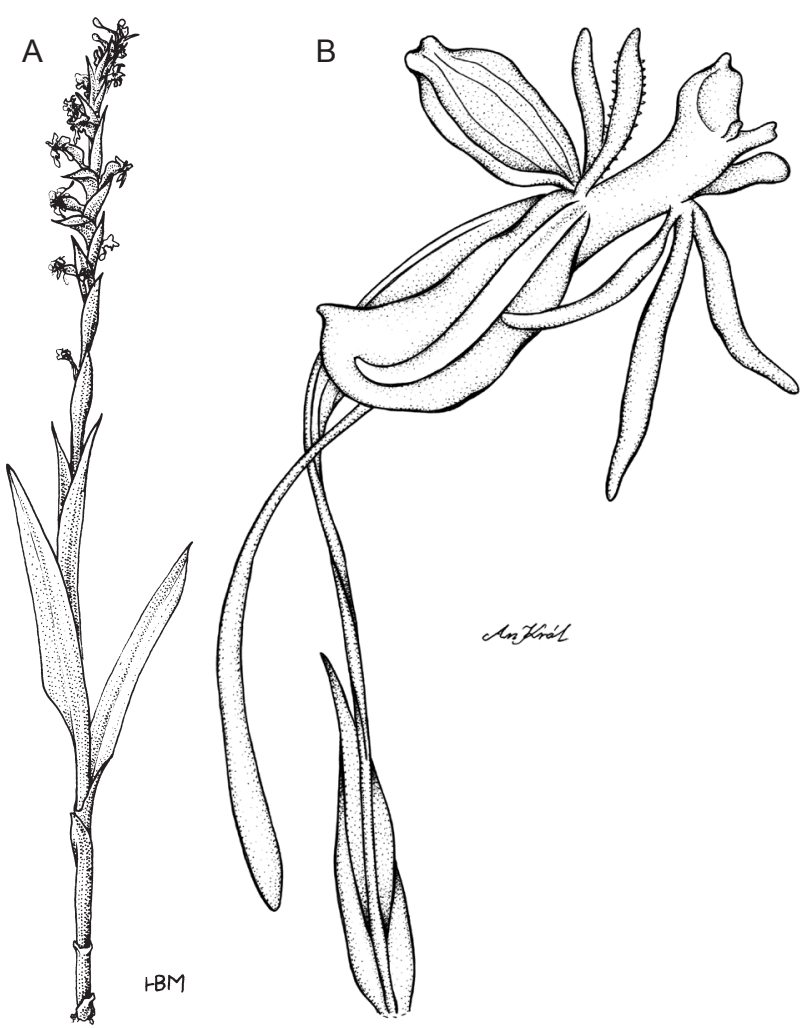

Fig. 12. Bilabrella macrostele (Summerh.) Szlach. \& Kras-Lap. Explanations: A- habit (drawn by H. B. Margonska from lectotype of Habenaria macrostele Summerh. - Norlindh \& Weimarck 4989, K); B - flower (drawn by A. Król from Wild 46228, AMES)

or obliquely oblong, concave, with involute margins, apiculus subapical, glabrous. Lip 3-lobed almost to the base, rather thick, glabrous; the middle lobe $6.7-8.5 \mathrm{~mm}$ long, 0.9-1.1 mm wide, linear, obtuse; lateral lobes 4.5-5.5 mm long, $0.5 \mathrm{~mm}$ wide, linear, subobtuse. Spur 13.5-20 mm long, ca. $0.8-1.1 \mathrm{~mm}$ in diameter, cylindrical in the basal part, inflated in the apical one, blunt, slightly twisted in the middle or not. Gynostemium stalked. Anther 2-2.7 mm long; connective apiculate; antherophores up to $1 \mathrm{~mm}$ long, delicate, upcurved. Stigmaphores club-like, massive, glabrous. Rostellum middle lobe distinctly shorter than connective. Auriculae very delicate, unequally bilobed (Figs 11-12). E c ology. Montane grassland, wet soil overlying rocks, seepage slopes. January-April, December.

D i s tribution. Democratic Republic of the Congo (Zaire), Rwanda, Burundi, Tanzania, Malawi, Zimbabwe, Mozambique, alt. 660-3000 m (Fig. 13).

Specimens examined. Democratic Republic of the Congo (Zaire) . Kasaï oriental: Lualaba, $44 \mathrm{~km}$ NNW from Kolwezi, clear forest, 1070 m, 24 Jan. 1983 (fl), Schaijes 1827 (BR!); Kivu: Mount Buzezu, 2400 m, 20 Dec. 1961 (fl), Hendrickx 4975bis (BR!); Mountains near E of Lake Kivu, 2400 m, Feb.-Mar. 1929 (fl), Humbert 7789 (BR!, K!, P!, UGDA-DLSz!). Rwanda. West: Karongi Distr., Prefecture Kibuye, Road Gisovu-Gatare, 
2200 m, 20 Mar. 1972 (fl), Troupin 14.515 (BR!). Burundi. Bujumbura Rural: Mount Heha, steppe with Eragrostis hispida, 336'S, 29³0'E, 2300 m, 5 Apr. 1980 (fl), Reekmans 8842 (K!); Cancuzo: Mt. Ngoma, 2500 m, 8 Feb. 1990 (fl), Arbonnier 99 (BR!); Mt. Ngoma, $3^{\circ} 12^{\prime}$ 'S, 293'ㄹ' 2500 m, 8 Feb. 1990 (fl), Arbonnier 222 (BR!); Karuzi: Moll Teza-Nyamugari, 2500 m, 28 Mar. 1992 (fl), Arbonnier 321 (BR!); Muramvya: Mount Teza, steppe, 2500 m, Reekmans 2380 (BR!); Yanza-Musumba, 19 Apr. (fl), Arbonnier 331 (BR!). Tanzania. Dar es Salaam: Ukonga, Lumakali River, in meadow, 3000 m, 4 Feb. 1914 (fl), Stolz 2490B part (K!); Iringa: Njombe Distr., Mbeya, Kitulo Sheep Project, grassland, 1200 m, 1 Mar. 1970 (fl), Tuller 112 (K!); Njombe, Lusitu, sandy soil among rocks, $660 \mathrm{~m}, 22$ Mar. 1989 (fl), Spurrier 67 (K!); Njombe, Luponde Estate, thin black soil on rock slabs (granite), $660 \mathrm{~m}, 13 \mathrm{Apr}$. 1989 (fl), Spurrier 69 (K!); Morogoro: Lusitu Ridge, black soil an seepage slopes among rocks, grass, 2220 m, 28 Apr. 1991 (fl), Spurrier 120 (K!). Malawi. Road above dam, grassy road margin, Mar. (fl), Ash 514 (K!); Central: Dedza Distr., Dedza Mt., grassland by summit, 2135 m, 23 Feb. 1982 (fl), Brummitt, Polhill \& Benda 16051 (K!); Dedza Distr., Dedza Mt., 2140-2230 m, 20 Mar. 1955 (fl), Exell, Mendonca \& Wild 1079 (BM!); Dedza Distr., Dedza Mt., between radis relay station a summit beacon, common in grassland, 2100 m, 3 Mar. 1977 (fl), Grosvenor \& Renz 1018 (K!); Dedza Distr., Dedza Mt., in montane grassland, 2000 m, 23 Feb. 1985 (fl), la Croix 668 (K!); Dedza Distr., Dedza Mt., marshy ground on the slopes, up to summit, $2340 \mathrm{~m}, 3$ Mar. 1974 (fl), Westwood 683 (K!); Mchinji Distr., Matutu, up road $\mathrm{nr} 706$, but on wet rock, 8 Apr. (fl), Nelsh 707 (K!); Southern: Mulanje Distr., Mulanje Mt., Chambe Plateau, grassland by top end of sky line, $1800 \mathrm{~m}$, 14 Feb. 1979 (fl), Blacmore, Brummitt \& Banda 424 (K!); Mulanje Distr., Mulanje Mt., Litchenya Plateau, wet flush on rocks at edge of Plateau, $1980 \mathrm{~m}, 17 \mathrm{Feb}$. 1982 (fl), Brummitt, Polhill \& Patel 15939 (K!); 1881 (fl), Buchanan 306 (K!); Mulanje Distr., Mt. Mulanje, just S of Sombani, 2160 m, 14 Jan. 1971 (fl), Hilliard \& Burtt 6255 (E!); Mulanje Distr., Mulanje. Lichenya Path, in granite heavy shade path, $1650 \mathrm{~m}, 19$ Mar. 1983 (fl), la Croix \& Killick 455 (K!); Zomba Distr., Zambia Plateau, below road to summit oposite Malosa saddle, wet flush on rocky hillside, 1900 m, 15 Mar. 1970 (fl), Brummitt 9140 (K! \& spirit.!); Zomba Distr., Top of Zomba,. 1881 (fl), Buchanan 910 (K!); Zomba Distr., Zomba Mt. Forest Nursery, shallow montane latosols, 1830 m, 9 Mar. 1955 (fl), Exell, Mendonca \& Wild 717 (BM!); Zomba Distr., Zomba Plateau, near Chingwe's Hole, in wet grassland, 1900 m, 26 Feb. 1977 (fl), Grosvenor \& Renz 973 (K!); Zomba Distr., Zomba Mt., Chingwe's Hole, in bog on seepage slopes in montane grassland, alt. 1800 m, 14 Feb. 1981 (fl), la
Croix 105 (K!); The same locality, 21 Mar. 1981 (fl), la Croix 128 (K!); The same locality, 13 Mar. 1982 (fl), la Croix 341 (K!); Zomba Distr., Top of Zomba Mts., in swamp, 25 Jan. 1918 (fl), McLoughlin 112 (K!); Zomba Distr., Zomba, rocks before final climb to Chinawe's Hole, seepage rock, 9 Mar. 1970 (fl), Moriarty 160 (K!); Zomba Distr., Zomba Plateau, in grassland, alt. 1800 m, 17 Feb. 1980 (fl), Morris 719 (K!); In montane grassland, alt. 1950 m, 24 Feb. 1980 (fl), Morris 736 (K!); In grassland, 1800 m, 1 Apr. 1980 (fl), Morris 871 (K!); The same locality, Morris 887 (K!); Zomba Distr., Zomba Mt., upward road, on seepage rocks with some grass, 1490 m, 31 Mar. 1978 (fl), Pawek 14166 (K!); Zomba Distr., Zomba Mt. Chingwe's Hole, Westwood 625 (K!); Zomba Distr., Zomba Mt., Westwood 630 (K!); Zomba Distr., Zomba Mt., 24 Mar. 1972 (fl), Westwood 665 (K!). Zimbabwe. Harare: Salisbury, Melsetter, Chimanimani Mts., upland Plateau, 10 Feb. 1954 (fl), Ball 214 (K!); Salisbury, Melsetter, Skyline 17 miles Sw of Melsetter, in wet highland vlei, 1500 m, 22 Feb. 1953 (fl), Crook 447 (41632) (BR!, K!); Salisbury, Melsetter, near "Skyline" Melsetter-Chipinga road, moist grassland by roadside, 28 Feb. 1956 (fl), Drummond S109 (K!); Salisbury, Melsetter, Mar. 1957 (fl), Goodier 198 (K!); Salisbury, Melsetter, Bhimanimani Mountains, Bundi Valley, near side of path through the valley, 1500 m, 5 Apr. 1969 (fl), Grosvenor 482 (K!); Salisbury, Umtali, Tumba, 1860 m, 18 Mar. 1956 (fl), Chase 6050 (BM!, K!); Salisbury, Umtali, Ergwa, in vlei, 1920 m, 2 Mar. 1954 (fl), Wild 4447 (K! \& spirit.); Vumba, 1740 m, 2 Mar. 1960 (fl), Heed 197 (BM!); Manicaland: Inyanga Distr., Mare dam, in open grassland, 1860 m, 4 Mar. 1969 (fl), Jacobsen 3724 (K!); Inyanga Distr., Nyanga, in campo montano, Mt. Inyangani summit, 2450 m, 14 Feb. 1931 (fl), Norlindh \& Weimarck 4989 (K!, BM!, BR!). Inyanga Distr., Troutbeck, North Downs, in marshy ground among rocks, 1980 m, 8 Mar. 1981 (fl), Philox, Leppard, Duri, Urayai 8920 (K!); Inyanga Distr., Mt. Inyangani, monte grassland, 2500 m, 7 Mar. 1981 (fl), Philox, Leppard, Duri, Urayai 8896 (K!); Inyanga Distr., Inyanga in vlei, 1800 m, 22 Feb. 1946 (fl), Wild 950 (14631) (K!); Makonis Distr., Scape 18", Chimbi Farm, 1560 m, 14 Feb. 1960 (fl), Chase 7271 a (K!); Melsetter Distr., east-facing slope of Mt. Peni, 1560 m, 16 Mar. 1981 (fl), Philox, Leppard, Duri, Urayai 8994 (K!); Umtali Distr., Nimalaja sange, Lagua, 3150 m, 2 Mar. 1954 (fl), Wild 46228 (AMES!, UGDA- DLSz! - fragment, drawing). Mozambique. Manica: Manica E Sofala, Tsetserra, grassland, on shallow soil over granite rocks, 2140 m, 7 Feb. 1955 (fl), Exell, Mendonca, Wild 241 (BM!).

N o t e s. Bilabrella macrostele is easily distinguishable species by its unique, stalked gynostemium, not found elsewhere in the genus. 
In 1984 Geerinck described Habenaria tournyana from Burundi. Comparing the type collections of this species and $H$. macrostele we found no differencies between them, hence we treat them as conspecific.

Habenaria macrostele was not included in Summerhayes' (1968b) account of the genus in F.T.E.A., but specimens are now known from southern Tanzania. Plants from this country, from Burundi, central Malawi and some from south Malawi have the basal leaf sheaths spotted with black, sometimes heavily. Unlike them, plants from Zimbabwe, and some from Malawi, have unspotted sheaths. The leaves of plants from Zimbabwe are usually wider. The flowers, however, are uniform thoughout the range (la Croix \& Cribb 1995).

\subsubsection{Section Papillabiae Szlach. \& Kras, sect. nov.}

Type species: Bilabrella arianae (Geerinck) Szlach. \& Kras [ $\equiv$ Habenaria arianae Geerinck].

Petals and lip pubescent. Gynostemium sessile.

A monotypic section.

2. Bilabrella arianae (Geerinck) Szlach. \& Kras, Richardiana 9(4): 158. 2009.

Habenaria arianae Geerinck in Geerinck \& Coutrez, Not. Taxon. Orchid. Afr. Centr., Habenaria: 7. 1977. - Geerinck, Bull. Jard. Bot. Belg. 50: 117. 1980. Geerinck, Distr. Pl. Afr. 18: 630. 1980. - Geerinck, Fl. Afr. Centr., Orchid. 1: 100. 1984. - la Croix et al., Orchid. Malawi: 69. 1991. - la Croix \& Cribb, Fl. Zambes., Orchid. 11: 88. 1995.

Type: Burundi, Lewalle 574 (holotype, BR!).

Habenaria sp. Williamson, Orchid. S. Centr. Africa: 63. 1977.

Tubers $1.3 \mathrm{~cm}$ long, $0.5 \mathrm{~cm}$ in diameter, ellipsoid or ovoid. Stem 13-23 cm tall, erect, slender, glabrous. Leaves 10-20, up to $5 \mathrm{~cm}$ long, 0.3-0.4 cm wide, linear to linear-lanceolate, acute, suberect, the upper ones smaller. Inflorescence 4-7 cm long, densely several- to many-flowered. Flowers light green. Floral bracts up to $17 \mathrm{~mm}$ long. Pedicel and ovary 9-14 mm long, glabrous. Dorsal sepal 3-6 mm long, 2-3 mm wide, oblong-elliptic, obtuse to shortly apiculate, convex, glabrous, erect. Petals bilobed nearly to the base, papillose; anterior lobe 5-10.5 mm long, $1 \mathrm{~mm}$ wide, linear-subulate, apiculate; posterior lobe 4-7 $\mathrm{mm}$ long, 0.7-2 $\mathrm{mm}$ wide, linearlanceolate, falcate to suberect, subacute. Lateral sepals 3-8 mm long, 2-4 mm wide, obliquely ovate, glabrous. Lip 3-lobed almost to the base, deflexed, fleshy, densely covered by hairs of various length; the middle lobe 4.5-8 $\mathrm{mm}$ long, $1 \mathrm{~mm}$ wide, linear, obtuse; lateral lobes 5-11 mm long, $1 \mathrm{~mm}$ wide, linear-lanceolate, acute, curved upwards at the apex. Spur 7-12 mm long, pendent, narrowly cylindrical, slightly inflated at the apex,

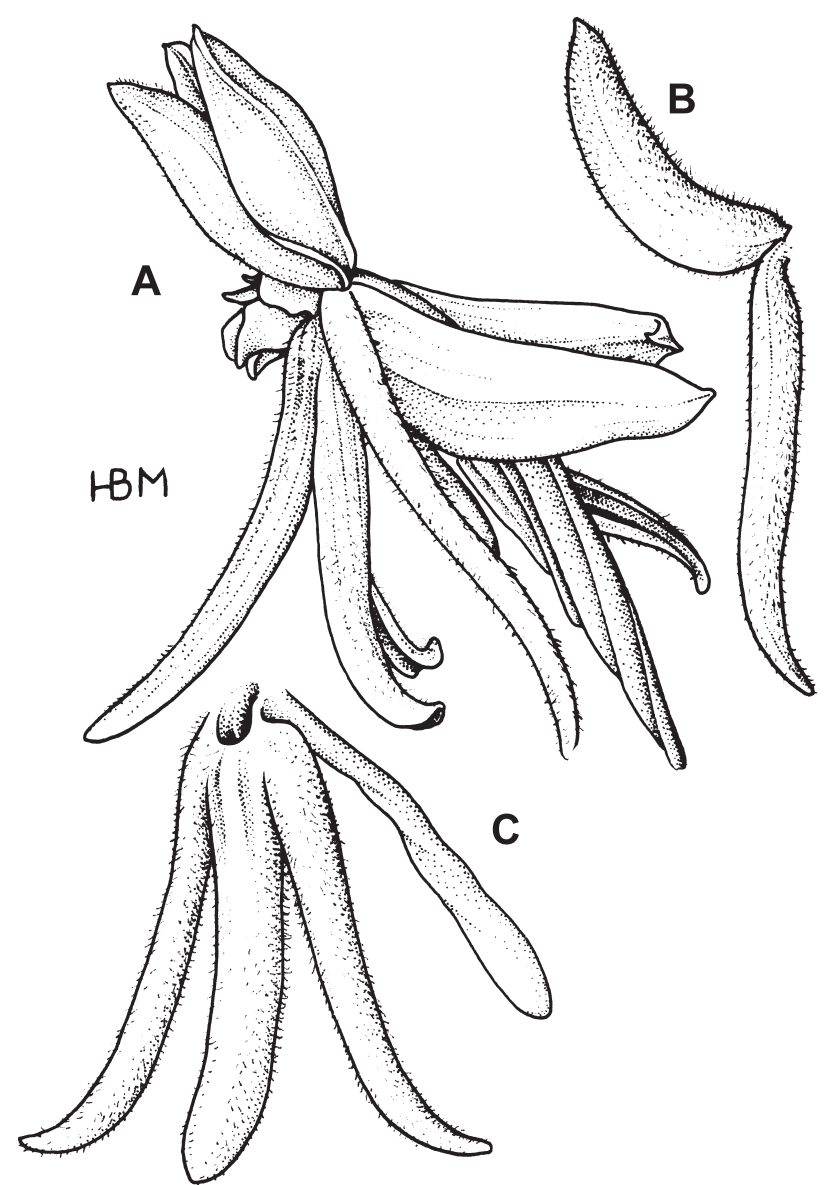

Fig. 14. Bilabrella arianae (Geerinck) Szlach. \& Kras Explanations: A - flower; B - petal; C - spur and lip (redrawn by H. B Margońska from Geerinck 1984)

blunt. Gynostemium sessile. Anther 2-3.1 mm long; connective truncate; antherophores $0.5-1.5 \mathrm{~mm}$ long, delicate, slightly upcurved. Stigmaphores $0.5-1.5 \mathrm{~mm}$ long, clavate. Rostellum middle lobe slightly shorter than connective. Auriculae delicate, small, bilobed in the upper half (Fig. 14).

E c o lo g y. Wet grassland, seepage areas with shallow soil overlying rocks. January-April.

Distribution. Congo, Democratic Republic of the Congo (Zaire), Burundi, Zambia, alt. 1420-2300 m (Fig. 15).

Specimens examined. Congo. Souree: 1600 m, 6 Jan 1969 (fl), Lisowski, Malaisse \& Symoens 869 (BR!). Democratic Republic of the Congo (Zaire): Haut-Katanga: Park Nat. Kundelungu, Katwa-Gombela, km 5, 10³4'S, 2750'E, 1640 m, 25 Jan. 1986 (fl), Bamps \& Malaisse 8318 (BR!); Kundelungu., env. near Loushi, near the village Kibunzi, Valley of Kapolo, 1420 m, 10 Jan. 1971 (fl), Lisowski 66.388 (BR!). Burundi. Bujumbura: Mt. Manga Mugongo, Lewalle 574 (BR!); Rushubi, 322'S, 29²8'E, 2000 m, 25 Mar. 1977 (fl), Reekmans 5902 (BR!); Bururi: Mont Bururi, 2300 m, 10 Feb. 1991 (fl), Arbonnier 192 (BR!); Rutovu pyramides near the spring of the Nil, $3^{\circ} 55^{\prime} \mathrm{S}, 29^{\circ} 50^{\prime} \mathrm{E}$, 
2100 m, 27 Feb. 1966 (fl), Lewalle 485 (BR!); Gitega: Arr. Bukirasazi, Estate Isabu-Luvironza, meadow with stones on small hill, $3^{\circ} 46 \mathrm{~S}, 29^{\circ} 49^{\prime} \mathrm{E}, 1760 \mathrm{~m}, 15 \mathrm{Feb}$. 1969 (fl), Delarge 71 (BR!); Mont Songa, meadow with stones, 1800 m, 4 Feb. 1967 (fl), Lewalle 1555 (BR!); Kayanza: Banga, meadow of Protea, $3^{\circ} 08^{\prime} \mathrm{S}, 29^{\circ} 37^{\prime} \mathrm{E}$, 2000 m, 16 Feb. 1966 (fl), Lewalle 449 (BR!); Muramvya: Mont Manga, 2300 m, 9 Mar. 1969 (fl), Delarge 102 (BR!). Zambia. North-western: near Kabompo River, rocky outcrop in wet Brachystegia woodland, Williamson \& Simon 1766.

Notes. Although almost all species of Bilabrella posseses more or less papillate or ciliate petals, only few of them has lip covered by various kind of hairs. To the last group belong $B$. arianae and $B$. williamsonii. $B$. arianae, however, is unique in the genus by having stiff hairs of various length on the whole lip and petals, wheras in $B$. williamsonii the lip and petals are minutely papillose. Additionally, the leaves of $B$. arianae are numerous, grass-like and erect. In $B$. williamsonii the lower 2-3 leaves are linear-lanceolate, widely spread, the upper ones are much smaller and erect.

B. arianae was given from Angola by la Croix \& Cribb (1995: 89), however, we have examined no specimens of $B$. arianae from this country.

\subsubsection{Section Diselloidae Szlach. \& Kras, sect. nov.}

Type species: Bilabrella diselloides (Schltr.) Szlach. \& Kras-Lap. [ $\equiv$ Habenaria diselloides Schltr.].

Lip lobes oblong to ligulate-lanceolate. Petals divided above the basal third or quarter. Gynostemium sessile.

A section of three species.

\section{Key to species}

1. Lip lateral lobes 3 times shorter than the middle one 3. B. robusta

1. Lip lateral lobes at least half as long as the middle one ...... 2

2. Anterior petal lobe lanceolate, apiculate, posterior lobe oblong-lanceolate, acute to acuminate. Lip middle lobe wider than laterals

4. B. diselloides

2. Anterior petal lobe more or less deltoid, acute, posterior lobe linear to narrowly oblong, obtuse. Lip middle lobe as wide as laterals

5. B. dives

3. Bilabrella robusta (Welw.) Szlach. \& Kras-Lap., Richardiana 3(3): 142. 2003. - Szlachetko et al., Orchid. West-Central Africa 1: 213. 2010.

Habenaria robusta Welw. ex Rchb. f., Flora 1: 102. 1867. - Rolfe, Fl. Trop. Afr. 7: 233. 1898. - Kraenzlin, Orchid. Gen. Sp.: 216. 1901.

Type: Angola, Welwitsch 695 (lectotype, designated by Szlachetko et al. [2010: 213], BM!; isolectotypes, $\mathrm{W}-\mathrm{R}$ !, $\mathrm{K}$ ! - fragment).

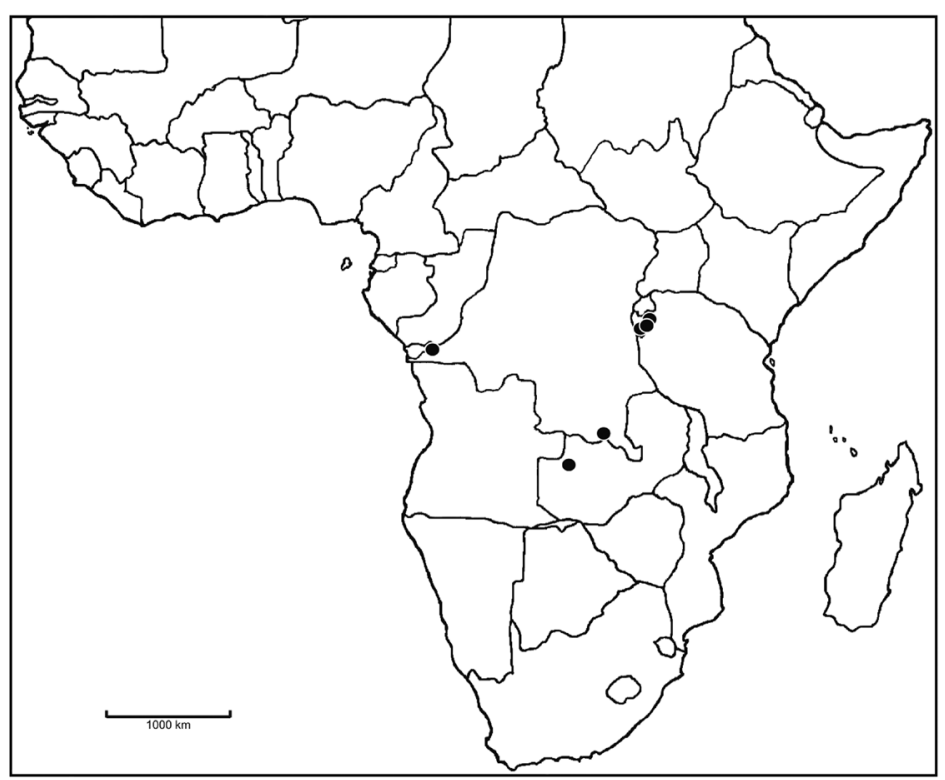

Fig. 15. Distribution of Bilabrella arianae (Geerinck) Szlach. \& Kras

Plants up to $140 \mathrm{~cm}$ tall, robust, erect, densely leafy. Leaves numerous, up to $24 \mathrm{~cm}$ long and $5 \mathrm{~cm}$ wide, lanceolate to ovate-lanceolate, acute, suberect, decreasing in size up the stem. Inflorescence up to $40 \mathrm{~cm}$ long, densely multi-flowered. Flowers relatively small. Ovary ca. $16 \mathrm{~mm}$ long, pedicel very short, 1-2 mm long. Dorsal sepal $5 \mathrm{~mm}$ long, $3 \mathrm{~mm}$ wide, oblong elliptic, obtuse, concave, erect. Petals bilobed distinctly above the base, densely ciliolate; anterior lobe $4 \mathrm{~mm}$ long, $0.8 \mathrm{~mm}$ wide, ligulate-lanceolate, falcate, obtuse; posterior lobe $3 \mathrm{~mm}$ long, $1 \mathrm{~mm}$ wide, linear-lanceolate, falcate, acuminate. Lateral sepals $7 \mathrm{~mm}$ long, $5 \mathrm{~mm}$ wide, obliquely oblong ovate or semiorbicular, with laterally placed apiculus, concave, deflexed. Lip 3-lobed above an undivided basal $2 \mathrm{~mm}$; the middle lobe 7-8 $\mathrm{mm}$ long, 1.3-1.5 $\mathrm{mm}$ wide, ligulateoblanceolate, obtuse; lateral lobes $2 \mathrm{~mm}$ long, $0.3 \mathrm{~mm}$ wide, filiform, obtuse, divergent. Spur $19 \mathrm{~mm}$ long, $1.8 \mathrm{~mm}$ in diameter at the apex, cylindrical, slightly swollen towards the apex, subacute, half twisted near the middle. Gynostemium sessile. Anther $3 \mathrm{~mm}$ long; connective apiculate; antherophores $2 \mathrm{~mm}$ long, slender, slightly upcurved. Stigmaphores ca. $2 \mathrm{~mm}$ long, massive, club-like, ciliate. Rostellum middle lobe shorter than connective. Auriculae unknown (Fig. 16). E c ology. Among brush-wood. January-March. D istribution. Angola, Zambia, Zimbabwe, alt. 1050-1440 m (Fig. 17).

Specimens examined. Angola. Malanje: Pungo Andongo, Sobate da Quibangi, Jan. 1834 (fl), Welwitsch 695 (BM!, K!, W-R!). Zambia. Eastern: Kalulu Forest Reserve, in tall grass wodland with occurrence of Julbernardia tomentosa trees, 9 Mar. 1962 (fl), 


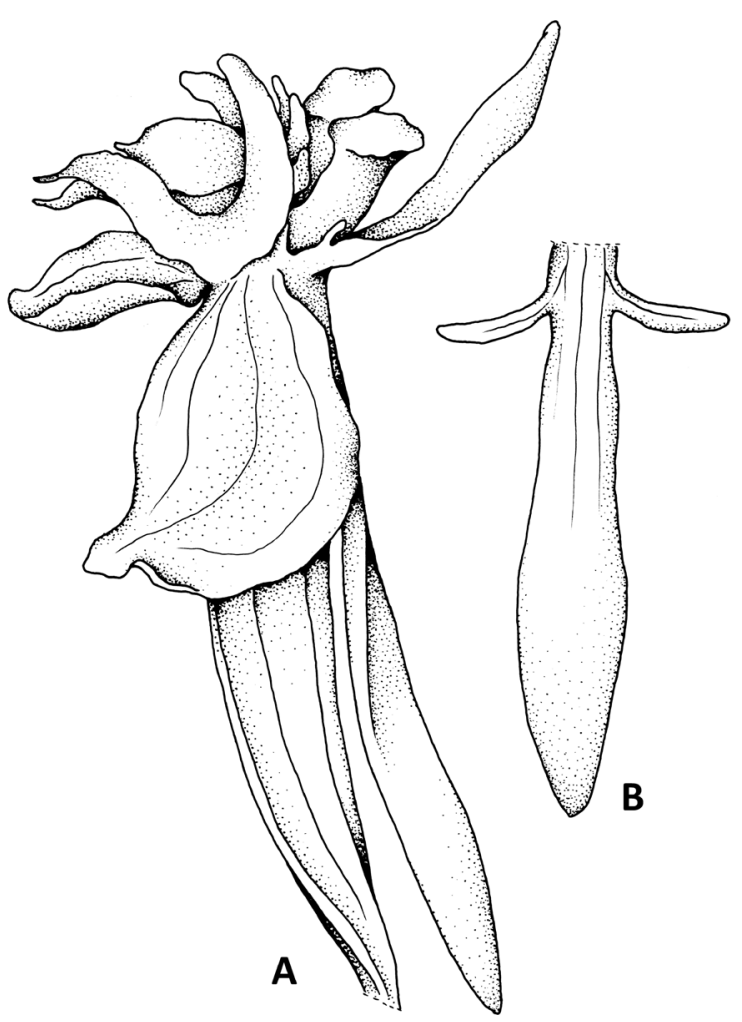

Fig. 16. Bilabrella robusta (Welw.) Szlach. \& Kras-Lap. Explanations: A - flower; B - lip (drawn by H. B. Margońska from isolectotype of Habenaria robusta Welw. - Welwitsch 695, W-R)

Morze 87 (K!); Southern: Mapanza S. Dry bush, 1050 m, 22 Feb. 1954 (fl), Robinson 550 (K!); 24 Jan. 1954 (fl), Robinson 2139 (K! \& spirit.). Zimbabwe. Harare: Salisbury Distr., Hartmann Hill, also collected in Karoi and Northern Umcukwes Distr, 1440 m, 29 Feb. 1952 (fl), Greatrex GHS 35789 (K! \& spirit.).

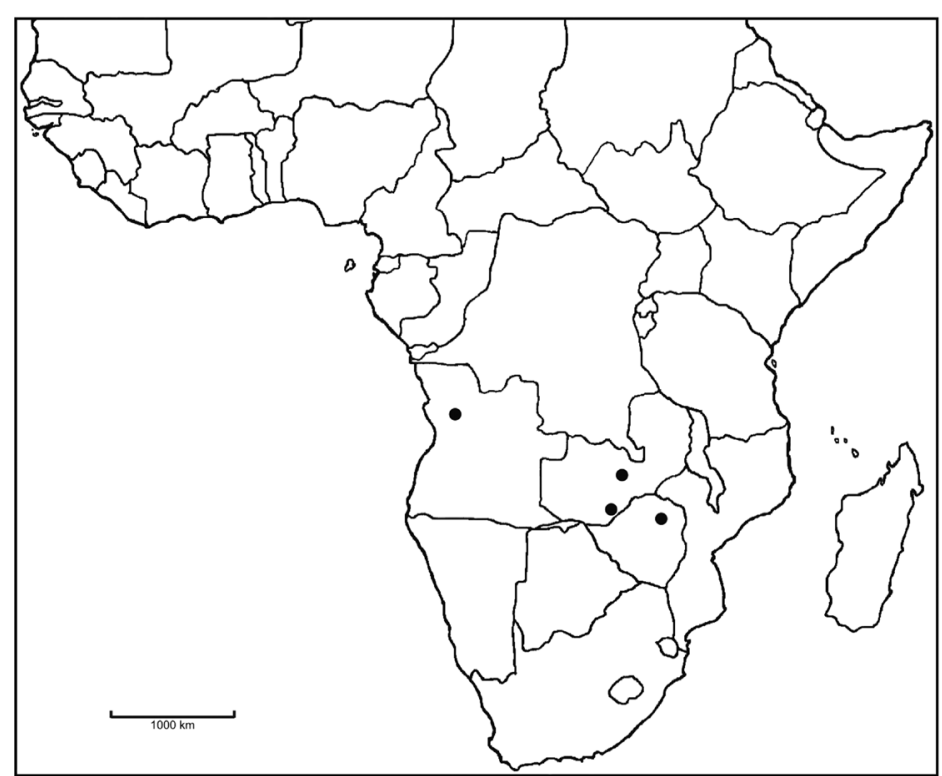

Fig. 17. Distribution of Bilabrella robusta (Welw.) Szlach. \& Kras-Lap.
N ot e s. This is rather curious species, easily distinguishable from other representatives of the genus. Very peculiar lip shape, and short and massive petals could suggest that the species has been described based on abnormal specimens, so often in this genus. Its taxonomic status requires further works.

We have examined type materials deposited at BM, $\mathrm{K}$ and W-R. In BM there is only a drawing of the plant with no other materials, in $\mathrm{K}$ - we found a fragment of the type specimen and in W-R Reichenbach's drawing of flower, gynostemium and floral parts (W-R 35830), natural size drawings of plant habit and flower (W-R $35831 \& 35829)$. Six flowers are attached to the latter sheet.

4. Bilabrella diselloides (Schltr.) Szlach. \& Kras-Lap., Richardiana 3(3): 140. 2003.

Habenaria diselloides Schltr., Bot. Jahrb. Syst. 53: 513. 1915. - Schlechter, Repert. Sp. Nov Regni Veg. 68: tab. 25. 1932. - Williamson, Orchid. S. Centr. Afr.: 56. 1977. - la Croix \& Cribb, Fl. Zambes., Orchid. 11: 90. 1995.

Type (hoc loco selectus): Tanzania, Stolz 2613 (B+; lectotype, K!; isolectotype, S not seen).

Tubers $1.1-1.5 \mathrm{~cm}$ long, $1.1-1.3 \mathrm{~cm}$ in diameter, globose or ellipsoid. Stem $14-30 \mathrm{~cm}$ tall, densely leafy. Leaves 7-15, the lowermost 3 sheath-like, white with green reticulate veining, the next 7-8 semi-erect, set close together, up to $15 \mathrm{~cm}$ long, $1 \mathrm{~cm}$ wide, lanceolate or linear, acute, decreasing in size upwards. Inflorescence $4.5-10 \mathrm{~cm}$ long, densely many-flowered. Flowers yellow green, papillose. Floral bracts to $15 \mathrm{~mm}$ long, ovate, acute. Ovary and pedicel erect; the pedicel

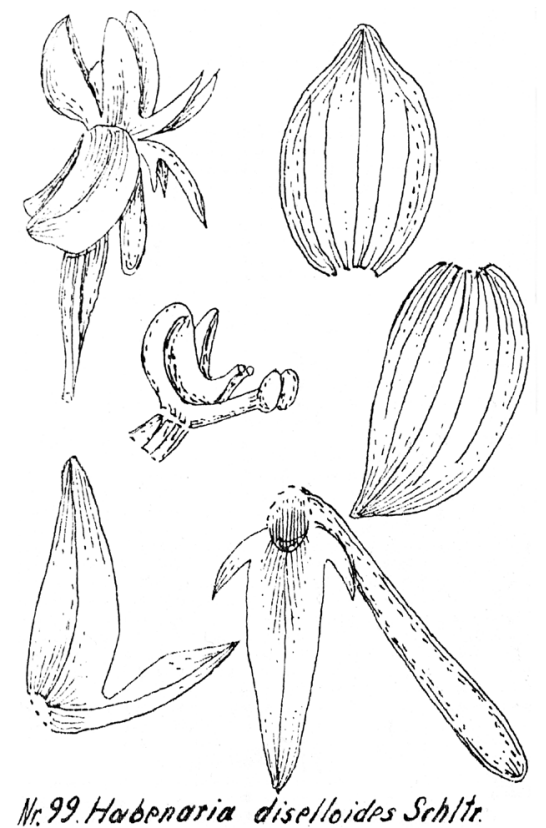

Fig. 18. Original Schechter (1932) drawing of Habenaria diselloides Schltr. 
$3 \mathrm{~mm}$ long, ovary ca. $10 \mathrm{~mm}$ long. Dorsal sepal 3-5 mm long, 2.5-3.5 mm wide, erect, elliptic-ovate, concave. Petals bilobed above $1.5 \mathrm{~mm}$ from the base, glabrous; anterior lobe 1-2.3 $\mathrm{mm}$ long, $0.4 \mathrm{~mm}$ wide, lanceolate, apiculate; posterior lobe 3-3.6 mm long, 1-2 mm wide, oblong-lanceolate to lanceolate, acute to acuminate, erect, lying inside dorsal sepal. Lateral sepals $4-5.5 \mathrm{~mm}$ long, 2.5-3.3 mm wide, spreading, obliquely ovate. Lip $5 \mathrm{~mm}$ long in total, 3-lobed above 1.5-2 $\mathrm{mm}$ long claw; the middle lobe 3-3.5 mm long, 1.5-2 mm wide, oblong- or lanceolate-triangular, acute; lateral lobes 1.4-2.5 mm long, 0.5-0.6 mm wide, linear, to obliquely linear-lanceolate, acute. Spur 4-7.5 mm long, swollen at the apex, parallel to the ovary. Gynostemium sessile. Anther $2 \mathrm{~mm}$ tall; connective triangular; antherophores $1 \mathrm{~mm}$ long, very delicate, upcurved. Stigmaphores 1-1.5 mm long, porrect, short and delicate. Rostellum middle lobe as long as connective. Auriculae small, ellipsoid (Figs 18 \& 19).

E c o 1 o g y. In grassland, wet pebbly grassy seepages, short grass between rocks. Flowering in February-April. Distribution. Tanzania, Malawi, alt. 1700$2250 \mathrm{~m}$ (Fig. 20).

Specimens examined. Tanzania. Cuthbert 28 (K!); Iringa: Njombe, Luponde Estate short grass between rocks, shallow turf overbying rock, $2160 \mathrm{~m}$, 14 Mar. 1991 (fl), Spurrier 114 (K!); Njombe, Bulongwa, Ukinga Mts, 2000 m, Mar. 1914 (fl), Stolz 2613 (K!). Malawi. Northern: Chisanga Falls, Nyika N Plateau, wet grasslnd in clearing in Brachystegia woodland, $30 \mathrm{~m}$ from falls. 15 Feb. 1983 (fl), Elias 2 (K!); Nkhata Bay Distr., S Vipya, Vipya Link Road, grassland, 1800 m, 24 Apr. 1974 (fl), Pawek 8549 (K!); Nyika Plateau, Zovochipolo, $3 \mathrm{~km}$ of Zambian rest house, in short wet grass between rocky outcrops, $2180 \mathrm{~m}, 25 \mathrm{Feb}$. 1982 (fl), Dowsett-Lemaire 276 (K!); Nyika, Chisanga Falls, in miombo woodland, 1700 m, 4 Mar. 1982 (fl), Dowsett-Lemaire 359 (K!); Nyika Nat. Park., 100 m past turn off to Chari above Dam 3, 2200 m, 8 Apr. 1984 (fl), la Croix 603 (K!); Nyika N Plateau, Chelinda Bridge, in grass, 2250 m, 26 Feb. 1987 (fl), la Croix 986 (K!); Nyika Nat. Park, 2250 m, 3 Mar. 1986 (fl), la Croix 999 (K!); Nyika Plateau, grassland, $2250 \mathrm{~m}$, 3 Apr. 1976 (fl), Phillips 1625 (K!); Nyika Plateau, in grassland, Feb. 1969 (fl), Williamson 387 (K!); Nyika Plateau, Apr. 1971 (fl), Williamson 2167 (K!); Rumphi Distr., Nyika Plateau, $25 \mathrm{~km}$ SE of Chelinda Camp, on road to Kasaramba, in short grass on damp rocks, also nearby grassland, 13 Mar. 1977 (fl), Grosvenor \& Renz 1194 (K!).

N o t e s. Bilabrella diselloides appears to be related to $B$. dives. Both share similar lip shape, but petals morphology and gynostemium structure are good criterion for separation both species. In $B$. diselloides anterior petal lobes are narrowly triangular, oblong-lanceolate to

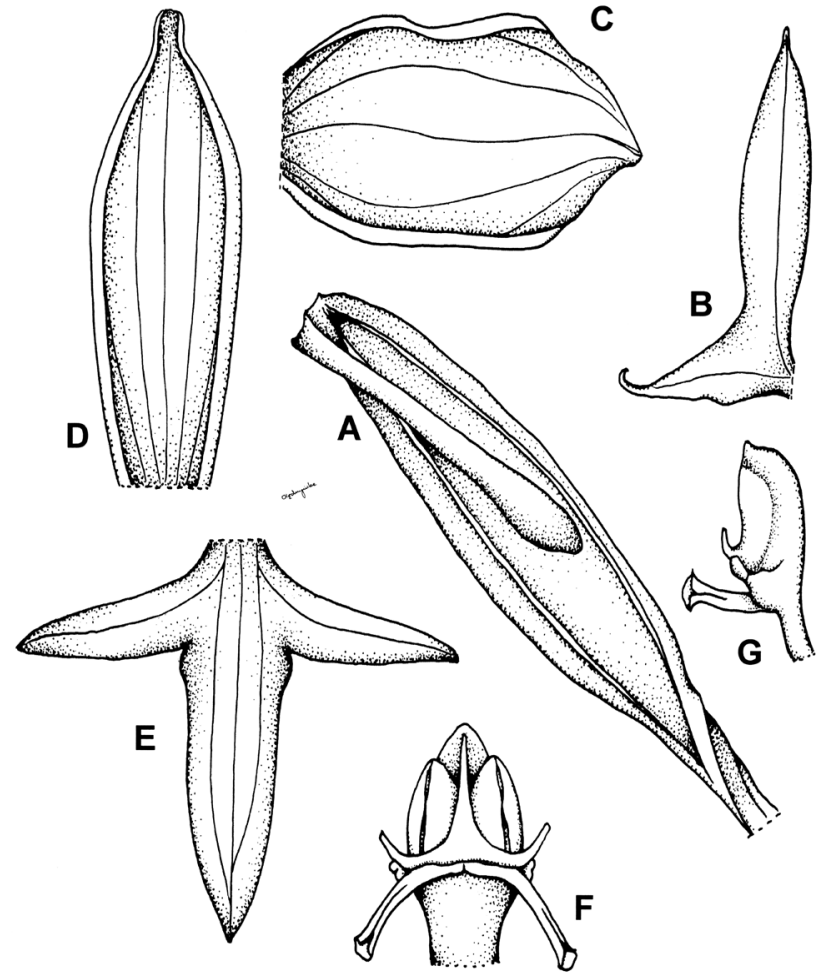

Fig. 19. Bilabrella diselloides (Schltr.) Szlach. \& Kras-Lap. Explanations: A - ovary and spur; B - petal; C - lateral sepal; D - dorsal sepal; E - lip; F - gynostemium, front view; G - gynostemium, side view (drawn by N. Olędrzyńska from lectotype of Habenaria diselloides Schltr. - Stolz 2613, K)

lanceolate, acute to apiculate, posterior lobes obliquely triangular, ovate-lanceolate to lanceolate, acute to acuminate and stigmaphores are short and very delicate. In $B$. dives anterior petal lobes are deltoid, ovate to ovate-lanceolate, posterior lobes narrowly oblong

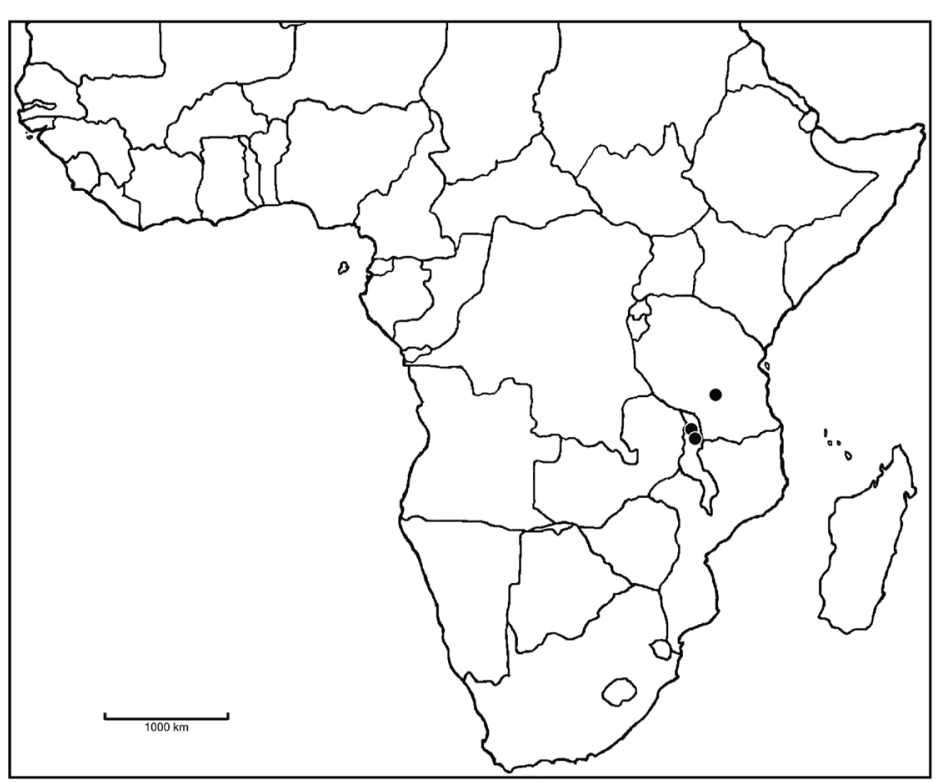

Fig. 20. Distribution of Bilabrella diselloides (Schltr.) Szlach. \& Kras-Lap. 


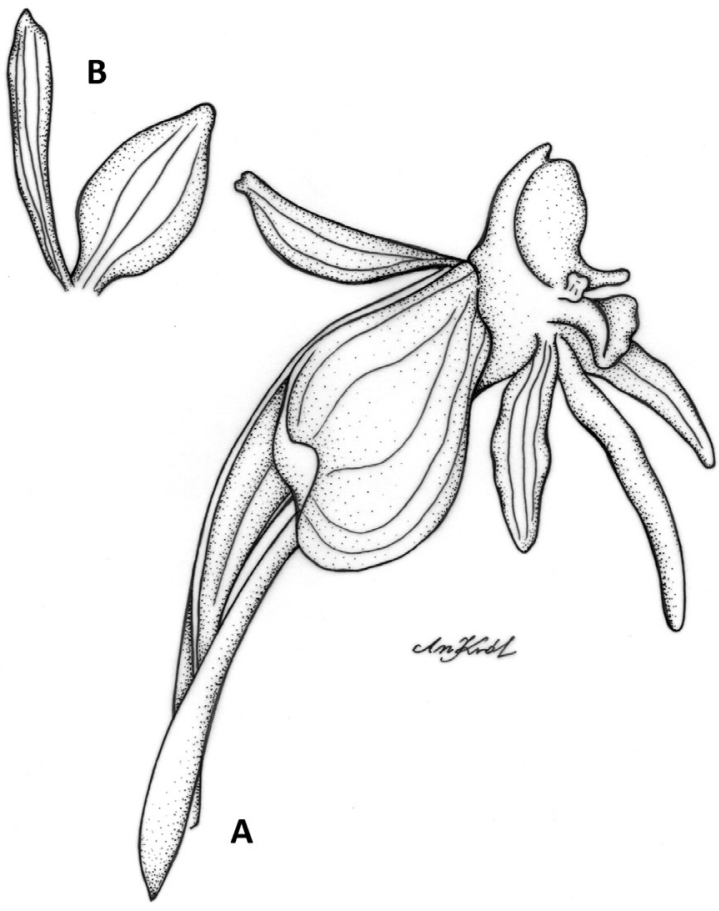

Fig. 21. Bilabrella dives (Rchb. f.) Szlach. \& Kras Explanations: A - flower; B - petal (drawn by A. Król from Medley Wood s.n., AMES)

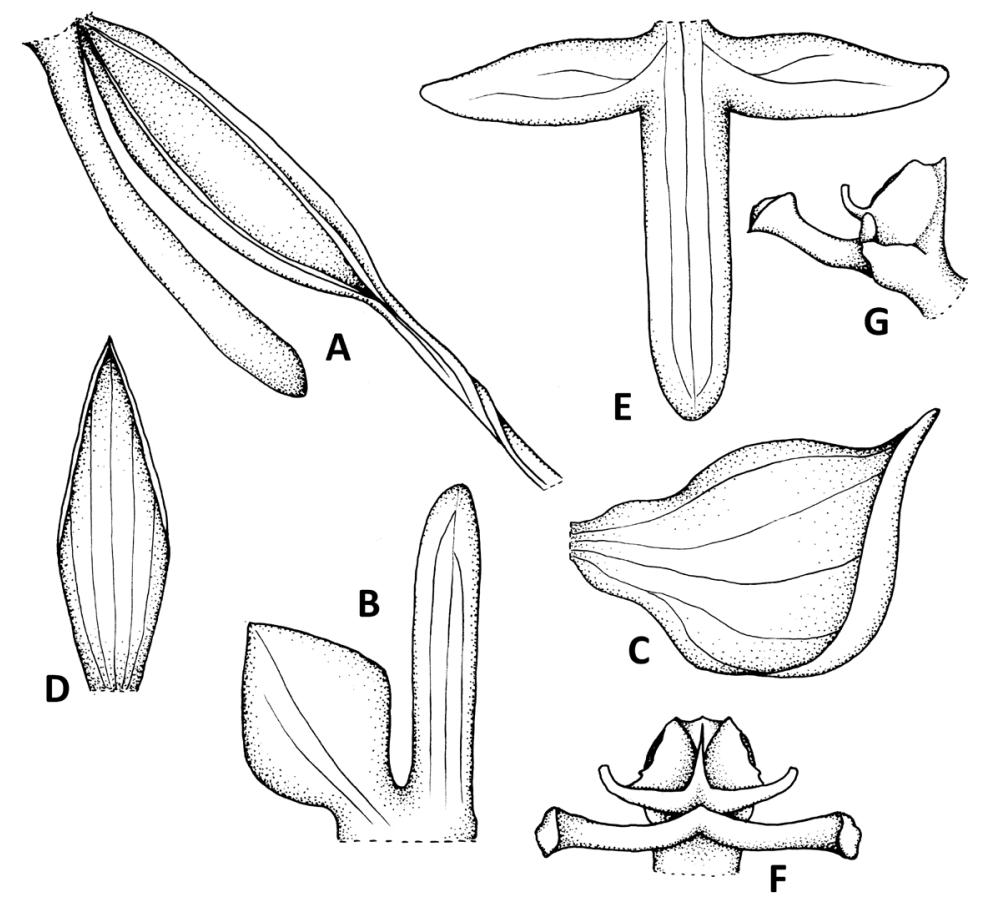

Fig. 22. Bilabrella dives (Rchb. f.) Szlach. \& Kras Explanations: A - ovary, pedicel, spur; B - petal; C - lateral sepal; D - dorsal sepal; E - lip; F - gynostemium, front view; G - gynostemium, side view (drawn by N. Olędrzyńska from lectotype of Habenaria dives Rchb. f. - Sanderson 501, K) to lanceolate, obtuse, puberulous and stigmaphores relatively long and massive.

Type material of Habenaria diselloides we examined had somewhat different form of lip with lateral lobes larger than those presented by Schlechter (1932) in his original illustration.

It has been suggested that $H$. diselloides is an abnormal form of $H$. stolzii (Williamson 1977).

5. Bilabrella dives (Rchb. f.) Szlach. \& Kras, Richardiana 6(4): 197. 2006.

Habenaria dives Rchb. f., Flora 50: 117. 1867. - Bolus, Icon. Orchid.Austro-Afr. 3: tab. 22. 1913. - Kraenzlin, Orchid. Gen. Sp.: 215. 1901. - Rolfe, Fl. Cap. 5(3): 128. 1913. - Stewart et al., Wild Orchid. S. Afr.: 89. 1982. - Linder \& Kurzweil, Orchids of Southern Africa: 124. 1999.

Type (hoc loco selectus): Republic of South Africa, Sanderson 501 (lectotype, K!; isolectotype, SAM not seen).

Plants 1-73 cm, slender to robust, leafy. Leaves 6-11, 8-11(24) cm long, suberect or the lower ones spread, lanceolate to oblong-lanceolate, acute to acuminate, decreasing in size upwards. Inflorescences lax to dense, many-flowered. Flowers white with sepals and ovary green. Floral bracts 10-17 mm long, leaf-like, concave, ovate-lanceolate, acuminate, minutely glandularly ciliolate, about as long as the pedicellate ovary. Pedicellate

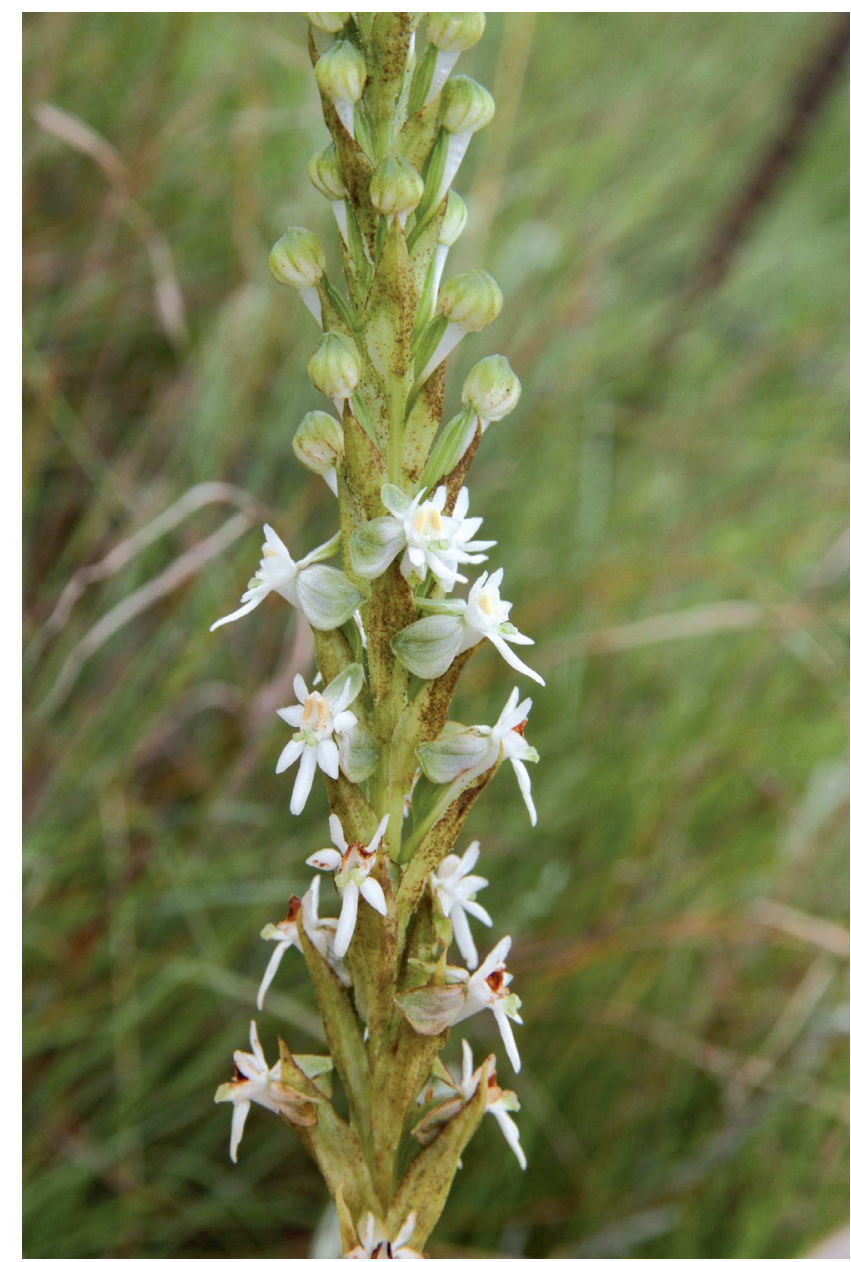

Fig. 23. Bilabrella dives (Rchb.f.) Szlach. \& Kras - inflorescence (phot. L. Grobler) 
ovary 15-20 mm long. Dorsal sepal 3-5 mm long, 1-2 mm wide, oblong-lanceolate to oblong-elliptic, obtuse to subacute, concave. Petals bilobed distinctly above the base; anterior lobe 1-3.3 mm long, $1.5 \mathrm{~mm}$ wide, deltoid, ovate to ovate-lanceolate, acute, glabrous or minutelly papillose; posterior lobe $3-5 \mathrm{~mm}$ long, 0.3-0.5 mm wide, linear to narrowly oblong, obtuse, puberulous. Lateral sepals 4-6.5 mm long, 3-4.5 mm wide, obliquely obovate, obtuse or acute, concave. Lip 3-lobed, porrect; the middle lobe 3-7 mm long, 0.7$1 \mathrm{~mm}$ wide, oblong to ligulate, obtuse; lateral lobes 2-5 $\mathrm{mm}$ long, $1 \mathrm{~mm}$ wide, spreading, obliquely ovate, oblong or lanceolate, subacute. Spur 9-15 mm long, tapered from a widened mouth, slightly inflated apically. Gynostemium sessile. Anther $2 \mathrm{~mm}$ tall; connective truncate, very shortly apiculate; antherophores less than $1 \mathrm{~mm}$ long, delicate, upcurved. Stigmaphores $2 \mathrm{~mm}$ long, rather massive. Rostellum middle lobe as long as connective. Auriculae small, ellipsoid (Figs 21-23).

E c o logy. In grassland, rocky soil. Flowering in January-December.

D is tribution. Congo, Republic of South Africa, alt. 15-1920 m (Fig. 24).

Specimens examined. Congo. Congo Central: Irogo, 1200 m, 8 Feb. 1895 (fl), Schlechter 6658 (C!, K!, W-R!, WU!). Republic of South Africa. Cape: Bizana Distr., $1 \mathrm{ml} \mathrm{W}$ from Port Edward port, 240 m, 12 Jan. 1955 (fl), Schelpe 5085 (BM!, K!); Cape, Acocks 13361 (AMES!, UGDA-DLSz! - drawing); Drakensberg, MacLear Farm 213, +/- 10 km N of Ugie, grassland, floodplane. Sandy clay soil, molteno, full

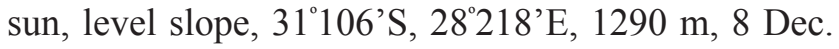
1994 (fl), Bester 2182 (K!); MacLear commonage +/- $5 \mathrm{~km} \mathrm{NW}$ on the road to Rhodes, grassland-vlei, poorly drained clay-sandy soil, molteno, partial sun, level slope, 31 047'S, 28297'E, 1410 m, 8 Feb. 1994 (fl), Bester 2177 (K!); Durban: 15 m, Jan. 1892 (fl), Medley Wood 1534 (AMES!, K!, W-R!); Natal, Durban, 60 m, 22 Jan. 1901 (fl), Medley Wood 8171 (E!); Natal, 9 Feb. 1905 (fl), Medley Wood s.n. (AMES!); Eastern Cape: Lysikisiki. L.P., grassy field above Magwe falls, 27 Jan. 1929 (fl), Galpin 9478 (K!); Transkei, Baziyn Mt. Mpolompo valley, grass slopes, $1320 \mathrm{~m}, 10 \mathrm{Feb}$. 1981 (fl), Hilliard \& Burtt 13937 (E!, K!); Umzimkulu, slope above Ntsikeni Plantation Forest, 3007.91'S, 29³3.25'E, upland grassland, $1600 \mathrm{~m}, 22$ Jan. 2002 (fl), Bytebier 2223 (K!); 07 State, 1862 (fl), Cooper 1099 (W-R!); Free State: Bernburg, 1050 m, Feb. (fl), Baur 632 (K!); Natal, Lotus River Distr., Hay field, Single Free Farm, open grassland, 1250 m, 10 Feb. 1964 (fl), Moll 612 (K!); Gauteng: Graskop Ivl. Distr. Pretoria, in wet ground beside streamlet leading to "Edge of the Berg”, 1560 m, 4 Mar. 1937 (fl), Buchanan 14455 (K!); Inanda, wood, ex herb. Schinz 54 (HBG!); KwazuluNatal: Natal, Sani Pass Hotel - Sani Pass, 29³7'S,

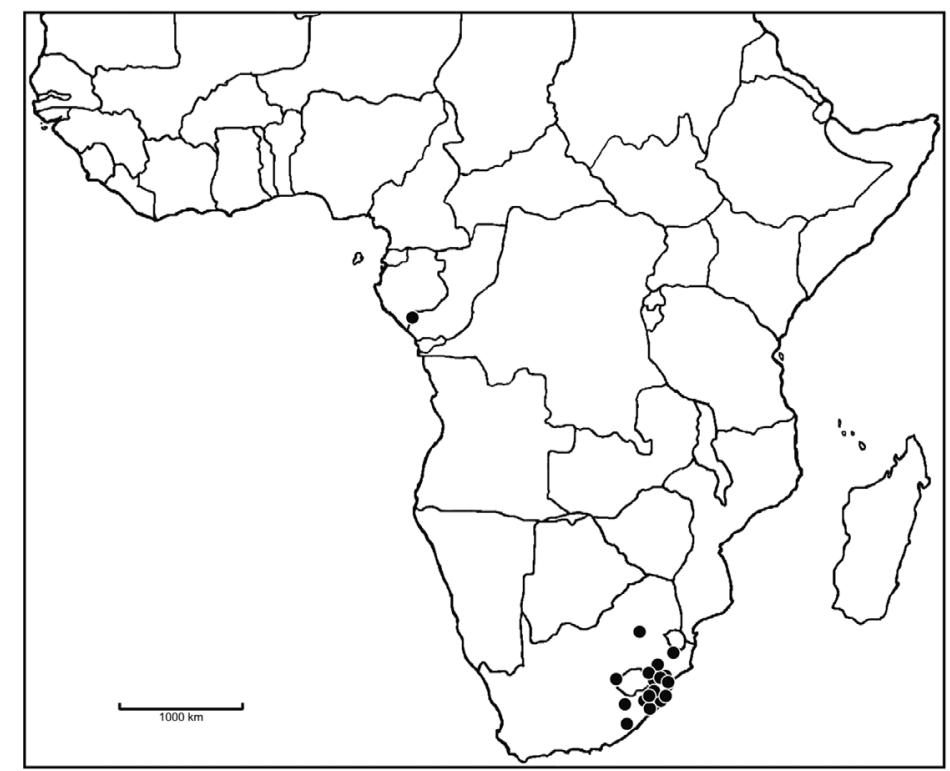

Fig. 24. Distribution of Bilabrella dives (Rchb.f.) Szlach. \& Kras

292'ㄹ' 1730 m, 5 Feb. 1982 (fl), Bamps 7149 (BR!); Natal, Buchanan 14 (W-R!); Natal, Nov. 1875 (fl), Buchanan 1315 (K!); Natal, Nov. 1875 (fl), Buchanan 1413 (K!); Natal, Greinying 45 (W-R!); Natal, 15 m, 5 Jan. 92 (fl), Medley Wood 1005 (BM!); Natal, near Nottingham Road, grassland, 1395 m, 8 Feb. 1954 (fl), Schelpe 4533 (BM!); Alexandra Distr, Natal, Station Dumisa - Farm Friedenau, 600 m, 14 Dec. 1908 (fl), Rudatis 548 (BM!, E!, K!, W!); Natal, Bergville Distr., Oliviershoek Pass, 1530 m, 13 Feb. 1955 (fl), Schelpe 5257 (BM!); Natal, Clermont, 5 Jan. 1892 (fl), Medley Wood 1015 (E!); Natal, KwaZulu, Congella Flats, Sanderson 501 (K! - drawing); Natal, Dargle, On the farm Kilgobbin, 1200 m, 10 Feb. 1973 (fl), Stewart 1665 (K!); Natal, Fields Hill, 300-600 m, 12 Dec. 1908 (fl), s.n. (E!); Natal, Greytown, Jan. 1935 (fl), Wylie s.n. (K!); iLembe Distr., Natal, Loteni nature Reserve, N facing slope, open grassland, $1530 \mathrm{~m}, 16 \mathrm{Jan} .1979$ (fl), Phelan 252 (K!); Natal, Inanda, July 1879 (fl), Medley Wood 437 (K!); Natal, Itafamasi, May \& June 1880 (fl), Eood 737 (K!); Natal, Farm Ehlatini, Karkloof Forest, Themeda grassland, in the lower lying area, $1200 \mathrm{~m}$, 14 Feb. 1965 (fl), Moll 3462 (2930) (K!); Natal, Kokstad Distr., Mt. Currie, neck between two peaks at summit, long grassveld, rocky red clay-loam, 29 Feb. 1979 (fl), Venter 5259 (K!); Upper Loteni valley, vicinity of Ash Cave, 1920 m, 5 Feb. 1985 (fl), Hilliard \& Burtt 18109 (E!); Mpedhle Distr., Natal, 2929AD, Natal, Orange Free Station, bonder, open grassy ground, 12 Feb. 1933 (fl), Kerr s.n. (K!); Natal, Feb. 1895 (fl), Schlechter s.n. (AMES!, UGDA-DLSz! - fragment, drawing); Natal, Polela Distr., Mt. Drakensberg Bamboo, on grassy slopes, alt. 1800 m, Apr. 1908 (fl), Dordge 5573 (K!); 
Natal, Poleta Distr., 4 miles N of Bulweron road to Impendhle, moist grassland, 1 Feb. 1957 (fl), Marais 1462 (K!); Natal, Port Edward, grassland, 18 Dec. 1930 (fl), Moos 19242 (BM!); Umkomanzi, 9 Feb. 1895 (fl), Krook 109 (W-R!); Umkomanzi, 9 Feb. 1895 (fl), Krook 139 (W-R!); Umkumanzi, 9 Feb. 1895 (fl), Penther 109 (W-R!); Natal, Underberg Distr., valley bottom of Umzimkulu above Drakensberg, Garden Hotel, grassland, alt. 1650 m, 27 Jan. 1975 (fl), Hilliard \& Burtt 7748 (E!; K!); Umzinyathi Distr., Natal, 11 miles inland from Scottburgh, 405 m, 5 Jan. 1950 (fl), Poynton 5 (E!); Natal, Weenen, prope Eastcourt, 25 Feb. 1895 (fl), Penther 139 (W-R!); Natal, Zulu-Land, 1865 (fl), Gerrard 383 (BM!); Natal, Mount West, Feb. 1895 (fl), s.n. (BM!, BR!, K!); Natal, Zybica, Louwsburg, Hala Nature Reserve, 1300 m, 24 Feb. 1984 (fl), Hilliard \& Burtt 17731 (E!); Sine loc., 18 Feb. 1902 (fl), Johnston 856 (E!); 1892 (fl), Sanders s.n. (BM!); Welwitsch 1513 (W-R!). Isuepe Distr. S.E. Tol., 24 Jan. 1950 (fl), Sidey 1971 (F!).

N o t e s. This species appears to be similar to Bilabrella diselloides, but can be easily separated by the form of petals and gynostemium, what we discuss above.

\subsubsection{Section Antenniferae Szlach. \& Kras, sect. nov.}

Type species: Bilabrella antennifera (A. Rich.) Szlach. $\&$ Kras [三 Habenaria antennifera A. Rich.].

Lip lobes filiform to narrowly lanceolate. Petals divided to the base. Gynostemium sessile.

A group of 14 species.

\section{Key to species}

1. Auriculae bilobed 2

1. Auriculae entire 3

2. Spur geniculate near the middle 6. B. tortilis

2. Spur not geniculate 7. B. isoantha

3. Spur more or less twisted 4

3. Spur not twisted 7

4. Spur twisted near the middle 8. B. ndiana

4. Spur twisted near the apex 5

5. Posterior petal lobe linear, subobtuse to subacute, ciliate 9. B. vollesenii

5. Posterior petal lobe filiform, obtuse, glabrous ....... 6 6. Leaves 8-11.5 × 0.3-0.6 cm, anterior petal lobe 6.9-9.5 $\times$ 0.3-0.6 mm, filiform, acute, glabrous ... 10. B. compta 6. Leaves 11-23 $\times 1.2-3 \mathrm{~cm}$, anterior petal lobe 7-13 $\times$ $1.3 \mathrm{~mm}$, narrowly lanceolate-linear, falcate, acuminate to caudate, papillose-puberulate ........ 11. B. marxiana 7. Spur subequal in length to the lip middle lobe ........ 8 7. Spur prominently longer than lip middle lobe ....... 9 8. Petals glabrous; anterior lobe 7-10 × 1-2 mm, oblong-lanceolate, obtuse; posterior lobe $6-8 \times 1 \mathrm{~mm}$; stigmaphores and antherophores slender, upcurved, cylindrical-clavate

12. B. stolzii
8. Petals ciliolate; anterior lobe $3-4 \times 1.2 \mathrm{~mm}$, oblongoblanceolate, acute; posterior lobe $3-4 \times 0.3 \mathrm{~mm}$; stigmaphores and antherophores short and massive

13. B. tisseranti

9. Anterior petal lobe glabrous ................................ 10

9. Anterior petal lobe ciliolate or papillose .............. 12

10. Posterior petal lobe glabrous ............. 14. B. woodii

10. Posterior petal lobe ciliolate ................................. 11

11. Leaves $13-15 \times 0.7 \mathrm{~cm}$, inflorescence $6-11 \mathrm{~cm}$ long, lip middle lobe $12 \mathrm{~mm}$ long, lateral lobes $10-11 \mathrm{~mm}$ long

15. B. stenophylla

11. Leaves $12-25 \times 2-5 \mathrm{~cm}$, inflorescence $15-40 \mathrm{~cm}$ long, lip middle lobe $10-17 \mathrm{~mm}$ long, lateral lobes $6-12 \mathrm{~mm}$ long

16. B. sochensis

12. Lip papillose

19. B. williamsonii

12. Lip glabrous

13

13. Petals minutely ciliolate, anterior lobe $4 \times 1.1 \mathrm{~mm}$, ligulate, rounded at the apex, posterior lobe $1.1 \times$ $0.8 \mathrm{~mm}$ wide, ovate to deltoid, obtuse, spur mouth widened

17. B. orangana 13. Petals ciliate, anterior lobe $13 \times 1-1.8 \mathrm{~mm}$, lanceolate, acute to acuminate, posterior lobe up to $4 \times 0.8 \mathrm{~mm}$, reflexed, linear-lanceolate, obtuse, often reduced in some plants, ciliate, spur mouth narrow ... 18. B. antennifera

6. Bilabrella tortilis (P. J. Cribb) Szlach. \& Kras-Lap., Richardiana 3(3): 143. 2003.

Habenaria tortilis P. J. Cribb, Kew Bull. 34: 323. 1979.

- la Croix \& Cribb, Fl. Zambes., Orchid. 11: 109. 1995.

Type: Tanzania, Cribb \& Grey-Wilson 10799 (holotype, $\mathrm{K}$ !).

Stem to $60 \mathrm{~cm}$ tall, erect, slender. Leaves $8-16,9 \mathrm{~cm}$ long, 0.4-1 cm wide, the lowermost sheathing, white with dark green reticulate veining; intermediate leaves semi-erect, linear-lanceolate to narrowly linear, acute, slightly folded. Inflorescence to $12 \mathrm{~cm}$ long, fairly densely many-flowered. Flowers green. Floral bracts 17-20 mm long, ovate-lanceolate, acuminate. Pedicel and ovary 13-20 mm long. Dorsal sepal $4 \mathrm{~mm}$ long, $2.5 \mathrm{~mm}$ wide, reflexed, elliptic-obovate, obtuse, cochleate. Petals 2-lobed almost to base; anterior lobe $8 \mathrm{~mm}$ long, 1-3 mm wide, oblong-lanceolate to lanceolate, obtuse, glabrous; posterior lobe 4.5-5 mm long, $0.5-1 \mathrm{~mm}$ wide, linear, obtuse, glabrous. Lateral sepals $7 \mathrm{~mm}$ long, $4 \mathrm{~mm}$ wide, deflexed, obliquely elliptic-obovate with a lateral apiculum. Lip 3-lobed almost to the base, all lobes pendent; the middle lobe $5-8 \mathrm{~mm}$ long, $0.5 \mathrm{~mm}$ wide, linear; lateral lobes 5-7 $\mathrm{mm}$ long, $0.5 \mathrm{~mm}$ wide, linear. Spur 8-11 mm long, more or less geniculate near the middle, swollen below the subacute or subobtuse apex. Gynostemium sessile. Anther $2 \mathrm{~mm}$ tall; connective obtuse, shortly apiculate; antherophores $3 \mathrm{~mm}$ long, the last $1 \mathrm{~mm}$ upturned, very slender. Stigmaphores c. $2 \mathrm{~mm}$ long, clavate, truncate at the apex. Rostellum 


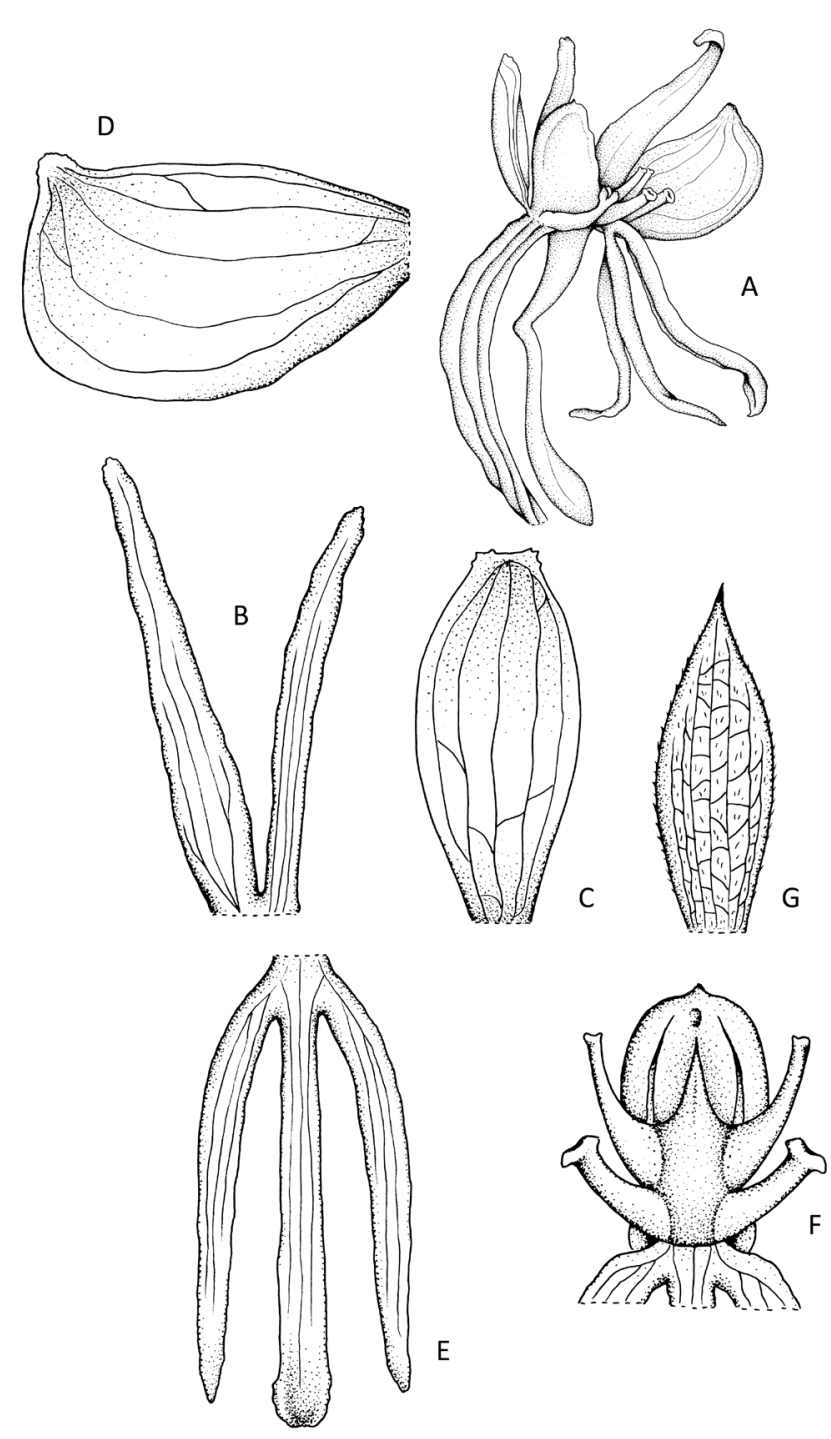

Fig. 25. Bilabrella tortilis (P.J. Cribb) Szlach. \& Kras-Lap.

Explanations: A - flower; B - petal; C - lateral sepal; D - dorsal sepal; E - lip; F - gynostemium, front view; G - bract (drawn by A. Król from holotype of Habenaria tortilis P.J. Cribb - Cribb \& Grey-Wilson 10799, K)

middle lobe distinctly shorter than connective. Auriculae deeply bilobed, distinctly stalked (Fig. 25).

E c o logy. Rocky hill, red sandy soil. FebruaryMarch.

Distribution. Tanzania, Zambia, alt. 1650$2200 \mathrm{~m}$ (Fig. 26).

$\mathrm{Spec}$ im en s ex a mined. Tanzania. Iringa: Njombe Distr. Near Matamba, at top edge of Chimala escarpment, 2200 m, 19 Feb. 1976 (fl), Cribb \& GreyWilson 10799 (K!). Zambia. Northern: Abercorn Distr., rocks on Subawanga Road near Kawimbe, in wet sandy gritty patches between flat rocks, 1650 m, 1 Mar. 1957 (fl), Richards 8417 (K!).

Notes. The unique character of this species is geniculate spur, not found in any other member of shortgynostemium Bilabrella, combined with stalked, deeply bilobed auriculae. The only other known species of $B i-$ labrella with similar form of the spur is B. urundiensis,

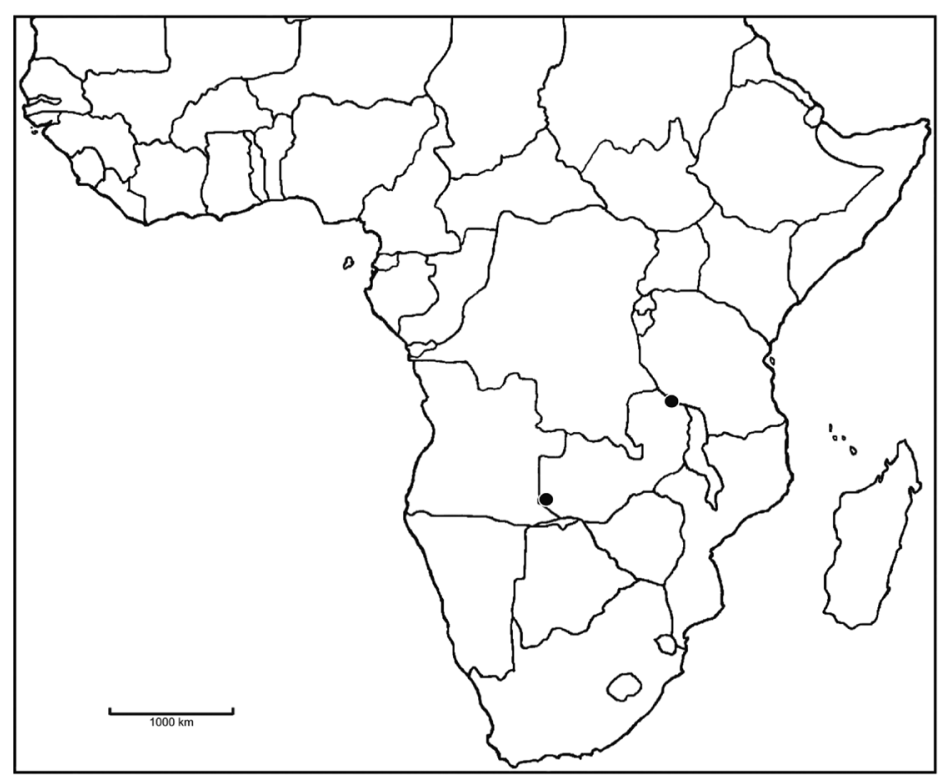

Fig. 26. Distribution of Bilabrella tortilis (P.J. Cribb) Szlach. \& Kras-Lap.

but it is easily distinguishable from $B$. tortilis by having slender, elongate gynostemium projections. $B$. tortilis appears to be somewhat similar to $B$. stolzii, $B$. retinervis and $B$. leucoceras and shares with the last two white basal sheaths on the stem which are reticulated with green. It may be distinguished from $B$. leucoceras by its much smaller flowers, shorter spurs and glabrous petal lobes (cf. Cribb 1989).

7. Bilabrella isoantha (Schltr.) Szlach. \& Kras-Lap., Richardiana 3(3): 141. 2003.

Habenaria isoantha Schltr., Bot. Jahrb. Syst. 53: 517. 1915. - Schlechter, Repert. Sp. Nov Regni Veg. 68: tab. 28. 1932. - Summerhayes, FTEA, Orchid. 1: 100. 1968 b.

Type: Tanganyika, Stolz 1148 (lectotype, designated by Summerhayes [1968b: 100], B!; isolectotypes, C!, K!, W-R!, S not seen).

Tubers $1 \mathrm{~cm}$ long, $0.75 \mathrm{~cm}$ in diameter, ellipsoid, tomentose. Stem 30-95 cm tall, glabrous, erect, rather slender to relatively stout, leafy throughout its length. Leaves 10-15, 8-15 cm long, 1.5-4 cm wide, the lowermost 1 or 2 reduced to sheaths, the middle $4-6$ spreading, lanceolate or oblong-lanceolate, acute, the upper ones smaller, adpressed to the stem, lanceolate, similar to the bracts. Inflorescence $8.5-30 \mathrm{~cm}$ long, rather closely 14- to many-flowered. Flowers suberect or curving outwards, whitish with green sepals, fragrant at night. Floral bracts 12-25 mm long, lanceolate, acuminate, thin and chaffy, the lower ones equalling or longer than the pedicel with ovary. Pedicel and ovary $16-20 \mathrm{~mm}$ long, slightly curved. Dorsal sepal 4-6 mm long, 1.8$3 \mathrm{~mm}$ wide, reflexed, narrowly elliptical to narrowly 


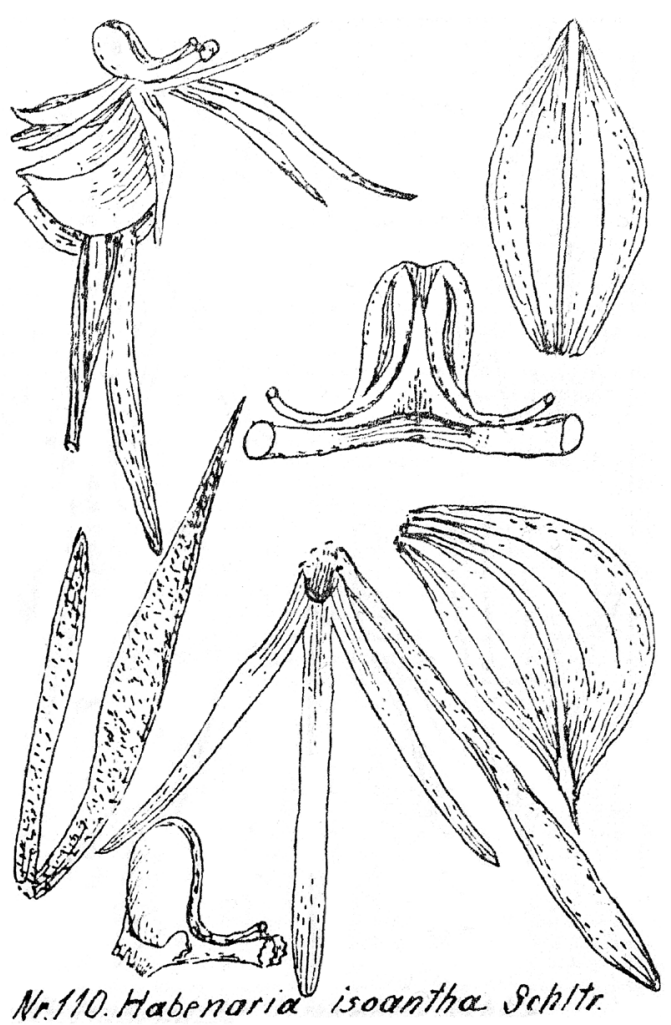

Fig. 27. Original Schlechter (1932) drawing of Habenaria isoantha Schltr.

obovate, obtuse to subacute, convex. Petals bilobed nearly to the base; anterior lobe $7 \mathrm{~mm}$ long, $1.2-1.5 \mathrm{~mm}$ wide, spreading forwards, obliquely lanceolate, acute or subacute, glabrous; posterior lobe 4-5(7) mm long, 0.2-0.5 mm wide, recurved or reflexed, linear, ciliolate.

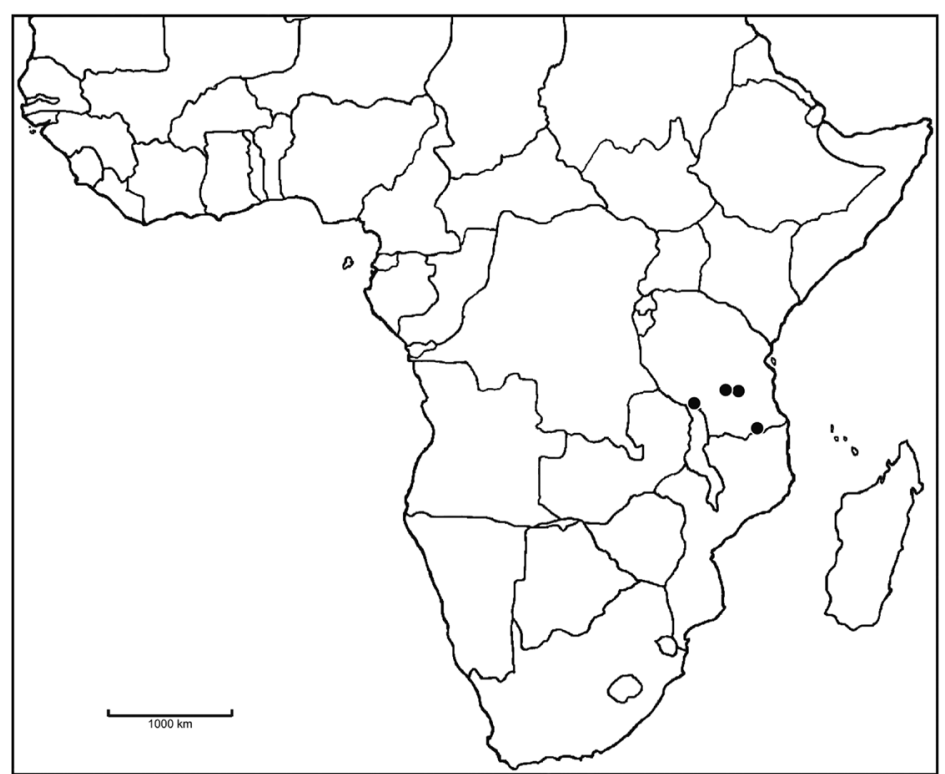

Fig. 29. Distribution of Bilabrella isoantha (Schltr.) Szlach. \& Kras-Lap.

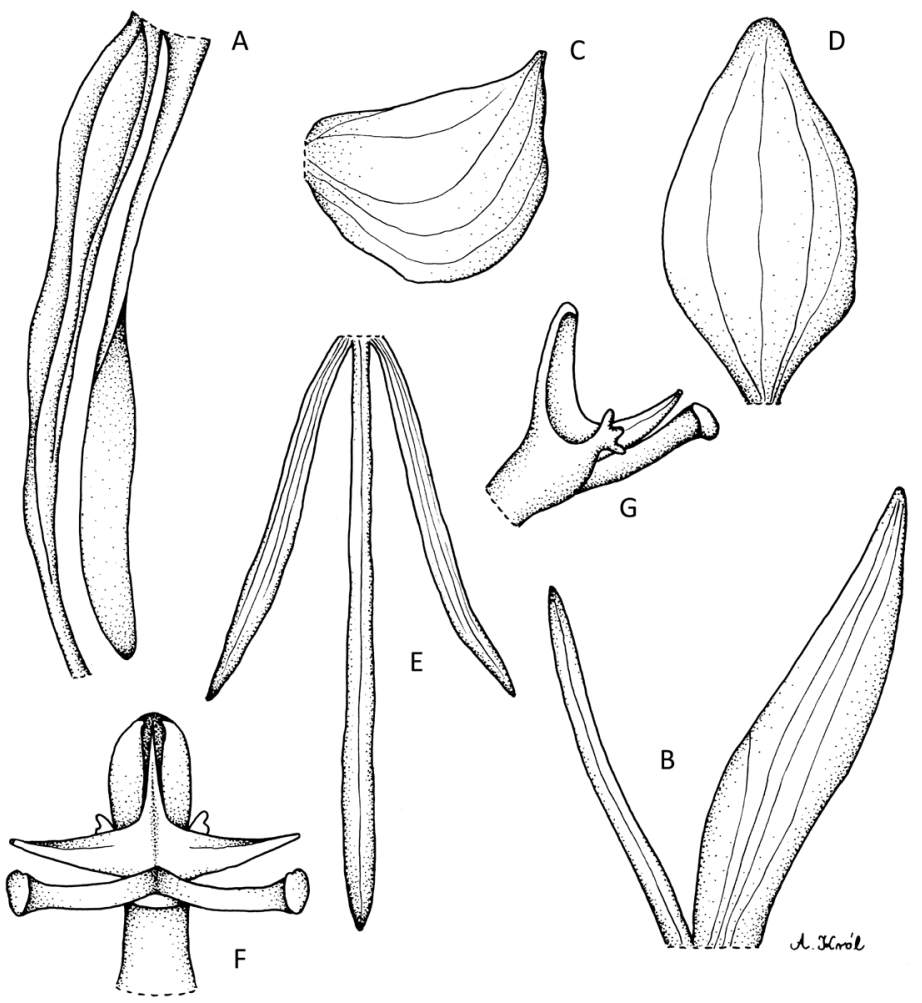

Fig. 28. Bilabrella isoantha (Schltr.) Szlach. \& Kras-Lap. Explanations: A - ovary, pedicel, spur; B - petal; $\mathrm{C}$ - lateral sepal; D - dorsal sepal; E-lip; F- gynostemium, front view; G - gynostemium, side view (drawn by A. Król from isolectotype of Habenaria isoantha Schltr. - Stolz 1148, C)

Lateral sepals 5.5-7 $\mathrm{mm}$ long, 3.5-5 $\mathrm{mm}$ wide, deflexed, obliquely semi-orbicular, the apiculum lateral. Lip deflexed, 3-lobed nearly to the base; the middle lobe 7.5-8.5 mm long, $0.6-1 \mathrm{~mm}$ wide, linear to narrowly ligulate, obtuse; lateral lobes $6-8 \mathrm{~mm}$ long, $0.7-1 \mathrm{~mm}$ wide, linear, obtuse or tapering in the apical part. Spur 13-17 mm long, straight or somewhat incurved, twisted in the middle, the apical half slightly swollen, subblunt. Gynostemium sessile. Anther $2.5 \mathrm{~mm}$ tall; connective rounded; antherophores 1.5-2 mm long, slender, upcurved. Stigmaphores 2-3 mm long, cylindrical, truncate. Rostellum middle lobe shorter than thecae. Auriculae delicate, distinctly bilobed, lobes very unequal in size (Figs 27-28).

E c o log y. On savannah, grey light soil. March-April. D i s tri bu ti on. Tanzania, alt. 1000-1575 m(Fig. 29). $\mathrm{S} p$ e c i m e $\mathrm{s}$ e $\mathrm{x}$ a $\mathrm{m}$ in ed. Tanzania. Iringa: Iringa town, $7^{\circ} 46^{\prime} \mathrm{S}, 35^{\circ} 42^{\prime} \mathrm{E}$, at pools in eroded landscape, 1575 m, 13 Mar. 1985 (fl), Pettersson, Hedren, Kibuwa 17 (K!); Mbeya: Rungwe Mt., Mamboneke, 1350 m, 1 Mar. 1912 (fl), Stolz 1148 (B!, K!); Ruvuma: Modasa Hovsing Sire, in shade - grey light soil, Brachystegia woodland, 1000 m, 6 Apr. 1986 (fl), Leyser 120 (K! \& sprit.).

N ot e s. This species appears to be closely related to Bilabrella ndiana. Both can be separated by the form of petals, especially anterior lobe. It is decurved and 
spreading, narrowly lanceolate to linear-lanceolate, acute, glabrous in $B$. ndiana, and spreading forwards, obliquely lanceolate, acute or subacute, ciliolate in B. isoantha. From similar B. sochensis and B. marxia$n a, B$. isoantha differs in having smaller flowers and differently proportioned petals.

8. Bilabrella ndiana (Rendle) Szlach. \& Kras-Lap., Richardiana 3(3): 141. 2003.

Habenaria ndiana Rendle, J. Linn. Soc., Bot. 30: 393. 1895. - Rolfe, Fl. Trop. Afr. 7: 239. 1898. - Summerhayes, FTEA, Orchid. 1: 101. 1968b. - la Croix et al., Orchid. Malawi: 76. 1991. - la Croix \& Cribb, Fl. Zambes., Orchid. 11: 100. 1995. - Stewart \& Campbell, Orchids of Kenya: 138. 1996.

Type (as holotype): Kenya, Gregory s.n. (lectotype, designated by Summerhayes [1968b: 101], BM!; isolectotype: $\mathrm{K}$ !).

Habenaria ingrata Rendle, J. Bot. 33: 279. 1895.

Type (Summerhayes 1968b, as holotype): Uganda, Scott Elliot 7198 in part (lectotype, designated by Summerhayes [1968b: 101], BM!; isolectotype, K!). Habenaria similis Schltr., Bot. Jahrb. Syst. 38: 147. 1906.

\section{Type: Tanzania, Holst $8958(\mathrm{~B}+)$.}

Tubers 2-3 cm long, 1-1.5 $\mathrm{cm}$ in diameter, ovoid or ellipsoid, tomentose. Stem 30-90 cm tall, erect, slender to rather stout, leafy throughout its length. Leaves 4-16, $8-20 \mathrm{~cm}$ long and $0.7-2 \mathrm{~cm}$ wide, the lowermost $1-3$ reduced to sheaths, sometimes whitish with green reticulate veins, the middle 5-7 suberect or spreading, linear or lanceolate-linear, acute, the upper ones adpressed to the stem, lanceolate, acuminate, often overlapping, similar to the lower bracts. Inflorescence $10-25 \mathrm{~cm}$ long, rather densely 10- to many-flowered. Flowers suberect or curving outwards, green or green and white. Flower bracts 10-30 $\mathrm{mm}$ long, lanceolate, acuminate, rather thin and scarious, usually shorter than the pedicel with ovary. Pedicel 18-20 mm long, ovary 8-12 $\mathrm{mm}$ long, straight or curved. Dorsal sepal 4-6 mm long, 1-3 mm wide, reflexed, narrowly elliptical to obovate, obtuse to subacute, convex. Petals bilobed to the base; anterior lobe 7.5-12 mm long, 1-1.5 mm wide, decurved and spreading, narrowly lanceolate to linear-lanceolate, acute, glabrous; posterior lobe 4-6.5 mm long, $0.5 \mathrm{~mm}$ wide, recurved, linear, ciliolate. Lateral sepals $6-.5 \mathrm{~mm}$ long, 3-5 mm wide, deflexed, obliquely semi-orbicular or obovate, laterally apiculate. Lip deflexed, 3-lobed to the base; the middle lobe $8.5-11.5 \mathrm{~mm}$ long, $1 \mathrm{~mm}$ wide, incurved, linear, obtuse; lateral lobes nearly as long as the middle lobe, $1 \mathrm{~mm}$ wide, lanceolate-linear, tapering towards the apex, pendant. Spur 15-25 mm long, parallel to the ovary, twisted in the middle, swollen in the apical half. Gynostemium sessile. Anther 3-4.5 mm tall;

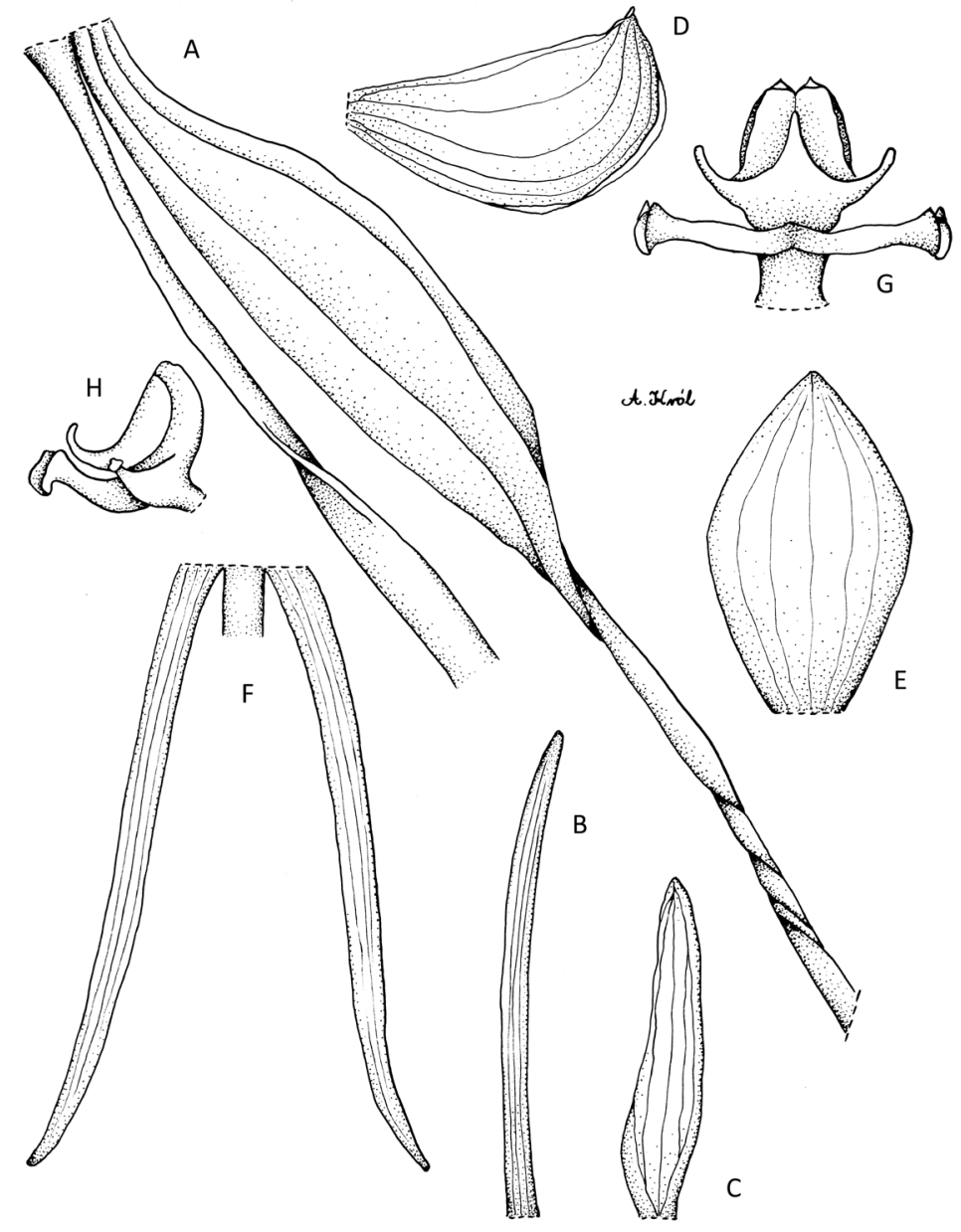

Fig. 30. Bilabrella ndiana (Rendle) Szlach. \& Kras-Lap. Explanations: A - ovary, pedicel, part of spur; B - anterior petal; $\mathrm{C}$ - posterior petal; D - lateral sepal; E - dorsal sepal; F - lip; G - gynostemium, front view; $\mathrm{H}$ - gynostemium, side view (drawn by A. Król from lectotype of Habenaria ndiana Rendle - Gregory s.n., BM)

connective truncate or slightly notched; antherophores $2 \mathrm{~mm}$ long, delicate, upcurved. Stigmaphores 2-3 mm long, rather stout, cylindrical, truncate. Rostellum middle lobe shorter than thecae. Auriculae tiny, entire, subglobose (Fig. 30).

E c ol o gy. Grassland, open bush, brown loam, rocky soil. February-December.

D i s tributi on. Uganda, Kenya, Burundi, Tanzania, Malawi, alt. 1020-2700 m (Fig. 31).

S pecimens examined. Uganda. And Congo, Ruwenzori, 2700 m, May 1894 (fl), Scott Elliot 7198 part (BM)!. Kenya. Coastal: Chyulu-North, in grassland, 1560 m, 21 May 1938 (fl), Bally 7870 (K!); 21 Apr. 1938 (fl), Bally 7871 (K!); Teita Distr., Ndi Mt., Acacia scrub broken by gneiss hills, 1320 m, 1 Apr. 1893 (fl), Gregory s.n. (BM!, K!); Rift Valley: Near Nakuru, grassland near road, 1800 m, 3 Dec. 1974 (fl), Williams 74/44 (BR!, K!); Western: Mt. Elgon, 2010 m, 12 Nov. 1930 (fl), Lugard 110 (K!); SW slopes of Mt. Elgon, Kituwaba Farm, near Kitale in short grass, 1934 (fl), Tweedie 207 (K!); S Elgon, grassland near temporary 


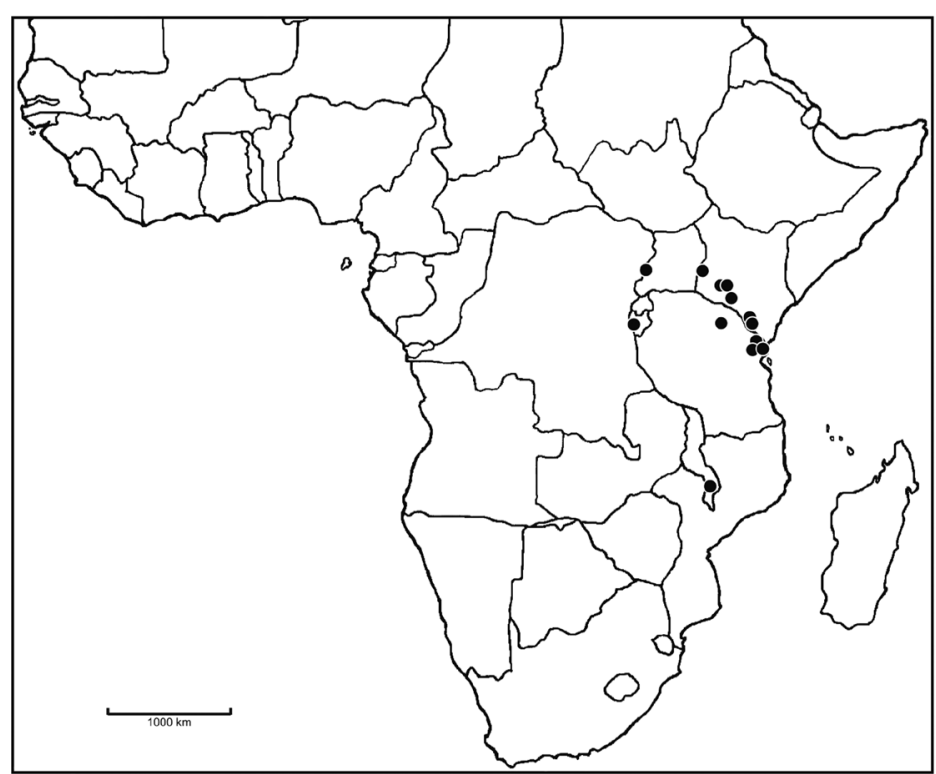

Fig. 31. Distribution of Bilabrella ndiana (Rendle) Szlach. \& Kras-Lap.

water, 2010 m, Aug. 1936 (fl), Tweedie 336 (K!); S Elgon, long grass in bush country, $2010 \mathrm{~m}$, Sep. 1937 (fl), Tweedie 404 (K!); NE Elgon, Svam sawmill, open bush, 2340 m, Sep. 1939 (fl), Tweedie 483 (K!); Kitosh Distr., Kuiwa River, in much grazed grassland in native cultivation with Combretum, Terminalia, Markhamia bushland on a dark reddish brown loam derived from gneiss, 1500 m, 25 July 1951 (fl), Greenway \& Doughty 8527 (K!); Londiani Distr., near Tonbut village, in wet vlei grassland, 2355 m, 7 July 1957 (fl), Swant G17 (K!). Burundi. Bujumbura: Bujumbura - Bugarama pl. 19., 25 Feb. 1992 (fl), Arbonnier 317 (BR!); Bugarama, 319'S, 2927'E, 10 Feb. 1991 (fl), Arbonnier 791 (BR!); Masailand, high open grassland, 18 Apr. 1943 (fl), Scott Elliot 276 (K!). Tanzania. Arusha: Ngorongoro crater, east slope, 1680 m, Apr. 1941 (fl), Bally 2430 (K!); Tanga: Lushoto Distr., Lushoto-Mombo road, 1 1 12 miles SW of Gare turn off. W Usambaras, small grassy area near large rock slabs at hilltop, 1300 m, 16 June 1953 (fl), Drummond \& Hemsley 2941 (K!); Tanga Distr., Sangarawe, in open Hyparrhenia-Themeda grassland, 1020-1080 m, 30 Aug. 1929 (fl), Greenway 1724 (K!); Tanga Distr., Sangarawe, West of Amani, grassland, mostly Hyparrhenia \& Themeda grass, 1020-1080 m, 21 July 1940 (fl), Moreau 211 (K!); Usambara Mts. (M'dola), mountain grassland, probably swampy in reins, 1050 m, 24 Aug. 1954 (fl), Faulkner 1491 (K!). Malawi. Southern: Zomba Distr., Zomba Plateau, 1700 m, 2 Apr. 1982 (fl), Burgess 15 (BR!); Zomba Distr., Zomba, A “up-road", on seepage slope among rocks, 1350 m, 6 Mar. 1983 (fl), la Croix 467 (K!).

N o t e s. According to Rendle (1895) this species approaches the Malagasy Habenaria incarnata, but has shorter stigmatic processes which do not exceed the antherophores; the shape of the sepals is also different. It is also near the Ethiopian H. humilior, but is a taller plant, and has narrower lateral petals; in the latter point it also differs from the nearly allied H. pedicellaris Rchb. f., and further in the length of the bracts (Rendle 1895).

In our opinion Bilabrella ndiana appears to be similar to $B$. isoantha and both can be distinguished by the petals. They are bilobed to the base in both, but anterior lobe of $B$. ndiana is narrowly lanceolate to linear-lanceolate, acute, glabrous and posterior lobe - linear, ciliolate. Anterior petal lobe of $B$. isoantha is obliquely lanceolate, acute or subacute, ciliolate and posterior lobe is linear, ciliolate.

Plants which appear to be abnormal forms of this species have been recorded from Elgon and other places. The petals and lip in these specimens are malformed but the gynostemium is usually normal (Summerhayes 1968b).

9. Bilabrella vollesenii (Thomas \& P. J. Cribb) Szlach. \& Kras-Lap., Richardiana 3(3): 143. 2003.

Habenaria vollesenii Thomas \& P. J. Cribb, Kew Bull. 51(1): 149. 1996. - Edwards, Demissew, Hedberg, Flora of Ethiopia and Eritrea 6: 221.1997. - Demissew, Cribb, Rasmusen, Field guide to Ethiopian Orchids: 98. 2004.

Type: Ethiopia, Gilbert, Sebsebe \& Vollesen 80620 (holotype, K!; isotype, ETH not seen).

Tubers 1.8-2.3 cm long, $0.8-1.2 \mathrm{~cm}$ wide, globose, ellipsoid or ovoid, sparsely tomentose. Stem $25-40 \mathrm{~cm}$ tall, erect, rather slender, leafy in lower half. Leaves 2-5, 5-11 cm long, 0.8-1.4 cm wide, suberect or more or less spreading, lanceolate to almost linear, acute, the largest towards the base, the upper ones appressed to the stem, much smaller, lanceolate, similar to the lower bracts. Inflorescence 12-14 cm long, rather laxly 20-35-flowered. Flowers spreading, green or greenish-white. Floral bracts 10-15 mm long, narrowly-lanceolate, acuminate, usually somewhat shorter than the pedicel with ovary. Pedicel with ovary 10-13 mm long, straight or more or less curved. Dorsal sepal 3.1-3.9 mm long, 1.7-2.2 mm wide, reflexed, elliptic, obtuse to acute, convex. Petals bilobed nearly to the base; anterior lobe 7.5-8.7 mm long, $0.2-0.5 \mathrm{~mm}$ wide, linear-subulate, subobtuse to subacute, ciliate; posterior lobe $3.5-4 \mathrm{~mm}$ long, $0.5 \mathrm{~mm}$ wide, erect, linear, subobtuse to subacute, ciliate. Lateral sepals 4-5.2 mm long, 2.3-3 mm wide, deflexed, obliquely oblong-obovate, subacute. Lip 3-lobed from an undivided base less than $1 \mathrm{~mm}$ long; the middle lobe 5.5-7 mm long, 0.6-1.2 mm wide, linear, rounded to obtuse; lateral lobes 6-8 mm long, $0.2-0.5 \mathrm{~mm}$ wide, lanceolate-linear, acute. Spur 10-14 mm long, curving away from ovary to pendent, filiform, slightly swollen in the apical half, twisted just above the apex. 
Gynostemium sessile. Anther $1.5 \mathrm{~mm}$ tall; connective rounded, shortly apiculate; antherophores $1 \mathrm{~mm}$ long, slender. Stigmaphores $2 \mathrm{~mm}$ long, cylindrical. Rostellum middle lobe shorter than thecae. Auriculae entire, slender, oblong-ellipsoid (Fig. 32).

E c o log y. Low woodland high bushland on side of hill with Acacia and Lannea rivae, open grassland and area with another plants, grey sandy soil. May-June.

Distribution. Ethiopia, alt. 1200-1320 m (Fig. 33).

Specimens examined. Ethiopia. Bale: $11 \mathrm{~km}$ from Bidre on road to Dello Mena (Maslo), Basement complex slope with open woodland with Acacia, Boswellia neglecta, Commiphora and Kirkia, open areas dominated by Erythrochlamys cufodontii, in shade and in open, 5'59'N, 39³4'E, 1320 m, 2 June 1988 (fl), Gilbert \& Sebsebe 8602 (K!); Sidamo: $3 \mathrm{~km}$ north of Bittata on Waddera - Negele road, 8 June 1968 (fl), Gilbert, Sebsebe \& Vollesen 80620 (K!); 10 km NW of Moyale, Acacia-Commiphora - bushland on grey sandy soil, 3'38'N, 3901'E, 1200 m, 22 May 1986 (fl), Mesfin \& Vollesen 4139 (K!).

Notes. According to Thomas \& Cribb (1996) it differs from Habenaria humilior in having non-reclinate locules, anterior lobes of petals not wider than posterior ones, lateral lobes of lip longer than middle lobe, shorter spur, shorter stigmaphores and antherophores. In our opinion this species may be confused with both Bilabrella compta and B. marxiana, which have spur likewise twisted near the apex. Posterior petal lobe of $B$. vollesenii is ciliate in contrast to both aforementioned species where it is glabrous.

10. Bilabrella compta (Summerh.) Szlach. \& Kras.Lap., Richardiana 3(3): 139. 2003. - Szlachetko et al., Orchid. of West-Central Africa 1: 223. 2010.

Habenaria compta Summerh., Kew Bull. 16: 276. 1962. - Williamson, Orchid. S. Centr. Afr.: 54. 1977. - la Croix \& Cribb, Fl. Zambes., Orchid. 11: 90. 1995.

Type: Zambia, Richards 5867 (holotype, K!; isotype, $\mathrm{K} !$ - spirit.).

Tubers 2-2.5 cm long, $1 \mathrm{~cm}$ in diameter, ellipsoid. Plants $37-45 \mathrm{~cm}$ tall, erect, slender, glabrous. Leaves $7-9,8-11.5 \mathrm{~cm}$ long, $0.3-0.6 \mathrm{~cm}$ wide, linear to linearlanceolate, acute to acuminate, erect to suberect, decreasing in size up the stem. Inflorescence $7-13 \mathrm{~cm}$ long, laxly or densely several- to many-flowered. Flowers pale green. Floral bracts $15-20 \mathrm{~mm}$ long, ovate-lanceolate, acuminate, reticulate. Pedicel and ovary 18-20 mm long, slender. Dorsal sepal 4.5-5 $\mathrm{mm}$ long, 1-1.5 mm wide, elliptic to oblong obovate, obtuse, reflexed, concave. Petals bilobed nearly to the base, glabrous; anterior lobe 6.9-9.5 mm long, 0.3-0.6 $\mathrm{mm}$ wide, filiform, acute; posterior lobe $3.9-6 \mathrm{~mm}$

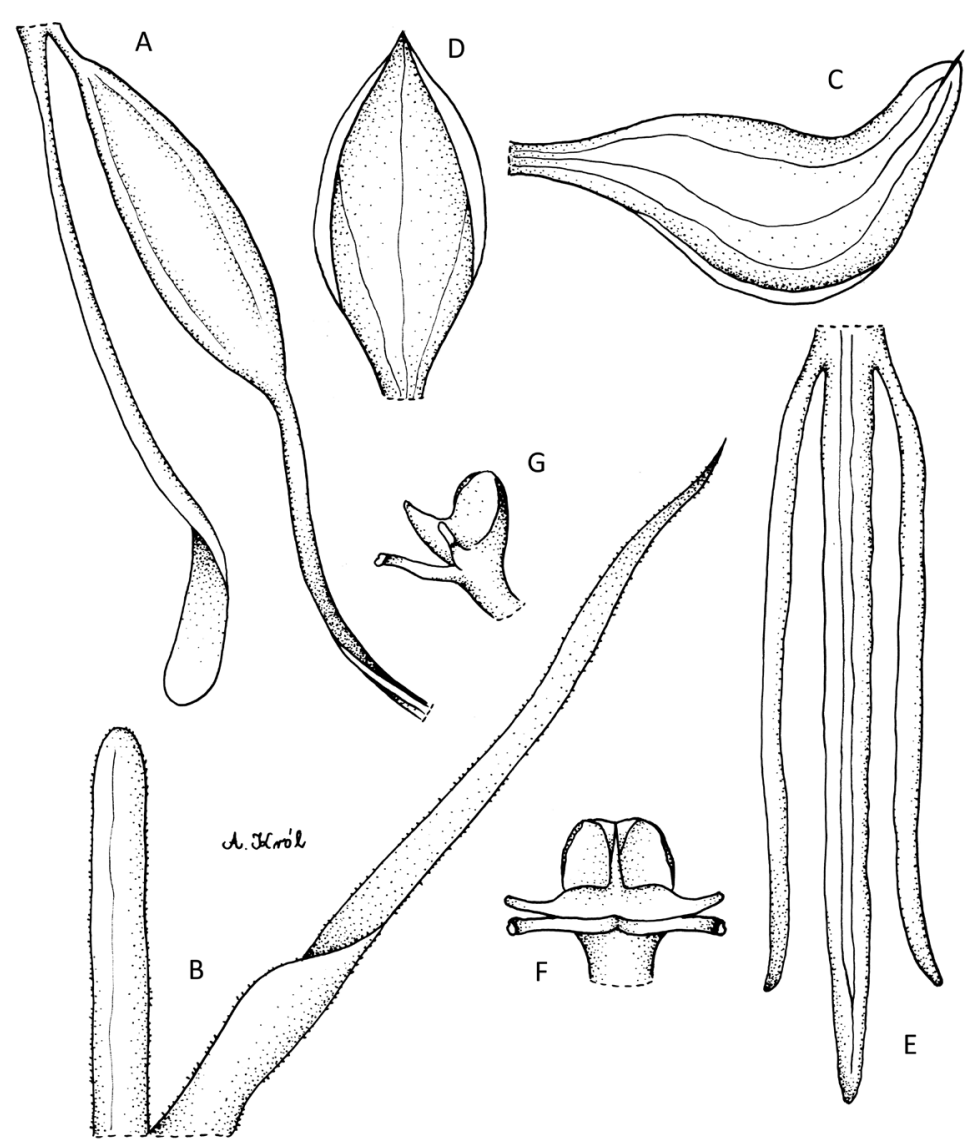

Fig. 32. Bilabrella vollesenii (Thomas \& P.J. Cribb) Szlach. \& Kras-Lap.

Explanations: A - ovary, pedicel, spur; B - petal; C - lateral sepal; D - dorsal sepal; E - lip; F - gynostemium, front view; $\mathrm{G}$ - gynostemium, side view (drawn by A. Król from holotype of Habenaria vollesenii Thomas \& P.J. Cribb - Gilbert, Sebsebe \& Vollesen 80620, K)

long, 0.2-0.7 $\mathrm{mm}$ wide, filiform, acute. Lateral sepals 5.8-6.7 mm long, 3-3.5 mm wide, obliquely oblongobovate, concave, laterally placed apiculus short. Lip

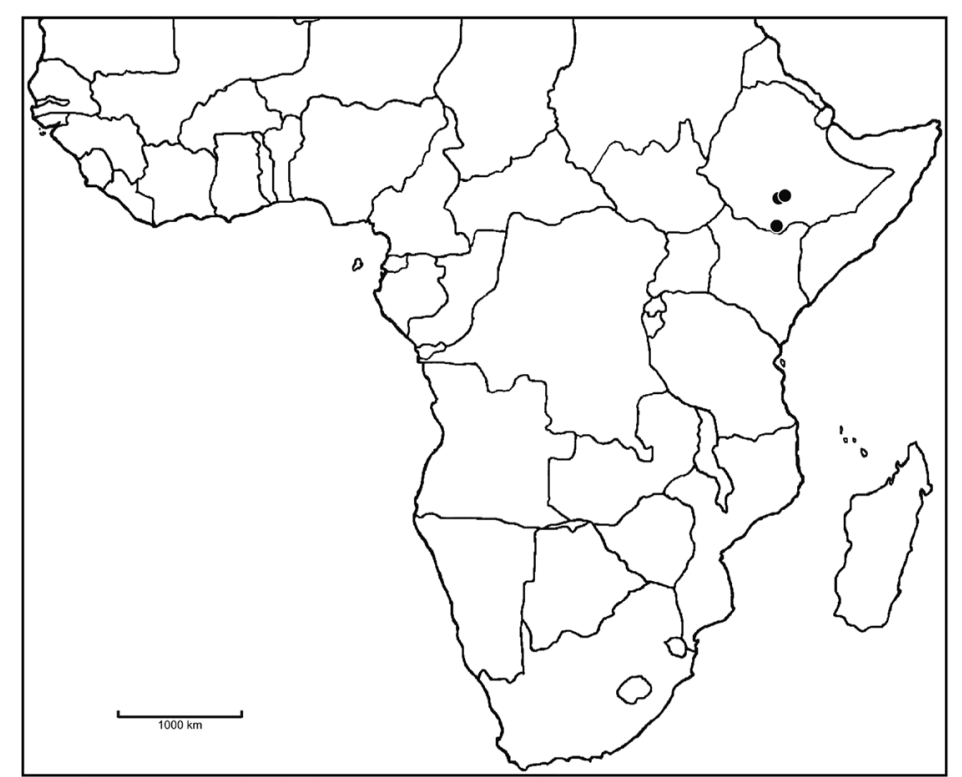

Fig. 33. Distribution of Bilabrella vollesenii (Thomas \& P..J.Cribb) Szlach. \& Kras-Lap. 


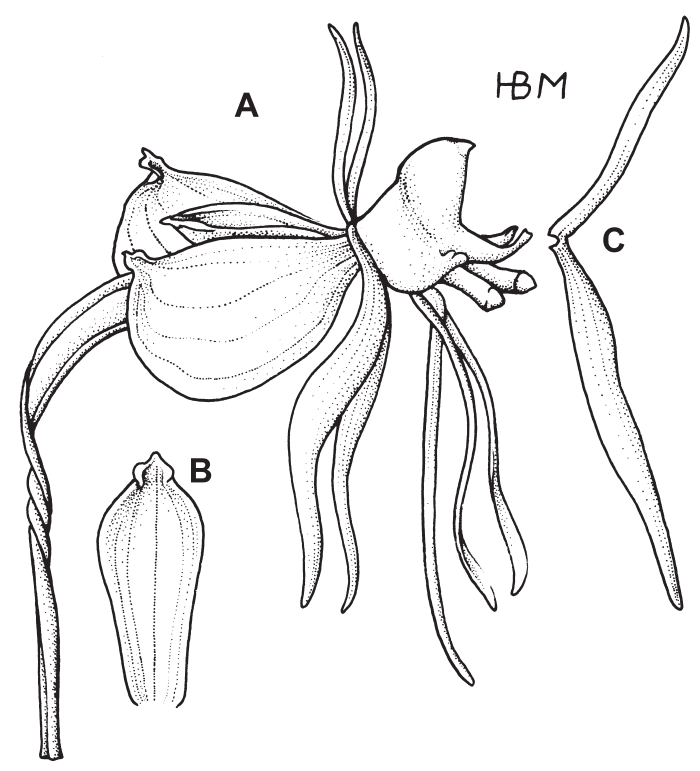

Fig. 34. Bilabrella compta (Summerh.) Szlach. \& Kras-Lap. Explanations: A - flower; B - dorsal sepal; C - petal (drawn by H. B. Margońska from holotype of Bilabrella compta Summerh. - Richards $5867, \mathrm{~K})$

3-lobed almost to the base; the middle lobe $9-11 \mathrm{~mm}$ long, $0.5 \mathrm{~mm}$ wide, linear-filiform, obtuse; lateral lobes 7-9.5 mm long, $0.5 \mathrm{~mm}$ wide, filiform, acute. Spur 25$30 \mathrm{~mm}$ long, narrowly cylindrical to filiform, slightly inflated and twisted at the subobtuse apex. Gynostemium sessile. Anther 3-3.5 mm tall; connective shortly apiculate; antherophores $2 \mathrm{~mm}$ long, upcurved. Stigmaphores 2-3.3 mm long, cylindrical. Rostellum middle lobe as long as thecae. Auriculae very tiny, shallowly bifid (Fig. 34). E c o lo g y. Terrestrial on marsh close to river banks. Flowering in August-November.

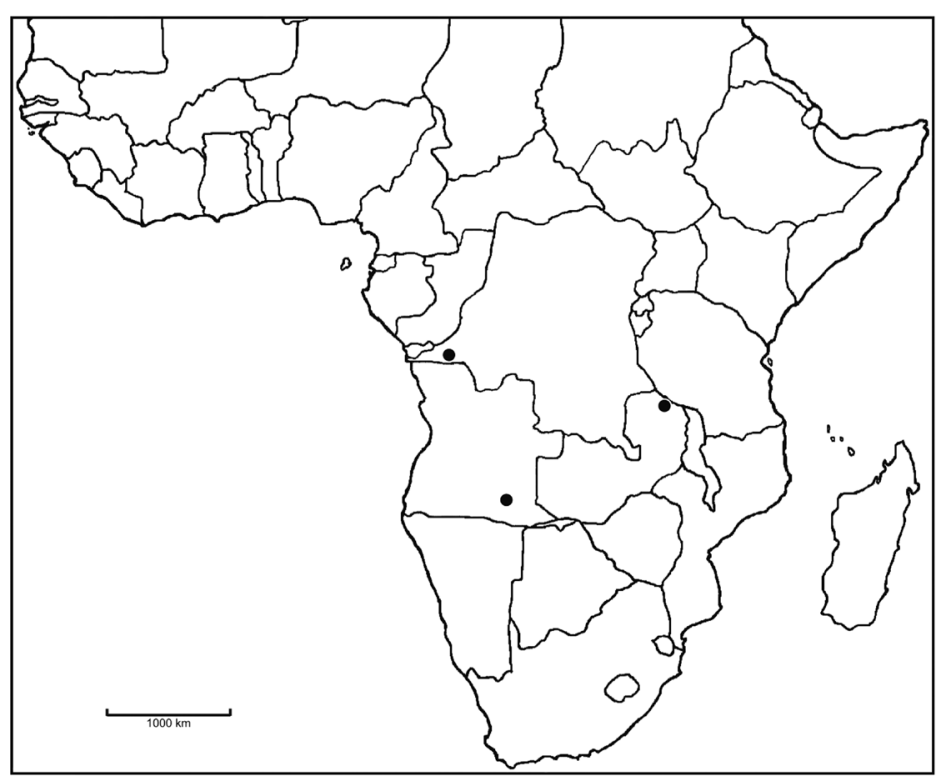

Fig. 35. Distribution of Bilabrella compta (Summerh.) Szlach. \& Kras-Lap.
Di stribution. Democratic Republic of the Congo (Zaire), Angola, Zambia, alt. 1100-1500 m (Fig. 35).

Specimens examined. Democratic Republic of the Congo (Zaire). Bas-Congo: Kimbangu, 1100 m, 19 Nov. 1899 (fl), Baum 425 (K!). Angola. KuandoKubango: Cubango, 1976 (fl), Schelpe s.n. Zambia. Northern: Mbala Distr. Kawimbe, marsh by Lumi River, 1500 m, 17 Aug. 1956 (fl), Richards 5867 (K! \& spirit.).

N o t e s. Bilabrella compta is similar to B. marxiana and can be easily confused with this species. It seems that both can be distinguished based on both some vegetative characters (e.g. leaves $8-11.5 \times 0.3-0.6 \mathrm{~cm}$ in B. compta vs $11-23 \times 1.2-3 \mathrm{~cm}$ in $B$. marxiana) as well as floral character, especially anterior petal lobe (filiform, glabrous in B. compta vs lanceolate-linear, papillosepuberulate in $B$. marxiana). It can be easily separated from $B$. vollesenii by glabrous posterior petal lobe (vs ciliate in B. vollesenii). Summerhayes (1962) describing Habenaria compta included to it Baum 425 collection from Angola, which is the type number of H. kubangensis Schltr. Summerhayes stated that original description and published reproduction of Schlechter's drawing of the latter show certain discrepancies with isotype specimen kept at COI. We have examined in details Baum 425 plants deposited at BM, HBG, K and W and compared them with the type specimen of $H$. compta and see no reason for combining them together. Both species differs clearly in the length of stigmaphores and antherophores, presence of the lip claw and width of anterior petals lobe. In results, occurrence of $B$. compta in Angola might be questionable. On the other hand, it cannot be excluded that Baum 425 collection consists of mixed taxa.

11. Bilabrella marxiana (Schltr.) Szlach. \& Kras, Richardiana 9(4): 159. 2009.

Habenaria marxiana Schltr., Bot. Jahrb. Syst. 53: 517. 1915. - Schlechter, Repert. Sp. Nov Regni Veg. 68: tab. 30. 1932.

Type (hoc loco selectus): Tanganyika, Stolz 1149 (lectotype, B!; isolectotypes, K!, W-R!, S not seen).

Stem $60-83 \mathrm{~cm}$ tall, erect, leafy, glabrous. Leaves $10-13,11-23 \mathrm{~cm}$ long, $1.2-3 \mathrm{~cm}$ wide, narrowly lanceolate, acute or acuminate, gradually transforms into bracts upwards. Inflorescence 15-30 cm long, densely multiflowered. Flowers whitish with greenish apices of perianth parts. Floral bracts 8-22 mm long, ovatelanceolate, acuminate, glabrous. Pedicellate ovary to $25 \mathrm{~mm}$ long, slender, glabrous. Dorsal sepal 5-6 mm long, 1.8-3 mm wide, elliptic to oblong elliptic, obtuse to acuminate, concave, recurved. Petals bilobed to the base; anterior lobe 7-13 $\mathrm{mm}$ long, up to $1.3 \mathrm{~mm}$ wide, narrowly lanceolate-linear, falcate, acuminate to 


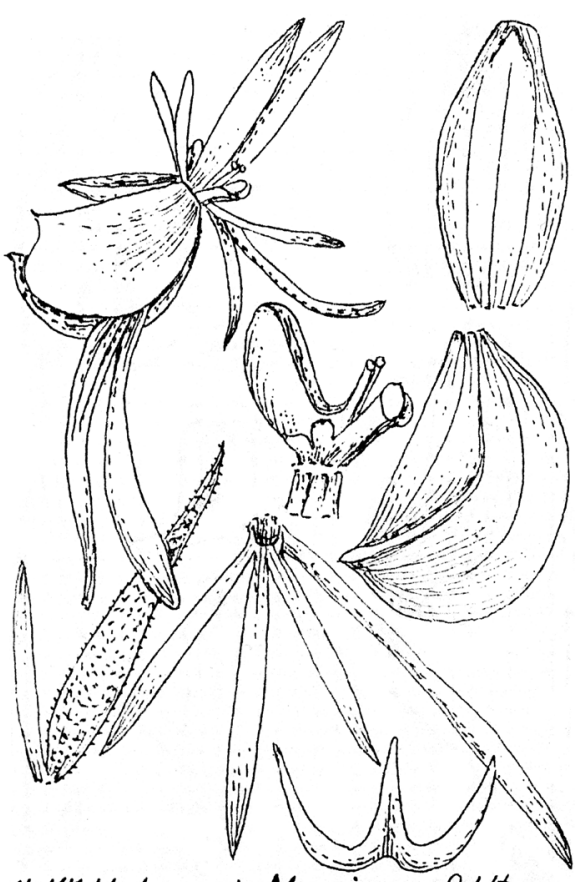

Nr.117. Habenaria Marxiana Schitr.

Fig. 36. Original Schlechter (1932) drawing of Habenaria marxiana Schltr.

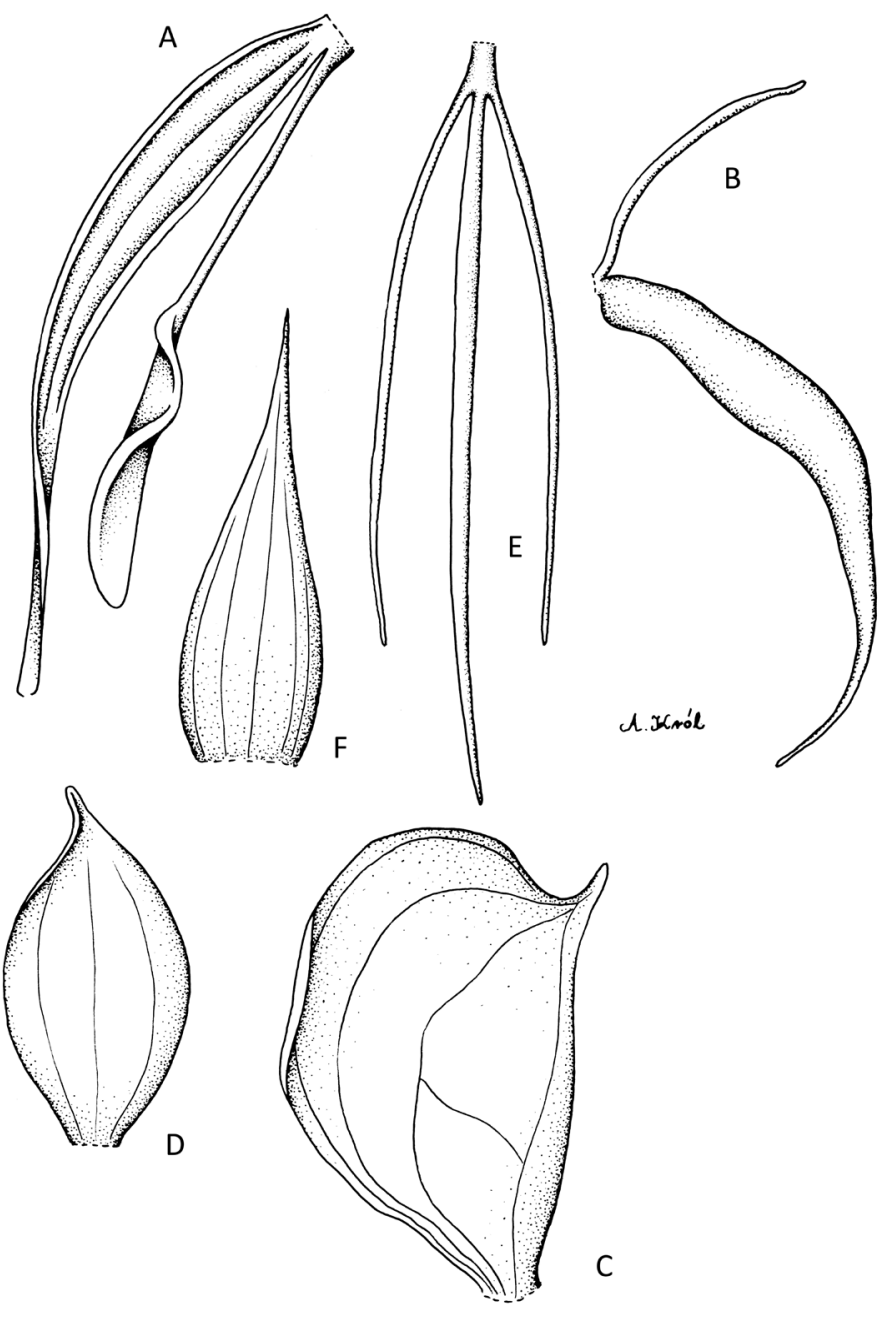

Fig. 37. Bilabrella marxiana (Schltr.) Szlach. \& Kras

Explanations: A - ovary, pedicel, spur; B - petal; C - lateral sepal; D - dorsal sepal; E - lip; F - floral bract (drawn by A. Król from isolectotype of Habenaria marxiana Schltr. - Stolz 1149, K)

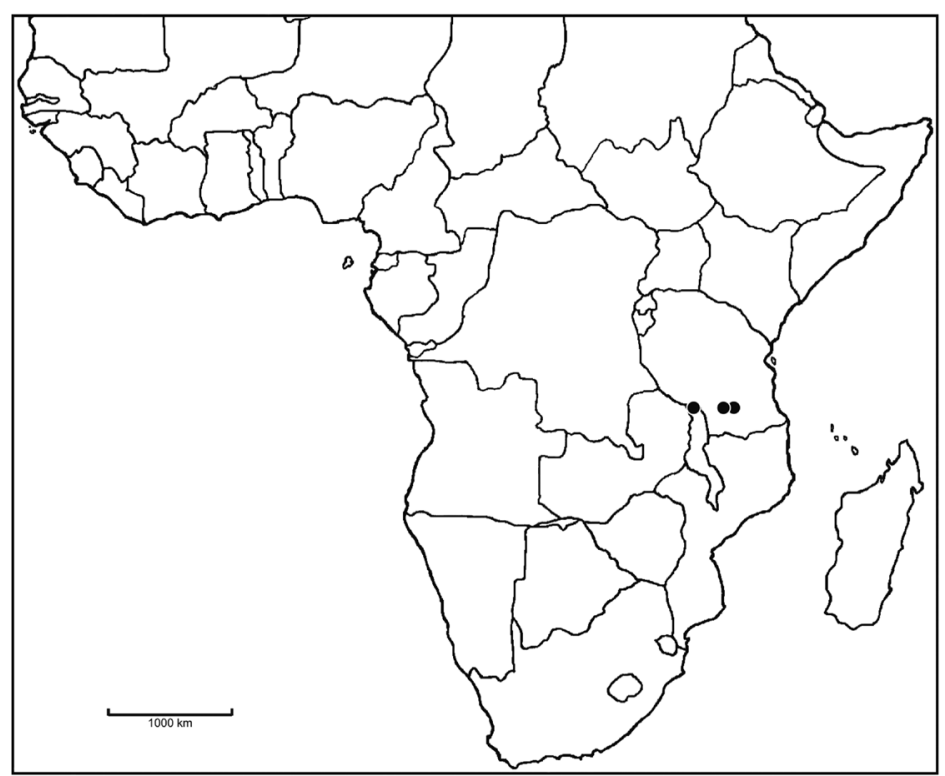

Fig. 38. Distribution of Bilabrella marxiana (Schltr.) Szlach. \& Kras

caudate, papillose-puberulate; posterior lobe $5-5.5 \mathrm{~mm}$ long, 0.2-0.4 $\mathrm{mm}$ wide, filiform, obtuse, glabrous. Lateral sepals 7-8.5 mm long, 5-6 mm wide, obliquely ovate, laterally apiculate, deflexed. Lip 3-lobed above the basal $1 \mathrm{~mm}$, lobes pendent; the middle lobe 10 $14.5 \mathrm{~mm}$ long, $0.3-0.8 \mathrm{~mm}$ wide, linear, acuminate; lateral lobes 8-10 $\mathrm{mm}$ long, $0.3-0.8 \mathrm{~mm}$ wide, linear to filiform, obtuse to acuminate. Spur 16-17.5 mm long, gently pendent, more or less twisted prominently below the middle and distinctly swollen, blunt. Gynostemium sessile. Anther $4 \mathrm{~mm}$ tall; connective rounded; antherophores $2 \mathrm{~mm}$ long, slender, upcurved. Stigmaphores $2 \mathrm{~mm}$ long, slender, clavate. Rostellum middle lobe as long as thecae. Auriculae entire, ellipsoid-ovoid (Figs 36-37).

E c o logy. Upland grassland. March-May. D istribution. Tanzania, alt. 1000-1350 m (Fig. 38).

$\mathrm{S} p$ e c i m e $\mathrm{s}$ e $\mathrm{x}$ a $\mathrm{m}$ in ed. Tanzania. Iringa: The land over Ruhudje, the Lupembe country, $\mathrm{N}$ of river, May 1931 (fl), Schlieben 797 (BM!); The land over Ruhudje, the Lupembe country, N of river, May 1931 (fl), Schlieben 884 (BM!); Mbeya: Near the Rungwa Mountain, near Wamboneke village, $1350 \mathrm{~m}, 1$ Mar. 1912 (fl), Stolz 1149 (B!, K!, W-R!); Morogoro: Mahenge, frequent in grass savanne, 1000 m, 30 Apr. 1931 (fl), Schlieben 2159 (BM!, MA!).

N o t e s . According to Schlechter (1915) his Habenaria marxiana is similar to $H$. sochensis, but has shorter spur and smaller perianth parts (Schlechter 1932). We can add here a differences in the petal lobes morphology. Bilabrella marxiana is somewhat similar to B. williamsonii, but has completely glabrous lip. Species very similar to $B$. marxiana is $B$. compta. They can be 


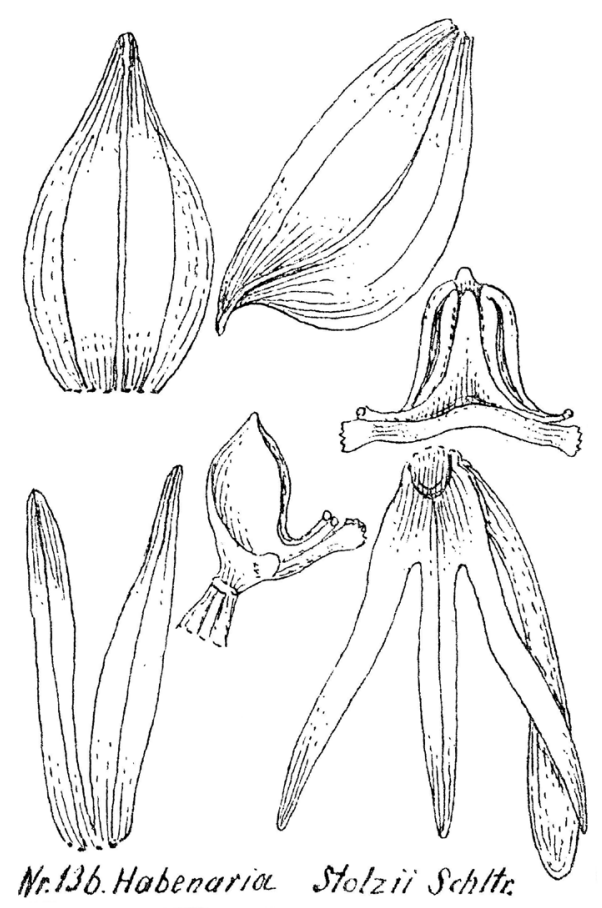

Fig. 39. Original Schlechter (1932) drawing of Habenaria stolzii Schltr.

separated by leaves $(8-11.5 \times 0.3-0.6 \mathrm{~cm}$ in $B$. compta vs $11-23 \times 1.2-3 \mathrm{~cm}$ in B. marxiana) and anterior petal lobe (filiform, glabrous in B. compta vs lanceolatelinear, papillose-puberulate in B. marxiana).

12. Bilabrella stolzii (Schltr.) Szlach. \& Kras-Lap., Richardiana 3(3): 142. 2003.

Habenaria stolzii Schltr., Bot. Jahrb. Syst. 38: 147. 1906, non Kraenzl., 1912. - Schlechter, Bot. Jahrb.

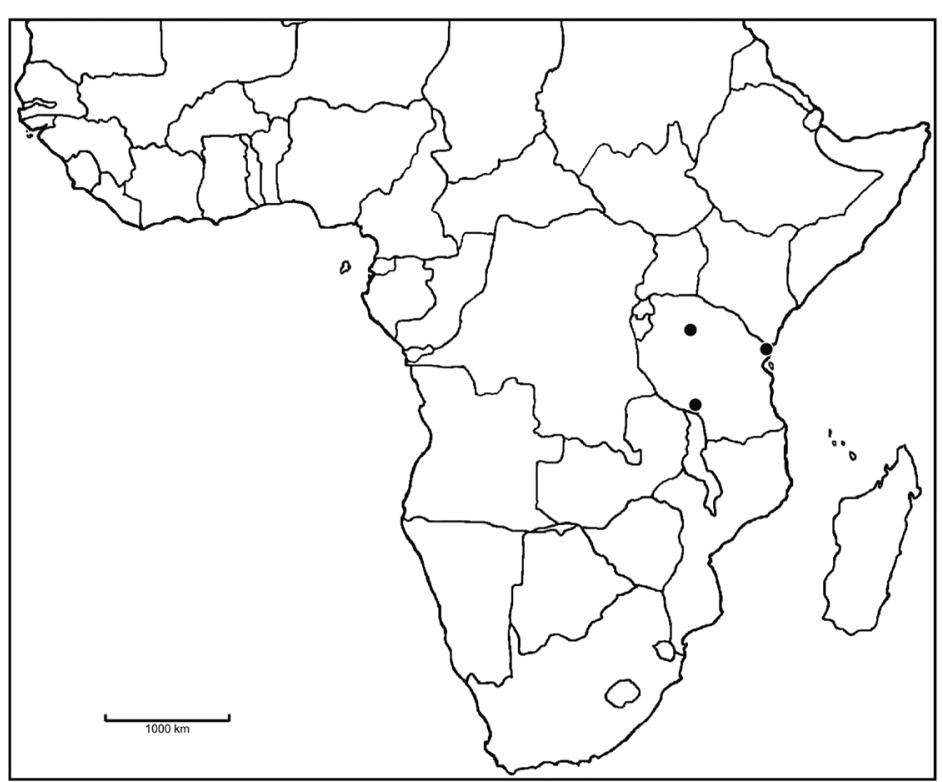

Fig. 41. Distribution of Bilabrella stolzii (Schltr.) Szlach. \& KrasLap.

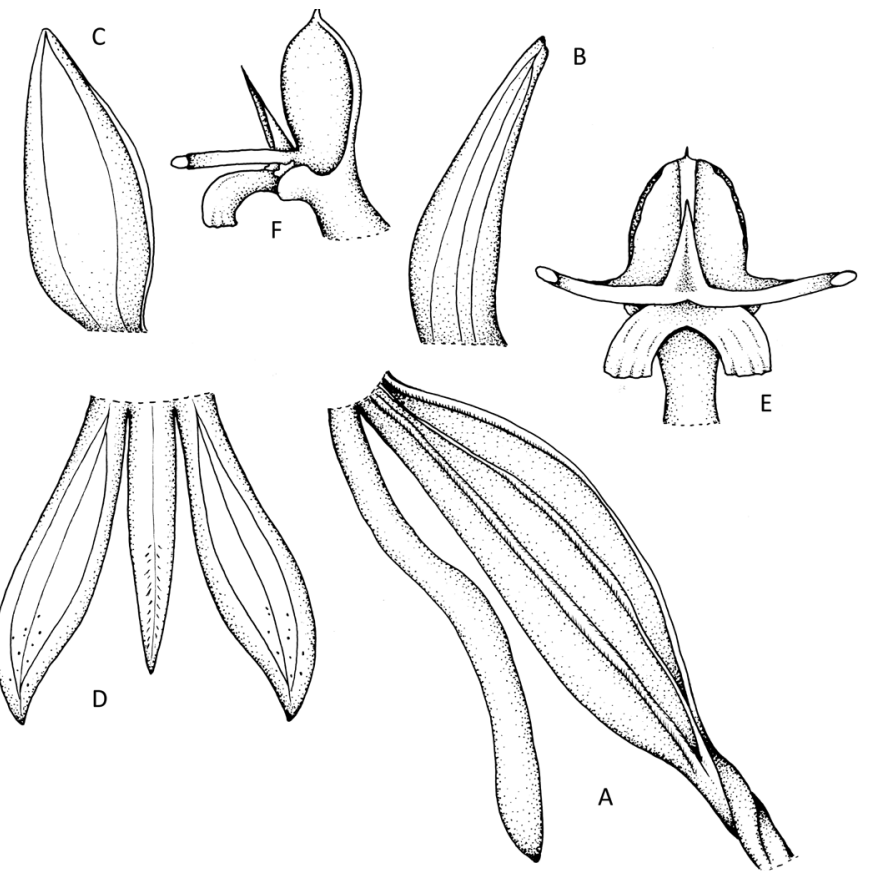

Fig. 40. Bilabrella stolzii (Schltr.) Szlach. \& Kras-Lap. Explanations: A - ovary, pedicel, spur; B - part of petal; C - dorsal sepal; D - lip; E - gynostemium, front view; F - gynostemium, side view (drawn by A. Król from Stolz 140, HBG)

Syst. 53: 518. 1915. - Schlechter, Repert. Sp. Nov Regni Veg. 68: tab. 34. 1932. - Summerhayes, FTEA, Orchid. 1: 99. 1968b.

Type : Tanzania, Stolz $2(\mathrm{~B}+)$.

Tubers $1.5-3.5 \mathrm{~cm}$ long, $0.75-1.5 \mathrm{~cm}$ in diameter, ovoid or ellipsoid, densely tomentose. Stem $20-85 \mathrm{~cm}$ tall, erect, slender to stout, leafy throughout its length. Leaves $13-17,10-14 \mathrm{~cm}$ long, $0.5-1.7 \mathrm{~cm}$ wide, the lowermost 2-3 reduced to sheaths, whitish with green reticulate veins, the middle $8-10$ erect or suberect, linear or very narrowly lanceolate, long tapering at the apex, the largest the upper leaves adpressed to the stem, lanceolate, very acute, similar to the bracts. Inflorescence 5-24 cm long, rather densely 8- to many-flowered. Flowers curved outwards, green. Floral bracts $10-25 \mathrm{~mm}$ long, thin in texture, lanceolate, acuminate, mostly longer than the pedicel with ovary. Pedicel with ovary 15-17 mm long, slender, curved. Dorsal sepal $7 \mathrm{~mm}$ long and 3-5 mm wide, reflexed, narrowly elliptical, obtuse. Petals bilobed nearly to the base, glabrous; anterior lobe 7-10 mm long, 1-2 mm wide, oblong-lanceolate, obtuse; posterior lobe $6-8 \mathrm{~mm}$ long, $\pm 1 \mathrm{~mm}$ wide, recurved, widely linear, obtuse. Lateral sepals $8 \mathrm{~mm}$ long, 3.5$5.5 \mathrm{~mm}$ wide, deflexed, obliquely semi-orbicular with the laterally placed apiculus. Lip decurved, 3-lobed from a basal undivided part up to $2 \mathrm{~mm}$ long; the middle lobe 6.5-10 mm long, $1 \mathrm{~mm}$ wide, linear-lanceolate, acute; lateral lobes $8-11 \mathrm{~mm}$ long, $2 \mathrm{~mm}$ wide, obliquely oblanceolate-ligulate, tapering towards the apex, acute. 
Spur 10-12.5 $\mathrm{mm}$ long, $1 \mathrm{~mm}$ in diameter, parallel to the ovary, narrowly cylindrical, apical half slightly swollen or not at all. Gynostemium sessile. Anther 3-4 mm tall; connective short apiculate; antherophores 1-2 mm long, slender, upcurved. Stigmaphores $3 \mathrm{~mm}$ long, cylindrical-clavate, truncate. Rostellum middle lobe rather massive, blunt, slightly shorter than thecae. Auriculae entire, small, subglobose (Figs 39-40).

E c o logy. In mountain meadow. May.

D is tribution. Tanzania, alt. 2020-2100 m (Fig. 41).

Specimens examined. Tanzania. Mbeya: Kyimbila Distr., North of lake Nyasa; from Ukinga, Bulongwa, in mountain meadow, 2100 m, 1915 (fl), Stolz K59 (K!); Mwanza: Near Isaka in Bundali, 2020 m, May 1900 (fl), Stolz 2 (B+); Near Isaka in Bundali, 2020 m, May 1900 (fl), Stolz 140 p.p. (HBG!); Near Njamjamica, Leedal 450 (K!).

N o t e s. This is the only species of the section Antenniferae with lip lateral lobes being more or less longer than the middle one. It is somewhat similar to Bilabrella tisseranti from West Africa, but differs from the latter by petals and gynostemium morphology. Petals of $B$. stolzii are glabrous, anterior lobe is 7-10 $\times 1-2 \mathrm{~mm}$, oblonglanceolate, obtuse and posterior lobe $6-8 \times 1 \mathrm{~mm}$ (vs petals ciliolate in $B$. tisseranti, with anterior lobe $3-4 \times$ $1.2 \mathrm{~mm}$, oblong-oblanceolate, acute and posterior lobe $3-4 \times 0.3 \mathrm{~mm})$. The stigmaphores and antherophores of $B$. stolzii is slender, upcurved, cylindrical-clavate, whereas in $B$. tisseranti they are exceptionally massive and short.

There are two different species bearing the name Habenaria stolzii - described by Schlechter (1906) and Kraenzlin (1912). We were not able to locate any type material of the Schlechter's species and based our opinion on diagnosis and Schlechter's drawings. No doubt, it represents the genus Bilabrella. Detailed examination of the type material of Kraenzlin's Habenaria stolzii kept at HBG led us to the conclusion that part of it should be classified in the genus Platycoryne, but other part is what we determined as H. stolzii sensu Schlechter. According to Summerhayes (1968b) H. diselloides Schltr. consists of abnormal specimens in which the petals are reduced and \pm antheriferous while the liplobes and spur are reduced. Consequently, he reduced it to the synonym of $H$. stolzii. We cannot accept this point of view and recognize both species as separate. Both of them differ one from another in the petals and lip lobes proportions.

13. Bilabrella tisseranti (Szlach. \& Olsz.) Szlach. \& Kras-Lap., Richardiana 3(3): 142. 2003. - Szlachetko et al., Orchid. of West-Central Africa 1: 219. 2010. Habenaria tisseranti Szlach. \& Olsz., Fl. Cam., Orchid. 34(1): 174. 1998.
Type: Central African Republic, Tisserant 2774 (holotype, P!).

Tubers single, up to $1.5 \mathrm{~cm}$ long and $0.5 \mathrm{~cm}$ in diameter, oval. Stem $60 \mathrm{~cm}$ tall, rather erect, delicate, glabrous. Leaves 9, up to $16 \mathrm{~cm}$ long, ca. $0.8 \mathrm{~cm}$ wide, grass-like, acute, erect, decreasing in size up the stem. Inflorescence $5.5 \mathrm{~cm}$ long, ca. 12-flowered, rather dense. Floral bracts $10 \mathrm{~mm}$ long, glabrous. Pedicel and ovary $15 \mathrm{~mm}$ long, glabrous. Dorsal sepal 3-4 mm long, $2 \mathrm{~mm}$ wide, oblong-obovate, with slightly bifid and thickened apiculus, cochleate, glabrous. Petals bilobed to the base, ciliolate, rather thin; anterior lobe 3-4 mm long, $1.2 \mathrm{~mm}$ wide, oblong-oblanceolate, acute; posterior lobe 3-4 mm long, $0.3 \mathrm{~mm}$ wide, linear, subobtuse. Lateral sepals $4 \mathrm{~mm}$ long, $3.2 \mathrm{~mm}$ wide, obliquely widely obovate, cochlate at the apex, with laterally placed, slightly thickened apiculus, glabrous. Lip 3-lobed above the short, undivided basal part ca. $0.7 \mathrm{~mm}$ long, thin, glabrous; the middle lobe 5-6 mm long, $0.3 \mathrm{~mm}$ wide, filiform, subacute; lateral lobes $3.5 \mathrm{~mm}$ long, $0.3 \mathrm{~mm}$ wide, filiform, slightly curved upwards. Spur $7 \mathrm{~mm}$ long, $0.7 \mathrm{~mm}$ in diameter at blunt apex, narrowly cylindrical, not swollen, parallel to the ovary. Gynostemium sessile. Anther $2 \mathrm{~mm}$ tall; connective truncate; stigmaphores equal in length to antherophores, both ca. $1.5 \mathrm{~mm}$ long, short and massive. Rostellum middle lobe

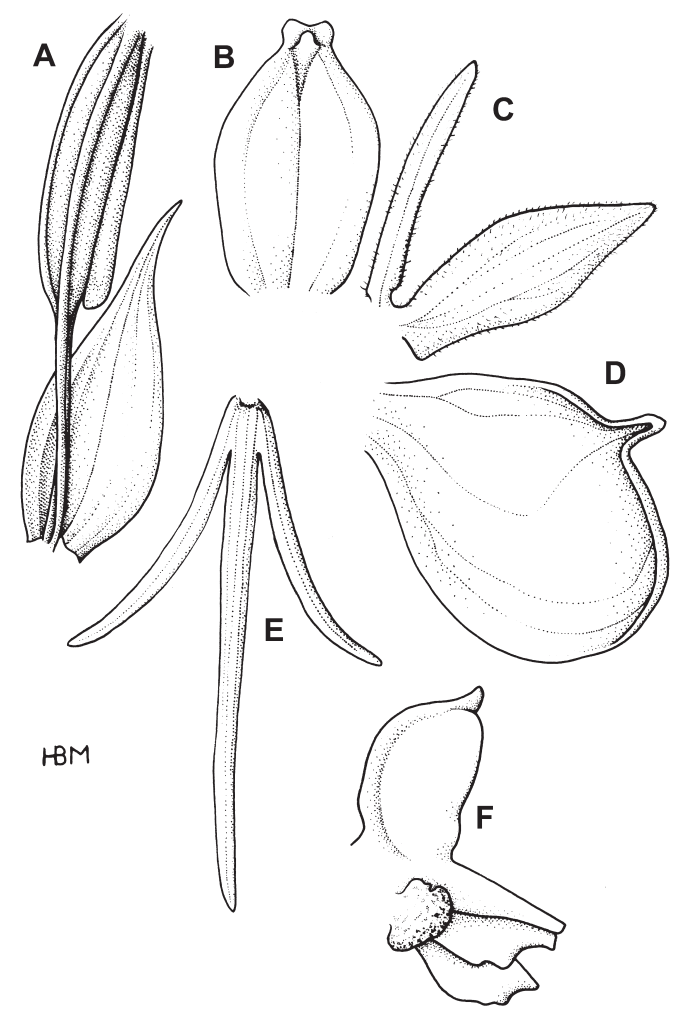

Fig. 42. Bilabrella tisseranti (Szlach. \& Olsz.) Szlach. \& Kras-Lap. Explanations: A - ovary, pedicel, spur and floral bract; B - dorsal sepal; C - petal; D - lateral sepal; E - lip; F - gynostemium, side view (drawn by H. B. Margońska from holotype of Habenaria tisseranti Szlach. \& Olsz. - Tisserant 2774, P) 


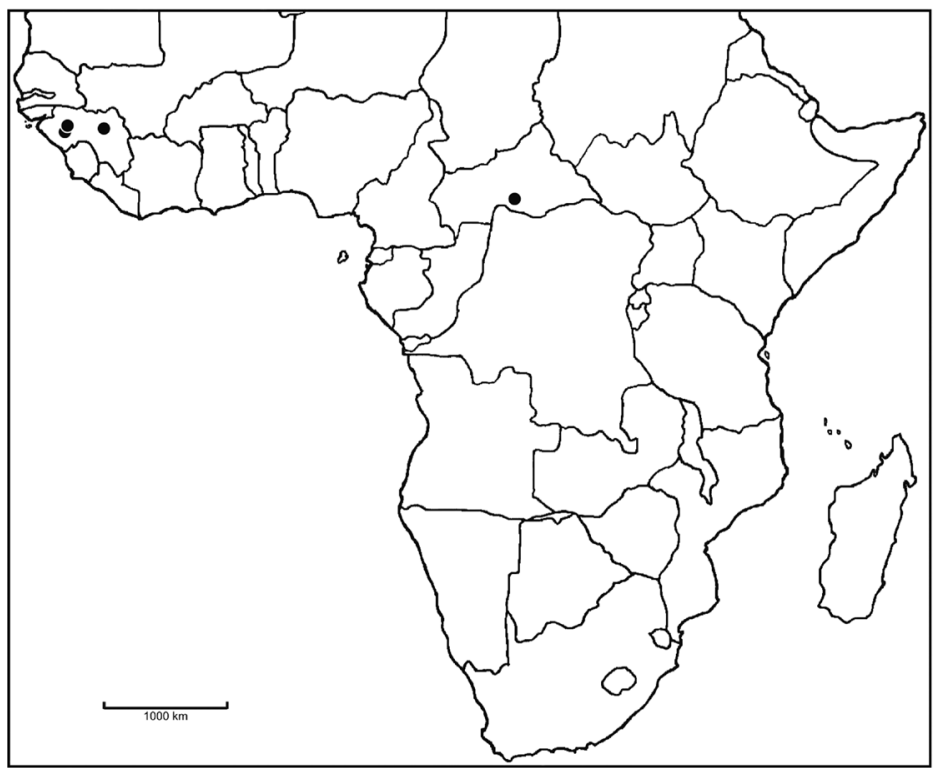

Fig. 43. Distribution of Bilabrella tisseranti (Szlach. \& Olsz.) Szlach. \& Kras-Lap.

shorter than thecae. Aucriles unlobed, orbicular, very large (Fig. 42).

E c o 1 og y. Grassland. June-July.

Distribution. Guinea, Central African Republic (Fig. 43).

S pecimens examined. Guinea. Kankan: Kouroussa, Conakry, Bambaya, July 1901 (fl), Pobeguin 759 (P!); Pita and environs, July 1909 (fl), Pobeguin 2171 (P!); Kindia: Environs de Kindia, Kinsom Plateau, July 1937 (fl), Jacques-Felix 1802 (K!, P!). Central African Republic. Lobaye: Ouaka, Pudjeyo, $60 \mathrm{~km} \mathrm{SE}$ Bambari, 30 June 1928 (fl), Tisserant 2774 (P!).

Notes. This species is similar somewhat to $B i-$ labrella stolzii from Tanzania. It has however ciliolate petals (vs glabrous), with prominently shorter and acute anterior $(3-4 \times 1.2 \mathrm{~mm}$ vs $7-10 \times 1-2 \mathrm{~mm})$ and posterior petals lobe $(3-4 \times 0.3 \mathrm{~mm}$ vs $6-8 \times 1 \mathrm{~mm})$. The gynostemium of $B$. tisseranti is exceptional in the genus by having very short and massive stigmaphores and antherophores.

14. Bilabrella woodii (Schltr.) Szlach. \& Kras, Richardiana 9(4): 159. 2009.

Habenaria woodii Schltr., Bot. Jahrb. Syst. 38: 149. 1906.

Type (hoc loco selectus): Republic of South Africa, Wylie sub Medley Wood 7601 (no. 9468 in Herb. Schltr.) $(\mathrm{B}+$; lectotype, $\mathrm{K} !-$ fragment \& drawing; isolectotypes, BOL not seen, NH not seen).

Stem 40-60 cm long, very slender to delicate, erect, leafy. Leaves $3-5$, to $15 \mathrm{~cm}$ long and $0.3 \mathrm{~cm}$ wide, linear, grass-like, acute, erect, gathered in the lower

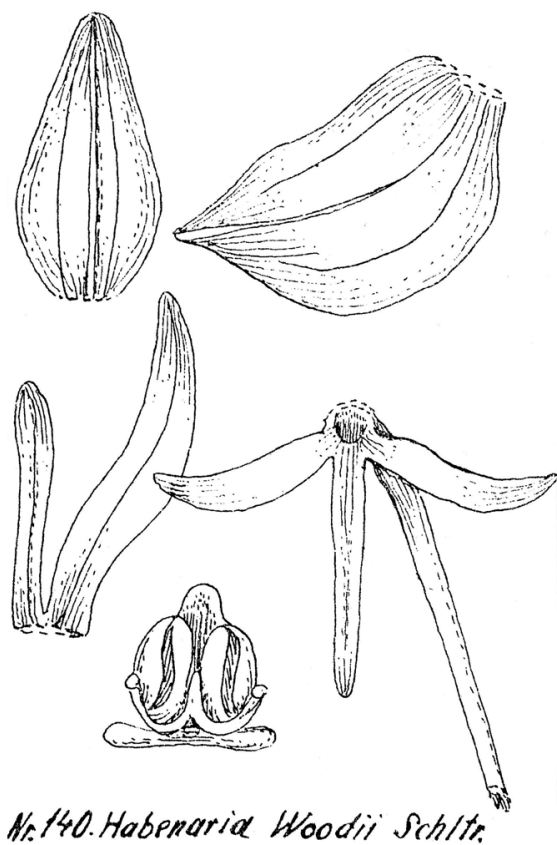

Fig. 44. Original Schlechter (1932) drawing of Habenaria woodii Schltr.

part of the stem, gradually decreasing in size upwards. Inflorescence to $8 \mathrm{~cm}$ long, 6-15-flowered, cylindrical to oblong, subdense. Floral bracts ca. $10 \mathrm{~mm}$ long, ovate-lanceolate, acuminate. Pedicellate ovary to $20 \mathrm{~mm}$ long, glabrous, distinctly longer than bracts. Dorsal sepal $3 \mathrm{~mm}$ long, $1 \mathrm{~mm}$ wide, elliptic-ovate, obtuse, concave, glabrous. Petals bilobed almost to the base, glabrous; anterior lobe $4 \mathrm{~mm}$ long, $0.5 \mathrm{~mm}$ wide, falcate, linear-ligulate to linear-oblanceolate, subobtuse; posterior lobe $3 \mathrm{~mm}$ long, $0.2 \mathrm{~mm}$ wide, obliquely linear, obtuse. Lateral sepals $4 \mathrm{~mm}$ long, $1.5-2 \mathrm{~mm}$

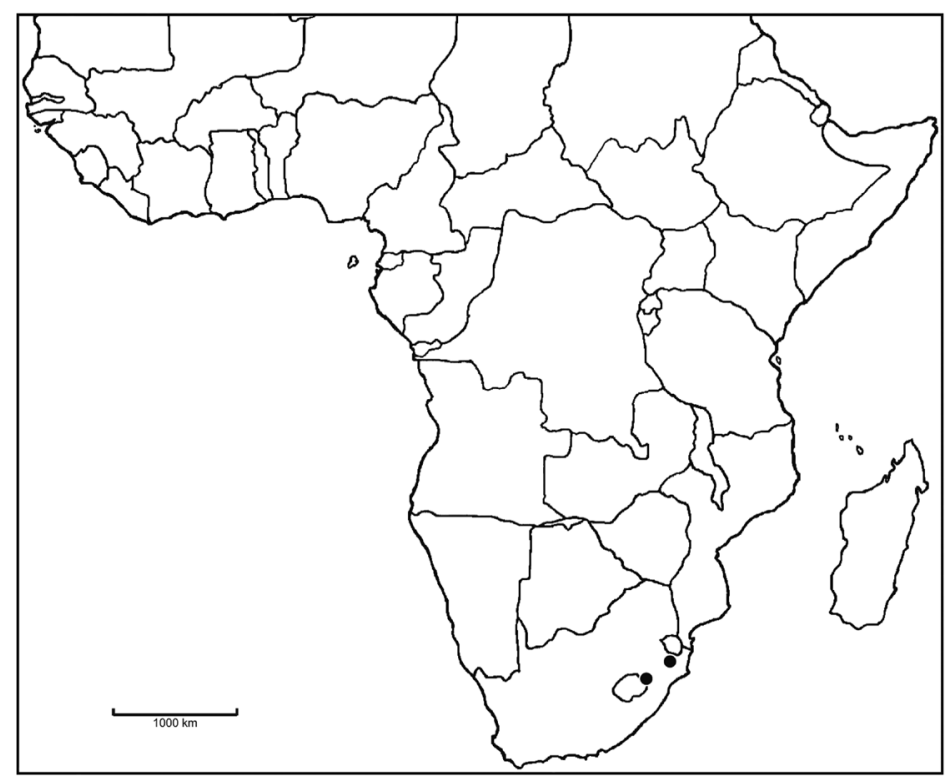

Fig. 45. Distribution of Bilabrella woodii (Schltr.) Szlach. \& Kras 
wide, obliquely elliptic-ovate, subacute, glabrous. Lip 3-lobed almost to the base, glabrous; the middle lobe $4 \mathrm{~mm}$ long, $0.5 \mathrm{~mm}$ wide, linear, obtuse; lateral lobes $3 \mathrm{~mm}$ long, 0.6-0.7 mm wide, oblong-lanceolate, obtuse, falcately divergent. Spur $18 \mathrm{~mm}$ long, subfiliform, apically inflated, blunt. Gynostemium sessile. Anther $2 \mathrm{~mm}$ long; connective triangular-ovate, blunt; antherophores $1.5 \mathrm{~mm}$ long, delicate, upcurved. Stigmaphores $1.5 \mathrm{~mm}$ long, clavate. Rostellum middle lobe half as long as thecae. Auriculae unknown (Fig. 44).

E c ology. Terrestrial in grass on black moist soil. April.

Distribution. Republic of South Africa, alt. 300$600 \mathrm{~m}$ (Fig. 45).

Specimens examined. Republic of South Africa. Kwazulu-Natal: Natal, Glade near Raphia grove, Maputa between grass in black moist soil, 18 Apr. 1968 (fl), Strey 8189 (K!); Ungoya, 300-600 m, Wylie sub Medley Wood 7601 (no. 9468 in Herb. Schltr.) (K!).

Notes. Schlechter (1906) describing Habnaria woodii compared it with $H$. dives. Both species differ, however, from each other in almost all floral segments morphology. Bilabrella woodii is unique in the genus in having very slender and delicate habit. In our opinion it is very similar with both $B$. sochensis and $B$. stenophylla, from which it can be distinguished by having completely glabrous petals. In $B$. sochensis and $B$. stenophylla posterior petal lobe is ciliolate.

15. Bilabrella stenophylla (Summerh.) Szlach. \& Kras, Richardiana 3(3): 142. 2003.

Habenaria stenophylla Summerh., Kew. Bull. 16: 293. 1962. - Summerhayes, FTEA, Orchid. 1: 102. 1968b.

Type: Tanganyika, Milne-Redhead \& Taylor 10384 (holotype, K!).

Stem $40-50 \mathrm{~cm}$ tall, erect, rather slender, leafy throughout its length. Leaves $12,13-15 \mathrm{~cm}$ long, $0.7 \mathrm{~cm}$ wide, the lowermost 2-3 reduced to sheaths which are white with green reticulate veining, the middle 5-6 suberect, linear, the upper ones smaller, adpressed to the stem, lanceolate, similar to the bracts. Inflorescence 6-11 cm long, rather densely 10-20-flowered. Flowers suberect, green with a whitish centre. Floral bracts 15-20 mm long, lanceolate, acuminate, rather thin and chaffy, finely toothed, shorter than the pedicel with ovary. Pedicel with ovary $20 \mathrm{~mm}$ long, straight or slightly curved. Dorsal sepal 6-7 mm long, $2 \mathrm{~mm}$ wide, reflexed, narrowly elliptical, rounded at apex, convex. Petals bilobed nearly to the base; anterior lobe 9-12.5 mm long, $2 \mathrm{~mm}$ wide, projecting forwards, lanceolate, subacute, glabrous; posterior lobe 6-8 $\mathrm{mm}$ long, $0.5 \mathrm{~mm}$ wide, recurved, linear, shortly ciliolate. Lateral sepals $8 \mathrm{~mm}$ long, $4 \mathrm{~mm}$ wide, deflexed, obliquely semi-

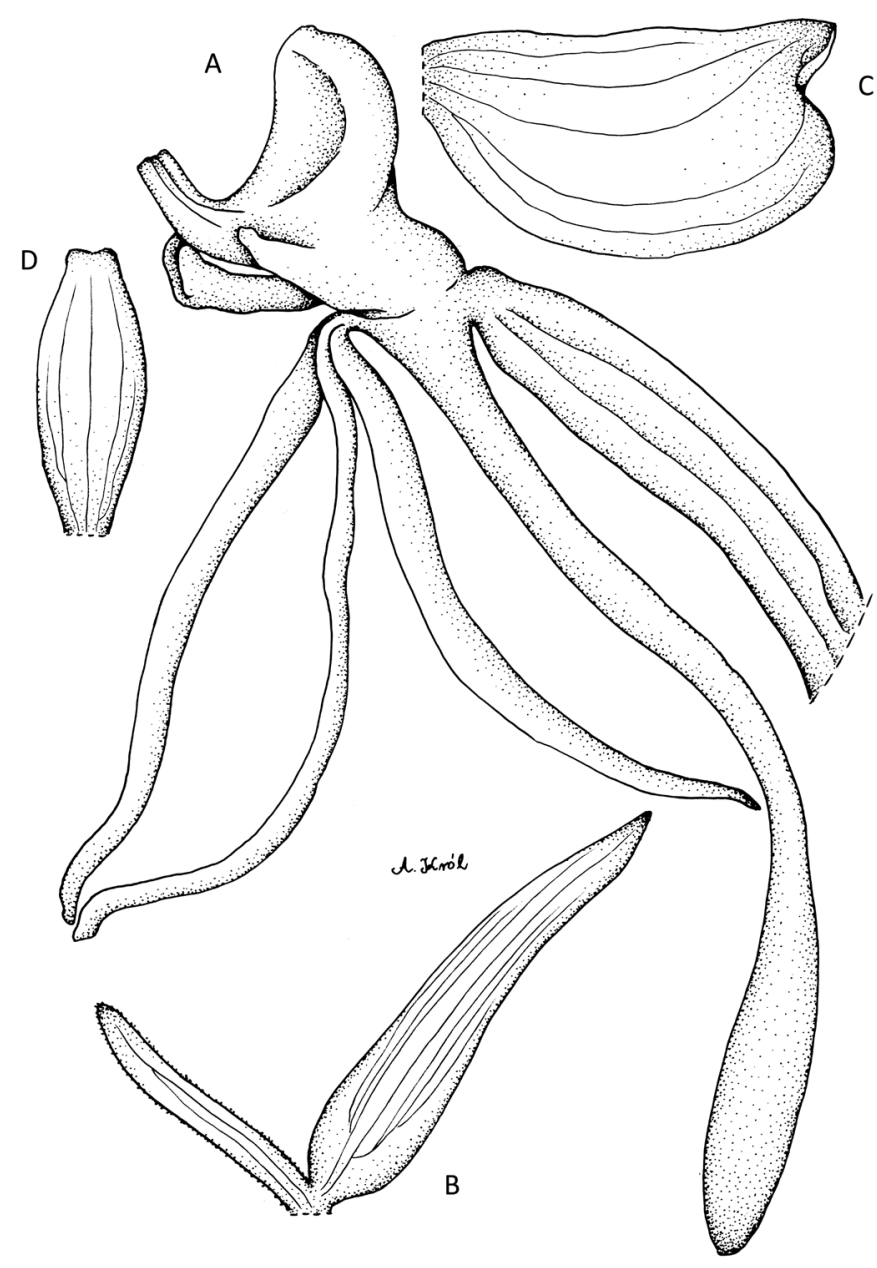

Fig. 46. Bilabrella stenophylla (Summerh.) Szlach. \& Kras Explanations: A - flower; B - petal; C - lateral sepal; D - dorsal sepal (drawn by A. Król from holotype of Habenaria stenophylla Summerh. - MilneRedhead \& Taylor 10384, K)

orbicular, the apiculum lateral. Lip deflexed, 3-lobed nearly to the base; the middle lobe $12 \mathrm{~mm}$ long, $0.5 \mathrm{~mm}$ wide, incurved, linear, obtuse; lateral lobes $10-11 \mathrm{~mm}$ long, a little wideer than the middle lobe, lanceolatelinear, acute. Spur 16-19 mm long, incurved, swollen in apical half or third. Gynostemium sessile. Anther $4.5 \mathrm{~mm}$ tall, antherophores $1.5 \mathrm{~mm}$ long; stigmaphores $3 \mathrm{~mm}$ long (Fig. 46).

E c o lo gy. Grassland, in meadow. January-May.

D i s tributi on. Democratic Republic of the Congo (Zaire), Tanzania, alt. 690-3000 m (Fig. 47).

Specimens examined. Democratic Republic of the Congo (Zaire). Haut-Katanga: Shaba Region, Kipopo, 13,5 km north-west of Lubumbashi, seasonally flooded grassland, 1240 m, 8 Jan. 1983 (fl), Schaijes 1791 (K!). Tanzania. Iringa: Makete Distr., Kitulo Plateau. High alitutde grassland between Kitulo and Matamba, 0905'S, 3350'E, 2600 m, 30 Mar. 1987 (fl), Lovett \& Congdon 1858 A (K!); Njombe, Lusitu Ridge, on better soil on rocky ridge, 690 m, 17 Apr. 1989 (fl), Spurrier 90 (K!); The same locality, dry grassland, 


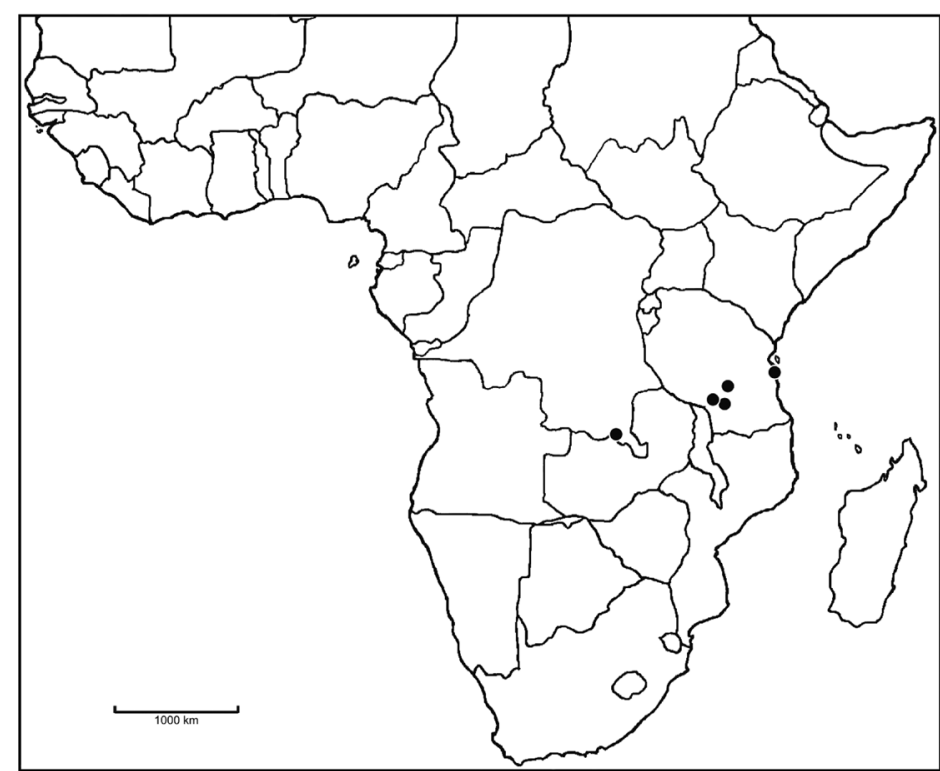

Fig. 47. Distribution of Bilabrella stenophylla (Summerh.) Szlach. \& Kras

poor soil, 2220 m, 28 Apr. 1991 (fl), Spurrier 121 (K!); Pwani: Ukonga, Lumakali River, in meadow, 3000 m, 4 Feb. 1914 (fl), Stolz 2490B part (K!); Ruvuma: Songea Distr., Matango Hills, Lupembe Hill, 20 May 1956 (fl), Milne-Redhead \& Taylor 10384 (K! - spirit.).

Notes. This species can be misidentified with $B i$ labrella sochensis as both have very similar form of the flowers. B. stenophylla however, has narrower leaves $(13-15 \times 0.7 \mathrm{~cm}$ vs $12-25 \times 2-5 \mathrm{~cm}$ in $B$. sochensis $)$, prominently shorter inflorescence $(6-11 \mathrm{~cm}$ vs $15-40 \mathrm{~cm}$ long) and different proportion between lip middle and lateral lobes (middle lobe $12 \mathrm{~mm}$ long, lateral lobes 10$11 \mathrm{~mm}$ long vs lip middle lobe $10-17 \mathrm{~mm}$ long, lateral lobes 6-12 mm long).

A striking feature consists of the cataphylls at the base of the stem which, as in the very different Bilabrella retinervis, are whitish with a distinct network of green veins. These are quite lacking in the two allies named above, the cataphylls, when present, being the same colour as the other leaf-sheaths. The specimens collected by Stolz were mixed with plants of B. leucoceras and $B$. kyimbilae, both of which can be distinguished readily from B. stenophylla.

16. Bilabrella sochensis (Rchb. f.) Szlach. \& Kras-Lap., Richardiana 3(3): 142. 2003.

Habenaria sochensis Rchb. f., Otia Bot. Hamburg.: 100. 1881. - Rolfe, Fl. Trop. Afr. 7: 239. 1898. - Summerhayes, FTEA, Orchid 1: 98. 1968b. - Grosvenor, Excelsa 6: 83. 1976. - Williamson, Orchid. S. Central Afr.: 55. 1977. - la Croix \& Cribb, Fl. Zambes., Orchid. 11: 105. 1995.

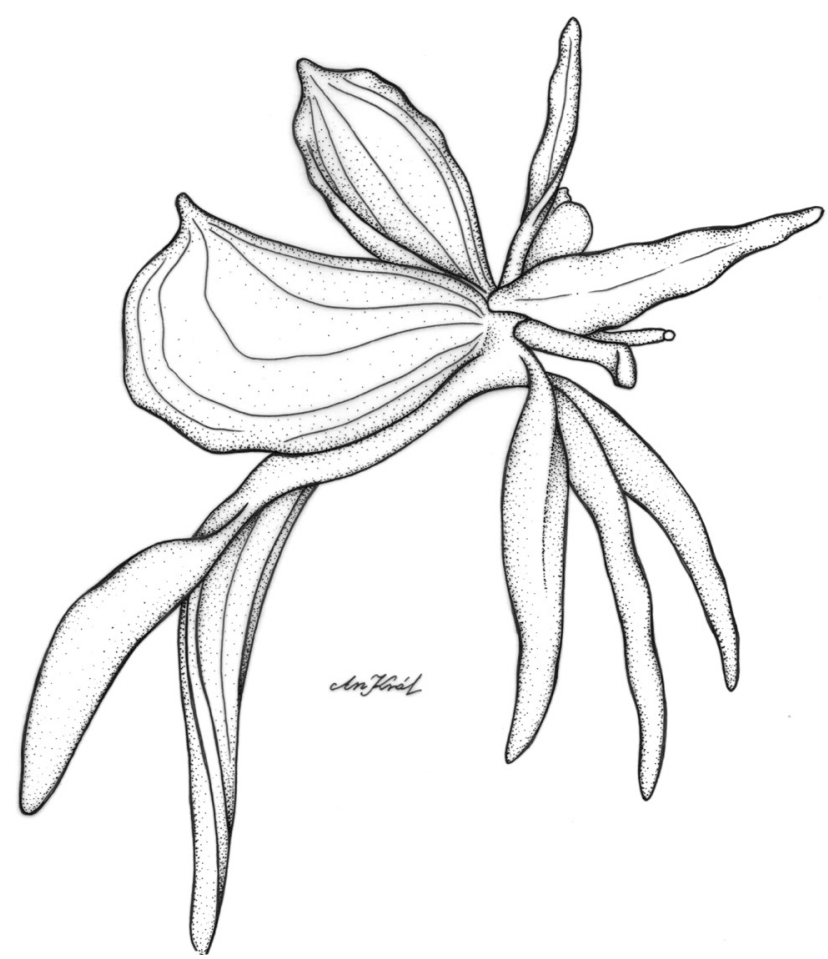

Fig. 48. Bilabrella sochensis (Rchb. f.) Szlach. \& Kras-Lap. - flower (drawn by A. Król from Bullock 3686, AMES)

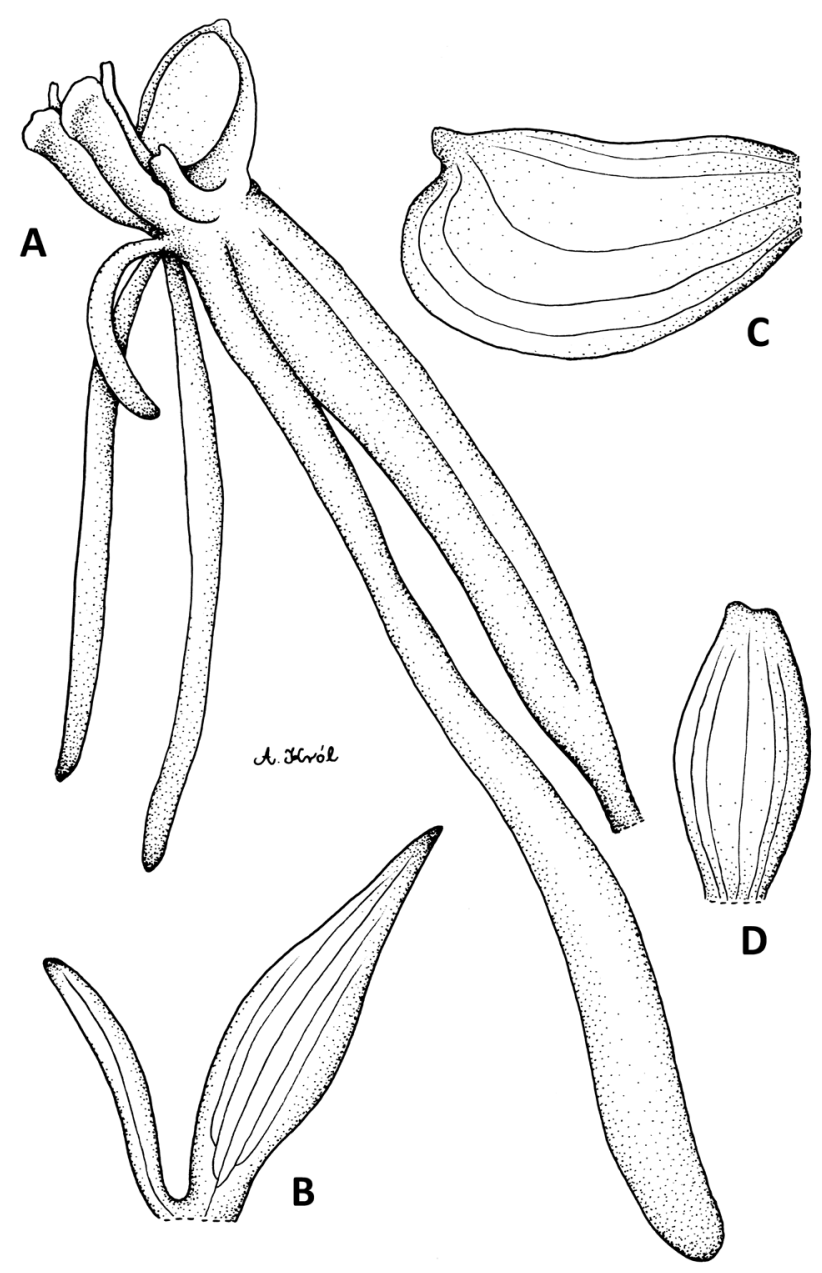

Fig. 49. Bilabrella sochensis (Rchb. f.) Szlach. \& Kras-Lap. Explanations: A - ovary, gynostemium, lip and spur; B - petal; C - lateral sepal; D - dorsal sepal (drawn by A. Król from lectotype of Habenaria sochensis Rchb. f. - Kirk s.n., K) 
Type (Summerhayes 1968b, as holotype): Malawi, Kirk s.n. (lectotype, designated by Summerhayes [1968b: 98], K!; drawing, W!).

Tubers 3-4.5 $\mathrm{cm}$ long, $1-2 \mathrm{~cm}$ in diameter, ovoid or ellipsoid, tomentose. Stem 60-200(300) cm tall, erect, robust, leafy throughout its length. Leaves 11$16,12-25 \mathrm{~cm}$ long and $2-5 \mathrm{~cm}$ wide, the lowermost 2 reduced to sheaths, recurved, the middle 6-7 suberect or spreading, lanceolate or oblong-lanceolate, acute, the upper ones smaller, adpressed to the stem, lanceolate, similar to the bracts. Inflorescence $15-40 \mathrm{~cm}$ long, rather loosely to subdensely many-flowered. Flowers curving outwards, green or yellow-green, fragrant unpleasant at night. Floral bracts $15-35 \mathrm{~mm}$ long, thin and chaffy, lanceolate, acuminate, nearly as long as the pedicel with ovary. Pedicel with ovary 20-30 mm long, slightly curved. Dorsal sepal 4-7 mm long, 2-3 mm wide, elliptic, obtuse, cochleate, reflexed. Petals bilobed to the base; anterior lobe $8-11 \mathrm{~mm}$ long, 2-3.5 $\mathrm{mm}$ wide, spreading forwards, lanceolate or oblong-lanceolate, acuminate, acute or subacute, almost glabrous; posterior lobe 5-6.5 mm long, scarcely $1 \mathrm{~mm}$ wide, recurved, linear, obtuse, ciliolate. Lateral sepals $6.5-10 \mathrm{~mm}$ long, 4-5.5 $\mathrm{mm}$ wide, deflexed, obliquely semi-orbicular to oblong-obovate, with laterally placed apiculus. Lip deflexed, 3-lobed almost to the base; the middle lobe 10-17 mm long, 0.5-1.5 mm wide, incurved, linear, subacute to subobtuse; lateral lobes $6-12 \mathrm{~mm}$ long, 0.5-1.5 mm wide, linear-lanceolate to linear, subacute. Spur 17-25 mm long, parallel to the ovary, narrowly cylindrical, swollen in the apical third. Gynostemium sessile. Anther 2.5-4.5 mm tall; connective rounded; antherophores 2-5 mm long, slender, upcurved. Stigmaphores 3-4 mm long, massive, cylindrical, truncate. Rostellum middle lobe shorter than thecae. Auriculae small, bifid in the upper half (Figs 48-49).

Ecology. Grassland, Eucalyptus, Uapaca and Brachystegia woodland, especially near streams, sometimes in shade, loamy, brown and black dambo soils. February-July.

D istributi on. Tanzania, Zambia, Malawi, Zimbabwe, alt. 800-2450 m (Fig. 50).

S pecimens examined. Tanzania. Iringa: Njombe, Dansland Hydro, black dambo soil but not in a seasonally flooded area-open grassland, $1920 \mathrm{~m}$, 18 May 1991 (fl), Spurrier 124 (K!); Mbeya: Ilembo Umalila, grassland, 1950 m, 10 Apr. 1970 (fl), Leedal 441 (K!); Mbasi, in wet ground, $1500 \mathrm{~m}$, Apr. 1935 (fl), Polock 4 (K!); Mbeya Town, 1740 m, 23 Apr. 1972 (fl), Nicholson 238 (K!); Ruvuma: Songea Distr., just E of R. Likuyu and about 1,5 km W of Ruanda turnoff, in grassland on slope between BrachystegiaUapaca woodland and river, 800 m, 12 Apr. 1956 (fl), Milne-Redhaed \& Taylor 9583 (K! \& spirit.); Songea

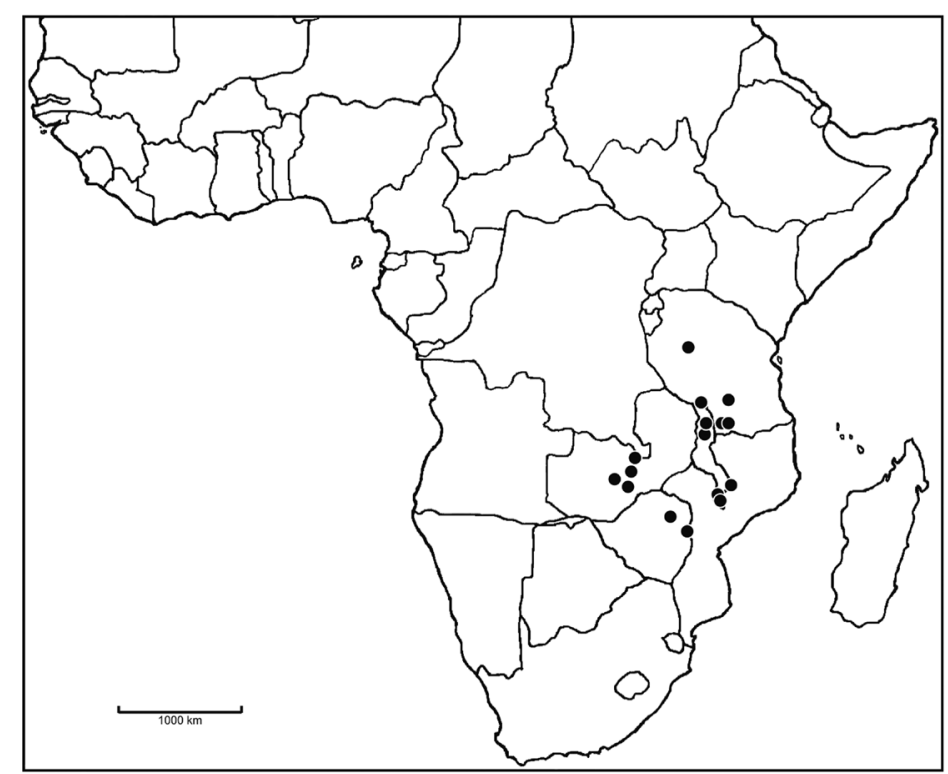

Fig. 50. Distribution of Bilabrella sochensis (Rchb.f.) Szlach. \& Kras-Lap.

Distr., about $3 \mathrm{~km} \mathrm{N.E.} \mathrm{of} \mathrm{Kigonsera,} \mathrm{in} \mathrm{open} \mathrm{speces}$ in Brachystegia-Uapaca woodland on red brown loam among grass, 970 m, 15 Apr. 1956 (fl), Milne-Redhaed \& Taylor 9727 (K! \& spirit.); Tabora: Ibeya, growing in shade in dense vegetation, 1650 m, 7 May 1944 (fl), Moreaus 289 (K!); Tanga: Ufinga Distr., 13 Feb. 1951 (fl), Bullock 3686 (AMES!, K! \& spirit, UGDA-DLSz! - fragment, drawing). Zambia. Central: Broken Hill. "Chipya" woodland with tall bracken on rich, loamy soils, 27 Feb. 1961 (fl), Morze 62 (K!); Mumbwa, road, July 1912 (fl), Macaulay 1173 \& 1173bis (K!); Freuqently common in miombo woodland on laterite, 18 Feb. 1968 (fl), Mutimushi 2461 (K!); Copperbelt: Kitwe, 27 Feb. 1971 (fl), Fanshawe 11.163 (K!); Lusaka: Btilanga Fish Farm, on river bank below dam apillway, brown soil, 1185 m, 3 Mar. 1963 (fl), Lusaka National History blub 237 (K!); Chunga Riunes, 6 miles exi Lusaka on Broken Hill, 1350 m, 9 Mar. 1958 (fl), Beuson 232 (BM!); 8 miles E of Lusaka, tall grass, 1260 m, 15 Mar. 1956 (fl), King 358 (K!). Malawi. Central: Kasungu Distr., Mpeni near Bvumbwe, among long grass by roadside, $1200 \mathrm{~m}, 30$ Mar. 1982 (fl), la Croix 303 (K!); Northern: Mzimba Distr., 7 miles N of Mperembe, by roadside in Brachystegia woodland, 1280 m, 10 Mar. 1978 (fl), Pawek 14025 (K!); Nyika Plateau, near Dembo Bridge. Grassland, 2150-2450 m, 18 Mar. 1982 (fl), Dowsett-Lemaire 356 (K! \& spirit.); $\mathrm{S}$ Viphya, Chikangawa, below Kasito resthouse, by rock slabs at food of Protea slope, 1700 m, 7 Mar. 1987 (fl), Cornelius (la Croix) 1004 (K!); South Viphya, Chikangawa (Kasitu), in rank grassland near rocks, $1700 \mathrm{~m}$, 22 Feb. 1983 (fl), Dowsett-Lemaire 632 (K!); Viphya Plateau, Luwama Dam road, by roadside, $1200 \mathrm{~m}$, 22 Mar. 1983 (fl), la Croix 471 (K!); S Viphya Plateau, 
beside Chikangawa-Nzuzu road, in grassland, $1650 \mathrm{~m}$, 4 Apr. 1983 (fl), la Croix 586 (K!); S Viphya, MzuzuMazembe road near bridge over Luchilemu R. Edge of Brachystegia woodland by roadside, quite near river, 1300 m, 16 Mar. 1986 (fl), la Croix 823 (K!); Southern: Blantyre Distr., Behind Maone Estate, $2 \mathrm{~km}$ north-east Limbe, Eucalyptus plantation, 1200 m, 17 Mar. 1970 (fl), Brummitt 9183 (K!); Chikwawa Distr., Bvumbwe, in Eucalyptus plantation, 1100 m, 18 Mar. 1981 (fl), la Croix 120 (K!); Chikwawa Distr., Bvumbwe, towards edge of Eucalyptus wood, 7 Apr. 1982 (fl), la Croix 306 (K!); Chilwa Island, grassy summit, $1060 \mathrm{~m}, 11$ Mar. 1955 (fl), Exell, Mendonca \& Wild 801 (BM!, K!); Manganja country, Soche Hill, Kirk s.n. (K!); Zomba Distr., Limbe, Buru Da, in long grass, 1050 m, 25 Mar. 1984 (fl), la Croix 577 (K!). Zimbabwe. Harare: Salisbury, 19 Feb. 1933 (fl), Eyles 7288 (K!). Salisbury Distr., in bush and grassland of district, Greatrex $90 b$ (K!); Salisbury, Meyrick Park, in grassland, one of the few spp. found in long grass, 12 Mar. 1950 (fl), Greatrex 27210 (AMES!, K!, UGDA-DLSz! - fragment, drawing); Manicaland: Umtali Distr., Odzani Heights, 1500 m, 24 Mar. 1957 (fl), Chase 6374 (K!).

$\mathrm{N}$ o t e s. This species appears to be simillar to B. stenophylla, but they can be keyed out by the leaves measure $(13-15 \times 0.7 \mathrm{~cm}$ in B. stenophylla vs $12-25 \times 2-5 \mathrm{~cm}$ in $B$. sochensis), length of inflorescence (6-11 cm long in the former vs $15-40 \mathrm{~cm}$ in the latter) and different length of the lip lobes (subeqaul in B. stenophylla vs lip middle lobe prominently longer than lateral ones in B. sochensis). Additional differences between these two taxa are the size of rostellum and the shape of petal lobes. Sometimes we found the note on herbarium sheets that $B$. retinervis is similar to $B$. sochensis. These species differ from each other in the habit, spur shape, form of anterior petal lobes and gynostemium general architecture.

17. Bilabrella orangana (Rchb. f.) Szlach. \& Kras, Richardiana 9(4): 159. 2009.

Habenaria orangana Rchb. f., Otia Bot. Hamburg.: 101. 1881. - Kraenzlin, Orchid. Gen. Sp.: 214. 1901.

Type (hoc loco selectus): Republic of South Africa, Cooper 1096 (lectotype, W-R!).

Plants $20-30 \mathrm{~cm}$ tall, rather robust, erect, leafy. Leaves 6-8, up to $9 \mathrm{~cm}$ long and $2.8 \mathrm{~cm}$ wide, ovatelanceolate, acute, suberect, gradually decreasing in size upwards, the upper ones lanceolate to oblong-lanceolate, acute to acuminate, more or less adnate to the stem. Inflorescences up to $11 \mathrm{~cm}$ long, dense, many-flowered. Floral bracts to $20 \mathrm{~mm}$ long, leaf-like, concave, ovatelanceolate, acuminate, minutely glandular. Pedicellate ovary 15-17 mm long, slender. Dorsal sepal $4 \mathrm{~mm}$ long, 1.5-2 mm wide, oblong-ovate to oblong-elliptic, sub-

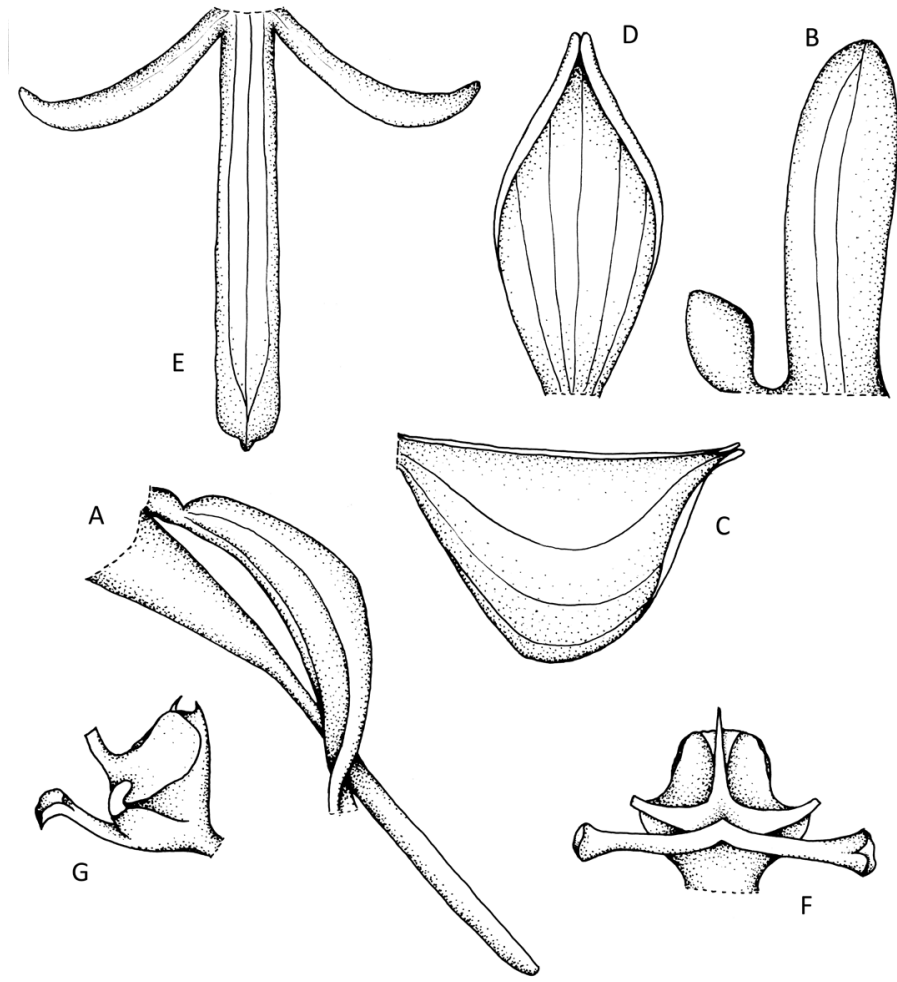

Fig. 51. Bilabrella orangana (Rchb. f.) Szlach. \& Kras Explanations: A - ovary and spur; B - petal; $\mathrm{C}$ - lateral sepal; D - dorsal sepal; E - lip; F - gynostemium, front view; G - gynostemium, side view (drawn by A. Król from lectotype of Habenaria orangana Rchb. f. - Cooper 1096, W-R)

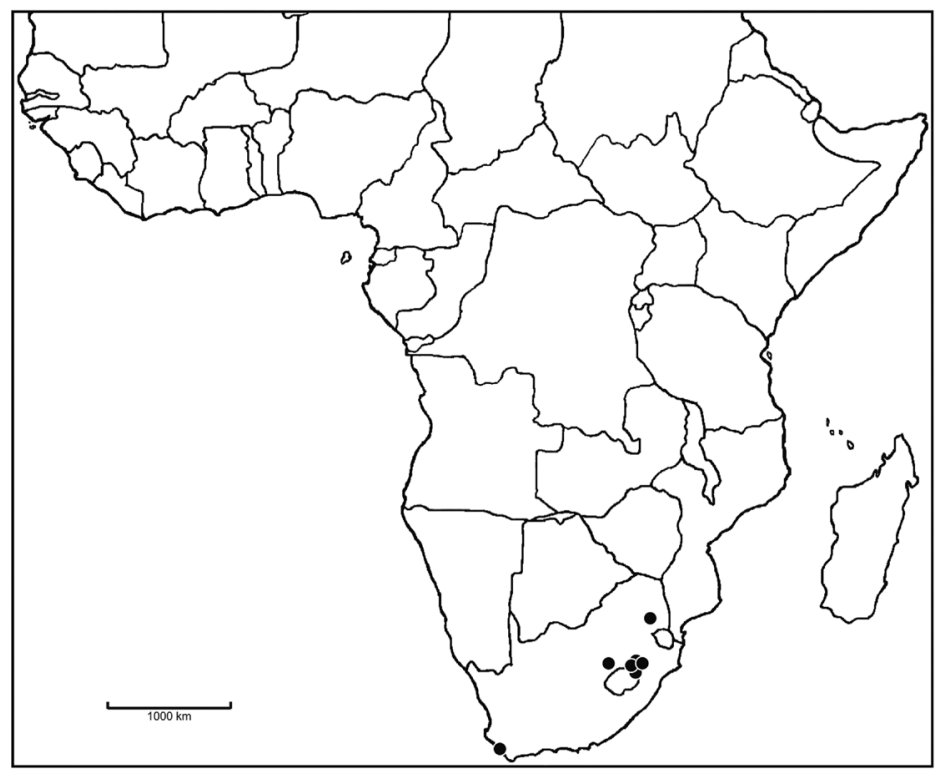

Fig. 52. Distribution of Bilabrella orangana (Rchb.f.) Szlach. \& Kras

obtuse to subacute, concave. Petals bilobed just above the base, minutely ciliolate; anterior lobe $4 \mathrm{~mm}$ long, $1.1 \mathrm{~mm}$ wide, ligulate, rounded at the apex; posterior lobe $1.1 \mathrm{~mm}$ long, $0.8 \mathrm{~mm}$ wide, ovate to deltoid, obtuse. Lateral sepals $5 \mathrm{~mm}$ long, $4 \mathrm{~mm}$ wide, obliquely ellipticobovate or elliptic-ovate, acute, concave. Lip 3-lobed to the base; the middle lobe $7 \mathrm{~mm}$ long, $1 \mathrm{~mm}$ wide, oblong to narrowly ligulate, obtuse, shortly apiculate; 
lateral lobes $4 \mathrm{~mm}$ long, $0.6 \mathrm{~mm}$ wide, spreading, falcately linear-lanceolate, subacute. Spur $13.5 \mathrm{~mm}$ long, tapering from a widened mouth, narrowly cylindrical. Gynostemium sessile. Anther $2 \mathrm{~mm}$ tall; connective shortly apiculate; antherophores less than $1 \mathrm{~mm}$ long, slender, upcurved. Stigmaphores $2 \mathrm{~mm}$ long, clavate, rather massive. Rostellum middle lobe subulate, as long as connective. Auriculae ellipsoid-falcate (Fig. 51). E c o logy. On marsh, in vlei. January-September. D i s tribution. Republic of South Africa, alt. 1350$2250 \mathrm{~m}$ (Fig. 52).

Specimens examined. Republic of South Africa. Free State: Orange Free State, 1862 (fl), Cooper 1096 (K! - photo); Free State Prov., Orange Free State, Cooper 1098 (W-R!); Kwazulu-Natal: Natal, Beyville Distr., Cathedral Peak Forest Research Sta, occasional in Themeda taiandra, grassveld in Catchment I, $1815 \mathrm{~m}$, 26 Jan. 1951 (fl), Killick 1353 (K!); Natal, Cathkni Park, Drakeneberg, grassy slopes, 1350-1500 m, 4 Feb. 1932 (fl), Galpin 11703 (K!); Harrismith O.R.C. Marsh, 2250 m, 19 Sep. 1905 (fl), Sankey 263 (K!); Natal, sources of Ingela River, Oliviers Hoek, wet places, $1500 \mathrm{~m}$, Apr. 1891 (fl), Allison 14 (K!); Western: Cape of Good Hope, Mar. 1873 (fl), Cooper 1093 (K!, W-R! part.); Transvaal, between Lydenburg and Sabie (Long Tom Pass), 1700-2000 m, 31 Jan. 1959 (fl), Werdermann \& Oberdieck 2094 (AMES!, K!, UGDA-DLSz! - fragment, drawing).

N o te s. It seems that this species is somewhat unique in this group by having wide spur mouth and peculiar posterior petal lobe, which is ovate to deltoid and obtuse. Considering habit and form of the petals, this species appears to be similar to Bilabrella dives. B. orangana can be distinguished from it, however, by narrow lateral lip lobes and rostellum middle lobe as long as connective. The status of both species requires further study.

18. Bilabrella antennifera (A. Rich.) Szlach. \& Kras, Richardiana 9(4): 158. 2009.

Habenaria antennifera A. Rich., Ann. Sci. Nat. Bot., Ser. 2(14): 268. 1840. - Edwards, Demissew, Hedberg, Fl. Ethiopia Eritrea 6: 221.1997. - Demissew, Cribb, Rasmusen, Field guide to Ethiopian Orchids: 100. 2004.

Type (hoc loco selectus): Ethiopia, Quartin Dillon s.n. (lectotype, $\mathrm{P}$ !, isolectotype, $\mathrm{W}$ !).

Habenaria simense Rchb. f., Linnaea 22: 859. 1849.

Type (hoc loco selectus): Ethiopia, Rueppel N4 (lectotype, W-R!; isolectotype, K?).

Habenaria leptobrachiata Ridley, Britten Journ. Bot. 24: 293. 1886.

Type (hoc loco selectus): Ethiopia, Schimper 1324 (in part) (lectotype, BM!, isolectotypes, $\mathrm{K}$ !, W!). Habenaria pantothrix Kraenzl. ex Engl., Abh. Preuss. Akad. Wiss 2: 181. 1892, nomen. \& in Bot. Jahrb. Syst. 16: 89. 1893.
Type (hoc loco selectus): Ethiopia, Schimper 1324 (in part) (B+; lectotype, HBG!; isolectotypes, $\mathrm{K}$ !, W!).

An erect terrestrial herb up to $45 \mathrm{~cm}$ high. Tubers up to $2.5 \mathrm{~cm}$ long, $1.3 \mathrm{~cm}$ in diameter, ovoid to ellipsoid. Stem leafy throughout its length, erect, rather robust. Leaves 4-7, the largest 4-14 cm long, 1.4-3.5 cm wide, ovate to elliptic-lanceolate, acute, often folded together and curved, gradually decreasing in length and width upwards. Inflorescence 4.5-20 cm long, cylindrical, subdensely to densely many-flowered. Flowers green, yellow-green or green and white. Floral bracts $14-18 \mathrm{~mm}$ long, mostly longer than the ovary, ovate-elliptic, acute or acuminate, glandular-pubescent. Pedicel and ovary 12-16 mm long, glabrous. Dorsal sepal 3.7-4.5 mm long, 1.5-2.5 mm wide, oblong obovate to narrowly elliptic, rounded or obtuse and more or less recurved at the apex, concave, lying over the gynostemium. Petals bilobed to the base, ciliate; anterior lobe $13 \mathrm{~mm}$ long, 1-1.8 mm wide, lanceolate, acute to acuminate; posterior lobe up to $4 \mathrm{~mm}$ long, $0.8 \mathrm{~mm}$ wide, reflexed, linear-lanceolate, obtuse, often reduced in some plants. Lateral sepals 6.5-7.5 mm long, 5-5.5 mm wide, reflexed, obliquely oblong-elliptic, laterally apiculate, obtuse, concave. Lip 3-lobed above the base; the middle lobe 6-9 $\mathrm{mm}$ long, 0.8-1.7 mm wide, linear to ligulate, acute; lateral lobes 5-7 mm long, 0.6-1 mm wide, linear, acute, pendent. Spur 9-12 mm long, club-shaped, straight or slightly bent in middle. Gynostemium sessile. Anther $2 \mathrm{~mm}$ long; connective massive, rounded; antherophores $2 \mathrm{~mm}$ long, very delicate, upcurved. Stigmaphores $2 \mathrm{~mm}$ long,

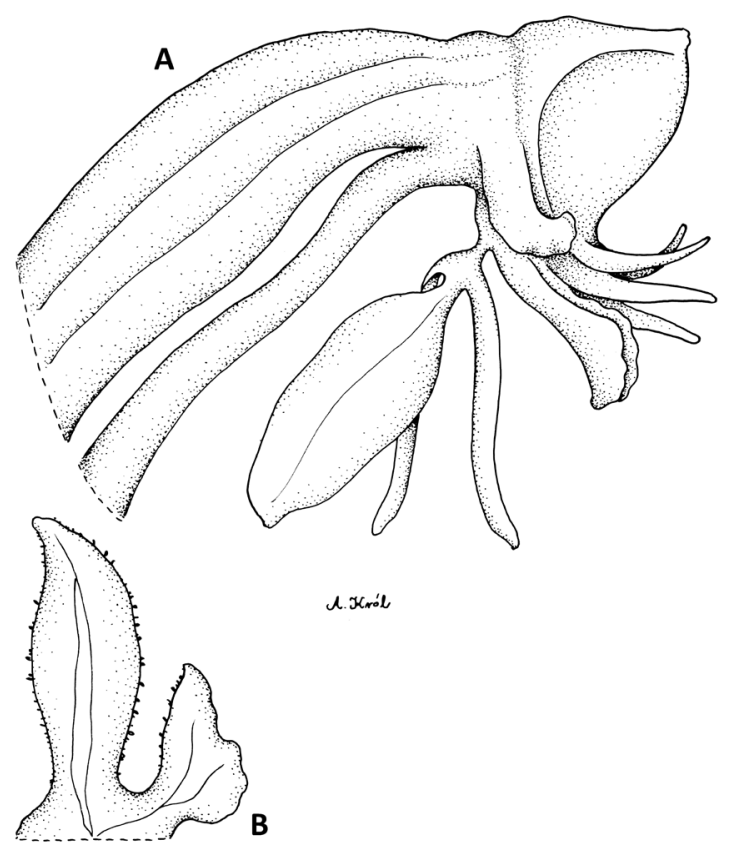

Fig. 53. Bilabrella antennifera (A. Rich.) Szlach. \& Kras Explanations: A - flower; B - petal (drawn by A. Król from isolectotype of Habenaria antennifera A.Rich. - Quartin Dillon s.n., W) 


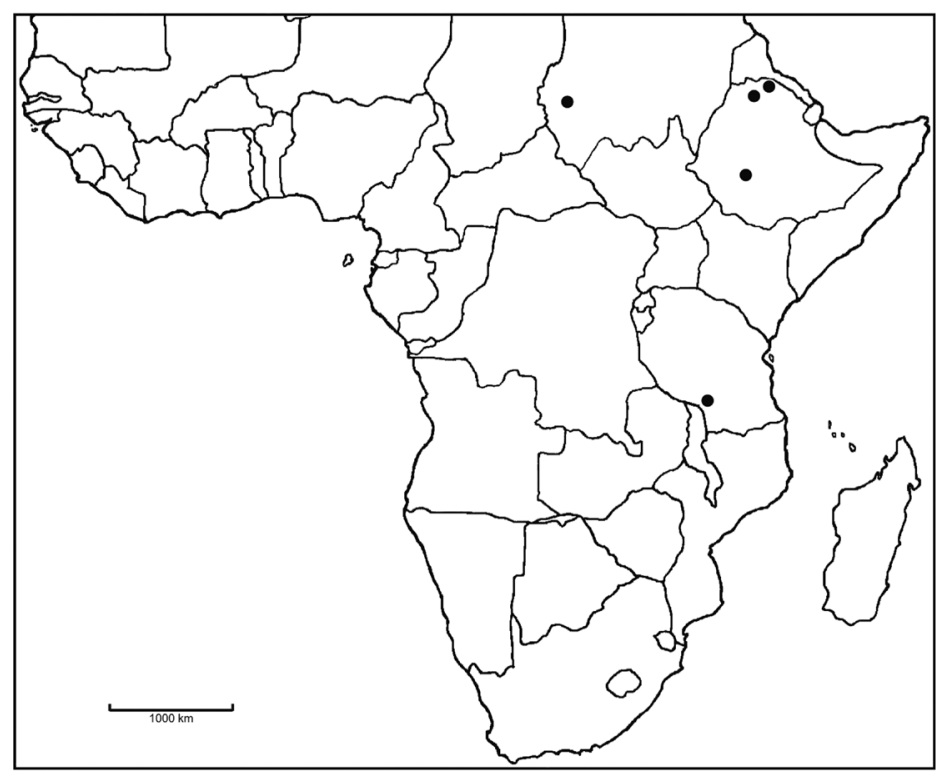

Fig. 54. Distribution of Bilabrella antennifera (A.Rich.) Szlach. \& Kras

narrowly cylindrical. Rostellum middle lobe shorter than connective. Auriculae very tiny, subglobose (Fig. $53)$.

Ecology. Grassland, open scrub, amongs rocks. March-October.

D i s tr i bu ti o n. Sudan, Ethiopia, Yemen, Tanzania, alt. 240-3200 m (Fig. 54).

S pecimens examined. Sudan. Darfur: Jebel Marra, Wadi Tongul, on volcanic soils in upland grassland occasionally, 2400 m, 8 Sep. 1982 (fl), Klug Hamburg BFH 689 (K!); Jebel Marra, upland meadow, $2280 \mathrm{~m}$, Wickens 2459 (K!); Jebel Marra, Towra Tonga, upland grassland, ash soils, 2250 m, 19 Sep. 1964 (fl), Wickens 2647 (K!); Jebel Marra, N of Towra Tonga, marsh, 2580 m, 21 Sep. 1964 (fl), Wickens 2690 (K!). Ethiopia. Amhara: Bagemder Prov., Simen, Rueppel N4 (W-R!); An way to Waima, on slopes, 240-270 m, 23 Aug. 1852 (fl), Schimper 607 (K!, W-R!); Kaffa: Mt. Maigudo, Kaffa, Ground orchid 9-12" with yellowish-green flowers, 24 Oct. 1954 (fl), Mooney 6153 (K! - spirit.); Tigray: Adua, Quartin Dillon s.n. (W!); Dschau Meda, 1863-8, Schimper 1324 (BM!, K!, W!). Yemen. In a dense colony with Satyrium, Disa pulchella, Habenaria lefebureana, in grassland at the summit of Jebel Taaga, 3200 m, 28 July 1977 (fl), Wood 1718 (K! - spirit.). Tanzania. Iringa: Njombe, Tanwat Plantation, faintly long grass, open hillside, red soil with Aloe nuttii \& A. laterclia, 1860 m, 26 Mar. 1989 (fl), Spurrier 66 (K!).

N o te s. Bilabrella antennifera appears to be similar to $B$. isoantha, but both species are easily separable one from another by completely different spur shapes and proportions in the form of petal lobes.
19. Bilabrella williamsoni (P. J. Cribb) Szlach. \& Kras, Richardiana 9(4): 159. 2009.

Habenaria williamsonii P. J. Cribb, Kew Bull. 32: 143. 1977.

Type: Malawi, Williamson 283 (holotype, K!; isotypes, SRGH not seen, drawing: K!).

Tubers single, up to $1.3 \mathrm{~cm}$ long and $0.5 \mathrm{~cm}$ in diameter, ovoid. Stem $23 \mathrm{~cm}$ tall, erect, slender, glabrous. Leaves 10-11, up to $6 \mathrm{~cm}$ long, ca. $0.8 \mathrm{~cm}$ wide, the median linear-lanceolate, acute, spread, decreasing in size up the stem. Inflorescence 2-6 cm long, 7-12-flowered, rather dense. Flowers green. Floral bracts $12 \mathrm{~mm}$ long, ovate-lanceolate, acuminate, pubescence outside. Pedicel and ovary $10 \mathrm{~mm}$ long, glabrous. Dorsal sepal $4 \mathrm{~mm}$ long, 3-4 mm wide, oblong-ovate to elliptic, obtuse, cochleate, glabrous. Petals bilobed to the base, papillose; anterior lobe $5 \mathrm{~mm}$ long, $1 \mathrm{~mm}$ wide, oblonglanceolate to linear-lanceolate, subacute to subobtuse; posterior lobe $4 \mathrm{~mm}$ long, $1 \mathrm{~mm}$ wide, linear, subobtuse. Lateral sepals $5 \mathrm{~mm}$ long, $3 \mathrm{~mm}$ wide, obliquely ovate, obtuse, slightly cochlate, glabrous. Lip 3-lobed to the base, papillose; the middle lobe $4.5 \mathrm{~mm}$ long, to $1 \mathrm{~mm}$ wide, linear, subobtuse; lateral lobes $5 \mathrm{~mm}$ long, $1 \mathrm{~mm}$ wide, linear, falcately pendent, subacute. Spur $7 \mathrm{~mm}$ long, narrowly cylindrical, pendent, slightly swollen in the apical third. Gynostemium sessile. Anther $2 \mathrm{~mm}$

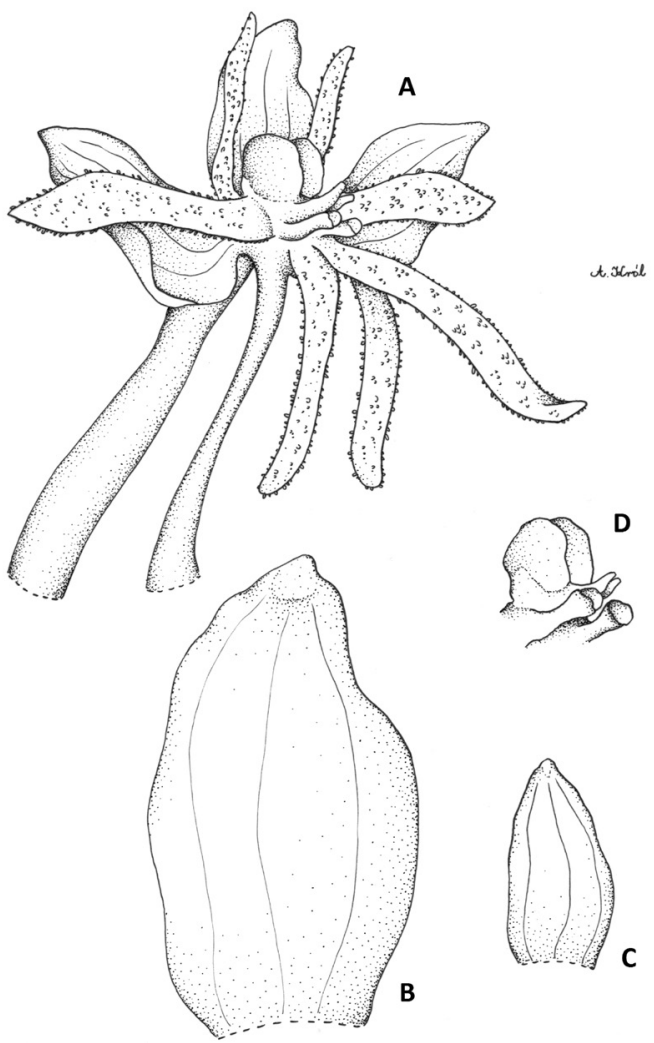

Fig. 55. Bilabrella williamsonii (P. J. Cribb) Szlach. \& Kras Explanations: A - flower; B - lateral sepal; C - dorsal sepal; D - gynostemium, side view (drawn by A. Król from holotype of Habenaria williamsoni P. J. Cribb - Williamson 283, K) 


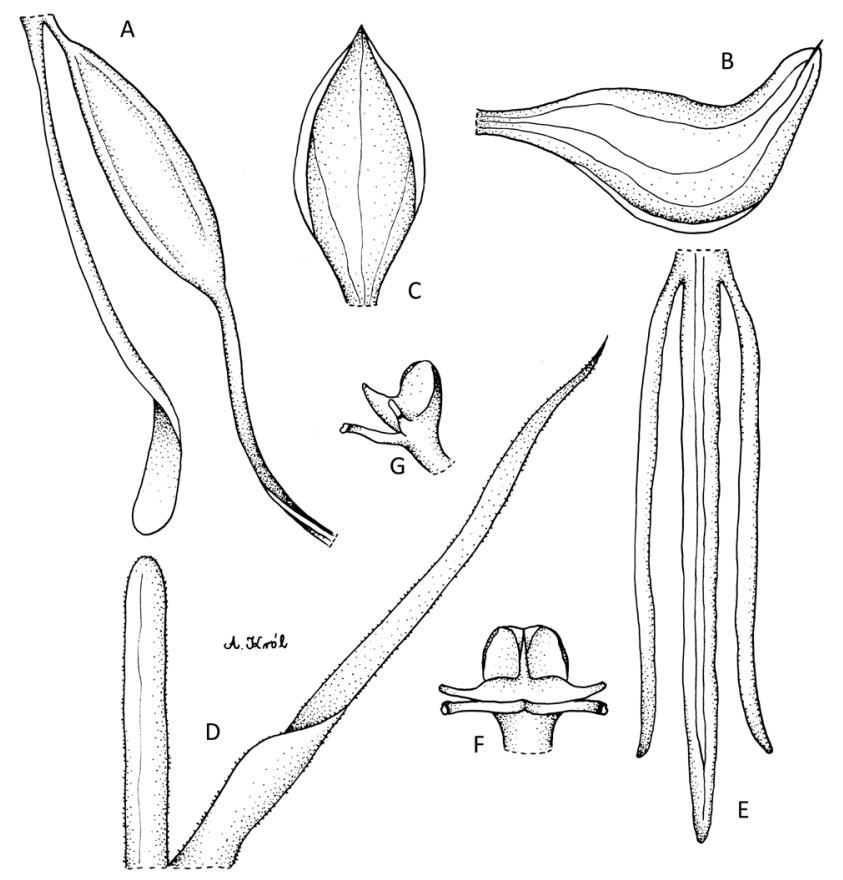

Fig. 56. Bilabrella williamsonii (P.J. Cribb) Szlach. \& Kras Explanations: A - pedicel, ovary and spur; B - lateral sepal; C - dorsal sepal; D - petal; E - lip; F-G - gynostemium, various views (drawn by A. Król from holotype of Habenaria williamsoni P.J. Cribb - Williamson 283, K)

tall; connective rounded; antherophores less than $1 \mathrm{~mm}$ long, delicate, upcurved. Stigmaphores ca. $1 \mathrm{~mm}$ long, clavate, truncate. Auriculae inconspicuous (Figs 55-56). Ec ology. Grassland. March-April.

Dis tribution. Burundi, Malawi, alt. 2285-2300 m (Fig. 57).

Specimens examined. Burundi. Bujumbura: Mount Heha, stony steppe with Eragrostis hispida, 3³6’'S, 2930'E, 2300 m, 5 Apr. 1980 (fl), Reekmans

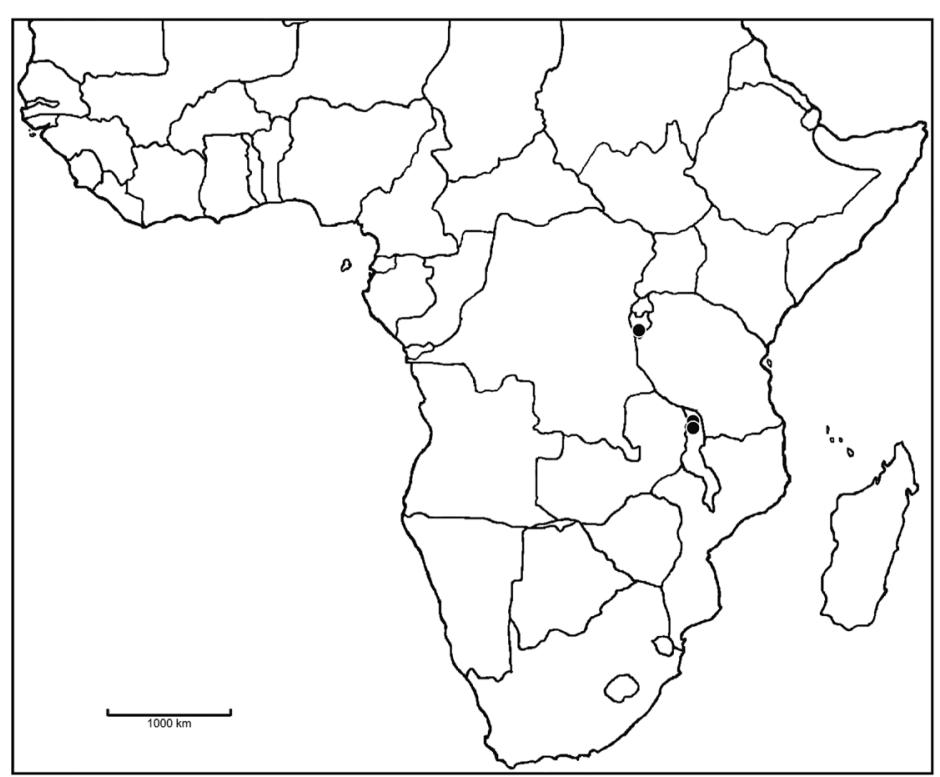

Fig. 57. Distribution of Bilabrella williamsoni (P.J.Cribb) Szlach. $\&$ Kras
8841 (BR!, K!). Malawi. Northern: Rumphi Distr., Nyika Plateau, Chelinda Bridge and Falls, in short grass above rocks, 13 Mar. 1977 (fl), Grosvenor \& Renz 1213 (K!); Nyika National Park, near Chelinda Bridge, in seepage area round rock in montane grassland, $2285 \mathrm{~m}$, 3 Mar. 1987 (fl), la Croix 1001 (K!); Nyika Plateau, 2300 m, Mar. 1967 (fl), Williamson 283 (K!).

$\mathrm{N}$ otes. Bilabrella williamsonii resembles in some respects $B$. arianae. In both species the lip is not glabrous - it is papillose in the the former, and hairy in the latter. Cribb (1977) describing Habenaria williamsonii placed it in the section Replicatae Kraenzl., stated however, that it is atypical in that most other members of the section have a reflexed dorsal sepal. Cribb (1977) noted that $H$. williamsonii may be better placed in section Productae Summerh. (now the genus Renzorchis Szlach. \& Olsz.) with $H$. singularis Summerh., which it resembles somewhat in flower shape but from which it differs vegetatively, in gynostemium architecture and in having more or less equally lobed petals. Acording to Cribb (1977) H. williamsonii is similar in having reticulate basal leaves with $H$. retinervis, but is a much smaller plant with flowers less then half the size of those of $H$. retinervis.

\subsection{Subgenus Bilabrella}

Gynostemium slender, elongate. Antherophores, stigmaphores and rostellophores longer than fertile part of anther. Caudilces longer than pollen mass.

A group with over 70 species.

\section{Key to sections and subsections}

1. Auriculae entire, not split into two lobules, sometimes irregularly sculpted sect. Bilabrella (2)

1. Auriculae more or less split into two parts sect. Biauriculatae (4)

2. Posterior petal lobes much wider than anterior one . subsect. Inaequilobae (B. inaequiloba)

2. Posterior petal lobes usually much narrower than anterior one 3

3. Anterior petal lobes at least 3 times wider than posterior one subsect. Bilabrella

3. Anterior petal lobes less than twice wider than anterior one subsect. Arachnoideae (78)

4. Lip lobed near the middle, both lateral lobes crossed below the middle lobe

subsect. Decapterae (B. decaptera)

4. Lip divided near the base 5

5. Both petal lobes lanceolate, oblong-lanceolate to oblanceolate, both lobes similar in form, usually anterior lobe smaller than posterior ones subsect. Martiales (99)

5. Both petal lobes dissimilar in form, anterior lobes usually larger than posterior ones 
6. Anterior petal lobes bilobulate subsect. Disparilae (103)

6. Anterior petal lobes entire 7

7. Petals bilobulate above basal $2-3 \mathrm{~mm}$ subsect. Dalzielae (106)

7. Petals bilobed to the base or so subsect. Biauriculatae (107)

\subsubsection{Section Bilabrella}

Auricles entire.

\subsubsection{Subsection Inaequilobae Szlach. \& Kras, subsect. nov.}

Type species: Bilabrella inaequiloba (Schltr.) Szlach. $\&$ Kras [三 Habenaria inaequiloba Schltr.].

Posterior petal lobes elliptic-ovate, anterior petal lobes subulate. Lip lateral lobes 3 times shorter than the middle one, divergent

A monotypic subsection.

20. Bilabrella inaequiloba (Schltr.) Szlach. \& KrasLap., Richardiana 6(4): 197. 2006.

Habenaria inaequiloba Schltr., Bot. Jahrb. Syst. 53: 514. 1915. - Schlechter, Repert. Sp. Nov Regni Veg. 68: tab. 27. 1932.

Type (hoc loco selectus): Tanzania, Stolz 713 (lectotype, B!; isolectotypes, $\mathrm{K}$ !, W-R!).

Stem 50-70 cm tall, slender to rather stout, erect, leafy, glabrous. Leaves 5-8, erect-patient, up to $18.5 \mathrm{~cm}$ long and $2.3 \mathrm{~cm}$ wide, linear-lanceolate, acute, gradually decreasing in size upwards. Inflorescence up to $20 \mathrm{~cm}$ long, subdensely 20-30-flowered. Floral bracts to

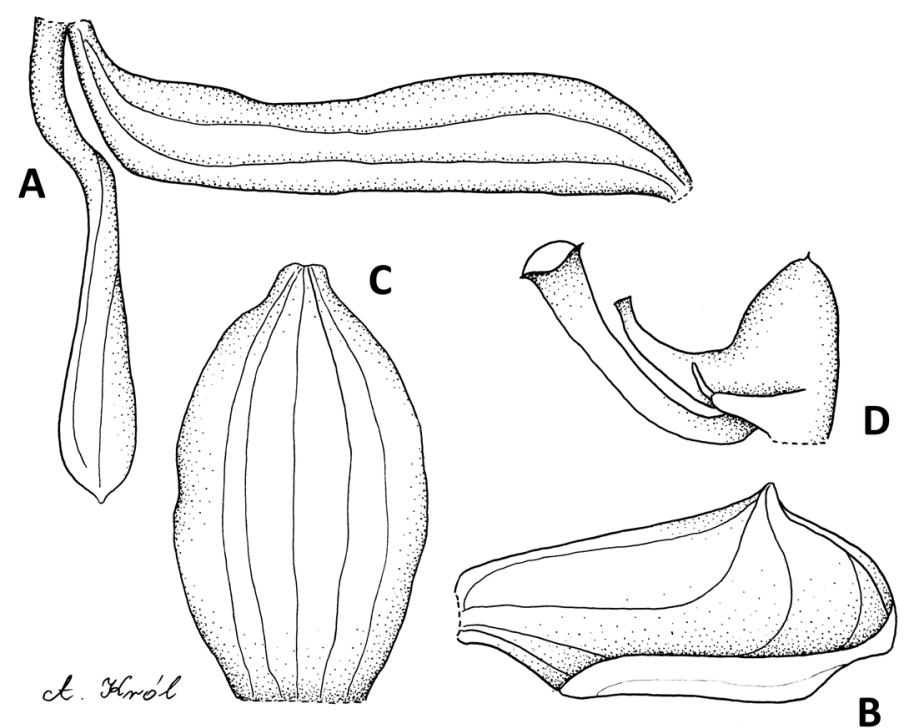

Fig. 59. Bilabrella inaequiloba (Schltr.) Szlach. \& Kras-Lap. Explanations: A - ovary and spur; B - lateral sepal; C - dorsal sepal; D gynostemium, side view (drawn by A. Król from isolectotype of Habenaria inaequiloba Schltr. - Stolz 713, W-R)

$20 \mathrm{~mm}$ long, elliptic-lanceolate, acuminate, more or less pudescent. Pedicellate ovary $20 \mathrm{~mm}$ long. Flowers greenish, brown tinged or stripped in the centre. Dorsal sepal 4-5 mm long, $3 \mathrm{~mm}$ wide, ovate, obtuse, reflexed. Petals bilobed nearly to the base; anterior lobe $5 \mathrm{~mm}$ long, $0.3 \mathrm{~mm}$ wide, subfiliform, acute; posterior lobe $4 \mathrm{~mm}$ long, $3 \mathrm{~mm}$ wide, obliquely rhomboid-ovate, obtuse. Lateral sepals 5-6.5 mm long, 4-4.5 mm wide, obliquely obovate, laterally apiculate, deflexed. Lip 3-lobed above the base; the middle lobe $5.5 \mathrm{~mm}$ long, $0.8 \mathrm{~mm}$ wide, linear-ligulate, obtuse; lateral lobes $1 \mathrm{~mm}$

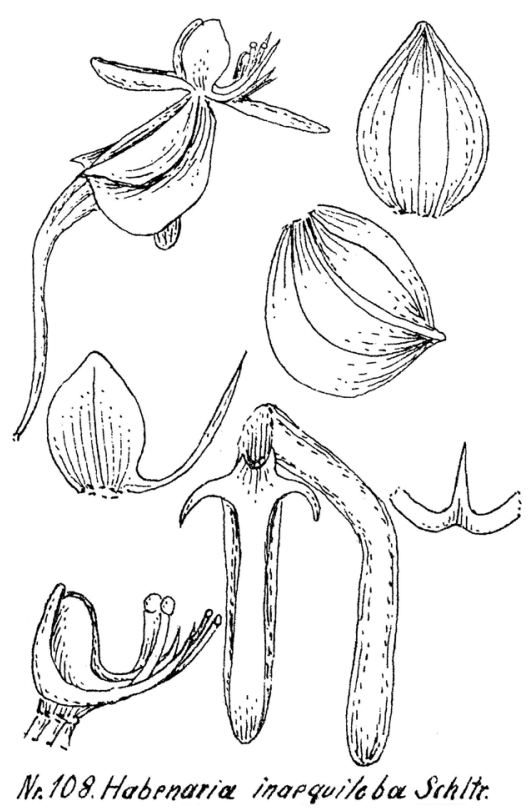

Fig. 58. Original Schlechter (1932) drawing of Habenaria inaequiloba Schltr.

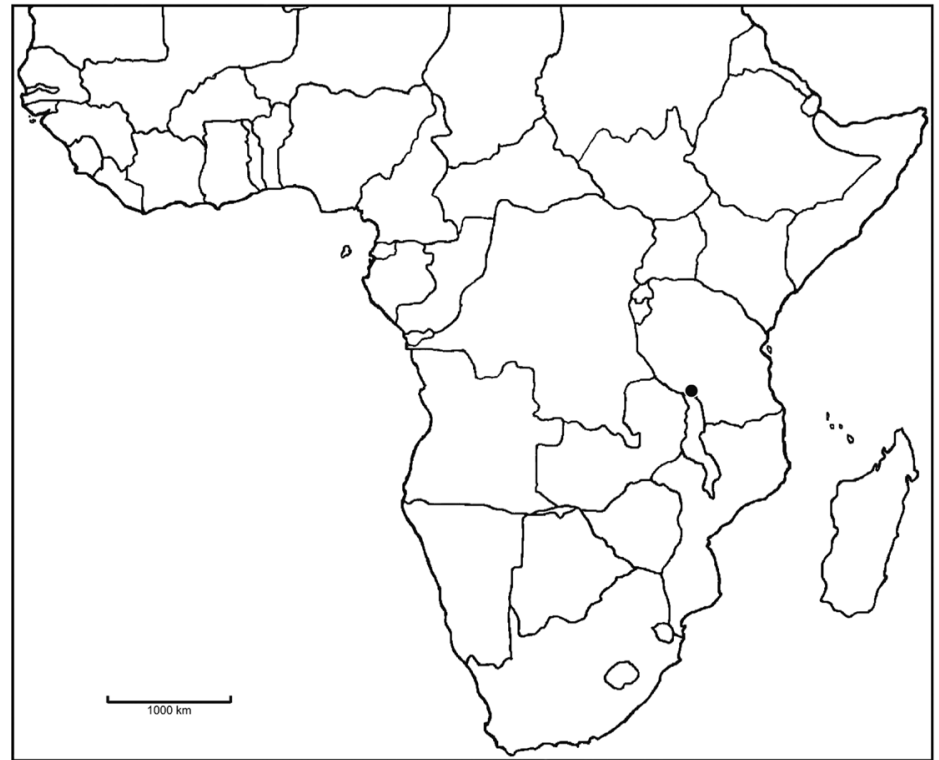

Fig. 60. Distribution of Bilabrella inaequiloba (Schltr.) Szlach. \& Kras-Lap. 
long, $0.3 \mathrm{~mm}$ wide, falcate-subulate, acute. Spur 7-9 mm long, cylindrical, obtuse, pendent. Anther $3 \mathrm{~mm}$ tall; connective rounded; antherophores $4 \mathrm{~mm}$ long, slender, gradually upcurved. Stigmaphores $4 \mathrm{~mm}$ long, narrowly clavate, truncate. Rostellum middle lobe slightly shorter than thecae. Auriculae digitate (Figs 58-59).

E c o lo gy. Upland grassland.

Distribution. Tanzania, alt. $1200 \mathrm{~m}$ (Fig. 60). Specimens examined. Tanzania. Mbeya: At mountain near Mwandemba, short way off Kyimbila, 1200 m, 1911 (fl), Stolz 713 (B!, K!, W-R!).

N otes. Schlechter (1915) compared this species to Habenaria peltastes. In our opinion, however, both species are somewhat similar in petal form only, differing clearly from one another in the lip morphology and spur form. It appears that $B$. ineaquiloba occupies rather an isolated position and is not closely related to any other species of the genus.

\subsubsection{Subsection Bilabrella}

Anterior petal lobe at least 3 times wider than posterior one.

\section{Key to species}

1. Spur distinctly shorter than pedicel and ovary ......... 2

1. Spur as long as or longer than pedicel and ovary ... 12

2. Spur prominently twisted near or below the middle .. 3

2. Spur not twisted .................................................... 5

3. Spur slightly swollen in the apical third or so .........

\section{B. incarnata}

3. Spur ampullaceous in the apical third 4

4. Spur forming a complete loop ..... 22. B. anaphysema

4. Spur without any loop .................... 23. B. confusa

5. Spur cylindrical ................................ 24. B. pauper

5. Spur filiform, apically slightly swollen ................... 6

6. Spur geniculate near the middle .... 25. B. urundiensis

6. Spur not geniculate ............................................... 7

7. Lip 3-lobed above prominent basal undivided part .. 8

7. Lip 3-lobed to the base ......................................... 9

8. Petals bilobed to the base, glabrous, anterior lobe $4 \times$ $0.3 \mathrm{~mm}$, lip middle lobe $7 \mathrm{~mm}$ long ... 26. B. pedicellaris 8. Petals bilobed to the base, ciliolate, anterior lobe 5.5-13 $\times 1-1.5 \mathrm{~mm}$, lip middle lobe 7-15 $\mathrm{mm}$ long ...... 27. B. ichneumonea

9. Anterior petal lobes linear-lanceolate, widest at the base. . 10

9. Anterior petal lobes oblong-elliptic, oblong-lanceolate or ligulate, widest near the basal third or so ........... 11

10. Petals glabrous ........................... 28. B. cornutella

10. Petals densely ciliate ................... 29. B. lisowskii

11. Petals posterior lobe papillose, spur 16-20 mm long 30. B. hochstetteriana

11. Petals posterior lobe ciliolate, spur $11.5-16.5 \mathrm{~mm}$ long. 31. B. replicata
12. Spur only slightly swollen near the apex, if any .... 13

12. Spur distinctly swollen in the apical part ........... 16 13. Anterior petal lobe $9-14 \mathrm{~mm}$ long ... 32. B. huillensis 13. Anterior petal lobes usually less than $9 \mathrm{~mm}$ long .. 14

14. Stigmaphores papillose 33. B. falcata

14. Stigmaphores glabrous 15

15. Anterior petal lobe glabrous, posterior one ciliolate all over 34. B. caffra 15. Anterior petal lobe ciliolate, posterior one ciliolate along margins only 35. B. falcicornis 16. Spur twisted 17

16. Spur not twisted ................................................ 18 17. Lateral sepals obliquely triangular-ovate, widest at the apex, anterior petal lobes twice wider than posterior one 36. B. humblotii

17. Lateral sepals obliquely oblong-ovate, widest just above the middle, anterior petal lobes ca 4-5 times wider than posterior one 37. B. humilior 18. Anterior petal lobes furculate apically, stigmaphores glabrous 38. B. nyikana 18. Anterior petal lobes entire, stigmaphores ciliolate 39. B. culcifera

21. Bilabrella incarnata (Lyall. ex Lindl.) Szlach. \& Kras-Lap., Richardiana 3(3): 140. 2003. Bonatea incarnata Lyall. ex Lindl., Gen. Sp. Orchid. P1.: 327. 1835.

Type: Madagascar, Lyall s.n. (K?).

Habenaria incarnata (Lyall. ex Lindl.) Rchb. f., Flora 48: 180. 1865. - Kraenzlin, Orchid. Gen. Sp.: 223. 1901. - Perrier de la Bathie, Fl. Madagasc. 1: 55. 1939. - Puy et al., Orchids of Madagascar: 153. 1999.

Habenaria rutenbergiana Kraenzl., Abh. Naturwiss. Vereine Bremen 7: 258. 1882.

Type (hoc loco selectus): Madagascar, Rutenberg s.n. (B+; lectotype, HBG!).

Habenaria diptera Schltr., Beih. Bot. Centralbl. 39(2): 316. 1916.

Type: Madagascar, Perrier de la Bathie $34(\mathrm{~B}+)$.

Tubers 2, ovoid to oblong. Plants $40-90 \mathrm{~cm}$ tall, stout, erect. Leaves 5-12, up to $17 \mathrm{~cm}$ long, 1-2.5 $\mathrm{cm}$ wide, linear to linear-lanceolate, acute, decreasing gradually upwards. Inflorescence 10-20 cm long, 20-40-flowered, rather dense. Flowers greenish to yellowish. Floral bracts 20-30 mm long, lanceolate, acuminate, glabrous or ciliolate. Pedicellate ovary $25-30 \mathrm{~mm}$ long, glabrous. Dorsal sepal 6-7 mm long, 2-2.2 $\mathrm{mm}$ wide, oblong to narrowly elliptic, obtuse, concave, 3-5-nerved. Petals bilobed nearly to the base; anterior lobe $6.5-7 \mathrm{~mm}$ long, 1.5-2 mm wide, oblong-lanceolate to linear-lanceolate, acuminate, glabrous; posterior lobe $6 \mathrm{~mm}$ long, $0.4 \mathrm{~mm}$ wide, linear to linear-filirom, subobtuse, ciliate. Lateral sepals 8-10 mm long, 5-6 mm wide, semi-obovate to obliquely oblong-obovate, concave, laterally apiculate. 


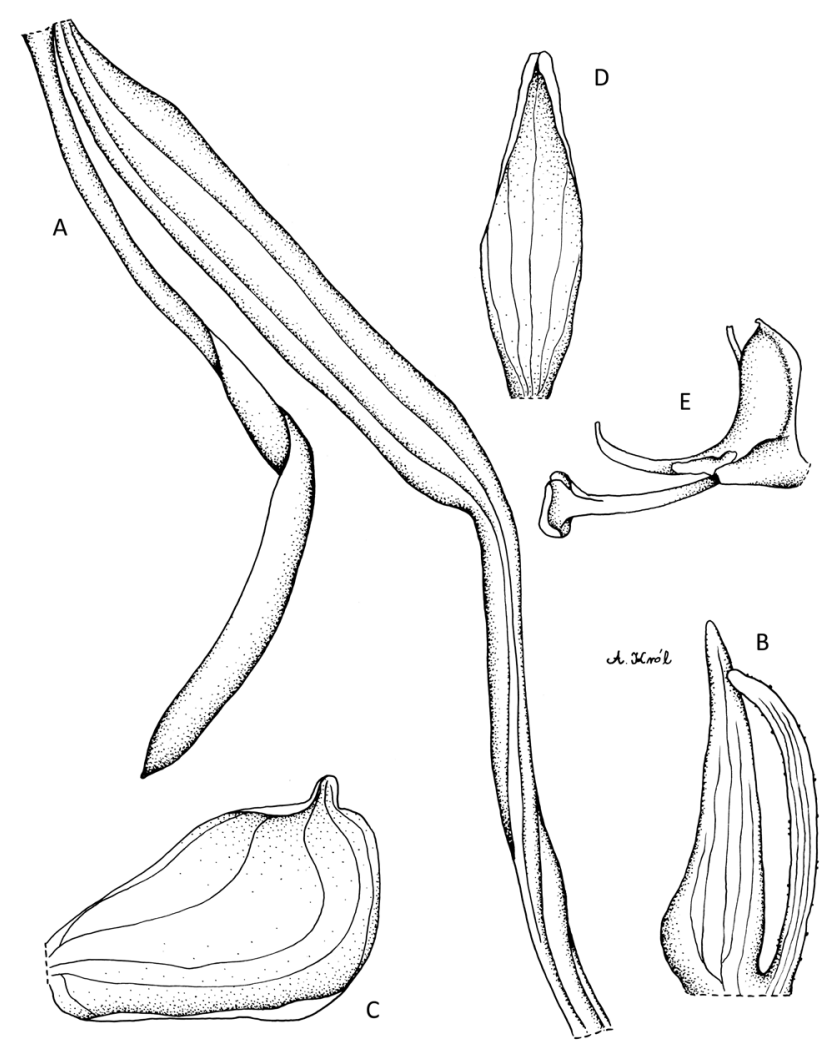

Fig. 61. Bilabrella incarnata (Lyall. ex Lindl.) Szlach. \& Kras-Lap. Explanations: A - ovary, pedicel, spur; B - petal; C - lateral sepal; D - dorsal sepal; E - gynostemium, side view (drawn by A. Król from Baron 213, E)
A

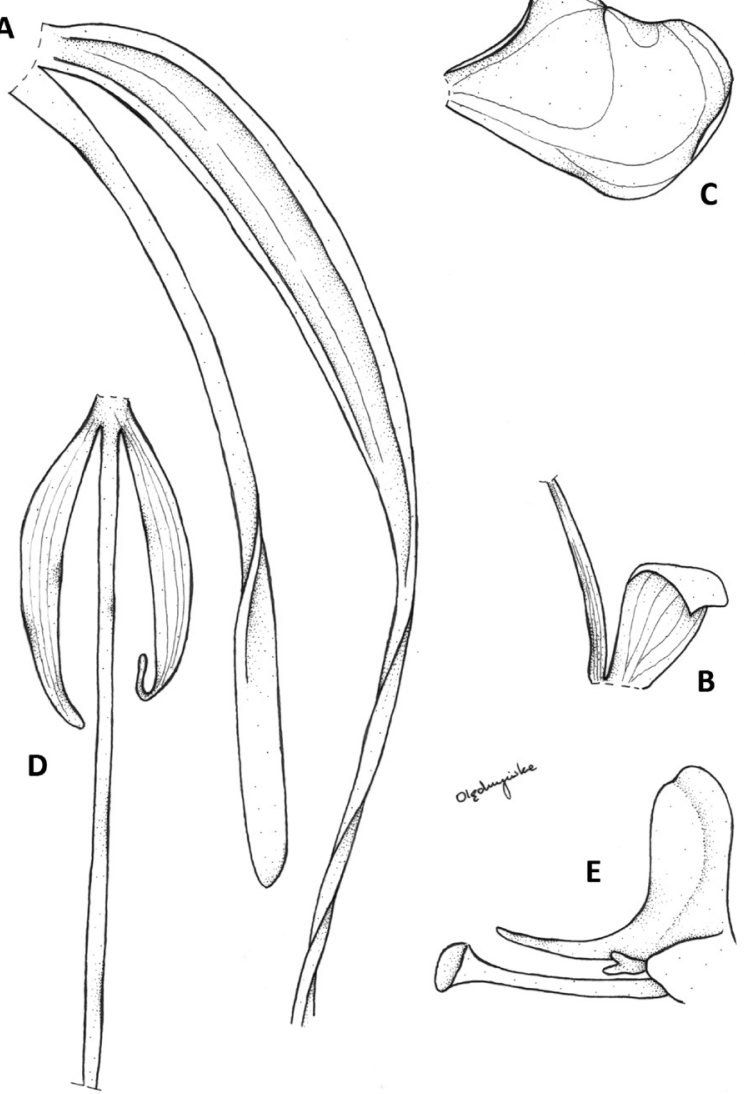

Fig. 62. Bilabrella incarnata (Lyall. ex Lindl.) Szlach. \& Kras-Lap. Explanations: A - ovary, pedicel, spur; B - petal; C - lateral sepal; D - lip; E - gynostemium, side view (drawn by N. Olędrzyńska from Scott-Elliot 2576, BM)
Lip 3-lobed above the base; the middle lobe 11-16 mm long, $0.4 \mathrm{~mm}$ wide, filiform; lateral lobes $9-10 \mathrm{~mm}$ long, $1 \mathrm{~mm}$ wide, obliquely linear or oblong-lanceolate, acute to acuminate, pendent. Spur 22-27 mm long, narrowly cylindrical, twisted near the middle, swollen in the apical half, blunt. Anther 3-4 mm tall; connective rounded, rather massive; antherophores 3-4 mm long, delicate. Stigmaphores 4-5.5 mm long, narrowly cylindrical, clavate and truncate apically. Rostellum middle lobe slightly longer than connective. Auriculae oblong-ellipsoid (Figs 61-62).

E c o logy. In grassland, forest and woodland margins, praires, margins and edges of forest. FebruaryDecember.

D is tribution. Madagascar and Comoros (Puy et al. 1999), alt. sea level-1530 m (Fig. 63).

Specimens examined. Madagascar. Antananarywa: Antananarivo, Ankazobe, Kiangara, Borikely, Tampoketsa d'Ankazobe, forest, 17'55'27'S, 4755'15"E, 1530 m, 19 Mar. 1999 (fl), Labat, Haevermans, Randrianjohany \& Rabenantoandro 2972 (K!); Fianarantsoa: Imerina, 26 Feb. 1928 (fl), Decary 6165 (K!); Imerina, Oct. 1885 (fl), Fox 42 (K!); Toliara: Cheifly in Betsileo-land, July 1880 (fl), Scott Elliot 2576 (BM!, E!, K!); Central part of the island, July 1880 (fl), Baron 213 (K!); Central part of the island, Dec. 1883 \& June 1889 (fl), Baron 2253 (K!); Sep. 1891 (fl), Baron 6092 (K!); Sine loc., Lyall 179 (K!); Sine loc., Pettersson \& Nilsson 209 (K! \& spirit.); Sine loc., Pettersson \& Nilsson 2676 (BM!, E!).

Notes. Reichenbach (1865) describing his Habenaria incarnata compared it with $H$. sochensis and $H$. isoantha. $H$. incarnata differs from the former by having twisted spur, obliquely linear or oblong-lanceo-

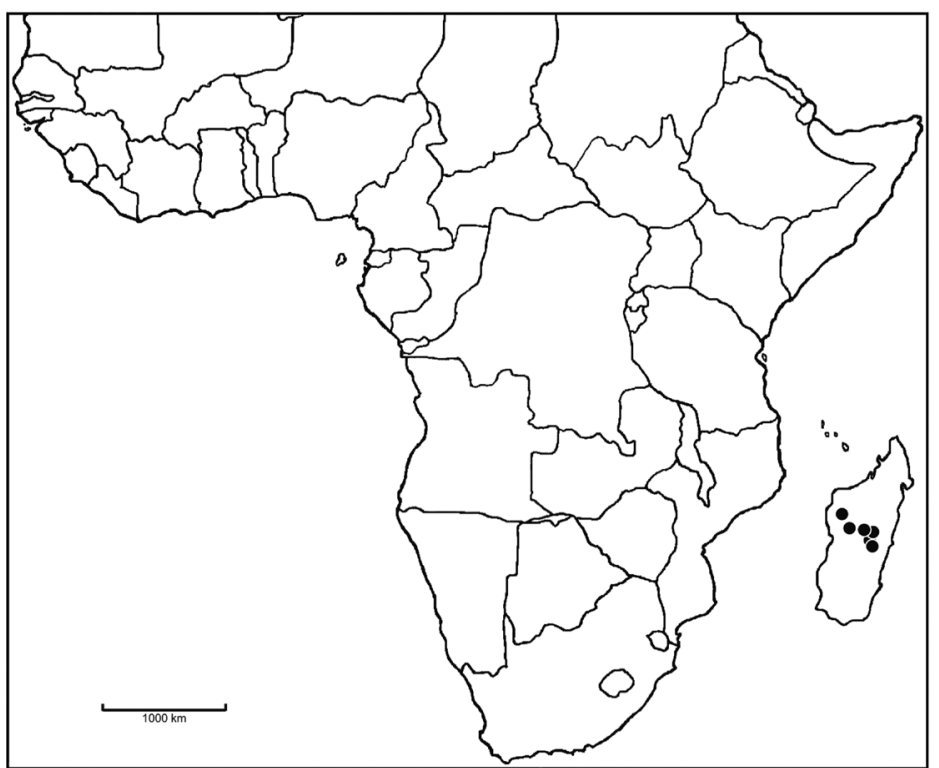

Fig. 63. Distribution of Bilabrella incarnata (Lyall. ex Lindl.) Szlach. \& Kras-Lap. 
late lip lateral lobes and different proportion between anther fertile part and anther and stigma projections. Additionally it can be separated from $H$. isoantha by the form of petal lobes.

Bilabrella incarnata can be easily separated from other members of this subsection having twisted spur by its length - it is the only species in which the spur is shorter than pedicellate ovary.

22. Bilabrella anaphysema (Rchb. f.) Szlach. \& KrasLap., Richardiana 3(3): 139. 2003. - Szlachetko et al., Orchid. of West-Central Africa 1: 214. 2010. - Rolfe, Fl. Trop. Afr. 7: 235. 1898. - Kraenzlin, Orchid. Gen. Sp.: 225. 1901. - Suessenguth \& Merxmuller, Contrib. Fl. Marandellas Distr.: 83. 1951. - Robyns \& Tournay, Fl. Parc Natl. Albert 3: 432. 1955. - Saeger, Exploration du Parc National de la Garamba: 251. 1956. - Goodier \& Phipps, Kirkia 1: 52. 1961. - Summerhayes, FTEA, Orchid. 1: 95. 1968b. - Grosvenor, Excelsa 6: 83. 1976. - Williamson, Orchid. S. Centr. Afr.: 56. 1977. - Geerinck, Fl. Afr. Centr., Orchid. 1: 105. 1984. - la Croix \& Cribb, Fl. Zambes., Orchid. 11: 88. 1995.

Habenaria anaphysema Rchb. f., Flora 50: 101. 1867.

Type: Angola, Welwitsch 744 (lectotype, designated by Szlachetko et al. [2010: 214], W-R!; isolectotypes, BM!, LISU not seen, drawing: K!).

Tuber $0.7-1.5 \mathrm{~cm}$ long, $0.5-1 \mathrm{~cm}$ in diameter, ellipsoid. Stem $15-60 \mathrm{~cm}$ tall, erect, slender, glabrous. Leaves 6-12, 5-12 cm long, 0.3-1.1 cm wide, linear or narrowly linear, acute, suberect to spreading, the upper ones much smaller. Inflorescence $5-23 \mathrm{~cm}$ long, 8-24-flowered, rather loose to subdense. Flowers green, sometimes fragrant at night. Floral bracts $5-10 \mathrm{~mm}$ long, ovate-lanceolate, acuminate. Pedicel and ovary 12-14 mm long, glabrous. Dorsal sepal 5-6.5 mm long, 2-3.5 mm wide, elliptic, concave, obtuse, glabrous, reflexed. Petals bilobed nearly to the base; anterior lobe $8-12 \mathrm{~mm}$ long, 1-2 mm wide, obliquely narrowly lanceolate, acute to acuminate, curved downward, almost glabrous or ciliolate at the base only; posterior lobe 4-5 mm long, 0.3-0.5 mm wide, linear to filiform, acute, recurved, rarther thin, ciliolate. Lateral sepals $7 \mathrm{~mm}$ long, 4.5-5 mm wide, obliquely obovate to elliptic, concave, with prominent lateral apiculus, glabrous. Lip 3-lobed from an undivided basal part 1-2 mm long, deflexed; the middle lobe 8-10.5 mm long, 0.5-0.8 mm wide, linear-lanceolate, acute or obtuse; lateral lobes 4-6.5 mm long, $0.5 \mathrm{~mm}$ wide, linear, acute, pendent. Spur 7-12 mm long, forming a complete loop in the apical part, distinctly swollen in the apical quarter, ampullaceous, $1.5 \mathrm{~mm}$ in diameter, blunt. Anther $2.5 \mathrm{~mm}$ long; connective truncate or slightly notched apically; antherophores $4 \mathrm{~mm}$ long, slender. Stigmaphores 3-4 mm long, narrowly cylindrical. Rostellum

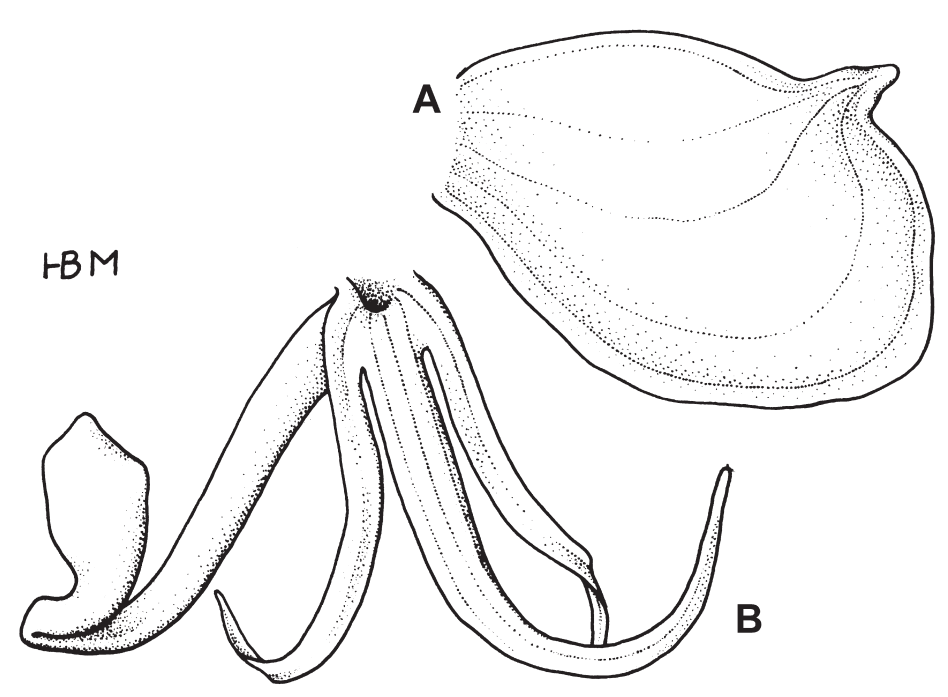

Fig. 64. Bilabrella anaphysema (Rchb. f.) Szlach. \& Kras-Lap. Explanations: A - lateral sepal; B - lip and spur (drawn by H. B. Margońska from lectotype of Habenaria anaphysema Rchb. f. - Welwitsch 744, W-R)

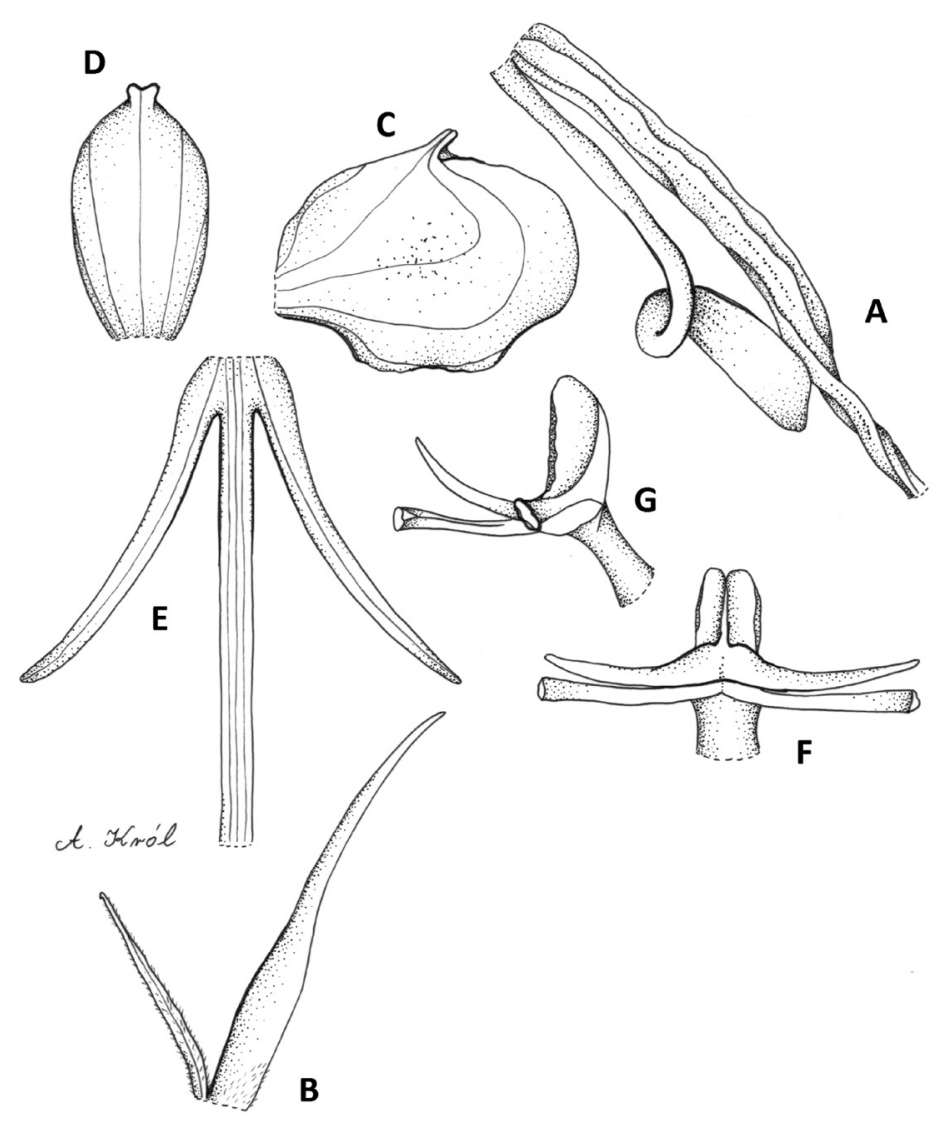

Fig. 65. Bilabrella anaphysema (Rchb. f.) Szlach. \& Kras-Lap. Explanations: A - ovary, pedicel, spur; B - petal; C - lateral sepal; D dorsal sepal; E - lip; F - gynostemium, front view; G - gynostemium, side view (drawn by A. Król from Habenaria anaphysema Rchb. f. - Lisowski, Malaisse \& Symoens 13.112, UGDA-DLSz)

middle lobe shorter than thecae. Auriculae oblongellipsoid (Figs 64-65).

E c o log y. Terrestrial in swamp and seasonally flooded grassland, perennially very wet upland bog, savannah. Flowering in September-August. 


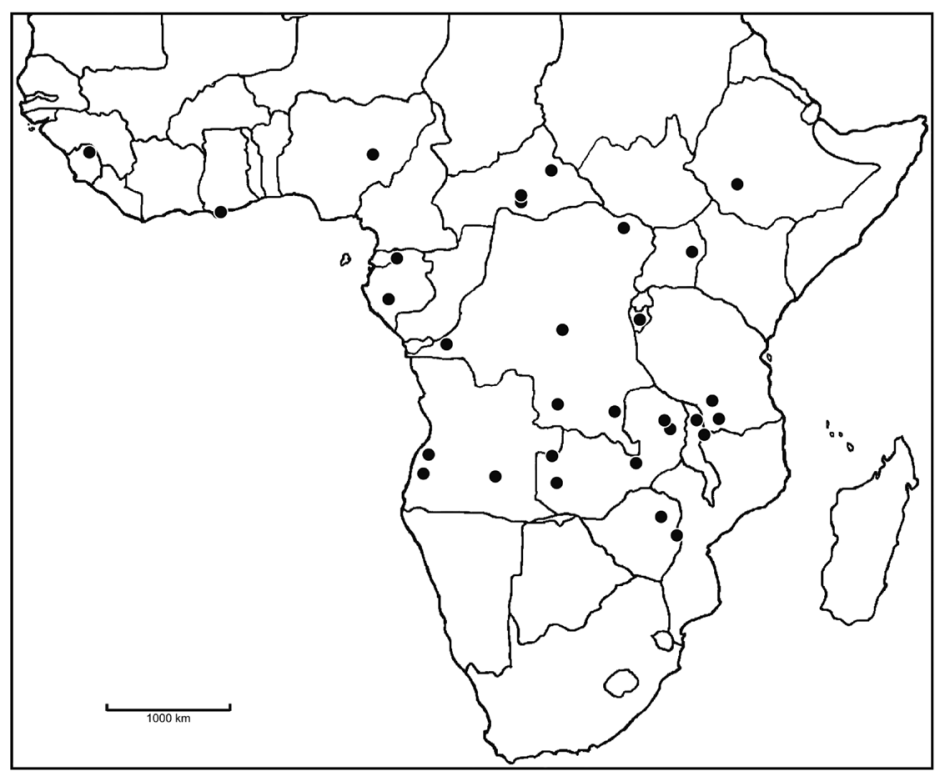

Fig. 66. Distribution of Bilabrella anaphysema (Rchb.f.) Szlach. \& Kras-Lap.

Distribution. Sierra Leone, Ghana, Nigeria, Central African Republic, Ethiopia, Gabon, Congo, Democratic Republic of the Congo (Zaire), Uganda, Burundi, Tanzania, Angola, Zambia, Malawi, Zimbabwe, alt. 7,5-4200 m (Fig. 66).

S pec imens ex a m ined. Sierra Leone. Northern: Koinadugu Distr., Loma Mts., high altitude grassland, 14 Sep. 1945 (fl), Jaeger 1663 (K!) (as H. genuflexa). Ghana. Western: Sekondi-Takoradi Distr., Takoradi, 7.5 m, Aug. 1941 (fl), Bunting 70 (BM!); SekondiTakoradi Distr., Takoradi, 7.5 m, Aug. 1941 (fl), Vigne 4785 (BM!). Nigeria. Northern \& Plateau: In swamp, 1290 m, Aug. 1962 (fl), King 175 (K!) (as H. genuflexa). Central African Republic: Haute-Kotto: Upper Kotto River, Pawa River marsh, tributary of Banga River, $90 \mathrm{~km} \mathrm{~W}$ of Yalinga (Cribb \& Fay 1986), 23 Aug. 1921 (fl), Le Testu 3126 (BM!); Ouaka: 60 km N de Bambari (Cribb \& Fay 1986), Le Testu 1575 (K!); Riv. Gumbura, 60 km N. Bambari, 7 Apr. 1924 (fl), Le Testu 1.975 (BM!); 30 km S Ippy (Cribb \& Fay 1986), Le Testu 1508 (K!). Ethiopia. Amhara: Waka and Chad, Le Testu 1.908 (BM!); Waka and Chad, 7 Apr. 1924 (fl), Le Testu 1.975b (BM!); Waka and Chad, 8 Oct. 1931 (fl), Le Testu 2.935 (BM!). Gabon. Ngounie: Ngounye Mountain, 17 Nov. 1924 (fl), Le Testu 5.061 (BM!, K!) (as H. genuflexa); Woleu-Ntem: Between R. Waleu \& R. Ntem. "pelonses du rocher de Ngolayop", 14 May 1933 (fl), Le Testu s.n. (K!); Circonscription around WoleuNtem, 12 May 1933 (fl), Le Testu 9.120 (BM!, K!) (as H. genuflexa). Democratic Republic of the Congo (Zaire). Kinhasa: Kisantu, Luvu (Mayombe), Savannah, 26 Dec. 1952 (fl), Callens 3811 (K!); Haut-Lomami: Kundelungu Plateau, $2 \mathrm{~km}$ from the source of Lutshipuka,
1600 m, 8 Jan. 1971 (fl), Lisowski, Malaisse \& Symoens 13.112 (BR!, UGDA-DLSz!); Lomami, Daneloj 372 (BRLU!); 1988 (fl), Sita \& Moutsambote s.n. Uganda. Eastern: Katakwi Distr., Angole. Itema country at Bugongi in grass savannah, fairly frequent, $1500 \mathrm{~m}, 19$ Feb. 1930 (fl), Snowden 1649 (BM!). Burundi. Bururi: Colline Rwamunyinya, grass with Exotheca and Eragrostis, 400'S, 294'ㄹ, 2000 m, Reekmans 8698 (K!) (as H. genuflexa). Tanzania. Iringa: Njombe Distr., Mwakete-Njombe Road, in wet swamp among grass, 2100 m, 17 Jan. 1957 (fl), Richards 7848 (K!); Njombe Distr., Dansland, dambo - fairly damp situation - with H. schimperiana in thin grass, 2160 m, 29 Jan. 1989 (fl), Spurrier 48 (K!) (as H. genuflexa); Ruvuma: Songea Distr., Ulamboni Valley about $11 \mathrm{~km}$ W of Songea in grey sand, seasonally flooded grassland, at present reasonably dry, 960 m, 31 Dec. 1955 (fl), Milne-Redhaed \& Taylor 7977 (AMES!, BR!, K!, K! - spirit.). Angola. Benguella: Country of the Ganguellas and Ambuellas, Gossweiler 3307 (K!); Huila: Between Humpata and Nene, 1250-1800 m, Apr. 1860 (fl), Welwitsch 744 (BM!, K! - drawing, LISU (fide Summerhayes 1968b), W-R!); Rio Nene, Near Humpata, 2000 m, 16 May 1937 (fl), Exell \& Mendonca 1984 (K!); Kuando-Kubango: Rio Quiriri, 7 Nov. 1906 (fl), Gossweiler 3910 (K!). Zambia. Central: Mkushi, dambo, Dec. 1967 (fl), Williamson 645 (K!); Northern: Kasama Distr., 8 km N of Kasama, 6 Dec. 1960 (fl), Robinson 4153 (BR!, K! \& spirit.); Mporokoso Distr., Kalungwishi Headwaters at Lipangala (Mipangala) confluence, Kashama (Bemba), peaty dambo, 9 92 $52.6^{\prime} \mathrm{S}, 30^{\circ} 21.5^{\prime} \mathrm{E}, 1540 \mathrm{~m}, 8 \mathrm{Jan} .2000$ (fl), Bingham \& Beel 12050 (K!); Lake Young, Shiwa $\mathrm{Ngandu}$, in swamp grassland on lake shore, $1350 \mathrm{~m}$, 14 Jan. 1959 (fl), Richards 10737A (K!); NorthWestern: Balovale, permanent seepage, edge of dambo Kucheka Valley, 1 Nov. 1961 (fl), Holmes 0312 (K!); Western: Milimo Dambo, 6 miles on Abercon Road ex Kasama, marshy ground, 4200 m, Dec. 1953 (fl), Benson 59 (BM!); The same locality, Benson 60 (BM!). Malawi. Northern: Nkhata Bay Distr., Mzenga Estate, in dambo, 600 m, 7 Jan. 1987 (fl), la Croix 927 (K!); Nyika Plateau, Zovo-chipolo perennial bog, $2 \mathrm{~km} \mathrm{NE}$ of Zambian Rest House, 2100 m, 7 Jan. 1982 (fl), DowsettLemaire 195 (K!); 15 Jan. 1982 (fl), Dowsett-Lemaire 233 (K!). Zimbabwe. Harare: Salisbury, Luelzelter Distr., Chimanimani Mts., Bundi upper Plateau, in valley, 1560-1650 m, 30 Jan. 1958 (fl), Hall 219 (K!); Salisbury, Marandellas Distr., in vlei, 1440 m, 20 Jan. 1952 (fl), Corby 759 (35973) (K!); Salisbury, Melsetter, Chimanimani Mts., Stonghenge Plateau, boggy area at the edge of Themeda veld, $1650 \mathrm{~m}, 1$ Feb. 1957 (fl), Phipps 369 (K!); Salisbury, Mkushi, in dambo, $1200 \mathrm{~m}$, 5 Jan. 1958 (fl), Robson 2646 (K!); Manicaland: Melsetter Distr., Chimanimani Mts., in damp sponge, Jan. 1954 (fl), Ball 188 (K!). Sine loc., Welwitsch 70 (HBG!). 
Notes. This is the only member of the genus with spur forming a complete loop. Somewhat similar to this species is $B$. confusa with ampullaceous spur apex, which does not form, however, a complete loop. Additionally, petals of $B$. confusa are ciliolate on both lobes, whereas in $B$. anaphysema only posterior petal lobe is ciliolate. The anterior one can either be almost glabrous or ciliolate at the base only.

23. Bilabrella confusa (Rolfe) Szlach. \& Kras, Richardiana 9(4): 158. 2009.

Habenaria confusa Rolfe, Fl. Trop. Afr. 7: 241. 1898.

Type (Szlachetko 2007): Angola, Welwitsch 681 (lectotype, designated by Szlachetko [2007], BM!; isolectotypes, $\mathrm{K}$ !, P!, W-R!, COI not seen).

Tuberoids $2.5 \mathrm{~cm}$ long, $1 \mathrm{~cm}$ in diameter, ellipsoid. Stem $45-75 \mathrm{~cm}$ high, erect, rather slender, glabrous. Leaves 3-4, 11-17 cm long, 0.8-1.5 cm wide, linear-lanceolate, acute, on the lower part of the stem, decreasing in size upwards. Inflorescence 7-13 cm long, laxly 11-17-flowered. Flowers green. Floral bracts 7-10 mm long, ovate-lanceolate, acute to acuminate, glabrous. Pedicel 10-15 mm long, ovary $7 \mathrm{~mm}$ long, glabrous. Dorsal sepal $5 \mathrm{~mm}$ long, 2-3 mm wide, elliptic-oblong, subacute to subobtuse, concave. Petals bilobed to the base, ciliolate; anterior lobe $7.5 \mathrm{~mm}$ long, $0.5 \mathrm{~mm}$ wide, linear-lanceolate, acuminate; posterior lobe $3.5 \mathrm{~mm}$ long, $0.2 \mathrm{~mm}$ wide, filiform, subobtuse to acute. Lateral sepals $5.5-7 \mathrm{~mm}$ long, $3.5-4 \mathrm{~mm}$ wide, obliquely elliptic-obovate to semi-orbicular, concave, with laterally placed apiculus. Lip 3-lobed above the basal $2 \mathrm{~mm}$; lateral lobes $6 \mathrm{~mm}$ long, 0.3-0.4 mm wide, linearfiliform, acute, pendent; the middle lobe $9 \mathrm{~mm}$ long,

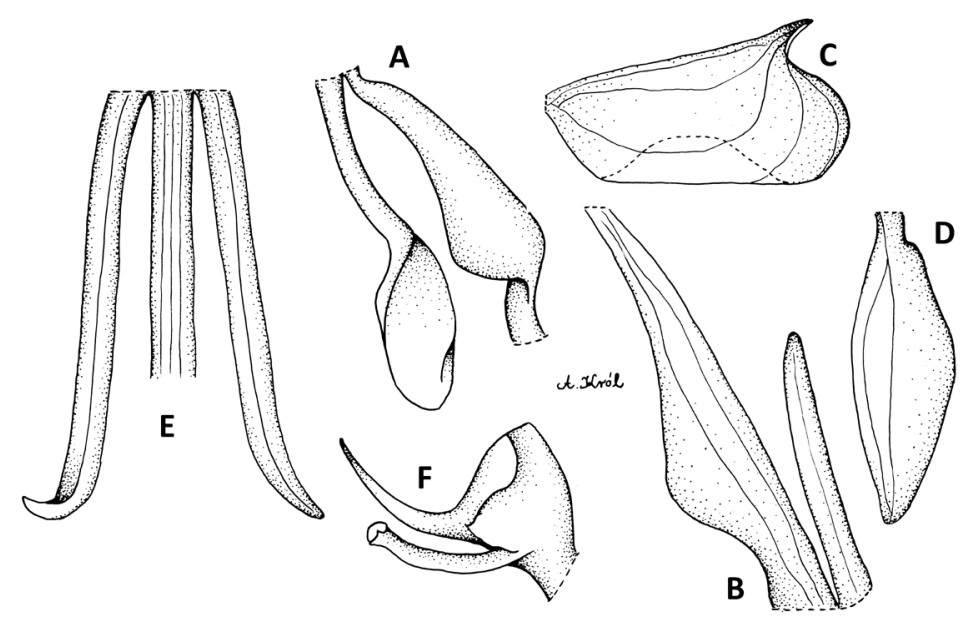

Fig. 67. Bilabrella confusa (Rolfe) Szlach. \& Kras

Explanations: A - ovary, pedicel, spur; B - petal; C - lateral sepal; D dorsal sepal; E - lip; F - gynostemium, side view (drawn by A. Król from isolectotype of Habenaria confusa Rolfe - Welwitsch 681, K)

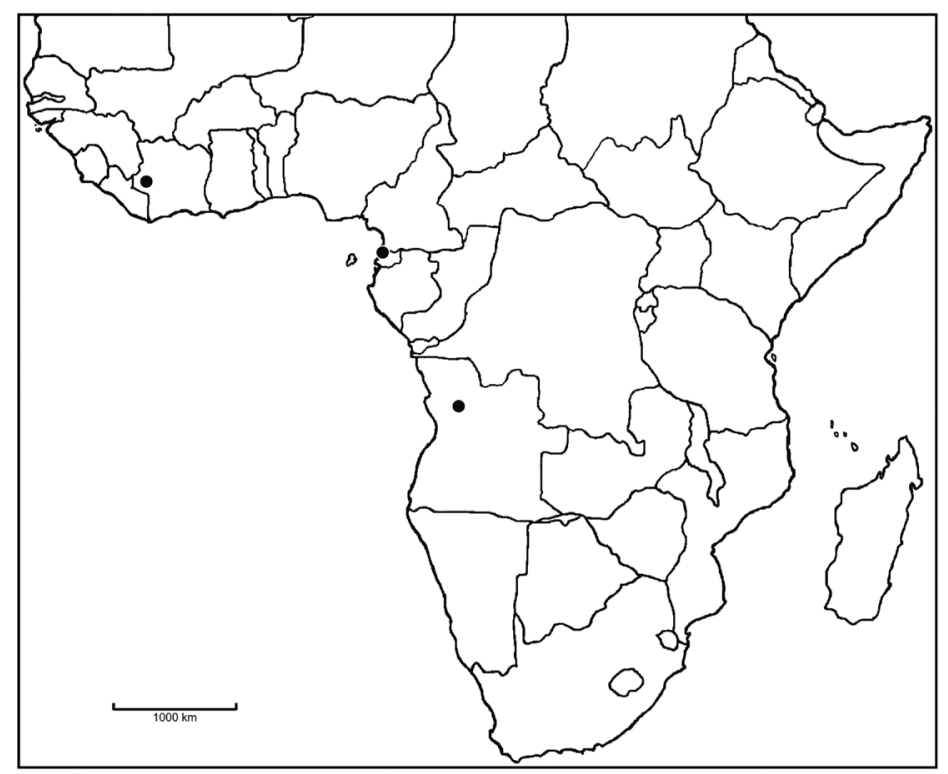

Fig. 68. Distribution of Bilabrella confusa (Rolfe) Szlach. \& Kras

0.3-0.5 mm wide, linear-filiform, acute. Spur 8.5-11 mm long, narrowly cylindrical, half twisted near apical third, oblong-saccate above, obtuse to subobtuse. Anther $2 \mathrm{~mm}$ long; connective shortly triangular; antherophores $4 \mathrm{~mm}$ long, slender, gently upcurved. Stigmaphores $3.5 \mathrm{~mm}$ long, cylindrical, truncate, distinctly shorter than antherophores. Rostellum middle lobe shorter than thecae. Auriculae tiny, digitate (Fig. 67).

E c o logy. In fissures of rocks. January-December. D i stribution. Ivory Coast, Equatorial Guinea, Angola, alt. 720-1140 m (Fig. 68).

$\mathrm{S} p$ e cimens examined. Ivory Coast. DixHuit Montagnes: Man Department, Road to the Mont Tonkui, 5 Dec. 1962 (fl), Jangoua 680 (BRLU!). Equatorial Guinea. Litoral: Ayamiken, Rio Campo, $2^{\circ} 07^{\prime} \mathrm{N}$, $10^{\circ} 01$ 'E, 30 May 2000 (fl), Perez Viso 2817bis (MA!). Angola. Malanje: Pungo Adongo, near the River Pedra de Sougue, in fissures of rocks, 720-1140 m, Jan. 1857 (fl), Welwitsch 681 (BM!, K!).

N ot e s. According to Rolfe (1898) it is more robust plant than B.ichneumonea, with which it can be confused; the leaves are wider, the flowers much larger, and the spur much more strongly clavate. $B$. confusa is somewhat similar to $B$. incarnata, but differs in having shorter spur, different form of lip and petal lobes, and in longer antherophores than stigmaphores. B. confusa appears to be similar to $B$. anaphysema. The latter, however, has a spur forming a complete loop and petals with ciliolate posterior lobe, but with almost glabrous or only basally ciliolate anterior one.

24. Bilabrella pauper (Summerh.) Szlach. \& Kras-Lap., Richardiana 3(3): 142. 2003. - Szlachetko et al., Orchid. of West-Central Africa 1: 223. 2010. 
Habenaria pauper Summerh., Kew Bull. 1932: 341. 1932. - Summerhayes, FWTA, ed. 1, 2: 412. 1936. Saeger, Exploration du Parc National de la Garamba: 255. 1956. - Summerhayes, FWTA, ed. 2, 3: 196. 1968a. - Summerhayes, FTEA, Orchid. 1: 84. 1968b. - Geerinck \& Coutrez, Not. Taxon. Orchid. Afr. Centr., Habenaria: 10. 1977. - Geerinck, Fl. Afr. Centr., Orchid. 1: 116. 1984. - Szlachetko \& Olszewski, Fl. Cam., Orchid. 34(1): 169. 1998.

Type (Summerhayes 1968b, as holotype): Cameroon, Maitland 1562 (lectotype, designated by Summerhayes [1968b: 196], K!).

Tubers $1.5 \mathrm{~cm}$ long, $0.5 \mathrm{~cm}$ in diameter, narrowly ellipsoid. Stem $25-65 \mathrm{~cm}$ tall, erect, slender, glabrous. Leaves 6-12, 5-20 cm long, 0.6-1.2 cm wide, grass-like, acute, suberect, decreasing in size up the stem. Inflorescence 3-14 cm long, 5-40-flowered, rather dense to lax. Flowers white or greenish-white. Floral bracts $5-13 \mathrm{~mm}$ long, ovate-lanceolate, acuminate, along margins and inwards sparsely glandular. Pedicel and ovary 8-14 mm long, glabrous. Dorsal sepal 3-4 mm long, 1.7-2 mm wide, elliptic, oblong-obovate, apiculate to obtuse, cochleate, glabrous, erect or reflexed. Petals bilobed to the base, rather fleshy, ciliate; anterior lobe $2.7-3 \mathrm{~mm}$ long, 1-1.6 mm wide, widely ovate-oblanceolate or obcuneate, sometimes shallowly bilobed at the apex; posterior lobe 2.5-4 $\mathrm{mm}$ long, 0.3-0.5 mm wide, linear, obtuse. Lateral sepals 3-5 mm long, $3 \mathrm{~mm}$ wide, obliquely obovate or semi-orbicular, apical margin involute, apiculus laterally placed, glabrous. Lip 3-lobed above the basal undivided part ca. $1 \mathrm{~mm}$ long, rather

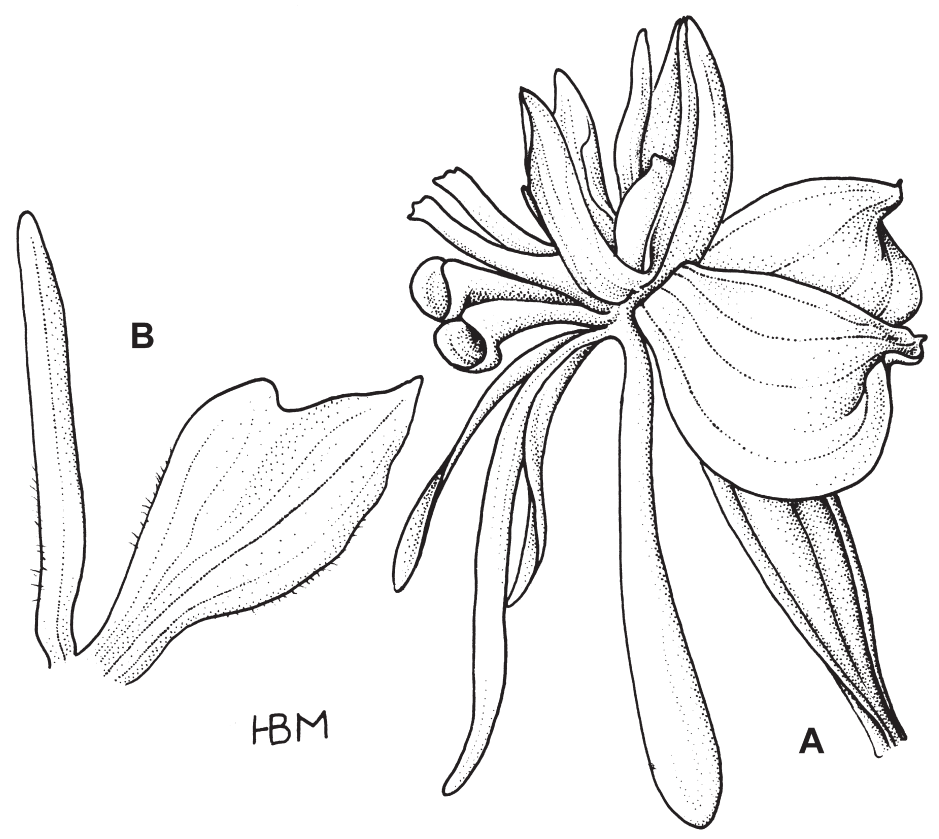

Fig. 69. Bilabrella pauper (Summerh.) Szlach. \& Kras-Lap. Explanations: A - flower; B - petal (drawn by H.B. Margońska from lectotype Maitland $1562, \mathrm{~K}$ )

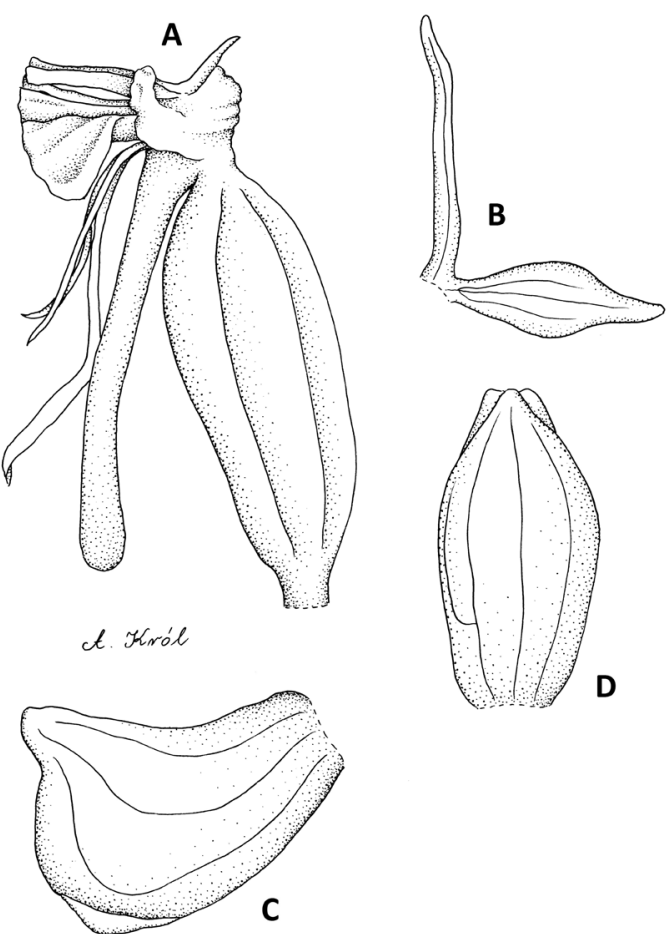

Fig. 70. Bilabrella pauper (Summerh.) Szlach. \& Kras-Lap. Explanations: A - ovary, gynostemium, spur and lip; B - petal; C - lateral sepal; D - dorsal sepal (drawn by A. Król from lectotype of Habenaria pauper Summerh. - Maitland 1562, K)

fleshy, delicate, glabrous; the middle lobe $5-6.5 \mathrm{~mm}$ long, 0.3-0.5 mm wide, linear, obtuse to acute; lateral lobes 3-4 mm long, $0.2 \mathrm{~mm}$ wide, filiform, subobtuse, pendent. Spur 6-8.2 $\mathrm{mm}$ long, $0.6 \mathrm{~mm}$ in diameter, cylindrical, with a slightly swollen apex, sometimes slightly twisted, parallel to the ovary. Anther 1.5-3.5 mm long; connective rounded; antherophores ca. 2.5-3.5 mm long, nearly as long as stigmaphores. Stigmaphores very massive, much widening towards the apex. Rostellum middle lobe shorter than thecae. Auriculae ellipsoid (Figs 69-71).

E c o lo gy. Humid savannah, marshy ground. MayDecember.

D i s tr ibut i o n. Guinea, Nigeria, Cameroon, Democratic Republic of the Congo (Zaire), Uganda, Rwanda, Burundi, Tanzania, alt. 700-1650 m (Fig. 72).

Specimens examined. Guinea. Labé: Sere bowal, abnormal form, Oct. 1898 (fl), Maclaud 232 (P!); Mamou: Dalaba Prefecture, Dalaba, abnormal form, July 1912 (fl), Caille 2 (P!); Mamou Prefecture, Timbo, Fouta Djallon, humid bowal, 22 Sep. 1958 (fl), Adam 14579 (P!); Mamou Prefecture, Boval, Timbo, abnormal form, 10 Aug. 1898 (fl), Maclaud 270 (P!). Nigeria. Northern: Adamawa State, Mambila Plateau, Mayo Daga, grassland slope, $7^{\circ} \mathrm{N}, 11^{\circ} 10^{\prime} \mathrm{E}, 1650 \mathrm{~m}, 2$ July 1958 (fl), Chapman l (K!). Cameroon. Adamawa: Takouli-mayo, near confluence mayo, $20 \mathrm{~km}$ N Banyo, 5 June 1967 (fl), Letouzey 8505 (P!); East: Betare Oya, July 1939 (fl), Jacques-Felix 4583 (K!, P!); Northwest: 


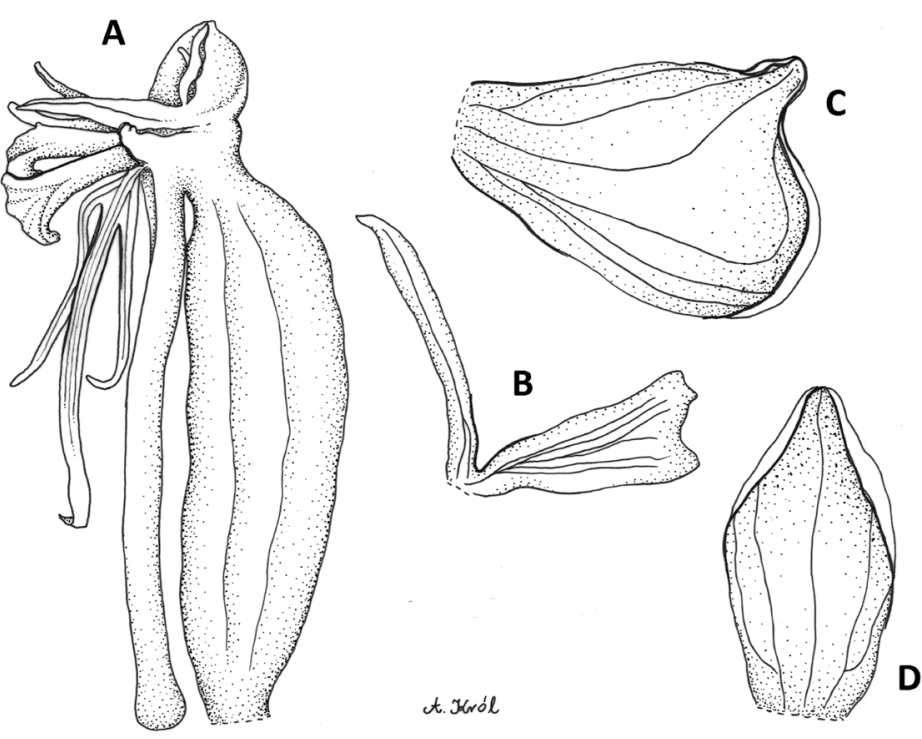

Fig. 71. Bilabrella pauper (Summerh.) Szlach. \& Kras-Lap. Explanations: A - ovary, gynostemium, spur and lip; B - petal; C - lateral sepal; D - dorsal sepal (drawn by A. Król from Jacques-Felix 4583, P)

Bamenda, 1600 m, May 1931 (fl), Maitland 1562 (BR!, K!). Democratic Republic of the Congo (Zaire). HautUele: Garamba National Park, Ndiwili, 23 May 1951 (fl), Saeger 001183 (BR!); Garamba National Park, 700-800 m, 3 July 1952 (fl), Troupin 1367 (BR!); Garamba National Park, main road, 700-800 m, 17 July 1952 (fl), Troupin 1568 (BR!, K!). Garamba National Park, main road, $30 \mathrm{~km}$ of Garamba valley, 700-800 m, 6 Aug. 1952 (fl), Troupin 1778 (BR!, K!); NordUbangi: Buda (territoire Basobolo), 8 July 1957 (fl), Evrard 2.444 (BR!). Uganda. West Nile: 2 miles NW Manacha West Camp, marshy ground, on the side of a hill west, 1380 m, 21 July 1953 (fl), Chancellor 16 (K!). Rwanda. South: Huye Distr., Mugombwa, 23 Dec. 1990 (fl), Arbonnier 152 (BR!); 27 Dec. 1992 (fl), Arbonnier 378 (BR!). Burundi. Bururi: Nyanza lake, plain, bog, 830 m, 22 Dec. 1969 (fl), Lewalle 4230 (BR!). Tanzania. Kigoma: Kazulu, 1440 m, 22 Dec. 1930 (fl), Rounee D2 (K!).

N o t e s. Bilabrella pauper is very characteristic by its peculiar gynostemium structure, especially very massive stigmaphores. According to Summerhayes (1932) it appears to be closely related to Habenaria welwitschii, but has cylindrical spur, shorter than pedicel and ovary. Additionally, both species are easily distinguishable by the form of petals. B. pauper is somewhat similar to $B$. inaequiloba, but has distinctly formed lip and petals.

25. Bilabrella urundiensis (Summerh.) Szlach. \& Kras, Richardiana 9(4): 159. 2009.

Habenaria urundiensis Summerh., Kew Bull. 16: 301. 1962.

Type: Burundi, Germain 6531 (holotype, K!).

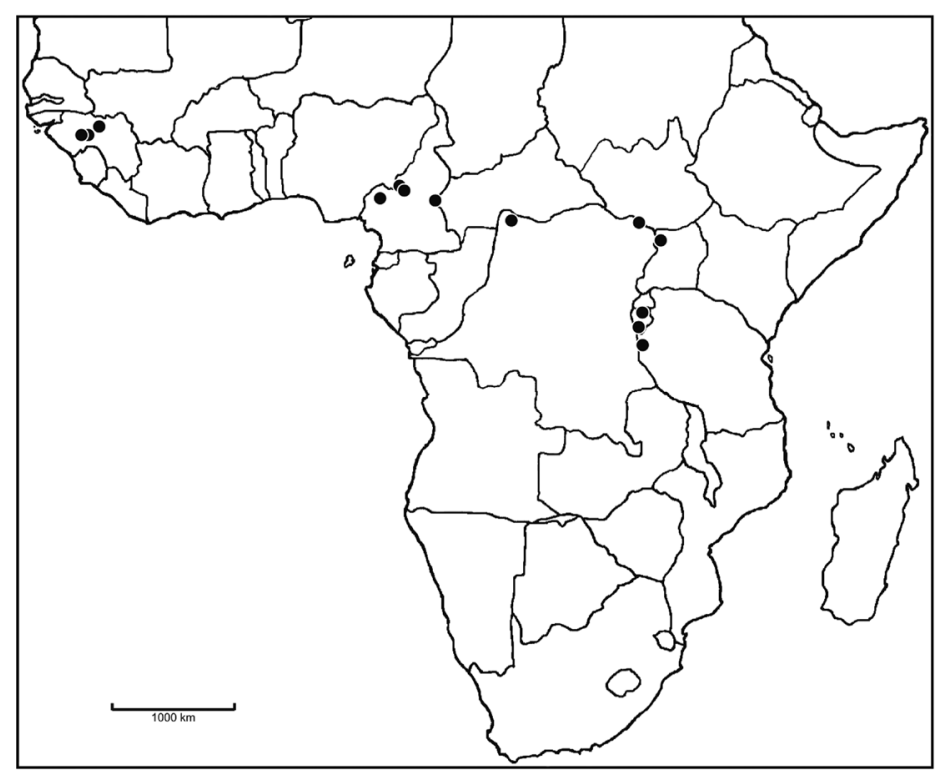

Fig. 72. Distribution of Bilabrella pauper (Summerh.) Szlach. \& Kras-Lap.

Tubers 1-2.5 cm long, $0.8-1.3 \mathrm{~cm}$ wide, ovoid to ellipsoid. Stem $40-100 \mathrm{~cm}$ tall, erect to lightly flexuose, leafy. Leaves 9-12, to $20 \mathrm{~cm}$ long and $1 \mathrm{~cm}$ wide, 4-7 intermediate linear or linear-lanceolate, acuminate, gradually decreasing upwards. Inflorescence $10-20 \mathrm{~cm}$ long, densely multiflowered. Flowers green. Floral bracts lanceolate, acuminate. Pedicel and ovary 10$16 \mathrm{~mm}$ long, erect or arcuate. Dorsal sepal 5-6 mm

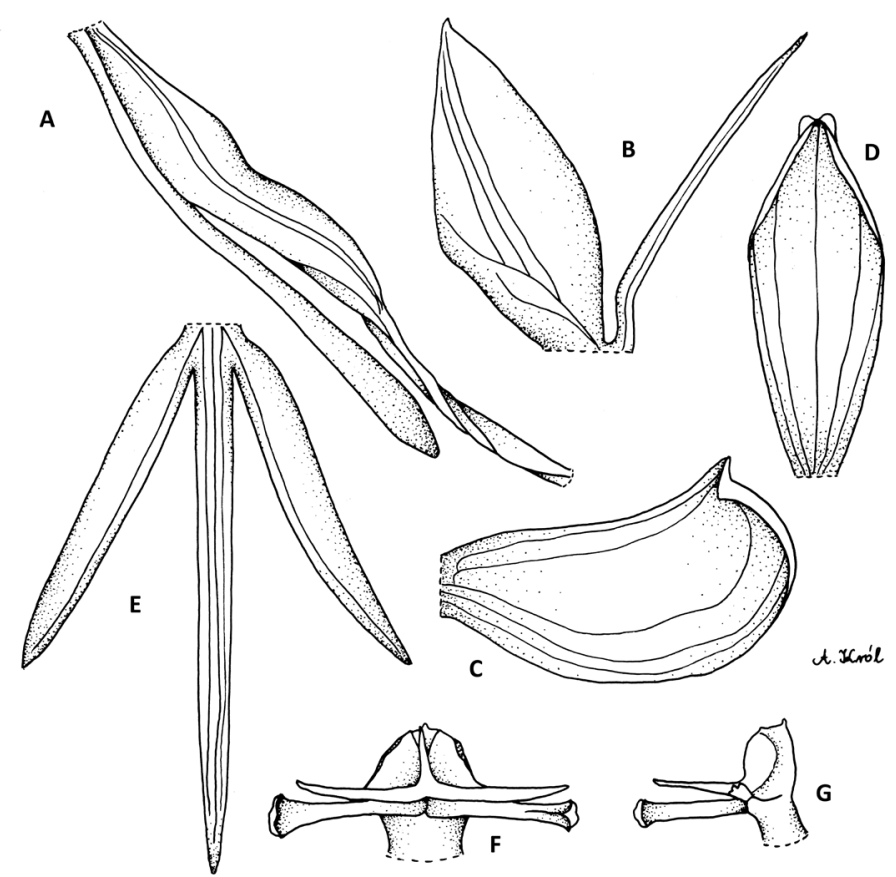

Fig. 73. Bilabrella urundiensis (Summerh.) Szlach. \& Kras Explanations: A - ovary, pedicel, spur; B - petal; C - lateral sepal; D - dorsal sepal; E - lip; F - gynostemium, front view; G - gynostemium, side view (drawn by A. Król from holotype of Habenaria urundiensis Summerh. Germain 6531, K) 


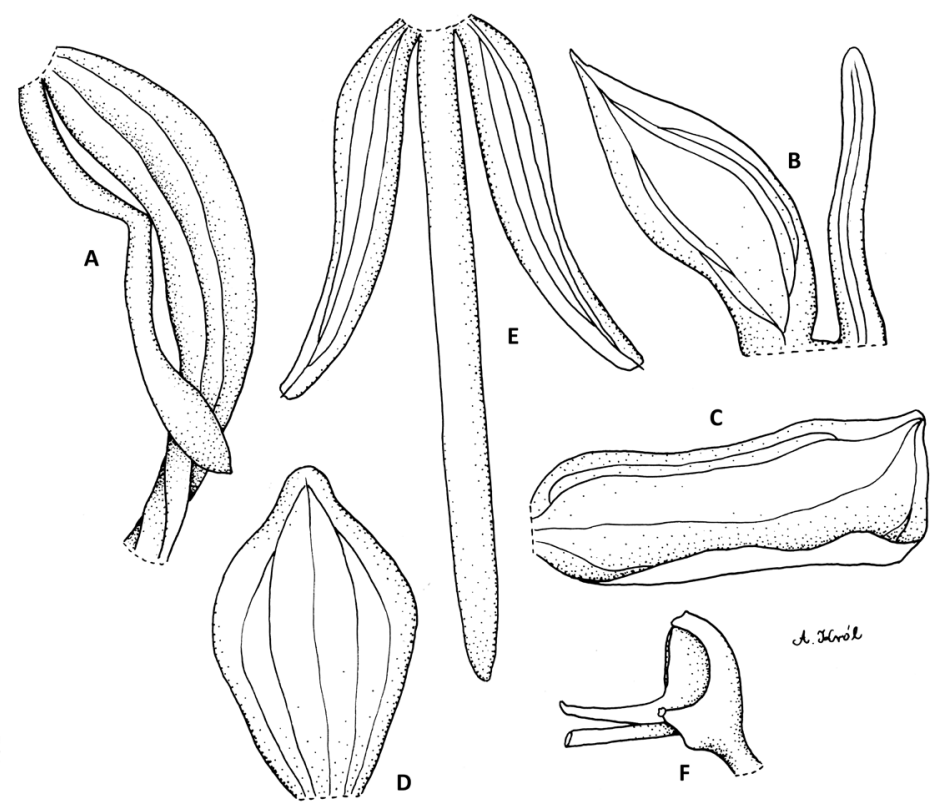

Fig. 74. Bilabrella urundiensis (Summerh.) Szlach. \& Kras Explanations: A - ovary, pedicel, spur; B - petal; C - lateral sepal; D - dorsal sepal; E - lip; F - gynostemium, side view (drawn by A. Król from holotype of Habenaria urundiensis Summerh. - Germain 6531, K)

long, 2-2.5 mm wide, oblong elliptic to obovate, shortly apiculate, obtuse, concave, reflexed, 5-nerved. Petals bilobed to the base; anterior lobe 5-6 $\mathrm{mm}$ long, 2-3 $\mathrm{mm}$ wide, lanceolate-elliptic to ovate-lanceolate, subacute to acute, subcarnose, multinerved; posterior lobe $5 \mathrm{~mm}$ long, 0.3-0.5 mm wide, linear or filiform, ciliolate, reflexed or incurvate. Lateral sepals 5-6 mm long, 3-4 mm wide, obliquely obovate to semi-orbiculate, obtuse, concave, reflexed, 5-nerved. Lip deflexed or porrect, 3-lobed above the basal $1 \mathrm{~mm}$; the middle lobe $5-8 \mathrm{~mm}$

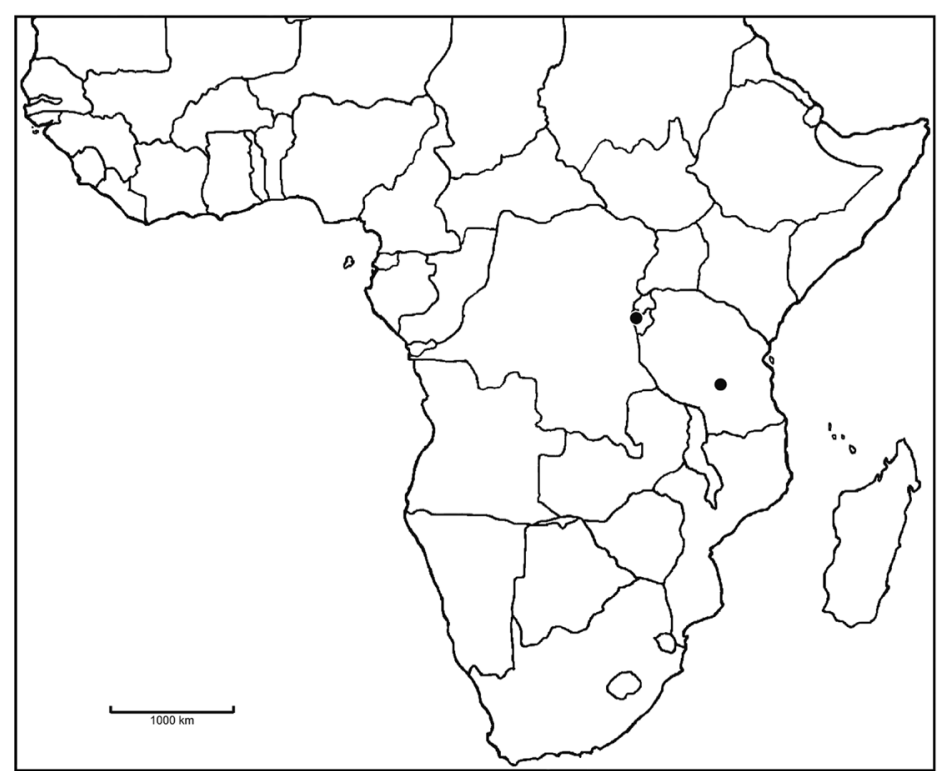

Fig. 75. Distribution of Bilabrella urundiensis (Summerh.) Szlach. \& Kras long, $0.5 \mathrm{~mm}$ wide, ligulate-linear, obtuse; lateral lobes 3.5-5 mm long, 0.5-1 mm wide, linear-lanceolate, subacute. Spur 10-13 mm long, pendent, geniculate near the middle, in basal half or third cylindrical, apically clavate inflated, subobtuse. Anther $2 \mathrm{~mm}$ tall; connective shortly apiculate; antherophores $3 \mathrm{~mm}$ long, straight. Stigmaphores $3 \mathrm{~mm}$ long, narrowly cylindrical, truncate. Rostellum middle lobe as long as connective. Auriculae tiny, irregularly 3-lobed (Figs 73-74).

E c ology. Hyparrhenia savannah and LoudetiaTerminalia savannah, dry woodland with Uapaca, Philippia, Isoberlinia, Brachystegia, Combretum. February-March.

Di stribution. Democratic Republic of the Congo (Zaire), Tanzania, alt. $1770 \mathrm{~m}$ (Fig. 75).

Specimens examined. Democratic Republic of the Congo (Zaire). Nord-Kivu: Urundi, Ruzizi plain, in Hyparrhenia savannah, Feb. 1950 (fl), Germain 6038 (K!); Rwagarika region, Ruzizi plain, in Loudetia-Terminalia savannah, Mar. 1950 (fl), Germain 6531 (K!). Tanzania. Iringa: Great North Road, Iheme, 19 miles $\mathrm{S}$ of Iringa, rocky hill, red sandy soil, with mixed dry woodland - Uapaca, Philippia, Isoberlinia, Brachystegia, Combretum, 1770 m, 24 Feb. 1962 (fl), Polhill \& Paulo $1598(\mathrm{~K} !)$.

Notes. This species appears to be allied to B. pauper, but differs by the characters given in the descriptions. The most obvious features are the much longer inflorescense, the relatively wide anterior petal lobe and the longer, more slender spur. It is also similar to B. falcicornis, especially in the general gynostemium structure and lip morphology, but has much larger flowers and all perianth segments. Taxonomic status of both of these species requires field studies. The geniculate spur $B$. urundiensis shares with $B$. tortilis, but both have different gynostemium (slender projections in the former vs short in B. tortilis).

26. Bilabrella pedicellaris (Rchb. f.) Szlach \& Kras, Richardiana 9(4): 159. 2009.

Habenaria pedicellaris Rchb. f., Otia Bot. Hamburg.: 100. 1881. - Rendle, J. Linn. Soc., Bot. 30: 393. 1895. - Rolfe, Fl. Trop. Afr. 7: 244. 1898. - Kraenzlin, Orchid. Gen. Sp.: 221. 1901. - Robyns \& Tournay, Fl. Spermat. Parc Natl. Albert 3: 434. 1955.

Type: Ethiopia, Schimper 1369a (lectotype, designated by Szlachetko et al. [2010: 230], W-R!; isolectotype, $\mathrm{K}$ ! - drawing).

Habenaria pedicellaris Rolfe, Fl. Trop. Afr. 7: 244. 1898, quoad spec. volkensii, non Rchb. f..

Type: Ethiopia, Schimper 1369 b (syntype, W!) \& Tanzania, Volkens 2321 (syntype, BM!).

Tuberoids $1-3 \mathrm{~cm}$ long, $0.5-1.5 \mathrm{~cm}$ wide, ellipsoid. Stem $30-50 \mathrm{~cm}$ tall, erect, rather slender, glabrous. 


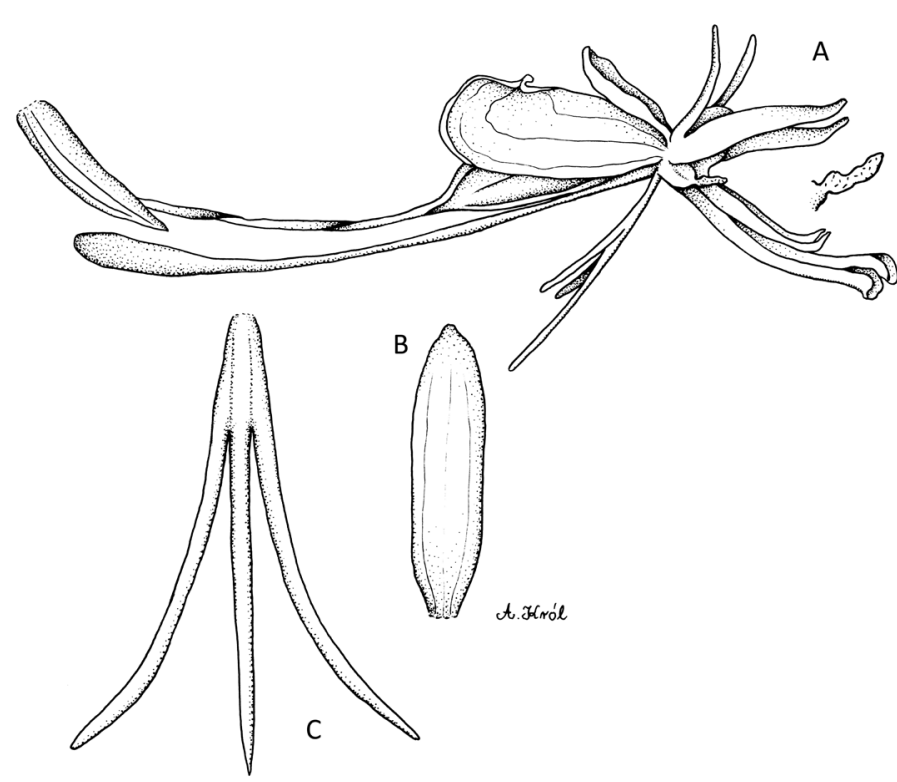

Fig. 76. Bilabrella pedicellaris (Rchb. f.) Szlach \& Kras Explanations: A - flower and auricula; B - dorsal sepal; C - lip (drawn by A. Król from lectotype of Habenaria pedicellaris Rchb.f. - Schimper $1369 a, \mathrm{~W}-\mathrm{R})$

Leaves $5-7$, up to $10 \mathrm{~cm}$ long and $1.2 \mathrm{~cm}$ wide, linearlanceolate, acute, spreading, decreasing in size upwards. Inflorescence up to $10 \mathrm{~cm}$ long, laxly 13-20-flowered. Floral bracts 8-13 mm long, ovate-lanceolate to oblonglanceolate, acuminate, thin, membraneous. Ovary and pedicel up to $23 \mathrm{~mm}$ long, slender. Dorsal sepal $4 \mathrm{~mm}$ long, $1.2 \mathrm{~mm}$ wide, oblong to narrowly elliptic, obtuse, cochleate. Petals bilobed to the base; anterior lobe $4 \mathrm{~mm}$ long, $0.3 \mathrm{~mm}$ wide, filiform, blunt, glabrous; posterior lobe 5.5-6 mm long, $1 \mathrm{~mm}$ wide, linear-lanceolate, acuminate, glabrous. Lateral sepals $6.5 \mathrm{~mm}$ long, $3 \mathrm{~mm}$ wide, obliquely oblong-obovate, with laterally placed, acute apiculus, cochleate. Lip 3-lobed above the basal $2.5 \mathrm{~mm}$; the middle lobe $7 \mathrm{~mm}$ long, $0.4 \mathrm{~mm}$ wide, narrowly linear, subacute; lateral lobes $6.5 \mathrm{~mm}$ long, $0.4 \mathrm{~mm}$ wide, narrowly linear, subacute, pendentspreading. Spur to $20 \mathrm{~mm}$ long, filiform, clavate at the blunt apex, parallel to pedicel and ovary. Anther $3 \mathrm{~mm}$ long; connactive obtuse; antherophores $4.5 \mathrm{~mm}$ long, slender, abruptly upcurved at the apex only. Stigmaphores $6.5 \mathrm{~mm}$ long, narrowly cylindrical, slightly sigmoid apically, glabrous. Rostellum middle lobe as long as connective. Auriculae digitate, somewhat irregular (Fig. 76).

E c o l o g y. In swamp. Flowering in January-October. Distribution. Ethiopia, Uganda, Tanzania, Zambia, Zimbabwe, alt. 900-3000 m (Fig. 77).

Specimens examined. Ethiopia. $1740 \mathrm{~m}, 20$ Oct. 63 (fl), Schimper 1369 (BM!). Uganda. Eastern: Sironko Distr., near Bulambuli, Mt. Elgon, 2700-3000 m, 14 July 1924 (fl), Snowden 915 (K! - drawing); Limona, escarpment, in swamp, 2220-2250 m, Dummer

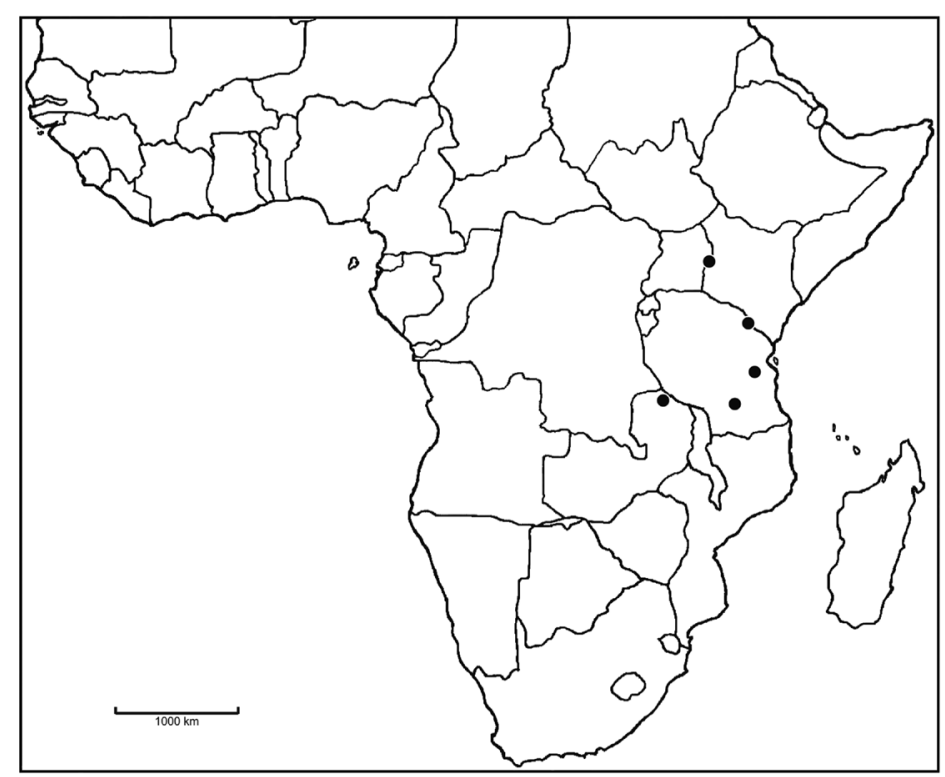

Fig. 77. Distribution of Bilabrella pedicellaris (Rchb.f.) Szlach. \& Kras

16 (E!). Tanzania. Kilimanjaro: Marangu, $1450 \mathrm{~m}$, May 1893 (fl), Volkens 272 (BM!); Marangu, 1550 m, June 1894 (fl), Volkens 2321 (BM!, WU!); Morogoro: Mahenge, Sali, Ugougo, 1200 m, 23 Mar. 1931 (fl), Schlieben 1958 (BM!, BR!); Uluguru Mts., Kikue, 1400 m, 10 Apr. 1933 (fl), Schlieben 3758 (BM!). Zambia. Northern: Abercon Distr, 1500 m, Jan. 1933 (fl), Gamwell 130 (BM!). Zimbabwe. Lippu Buyi, 900 m, 19 Apr. 1907 (fl), Swynnerton 744 (BM!).

Notes. Rather easily distinguishable species by having stigmaphores prominently longer than antherophores and lip with distinct basal claw. Bilabrella pedicellaris can be misidentified with $B$. ichneumonea from which it differs by i.a. glabrous petals with anterior lobe being $4 \times 0.3 \mathrm{~mm}$ (vs petals ciliolate, anterior lobe 5.5-13 $\times 1-1.5 \mathrm{~mm}$ in B. ichneumonea), and shorter lip middle lobe ( $7 \mathrm{~mm}$ vs $7-15 \mathrm{~mm}$ ).

27. Bilabrella ichneumonea (Sw.) Szlach. \& Kras-Lap., Richardiana 3(3): 140. 2003. - Szlachetko, Orchid. Ivory Coast: 42. 2008. - Szlachetko et al., Orchid. of West-Central Africa 1: 229. 2010.

Habenaria ichneumonea (Sw.) Lindl., Gen. Sp. Orchid. Pl.: 313. 1835. - Rolfe, Fl. Trop. Afr. 7: 240. 1898. Kraenzlin, Orchid. Gen. Sp.: 320. 1901. - Troupin, Fl. Spermat. Parc Nat. Garamba 1: 252. 1956. - Summerhayes, FWTA, ed. 2, 3: 196. 1968a. - Summerhayes, FTEA, Orchid. 1: 94. 1968b. - Grosvenor, Excelsa 6: 83. 1976. - Geerinck \& Coutrez, Not. Taxon. Orchid. Afr. Centr., Habenaria: 9. 1977. - Williamson, Orchid. S. Centr. Afr.: 56. 1977. - Geerinck, Fl. Afr. Centr., Orchid. 1: 107. 1984 - la Croix et al., Orchid. Malawi: 
72. 1991. - la Croix \& Cribb, Fl. Zambes., Orchid. 1: 97. 1995. - Stewart \& Campbell, Orchids of Kenya: 135. 1996. - Edwards, Demissew, Hedberg, Flora of Ethiopia and Eritrea 6: 220. 1997. - Szlachetko \& Olszewski, Fl. Cam., Orchid. 34(1): 175. 1998. - Linder \& Kurzweil, Orchids of Southern Africa: 130. 1999. - Perez-Vera, Orchid. Cote d'Ivoire: 374. 2003. - Jacques-Georges, Extrait de la Flore descriptive des Monts Nimba 5: 1700. 1981-1983. - Demissew, Cribb, Rasmusen, Field guide to Ethiopian Orchids: 97. 2004.

Orchis ichneumonea Sw. in Schrad., Neues J. 1: 21. 1805.

Type: Sierra Leone, Afzelius s.n. (lectotype, designated by Szlachetko et al. [2010: 229], W-R!; isolectotypes, BM!, UPS not seen, drawings: K!, W-R!).

Tubers $0.7-2.5 \mathrm{~cm}$ long, $0.4-1.2 \mathrm{~cm}$ in diameter, ellipsoid or ovoid. Stem $15-85 \mathrm{~cm}$ tall, erect, delicate, glabrous. Leaves 5-12, 7-21 cm long, 0.4$1.2 \mathrm{~cm}$ wide, grass-like, acute, erect, decreasing in size up the stem. Inflorescence 6-22 cm long, laxly or densely few- to many-flowered. Flowers green or green with white centre. Floral bracts 5-17 mm long, glandular inwards. Pedicel and ovary 15-30 mm long, glabrous. Dorsal sepal 3-5.5 mm long, 1.2-2.5 mm wide, ovate, subobtuse, cochleate, glabrous. Petals bilobed to the base, ciliolate; anterior lobe $5.5-13 \mathrm{~mm}$ long, 1-1.5 mm wide, lanceolate with filiform apex, rather thick, fleshy, more or less auriculate basally and here the widest; posterior lobe $3-4.5 \mathrm{~mm}$ long, 0.2$0.3 \mathrm{~mm}$ wide, filiform, thin, delicate. Lateral sepals 4-8.5 mm long, 3-5 mm wide, obliquely elliptic or broadly obovate, cochleate at the apex, with laterally placed apiculus ca. $0.5 \mathrm{~mm}$ long, glabrous. Lip 3-lobed above the undivided basal part ca. 1.5-4 mm long, rather thick, fleshy, glabrous; the middle lobe 7-15 mm long, 0.3-0.5 mm wide, filiform; lateral lobes 6-13 mm long, 0.2-0.4 mm wide, filiform, all lobes pendent. Spur 10-25 mm long, $0.3 \mathrm{~mm}$ in diameter at the base, $1.2 \mathrm{~mm}$ in diameter at the apex, slightly incurved, narrowly cylindrical, apical quarter swollen, blunt. Anther 1.2-2.5 mm tall; connective truncate; antherophores 3.5-7 mm long, very slender, upcurved at the apex. Stigmaphores 3.5-7 mm long, narrowly cylindrical, erect. Rostellum middle lobe shorter than thecae. Auriculae entire (Figs 78-79).

E c o lo g y. Damp grassland, swamp, seasonally wet grassland on verge of perennially wet bogs, sometimes in shallow, standing water. January-December.

D is tribution. Senegal, Guinea, Sierra Leone, Ivory Coast, Nigeria, Ethiopia, Cameroon, Central African Republic, Gabon, Democratic Republic of the Congo (Zaire), Uganda, Tanzania, Angola, Zambia, Malawi, Zimbabwe, Botswana, alt. 52,5; 590-1900 m (Fig. 80).

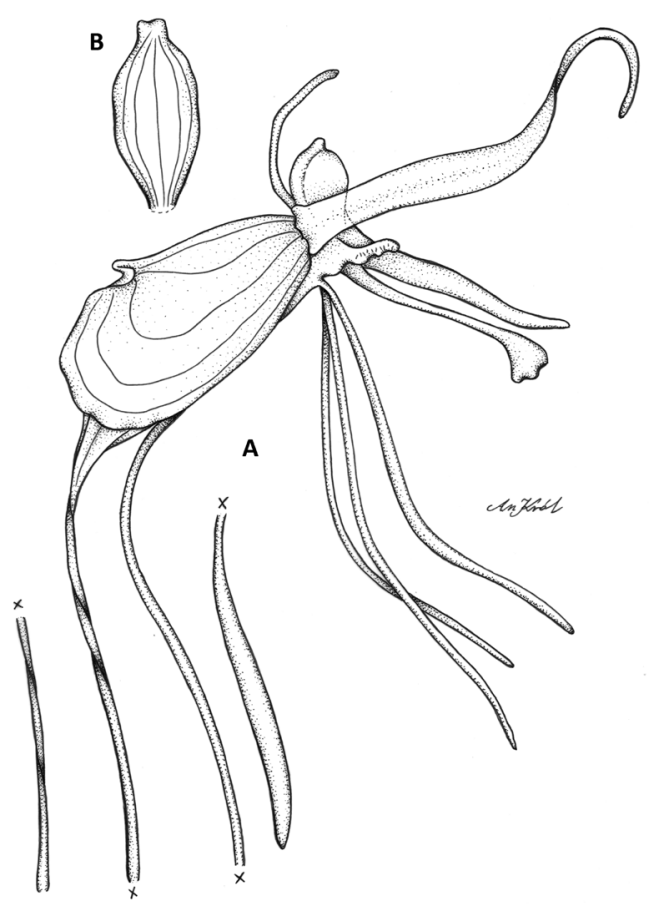

Fig. 78. Bilabrella ichneumonea (Sw.) Szlach. \& Kras-Lap. Explanations: A - flower; B - dorsal sepal (drawn by A. Król from Richards 16195, AMES)

Specimens examined. Senegal. Ziguinchor: Ziguinchor (Casamance), on edge of an old forested area, very uniform grassland, sandy soil liable to same flooding in wet season but probably very dry at other times, 4 Sep. 1962 (fl), Widebent 93 (K!). Guinea. Faranah: Kalia, marsh around ravine of the Ditinn River, July 1912 (fl), s.n. (P!); Kouria to Irebeleya, Sep, Chevalier s.n. (P?); Kouroussa, Conakry, Teliko, July 1901 (fl), Pobeguin 755 (P!); Kankan: Dalaba, Plaine, July 1912 (fl), Caille 8 (P!); Leyele Falls, near lake Fouta Djallon, on poor peaty soil, 20 July 1962 (fl), Adam 302 (K!); Timbi-Fouta Djallon, Adam 14745 (K!); Kindia: Kindia, Aug. 1954 (fl), Jacques-Felix 7038 (K!, P!); Sine loc., Maclaud 229 (P!); Nzérékoré: Macenta, 600-750 m, 14-15 Oct. 1947 (fl), Baldwin 9802 (K!); Below Monts Nimba, June 1945 (fl), Schnell 3027 (K!). Sierra Leone. Western Area Rural: Waterloo, Foni flats, Deighton 2064 (K!); Wellington, in wet soil among Cyperaceae in clearing, Aug. 1962 (fl), Harvey $56(\mathrm{~K} !) ; 2$ miles along road west from Waterloo, marsh on laterite, $52.5 \mathrm{~m}, 22$ Aug. 1958 (fl), Melville \& Hooker 280 (K!); Sine loc., Afzelius s.n. (W-R!). Ivory Coast. Dix-huit Montagnes: Danané, Nozeran s.n. (P not seen, Cribb \& Perez-Vera 1975); Moyen-Cavally: Mt. Tonkoui, Aké Assi 2630 (UCJ not seen, Cribb \& Perez-Vera 1975). Nigeria. Northern: Plateau State, Bauchi Plateau, Vom, 1350-1500 m, Dec. 1922 (fl), Young s.n. (K!). Ethiopia. Dire Dawa: Hoga Prov., Addis-Jimma $40 \mathrm{~m}$ from Jimma, grassland, weak draired soil, 1680 m, 10 Sep. 1962 (fl), Thomas 86 (K!); 


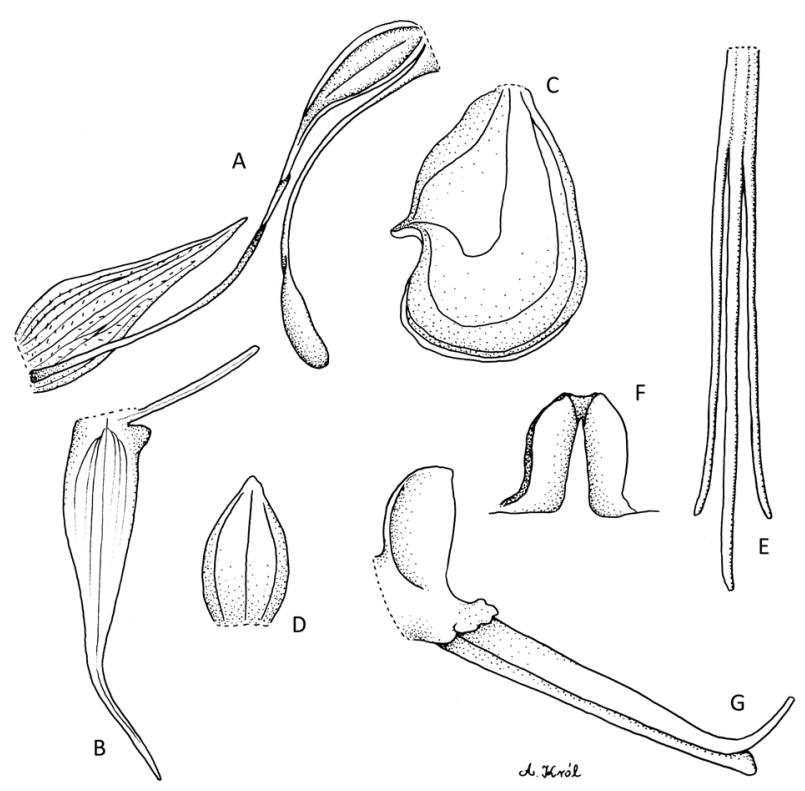

Fig. 79. Bilabrella ichneumonea (Sw.) Szlach. \& Kras-Lap. Explanations: A - ovary, pedicel, spur, floral bract; B - petal; C - lateral sepal; D - dorsal sepal; E - lip; F - gynostemium, front view; G - gynostemium, side view (drawn by A. Król from isolectotype of Orchis ichneumonea Sw. - Afzelius s.n., BM)

Between Tigre and Begemder, Schmiper 1364 (W-R!); Between Tigre and Begemder, 1740 m, 20 Oct. 1963 (fl), Schimper 1369 (BM!). Cameroon. East: $20 \mathrm{~km}$ W Ibol, 40 km E Ngaoundere, Station Bowol, 27 June 1976 (fl), Fotius 2530 (P!). Central African Republic. Bamingui-Bangoran: Niao River, $4 \mathrm{~km} \mathrm{~S}$ of Sakala

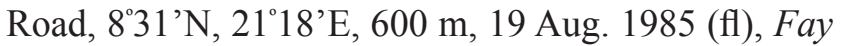
7419 (K!, MO not seen.); Haute-Kotto: Haute Kotto, 27 Sep. 1921 (fl), Becqueathed 1967 (BM!); HauteKotto, Obangui-Chari, $70 \mathrm{~km} \mathrm{~W}$ of Yalinga, 1 June 1922 (fl), Le Testu 3.923 (BM!); Manova-Gounda-St. Floris Natl. Park., Pende Creek, $3 \mathrm{~km} \mathrm{~S}$ of Koumbala

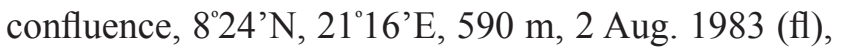
Fay 5639 (K!). Oubangui-Chari, Haute-Kotto, Yalinga, 27 Sep. 1921 (fl), Le Testu 3.296 (BM!, P!). Gabon: Sine loc., 30 Dec. 1908 (fl), Le Testu 1924 (BM!, BR!); Sine loc., Soyaux 211 (HBG!). Democratic Republic of the Congo (Zaire). Haut-Katanga: Katanga, on Lubumbashi Valley, 21 Dec. 1923 (fl), Heischberg 163 (K!); Haut-Shaba, Kundelungu Plateau, near Lualala Port, steppe, 1600 m, 21 Apr. 1971 (fl), Lisowski 66294 (UGDA-DLSz!); Haut-Shaba, Plateau of Kundelungu, Env. 18,4 km S.E. from Ponte the Lualala, 10'19'40"S, 27'52’40”'E, 1680 m, 21 Apr. 1971 (fl), Lisowski 66326 (UGDA-DLSz!); Shaba Region, 50 km WS of Kolwezi in the Mountain Vamba road, in flooded grassland, 1355 m, 26 Dec. 1982 (fl), Schaijes 1724 (K!); 1988 (fl), Sita \& Moutsambote s.n.; Haut-Lomami: SandoaEast, Feb. 1932 (fl), Overlaet 1269 (K!); Haut-Uele: Garamba National Park, Ukwa, 700-800 m, 1 May 1950 (fl), Noirfalise 217 (K!); Tukpuro (Kele), laterite soil,

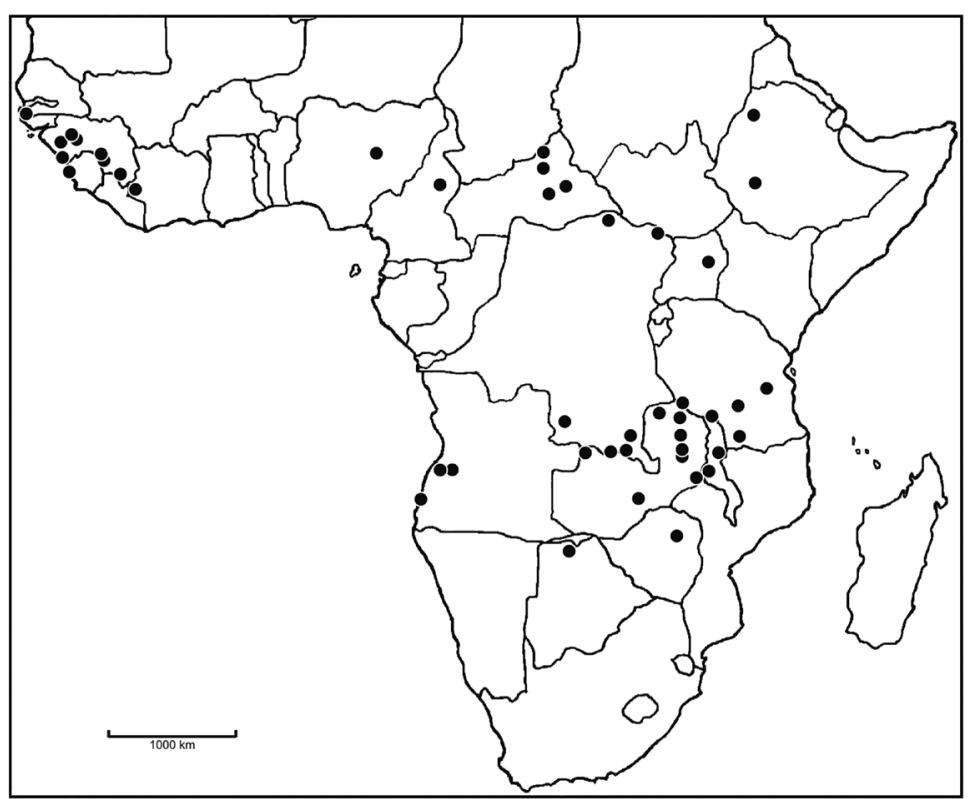

Fig. 80. Distribution of Bilabrella ichneumonea (Sw.) Szlach. \& Kras-Lap.

13 July 1954 (fl), Gerrard 1480 (K!). Uganda. Eastern: Soroti Distr., Serere. Rapai, 1110 m, 1926 (fl), Maitland 1308 A (K!). Tanzania. Iringa: Southern Highlands, Mufinoi, swamp near Irunda Hill, near grassland area, 1900 m, 1 Mar. 1985 (fl), Keeley PK37 (K!). Mbeya: Mbozi Distr., $60 \mathrm{~km}$ from Mbeya on Tunduma road, on left of road, in swampy grassland, 1700 m, 12 Feb. 1979 (fl), Cribb, Grey-Wilson \& Mwasumbi 11409 (K!). Ruvuma: Matagoro Mts., numerous on summili, 2 Apr. 1918 (fl), MacLoughlin 60 (K!); Songea Distr., Kwamponjore Valley, in boggy grassland, growing in water about $8 \mathrm{~cm}$ deep, 1000 m, 26 Apr. 1956 (fl), Milne-Redhead \& Taylor 9927 (AMES!, K!). Songea Distr., Luhila, Luhila Lake, on acid sandy soil on grassy plains, 18 Sep. 1956 (fl), Semsei 2478 (K!); Lazulu, in dark marshy alluvial soil, marsh, 1350 m, 12 Jan. 1931 (fl), Rounee D3 (K!). Angola. Benguela: Ganda, Alto Catumbela, $269 \mathrm{~km}$ miland, in swampy ground, $1350 \mathrm{~m}$, Jan. 1941 (fl), Fanlkner A431 (K!); Country of the Ganguellas and Ambuellas, Gossweiler 2344 (K!); Namibe: Rio Cubal, Nov. 1899 (fl), Baum 425b (K!). Zambia. Central: Chabuzga R, 100-129 km E of Lusaka, Chakwenga Headwaters in pernamently wet dambo, 25 Aug. 1963 (fl), Robinson 5637 (BRLU!, K!); Eastern: $30 \mathrm{~m} \mathrm{~W}$ of Chipata, 3 July 1974 (fl), Williamson \& Gassner 2294 (AMES!, K!); The same locality, Williamson \& Gassner 2295 (K!); Northern: Abercorn Distr., Mbulu River Abercorn, at edge of M'bulu riverine forest in swamp, 2 Feb. 1963 (fl), Richards 15978 (K!); Kabwe Distr., Mpunde Mission, $53 \mathrm{~km}$ NW from Kabwe, Kelongwe River, dambo grassland, 1406'S, 28 06'E, 20 Jan. 1973 
(fl), Kornaś 3033 (K!); Kasama Distr., Mungwi-Kasama Road, almost in water among grass and rocks in swamp, 1320 m, 26 Feb. 1962 (fl), Richards 16195 (AMES!, K!, UGDA-DLSz! - fragment, drawing); Mbala Distr., Sais Valley, marsh, edge of mushitu, wet grass, $1500 \mathrm{~m}$, 20 July 1970 (fl), Sanane 1311 (AMES!, K!); Mpika Distr., $57 \mathrm{~km}$ SW of Mpika, swamp, 12 ${ }^{\circ} 15^{\prime} \mathrm{S}, 31^{\circ} 10^{\prime} \mathrm{E}$, 1450 m, 13 Aug. 1966 (fl), Gillett 17443 (K!); 148 km $\mathrm{S}$ of Mpika, seasonally damp sand on foot of gratzite, 2 Mar. 1962 (fl), Robinson 4967 (K!); E of Shiwa Ngandu, in a moorland drainage peat bog in ass. with Cyperaceae, Lycopodium, Utricularia spp., Drosera sp. and Eriocaulon spp., 1500 m, 24 July 1938 (fl), Greenway 5498 (K!); Shiwa Ngandu, 5 Aug. 1938 (fl), Hanham s.n. (BM!); North-Western: Mwinilunga Distr., Sinkabolo Dambo, in boggy grassland not in the wettest parts, 9 Dec. 1937 (fl), Milne-Redhead 3570 (AMES!, K!); Mwinilunga Distr., Dambo NE of Dobeka Bridge, in boggy grassland, 11 Dec. 1937 (fl), Milne-Redhead 3570A (K!); Mwinilunga Distr., Zambezi, $6 \mathrm{~km}$ N of Kalene Hill Mission, 22 Feb. 1975 (fl), Williamson \& Gassner 2465 (K!). Malawi. Central: Kasungu Distr., Kasungu, 22 Aug. 1956 (fl), Jackson 2034 (K!); Northern: Nkhata Bay Distr., Kawalazi, in dambo, 600 m, 3 Feb. 1986 (fl), la Croix 796 (K!); Mzimba Distr., Mzuzu, 2 miles W at Katoto, in bog among short grasses, 1350 m, 25 July 1976 (fl), Pawek 11491 (K!). Zimbabwe. Harare: In many sandy vleis in the Salisbury Distr., 24 Feb. 1945 (fl), Greatrex 26 (K! \& icono!); Salisbury Distr., in wet vleis, 25 Feb. 1945 (fl), Greatrex 86 (K!); Salisbury Distr., 12 miles NE of City, in vlei, 1260 m, 31 Dec. 1958 (fl), Greatrex 91703 (K!), Victoria, 1909-1912 (fl), Monro 1786 (BM!); East Africa, Ipornlore Farm, Gutz 63 (HBG!). Botswana. Northern: Dabonga Boat Harbour, in shallowly but permanently inudated by water among various sedges and grasses and Ficus verruculosa, $19^{\circ} 06^{\prime}$ 'S, 23 $08^{\prime} 6^{\prime \prime} \mathrm{E}$, 21 July 1973 (fl), Smith 675 (K!); North-Western: Off edge of Mboma-Gadikwe channel, in perannially inundated floodplain, in back swamp $20 \mathrm{~cm}$ deep among stunted Miscanthidium \& in clumps of Eriochrysis pallida grass, 19¹1'4"S, 23\%15'75'E, 21 June 1979 \& 3 July 1979 (fl), Smith 2798 (K!).

N o te s. Bilabrella ichneumonea is apparently similar to both $B$. cribbiana and $B$. cornutella. It has however, lip 3-lobed from an undivided basal $2 \mathrm{~mm}$, with pendent lobes, lateral lobes slightly shorter than the middle one, and stigmaphores $4.6 \mathrm{~mm}$ long. Both $B$. cribbiana and B. cornutella are characterized by lip 3-lobed almost to the base, lateral lobes much shorter than the middle one and divergent, and stigmaphores $7 \mathrm{~mm}$ long.

It appear that $B$. ichneumonea is also very similar to $B$. pedicellaris and can be easily confused with this species. It has ciliolate petals (vs glabrous), longer anterior petal lobe $(5.5-13 \times 1-1.5 \mathrm{~mm}$ vs $4 \times 0.3 \mathrm{~mm})$ and longer lip middle lobe (7-15 $\mathrm{mm}$ vs $7 \mathrm{~mm}$ long) than B. pedicellaris. Szlachetko et al. (2010) amalgamated both species.

Isolectotype specimen of Orchis ichneumonea at W-R consists of a single, partially destroyed specimen glued to the sheet, having a single flower in very bad condition.

Gutz 63 collection at HBG represents peloric form of this species.

28. Bilabrella cornutella (Summerh.) Szlach. \& KrasLap., Richardiana 3(3): 139. 2003.

Habenaria cornutella Summerh., Kew Bull. 1938: 144. 1938. - Summerhayes, FTEA, Orchid. 1: 87. 1968b. Geerinck \& Coutrez, Not. Taxon. Orchid. Afr. Centr., Habenaria: 8. 1977. - Geerinck, Fl. Afr. Centr., Orchid. 1: 118. 1984. - Szlachetko \& Olszewski, Fl. Cam., Orchid. 34(1): 176. 1998.

Type (as holotype): Uganda, Eggeling 571 (lectotype, designated by Summerhayes [1968b: 87], K!; drawing: $\mathrm{K}$ !).

Roots very long, up to $30 \mathrm{~cm}$, subterranean stolons long, slender. Stem 30-80 cm tall, erect, rather delicate, glabrous. Leaves 7-10, 10-28 cm long, up to $1(1.2) \mathrm{cm}$ wide, linear or linear-lanceolate, acute, erect or suberect, decreasing in size up the stem. Inflorescence 6-16 cm long, 8-30-flowered, rather lax. Flowers green. Floral bracts $8-15 \mathrm{~mm}$ long, pubescent on both sides. Pedicel and ovary 15-23 mm long, rather erect; pedicel glabrous; ovary minutely papillate. Dorsal sepal 5-6.5 mm long, 2-3 mm wide, elliptic, obtuse, cochleate, glabrous. Petals bilobed to the base, glabrous; anterior lobe $8.5-15.5 \mathrm{~mm}$ long, 1-1.5 mm wide, lanceolate or

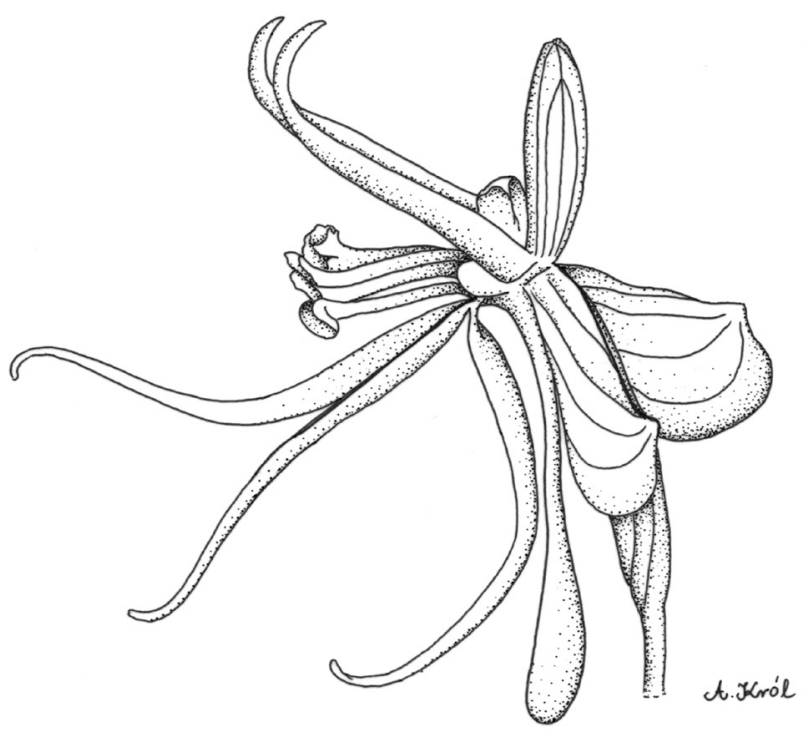

Fig. 81. Bilabrella cornutella (Summerh.) Szlach. \& Kras-Lap. flower (drawn by A. Król from lectotype of Habenaria cornutella Summerh. - Eggeling 571, K) 


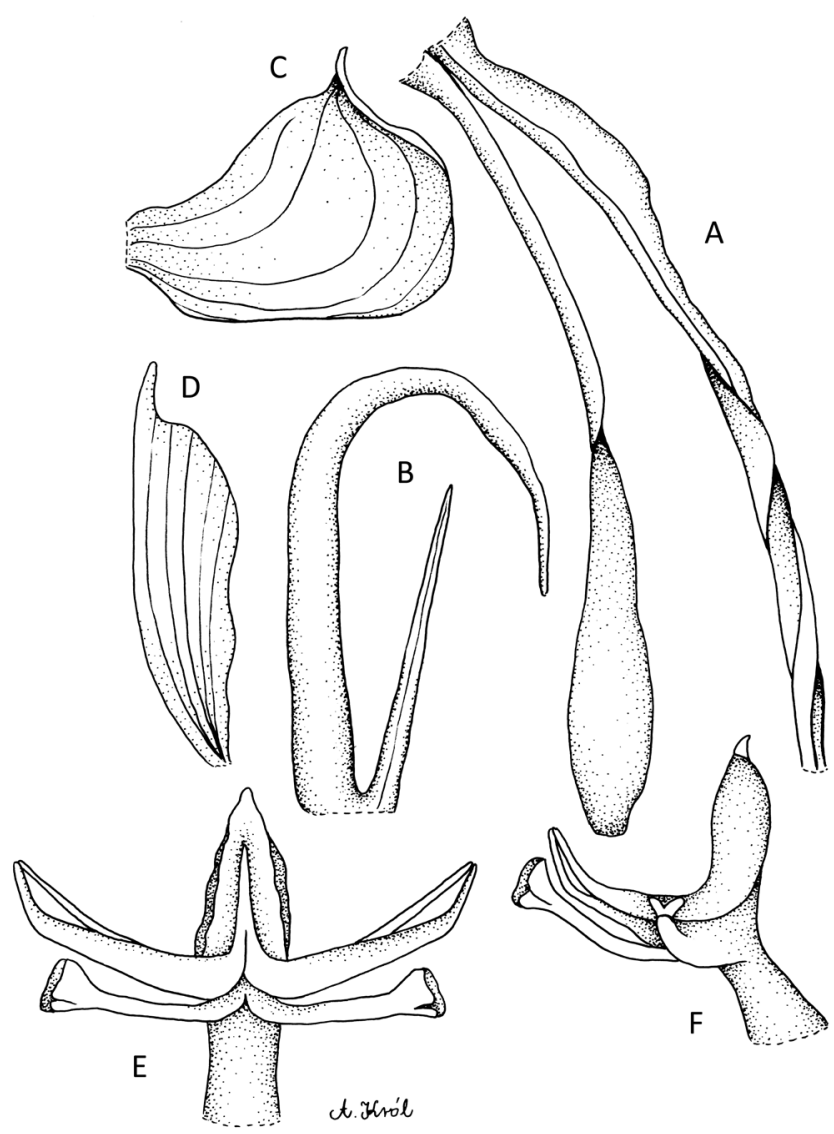

Fig. 82. Bilabrella cornutella (Summerh.) Szlach. \& Kras-Lap. Explanations: A - ovary, pedicel, spur; B - petal; C - lateral sepal; D dorsal sepal; E - gynostemium, front view; F - gynostemium, side view (drawn by A. Król from Habenaria cornutella Summerh. - Lisowski 66325, UGDA-DLSz)

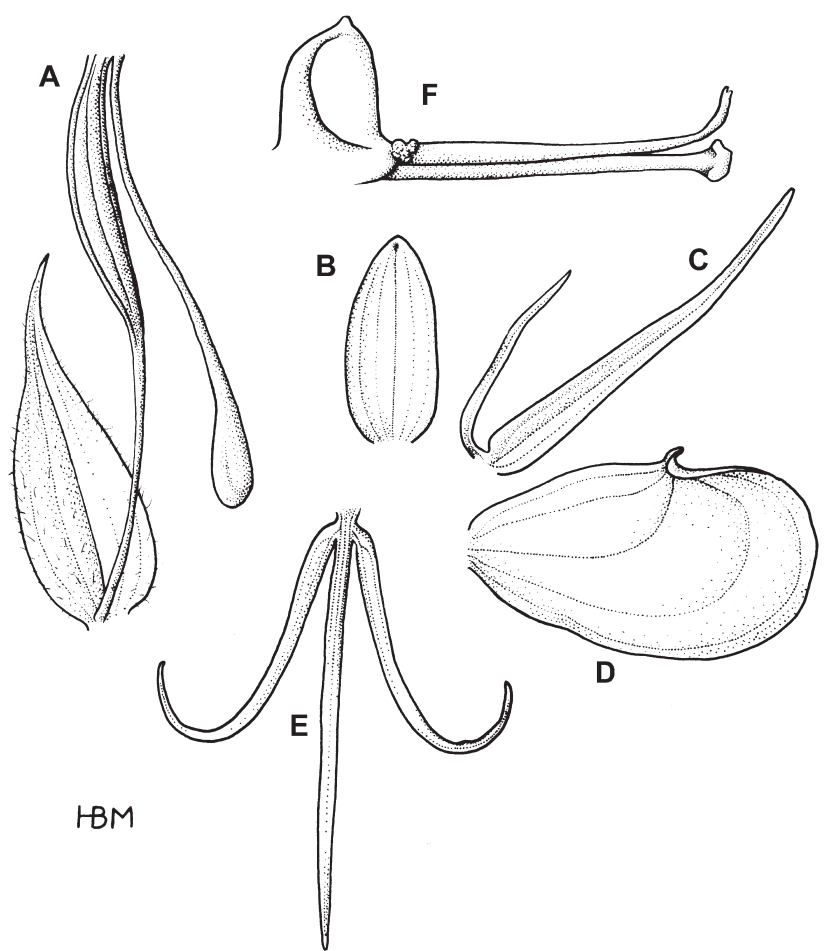

Fig. 83. Bilabrella cornutella (Summerh.) Szlach. \& Kras-Lap. Explanations: A - ovary, pedicel, spur and bract; B - dorsal sepal; C - petal; D - lateral sepal; E - lip; F - gynostemium, side view (drawn by H.B. Margońska from Habenaria cornutella Summerh. - Letouzey 5500, P) obliquely lanceolate, subulate above, rather thick and fleshy, widest at the base; posterior lobe 5-6 $\mathrm{mm}$ long, $0.2-0.3 \mathrm{~mm}$ wide, filiform, erect, thin. Lateral sepals 7-10 mm long, 4-6 mm wide, obliquely oblong-elliptic, to widely obovate, cochleate, with laterally placed apiculus 0.5-0.8 mm long, glabrous. Lip 3-lobed above the very short undivided basal part, ca. $0.5-1 \mathrm{~mm}$ long, rather fleshy, glabrous; the middle lobe 12-14 mm long, 0.5-1 mm wide, linear or filiform, acute; lateral lobes ca. $11 \mathrm{~mm}$ long, 0.3-0.6 mm wide, linear-lanceolate or filiform, upcurved or sometimes pendent. Spur 12-19 mm long, $0.3-0.8 \mathrm{~mm}$ in diameter near the middle, $1.6-2 \mathrm{~mm}$ in diameter at the apex, narrowly cylindrical, swollen club-shaped in the apical half to quarter, blunt. Anther $2.5 \mathrm{~mm}$ tall; connective shortly apiculate; antherophores $7 \mathrm{~mm}$ long, apically abruptly upcurved. Stigmaphores $7 \mathrm{~mm}$ long, very slender, truncate apically. Rostellum middle lobe shorter than thecae. Auriculae small, entire, subglobose (Figs 81-83).

E c o lo gy. Swamp with Sphagnum and Miscanthidium, open marsh. Flowers throughout the year.

Distribution. Cameroon, Central African Republic, Congo, Democratic Republic of the Congo (Zaire), Uganda, Rwanda, Burundi, alt. 900-1200 m (Fig. 84).

S pecimen s ex a mined. Cameroon. East: $17 \mathrm{~km}$ SSW Koso, 60 km SSW Batouri, 26 July 1963 (fl), Letouzey 5500 (P!); South: Bitye, River Ja, 13 Sep. 1918 (fl), Bates 1439 (BM!). Central African Republic. Oubangui Chari, between Yalinga and Bia, Upper Kotto River, 28 July 1922 (fl), Le Testu 4029 (BM!, BR!, K!, P!). Congo. Pool: Road to Gamakala, $40 \mathrm{~km} \mathrm{~N}$ of Brazaville, 31 Jan. 1969 (fl), Attims 75 (P!); Lefini, NE

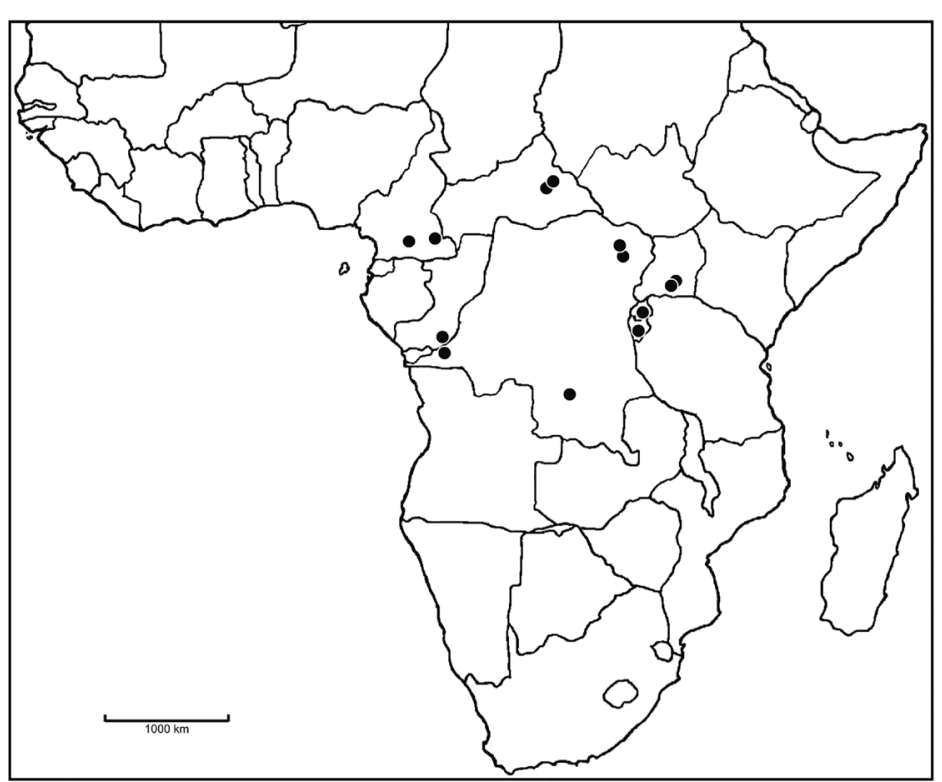

Fig. 84. Distribution of Bilabrella cornutella (Summerh.) Szlach. \& Kras-Lap. 
of Brazaville, road to Douvres, village of Gamakala, 22 June 1960 (fl), Descoings 5911 (P!). Democratic Republic of the Congo (Zaire). Haut-Lomami: Keyborg - valley Kimilolo, 2 Jan. 1957 (fl), Detilleux 389(BR!); Haut-Uele: Niangara - Wamba, July 1931 (fl), Lebrun 3254 (BR!, K!); Between Yubr and Betongwe, $50 \mathrm{~km}$ $\mathrm{N}$ from Wamba, swamp near River Maika, $1000 \mathrm{~m}$, 15 July 1937 (fl), Louis 4447 (BR!); Between Yubr and Betongwe, $50 \mathrm{~km} \mathrm{~N}$ from Wamba, swamp near River Maika, 1000 m, 15 July 1937 (fl), Louis 4449 (BR!); Kongo Central: Kisantu, between Mts Mbomboat and Kimfurto, 9 Jan. 1959 (fl), Pauwals 1229 (BR!). Uganda. Central: Kampala Distr., Mengo, Kyagwe, Namanve Swamp, in Saccharum patches, 1170 m, Mar. 1932 (fl), Eggeling 571 (K!); Kampala Distr., Bugaba, outer margin of Miscanthidium swamp, 14 Sep. 1967 (fl), Lock s.n. (K! - drawing); Masaka Distr., East side of Lake Nanbugabo, Sphagnum bog, 1140 m, 6 Dec. 1953 (fl), Drummond \& Hemsley 4647 (K!); Masaka Distr., Lake Nabugabo, Masaka, open marsh, 1200 m, Apr. 1935 (fl), Synge S.1951 (BM!, K!); Wakiso Distr., Entebbe, swamp, 900 m, Sep. 1905 (fl), Broun 330 (K!); 1200 m, Dec. 1915 (fl), Dummer 2686 (K!). Rwanda. Sine loc., Bossche s.n. (BR!). Burundi. Kinyinya Moso Umudi, 2 July 1952 (fl), Michel 3178 (BR!).

Notes. This species is very similar to Bilabrella lisowskii but can be easily separated from the latter by having glabrous petals. It can be misidentified with $B$. ichneumonea. In the latter, however, the petal ante- rior lobes are widest distinctly above the base and lip is 3-lobed above the basal undivided part about $2 \mathrm{~mm}$ long. In B. cornutella the petal anterior lobes are widest at the base and the lip is 3-lobed nearly at the base.

29. Bilabrella lisowskii (Szlach.) Szlach. \& Kras-Lap., Richardiana 3(3): 141. 2003. - Szlachetko et al., Orchid. of West-Central Africa 1: 233. 2010.

Habenaria lisowskii Szlach., Fragm. Flor. Geobot. 40(2): 721-723, fig. 3. 1995. - Szlachetko \& Olszewski, Fl. Cam., Orchid. 34(1): 178. 1998.

Type: Congo, Lisowski C-239 (holotype, UGDADLSz!).

Stem $56-58 \mathrm{~cm}$ tall, erect, delicate, glabrous. Leaves 4-6, up to $16 \mathrm{~cm}$ long and $1 \mathrm{~cm}$ wide, linear-lanceolate, acute; the upper 3-4 diminishing rapidly in size to the bract-like leaflets. Inflorescence 11-12 cm long, 9-16-flowered, rather loose. Floral bracts $12 \mathrm{~mm}$ long, densely glandular on both sides. Pedicel and ovary up to $25 \mathrm{~mm}$ long. Dorsal sepal $5 \mathrm{~mm}$ long, $3.2 \mathrm{~mm}$ wide, obovate, cuspidate, cochleate towards the apex, glabrous. Petals bilobed to the base, densely ciliate; anterior lobe $9 \mathrm{~mm}$ long, $2 \mathrm{~mm}$ wide, obliquely lanceolate with subulate apex, widest in the lower third; posterior lobe $5.5 \mathrm{~mm}$ long, $0.2 \mathrm{~mm}$ wide, filiform or linear, acute. Lateral sepals $8 \mathrm{~mm}$ long, $4.5 \mathrm{~mm}$ wide, obliquely obovate-elliptic, concave, with lateral apiculus, glabrous. Lip 3-lobed above the very short basal undivided part,

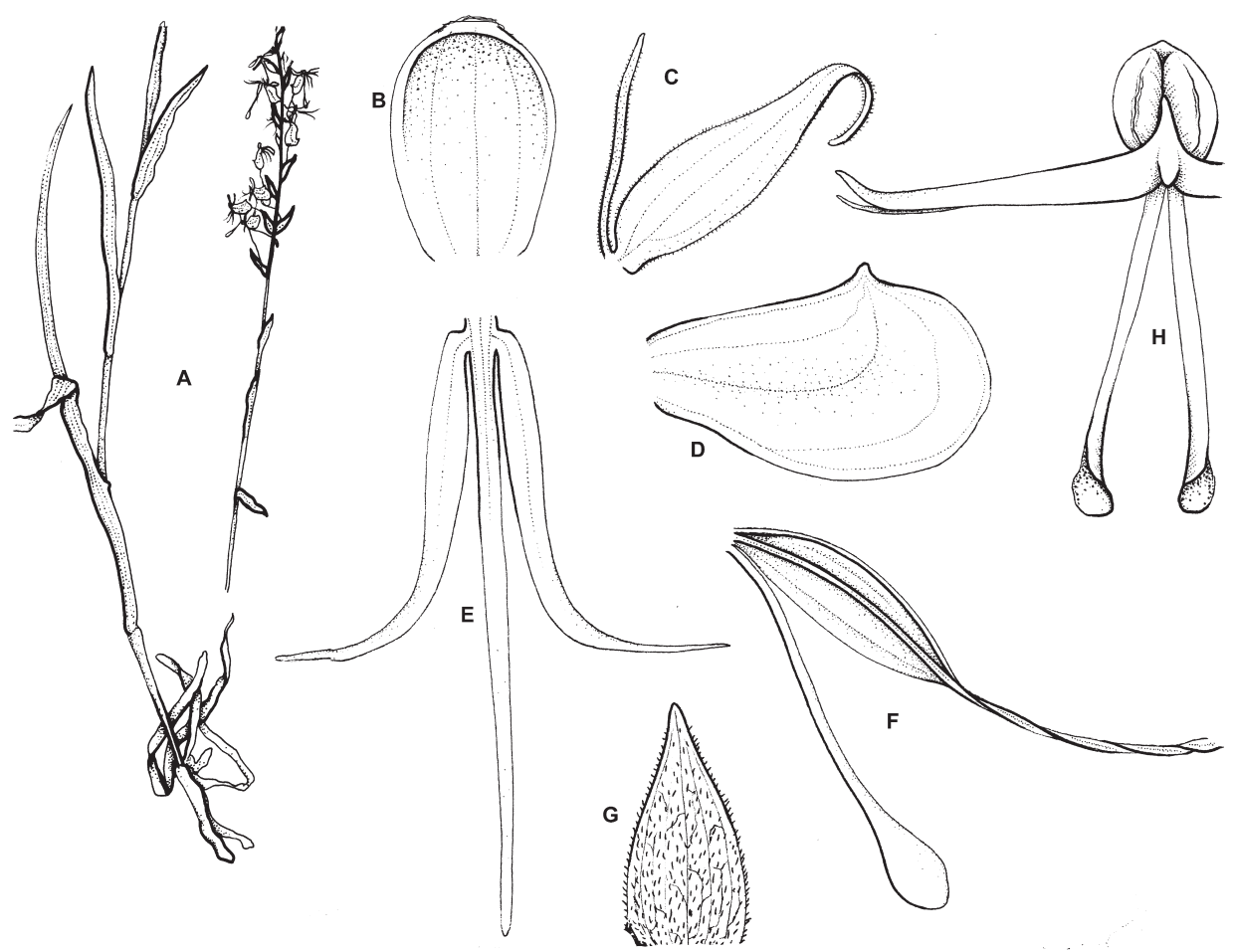

Fig. 85. Bilabrella lisowskii (Szlach.) Szlach. \& Kras-Lap.

Explanations: A - habit; B - dorsal sepal; C - petal; D - lateral sepal; E - lip; F - ovary, pedicel, spur; G, bract; H - gynostemium, front view (drawn by D L. Szlachetko from holotype of Habenaria lisowskii Szlach. - Lisowski C-239, UGDA-DLSz) 


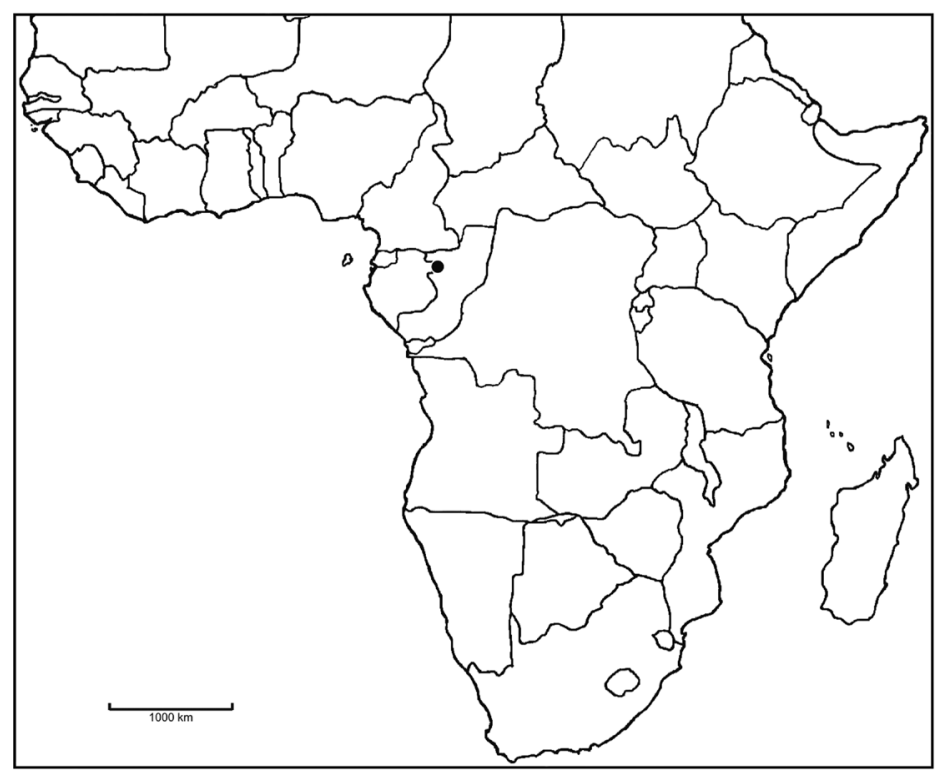

Fig. 86. Distribution of Bilabrella lisowskii (Szlach.) Szlach. \& Kras-Lap.

somewhat fleshy; the middle lobe $13 \mathrm{~mm}$ long, $0.5 \mathrm{~mm}$ wide, linear, acute; lateral lobes 10-11 mm long, $0.7 \mathrm{~mm}$ wide, lanceolate-falcate, acute. Spur $15 \mathrm{~mm}$ long, $1.8 \mathrm{~mm}$ in diameter at the apex, narrowly cylindrical, distinctly swollen in the apical quarter, blunt, pendent. Anther ca. $3 \mathrm{~mm}$ tall; connective broadly triangular, acute; antherophores ca. $7 \mathrm{~mm}$ long, slender, abruptly upcurved at the apex. Stigmaphores ca. $7 \mathrm{~mm}$ long, slender, narrowly cylindrical. Rostellum middle lobe ca. half as long as connective. Auriculae entire (Fig. 85).

Ecology. Damp places.

Distribution. Not known elsewhere (Fig. 86).

Specimens examined. Congo. Salines de Mbouebé, Lisowski C-239 (UGDA-DLSz!).

N o te s. It is similar to Bilabrella cornutella, but in contrast to this species its petals are densely ciliate.

30. Bilabrella hochstetteriana (Kraenzl. \& Schltr.) Szlach. \& Kras, Richardiana 9(4): 158. 2009.

Habenaria hochstetteriana Kraenzl. \& Schltr., Orchid. Gen. \& Sp. 1: 210. 1898. - Kraenzlin, Orchid. Gen. Sp.: 210. 1901.

Type (hoc lococ selectus): Djeladjeranne, Schimper III. 1645 b (lectotype, P!; isolectotypes, BR!, K!, S not seen, $\mathrm{W}$ !).

Tubers ovoid. Stem up to $70 \mathrm{~cm}$ tall, slender, leafy. Leaves 5-8, up to $20 \mathrm{~cm}$ long, 0.9-1.8 cm wide, gathered in the lower part of the stem, narrowly linear-lanceolate, acute, erect-spreading, decreasing in size gradually upwards, the basal ones sheath-like. Inflorescence $7-10 \mathrm{~cm}$ long, laxly multiflowered. Floral bracts 10$19 \mathrm{~mm}$ long, ovate-lanceolate, acute, scarious, shorter than pedicel and ovary. Pedicel and ovary $25 \mathrm{~mm}$ long.
Dorsal sepal $5 \mathrm{~mm}$ long, $2 \mathrm{~mm}$ wide, oblong-ovate or oblong-elliptic, obtuse, reflexed. Petals bilobed to the base; anterior lobe 7.5-9 mm long, 1.5-2 mm wide, oblong- or ligulate-lanceolate, acute, glabrous or sparsely papillose; posterior lobe 4-4.5 mm long, 0.4$0.5 \mathrm{~mm}$ wide, linear-filiform or linear, acute, papillose. Lateral sepals $7 \mathrm{~mm}$ long, $4 \mathrm{~mm}$ wide, cuneate-obovate, rounded at the apex with laterally placed apiculus, concave. Lip 3-lobed above very short claw; the middle lobe 9-10 mm long, 0.5-1 mm wide, linear, acute; lateral lobes 4-6 mm long, 0.4 mm wide, linear, acute, pendent. Spur 16-20 mm long, filiform, swollen in apical third, blunt, parallel to the ovary. Anther $2 \mathrm{~mm}$ long; connective rounded; antherophores $4 \mathrm{~mm}$ long, slender, gently upcurved apically. Stigmaphores $5 \mathrm{~mm}$ long, narrowly cylindrical, truncate, glandular. Rostellum middle lobe distinctly shorter than connective. Auriculae small, very obscurely bifid (Figs 87-88).

E c o logy. In wet meadow, among tussocks of Exotheca, stoney ground and volcanic sand. MarchDecember.

D i s tributi o n. Ethiopia, Uganda, Kenya, Tanzania, alt. 600-2550 m (Fig. 89).

Specimens examined. Ethiopia. Tigray: Djeladjeranne, Schimper III, 1645 b (BR!, K!, P!, W!). Uganda. Lake Karenge, 13 Nov. 1903 (fl), Bagshawe

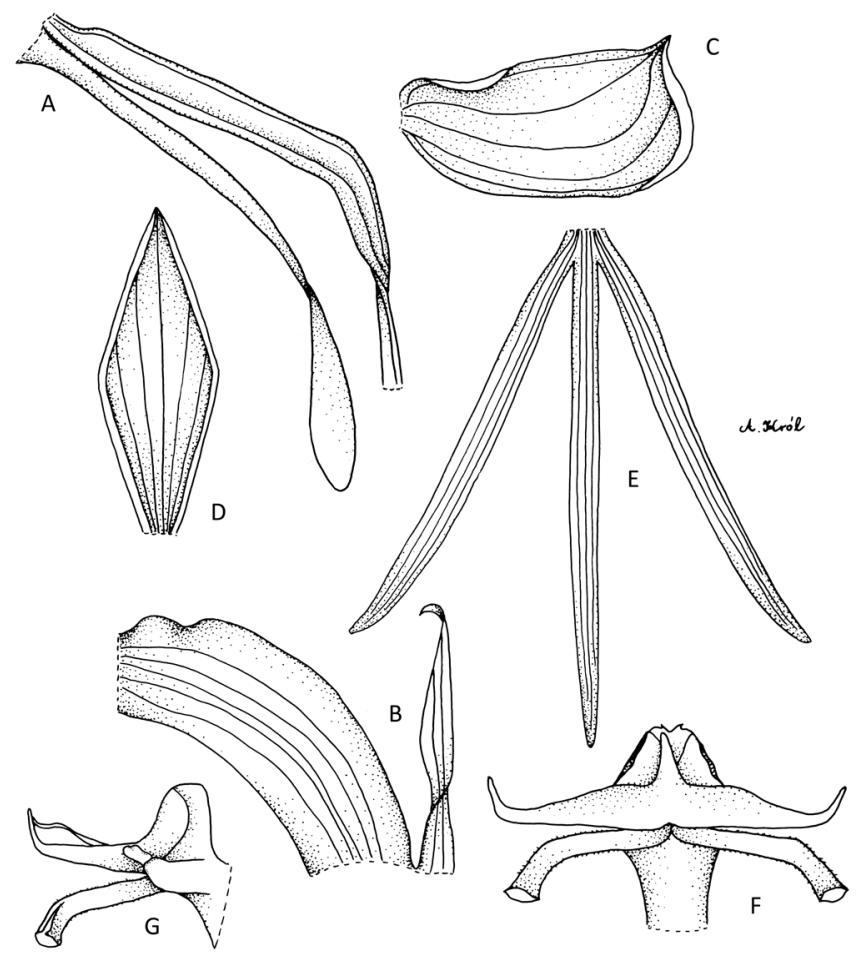

Fig. 87. Bilabrella hochstetteriana (Kraenzl. \& Schltr.) Szlach. \& Kras

Explanations: A - ovary, pedicel, spur; B - petal; C - lateral sepal; D - dorsal sepal; E - lip; F - gynostemium, front view; $\mathrm{G}$ - gynostemium, side view (drawn by A. Król from isolectotype of Habenaria hochstetteriana Kraenzl. \& Schltr. - Schimper III.1645, BR) 


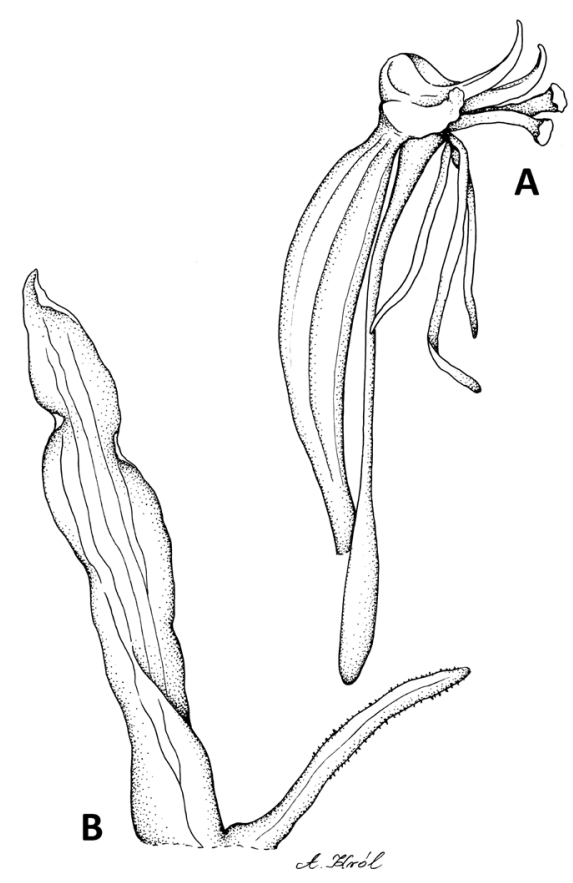

Fig. 88. Bilabrella hochstetteriana (Kraenzl. \& Schltr.) Szlach. \& Kras

Explanations: A - ovary, gynostemium, spur and lip; B - petal (drawn by A. Król from isolectotype of Habenaria hochstetteriana Kraenzl. \& Schltr. - Schimper III.1645b, K)

s.n. (BM!). Eastern: Kapchorwa Distr., Mt. Elgon, Butandiga, 600 m, Aug. 1934 (fl), Hansfort S. 797 (BM!); Mbale Distr., Birunga. Mts., Kigezi, wet meadow on slopes of Mgahinga Mt., 2100-2250 m, 12 Mar. 1934 (fl), Longfield 85 (BM!). Kenya. Rift Valley: Naivasha Prov., Aberdare Mts., Kinangop, open short grassland, 2550 m, Oct. 1934 (fl), Synge S. 1119 a (BM!). Tanzania.

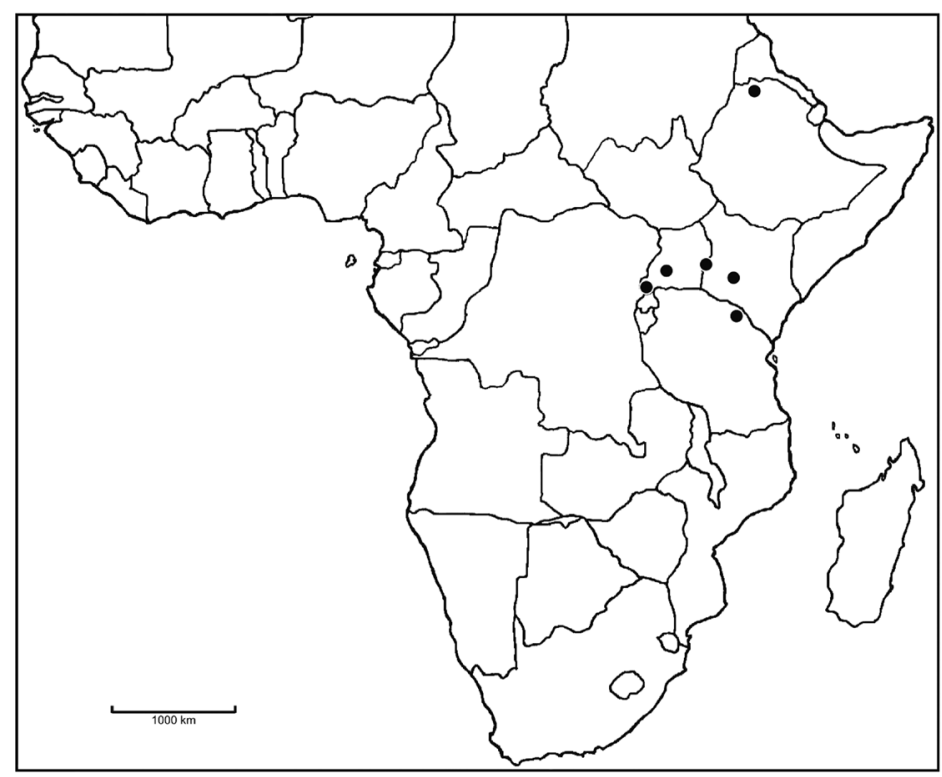

Fig. 89. Distribution of Bilabrella hochstetteriana (Kraenzl. \& Schltr.) Szlach. \& Kras
Arusha: Engare Nanyuki River, Momela, on rough stoney ground, shallow soil of volcanic sand, among tussocks of Exotheca, 1524 m, 10 Dec. 1968 (fl), Richards 23314 (K!).

$\mathrm{N}$ o t e s. Apparently similar to B. limnophila, but has different spur and completely different gynostemium. This species is sometimes treated as conspecific with both $B$. humilior and B. replicata. In our opinion however, it differs from both species mentioned by having glandular stigmaphores and rostellum middle lobe distinctly shorter than connective. Additionally, in $B$. humilior the spur is longer than pedicel and ovary, and in $B$. replicata posterior petal lobes are ciliolate.

31. Bilabrella replicata (Hochst. ex A. Rich.) Szlach. \& Kras, Richardiana 9(4): 159. 2009.

Habenaria replicata Hochst. ex A. Rich., Tent. Fl. Abyss. 2: 296. 1851.

Type (Szlachetko et al. 2010): Ethiopia, Schimper 1645 a (lectotype, designated by Szlachetko et al. [2010: 235], P!; isolectotypes, BM!, K!, S not seen, W!, W-R!).

Tuber $1.5 \mathrm{~cm}$ long, $0.8 \mathrm{~cm}$ in diameter, ovoid or ellipsoid. Stem tp $80 \mathrm{~cm}$ tall, erect, rather delicate, glabrous. Leaves $6-8$, to $13 \mathrm{~cm}$ long and to $1.1 \mathrm{~cm}$ wide, linear-lanceolate or narrowly lanceolate, spread, decreasing in size up the stem. Inflorescence $8-11 \mathrm{~cm}$ long, 12-20-flowered, rather lax. Flowers greenish-white. Floral bracts 8-14 mm long, oblong-lanceolate to ovatelanceolate, acuminate, glabrous. Pedicel and ovary 15-24 mm long, glabrous. Dorsal sepal 5-7.5 mm long, $2.5 \mathrm{~mm}$ wide, elliptic to elliptic-ovate, obtuse, slightly cochleate, glabrous, deflexed. Petals bilobed to the base; anterior lobe 8-9 mm long, 1.5-2 mm wide, obliquely oblong-lanceolate, subacute to apiculate, glabrous; posterior lobe $4.5 \mathrm{~mm}$ long, $0.3 \mathrm{~mm}$ wide, linear-filiform, thin, delicate, ciliolate. Lateral sepals 6.5-7.5 mm long, 4-5 mm wide, obliquely oblong-elliptic, concave, with laterally placed short, obtuse apiculus, glabrous. Lip 3-lobed to the base, glabrous, deflexed; the middle lobe 8-9 mm long, 0.7-0.9 mm wide, linear, lanceolate above the middle, acute; lateral lobes $5.5 \mathrm{~mm}$ long, $0.5-0.7 \mathrm{~mm}$ wide, linear, acuminate, pendent. Spur 11.5-16.5 mm long, narrowly cylindrical, moderately swollen in the apical half or third, and here slightly swollen, subobtuse, pendent. Anther 1.5-3 mm long; connective truncate to shallowly notched; antherophores 3-4.5 mm long, slender, upcurved abruptly at the apex. Stigmaphores 3-5 mm long, cylindrical, truncate apically. Rostellum middle lobe subulate-filiform, slightly longer than connective. Auriculae rather small, irregular.

E c o logy. No records. August-October.

D istribution. Ethiopia (Fig. 90).

S pecimen s examined. Ethiopia. Tigray: Djeladjeranne, 10 Aug. 1840 (fl), Schimper 1645a (BM!, 


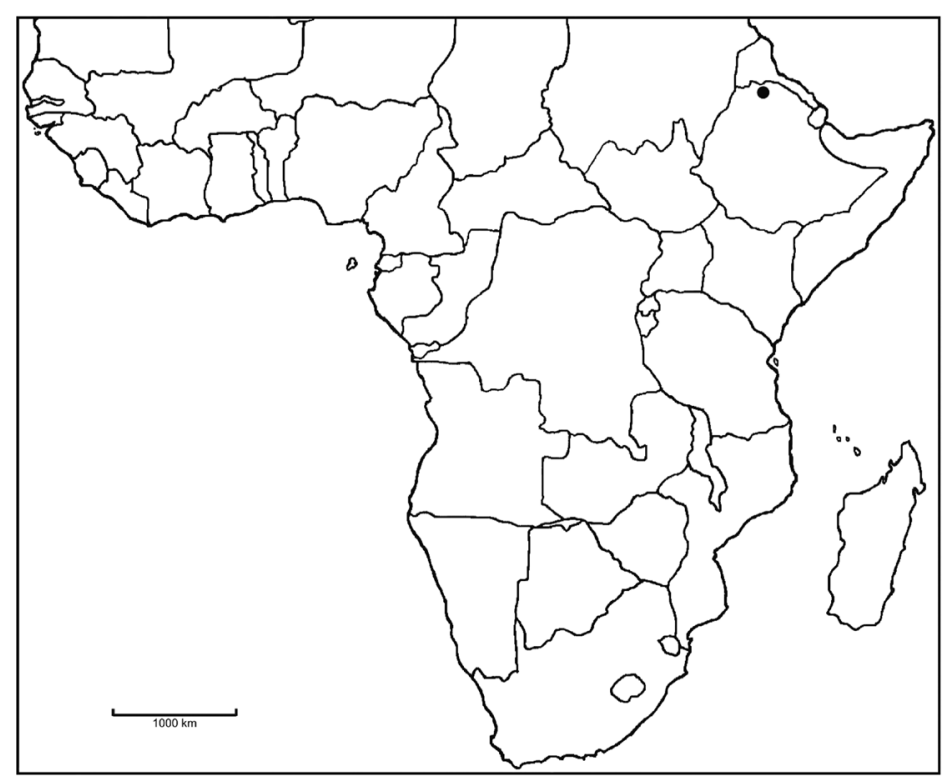

Fig. 90. Distribution of Bilabrella replicata (Hochst. ex A. Rich.) Szlach. \& Kra

K!, P!, W!, W-R!); Oct. 1908 (fl), Brockman 194 (K!). $\mathrm{N}$ o t e s. It appears to be similar to $B$. hochstetteriana, from which it is easily distinguishable by ciliolate petals posterior lobe (vs papillose) and shorter spur (11.5$16.5 \mathrm{~mm}$ long vs $16-20 \mathrm{~mm}$ long).

Isolectotype number kept at W-R is unusual in the flower structure - its lip is unlobed, lanceolate, acute and petals consist of two narrowly triangular lobes.

32. Bilabrella huillensis (Rchb. f.) Szlach. \& Kras-Lap., Richardiana 3(3): 140. 2003. - Szlachetko et al., Orchid. of West-Central Africa 1: 234. 2010.

Habenaria huillensis Rchb. f., Flora 48: 179. 1865. Rolfe, Fl. Trop. Afr. 7: 240. 1898. - Kraenzlin, Orchid. Gen. Sp.: 220. 1901. - Andrews, Flow. Pl. A.E. Sudan 3: 323. 1956. - Summerhayes, FWTA, ed. 2, 3: 196. 1968a. - Summerhayes, FTEA, Orchid. 1: 96. 1968b. - Geerinck \& Coutrez, Not. Taxon. Orchid. Afr. Centr., Habenaria: 9. 1977. - Reekmans, Lejeunia, Nov ser. 100: 45. 1980. - Geerinck, Fl. Afr. Centr., Orchid. 1: 113. 1984. - la Croix \& Cribb, Fl. Zambes., Orchid. 1: 96. 1995. - Stewart \& Campbell, Orchids of Kenya: 134. 1996. - Szlachetko \& Olszewski, Fl. Cam., Orchid. 34(1): 186. 1998.

Type: Angola, Welwitsch 724 (lectotype, designated by Szlachetko et al. [2010: 234], BM!; isolectotypes, $\mathrm{K}$ !, W-R!).

Tuber 1-3 cm long, ca. $1 \mathrm{~cm}$ in diameter, ovoid or ellipsoid. Stem $30-110 \mathrm{~cm}$ tall, erect, stout or realtively delicate, glabrous. Leaves 8-16, 8-37 cm long, up to 1.5 (occasionally 2) cm wide, linear-lanceolate, acute, suberect, decreasing in size up the stem. Inflorescence
6-27 cm long, 8-50-flowered, rather lax to dense. Flowers green or yellowish-green with whitish centre. Floral bracts 10-25 mm long, glabrous or in the lower half densely glandular along margins and inside. Pedicel and ovary 15-20 mm long, glabrous. Dorsal sepal 4.5$7.5 \mathrm{~mm}$ long, $1.5-3 \mathrm{~mm}$ wide, oblong-lanceolate to oblong-obovate, apiculate, truncate or obtuse at the apex, cochleate, glabrous. Petals bilobed to the base; anterior lobe 9-14 mm long, 1-2.5 mm wide, obliquely lanceolate, linear- or oblong-lanceolate, apiculate, rather thick, glabrous or rarely papillose; posterior lobe $4.5-7.5 \mathrm{~mm}$ long, 0.2-0.6 mm wide, linear or filiform, thin, delicate, ciliolate, sometimes glabrous. Lateral sepals $6.5-10 \mathrm{~mm}$ long, 3-5 mm wide, obliquely oblong-elliptic, concave, with laterally placed short, erect apiculus, glabrous. Lip 3-lobed above the basal undivided part 0.5-1 $\mathrm{mm}$ long, thick, fleshy, glabrous; the middle lobe 9-14 mm long, 0.9-1.2 mm wide, linear-lanceolate, acute; lateral lobes 6-13.5 mm long, 1-1.2 mm wide, obliquely narrowly lanceolate, acuminate to apiculate, upcurved. Spur 1220 (rarely 30) $\mathrm{mm}$ long, $0.8-1 \mathrm{~mm}$ in diameter in the basal part, $1.5 \mathrm{~mm}$ in diameter at the apex, moderately swollen in the apical half, subacute, pendent. Anther 1.5-2.5 mm long; connective truncate; antherophores (3.5)5-8 mm long, slender, slightly upcurved. Stigmaphores of the same length, relatively massive, straight, truncate. Rostellum middle lobe subulate, longer than connective. Auriculae rather small, obscurely bifid or entire (Figs 91-95).

E c o logy. Terrestrial in grassland and marsh, with Brachystegia boehmii, rocky and laterite soil. JanuaryOctober.

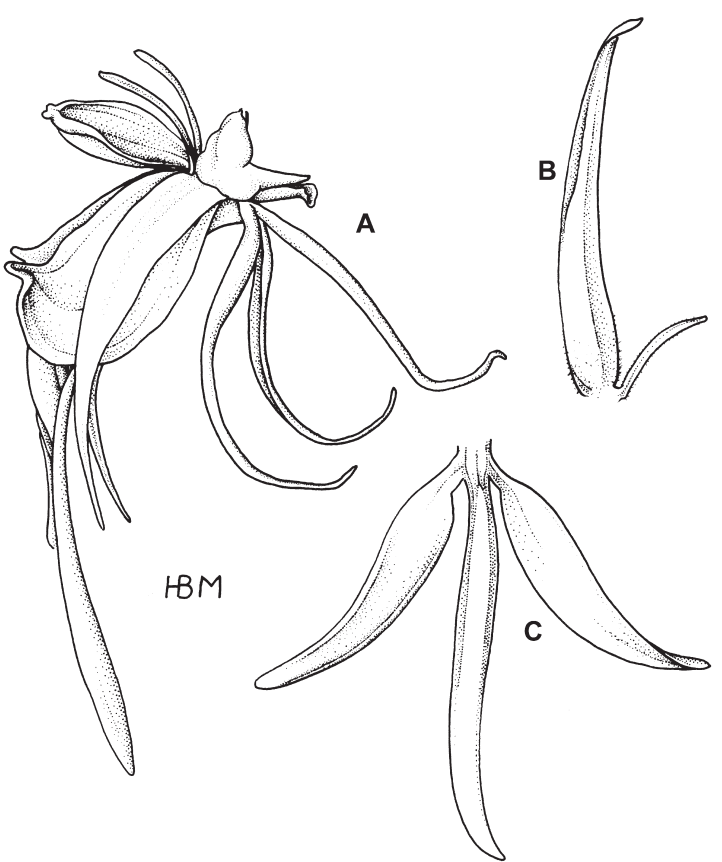

Fig. 91. Bilabrella huillensis (Rchb. f.) Szlach. \& Kras-Lap. Explanations: A - flower; B - petal; C - lip (drawn by H. B. Margońska from isolectotype of Habenaria huillensis Rchb. f. - Welwitsch 724, W-R) 


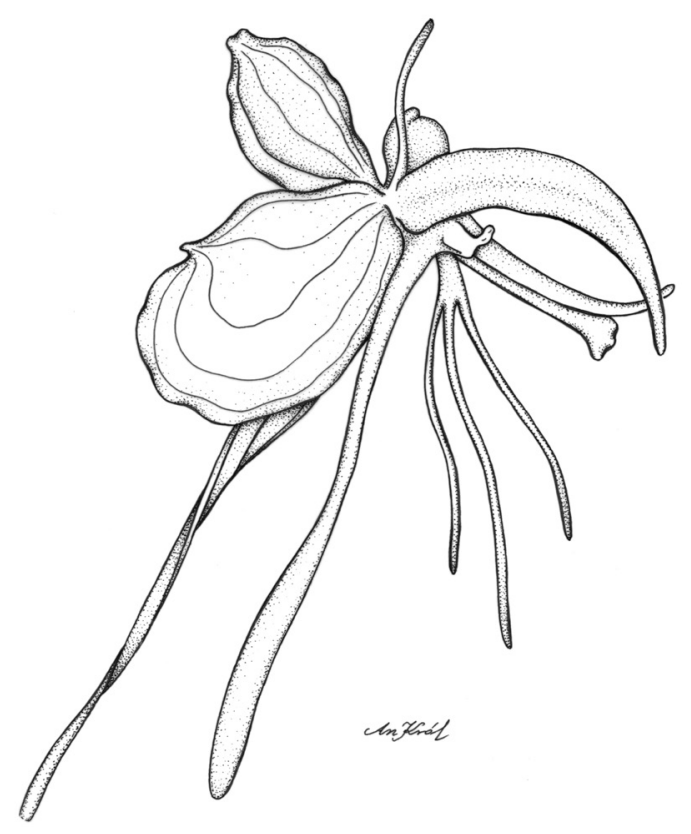

Fig. 92. Bilabrella huillensis (Rchb. f.) Szlach. \& Kras-Lap. - flower (drawn by A. Król from Bolus 477, AMES)

D i stribution. Chad, Sudan, Ghana, Nigeria, Cameroon, Central African Republic, Congo, Democratic Republic of the Congo (Zaire), Uganda, Burundi, Kenya, Angola, Zambia, Malawi, Zimbabwe, Republic of South Africa, alt. 165-2250 m (Fig. 96).

Specimens examined. Chad. Terr. of Chari, Mondjia and Krébédje villages, near Fort Sibut, 4 Nov, 1902 (fl), Chevalier 6052 (P!). Sudan. Equatorial: Kagelu Station, vlei, in small swamp on western boundary, 22 Sep. 1937 (fl), Myers 7761 (AMES!, K!); North Darfur: Golol proposed forest reserve, foot of Jebel Marra, in new plantation, on old cultivation area with terraces, over basalt, Anogeissus dominant, rare, $12^{\circ} 54^{\prime} \mathrm{N}, 23^{\circ} 51^{\prime} \mathrm{E}, 10$ Sep. 1960 (fl), Jackson 4046 (K!). Ghana. Volta: Akan Wawa Distr., Mountain summit $\mathrm{E}$ of Ahamansu village, on Togo frontier, amongst bunchgrasses in shallow stony soil undisturbed except

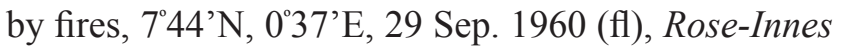
31331 (K!); On road by football park to Genui, $900 \mathrm{~m}$, 2 Aug. 1952 (fl), Westwood 27 (K!); 840 m, July 1954 (fl), Westwood 27A (K!). Nigeria. Bauchi: Northern Part, Lame Distr., Rahama, in grass with scrub, $810 \mathrm{~m}$, 20 Aug. 1954 (fl), Summerhayes 29 (K!); The same locality, among tall grasses, 12 Sep. 1948 (fl), Summerhayes s.n. (FHI 22442) (K!); Nabatum, 1320 m, Oct. 1957 (fl), King 33B (K!); Rocks, Sep. 1930 (fl), Lely P732 (K!); Kaduna: Northern Part, near Zaria, 12 Sep. 1929 (fl), Bryant s.n. (K!); Oyo: Ibadan Fuel Plantation, along ride in Cassia plantation, just below Forestry HQ office, 3 Sep. 1948 (fl), Keay s.n. (FHI 22440) (K!); Plateau: Northern Part, Jos Plateau, 1946 (fl), BattenPoole 183 (K!); Owo, on rocks, 1140 m, Sep. 1957 (fl),
King 32 (K!); Plateau, 1140 m, Oct. 1957 (fl), King 33 (K!). Cameroon. East: Nangue Mountain, Ngaoundere and Meiganga, 18 Sep. 1967 (fl), Jacques-Felix 8211 (P!); N side of Ngaoundere Plateau, 20 Sep. 1967 (fl), Jacques-Felix 8221 (P!). Central African Republic. Bamingui-Bangoran: Manovo-Gounda-St. Floris Natl. Park, $0.5 \mathrm{~km}$ N of Camp Koumbala on Kpana Creek, $0.5 \mathrm{~km}$ upstream of confluence of Koumbala Creek, $8^{\circ} 29^{\prime} \mathrm{N}, 21^{\circ} 13^{\prime} \mathrm{E}, 595$ m, 12 Dec. 1983 (fl), Fay 5829 (K!). Congo. Valley Tomi, 7 Sep. 1902 (fl), Chevalier 5375 (P!). Democratic Republic of the Congo (Zaire). Haut-Katanga: N Elisabethville, in marshes, $1400 \mathrm{~m}$, Jan. 1939 (fl), s.n. 73 (K!); Haut Katanga, 26 Jan. 1924 (fl), Hirschberg 187 (K!); Kipushi, vlei, 27 Jan. 1908 (fl), Kassner 2414 (HBG!); Kipushi, 10 Mar. 1970 (fl), Lisowski 65767 (UGDA-DLSz!); Kipopo, Lubumbashi, 12 Feb. 1969 (fl), Lisowski 65746 (UGDA-DLSz!); Lubumbashi, Kasapa, ain forest, 1220 m, 16 Jan. 1969 (fl), Lisowski 65560 (UGDA-DLSz!); Lubumbashi, 16 Jan. 1961 (fl), Lisowski 65760 (UGDA-DLSz!); Near Tsalamalala village, $12 \mathrm{~km} \mathrm{~W}$ from L'she, 1 Jan. 1970 (fl), Lisowski 65765 (UGDA-DLSz!); Haut-Lomami: Kiankwali, Biano Plateau, 5 May 1957 (fl), Dovigneaud 30270 (BRLU!); Lomami, May 1984 (fl), Dandus 355 (BRLU!); With Brachystegia boehmii on laterite ground, 26 Jan. 1960 (fl), Dovigneaud 5254 (BRLU!);

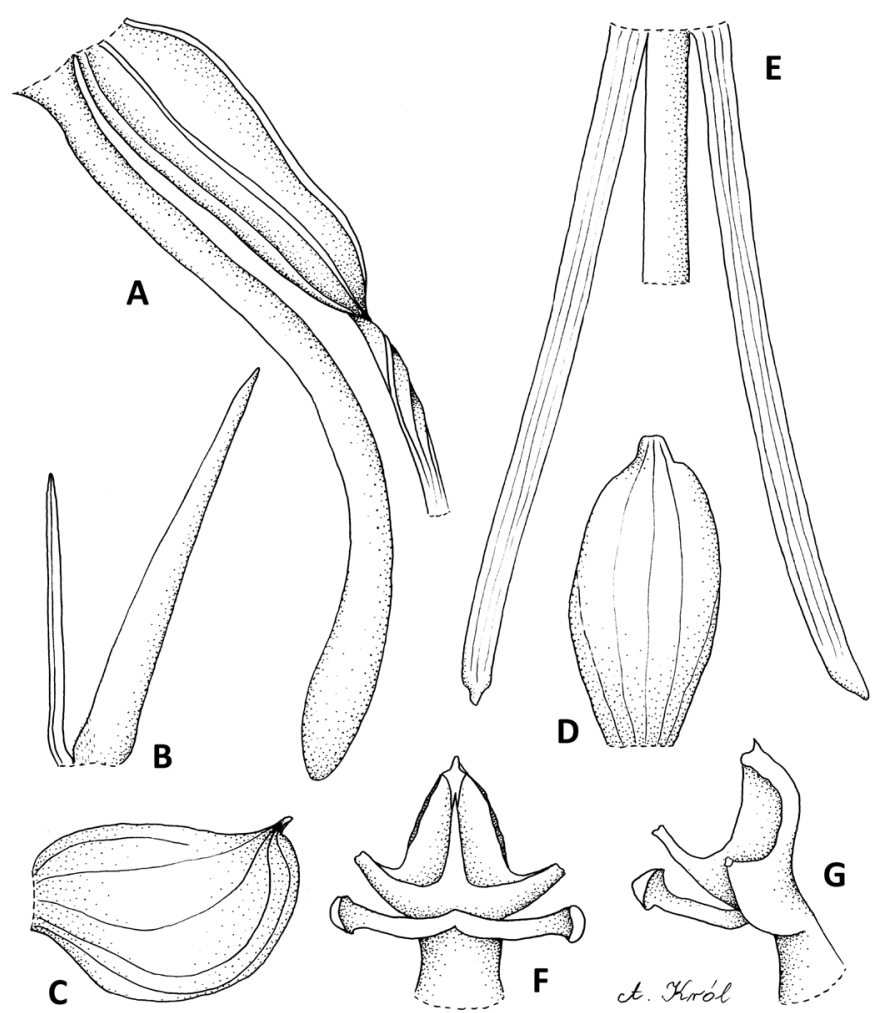

Fig. 93. Bilabrella huillensis (Rchb. f.) Szlach. \& Kras-Lap. Explanations: A - ovary, pedicel, spur; B - petal; C - lateral sepal; D dorsal sepal; E - lip; F - gynostemium, front view; G - gynostemium, side view (drawn by A. Król from lectotype of Habenaria huillensis Rchb. f. - Welwitsch 724, BM) 
Kasaï Oriental: Lupaka, 6 Feb. 1908 (fl), Kassner 2458 (HBG!); Nord Kivu: Busumbura - Bugarama, 1 Jan. 1993 (fl), Arbonnier 383 (BR!); Sine loc., Feb. 1908 (fl), Kassner 3564 (HBG!). Uganda. Northern: Arua Distr., West Nile subregion, between Chei Hill and Kaia River, Aringa Country, Sep. 1937 (fl), Eggeling 3407 (K!); Arua Distr., Valley $1 / 2$ mile $\mathrm{S}$ of Maracha West Camp, near streams and also of hills on shallow soil on rocky outcrops, 1380 m, 3 Aug. 1953 (fl), Chancellor 95 (K!); Kotido Distr., Karamoja subregion, Dodoth Sangaar River, near Lolelia, SE of Rom, savannah, $1200 \mathrm{~m}$, Sep. 1950 (fl), Eggeling 5997 (K!); Moroto Distr., Mt. Moroto, grassland on steep slopes, 2250 m, 5 Sep. 1956 (fl), Hardy \& Bally 10761B (K!); Western: Bushenyi Distr., Farm Institute, black soil, dry, 900 m, Dec. 1994Jan 1995 (fl), Spurrier V.29 (K!); In grass, woodland, 15 Aug. 1969 (fl), s.n. 6153 (K!); Hoboko, near Nile, grassland, Aug. 1938 (fl), Hazel 668 (AMES!, K!). Burundi. Gitega: Road Gitega-Karuzi, Rusi, 1800 m, 28 Dec. 1966 (fl), Lewalle 1397 (BR!); Rutana: Rutana rocky steppe, 30 Jan. 1976 (fl), Reekmans 4710 (C!); Ruyigi: Kinyinya, 27 Dec. 1992 (fl), Arbonnier 377 (BR!). Kenya. Coast: Taita Hills, in grassland at foot of precipice, 1200 m, 19 May 1931 (fl), Napier 1144 (K!); Nyanza: Trano-Nzoia, Kiminini, 1770 m, Sep. 1944 (fl), Tweedie 628 (K! - spirit.); Western: E Mt.

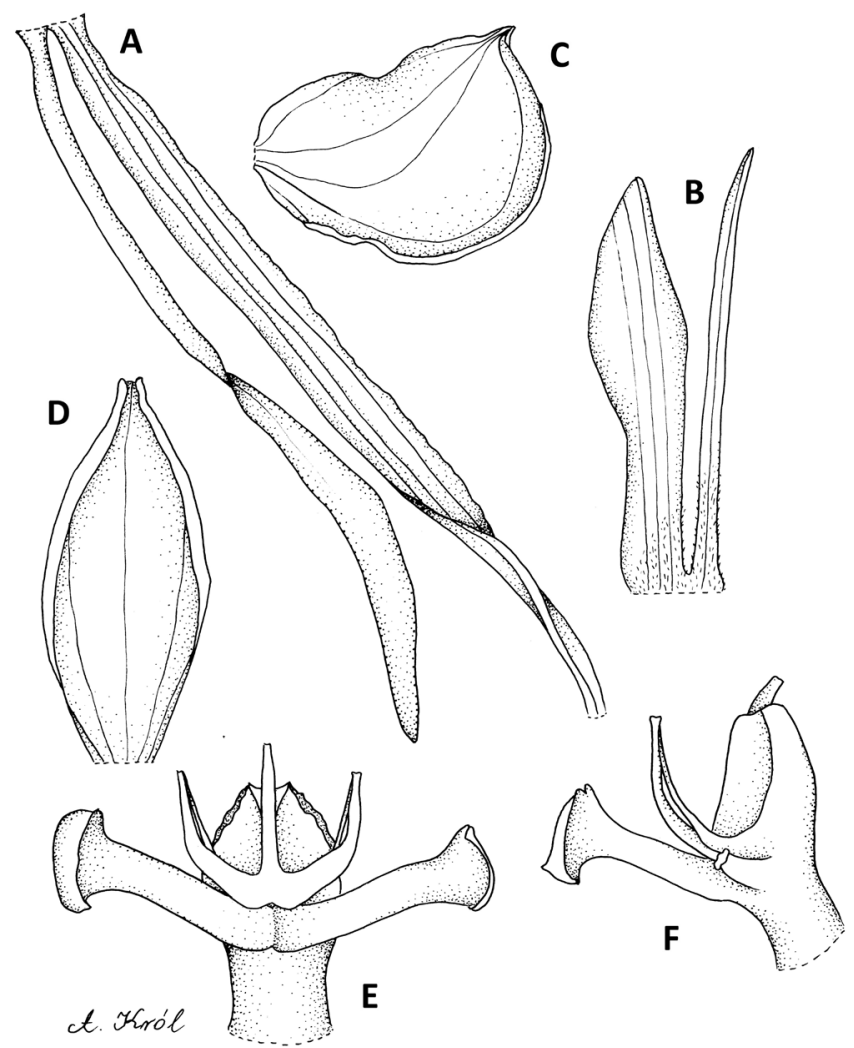

Fig. 94. Bilabrella huillensis (Rchb. f.) Szlach. \& Kras-Lap. Explanations: A - ovary, pedicel, spur; B - petal; C - lateral sepal; D - dorsal sepal; E - gynostemium, front view; F - gynostemium, side view (drawn by A. Król from Habenaria huillensis Rchb. f. - Lisowski 65767, UGDA-DLSz)

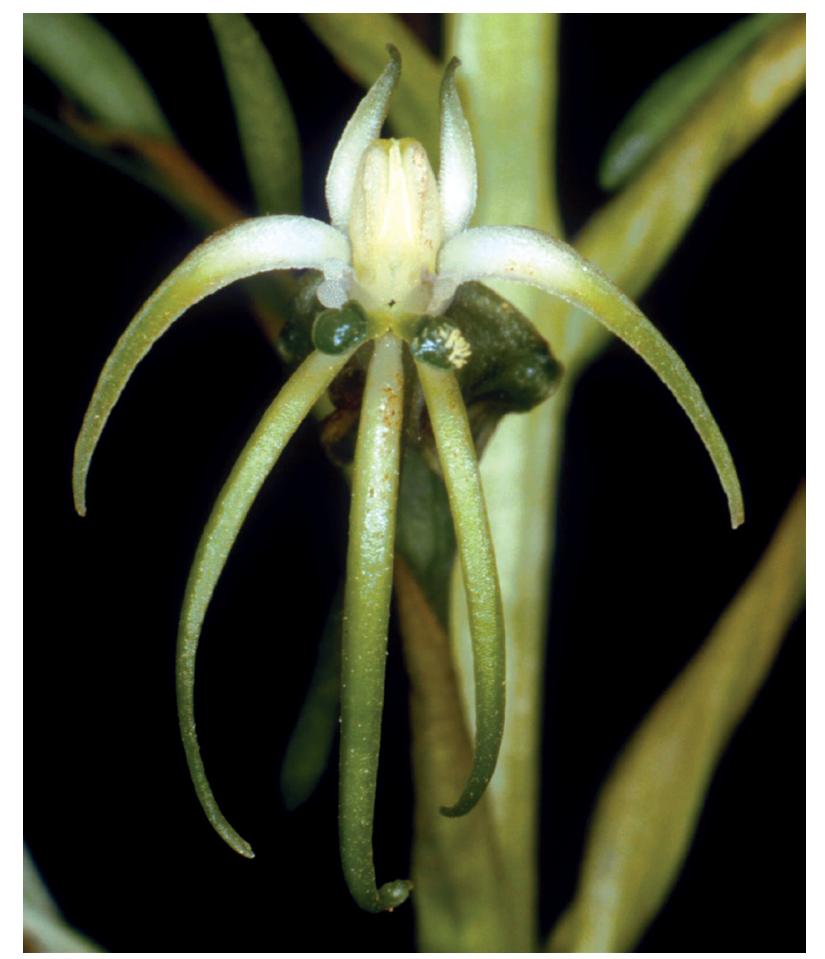

Fig. 95. Bilabrella huillensis (Rchb.f.) Szlach. \& Kras-Lap. - flower close-up (phot. L. Grobler)

Elgon, in open stony grassland, $2250 \mathrm{~m}, 1$ Oct. 1955 (fl), s.n. 224 (K!). Angola. Benguela: Oyera Chara, near Babaera, 10 Dec. 1959 (fl), Stopp BO158 (K!); Huila: Between Monino R. and lake of Ivantala (fide Rolfe 1898) Welwitsch 724c.; Huila, s.n. 14 (K!); Antunes 14 (K! - part); Malange: Feb. 1880 (fl), Mechow 437 (W-R!); South Kuanza: Lopollo ad Catumba, Mar. 1860 (fl), Welwitsch 724b (W-R!). Zambia. Northern: Abercorn Distr., road to Uningi Pans, on open grassland

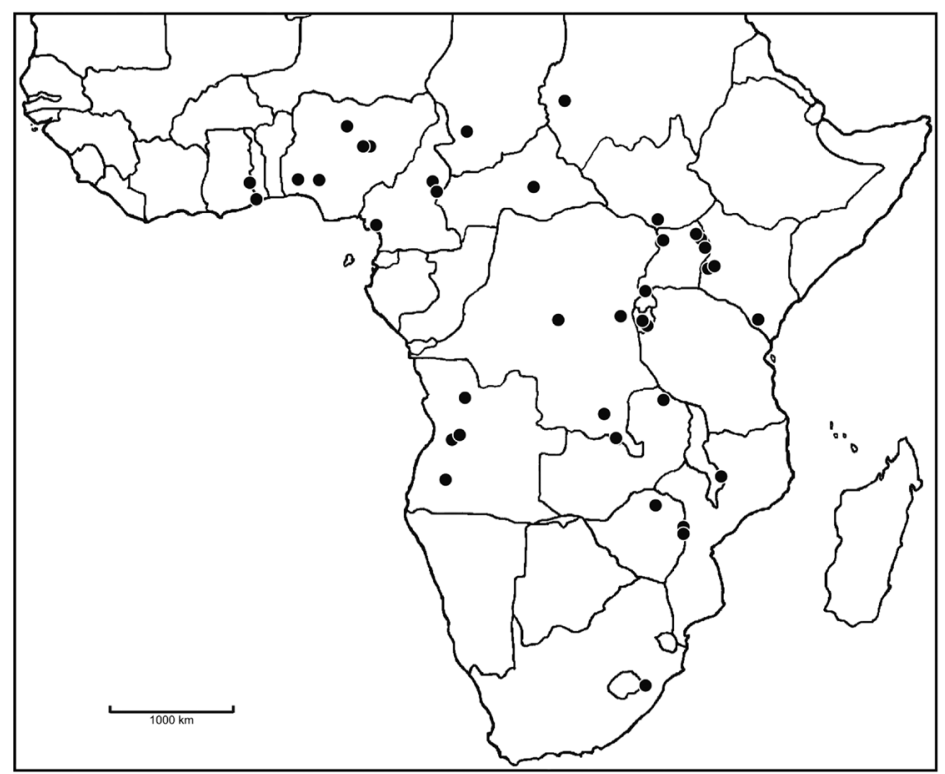

Fig. 96. Distribution of Bilabrella huillensis (Rchb.f.) Szlach. \& Kras-Lap. 
and marshy land, 1500 m, 12 Feb. 1967 (fl), Richards $22080 a$ (K!); Abercorn, Kwimbe dambo, in drainage ditch of dambo, $08^{\circ} 50^{\prime} \mathrm{S}, 31^{\circ} 33^{\prime} \mathrm{E}, 165 \mathrm{~m}, 25$ May 1967 (fl), Robertson 626 (K!). Malawi. Southern: Fort Schalin Distr., Namwera Hills, Mt. Ugugu, Brachystegia woodland, 1200 m, 21 Jan. 1971 (fl), Hillard \& Burtt 6302 (E!). Zimbabwe. Manicaland: Inyanga, 1700 m, 20 Jan. 1931 (fl), Norlindh \& Weimarck 4488 (BM!); Umtali Distr, 1080 m, 21 Mar. 1955 (fl), Chase 50818 (K!); Umtali Distr., Odanri River Valley, 1915 (fl), Teague 341 (K!); Mashonaland West: Lomagundi Distr. $22 \mathrm{~km}$ from Umvukwes to Sipolilo, woodland at foot of kopje, 10 Feb. 1982 (fl), Brummitt \& Drummond 15855 (K!). Republic of South Africa. Kwazulu-Natal: Impendle Distr., Loteni Nature Reserve, 1530 m, 31 Jan. 1879 (fl), Phelan 286 (E!); Girqualand, Kokstad, 1500 m, Feb. 1883 (fl), Bolus 477 (AMES!, BM!, K!, UGDADLSz! - fragment, drawing, W!).

N o t e s. This species is one of few of this group with spur almost equally thick along the entire length. It is similar to B. falcata, B. caffra and B. falcicornis, but despite all of them it has the longest anterior petal lobes reaching $14 \mathrm{~mm}$. In all aforementioned species this part of petal is much shorter, only occasionally exceeding $9 \mathrm{~mm}$.

33. Bilabrella falcata (G. Will.) Szlach. \& Kras.-Lap., Richardiana 3(3): 140. 2003. - Szlachetko et al., Orchid. of West-Central Africa 1: 221. 2010.

Habenaria falcata G.Will., P1. Syst. Evol. 134: 54. 1980. - la Croix \& Cribb, Fl. Zambes., Orchid. 11: 93. 1995.

Type: Zambia, Williamson \& Simon 1775 (holotype, SRGH not seen; isotype, $\mathrm{K}$ !).

Tubers ovoid to ellipsoid. Plants ca. $45 \mathrm{~cm}$ tall, erect, rather slender. Leaves ca. 11, 3-4 of them gathered at the base, $7-10 \mathrm{~cm}$ long, $0.7-1 \mathrm{~cm}$ wide, linear-lanceolate, acute, white with green reticulate veining, decreasing in size up the stem. Inflorescence to $15 \mathrm{~cm}$ long, fairly laxly 16-30-flowered. Flowers green. Floral bracts to $10 \mathrm{~mm}$ long, ovate-lanceolate, acuminate. Pedicel and ovary $20 \mathrm{~mm}$ long, slender, glabrous. Dorsal sepal $5 \mathrm{~mm}$ long, $2 \mathrm{~mm}$ wide, reflexed, elliptic to narrowly obovate, apiculate, obtuse to acute, concave. Petals bilobed to the base; anterior lobes $6-9.5 \mathrm{~mm}$ long, $1.1 \mathrm{~mm}$ wide, narrowly lanceolate, acuminate, reflexed, the apex upturned, glabrous; posterior lobe $5 \mathrm{~mm}$ long, $0.6 \mathrm{~mm}$ wide, filiform, obtuse to acute, erect, ciliolate. Lateral sepals $6.5-8 \mathrm{~mm}$ long, 3.5-5 mm wide, obliquely obovate to elliptic, shortly apiculate, reflexed. Lip pendent, 3-lobed to the base, all lobes slightly upcurved apically; the middle lobe $8-11 \mathrm{~mm}$ long, ca. $0.8 \mathrm{~mm}$ wide, linear-filiform, obtuse; lateral lobes 9-12 mm long, ca. $0.8 \mathrm{~mm}$ wide, linear-filiform, pendent, acute. Spur 22-30 mm long, incurved, slender, cylindrical-filiform, slightly swol-

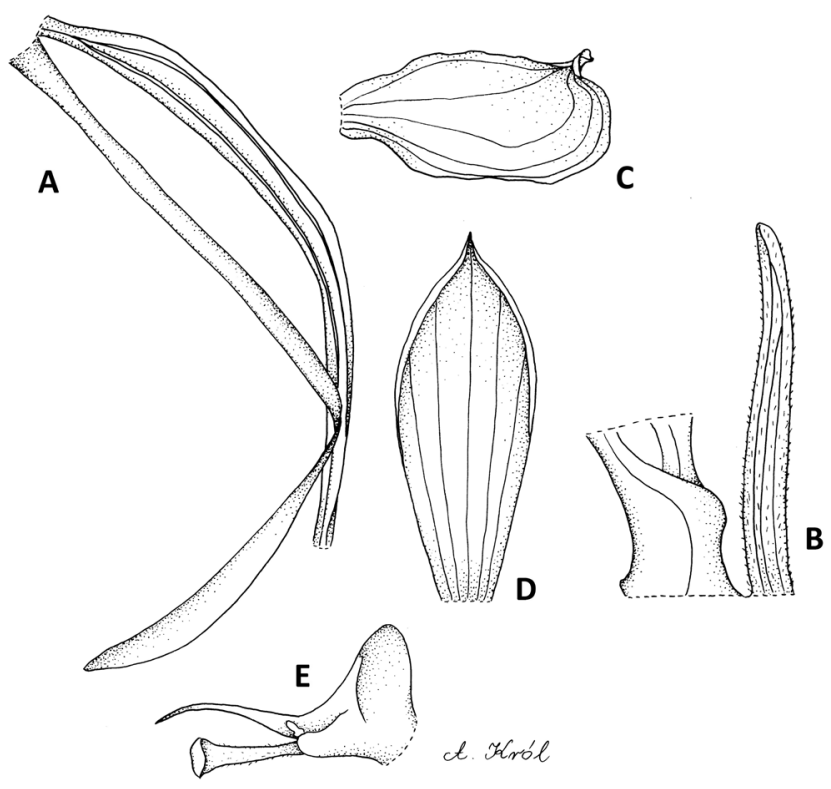

Fig. 97. Bilabrella falcata (G. Will.) Szlach. \& Kras-Lap. Explanations: A - ovary, pedicel, spur; B - petal; C - lateral sepal; D - dorsal sepal; E - gynostemium, side view (drawn by A. Król from isotype of Habenaria falcata G. Will. - Williamson \& Simon 1775, K)

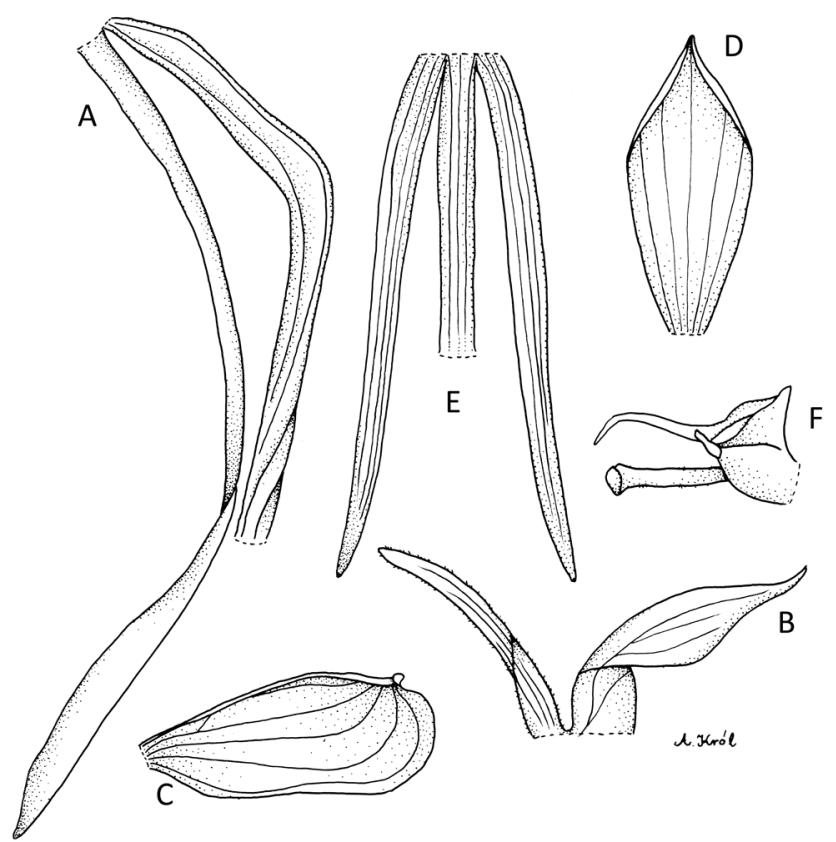

Fig. 98. Bilabrella falcata (G. Will.) Szlach. \& Kras-Lap. Explanations: A - ovary, pedicel, spur; B - petal; C - lateral sepal; D - dorsal sepal; E - lip; F - gynostemium, side view (drawn by A. Król from Habenaria falcata G. Will. - Williamson \& Simon 1775, K)

len below the apex if any, somewhat twisted or not. Gynostemium shortly stalked. Anther $1.8 \mathrm{~mm}$ long; connective obtuse; antherophores $3.5 \mathrm{~mm}$ long, very slender, gently curved down. Stigmaphores $3 \mathrm{~mm}$ long, narrowly cylindrical, truncate, papillose below receptive surface. Auriculae obscurely 3-lobed (Figs 97-98).

E c o logy. Sandy grassland. December. D i stribution. Democratic Republic of the Congo (Zaire) and Zambia, alt. 1500 m (Fig. 99). 


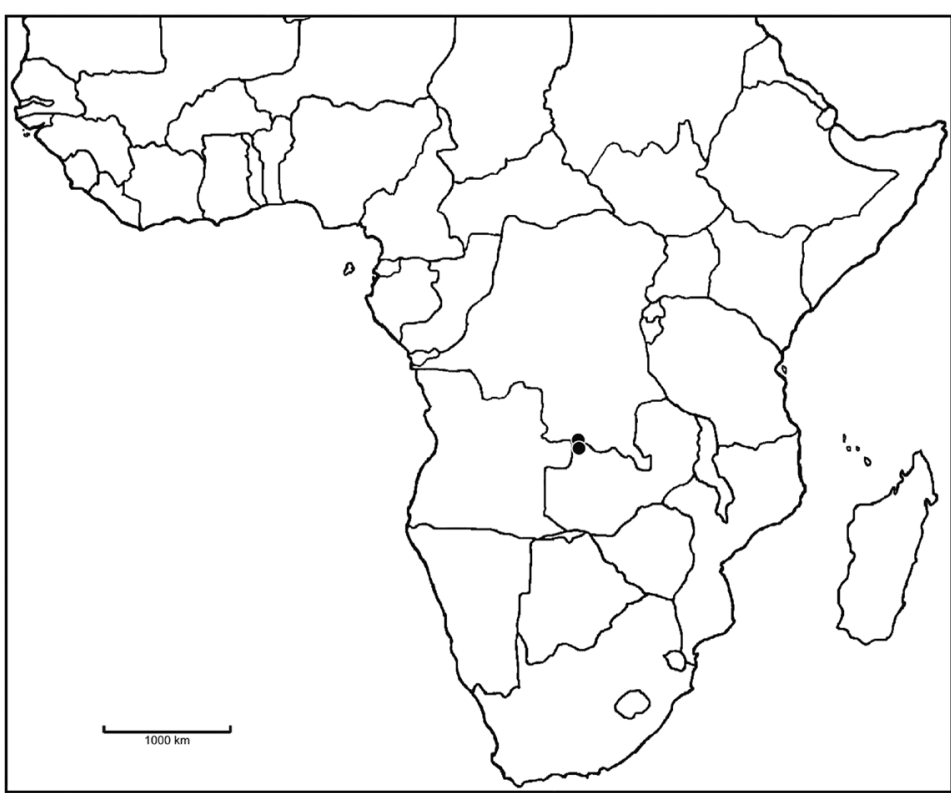

Fig. 99. Distribution of Bilabrella falcata (G.Will.) Szlach. \& Kras-Lap.

Specimens examined. Democratic Republic of the Congo (Zaire). Namibia Plateau, Shaba Region, 11,5 km SW of Koluwezi. Grassland, 1500 m, 25 Dec. 1982 (fl), Schaijes 1681 (K! \& spirit.). Zambia. NorthWestern: Mwinilunga Distr., $40 \mathrm{~km}$ E of Mwinilunga, Dec. 1969 (fl), Williamson \& Simon 1775 (K!).

N o t e s. This species has a very peculiar gynostemium architecture - it is distinctly stalked, like Bilabrella macrostele. Unlike the latter, however, stigmaphores and antherophores of $B$. falcata are elongate and slender, much longer than anther fertile part. The unique character of this species are papillose stigmaphores below receptive surface. $B$. falcata is probably related to $B$. huillensis, but differs in that the whole plant plus the leaves are shorter, the petal lobes are always glabrous and the middle lobe of the labellum is shorter than the lateral lobes and apically rounded. This taxon also has a longer falcate spur and a slightly stalked gynostemium. $B$. falcata appears to be also related to $B$. disparalis from which it can easily be distinguished by the lack of small lobes on the anterior petal lobe and the nontwisted falcate spur. In $B$. disparalis the auriculae are very much smaller and the stigmatic processes diverge (cf. Williamson 1980).

34. Bilabrella caffra (Schltr.) Szlach. \& Kras, Richardiana 9(4): 158. 2009.

Habenaria caffra Schltr., Ann. Transvaal. Mus. 10: 242. 1924. - la Croix \& Cribb, Fl. Zambes., Orchid. 11: 89. 1995 (as H. falcicornis var. caffra (Schltr.) Renz \& Schelpe).

Type: Republic of South Africa, Culver 28 (B+; BOL not seen).
Habenaria tetrapetala Kraenzl. ex Rolfe, Fl. Cap. 5(3): 131. 1913, non (Lind1.) Rchb. f., 1865.

Type: Republic of South Africa, Culver 28 (B+; BOL not seen).

Tuberoids $1.5-2 \mathrm{~cm}$ long, $1 \mathrm{~cm}$ in diameter, ovoid to ellipsoid. Stem $30-45 \mathrm{~cm}$ tall, erect, slender to rather stout, leafy. Leaves 6-10, the lower ones $4-11 \mathrm{~cm}$ long and $0.4-0.7 \mathrm{~cm}$ wide, linear or linear-lanceolate, with fine reticulate veining, suberect-spreading, upper leaves grading into bracts. Inflorescence $4-19 \mathrm{~cm}$ long, rather laxly 6-30-flowered. Flowers green and white. Floral bracts $12-30 \mathrm{~mm}$ long, oblong- to ovate-lanceolate, acuminate, sparsely glandular, finely reticulate, usually equalling or slightly shorter than pedicel and ovary. Pedicel and ovary 18-25 mm long, slender, glabrous. Dorsal sepal 4.5-6 mm long, 2-4 mm wide, ovate, erect, convex. Petals 2-lobed almost to the base; anterior lobe $6.5-7 \mathrm{~mm}$ long, 2-3 $\mathrm{mm}$ wide, ovatelanceolate or lanceolate, somewhat oblique, subobtuse to subacute, glabrous; posterior lobe $4-5.7 \mathrm{~mm}$ long, 0.5-0.9 mm wide, linear-falcate, obtuse to subacute, ciliolate. Lateral sepals 5.5-8 $\mathrm{mm}$ long, 4-5.5 mm wide, deflexed, obliquely ovate to oblong-ovate, with lateral apiculus. Lip 3-lobed almost to the base; the middle

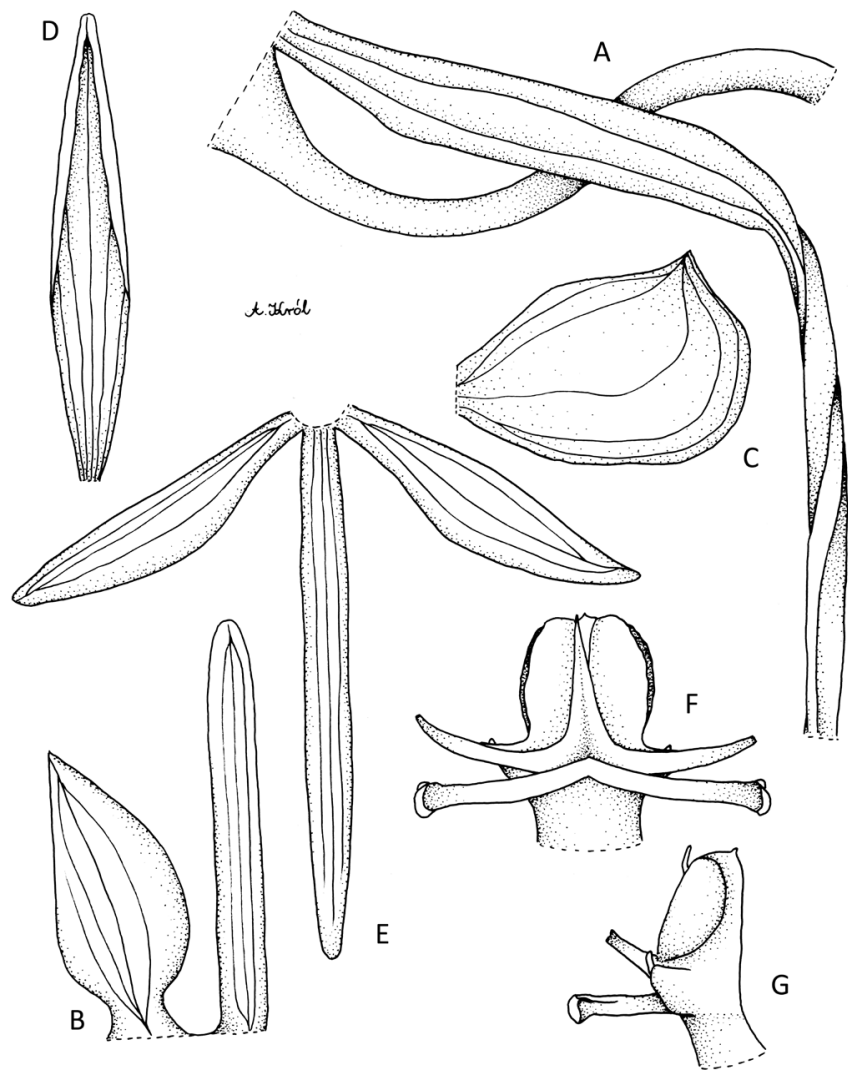

Fig. 100. Bilabrella caffra (Schltr.) Szlach. \& Kras Explanations: A - ovary, pedicel, spur; B - petal; C - lateral sepal; D - dorsal sepal; E - lip; F - gynostemium, front view; $\mathrm{G}$ - gynostemium, side view (drawn by A. Król from Habenaria tetrapetala Kraenzl. ex Rolfe - Tyson $1071, \mathrm{E})$ 


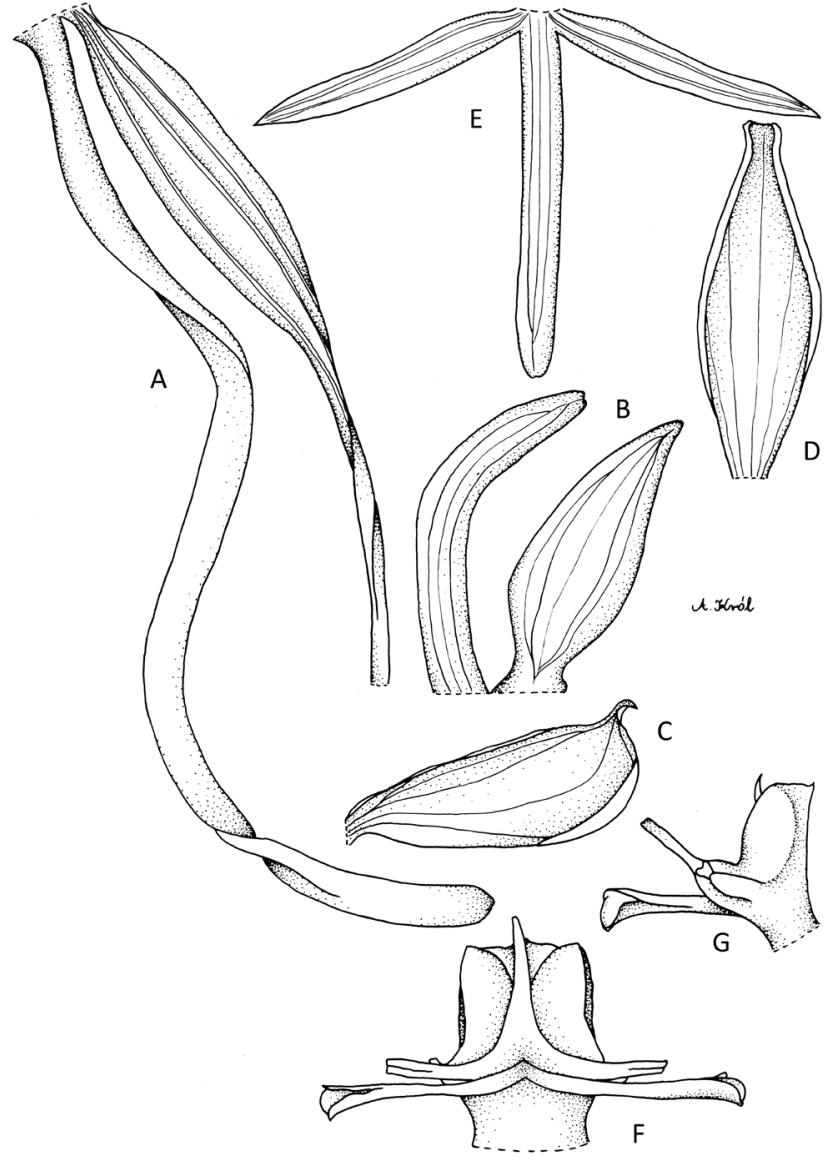

Fig. 101. Bilabrella caffra (Schltr.) Szlach. \& Kras Explanations: A - ovary, pedicel, spur; B - petal; C - lateral sepal; D - dorsal sepal; E - lip; F - gynostemium, front view; G - gynostemium, side view (drawn by A. Król from Pooley 2205, E)

lobe $10 \mathrm{~mm}$ long, 0.9-1.5 mm wide, ligulate-linear, obtuse; lateral lobes 6.5-7 mm long, 0.9-1.5 mm wide, linear-lanceolate to oblong, acute or obtuse. Spur 30$37 \mathrm{~mm}$ long, incurved, slender, slightly twisted or not.

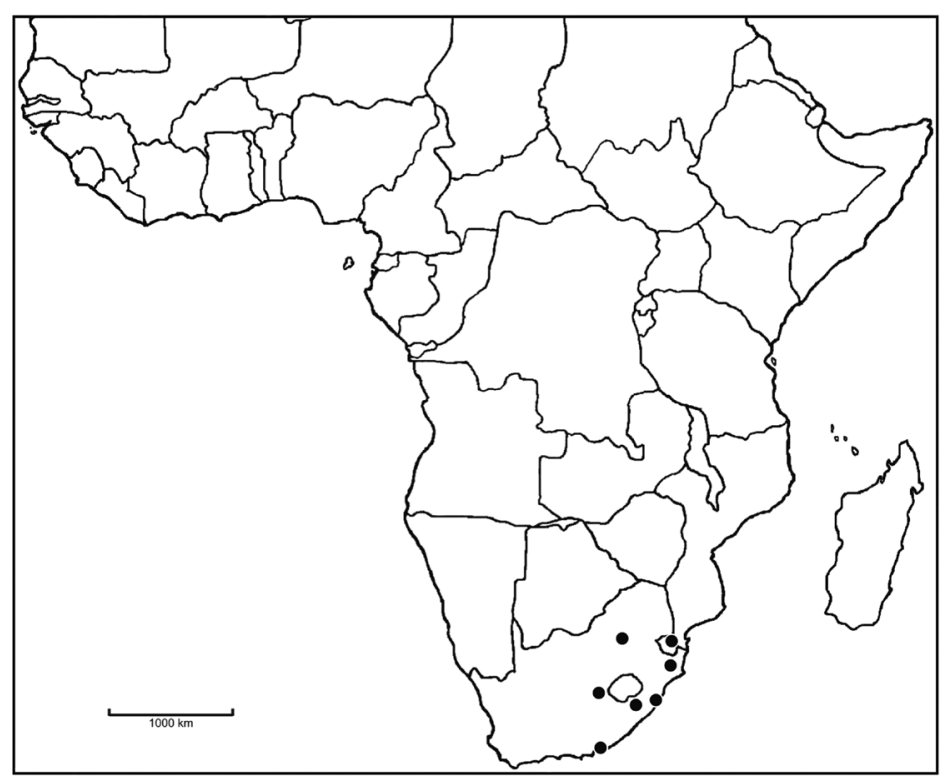

Fig. 102. Distribution of Bilabrella caffra (Schltr.) Szlach. \& Kras
Anther $2 \mathrm{~mm}$ high; connective truncate; antherophores. $2 \mathrm{~mm}$ long, slender. Stigmaphores $3 \mathrm{~mm}$ long, slender, narrowly cylindrical. Rostellum middle lobe $2 \mathrm{~mm}$ long, subulate, longer than connective. Auriculae irregularly lobed (Figs 100-101).

E c o lo gy. Terrestrial in dry and well-drained grassland. December-February.

Distribution. Republic of South Africa, Swaziland, alt. 300-1600 m (Fig. 102).

Specimens examined. Republic of South Africa. Eastern Cape: Grahamstown, Pandoland, St. Andreus Statio, 300 m, Jan. 1886 (fl), Tyson 3080 (K!); Gauteng: Johannesburg, between Ngome and Nongoma at the source of the Vama, $2731 \mathrm{CD}$, frequent in dry well-drained grassland, 750 m, 31 Dec. 1943 (fl), Linder 1943 (K!); Kwazulu-Natal: Congella Flats, 1860 (fl), Sanderson 500 (K!); Hlabisa Distr., Monzi Road, marsh, 21 Nov. 1978 (fl), Pooley 2205 (K!); Louwsberg, Itala Nature Reserve, 1300 m, 22 Feb. 1984, Hilliard \& Burtt 17730 (E!); Natal, Istapa, Jan. 1895 (fl), Schlechter s.n. (K!); Kokstad, East Griqualand, 1200 m, 1883 (fl), Tyson 1071 (BM!, K!); E Girqualand, growing in open grassland, 25 Feb. 1980 (fl), Boardman 338 (E!); E Transvaal, Blouboscop Ruins, growing on a dry hillside on a SE aspect, 1600 m, 25 Feb. 1978 (fl), Richardson 69 (E!); Sine loc., Tyson 1672 (E!). Swaziland. Stegi: Isateki Beacon, 8 Feb. 1957 (fl), Compton 26595 (K!); Nongwan Falls, 5 Jan. 1930 (fl), Brown 18101 (K!).

N o t e s . As Rolfe (1913) pointed out Bonatea tetrapetala (=Habenaria tetrapetala) is identical with Habenaria falcicornis. Under these circumstances Rolfe could not simply accept a species, which has been often mixed up with this plant, the name $H$. tetrapetala, but he should have given a new name to it. First of all, $H$. tetrapetala would only lead to new confusion, and besides Kraenzlin has added to his plant the name Bonatea tetrapetala as a synonym, and therefore his plant is as much a mixture as with other authors, we see only the one right way, that is to re-name the $H$. tetrapetala, as understood by Rolfe in the Flora Capensis. The plant should, as it is widely spread in the Kaffir countries, bear the name Habenaria caffra (Schlechter 1924).

B. caffra can be separated from B. falcicornis by petal lobes details - anterior petal lobe is glabrous and posterior one ciliolate all over in the former, and anterior petal lobe is ciliolate in the latter and posterior one ciliolate along margins only.

35. Bilabrella falcicornis Lindl., Bot. Reg. 20: t. 1701. 1835.

Type (hoc loco selectus): Cape Province, Burchell 5178 (lectotype, K!).

Habenaria falcicornis (Lindl.) Bolus, J. Linn. Soc., Bot. 19: 340. 1882. - Kraenzlin, Orchid. Gen. Sp.: 214. 1901. 


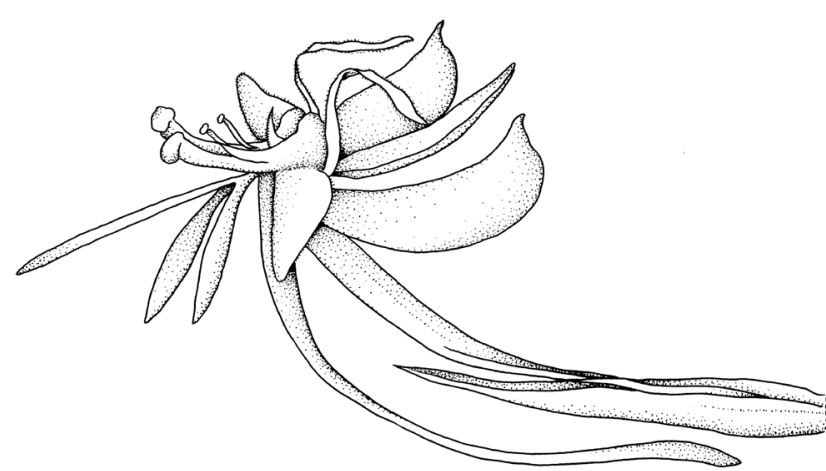

ct. Fról

Fig. 103. Bilabrella falcicornis Lindl. - flower (redrawn by A. Król from lectotype of Bonatea bilabrella Lindl. - Hutton s.n., K)

- Rolfe, Fl. Cap. 5(3): 130. 1913. - Grosvenor, Excelsa 6: 83. 1976. - Stewart et al., Wild Orchid. S. Afr.: 91. 1982. - la Croix \& Cribb, Fl. Zambes., Orchid. 11: 89. 1995. - Linder \& Kurzweil, Wild Orchids of Southern Africa: 125. 1999.

Bonatea bilabrella Lindl., Gen. Sp. Orchid. P1.: 328. 1835.

Type (hoc loco selectus): Republic of South Africa, Hutton s.n. (lectotype, K!).

Habenaria bilabrella (Lind1.) Kraenzl., Bot. Jahrb. Syst. 16: 86. 1893.

Bonatea tetrapetala Lindl., Compan. Bot. Mag. 2: 208. 1836.

Type (hoc loco selectus): Republic of South Africa, Bolus s.n. (lectotype, K!).

Habenaria tetrapetala (Lindl.) Rchb. f., Flora 48: 180. 1865, non Kraenzl. ex Rolfe, Fl. Trop. Afr. 5: 131. 1912. Habenaria mundtii Kraenzl., Bot. Jahrb. Syst. 16: 79. 1893.

Type (hoc loco selectus): Cape Province, Mundt \& Maire s.n. (B+, lectotype, $\mathrm{K} !)$.

Tubers to $5 \mathrm{~cm}$ long, $2.5 \mathrm{~cm}$ wide. Stem $22-90 \mathrm{~cm}$ tall, erect, relatively slender. Leaves 8-10, 8-18 cm long, to $1 \mathrm{~cm}$ wide, linear-lanceolate to lanceolate, acute, gradually decreasing in size upwards. Inflorescence 7-15 cm long, rather lax, many-flowered. Flowers white with green ovary, tips of the perianth lobes and stigmas. Floral bracts to $20 \mathrm{~mm}$ long, lanceolate to oblong-lanceolate, acuminate. Pedicel and ovary up to $30 \mathrm{~mm}$ long, slender. Dorsal sepal 4.5-6.5 mm long, 2-3 mm wide, elliptic to narrowly oblong, obtuse, cochleate. Petals bilobed to the base; anterior lobe 2.5-8 $\mathrm{mm}$ long, $2.5 \mathrm{~mm}$ wide at the base, spreading, obliquely ovate to lanceolate, subobtuse, ciliolate; posterior lobe 4-7 $\mathrm{mm}$ long, up to $0.8 \mathrm{~mm}$ wide, erect, linear to linear-oblanceolate, acute, ciliolate on margins. Lateral sepal 6-8 $\mathrm{mm}$ long, $4 \mathrm{~mm}$ wide, semi-obovate to semi-orbicular, with laterally placed subacute apiculus,

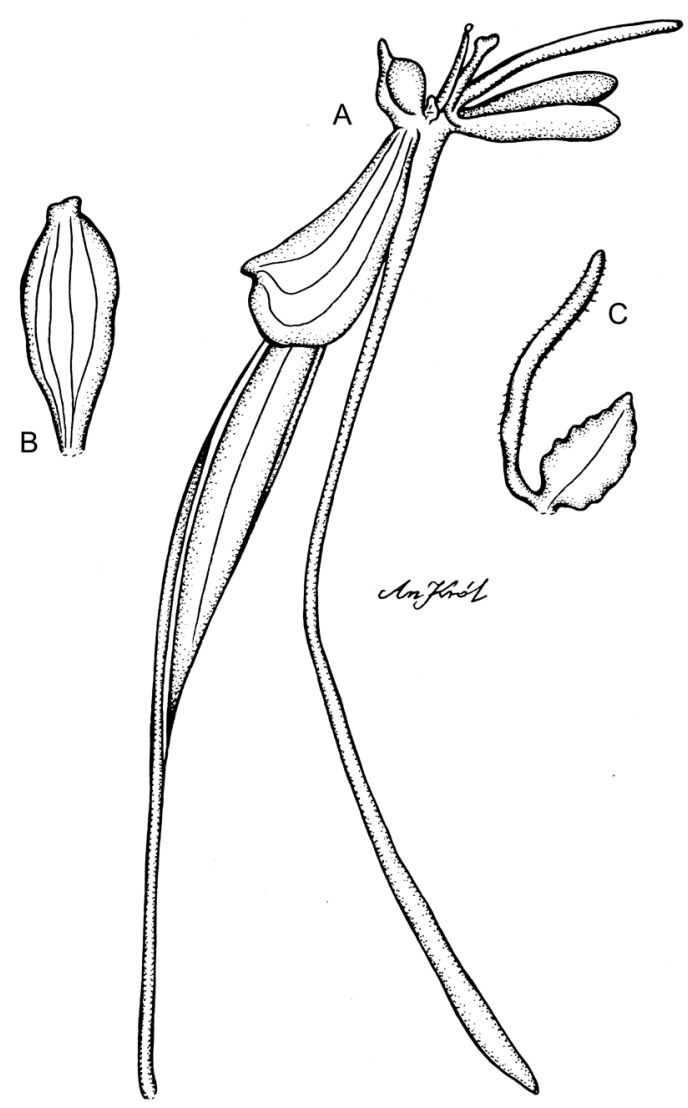

Fig. 104. Bilabrella falcicornis Lindl.

Explanations: A - flower; B - dorsal sepal; C - petal (drawn by A. Król from MacOwan s.n., AMES)

cochleate. Lip porrect, 3-lobed above undivided $1 \mathrm{~mm}$; the middle lobe 7-10(12) $\mathrm{mm}$ long, $1 \mathrm{~mm}$ wide, oblong ligulate to linear, obtuse; lateral lobes 5-9.5 mm long, to $1.7 \mathrm{~mm}$ wide, obliquely oblong-lanceolate to ellipticlanceolate, obtuse, widely spread. Spur 23-43 mm long, narrowly cylindrical-filiform, incurved, slightly inflated in the apical part if any, blunt. Anther $2.5 \mathrm{~mm}$ high; connective shallowly notched; antherophores $3 \mathrm{~mm}$ long, delicate, slightly upcurved. Stigmaphores $5 \mathrm{~mm}$ long, narrowly cylindrical, truncate. Rostellum middle lobe as long as connective. Auriculae entire, medium-sized (Figs 103-104).

E c o logy. In open grass in boggy land, clay soil. December-July.

D istribution. Zimbabwe, Republic of South Africa, alt. 100-1800 m (Fig. 105).

S pecimens examined. Zimbabwe. Harare: Salisbury, Inyanga Distr., 1800 m, Feb. 1956 (fl), Drewe s.n. (57833) (K!); Salisbury, Melsetter Distr., Chimanimani, grassland, 1500 m, 21 Feb. 1954 (fl), Ball 236 (46247) (K!). Republic of South Africa. Cape: Burchell 5178 (K!); Summit Long Hill, 1290 m, 5 Mar. 1893 (fl), Scott Elliot s.n. (K!); Cape Prov., Mundt \& Maire s.n. (K!); Eastern Cape: Drakensberg, MacLear Farm Feltham, $11 \mathrm{~km} \mathrm{~N}$ of Ugie, grassland, well drained sandy-clay soil, elliot, fun sun, level slope, on side of 


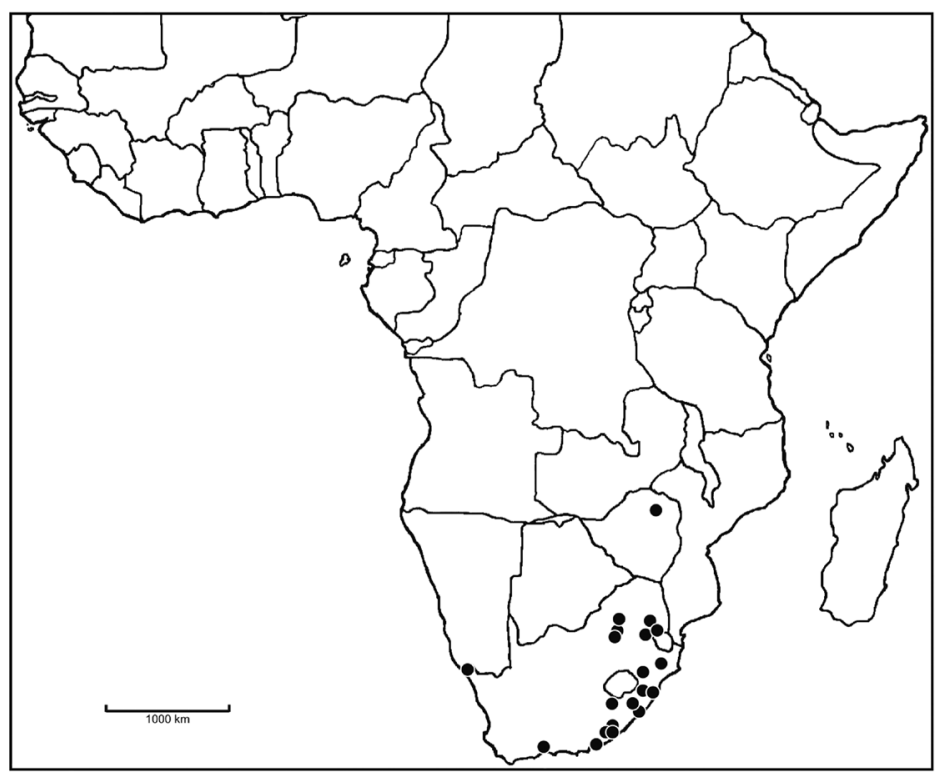

Fig. 105. Distribution of Bilabrella falcicornis Lindl.

road, 31'99'S, 28217'E, 1260 m, 7 Feb. 1994 (fl), Bester 2170 (K!); Drakensberg, MacLear Farm 185, 18 km NNW of Ugie, grassland - floodplain, clay soil, NE gentle slope, burnt 9 months ago, 31 $1^{\circ} 107^{\prime} \mathrm{S}$, 28 $214^{\circ} \mathrm{E}$, 1500 m, 8 Feb. 1994 (fl), Bester 2195 (K!); East London, May 1869 (fl), Halleck s.n. (W!); Stutterheim, red Ridge, common in Mistbelt grassland, Mar. 2000 (fl), Edwards \& Potgieter 1898 (K!); Eastern Frontier, Hutton s.n. (BM!); Kentani Distr., 1200 m, Feb. 1911 (fl), Pegler 347 (BM!, K!); Paisons Vlei, 10 miles Cape road, Port Elizabeth, open grass in boggy land, $27 \mathrm{Feb}$. 1932 (fl), Long 536 (K!); King Williams Town, near Kei Road, in grass veld, 20 Feb. 1961 (fl), Ranger 267 (K!); Free State: Transvaal, Donkerhoek, $15 \mathrm{~km}$ from Bronkhorst spruit on road from Pretoria, 8 Mar. 1978 (fl), Venter 03010 (04010) (K!); Orange River, 1921 (fl), Scott Elliot s.n. (BM!); Gauteng: Johannesburg Hillside, 1500 m, Feb./Mar. 1894 (fl), Galpin 1400 (K!); Johannesburg, 8 Mar. 1918 \& 25 Feb. 1920 (fl), Moss 7565 (BM!, K!); Transvaal, Pretoria Distr., Near Rietvlei (P'a-Dehmans road), among grass in low marshy place in veld, 26 Feb. 1954 (fl), Meelise 9247 (K!); Transvaal, Merwe Station, Mar. 1908 (fl), Reck 6861 (K!); Kwazulu-Natal: Alexandra Distr., Station Dumisa, 750 m, 14 Jan. 1911 (fl), Rudatis 1302 (BM!, E!); Alexandra Distr., Station Dumisa, 750 m, 14 Jan. 1911 (fl), Rudatis 1304 (W!); Entonjaneni Distr., 8 miles $\mathrm{W}$ from Melmoth, in grassland, rare, $930 \mathrm{~m}, 28 \mathrm{Jan}$. 1955 (fl), Schelpe 5186 (BM!); Ixopo, 16 July 1893 (fl), Schlechter s.n. (BR!); Ixopo, Jan. 1895 (fl), Scott Elliot s.n. (BR!, K!); Klipriver Distr., 2 Mar. 1895 (fl), Penther 71 (W!); Klipriver Distr. near Colenso, 27 Feb. 1895 (fl), Penther 342 (W!); Apr. 1903 (fl), Prior. s.n. (K!); Natal, Generd 583 (W!); Natal, 1500 m, Feb. 1883 (fl), s.n. (K!); 1867 (fl), Bowhen s.n. (K!); Port Natal, Scott Elliot s.n. (K!); Kwazulu-Natal, 1930 (fl), Sanderson 560 (K! - drawing); Natal Botanic Gardens, $100 \mathrm{~m}$, 1883 (fl), Wood 696 (BM!, E!); Kwazulu-Natal, in damp ground, 1884 (fl), Wood 3088 (K!); Port Edward, grassland, 20 Dec. 1930 (fl), Moss 19243 (BM!); Girqualand East, in monte Insirwa, 22 Jan. 1895 (fl), Penther 136 (W!); Girqualand East, Insizwa, 28 Jan. 1895 (fl), Penther 161 (W!); East Griqualand, Humid valleys North Koksladt, Mar. 1883 (fl), Scott Elliot s.n. (E!); Hills St. Bosch, 210 m, Feb. 1921 (fl), Fourcade 1135 (K!); Mpumalanga: Lydenburg Distr., Eendekraal, grassland, 19 Mar. 1962 (fl), Strey 4116 (K!); Transvaal, Lake Chrissie, 26 Feb. 1928 (fl), Moss 16497 (K!); Transvaal, Barberton, 930 m, Rogers 30257 (K!); Northern Cape: East, Ad Nalogha, 20 Jan. 1895 (fl), Penther 172 (W!); North West: Soutpan, Cooper s.n. (W!); Western Cape: Between Capetown and Georgetown, 1885 (fl), Scott Elliot s.n. (BM!); Near George, 200 m, 3 Mar. 1893 (fl), Schlechter 2325 (BM!, K!, WU!); Barrina, Baur 816 (K!); Sine loc., Drege 4566 (K!); Sine loc., Drege 64 (W!); Sine loc., Hutton s.n. (K!); Sine loc., Reichenbach Herb. Orchid. No. 35855. s.n. (W!); Sine loc., Reichenbach Herb. Orchid. No. 38994. s.n. (W!); Sine loc., Aug. 1888 (fl), Scott Elliot s.n. (E!); Sine loc., Mac Owan s.n. (AMES!).

N o t e s. Kraenzlin (1901: 221-222) described South African plants (mentioning Culver 28 from Barberton) under the name 'Habenaria tetrapetala Rchb. f.'. Kraenzlin's description and the specimen cited do not match the type of $H$. tetrapetala (Lindl.) Rchb. f., which is considered to be synonymous with $H$. falcicornis (Lind1.) H. Bol. Consequently, Rolfe (1913: 131) considering that Kraenzlin's description applied to a new species, treated it as ' $H$. tetrapetala Kraenzlin'. Schlechter (1924: 242) proposed the new name $H$. caffra to avoid nomenclatural confusion, which is in accordance with Article 64.1 of the International Code of Botanical Nomenclature (Greuter et al. 1987).

Plants with the lower petal lobe lanceolate and distincly longer than the upper were separated as $B$. caffra from those with a widely ovate lower petal lobe which was shorter than the upper, and which were placed in B. falcicornis; in addition plants recognized as B. caffra are generally more robust than those placed in B. falcicornis and the spur is usually longer. Plants from the Transvaal with this combination of characters do indeed appear distinct from southern form placed in B. falcicornis, but the apparent difference is spur length is not entirely consistent, and certain specimens from southern Natal and the eastern Cape Province can scarcely be assigned to either entity on the basis of petal shape. We has not observed any differences in the form of the gynostemium or remaining perianth segments, although the lip lateral lobes tend to be more falcately 
lanceolate in Transvaal specimens referred to B. caffra. The weak morphological discontinuites in the perianth discussed above, and the largely allopatric ranges of the two entities involved indicate that status of both of this taxa require further studies.

36. Bilabrella humblotii (Rchb. f.) Szlach. \& Kras, Richardiana 9(4): 158. 2009.

Habenaria humblotii Rchb. f., Flora 68: 535. 1885. Kraenzlin, Orchid. Gen. Sp.: 226. 1901.

Type (hoc loco selectus): Comoros, Humblot 426 (lectotype, W-R!; isolectotype, BM!).

Tuberoids to $2.5 \mathrm{~cm}$ long, $1.5 \mathrm{~cm}$ wide, ellipsoid to ovoid. Stem up to $80 \mathrm{~cm}$ tall, leafy, erect, rather slender. Leaves 10-13, up to $14 \mathrm{~cm}$ long and $2 \mathrm{~cm}$ wide, lanceolate, narrowly lanceolate to linear-lanceolate, acute, suberect, decreasing in size upwards. Inflorescence $10-20 \mathrm{~cm}$ long, loosely multi-flowered. Flowers greenish. Floral bracts up to $25 \mathrm{~mm}$ long, usually half as long as pedicel and ovary, oblong-lanceolate to ovatelanceolate, acuminate. Pedicel and ovary about $20 \mathrm{~mm}$ long. Dorsal sepal $6 \mathrm{~mm}$ long, $2.5 \mathrm{~mm}$ wide, oblong obovate to oblong-elliptic, shortly apiculate, acute to obtuse, concave, reflexed. Petals bilobed; anterior lobe linear, twice wider than posterior one, obtuse; posterior

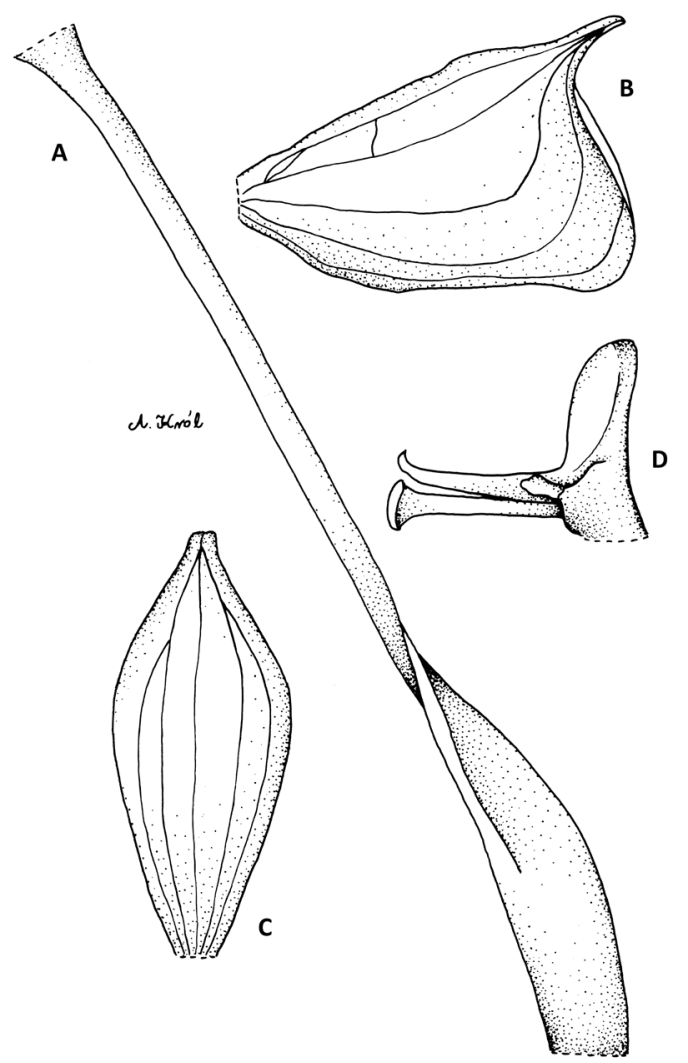

Fig. 106. Bilabrella humblotii (Rchb. f.) Szlach. \& Kras Explanations: A - spur; B - lateral sepal; C - dorsal sepal; D - gynostemium, side view (drawn by A. Król from isolectotype of Habenaria humblotii Rchb. f. - Humblot 426, BM)

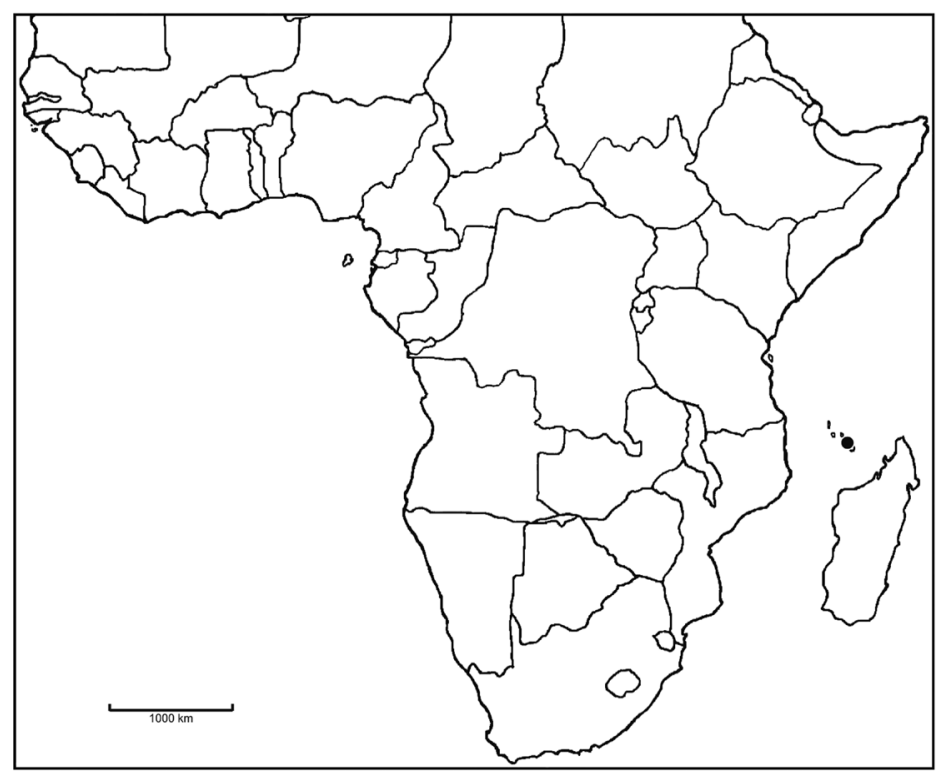

Fig. 107. Distribution of Bilabrella humblotii (Rchb.f.) Szlach. \& Kras

lobe linear. Lateral sepals $8 \mathrm{~mm}$ long, 5.5-6 mm wide, obliquely triangular-ovate, widest at the apex, with laterally placed apiculus, reflexed, concave. Lip 3-lobed, all lobes subsimilar and subequal, linear, acute. Spur 18-20 mm long, filiform, distinctly swollen and clavate towards the apex, twisted near the apex. Anther ca. $2.5 \mathrm{~mm}$ long; connective obtuse; antherophores $3 \mathrm{~mm}$ long, slender, slightly upcurved apically. Stigmaphores ca. $3 \mathrm{~mm}$ long, narrowly cylindrical, truncate at the apex. Rostellum middle lobe shorter than connective. Auriculae medium-sized, entire (Fig. 106).

E c o logy. No records.

Distribution. Endemic to Comoros (Fig. 107).

Specimens examined. Comoros. Sine loc., Humblot 426 (BM!).

$\mathrm{N}$ o t e s. Unfortunately, materials we examined were partly distroyed. B. humblotii can be confused with $B$. humilior described below. Both species can be distinguished by the form of lateral sepals and length/ width ratio of anterior petal lobes. Anterior petal lobes are prominently narrower in $B$. humblotii. Its status requires further studies.

37. Bilabrella humilior (Rchb. f.) Szlach. \& Kras-Lap., Richardiana 3(3): 140. 2003. - Szlachetko et al., Orchid. of West-Central Africa 1: 235. 2010.

Habenaria humilior Rchb. f., Otia Bot. Hamburg.: 100. 1881. - Rolfe, Fl. Trop. Afr. 7: 236. 1898. - Kraenzlin, Gen. \& Sp. Orchid. Pl: 223. 1901. - Summerhayes, Kew Bull 16: 283. 1962. - Tweedie, Journ. E.A. Nat. Hist. Soc. 24: 26. 1964. - Summerhayes, FTEA, Orchid. 1: 91. 1968b. - Piers, Orchid. E. Afr.: 29. 1968. - Grosvenor, Excelsa 6: 83. 1976. - Williamson, Orchid. S. Centr. Afr.: 53. 1977. - Geerinck, Fl. Afr. Centr., 
Orchid. 1: 113. 1984. - la Croix et al., Orchid. Malawi: 71. 1991. - la Croix \& Cribb, Fl. Zambes., Orchid. 11: 96. 1995. - Edwards, Demissew, Hedberg, Flora of Ethiopia \& Eritrea 6: 220. 1997. - Linder \& Kurzweil, Flora of Southern Africa: 129. 1999. - Demissew, Cribb, Rasmusen, Field guide to Ethiopian Orchids: 96. 2004.

Type: Ethiopia, Schimper 1373 (lectotype, designated by Szlachetko et al. [2010: 235], W-R!; isolectotypes, BM!, BR!, E!, K!, S not seen).

Habenaria humilior sensu Geerinck, Fl. Afr. Centr., Orchid. 1: 113. 1984, non Rchb. f..

Tuber 1-2 cm long, $0.7-1.5 \mathrm{~cm}$ in diameter, globose, ovoid or ellipsoid. Stem $12-70 \mathrm{~cm}$ tall, erect, rather stout or realtively delicate, glabrous. Leaves 7-13, 6-23 cm long, up to 1.7 (rarely 2.3 ) $\mathrm{cm}$ wide, linear-lanceolate or narrowly lanceolate, acute, suberect, decreasing in size up the stem. Inflorescence $2-25 \mathrm{~cm}$ long, 3- to many-flowered, rather lax to dense. Flowers green or greenish-white. Floral bracts 10-20 mm long, oblonglanceolate, acuminate, glabrous. Pedicel and ovary 15-22 mm long, glabrous. Dorsal sepal 4-6.5 mm long, $1.5-3 \mathrm{~mm}$ wide, oblong-ovate to oblong-elliptic, obtuse, slightly cochleate, glabrous, deflexed. Petals bilobed to the base; anterior lobe $6-13.5 \mathrm{~mm}$ long, $1.5-3 \mathrm{~mm}$ wide, obliquely oblong-lanceolate to ligulate-lanceolate, subacute to subobtuse, glabrous; posterior lobe 4-6 mm long, 0.4-0.6 mm wide, linear-filiform, thin, delicate,

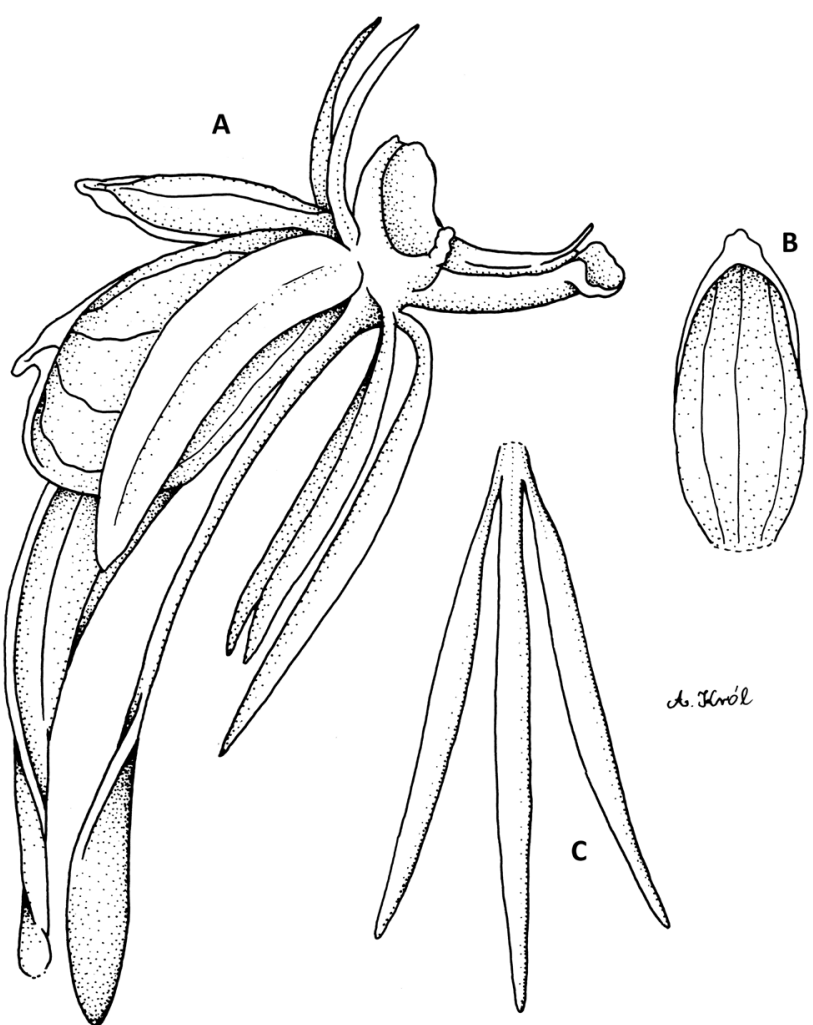

Fig. 108. Bilabrella humilior (Rchb. f.) Szlach. \& Kras-Lap. Explanations: A - flower; B - dorsal sepal; C - lip (drawn by A. Król from lectotype of Habenaria humilior Rchb. f. - Schimper 1373, W-R) ciliolate. Lateral sepals 6-9.5 mm long, 3-5 mm wide, obliquely oblong-obovate, widest just above the middle, concave, with laterally placed short, erect apiculus, glabrous. Lip 3-lobed above the basal undivided part $1.5 \mathrm{~mm}$ long, glabrous, deflexed; the middle lobe 6-11.5 mm long, 0.5-1 mm wide, linear, acute; lateral lobes 5-9 mm long, 0.5-1 mm wide, linear, acuminate, pendent. Spur 15-25 mm long, $1.8 \mathrm{~mm}$ in diameter at the apex, swollen in the apical half or third and twisted, subobtuse, pendent. Anther 1.5-3 mm long; connective rounded; antherophores 3-4.5 mm long, slightly upcurved towards the apex. Stigmaphores 3-5 mm long, rather massive, slightly upcurved, truncate. Rostellum middle lobe longer than connective. Auriculae rather small, entire (Figs 108-112).

E c ology. Montane grassland, grassy dambos, damp places in woodland clearings, seasonally flooded grassland, perenially wet bog and moist depressions or cracks in rocks, shallow poorly drained soil over rocks. January-December.

Distribution. Sudan, Ethiopia, Democratic Republic of the Congo (Zaire), Uganda, Kenya, Nairobi, Rwanda, Tanzania, Zambia, Malawi, Zimbabwe, alt. 900-3000 m (Fig. 113).

Specimens examined. Sudan. East Equatorial: Tugu Yangu (Latuka), Imatong Hills, 1500-1800 m, 12 June 1939 (fl), Andrews 1838 (K!); North Darfur: Jebel Marra, path from Taurotonga to Kalokitting,

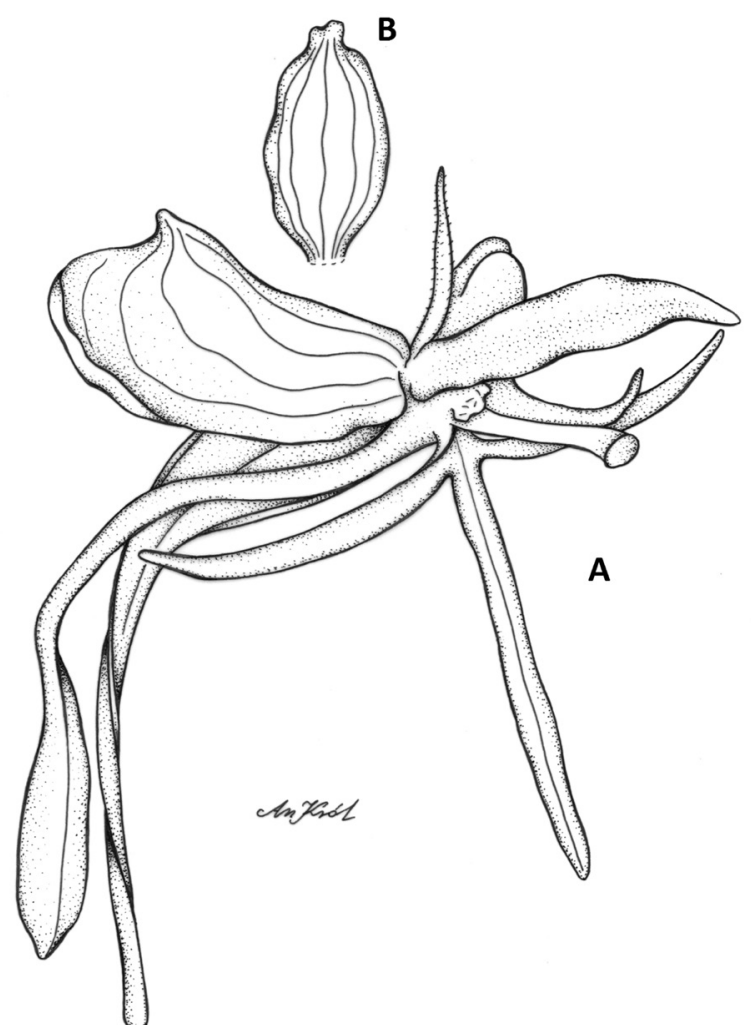

Fig. 109. Bilabrella humilior (Rchb. f.) Szlach. \& Kras-Lap. Explanations: A - flower; B - dorsal sepal (drawn by A. Król from Richards 22107, AMES) 


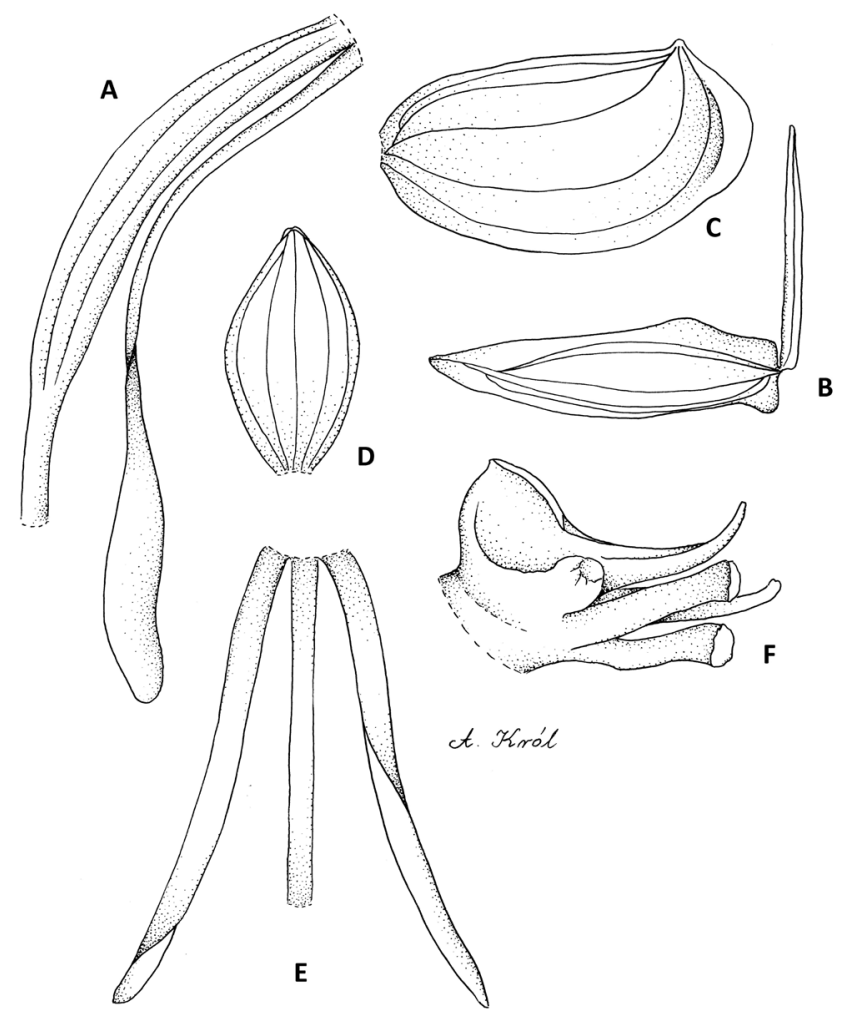

Fig. 110. Bilabrella humilior (Rchb. f.) Szlach. \& Kras-Lap. Explanations: A - ovary, pedicel, spur; B - petal; C - lateral sepal; D - dorsal sepal; E - lip; F - gynostemium, side view (drawn by A. Król from isolectotype of Habenaria humilior Rchb. f. - Schimper 1373, BM)

in grassland, $12^{\circ} 52^{\prime} \mathrm{N}, 24^{\circ} 17^{\prime} \mathrm{E}, 2000 \mathrm{~m}, 15$ Sep. 1960 (fl), Jackson 4074 (K!); Jebel Marra, Gor Lambang, marsh, 1890 m, 18 Sep. 1964 (fl), Wickens 2600 (K!). Ethiopia. Addis Ababa: About 200 km SW of Addis

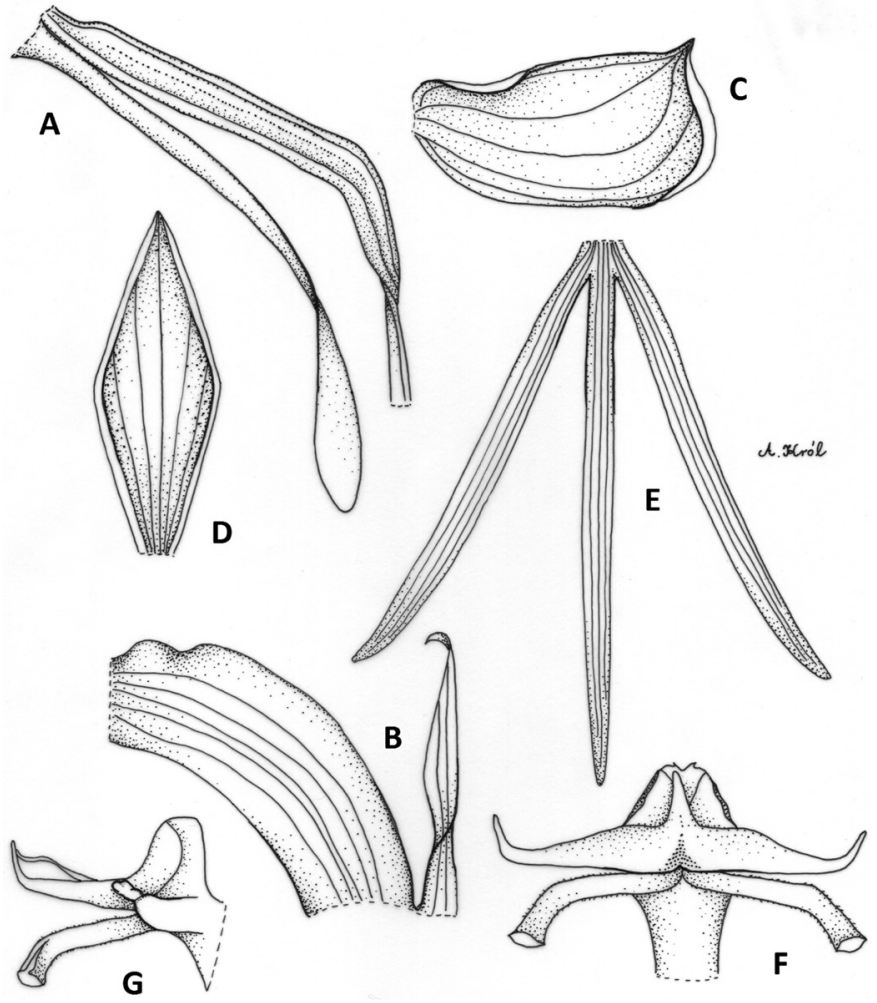

Fig. 111. Bilabrella humilior (Rchb. f.) Szlach. \& Kras-Lap. Explanations: A - ovary, pedicel, spur; B - petal; C - lateral sepal; D - dorsal sepal; E - lip; F-G - gynostemium, various views (drawn by A. Król from Schimper III.1649, BR)

Ababa, along road to Jimma, open woodland, brown loamy soil, open grassy places, 2000 m, 9 Aug. 1965 (fl), De Wilde 7502 (K!); About $200 \mathrm{~km} \mathrm{SW}$ of Addis Ababa, along road to Jimma, open grassy place in open

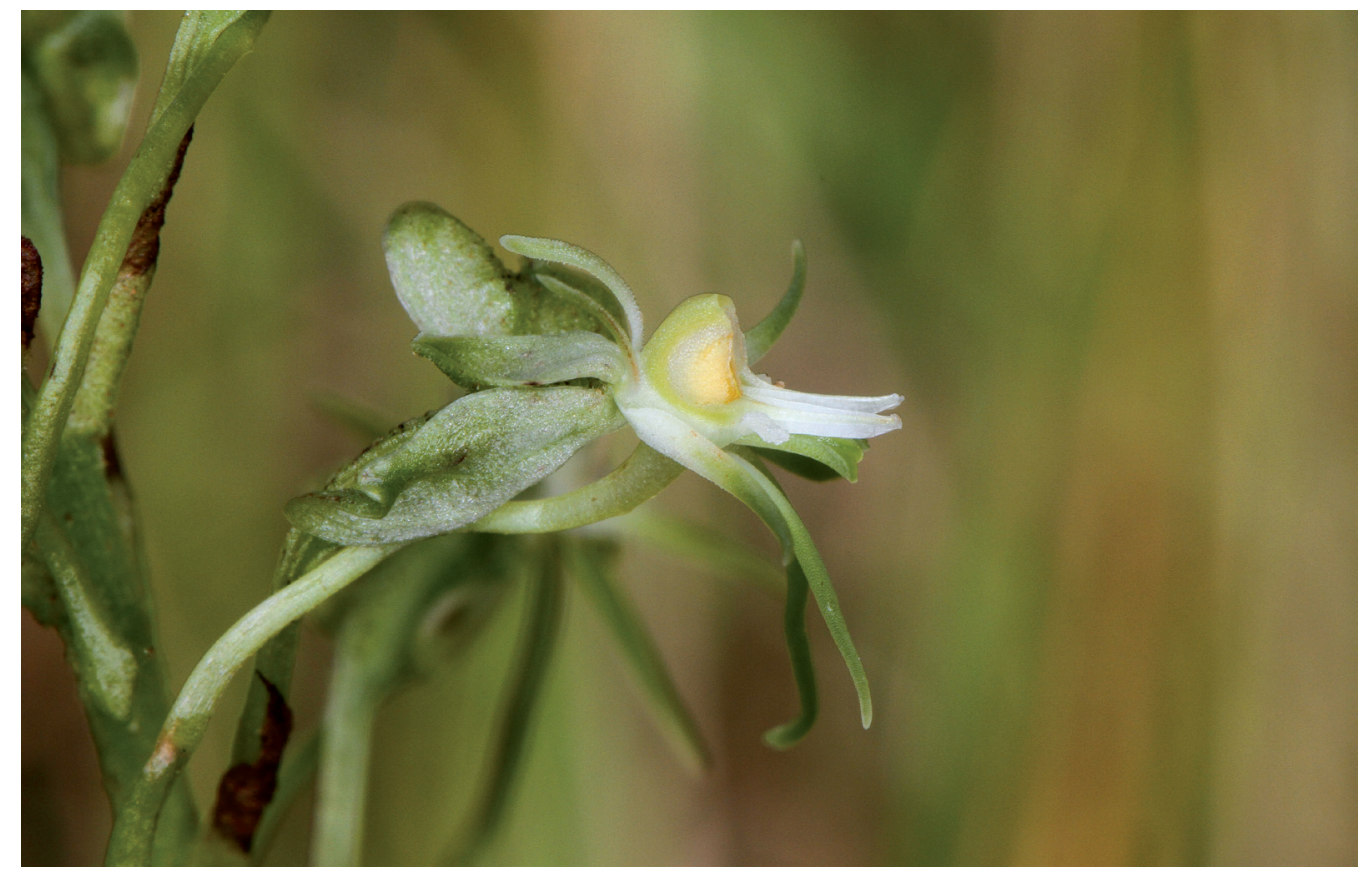

Fig. 112. Bilabrella humilior (Rchb.f.) Szlach. \& Kras-Lap. - flower close-up (phot. L. Grobler) 


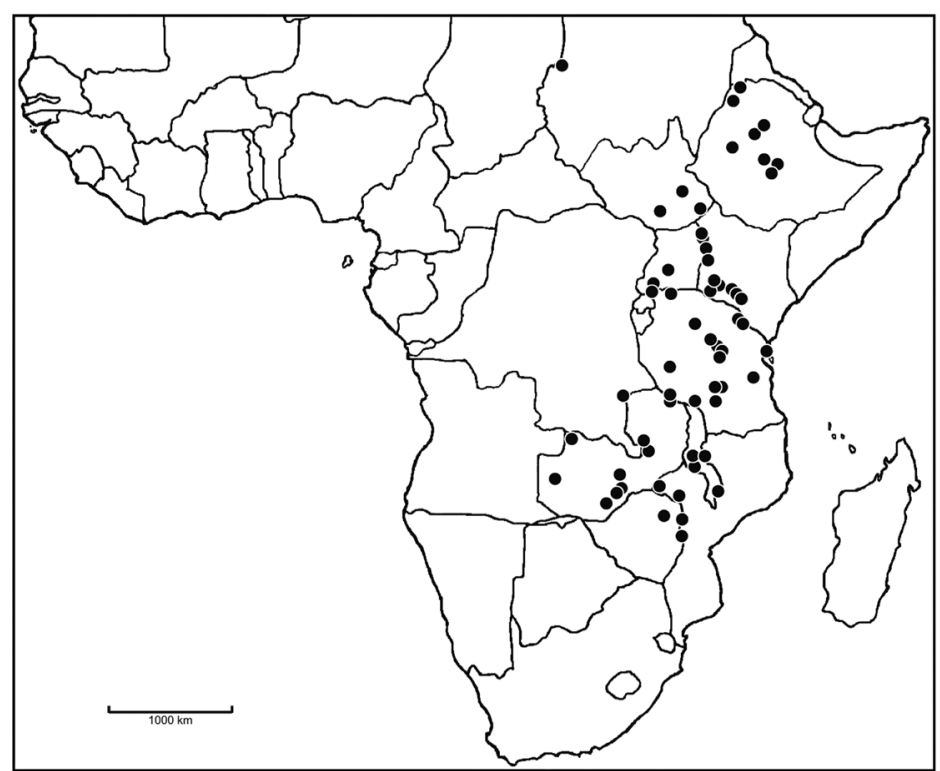

Fig. 113. Distribution of Bilabrella humilior (Rchb.f.) Szlach. \& Kras-Lap.

woodland, 2000 m, 9 Aug. 1965 (fl), De Wilde 7503 (K!); Entoto Hills, above Addis Ababa, in wet flusches between cultivated fields, 3000 m, 26 Sep. 2003 (fl), Edwards s.n. (K!); Shoa Prov. Bichoftu, occasional in scrub, 1800 m, 6 Sep. 1952 (fl), Curle \& Schelpe 13 (BM!); Shoa Prov. Bichoftu, in steep grassland, rare, 2100 m, 13 Sep. 1952 (fl), Curle \& Schelpe 57 (BM!); Wollega Region, $41 \mathrm{~km}$ on Ghimbi-Asosa road, earth banks or steep grassy slopes, $9^{\circ} 24^{\prime} \mathrm{N}, 35^{\circ} 35^{\prime} \mathrm{E}, 2050 \mathrm{~m}$, 15 Sep. 1975 (fl), Gilbert \& Thulin 783 (K!); Amhara: Dangila, $40 \mathrm{~km} \mathrm{~S}$ of Lake Tana, $2010 \mathrm{~m}, 1926$ (fl), Cheesman s.n. (BM!); Harari: Bale, Outskirts of Dello Mena (Maslo on many maps), near Saw Mill on Goba road, thickets of evergreen shrubs and battered remnants of Combretum, Pappea and Terminalia on gentle slope with red soil, $6^{\circ} 25^{\prime} \mathrm{N}, 39^{\circ} 48^{\prime} \mathrm{E}, 1350 \mathrm{~m}$, 31 May 1988 (fl), Gilbert \& Sebsebe $8550 U$ (K!); Oromiya: Arussi Prov., near Shashamane, along road to „Petrinelli”, 7¹5'N, 38 35'E, 1890 m, 19 Aug. 1961 (fl), Ouren 20791 (K!); $3 \mathrm{~km}$ along the track of Genale Daria, turning off $20 \mathrm{~km} \mathrm{~N}$ of Neghelle on the NeghelleKebra Mengist road, 1400 m, 24 May 1982 (fl), Friss, Tadesse \& Vollesen 3267 (C!); About $50 \mathrm{~km} \mathrm{NE}$ of Jimma, along road to Addis Ababa, grassy marshy place, blackish clay soil, 1800 m, 19 Aug. 1965 (fl), De Wilde 7829 (C!, K!); Kaffa Prov., about $9 \mathrm{~km}$ along the road Jimma to Bonga, sloping hills with coarse grasses (various height) herb and shrubs, shrubvegetation sometimes closed and forming thickets, dark, rather loamy reddish-brow soil, 7³7’ $\mathrm{N}, 36^{\circ} 45^{\prime} \mathrm{E}, 1650 \mathrm{~m}$, 9 July 1969 (fl), De Wilde 5419 (K!); Tigray: Tigre near Begemder, Schimper 1373 (W-R!); $170 \mathrm{~km}$ from Mojo, road to Shashemene fields, Acacia woodland, grassvegetation and some other shrubs, black loamy soil, $1850 \mathrm{~m}, 6$ Sep. 1967 (fl), Westphal \& Stevels 1679 (K!); Valley Arahagne, Quartin-Dillon \& Petit s.n. (P!). Democratic Republic of the Congo (Zaire). Haut-Katanga: Kabango, in steppe with Aeanthus, 2 Nov. 1914 (fl), s.n. (K!); River Mulungwe, 1910 (fl), Chaessens 638 (K!); Bequaert 6196 (K!). Uganda. Eastern: Kapchorwa Distr., Mt. Elgon, Butandiga, damp rocks near camp with very shallow soil covering, 2100 m, 5 Aug. 1934 (fl), Hansford 797 (K!); Kapchorwa Distr., Mt. Elgon, Buginyanga, short grassland, 1950 m, 18 July 1924 (fl), Snowden 924 (K!); Kapchorwa Distr., Sebei, shallow soil (over rock) supporting grass pasture, $1980 \mathrm{~m}$, 27 Aug. 1956 (fl), Noman 288 (K!); Northern: Moroto Distr., Mt. Minbane, grassland, Apr. 1960 (fl), Wilson 976 (K!); Western: Ankole, near Bugamba, Nov. 1940 (fl), Eggeling 4230 (K!); Ntungamo Distr., Lake Karenge, 13 Mar. 1903-4 (fl), Bagshawe s.n. (BM!); Ankole Igorora, Mitne, short grassland, $1680 \mathrm{~m}$, Nov. 1938 (fl), Bagshawe 461 (K!); Karamoja Region: Napak, grassland over wet rocks, 2250 m, 28 May 1940 (fl), Thomas 3650 (K!); Mt. Moroto, in grassland on steep slopes, 2250 m, 5 Sep. 1956 (fl), Bally \& Hardy 10761 (BM!). Kenya. Central: Kabete, in damp loeahilies after rainy season, 1930 (fl), Mettam 168a (K!); Kiambu Distr., 1800 m, May 1941 (fl), Bally 131478 (K!); Kiambu, 1710 m, June 1932 (fl), Vapin 2101 (K!). Nairobi. Kileleshwa, Kenya Girls High School, in grassland, 18 May 1961 (fl), Parsons EA12365 (K!); Nyanza: Cherangari Hills, Kaibibich, steep, grazed slope with thin sandy soil over guootzite with Satyrium schimperi, Heliedrysum, etc., $1^{\circ} 13^{\prime} \mathrm{N}, 35^{\circ} 16^{\prime} \mathrm{E}, 2550 \mathrm{~m}$, 5 Sep. 1969 (fl), Mabberley \& McCall 59 (K!); Kisii, 1530 m, June 1932 (fl), Vapier 3095 (K!); Rift Valley: Londiani, in open grassland, generally badly drained and boggy in rains, not common, rainy season, $2250 \mathrm{~m}$, 3 Aug. 1951 (fl), Davis 10 (K!); Nakuru-Eldoret road, Reservoir $80 \mathrm{~km}$ from Nakuru, Murram scrape by road, plants from wet flush or from thickets at margin with Rubus, Acacia mearnsii \& Vernonia spp., $0^{\circ} 01^{\prime} \mathrm{N}$, 353'ㄹ, 2730 m, 14 Oct. 1981 (fl), Gilbert \& Tadessa 6813 (K!); Turkana Distr., Kapenguria, grassland, 1890 m, July 1935 (fl), Thorold 2738 (K!); Uasin Gishu Distr., Eldoret, Oldan Sapuk, on a very shallow wet soil on rock pavements with Eragrostis "ciliaris" Loudetia, Melastomaceae a yellow Hyparrhenia, Exotheca, Heteropogon grassland, 2400 m, 22 July 1951 (fl), Greenway 8513 (K!); West Pokot Distr., Kapenguria, 1944 (fl), Hale B4077 (K!); Western: Kipkarren Distr., S of Elgon, rock pools and damp ground, Brodhurst Hill 27 (K!); Gazita River, 1875 m, Jan. 1940 (fl), Copley B599 (K!); Trans-Nzoia Distr., Elgon, lower foothills of Mt. Elgon, on rocky onterops near dam, 2010 m, 20 July 1957 (fl), Symes 164 (K!); Trans-Nzoia Distr., Elgon, on shallow wet soil over rock, 2010 m, June 1931 (fl), 
Tweedie 13 (K!). Rwanda. Kigali: Mibilizi, $14 \mathrm{~km} \mathrm{~S} \mathrm{of}$ Karama (Bugesera), savannah, rocky soil, 1450 m, 19 May 1978 (fl), Troupin 15938 (K!). Tanzania. Arusha: Momella, Arusha National Park, Apr. 1968 (fl), Greenway \& Kanui 13942 (K!); Iringa: Iheme, Apr. 1937 (fl), Ward 29 (K!); Iringa Distr., Nunda, open fireswept Protea-grassland with scattered parinari, $8^{\circ} 15^{\prime} \mathrm{S}, 35^{\circ} 28^{\prime} \mathrm{E}, 1750 \mathrm{~m}, 20$ Apr. 1991 (fl), Bidgood \& Congdon 2207 (K!); T7, Iringa Distr., main road between Sao Hill and Lugoda turn off, wide roadside verge, 21 Mar. 1986 (fl), Bidgood \& Keeley 385 (K!); T7, Iringa Distr., Southern Highlands, Mufindi, Nyamala, in bracken scrub grassland, 2010 m, May 1951 (fl), Eggeling 6107 (K!); Mufindi, Soa Hill, in long grass on roadside, 1800 m, 3 Apr. 1987 (fl), Leyn 152 (K!). Mufindi Distr., Lake Ngwazi, Dam end, Brachystegia - Parinari curatellifolia stunted woodland with canopy $2 \mathrm{~m}$ tall, with densely vegegated termite mounds with Croton, Apodytes dimidiata canopy 4-8 m tall, covered by roadside, $08^{\circ} 30^{\prime} \mathrm{S}, 35^{\circ} 15^{\prime} \mathrm{E}, 1830 \mathrm{~m}$, 9 Apr. 1986 (fl), Lovett 622 (K!); Mufindi Distr., Iringa, few km N of James' corner on Mbeya-Iringa road, roadside near swamp, $8^{\circ} 28^{\prime} \mathrm{S}, 35^{\circ} 02^{\prime} \mathrm{E}, 1900 \mathrm{~m}$, Pettersson, Hedren \& Kibuwa 714 (K!); Mufindi, grassland with scattered bushes below Superintendants house, Lugoda tea estate, 10 May 1968 (fl), Renvoize 2080 (AMES!, K!); Ngwazi, Mbeya-Iringa Road, annualls flooded grass, 1200 m, 6 Mar. 1970 (fl), Nicholson 149 (K!); T7, Lake Ngwazi, Mufindi, Miombo woodland, 1830 m, 18 Apr. 1982 (fl), Mwasumbi \& Mdemu 12223 (K!); Njombe Distr., Lihusosa swamp $17 \mathrm{~km} \mathrm{~N}$ of Njombe on Makumbako road, secondary grassland, 9'10'S, 34 45'E, 1800 m, 5 Apr. 1985 (fl), Pettersson, Hedren \& Kibuwa 654 (K!); Njombe, Tanwat, roadside edge in wattle plantation, full sun, 1700 m, 20 Apr. 1989 (fl), Spurrier 91 (K!); Sao Hill Plantation, in open grassy rich in pine plantation, 1800 m, 23 Feb. 1989 (fl), Spurrier 58 (K!); Sao Hill by roadside, 2000 m, 29 Mar. 1986 (fl), Keeley 64 (K!); Kagera: Bukoba Distr., Mshamba, 1350 m, Dec. 1931 (fl), Haarer 2370 (K!); Bukoba Distr., Nyashozi, Karagwe, 1500 m, Dec. 1931 (fl), Haarer 2430 (AMES!, K!); Kilimanjaro: T7, Moshi Distr., Kirdoroko Upare Country, 1500 m, 28 Oct. 1925 (fl), Haarer B2 (K!); Manyara: Mbulu Distr., Mbulumbul, Salt Pan Area, rare in Bothriochloa insculpta, upland grassland on a black saline clay of volcanic origin and associated with Oxalis, Orthosiphon, Indigofera glabra and Ajuga, 1530 m, 18 June 1946 (fl), Greenway 7771 (K!); Mbeya: Mbozi Distr., Songwe, $3 \mathrm{~km} \mathrm{~N}$ of Mbeya to Tunduma road, above Songwe River, on limestone, amongst grasses in Combretum, Terminalia, Bauhinia scrub woodland, $1350 \mathrm{~m}$, 12 Feb. 1979 (fl), Cribb, Grey-Wilson \& Mwasumbi 11423 (K!); T7, near Sao Hill Saw Mills, 141 km E of Mbeya on Iringa Road, grassland, 2 May 1975 (fl),
Hepper, Field \& Mhoro 5250 (K!); Morogoro: NW Uluguru Mts. Shambas above "Morningside", $1380 \mathrm{~m}$, 17 July 1972 (fl), Mabberley 1188 (K!); Rukwa: Tatanda Mission, near Zambian frontier on MbalaSumbawanga road, 9 June 1980 (fl), Hooper, Townsend \& Mwasumbi 1917 (K!); Shinyanga: Near Shinyanga, with Acacia on cotton soil, Apr. 1932-3 (fl), Bax 408 (AMES!, K!). Zambia. Central: Broken Hill, Chibwe P.F.A., on edge of rhe dambo in Julbernardia-Brachystegia woodland, 22 Jan. 1961 (fl), Morze 53A (K!); Serenje Distr., Kundalila Falls (53 km ENE Serenje), dambo near camp-site, 1500 m, 4 Feb. 1973 (fl), Schelpe 2914 (K!); Serenje Distr., Kundalila Falls, 15 km Se of Kanona, dambo above the falls, Jan. 1972 (fl), Williamson 2154 (K!); Serenje Distr., Kundalila falls, Feb. 1971 (fl), Williamson 2227 (K!); Eastern: 30 miles South of Nyaka, sandy dambo between rocks, Feb. 1969 (fl), Williamson 1394 (K!); Lusaka: Mazabuka Distr., Siamambo Forest Reserve, in wet dambo in front of Rest House, 30 Jan. 1960 (fl), White 6594 (K!); 35 miles south of Lusaka, in seasonally wet dambo, Feb. 1967 (fl), Williamson 143 (K!); Northern: Abercorn Distr., Kali Dambo, in very wet peaty marsh, 1500 m, 7 May 1952 (fl), Richards 1801 (K!); Abercorn Distr., marsh far end Lake Chilla, in peaty damp marsh, among rough grass, 1500 m, 29 Jan. 1955 (fl), Richards 4274 (K! \& spirit.); Abercorn Distr., rocks beyond Kawimbe, in grassland with a few trees, sandy soil, $1740 \mathrm{~m}, 20 \mathrm{Feb}$. 1967 (fl), Richards 22107 (AMES!, K!, UGDA-DLSz! - fragment, drawing); Abercorn Distr., swamp Lumi River, in wet muddy rough pasture, 1680 m, 31 May 1957 (fl), Richards 9942 (K!); Kawimbi-Lepersettlement, in short grass, sandy soil, 1500 m, 15 Feb. 1955 (fl), Richards 4490 (AMES!, K!); Kawimbi, pasture beyond fish ponds, among grass, peaty grey soil, 1500 m, 20 May 1955 (fl), Richards 5799 (K!); Swamp by Chambesi River Pontoon, in wet sandy clay among grass, 1200 m, 20 Jan. 1959 (fl), Richards 10765 (K!); Mbala Distr., Zomba Plain, edge of deep datich, dark loam wet soil, 1500 m, 15 May 1969 (fl), Sanane 730 (K!); North-Western: Mwinilunga Distr., just E of Malanchi Farm, dambo, 9 Jan. 1957 (fl), Robinson 2114 (K! - spirit.); Southern: Choma Distr., collecting in Simwamimuzuma National Forest No 180 on the Mabwingombe Hills in Uapaca woodland, 16 $56^{\prime} 15^{\prime}$ 'S, 27¹8'33”'E, 1200 m, Mar. 1997 (fl), Luwiika, Harder, Schmidt \& Zimba 624 (K!). Malawi. Central: Lilongwe Distr., Dzalanyama Forest Reserve, valley NW of Kamuzu hill, on flat rock and shallow gravel in Brachystegia-Julbernardia woodland, with Xerophyta, etc., 1420 m, 24 Feb. 1982 (fl), Brummitt, Polhill \& Banda 16079 (K!); Nkawa, in damp meady places, 14 Jan. 1936 (fl), Benson 11 (K!); Ntchisi Distr., $1 \mathrm{~km}$ from Chintembwe, Mission towards the Ntchisi-Dowa road, short damp grassland on crystaline rock, 1525 m, 7 Mar. 
1982 (fl), Brummitt, Polhill \& Banda 16369 (C!, K!); Kota Kota S. Soni Road, in open glade, wet underfoot, 22 Feb. 1953 (fl), Jackson 1094 (K!); Southern: Mulanje Distr., Mount Mulanje, alt. 1800-2100 m, 1897 (fl), Adamson 437 (E!); Mulanje Distr., Mount Mulanje, marshy places, 2400 m, 6 Apr. 1906 (fl), Adamson 449 (E!, K!). Mulanje Distr., Madzeka basin, a Little Ruo Corze, in damp places in woodland, clearings a in Plateaux grassland, 900-2100 m, 8 Apr. 1974 (fl), Allen 532 (K!); Mulanje Distr., Mulanje Mt., path from Tuchila Hut to head of Ruo Basin, shrubby hillside (Helichrysum, Vellozia, Kotschya, etc.), 1980-2130 m, 6 Apr. 1970 (fl), Brummitt 9644 (K!); Mulanje Distr., Mulanje Mt. Chambe Plateau, in high grassland, Apr. 1991 (fl), Jankins 119 (K!); Mulanje Distr., Mulanje Mt., Chuzana, Unfage slopes, 2040 m, 26 Mar. 1984 (fl), Johnston-Stewart 296 (K!); Mulanje Distr., Modzeka, rocks, montane grassland, 1950 m, 6 Apr. 1980 (fl), Morris 926 (K!). Zimbabwe. Harare: Salisbury, Ruwa River tributary stream, in well drained, stony ground, high above the River bank, 31 Jan. 1971 (fl), Linley 610 (K!); Manicaland: Inyanga, 1550 m, 4 Feb. 1931 (fl), Norlindh \& Weimarck 4835 (K!); Melsetter Distr., "Settler", at edge of vlei, 1500 m, 16 Feb. 1954 (fl), Ball 252 (K!); Melsetter Distr., "Settler" edge of vlei, 1500 m, 12 Feb. 1954 (fl), Ball 46249 (K!); Melsetter Distr., "Sibu" 10 miles W of Melsetter, in hillside grassland, vlei edges (dry), 31 Jan. 1953 (fl), Crook 445 (K!); Marienland, Nyumkwana valley, in high grassland, 1350 m, Feb. 1935 (fl), Gilliland 1539 (K!). Sine loc., Quartin Dillon s.n. (W-R!).

N otes. Summerhayes (1962) concluded that the flowers of $H$. rehmannii and $H$. tetrapetaloides were abnormal, but was unable to connect them with any normally developed specimens. In each case the lower petal lobe is partially suppresed and sometimes forked, but usually papillose basally, and the lip side lobe are reduced and tooth-like. A collection from Cathedral Peak in KwaZulu-Natal resembles the type of $H$. rehmannii exactly and a second collection from the same locality has the lip lateral lobes tooth-like but the lower petal lobes only slighty smaller than is typical for H. humilior. In other respects these collections agree very well with normal specimens of $H$. humilior, particulary in the structure of the gynostemium and in shorter, twisted spur. The proportionately long anther canals and stigmas and the twisted spur preclude them from being incluted in H. falcicornis (Linder \& Kurzweil 1999). In our opinion B. humilior is similar to B. humblotii and both species can be easily confused.

38. Bilabrella nyikana (Rchb. f.) Szlach. \& Kras-Lap., Richardiana 3(3): 141. 2003.

Habenaria nyikana Rchb. f., Otia Bot. Hamburg.: 100. 1881. - Rolfe, Flor. Trop. Afr. 7: 244. 1898. - Sum- merhayes, FTEA, Orchid. 1: 97. 1968b. - Grosvenor, Excelsa 6: 83. 1976. - Renz \& Schelpe, South Afr. Orchid. Journ. 11(1): 5. 1980. - Stewart, Linder \& Schelpe, Wild Orchids of Southern Africa: 91. 1982. - la Croix et al., Orchid. Malawi: 76. 1991. - la Croix \& Cribb, Fl. Zambes., Orchid. 11: 102. 1995. - Linder \& Kurzweil, Wild Orchids of Southern Africa: 128. 1999.

Type: Mozambique, Kirk s.n. (lectotype, designated by Summerhayes [1968b: 97], K!; drawing, W-R!). Habenaria elatior Schltr., Ann. Transvaal Mus. 10: 243. 1924.

Type: Transvaal, Schlechter 4779 (syntype, B+) \& Kwazulu-Natal, Wood 9374 (syntype, B+).

Tubers 1.5-2.5 cm long, $1 \mathrm{~cm}$ in diameter, ellipsoid, densely tomentose. Stem $40-150 \mathrm{~cm}$ tall, erect, stout, leafy throughout its length. Leaves $10-24,7-35 \mathrm{~cm}$ long, 1-2 cm wide, the lowermost sometimes reduced to a sheath, lower 6-10 suberect or less frequently spreading, linear or lanceolate-linear, acute, the upper ones smaller, closely adpressed to the stem and often overlapping, lanceolate, acuminate. Inflorescence 10-35 $\mathrm{cm}$ long, loosely or rather densely 8- to many-flowered. Flowers half-spreading, green with pale or white centre. Flower bracts $15-30 \mathrm{~mm}$ long, lanceolate, acuminate, thin and scarious, denticulate, the lower ones often equalling the pedicel with ovary. Pedicel with ovary 20-30 mm long, nearly straight or somewhat curved. Dorsal sepal 5-7.5 $\mathrm{mm}$ long, 2-3 $\mathrm{mm}$ wide, reflexed,

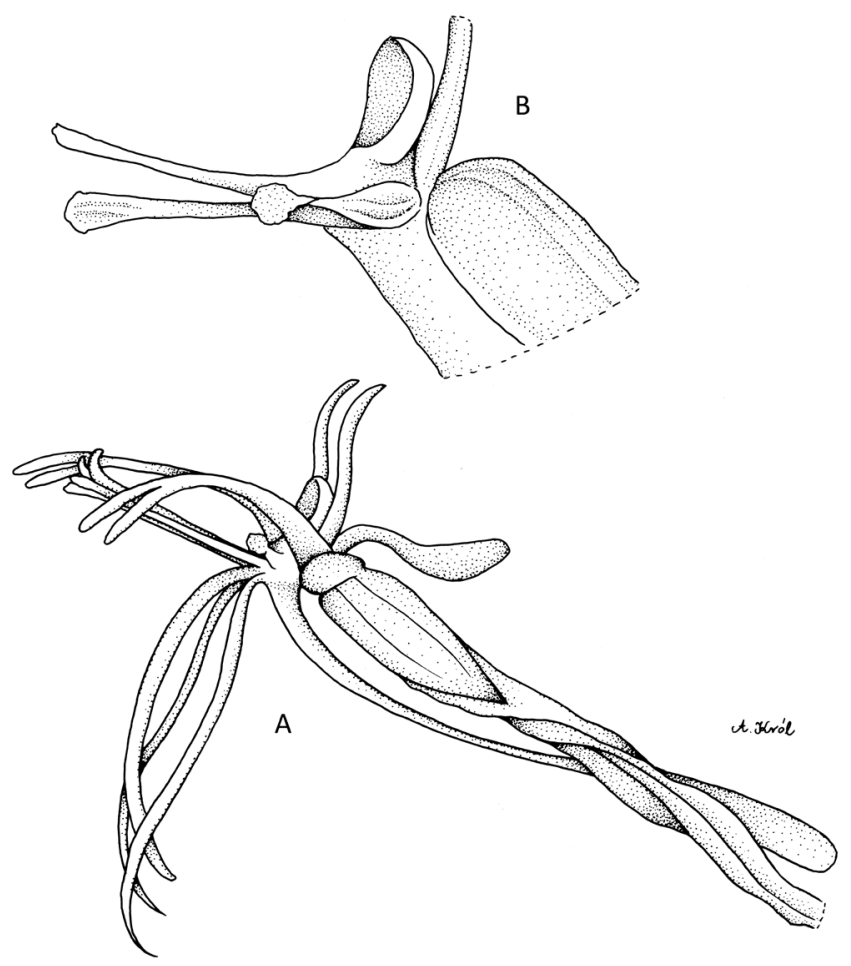

Fig. 114. Bilabrella nyikana (Rchb. f.) Szlach. \& Kras-Lap. Explanations: A - flower; B - gynostemium, side view (redrawn by A. Król from lectotype of Habenaria nyikana Rchb. f. - Kirk s.n., K) 


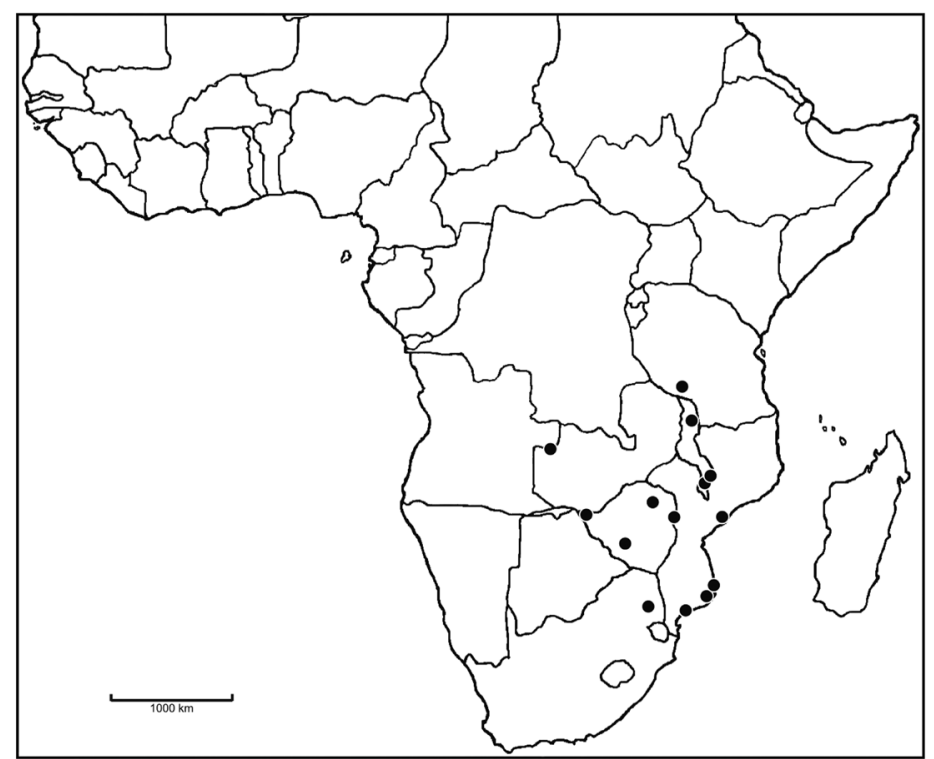

Fig. 115. Distribution of Bilabrella nyikana (Rchb.f.) Szlach. \& Kras-Lap.

narrowly elliptical, obtuse, convex. Petals bilobed nearly to the base; anterior lobe 9.5-14 mm long, 1.5$2.5 \mathrm{~mm}$ wide, spreading forwards, elongate-lanceolate, furculate apically, glabrous or ciliolate and pubescent; posterior lobe $5.5-7.5 \mathrm{~mm}$ long, scarcely $0.5-1 \mathrm{~mm}$ wide, recurved, linear, obtuse, ciliolate. Lateral sepals 8.5-10.5 mm long, 5-7 mm wide, deflexed, obliquely obcordate with marked lateral apiculum. Lip spreading downwards, 3-lobed nearly to the base; the middle lobe 11-15 mm long, $1 \mathrm{~mm}$ wide, incurved, linear, obtuse; lateral lobes 10-13 mm long, scarcely $1 \mathrm{~mm}$ wide, lanceolate-linear, acute. Spur 15-30 mm long, parallel to the ovary or upcurved, swollen in apical half, not twisted. Anther 2.5-4 mm tall; connective rounded; antherophores 3.5-6.5 mm long, slender, straight. Stigmaphores 4.5-6 mm long, slender, cylindrical, glabrous, truncate. Rostellum middle lobe shorter than thecae. Auriculae relatively large, subglobose (Fig. 114).

E c o logy. In bush along riverbanks, water-logged ground, warmbaths, grassland, Brachystegia woodland, in wet vlei. February-August.

D istribution. Tanzania, Zambia, Malawi, Mozambique, Zimbabwe, Republic of South Africa, alt. 600-1740 m (Fig. 115).

Specimens examined. Tanzania. Rukwa: Sumbawanga Distr., Ufipa, Chikanda, Lake Rukwa, locally common in badly drained, short, mixed grass areas atextreme edge of flood plain, 8 Feb. 1938 (fl), Midelwre 1481 (K!); Aug. 1858 (fl), Kirk 10/83 (K!). Zambia. North-Western: Zambezi, Bequeathed 311 (BM!); Zambezi, Sombu, Le Testu 311 (BM!); Dambo, Le Testu 911 (K!). Malawi. Northern: Nkhata Bay Distr., Vipya Plateau, Vipya Link road, turn off to Kawandama, grassland, 1740 m, 25 Mar. 1977 (fl), Pawek 12525 (K!); Southern: Blantyre Distr., Bvumbwe, growing at edge of Eucalyptus wood, 1200 m, 1 Apr. 1982 (fl), la Croix 304 (K!); Mulanje Distr., Likhubula valley, in Barchystegia woodland, 870 m, 2 Mar. 1981 (fl), la Croix 112 (K!); Mulanje Distr., Likhubula valley, Brachystegia woodland, 800 m, 6 Mar. 1981 (fl), la Croix 113 (K!); Thyolo Distr., Branbue, in grass at edge of gums, 1150 m, 9 Mar. 1985 (fl), la Croix 675 (K!). Mozambique. Inhambane: Massinga, Pomene, $4 \mathrm{~km}$ from Mata, 16 July 1981 (fl), Koning \& Hiemstra 9049 (K!); Zambezia: Mouth of R. Zambesi, Nyika Island, Aug. 1862 (fl), Kirk s.n. (K!, W-R!). Zimbabwe. Bulawayo: Matopo Hills, in damp ground, $1500 \mathrm{~m}$, Mar. 1907 (fl), Eyles 1037 (BM!); Harare: Marandellas Distr., in semi wet vlei, 5 Apr. 1950 (fl), Wild 3260 (27363) (K! \& spirit.); Salisbury, Spelonken Farm, between Christon Bank and Majoe Dam, in very wet vlei, 28 Mar. 1977 (fl), Grosvenor \& Renz 1298 (K!); Manicaland: Umtali Distr., SW partion of Municipal Sandpits area, Darlington Sulrub, 1080 m, 24 Mar. 1963 (fl), Chase 7908 (K!); Umtali Distr., 1110 m, 28 Feb. 1963 (fl), Chase 7958 (K!); Umtali Distr., Inyamatshura Mts., Murambi Garden, 1110 m, 21 Mar. 1963 (fl), Chase 7966 (K!); Masvingo: Victoria, Hillingtale neer Tokwe crossing on road to Beit Bridge, $1050 \mathrm{~m}$, Mar. 1958 (fl), Dale 10 (84998) (K! \& spirit.). Republic of South Africa. Limpopo \& Mpumalanga: Transvaal, Kruger National Park Distr., in water-logged ground, 600 m, 10 Apr. 1953 (fl), Schyff 2640 (K!); Bushveld, warmbaths, 7 Apr. 1928 (fl), Moss 16628 (K!).

$\mathrm{N}$ otes. It is similar to Bilabrella culcifera. Both species share similar form of perianth segments, but in $B$. nyikana the spur is usually more or less parallel to the ovary and stigmaphores are glabrous, in B. culcifera spur is upcurved and stigmaphores ciliolate. It seems that the most striking character of $B$. nyikana are furculate anterior petal lobes.

39. Bilabrella culcifera (Rolfe) Szlach. \& Kras, Richardiana 9(4): 158. 2009.

Habenaria culcifera Rolfe, J. Linn. Soc., Bot. 33: 278. 1895.

Type: Uganda, Scott Elliot 7854 (lectotype, designated by Szlachetko et al. [2010: 235], BM!; isolectotype, $\mathrm{K}$ !).

Stem up to $40 \mathrm{~cm}$ tall, erect, slender, glabrous. Leaves 6-8, up to $15 \mathrm{~cm}$ long and $1.3 \mathrm{~cm}$ wide, the lower ones vaginate, linear-lanceolate, acute, erect-spreading, decreasing in size upwards. Inflorescence up to $15 \mathrm{~cm}$ long, laxly multiflowered. Floral bracts $15 \mathrm{~mm}$ long, ovate-lanceolate, acuminate, thin, membraneous. Ovary and pedicel up to $20 \mathrm{~mm}$ long, slender. Dorsal sepal $5 \mathrm{~mm}$ long, $2 \mathrm{~mm}$ wide, oblong obovate to narrowly 


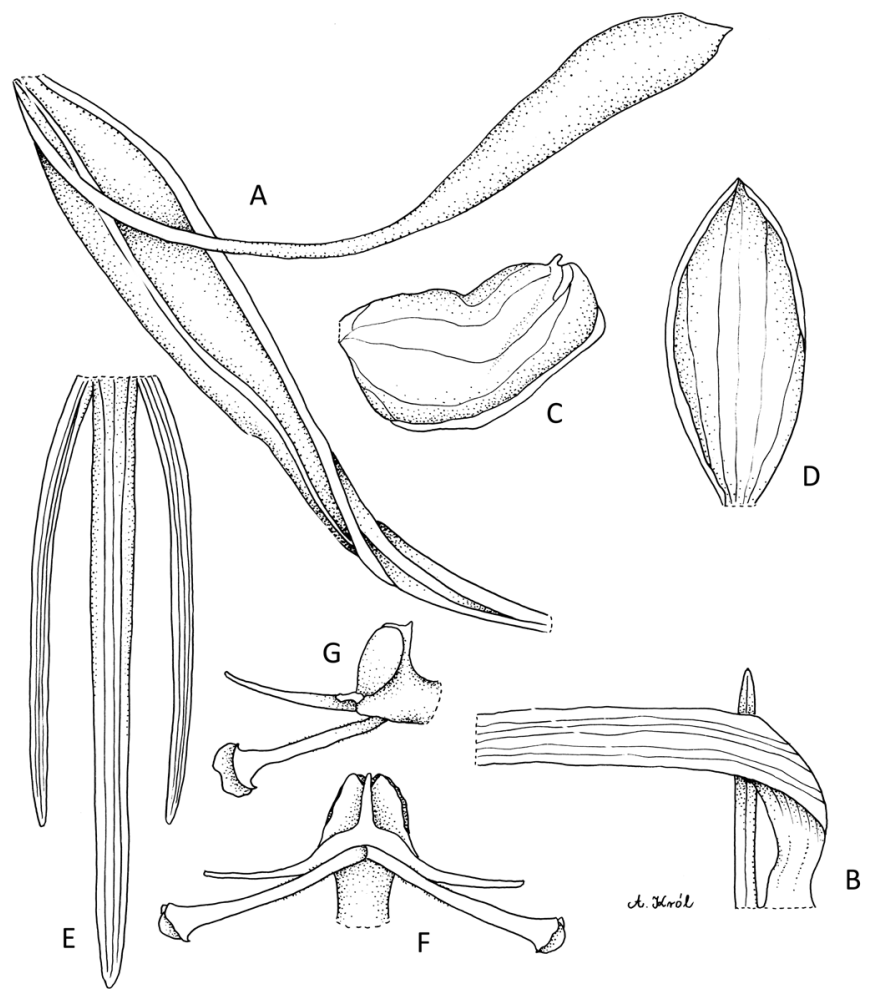

Fig. 116. Bilabrella culcifera (Rolfe) Szlach. \& Kras Explanations: A - ovary, pedicel, spur; B - petal; C - lateral sepal; D - dorsal sepal; E - lip; F - gynostemium, front view; $\mathrm{G}$ - gynostemium, side view (drawn by A. Król from lectotype of Habenaria culcifera Rolfe - Scott Elliot 7854, BM)

elliptic, obtuse, 3-nerved, cochleate. Petals bilobed to the base; anterior lobe $1.5 \mathrm{~mm}$ long, $0.3 \mathrm{~mm}$ wide, filiform, blunt, glabrous; posterior lobe $7 \mathrm{~mm}$ long, $1.1 \mathrm{~mm}$ wide, linear-lanceolate, acuminate, glabrous. Lateral sepals $6.5 \mathrm{~mm}$ long, $4 \mathrm{~mm}$ wide, obliquely obovate to oblong-elliptic, with laterally placed, acute

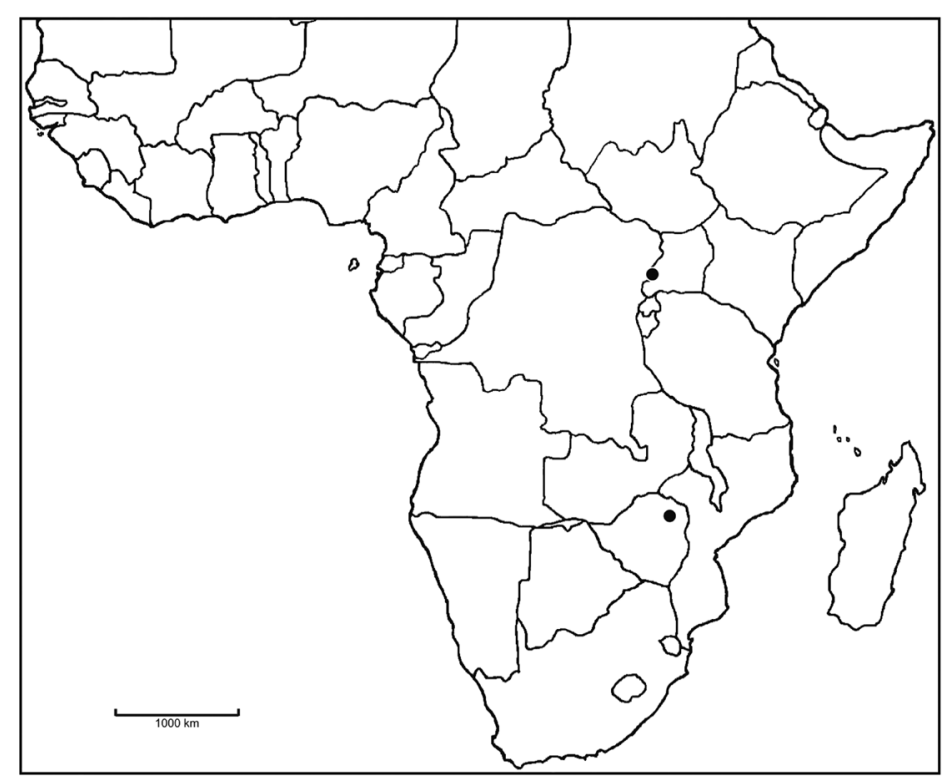

Fig. 117. Distribution of Bilabrella culcifera (Rolfe) Szlach. \& Kras apiculus. Lip 3-lobed just above the base; the middle lobe $11 \mathrm{~mm}$ long, $0.6 \mathrm{~mm}$ wide, narrowly linear, subobtuse to subacute; lateral lobes $8 \mathrm{~mm}$ long, $0.4 \mathrm{~mm}$ wide, narrowly linear, subacute, pendent. Spur 22-24 $\mathrm{mm}$ long, filiform, clavate at the acute apex, upcurved, not twisted. Anther $2.5 \mathrm{~mm}$ long; connective truncate or shallowly notched; antherophores $5 \mathrm{~mm}$ long, slender, delicate, straight. Stigmaphores $5 \mathrm{~mm}$ long, ciliolate, cylindrical, much swollen and truncate apically. Rostellum middle loobe as long as connective. Auriculae small, digitate (Fig. 116).

E c o logy. Hillsides. December.

Distribution. Democratic Republic of the Congo (Zaire), Uganda, Zimbabwe, alt. 2000 m (Fig. 117).

$\mathrm{S}$ pecimens examined. Democratic Republic of the Congo (Zaire). Gisuzu, Lalupungu, swamp, 12 Dec. 1951 (fl), Michel, Reed 901 (MO!). Uganda. Bundibugyo: Ruwenzori, Hillsides, 2000 m, Scott Elliot 7854 (BM!, K!). Zimbabwe. Harare: Salisbury, Dec. 1897 (fl), Rand 269 (BM!).

Notes. It appears to be closely related to aforementioned Bilabrella nyikana from which it differs by having entire, unlobed anterior petal lobes and ciliolate stigmaphores. Additionally its spur is permanently upcurved, what can be observed in B. nyikana only occasionally.

The other species having not glabrous stigmaphores is $B$. hirsutitrunci. In this species, however, stigmaphores are hirsute, and in B. culcifera ciliolate. Both species differs easily one from another in the flower parts morphology as well.

\subsubsection{Subsection Arachnoideae Szlach. \& Kras, subsect. nov}

Type species: Bilabrella schimperiana (Hochst. ex A. Rich.) Szlach. \& Kras-Lap. [三 Habenaria schimperiana Hochst. ex A. Rich.].

Anterior petal lobe usually wider than posterior one, occasionally half width as posterior one.

A group of about 10 species.

\section{Key to species}

1. Stigmaphores pubescent 40. B. hirsutitrunci

1. Stigmaphores glabrous or papillate 2

2. Spur ampullaceous in the apical third or so ........... 3

2. Spur neither twisted nor ampullaceous .................. 4

3. Spur twisted and geniculate near the middle

41. B. schimperiana

3. Spur parallel to the ovary, not twisted

42. B. cataphysema

4. Spur with acute apex 5

4. Spur blunt at the apex ......................................... 6

5. Leaves $10-28 \times 0.6-2.5 \mathrm{~cm}$, petals glabrous

43. B. weberana 


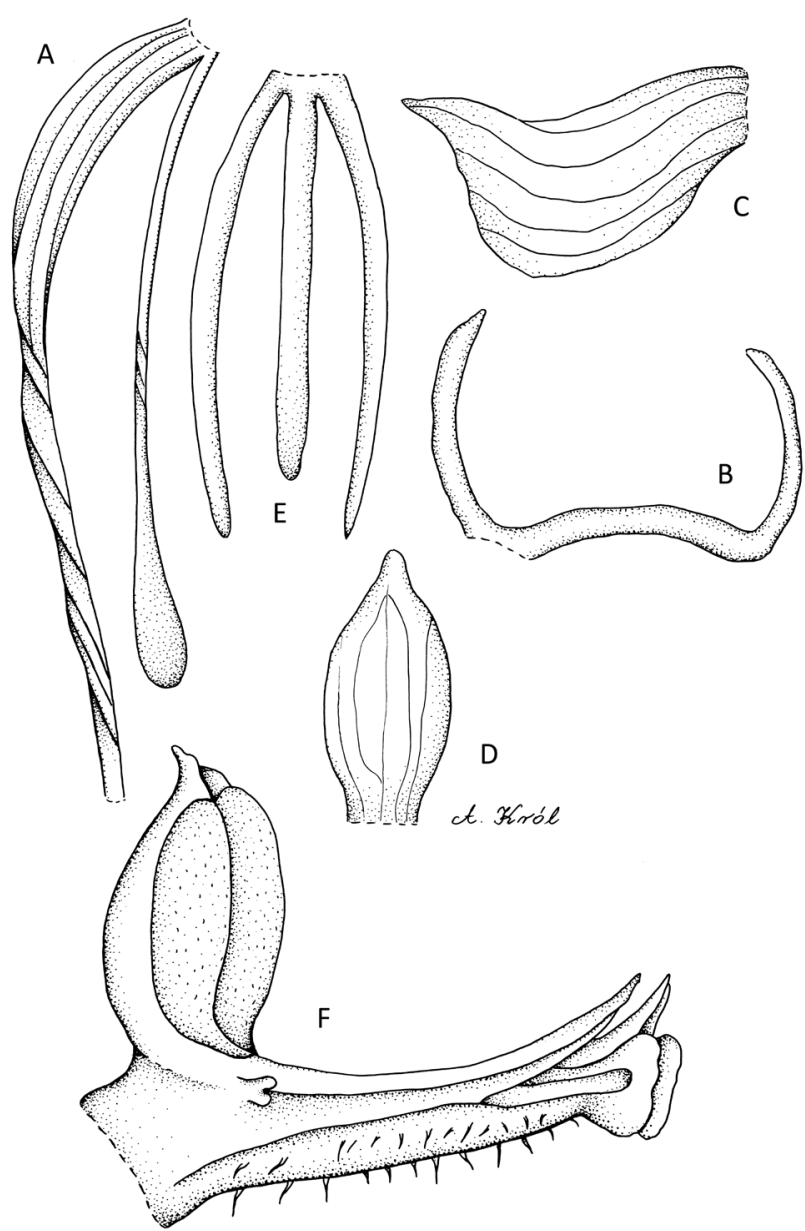

Fig. 118. Bilabrella hirsutitrunci (G. Will.) Szlach. \& Kras-Lap. Explanations: A - ovary, pedicel, spur; B - petal; C - lateral sepal; D - dorsal sepal; E - lip; F - gynostemium, side view (redrawn by A. Król from Habenaria hirsutitrunci G. Will. - Williamson \& Drummond 1973, SRGH)

5. Leaves 4-6 $\times 0.9-1.2 \mathrm{~cm}$, petals papillose

44. B. linguiformis

6. Petals papillose 7

6. Petals glabrous .................................................. 8

7. Both petals lobes similar in size and form ......

45. B. subaequalis

7. Anterior petal lobes much longer than posterior one 46. B. hebes

8. Dorsal sepal obscurely 3 -lobed just above the middle, lip 3-lobed to the base

47. B. orthocaulis

8. Dorsal sepal entire, lip 3-lobed above undivided basal claw ...... 9 9. Leaves up to $9 \times 2-5 \mathrm{~cm}$, inflorescence up to $30 \mathrm{~cm}$ long, gynostemium stalked .... 48. B. ugandensis 9. Leaves 5-17 $\times$ 0.5-1.7 cm, inflorescence $11-15 \mathrm{~cm}$ long, gynostemium sessile

49. B. galpinii

40. Bilabrella hirsutitrunci (G. Will.) Szlach. \& KrasLap., Richardiana 3(3): 140. 2003.

Habenaria hirsutitrunci G. Will., Pl. Syst. Evol. 134: 54. 1980. - la Croix et al., Orchid. Malawi: 70. 1991. - la Croix \& Cribb, Fl. Zambes., Orchid. 11: 94. 1995.
Type: Zambia, Williamson \& Drummond 1973 (holotype, SRGH not seen).

Habenaria sp. Williamson, Orchid. S. Centr. Africa: 53. 1977.

Stem $25-40 \mathrm{~cm}$ tall, slender, leafy. Leaves $7-8$, the lowest $3 \pm$ sheathing, the upper ones grading into the bracts, the middle ones semi-erect, $5-15 \mathrm{~cm}$ long, 0.4$1.9 \mathrm{~cm}$ wide, linear. Inflorescence $9-13 \mathrm{~cm}$ long, fairly densely several- to many-flowered. Flowers green. Floral bracts to $25 \mathrm{~mm}$ long, leafy. Pedicel and ovary erect, pedicel $15-16 \mathrm{~mm}$ long, ovary $8-10 \mathrm{~mm}$ long. Dorsal sepal 4-5 mm long, 1-3 mm wide, ovate, erect. Petals 2-lobed almost to base; posterior petal $4 \mathrm{~mm}$ long, $0.5 \mathrm{~mm}$ wide, narrowly linear, erect; anterior petal 14-18 mm long, ca. $1 \mathrm{~mm}$ wide at base, curving sideways and up, becoming filiform. Lateral sepals 6-8 $\mathrm{mm}$ long, 3-4.5 mm wide, reflexed, oblique with a lateral apiculum, usually rather brown and withered by flowering time. Lip 3-lobed to ca. $1 \mathrm{~mm}$ from base; the middle lobe 10-12 mm long, 0.9-1 mm wide, narrowly linear, obtuse; lateral lobes 12-15 mm long, $1 \mathrm{~mm}$ wide, narrowly linear, acute. Spur 16-23 mm long, slender but very swollen in the apical $5 \mathrm{~mm}$, parallel to the pedicel and ovary and tucked into the bracts. Anther 2-4 mm tall; connective triangularly apiculate; antherophores 4-5 mm long, slender, gently upcurved towards the apex. Stigmaphores 4-5.5 mm long, projecting forwards but diverging, truncate at apex, hirsute. Rostellophores 4-5 mm long, hirsute, lying above stigmatic arms. Rostellum middle lobe shorter than thecae. Auriculae inconspicuous (Fig. 118).

E c ology. Short grassland, seasonally wet sandy grassland plains, montane grassland, laterite rocks. February-April.

D i stribution. Zambia, Malawi, alt. 1500-2200 m (Fig. 119).

Specimens examined. Zambia. Northern: Abercorn Distr., Abercorn-Chilongowelo Road, in sandy damp ground among laterite rocks, $1650 \mathrm{~m}, 14$ Feb. 1960 (fl), Richards 12473 (K!); Abercorn Distr., road Uningi Pans, in short grass sandy, 1500 m, 5 Mar. 1965 (fl), Richards 19729 (K!); Bank of Luangwa River, 51,2 km south of Mporokoso, single, wet upland grassland, Feb. 1970 (fl), Williamson \& Drummond 1973 (SRGH not seen); Nyika Plateau, near rest house, in short grass near rocks, where locally common in Mar, $2150 \mathrm{~m}$, 11 Mar. 1982 (fl), Dowsett-Lemaire 355 (K!). Malawi. Northern: Nyika Plateau, Zovo-Chipolo grassland, often in short grass near rocky outcrops, widespread, 2200 m, 5 Mar. 1982 (fl), Dowsett-Lemaire 338 (K!). Nyika N Plateau, Zovo Chipolo, in montane grassland, 2100 m, 3 Mar. 1983 (fl), Elias 12 (K!); 8 Mar. 1983 (fl), Elias 14 (K!); Nyika National Park, beside turning circk, 2100 m, 9 Apr. 1984 (fl), la Croix 606 (K!). 


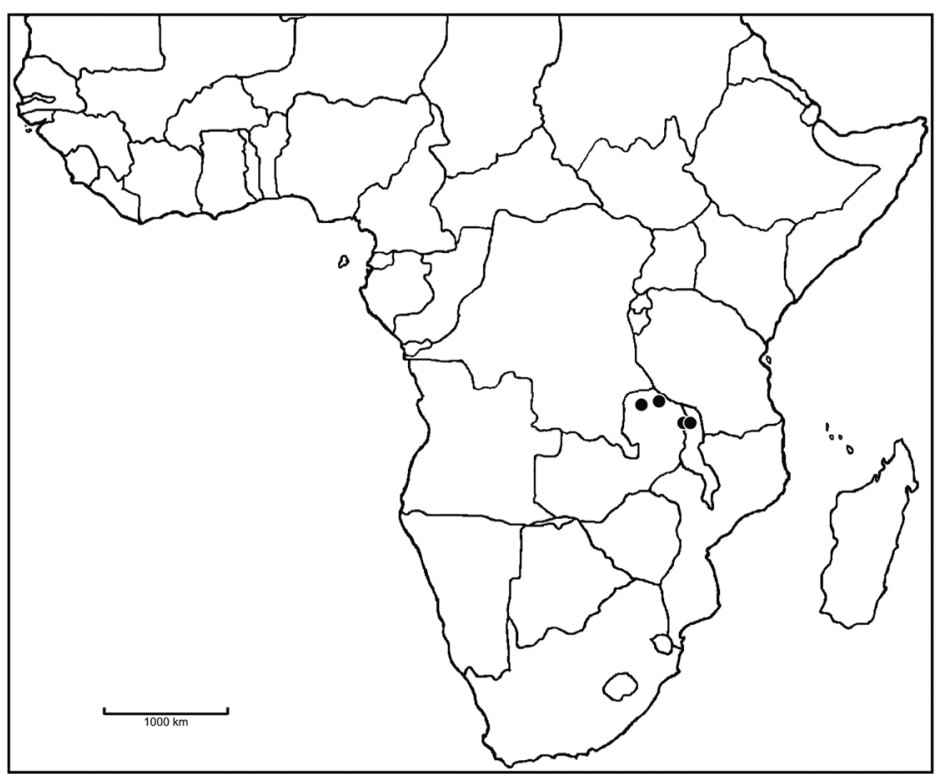

Fig. 119. Distribution of Bilabrella hirsutitrunci (G.Will.) Szlach. \& Kras-Lap.

Notes. Bilabrella hirsutitrunci is rather difficult to mistake with other species of the genus, especially by having hirsute stigmaphores and rostellophores, both characters unique in Bilabrella.

41. Bilabrella schimperiana (Hochst. ex A. Rich.) Szlach. \& Kras-Lap., Richardiana 3(3): 142. 2003. Szlachetko et al., Orchid. of West-Central Africa 1: 216. 2010.

Habenaria schimperiana Hochst. ex A. Rich., Tent. Fl. Abyss. 2: 295. 1851. - Kraenzlin, Orchid. Gen. Sp.: 226. 1901. - Rolfe, Fl. Trop. Afr. 7: 241. 1898. Summerhayes, Bot. Not. 1937: 186. 1937. - Robyns \& Tournay, Fl. Spermat. Parc Natl. Albert 3: 434. 1955. Andrews, Flow. Pl. A.E. Sudan 3: 323. 1956. - Copley, Tweedie \& Carroll, Journ. E. Afr. Nat. Hist. Soc. 24(4): 23. 1964. - Piers, Orchids E. Afr. 2: 34. 1968. - Summerhayes, FTEA, Orchid. 1: 89. 1968b. - Stewart in Agnew, Upl. Kenya Wild Fl.: 741. 1974. - Grosvenor, Excelsa 6: 83. 1976. - Geerinck \& Coutrez, Not. Taxon. Orchid. Afr. Centr., Habenaria: 11. 1977. - Williamson, Orchid S. Central Afr.: 51. 1977. - Reekmans, Lejeunia, Nov ser., 100: 45. 1980. - Renz \& Schelpe, South Afr. Orchid. Journ. 11(1): 10. 1980. - Stewart et al., Wild Orchid. South Afr.: 92. 1982. - Geerinck, Fl. Afr. Centr., Orchid. 1: 104-105. 1984. - la Croix et al., Orchid. Malawi: 78. 1991. - la Croix \& Cribb, Fl. Zambes., Orchid. 1: 94. 1995. - Stewart \& Campbell, Orchids of Kenya: 140. 1996. - Edwards, Demissew, Hedberg, Flora of Ethiopia \& Eritrea 6: 220. 1997. Szlachetko \& Olszewski, Fl. Cam., Orchid. 34(1): 164. 1998. - Linder \& Kurzweil, Orchids of Southern Africa: 130. 1999. - Demissew, Cribb, Rasmusen, Field guide to Ethiopian Orchids: 95. 2004. - Szlachetko, Orchid. Ivory Coast: 44. 2008.

Type: Ethiopia, Schimper 1210 (lectotype, designated by Szlachetko [2008: 44], P!; isolectotypes, BM!, $\mathrm{K}$ !, S not seen, W-R!).

Habenaria genuflexa Rendle, J. Bot. 33: 279. 1895.

Type: Uganda, Scott Elliot 7922 (lectotype, designated by Szlachetko [2007: 214], BM!, isolectotype, K!). Bilabrella genuflexa (Rendle) Szlach. \& Kras-Lap., Richardiana 3(3): 140. 2003.

Habenaria ichneumonea Kraenzl. in Durand \& De Wild., Bull. Jard. Bot. Belg. 38: 67. 1899, non (Sw.) Lindl.

Type: Tanzania, Descamps s.n \& Congo, Deveyre 500 (syntype, locality unknown).

Habenaria stenoloba Schltr., Bot. Jahrb. Syst. 38: 1. 1905.

Type: Gabon, Schlechter 12824 (B+; lectotype, designated by Szlachetko [2007], K!; isolectotypes, BM!, $\mathrm{BR}$ !).

Habenaria anaphysema Robyns \& Tournay, F1. Spermat. Parc Nat. Albert 3: 432. 1955, non Rchb. f.

Tubers 1-4 cm long, 1-2 $\mathrm{cm}$ in diameter, ellipsoid or ovoid. Stem $30-100 \mathrm{~cm}$ tall, erect, slender to rather stout, glabrous. Leaves 6-10, 7-28 cm long, 1-2 (rarely $3.7) \mathrm{cm}$ wide, linear to linear-lanceolate, acute, suberect or spreading, the upper ones smaller. Inflorescence 6-35 cm long, 4-30-flowered, or more, lax or rather dense. Flowers green with white central part, with an unpleasant smell. Floral bracts 10-30 mm long, ciliolate inside, usually much shorter than pedicel. Pedicel and ovary (20)25-42 mm long, glabrous. Dorsal sepal 6-8 mm long, 3.2-4 mm wide, oblong-elliptic, obtuse, convex, glabrous, reflexed. Petals bilobed to the base, rather thick; anterior lobe 9.5-18.5 mm long, 0.5-2 mm wide, linear-lanceolate to oblong-lanceolate, apiculate, spreading downwards, ciliate in the lower two third; posterior lobe 5-8 $\mathrm{mm}$ long, 0.3-1 $\mathrm{mm}$ wide, linear, falcate, acute, thinner than anterior lobe, completely ciliate. Lateral sepals 7.5-11 mm long, 5-8 $\mathrm{mm}$ wide, obliquely obovate or widely obovate, cochleate, glabrous, with ca. $0.8 \mathrm{~mm}$ long laterally placed apiculus. Lip 3-lobed above an undivided basal part 2-3 mm long, fleshy, glabrous; the middle lobe 10-17 mm long, 0.4-0.9 mm wide, linear-filiform, obtuse; lateral lobes $6.5-11 \mathrm{~mm}$ long, 0.3-0.7 mm wide, linear, acute, curved upwards. Spur 9-16 mm long, geniculate, sometimes straight, several times twisted near the middle, swollen in the apical half or third forming an ampulle, ca. $2 \mathrm{~mm}$ in diameter, blunt. Gynostemium in the basal third or quarter connate with the lip base. Anther 3-3.5 mm long; connective notched; antherophores 5-8 mm long, slender, gently upcurved. Stigmaphores 4-6.5 mm long, slender, gently upcurved, truncate at the apex. Rostellum middle lobe 


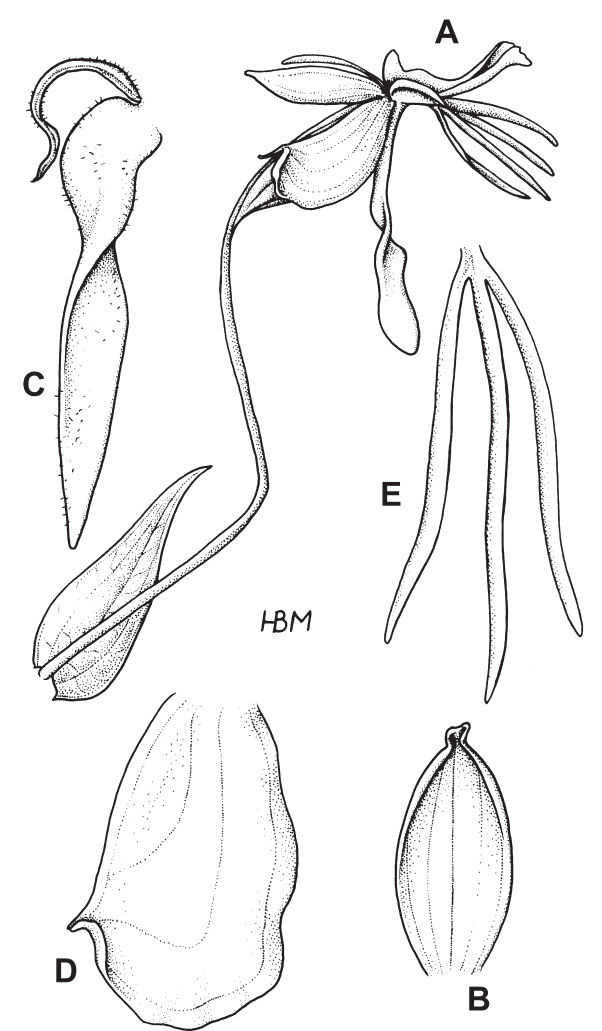

Fig. 120. Bilabrella schimperiana (Hochst. ex A. Rich.) Szlach. \& Kras-Lap.

Explanations: A - flower; B - dorsal sepal; C - petal; D - lateral sepal; E lip (drawn by H. B. Margońska from lectotype of Habenaria schmiperiana Hochst. ex A. Rich. - Schimper 1210, P)

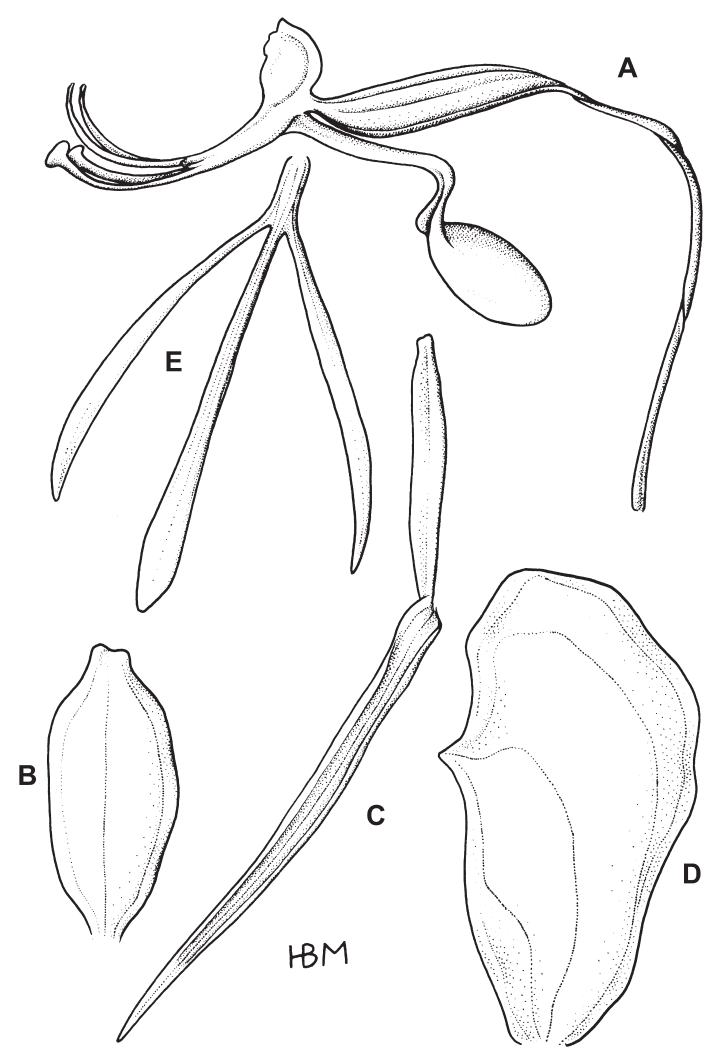

Fig. 121. Bilabrella schimperiana (Hochst. ex A.Rich.) Szlach. \& Kras-Lap.

Explanations: A - ovary, pedicel, spur and gynostemium, side view; B dorsal sepal; C - petal; D - lateral sepal; E - lip (drawn by H. B. Margońska from Habenaria genuflexa Rendle - Unknown collector, W-R)

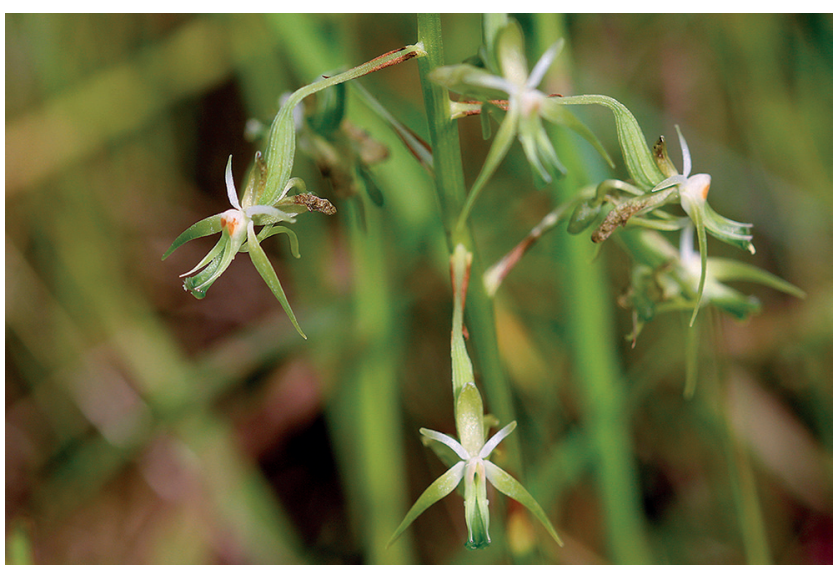

Fig. 122. Bilabrella schimperiana (Hochst. ex A. Rich.) Szlach. \& Kras-Lap. - inflorescense close-up (phot. R. Rawen)

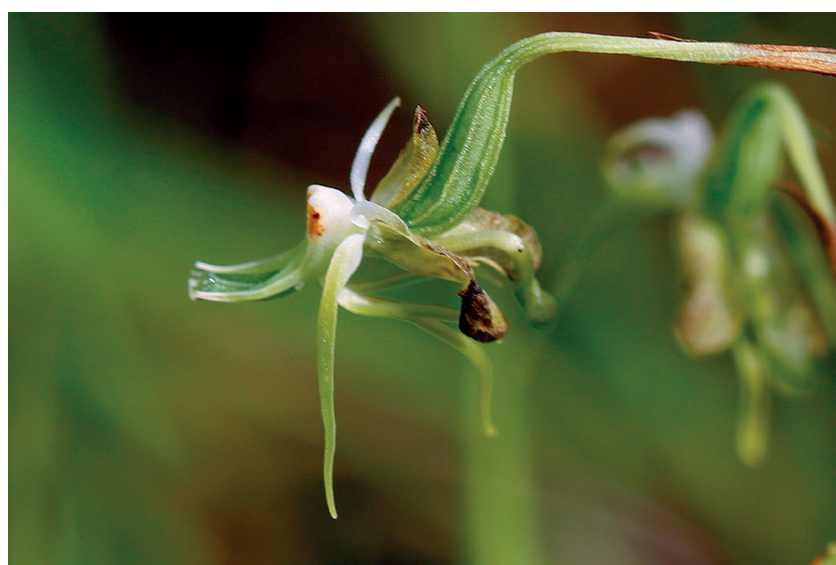

Fig. 123. Bilabrella schimperiana (Hochst. ex A. Rich.) Szlach. \& Kras-Lap. - flower close-up (phot. R. Rawen)

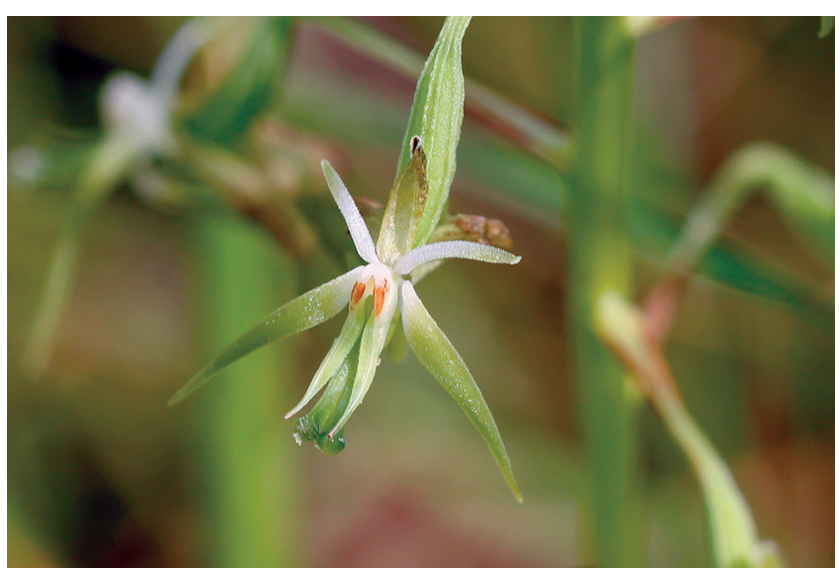

Fig. 124. Bilabrella schimperiana (Hochst. ex A. Rich.) Szlach. \& Kras-Lap. - flower close-up (phot. R. Rawen)

slightly shoretr than thecae. Auriculae small, entire, ellipsoid (Figs 120-124).

E c o log y. Swamp, wet grassland on badly drained soil, flooded grassland, marsh, clayish soil, granite. Throughout the year.

Distribution. Sudan, Eritrea, Guinea, Sierra Leone, Liberia, Ivory Coast, Burkina Faso, Ghana, Nigeria, Cameroon, Central African Republic, Ethiopia, Republic of Equatorial Guinea, Gabon, Congo, 


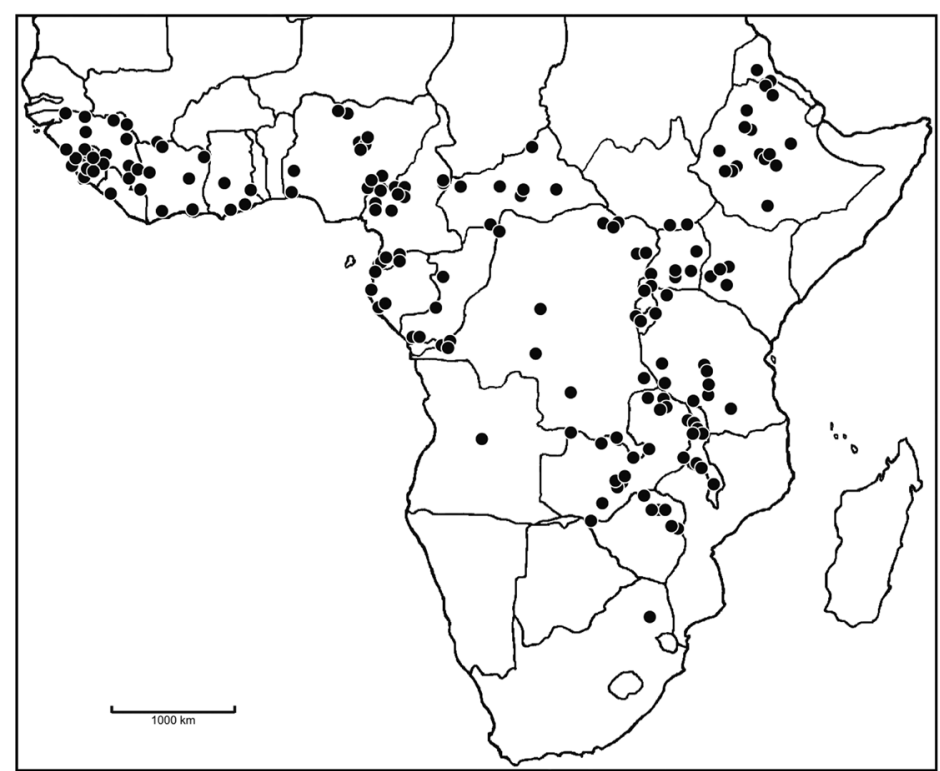

Fig. 125. Distribution of Bilabrella schimperiana (Hochst. ex A. Rich.) Szlach. \& Kras-Lap.

Democratic Republic of the Congo (Zaire), Uganda, Kenya, Burundi, Tanzania, Angola, Zambia, Malawi, Zimbabwe, Republic of South Africa, alt. 7,5; 120$2550 \mathrm{~m}$ (Fig. 125).

Specimens examined. Sudan. Equatoria West: Gilo, Imatong Mts., in burned-over grassland beside large rock slabs in mountain savannah, $1800 \mathrm{~m}$, 28 June 1947 (fl), MacLeay 103 (BM!); Yei Distr., Kajiko Valley, on almost bare gneiss outcrop, $1000 \mathrm{~m}$, 4 Sep. 1957 (fl), Jackson 3846 (K!). Eritrea. Andeba: 12 miles $\mathrm{E}$ of Keren, in unweeded sisal plantation, $14^{\circ} 47^{\prime} \mathrm{N}, 38^{\circ} 27^{\prime} \mathrm{E}, 1375$ m, 28 Aug. 1959 (fl), Mooney 8051 (K!); Southern: Scimenzana, Guna Guna, Pappi 641 (P!). Guinea. Boké: Bissan, Pussnbe, 21 Nov. 1942 (fl), s.n. 1391 (K!); Conakry: Tamara Island, Cote SO. Bank, Nov. 1924 (fl), Serand s.n. (P!); Faranah: Between Kouria and "Ymbo", 7 Oct. 1905 (fl), Caille 14966 (P!); Kankan: Kankan, Aug. 1954 (fl), JacquesFelix 7096 (K!, P!); Limbo, bank of the river, abnormal form, 17 Nov. 1898 (fl), Maclaud 94 (P!); Labé: Mali Region, 1500 m, Sep. 1954 (fl), Schnell 7072 (P!); Mamou: Dalaba Prefecture, Fouta Djallon, Adames 326 (K!); Plateau Dalaba-Diaguissa, 1100-1300 m, 8 Oct. 1907 (fl), Chevalier 18802 (K!, P!); Dalaba, 1200 m, Sep. 1954 (fl), Schnell 6816 (P!); Dalaba, Mount Tinka, 1200 m, 8 Oct. 1954 (fl), Schnell 7461 (P!); Mount Mamou (Bilimba), 1000 m, Sep. 1937 (fl), Jacques-Felix 1820 (K!); Timbo environs, Oct. 1937 (fl), Jacques-Felix 1888 (K!); Sokotoro, Timbo massif, Aug. 1898 (fl), Maclaud 241 (P!); Nzérékoré: Nzérékoré, Oct. 1937 (fl), Jacques-Felix 1951 (K!, P!); Mount Nimba, on crests, Sep. 1942 (fl), Schnell 1854 (K!); Schnell 3727 (K!). Sine loc., 1200 m, Oct. (fl),
Adam 24343 (P!); Big Rock, 1500 m, Nov. (fl), Adam 24677 (P!). Sierra Leone. Bombali: Binkolo, near Makeni, in damp soil on granite outcrop, 11 Sep. 1962 (fl), Harvey 155 (K!); Bonthe: Bonthe, in grassland on poor sandy soil, 10 Oct. 1946 (fl), Adames 82 (K!); In low grassfield, seen also in grass by path between Jama and Senihun and Njala, Deighton 3044 (K!); Gbap. Nongoba Bullom, in sandy soil among thatch grass, 11 Oct. 1951 (fl), Jordan 607 (K!); Mt. Oriel., Mar. 1915 (fl), Litchford 366 (K!); Kailahun: Kailahun, in short grass on hillside, 28 Sep. 1939 (fl), Deighton 3766 (K!); Kambia: Near Kambia Distr., Rolup, Sanda-Tenraron, in moist situation in shallow soil overlying laterite rock, 13 Sep. 1951 (fl), Jordan 539 (K!); Kenema: Masahun, in grassland, 11 Oct. 1992 (fl), Segerback 1551 (K!); 300 m, 7 Oct. 1914 (fl), Thomas 2894 (K!); Koinadugu: Loma Mts., damp places in high altitude grassland, 7 Sep. 1945 (fl), Jaeger 1552 (K!); Moyamba: Njala (Kori), 29 Sep. 1955 (fl), Pyne 4 (K!); Near Wonkifu (Tonko-Limba), in shallow soil overlying laterite rock, 2 Oct. 1953 (fl), Jordan 935 (K!); Port Loko: Rokupr quarry, Magbema Chiefdom, on shallow soil over rock, 10 Oct. 1951 (fl), Adames 252 (K!); Tonkolili: Bumbuna, 1800 m, 14 Oct. 1914 (fl), Thomas 3136 (K!); Bumbuna, 195 m, 14 Oct. 1914 (fl), Thomas 3216 (K!); 20 Oct. 1914 (fl), Thomas 3771 (K!); Western Area Rural: 2 miles along road west from Waterloo, marsh, 52.5 m, 22 Aug. 1958 (fl), Melville \& Hooker 278 (K!); SW of Hastings, secondary forest beneath trees, $120 \mathrm{~m}, 6$ Sep. 1958 (fl), Melville \& Hooker 445 (K!); 1828 (fl), Milton s.n. (BM!); Kanya, 1914 (fl), Thomas 3058 (K!); Kanya, 450 m, 7 Oct. 1914 (fl), Thomas 2978 (K!). Liberia. Montserrado County: Duport, moist sandy soil in grassy plam, 18 Nov. 1926 (fl), Linder 1506 (K!); Duport, about 8 miles east of Monrovia, former Porroh-bush, open grassy swamp, 6 Oct. 1966 (fl), Bos 2264 (K!); Duport, near Porrohbush (forest relic), near marsh, 10 Sep. 1962 (fl), Dillewijn 100 (K!); Nimba County: Sanokwele (Sanniquellie) Distr., on rock outcrop near Gbau, 22 Sep. 1947 (fl), Baldwin 9434 (K!); Mount Nimba, 19 Oct. 1975 (fl), Adam 29971 (K! - drawing); Grand Bassa, Dinklage 1768 (AMES!, UGDA-DLSz! - fragment, drawing) \& 2984 (AMES!, UGDA-DLSz! - fragment, drawing); Man, Chevalier 2555 (Summerhayes 1968b, not seen); Sassandra-Cavally, Chevalier 34172 (Summerhayes 1968b, not seen). Ivory Coast. BasSassandra: San-Pedro Dept., Orumbo-Boka, Aug. 1954 (fl), s.n. 6494 (P!); Dix-Huit Montagnes: Danané, 18 Oct. 1974 (fl), Perez-Vera 700 (ABI not seen, K!; P). Mt. Tonkoui, 24 Sep. 1969 (fl), Cremers 1148 (P!). Mt. Tonkoui, Oct. 1974 (fl), Perez-Vera 673 (ABI not seen, K!); Mt. Tonkoui, Aug. 1954 (fl), Schnell 6364 (P!); Savanes: Boundiali Dept. $64 \mathrm{~km}$ from Boundiali and Korhogo, 24 Aug. 1975 (fl), Perez-Vera 826 (K!); 
Zazan: $40 \mathrm{~km} \mathrm{~W}$ of Bania, $9^{\circ} 01^{\prime} \mathrm{N}, 3^{\circ} 27^{\prime} \mathrm{W}, 16 \mathrm{Sep}$. 1967 (fl), Geerling \& Bokdam 901 (WAG!, (along with H. chlorotica)); $40 \mathrm{~km} \mathrm{~W}$ of Bania, $9^{\circ} 01^{\prime} \mathrm{N}, 3^{\circ} 28^{\prime} \mathrm{W}$, 16 Sep. 1967 (fl), Geerling \& Bokdam 915 (WAG!). Burkina Faso. Cascades: Comoé Prov., Cliff Banfora, 20 Sep. 1958 (fl), Adam 15230 (K!); Adam 29966 (K! - drawing); Comoé Prov., Banfora-Solmarécageua, 20 Sep. 1958 (fl), Jaeger 5239 (K!); Comoé Prov., Banfora, 1 Sep. 1976 (fl), de Koning 7207 (WAG!); Comoé Prov., Banfora, 20 Sep. 1958 (fl), s.n. 5239 (K!).

Ghana. Central: Ankaful near Cape Coast, in damp grassland, with Anadelphia leptocoma, 21 Sep. 1962 (fl), Hall 2350 (K!); Greater Accra: Achimota, in damp grassland, 8 Sep. 1971 (fl), Hall 43107 (K!); Volta: Mile $29 \mathrm{~S}$ of Adidome road, moist grassland, 7 Sep. 1967 (fl), Hall 37048 (K!); Western: Takoradi, in swamp, 7.5 m, Aug. 1941 (fl), Vogne 4785 (K!); Tossekre, 15 Oct. 1956 (fl), Adam 12745 (K!); Tat and Gabon, Ashanti, at edges of nice swamp, Sep. 1954 (fl), Westwood 151 (K!). Nigeria. Bauchi: Northern Prov., Nom, Bauchi Plateau, 900-1350 m, Joung s.n. (K!); Northern Prov., Bauchi Prov., Lame Distr., Jawando, in rich black soil on rocky outcrop where surface water plentiful at this season, growing where grass was about a foot high, 960 m, 17 Aug. 1954 (fl), Summerhayes 21 (K!); Cross River: Obudu, SE State, grassland, $1400 \mathrm{~m}$, 6 Oct. 1975 (fl), Segerbade 1170 (K!); Eastern Prov., Ogoja Prov., Apkpo, 120 m, 4 Aug. 1955, Stone 33 (K!); With grass, on inselberg, 28 July 1968 (fl), Sanford 5109 (K!); Kaduna: Parakwai Hills, in open grassy seasonal swamp on top of inselberg, alt $800 \mathrm{~m}$ and also found on Mt. Kujama, 1000 m, 14 Aug. 1977 (fl), Sogerback 1291 (K!); Lagos: Colony Prov., Badagri Distr., other side of Badagri between the Lagoon and the sea, on white sands in Coconut Plantation, exposed to sea winds, 13 Aug. 1954 (fl), Onochie s.n. (FHI 33476) (K!); Oyo: Ibadan Distr., mile 119, rock hill on Oyo Road amongst grass, 26 July 1952 (fl), Savory 191 (K!); Plateau: Northern Prov., Jos Plateau, 1946 (fl), Batten-Poole 309 (K!); Jos, inselberg near Rest House, in grasses in moist soil in crevices between the granitic boulders, 15 Aug. 1946 (fl), Keay s.n. (FHI 20175) (K!); Northern Prov., Jos, 1290 m, Aug. 1950 (fl), King 47 (K!); Northern Prov., between Mongau and Kasima, S edge of Plateau, in grassland in valley in deep side, 900 m, 23 Sep. 1962 (fl), King 189 (K!); Northern Prov., Temaa, Kiumi, in grass at edge of granite, $450 \mathrm{~m}$, 30 Aug. 1955 (fl), Killiak 42 (K!); Kiumi F.R., near Jos, shallow damp ground on granite rock slopes, in open areas, abundant on SW facing slopes, $1200 \mathrm{~m}$, 24 July 1962 (fl), Lawlor \& Hall 29 (K!); Northern Prov., Panshanu Pass, ephemeral association on pocket of moist granite-derived soil on granite outcrop, $840 \mathrm{~m}, 20 \mathrm{Aug}$. 1962 (fl), Lawlor \& Hall 470 (K!); Northern Prov., Richa, Jos Plateau, grassland on ridge between 2 valleys, soil granitic, well drained, more or less dry at time, 1200 m, 8 Sep. 1962 (fl), Lawlor \& Hall 660 (K!); Northern Prov., Naraguta, 8 Aug. 1921 (fl), Lely 508 (K!); Northern Prov., on shallow wet rock soils, July 1930 (fl), Lely 1512 (K!); Northern Prov., 19 Aug. 1912 (fl), Nelson 16 (K!); Northern Prov., Jos Plateau, amongest grass dumps on rocks, 1200 m, 23 Aug. 1961 (fl), Westwood 254 (K!); Northern Prov., at foot of Mada Hills, 300 m, Sep. 1936 (fl), s.n. 152 (K!); Taraba: Sardauna Prov., Mambilla Plateau, in temporarily water logged shallow, peat ground on the edge of rocks, by Vet. rest house. L.I.B.C. Gembu, 1500 m, 28 July 1973 (fl), Chapman 18 (FHI 70818) (K!); Northern Prov., Mambila Plateau, Gembu, in shallow pocket of soil over rock, 1650 m, 5 July 1958 (fl), Chapman 1821 (K!); Igbooshere, open, wet land, in short grass, 10 Aug. 1952 (fl), Savory 267 (K!). Cameroon. Adamawa: Env. Meiganga, 1100 m, 13 Sep. 1967 (fl), Jacques-Felix 8073 (P!); Sine loc., Bates s.n. (BM!); E of Hossere Ngo, $80 \mathrm{~km}$ NW of Tibati, savannah, gneissoil, 9 Sep. 1962 (fl), Letouzey 5662 (K!); Pres Nianbaka, 5 km NNW Meiganga, 29 Sep. 1963 (fl), Letouzey 6041 (K!, P!); Central: Zabondo, $25 \mathrm{~km}$ NE de Linte, 30 Sep. 1966 (fl), Letouzey 8060 (P!); East: Mountain Nangue, Ngaoundere at Meiganga, 18 Sep. 1967 (fl), JacquesFelix 8212 (P!); Northwest: Bamenda, Wum, 1300 m, June 1931 (fl), Maitland 1640 (K!); Bamenda, Wum, 1300 m, June 1931 (fl), Maitland s.n. (K!); Piste EssuMunkep, near Kunyang, 90 km N Wum, granite, 11001200 m, 6 July 1975 (fl), Letouzey 1359 (K!); Lake Benakuma, 15 km W Wum, 900 m, 5 Aug. 1975 (fl), Letouzey 14177 (K!, P!); West: Pays Bamoun, Fou, Ban Fionmot, mountains, 18 Aug. 1970 (fl), CNAD 1909 (P!); Dschang, Baleveng, 15 Aug. 1970 (fl), CNAD 1969 (P!). Mango, 7 km S Poli, 24 July 1974 (fl), Fotius 2204 (P!); Top of Paix Montain, foothill of Nlonkou Mt., $4^{\circ} 55^{\prime} \mathrm{N}, 9^{\circ} 58^{\prime} \mathrm{E}, 1000 \mathrm{~m}, 14$ Sep. 1971 (fl), Leeuwenberg 8377 (K!, WAG!). Dschang, 1300-1400 m, 15-30 Aug. 1970 (fl), Mihinton 1969 (K!, P!); Dschang, 13001400 m, 15-30 Aug. 1970 (fl), Mihinton 2006 (K!, P!); Foumban-Foumbot, Montains, 16 Aug. 1970 (fl), Moussavou 1909 (K!, P!); Dschang, Baleveng, $1300 \mathrm{~m}$, 15 Aug. 1970 (fl), Moussavou 1969 (K!); Sine loc., 1891 (fl), Dusen s.n. (AMES!, UGDA-DLSz! - drawing). Central African Republic. Haute-Kotto: ManovoGounda-St. Floris National Park, Pende Creek, 14 km $\mathrm{S}$ of Koumbala confluence, $8^{\circ} 19^{\prime} \mathrm{N}, 21^{\circ} 14^{\prime} \mathrm{E}, 600 \mathrm{~m}$, 13 May 1984 (fl), Fay 6604 (K!); Manovo-Gounda-St. Floris National Park, Pende Creek, 14 km S of Koumbala confluence, $8^{\circ} 19^{\prime} \mathrm{N}, 21^{\circ} 14^{\prime} \mathrm{E}, 600 \mathrm{~m}, 13$ May 1984 (fl), Fay 6629 (K!); Pende Creek, 14 km S of Koumbala, moist meadow adjoming creek, hydromorphic, organically enriched loam, 600 m, 17 May 1984 (fl), Fay 6690 (K!); Yalinga region, 70 km W Yalinga, 1 June 1922 (fl), Le Testu 3923 (BM!, P!); Lobaye: Boukoko, 
around Ambenge River. 30 km N Bambari, 2 Aug. 1921 (fl), Tisserant 348 (P!); Boukoko, around Bangila, 1 July 1922 (fl), Tisserant 920 (P!); Boukoko, Plateau near Gumbum, Wamire, 7 Aug. 1924 (fl), Tisserant 1724 (P!); Nana-Grébizi: Oubangui-Chari, near Lakere, Yakete forest near Crampel Fort, Sep. 1857 (fl), Koechlin 4715 (P!); Ouaka: Oubangui-Chari, Plateau near R. Gumburu, $60 \mathrm{~km}$ N of Bambari, on laterite, 7 Aug. 1924 (fl), Falter \& Tisserant 1525 (K!); Oubangui, Bambari Region, Village Bangila, $30 \mathrm{~km}$ S Moronbas, Tisserant 403 (P!); Oubangui, Bambari Region, Village Bangila, 30 km S Moronbas, 1 Sep. 1922 (fl), Tisserant 636 (BM!, P!); Baidou River, 30 km S of Ippy, between Bambari and Bria, May 1925 (fl), (Cribb \& Fay 1986), Tisserant 1508 (P!); Plateau near Gumbum River, 60 km $\mathrm{N}$ of Bambari, 7 Aug. 1924 (fl), (Cribb \& Fay 1986), Tisserant 1525 (P!); Oubangui. Bambari Region, Plateau near Gumbum River, $60 \mathrm{~km} \mathrm{~N}$ Bambari, 7 Aug. 1924 (fl), Tisserant 1579 (P!); Oubangui, Bambari Region, near Bayedou, 30 km S Ippy, 10 Apr. 1924 (fl), Tisserant 1908 (P!); Ouham-Pendé: Oubangui-Chari, Bozoum, in savannah, $6^{\circ} 5^{\prime} \mathrm{N}, 16^{\circ} 5^{\prime} \mathrm{E}, 8$ Oct. 1931 (fl), Falter \& Tisserant 2935 (K!); Oubnagui, Bozoum, 8 Oct. 1931 (fl), Tisserant 2935 (BM!, K!, P!). Ethiopia. Addis Ababa: By Gibie River road bridge, grassland, thin soil, 1080 m, 11 Sep. 1962 (fl), Thomas 92 (K!); Kaffra, near Abalti, in moist grassland on reddish clay, $8^{\circ} 10^{\prime} \mathrm{N}, 37^{\circ} 34^{\prime} \mathrm{E}, 1900 \mathrm{~m}, 11$ Aug. 1962 (fl), Mooney 9265 (K!); About $20 \mathrm{~km}$ E of Lekemti, rocky, grassy hill slope, with brown loamy soil, 2000 m, 1 July 1965 (fl), De Wilde 7167 (C!, K!); About $200 \mathrm{~km} \mathrm{SW}$ of Addis Ababa, along road to Jimma, open, grassy place in open woodland, 2000 m, 9 Aug. 1965 (fl), De Wilde 7504 (K!); Shoa Prov., Square 58.D, on west bank of river Sibelo, naer Mulu Farm, in damp grassland, on black soil over volcanic rocks, 2100 m, 11 Sep. 1974 (fl), Ash 2600 (K!); Shoa Prov., Solulta. Square 58.D, on Gondar road, $25 \mathrm{~km}$ north of Addis Ababa, quite frequent in grass in drier parts of annually flooded grassland, 2500 m, 26 July 1975 (fl), Ash 3129 (K!); Shoa Prov., Square 70C, Koka Dam lake, west shore near Awash bridge, in rough grass, in clearning below Acacia woodland, alluvial tuff deposits of volcanic origin, $08^{\circ} 35^{\prime} \mathrm{N}, 39^{\circ} 02^{\prime} \mathrm{E}, 1450 \mathrm{~m}, 6$ Sep. 1976 (fl), Ash 3654 (K!); Gojam Prov., 3 km E of Giga, 74 km NW of Debra marcos along road to Dangila, along rocks, $30 \mathrm{~cm}$ above stream, 9 Aug. 1961 (fl), Burger 510 (K!); $40 \mathrm{~km}$ S of Lake Tana, Dangila, $2010 \mathrm{~m}, 5$ Sep. 26 (fl), Cheesman s.n. (BM!); Shoa Prov., Akaki, occasional in wet boulder strewn thorn savannah in black cotton soil, 2250 m, 6 Sep. 1952 (fl), Curle \& Schelpe 12 (BM!); S Shoa, Modjo, 1700 m, Aug. 1958 (fl), Hildebrandt 175 (WU!); Shoa, near Wolkitte, in open rough pature on heavy black clay, $8^{\circ} 15^{\prime} \mathrm{N}, 37^{\circ} 50^{\prime} \mathrm{E}, 1900 \mathrm{~m}, 11$ Aug. 1962 (fl), Mooney 9169 (K!); Amhara: Begemder,
Bahir Dahr. S end of Lake Tanga, marsh with several $\mathrm{cm}$ of water at tme of collection, dry at other times, many ssp, of grasses and sedges, $37^{\circ} 25^{\prime} \mathrm{N}, 11^{\circ} 31^{\prime} \mathrm{E}$, 1800 m, 6 Sep. 1973 (fl), Gilbert \& Getachew 3044 (K!); Gonder, Hericourt s.n. (P!); Begemder Prov. $10 \mathrm{~km}$ from Gonder, on road to Bahar Dar, wet meadow with open Eucalyptus plantation, 27 Sep. 1969 (fl), De Wilde \& Gilbert 272 (K!); Nr. Ejubi, 15 miles south of Debra Marcos, Gojjam, grazed bog, drying out in summer, 2100 m, 7 Sep. 1957 (fl), Hillier 905 (K!); Oromiya: About $10 \mathrm{~km}$ WSW of Jimma, along road to Bonga, open, grassy place on hill slope, $2000 \mathrm{~m}, 10 \mathrm{Aug}$. 1965 (fl), De Wilde 7552 (K!); Kaffa Prov., about 7 km along road Jimma to Bonga, gentle sloping open country with rather tall grasses, partly planted with young Eucalyptus, fertile, brovmish-red, rather humid soil, 7'37’N, 3645'E, 1600 m, 30 June 1969 (fl), De Wilde s.n. (K!); Sidamo, $1 \mathrm{~km} N$ of Harek'elo, along the road to Kebre Mengist, rocky outcrops with vegetation of Terminalia brownii, Acacia, Commiphora and Sesamothamnus rivae, in wet flushes, 1450 m, 24 May 1982 (fl), Friis, Tadesse \& Vollessen 3283 (C!, K!); Bahar Dar, Erdboden, 1800 m, Oct. 1967 (fl), Schuz Al (K!); Tigray: Chire Prov., Quartin-Dillon \& Petit 106 (P!, W-R!); Chire Prov., Quartin-Dillon \& Petit s.n. (P!, W-R!); Plowden s.n. (K!); "Tigre vers Begemder", Schimper 1378 (W-R!); 19 Aug. 1862 (fl), Schimper 2703 (BM!); About $5 \mathrm{~km} \mathrm{NW}$ of Makalle, passing the airport, treeless plain, partly cultivated with cerials (Barley, etc.), heavy brownish-black soil, $2050 \mathrm{~m}$, 6 Sep. 1970 (fl), De Wilde 6998 (K!); 34 km S of Enda Selassie, along the road to Gondar, rather flat plain with scattered shrub and treegray growth (Acacia, Ficus, etc.), in this season rather wet, heavy clayish soil, grasses and herbs forming the dominant vegetation, 1700 m, 11 Sep. 1970 (fl), De Wilde 7092 (K!). Woina, Schimper 596 (P!, W-R!). Heberok-Mettgelo, Schimper III no. 703 (HBG!)."Prope Dschomara", Schimper 1210; Aoderbati, Quartin-Dillon \& Petit s.n. (P!);

Republic of Equatorial Guinea. Río Muni, WeleNzas: Inselberg Ossap Anda, Region d'Oyem, 1'38'N, 11'37'78,8'E, 750 m, 11 Jan. 2003 (fl), Deman 45 (BRLU!); Inselberg Dumu, near the village Dumu, $1^{\circ} 22,2^{\prime}$ N, 11 19,19'E, 790 m, 6 June 2002, Parmentier \& Esomo 3636 (BRLU!); Inselberg d'Asoc, along road to village Asoc, a $15 \mathrm{~km}$ of Mongomo, $1^{\circ} 27^{\prime} \mathrm{N}, 11^{\circ} 20^{\prime} \mathrm{E}$, 790 m, 5 June 2002 (fl), Parmentier \& Esomo 692 (BRLU!). Gabon. Sine loc., Duparquet s.n. (P!); Estuaire: Cape Esterias, Dec. 1934 (fl), Klein 12b (P!); Cape Esterias, Dec. 1934 (fl), Klein 13 (P!); Plains, Dec. 1894 (fl), Leroy 30 (P!); Near Cape Esterias, $0^{\circ} 33^{\prime} \mathrm{N}$, 9²1'E, Dec. (fl), Reitsma J. M. \& B. 2758 (AMES!, LBV not seen, WAG!); Libreville, Dec. (fl), Schlechter 12824 (BM!, BR!, K! (the type of H. stenoloba)); Libreville, Dec. 1891 (fl), Thollon 163 (P!); Libreville, Dec. 
1891 (fl), Thollon 195 (P!); Libreville, Dec. 1891 (fl), Thollon 4069 (P!); Libreville, Dec. 1891 (fl), Thollon 4070 (P!); Sine loc., Thollon s.n. (AMES!); Near Libreville, 19 Dec. 1900 (fl), Klaine 2057 (P!); Near Libreville, 19 Dec. 1900 (fl), Klaine 2058 (P!); Sibang Farm, 11 Dec. 1881 (fl), Soyaux 346 (B+, BM!); Ngounié: About $1 \mathrm{~km} \mathrm{~N}$ of Doussala, $2^{\circ} 18^{\prime} \mathrm{S}, 10^{\circ} 35^{\prime} \mathrm{E}$, Dec. (fl), De Wilde, Arends \& de Bruijn 9160 (LBV, WAG!); Ogooué-Maritime: Near Ivinga, 2`50'S, 1006'E, Dec. (fl), van Nek 518 (LBV not seen, WAG!); Haut-Ogooue, 3,9 km from camp, savannah, 2'4'49'S, 14'03'50'E, 400 m, 30 Jan. 2004 (fl), Niangadouma \& Stone 409 (K!); Haute Ngounye, Echira, 17 Nov. 1924 (fl), Le Testu 5061 (K!, P!); Woleu-Ntem: Mount Miwa, Inselberg of $3 \mathrm{~km}$ from village of Kumassi, of $35 \mathrm{~km}$ from d'Oyem and Bitam, 0'50'N, 11'38'E, 29 Dec. 1999 (fl), Parmentier \& Nguema 548 (BRLU!); Coss, Bengo, 12 May 1933 (fl), Le Testu 9120 (BM!, K!, P!); Sine loc., De Wilde, Arends \& de Bruijn s.n. (BR!, P!); Leuga, Dec. 1908 (fl), Le Testu 1524 (BM!, BR!, P!); Leuga, Dec. 1908 (fl), Le Testu 1524a (BM!, BR!, P!). Congo. Kouilou: Savannah $18 \mathrm{~km}$ NW Dimonika, Mayombe, 4 Jan. 1969 (fl), Attims 20 (P!); Pool: Between Ngoma and Nyagezi, 3 Feb. 1927 (fl), Linder 2016 (K!); Plateaux: Kebara forest, hillside of Koukouya Plateau, 720 m, 5 Jan. 1970 (fl), Makany 1383 (P!); Plateau Koukouya, Gonbukila and Kebara, 720 m, 5 Jan. 1970 (fl), Makany 1383(!) (P!); "Congo. Gabon”, 1882 (fl), Thollon 12 (BM!, P!). Democratic Republic of the Congo (Zaire). Haut-Lomami: Lomami, Ditu, Dandon 112 (BRLU!); Gangala na Bodio (pres Faradge), savannah, 31 Aug. 1932 (fl), Hauman 489 (BRLU!); Ituri, env. $5 \mathrm{~km}$ of Bunia, road to Kosenyi, 6 Aug. 1975 (fl), Lisowski 41300 (UGDA-DLSz!); In vleis, 11 Jan. 1924 (fl), Heischberg 176 (K!); Marungu, Lake Buzanza, 1850 m, 20 Feb. 1970 (fl), Lisowski 10288 (UGDA-DLSz!); Marungu, Lake Buzanza, 20 Feb. 1970 (fl), Lisowski 10319 (UGDA-DLSz!); About $40 \mathrm{~km}$ East of Niangara on Dungu road, short grassland (with many sedges), on shallow soil on ironstone carapace, 30 July 1956 (fl), Keay 28 (AMES!, K!); Kinkele, 15 Dec. 1985 (fl), Kiss 35 (BR!, P!); Haut-Katanga: Ituri, Env. de Nduye, Mt. Mukonza, 8 Aug. 1975 (fl), Lisowski 40660 (UGDA-DLSz!); Katanga, Elisabethville, in some of marshes around Elisabethville, 1400 m, Jan. 1939 (fl), Russell 72 (K!); Haut-Uele: Amadi-Sbele, Sep. 1906 (fl), Seret 656 (AMES!, K!); Road between Gabu-Golu (Riv. Shari), Terr. Nioka, granitie, 1800 m, 12 Sep. 1947 (fl), Taton 594 (K!); Kinshasa: Kimuenza, waterside of the river Lukaya, 4'24'S, 1516'E, 12 Dec. 1982 (fl), Lejoly 82/1015 (BRLU!); Haut-Zaire: $17 \mathrm{~km}$ of BagbeleDungu, 800 m, 18 July 1950 (fl), Noirfalise 663 (AMES!, K!, UGDA-DLSz! - drawing) (as H. genuflexa); Kongo Central: Mbanza-Ngungu, 8 Jan. 1980 (fl), Lisowski 56051 (UGDA-DLSz!); The same locality, Lisowski 56856 (UGDA-DLSz!). Uganda. Central: Mpigi Distr., Kitala, 1800 m, Aug. 1931 (fl), Blake 2577 (K!); Raknda, Razhumbma, Kigezi, short grassland, 1500 m, Mar. 1946 (fl), Purseglove 2224 (K!); Eastern: Iganga Distr., Bunyiro, in Miscanthidium swamp at Mile 53 Masindi-Port Butiaba Road, July 1936 (fl), Eggeling 3054 (K!); Western: Bushenyi Distr., Makota Distr, 1170 m, Feb. 1905 (fl), Brown 142 (K!); Bushenyi Distr., near Isbcka, roadside between Iskcka and Ankole Estate, Fara laslitute, dry grassland - black soil, 17 Dec. 1994 (fl), Spurrier V.30 (K!); Kesese Distr., Ruwenzori, R. Ruimi (Wimi), Scott Elliot 7922 (BM!, K!, P!); 1650 m, 1913 (fl), Snowden 91 (K!); Bushenyi Distr., Ankole, Shema county at Kitakate, savannah on hill side, 1500 m, 24 Jan. 1929 (fl), Snowden 1275 (BM!, K!); Bushenyi Distr., Ankole, Shema country of Bugongi, in short grass savannah, fairly frequent, 1500 m, 17 Feb. 1930 (fl), Snowden 1649 (BM!, K!). Kenya. Eastern: Songet Forest Station, in damp grass glade in forest, on black-grey ill and clay, $0^{\circ} 2$ '20'S, 353'ㄹ, 2250 m, 8 Sep. 1952 (fl), Dyson 374 (K!); Nyanza: Londiani Distr., Londiani, open, soggy grassland, 2300 m, 18 Aug. 1949 (fl), Maas Geesteranus 5854 (F!, K!); Londiani, Rainfall 43", rainy season, in open grassland, generaly badly drained and boggy in rains, 2250 m, 15 July 1951 (fl), Davis 11 (BM!, K!); Forestry Department, Londiani, in open grassland, June 1932 (fl), Graham 2820 (901) (K!); Londiani, 2400 m, 3 Aug. 1956 (fl), Williams 11082 (K!); Rift Valley: Cherangani Hills, swamp near Kiptaberr Forest, swamp with Lobelia, Cyperus and Helichrysum, $1^{\circ} 70^{\prime} \mathrm{N}$, $35^{\circ} 20^{\prime} \mathrm{E}, 2550 \mathrm{~m}, 6$ July 1969 (fl), Mabberley \& McCall 3 (K!); Eldoret, 1800 m, June 1931 (fl), Harvey 1366 (K!); Forestry Department, Kinankop Plateau, grassland, 2550 m, June 1932 (fl), Dale 2846 (K!); K3 Transnzoia, Kitale Prison Farm, 5 km S Kitale, vlei with perennial grasses and sedges and many orchid species, Sristea, etc., 0 $0^{\circ} 5^{\prime} \mathrm{N}, 35^{\circ} 00^{\prime} \mathrm{E}, 1800 \mathrm{~m}, 8$ May 1971 (fl), Mabberley \& Tweedie 1120 (K!); Mabounde near Kitale, solitavly plant on heading of cultivation which must have been plonghed this year, $1830 \mathrm{~m}$, Aug. 1955 (fl), Tweedie 1349 (K!); Kitale, near Eldoret Trans Nzoia, marsh, low lying ground between two streams, open but growing amongst tall water-grass, $1860 \mathrm{~m}$, 29 June 1950 (fl), Wiltshire 5 (K!). Burundi. Bubanza: Rugazi, with Eragrostis olivacea, 3'14'S, 29'31'E, 1700 m, Mar. 1981 (fl), Reekmans 9818 (K!); Cibitoke: Rwagarika region, Ruzizi plain, savannah, Mar. 1950 (fl), Germain $6531 A$ (K!). Tanzania. T4. Iringa: Iringa Distr., Iringa town, at pools in eroded landscape, $7^{\circ} 6^{\prime} \mathrm{S}, 35^{\circ} 42^{\prime} \mathrm{E}$, 1575 m, Pettersson, Hedren \& Kibuwa 16 (K!); Iringa, Sao Hill Dambo, seasonally swamp, black soil, fairly long grass, 1800 m, 8 Jan. 1989 (fl), Spurrier 30 (K!); Dabaga Highland, Kibengu, 18 miles S of Dabaga, 
marshy straemside in upland grassland, $1920 \mathrm{~m}, 20 \mathrm{Feb}$. 1962 (fl), Polhill \& Paulo 1535 (K!); Southern Hilllands, Mufindi, Ngwazi, black soil in swamp above Ngwazi Dam, 1800 m, 2 Jan. 1989 (fl), Keeley 98 (K!); Mufindi Distr., near Sao Hill on the Iringa-Mbeya road, $90 \mathrm{~km} \mathrm{~W}$ Iringa, grassland close to wetland, $8^{\circ} 3$ 'S, 352'E, 1800 m, 1 Feb. 1997 (fl), Lewis 1393 (K!); Mufindi Distr., Ngwazi swamp, wet meadow grassland, $08^{\circ} 30^{\prime}$ 'S , 35 $15^{\circ}$ 'E, 1830 m, 27 Dec. 1986 (fl), Lovett 1188 (K!); Mufindi, wet meadow grassland at top end of lake Ngwazi, 08³1'S, 35¹0'E, 1830 m, 14 Dec. 1987 (fl), Lovett, Duncan \& Thomas 2817 (K!); Ruaha National Park, Magangwe R.P. at Isiki River, Combretum obovatum - thicket, 1320 m, 10 Mar. 1972 (fl), Bjornstad 1510 (K!); Kagera: Minziro Forest Reserve, W of Minziro Village along footpath to Mtukula in grassland at margin of small forest called Kabarokola, in grassland, 01'02’23“'S, 31'30’39”'E, 1130 m, 16 Mar. 2001 (fl), Festo \& Francis 1033 (K!); Kahororo Bukoba Distr, 1200 m, Feb. 1932 (fl), Haarer 2502 (K!); West Lake Prov., Muganza, Bushubi, Ngara, on grass, 1500 m, 25 Mar. 1960 (fl), Tanner 4798 (K!); Mbeya: T7. Southern Prov., Ngolezi, 1500 m, 16 Feb. 1970 (fl), Leedal 74 (K!); Between Murwa and Pungaluma hills, $2 \mathrm{~km}$ from Murwa, Acacia woodland, $8^{\circ} 50^{\prime} \mathrm{S}, 33^{\circ} 20^{\prime} \mathrm{E}$, 1200 m, 24 Feb. 1990 (fl), Lovett \& Kayombo 4243 (E!, K!); Mt. Mbeya, open grassland, 1200 m, 16 Mar. 1938 (fl), MacInnes 162 (BM!); Rukwa: Nkansi Distr., 8 km on Namanyere - Chala road, wet valley grassland on dark grey sandy loam, $7^{\circ} 32^{\prime}$ 'S, 31 $06^{\circ} \mathrm{E}, 1600 \mathrm{~m}, 3$ Mar. 1994 (fl), Bidgood, Mbago \& Vollesen 2582 (K!); T7. Ufipa Distr., Lake Kwela, low lying ill-drained soil near the lake, 1560 m, 19 Mar. 1950 (fl), Bullock 2669 (AMES!, K!); Ufipa Distr., Ufipa Plateau, Namakanga, 10 km west of Kaluko, on Tatanda dambo, 7 Feb. 1987 (fl), Moyer \& Sunane 190 (AMES!, K!, MO!); Ufipa Distr., Ufipa Plateau, Small dambo $1 \mathrm{~km}$ south of the Kanyere River on the main road to Sumbawanga, in a wet dambo, 28 Feb. 1987 (fl), Moyer \& Sunane 211 (AMES!, K!, MO!); Sumbawanga Distr., Ufipa, Malonje Plateau, in rough, rather swampy ground, $1800 \mathrm{~m}$, 15 Mar. 1957 (fl), Richards 8739 (K!); Ufipa Distr., Lake Kwela, in damp grassland, 1650 m, 11 Mar. 1959 (fl), Richards 11133 (K!); Ufipa Distr., Molo Village, Malonje Plateau, in wet grassland, 1800 m, 1 Jan. 1962 (fl), Richards 15852 (K!); Singida: Manyoni Distr., Kazikazi, W of Kimatinde, seasonally wet bog, 1260 m, 14 Jan. 1933 (fl), Burtt 4625 (K!). Angola. Bié: Cangengo Distr., 1903 (fl), Gossweiler 560 (K!, P!). Zambia. Central: Mkushi Distr., Mkushi River Hotel, from fairly dry dambo-occasional specimens only, 5 Jan. 1961 (fl), Holmes 0269 (K!); Serenje, wet swamp, Dec. 1966 (fl), Odgers 187 (K!); Kali Dambo, in wet bog, $1500 \mathrm{~m}$, 26 Jan. 1952 (fl), Richards 783 (K!); Eastern: Fort Jamenson Distr., 25 miles $\mathrm{N}$ of Fort Jamenson, black swamp soil, 1200 m, 14 Feb. 1959 (fl), King 463 (K!); Lusaka: Lusaka West, Nosark's farm, seasonally flooded grassland over dolomite, 15'24'3'S, $28^{\circ} 08^{\prime} 6^{\prime}$ 'E, 1200 m, 25 Feb. 2000 (fb), Bingham \& Peters 12132 (K!); 30 miles south of Lusaka, in dambo, Feb. 1967 (fl), Williamson 99 (K!); 30 miles south of Lusaka, in wet dambo, Feb. 1967 (fl), Williamson 257 (K!); Lusaka, University dambo, Feb. 1970 (fl), Williamson 1853 (K!); Kabwe, in dambo, Feb. 1969 (fl), Williamson 2055 (K!); Lusaka Distr., 43 km E of Lusaka, 9 Feb. 1975 (fl), Williamson \& Gassner 2376 (K!); Northern: Abercorn Distr., pans near Abercorn, in very damp peaty ground in pans, in standing water, $1500 \mathrm{~m}, 28 \mathrm{Feb} .1952$ (fl), Richards 888 (K!); Abercorn Distr., Ningi Pans, in wet marsh among long grasses, 1650 m, 12 Feb. 1960 (fl), Richards 12461 (AMES!, K!); 6 miles from Kasama on the Isoka Road, dambo, marshy ground, $1260 \mathrm{~m}$, Jan. 1955 (fl), Benson 106 (BM!); Marsh Lumi River, in wet marsh among long grass, $1500 \mathrm{~m}, 9$ Feb. 1955 (fl), Richards 4401 (K!); Mbala Distr., Zembe Plain, valley grassland, wet swamp, long grass, $1500 \mathrm{~m}$, 20 Jan. 1968 (fl), Richards 22939 (K!); Kasama Distr., $10 \mathrm{~km}$ E of Kasama, parenailly wet dambo, $11 \mathrm{Dec}$. 1960 (fl), Richards 4166 (K!); Kasama Distr., 10 km E of Kasama, in dambo, 4 Feb. 1961 (fl), Richards 4355 (K!); Kipoma Falls, Dec. 1967 (fl), Williamson 686 (K!); North-western: Solwezi Distr., 95 km E of Solwezi/ Mwinilunga road, near the Kambizhi Primaty School, Jirundu National Forestry Reserve (Botanical Reserve), rock outcrop in Jirundu plains/swamp, 11 '54'45"'S, 2529'10”'E, 1470 m, 17 Feb. 1995 (fl), Luwiika, Harder, Zimba \& Nawa 76 (K!); Mwinilunga Distr., Kalenda Dambo, in boggy grassland overlying "laterite", 1 Jan. 1938 (fl), Milne-Redhead 3907 (K!); Southern: Kalomo Distr., between Ghoma and Musuka, wet dambo, 1050 m, 14 Mar. 1962 (fl), Astle 1560 (K!); Mapanza, Siamasundu, dambo, 1050 m, 21 Feb. 1957 (fl), Richards 2136 (AMES!, K!, UGDA-DLSz! - fragment, drawing); Mazabuka, west of Monze African Township, very wet dambo (some roots actually in the water) in the area of the Miangama Stream, 8 Feb. 1963 (fl), Linley 314 (K!); Muckle Neuk, $12 \mathrm{~m} \mathrm{~N}$ of Choma, dambo, 1230 m, 14 Jan. 1954 (fl), Richards 462 (K!); Shiri Highlands, Dec. 1881 (fl), Buchanan 302 (K!). Malawi. Central: Dedza Mt., lower slopes of Dedza Mt., in marshy grassland, 1650 m, 24 Mar. 1972 (fl), Westwood 657 (K!); Lilongwe Distr., Bumba College, dambo grassland, 1170 m, 7 Feb. 1974 (fl), Allen 489 (K!); Lilongwe Distr., Dzalanyama Forest Reserve, few $\mathrm{km} \mathrm{S}$ of ranch house, sandy soil between rocks on stream banks on hill slopes, 1280 m, 26 Mar. 1977 (fl), Brummitt, Seyani \& Patel 14949 (K!); Chongoni Dzedza Distr., in dambo, 1300 m, 24 Feb. 1984 (fl), la Croix 573 (K!); Came up in pot with Nervilia petraea, collected Malosa, in Brachystegia woodland, E-facing 
slope, 1050 m, 14 May 1984 (fl), la Croix 632 (K!); Damp clay dambo among grasses, 1070 m, 8 Feb. 1959 (fl), Robson 1512 (BM!, K!); Northern: Mzimba Distr., Enukweni, in dambo, 1100 m, 30 Jan. 1986 (fl), la Croix 788 (K!); Mzimba Distr., $8 \mathrm{~km} \mathrm{~N}$ of Mpherembe, flooded grassland, grwing in 3 inch. of water, 1150 m, 2 Mar. 1987 (fl), la Croix 994 (K!); Mzimba Distr., Mzuzu waterworks, in bog, 1350 m, 24 Jan. 1976 (fl), Pawek 10736 (K!); Mzimba Distr., 8 miles N of Mzambazi, 9 miles $\mathrm{S}$ of Mperembe, in wet bottom, mostly past blooming, $1260 \mathrm{~m}, 10$ Mar. 1978 (fl), Pawek 14007 (K!); Mzimba Distr., 11 11/2 m NW Chikangawa, grassland, 1695 m, 17 Jan. 1979 (fl), Phillips 4645 (K!); Mzimba, Mjinge dambo, 13 Feb. 1972 (fl), Westwood 605 (K!); Rumphi Distr., 11 miles N of Rumphi junct, 1 mile SE on Cilungiro rd., nearly dry clay grassy bottom, 1100 m, 26 Feb. 1978 (fl), Pawek 13917 (K!); Nyika Plateau, below Dam, bog 300 yds., grassland, 2250 m, 3 Feb. 1976 (fl), Phillips 1130 (K!); Nyika Plateau, Chelindi valley bog near leopard forest i.e.e. of airfield, 2250 m, 2 Apr. 1976 (fl), Phillips 1622 (K!); Near Compand Chirundi, seapage, Mar. (fl), Welsh 192 (K!); Southern: Machinga Distr., Kankhomba Village Mtila Habour, in a grassland, 7 Mar. 1985 (fl), Kwatha \& Patel 22 (K!); Bvumbure, in dambo, $1100 \mathrm{~m}, 22 \mathrm{Feb}$. 1981 (fl), la Croix 109 (K!); Zomba Distr., Zomba Plateau, Bhingues Hole, Blue Swollen Bridge, marsh, 23 Feb. 1978 (fl), Taylor 14 (K!); Zomba Mts., Research plots, open grassland, 18 Feb. (fl), Welsh 652 (K!). Zimbabwe. Harare: Matombo Distr., Farm Basha Kobila, grassland, 1440 m, Feb. 1957 (fl), Miller 4105 (K!); Mjumi Distr., Salisbury, in wet plains of vleis, 1350 m, 7 Mar. 1947 (fl), Wild 1868 (16211) (K!); Salisbury, 1500 m, Feb. 1919 (fl), Eyles 1477 (BM!); Salisbury, vlei, Feb. (fl), Eyles 6930 (K!); Salisbury, in vlei, 10 Feb. 1946 (fl), Greatrex 25 (K!); Salisbury, Bleveland Dam, on damp ground, 19 Feb. 1933 (fl), Greatrex 7287 (K!); Salisbury, Domboshawa, 1500 m, 6 Feb. 1958 (fl), Leach 82891 (K!); Salisbury, north road, 11 Feb. 1929 (fl), Young s.n. (AMES!, K!); Victoria, 1909 (fl), Monro 865 (BM!); The same locality, 1909 (fl), Monro 904 (BM!); The same locality, 1909 (fl), Monro 956 (BM!); The same locality, 1909-12 (fl), Monro 1753 (BM!); Vicotria, 1909-12, Young s.n. (BM!); Manicaland: Makonis Distr., Scape 18", Chimbi Farm, 1560 m, 14 Feb. 1960 (fl), Chase $7271 b$ (K!); Makonis Distr., 1800 m, 9 Feb. 1931 (fl), Norlindh \& Weimarck 4933 (K!); Rusape Distr., in marsh, 25 Jan. 1949 (fl), Munch 161 (22667) (K!); Rusape Distr., Dunedin, in marsh, 5 Feb. 1950 (fl), Monro 230 (K!); Umtali Distr., on moist bank of Mpanda River above new bridge to R.C. Mission, 900 m, 5 Apr. 1959 (fl), Chase 7078 (K!); Mashonaland East: Goromonzi Distr., Damusi, Bhidamora Reserve, in vlei, 26 Feb. 1967 (fl), Grosvenor 304 (K!); Marandellas, Digglefold, semi-wet vlei, 20 Jan. 1949 (fl), Corly 355 (22856) (K!); Marandellas Distr., about 3 miles NE of Marandellas on Amika Farm, in vleis round Marandellas, 28 Jan. 1970 (fl), Grosvenor 502 (K!); 30 July 1920 (fl), Hislop 34 (K!); Mrewa Distr., Uzumba Tribal Trust Land, Mazoe Valley, 11 Feb. 1969 (fl), Mavi 942 (K!); Mashonaland West: Urungwe National Park, $271 \mathrm{~km}$ from Salisbury on Chirundu road, open savannah woodland, 1190 m, 20 Feb. 1981 (fl), Philox, Leppard \& Dini 8797 (K!); Urungwe Distr., $5 \mathrm{~km} \mathrm{~N}$ of Mwami on Nyasha Farm, in wet vlei, 3 Apr. 1974 (fl), Stidolph 230,201 (K!); Lomagundi Distr., Ayrshire North, in vleis, 24 Feb. 1964 (fl), West 4740 (K!); Matabeleland North: Victoria Falls, 900 m, 30 Jan. 1934 (fl), Davies s.n. (BM!); Victoria Falls National Park, in the rain forest area, wooded grassland, black clay soil, $880 \mathrm{~m}$, 7 Feb. 1980 (fl), Ncube 74 (K!); Matoba Distr. No. 46486, Salisbury, 1410 m, Feb. 1954 (fl), Miller 2193 (F!). Republic of South Africa. Mpumalanga: Transvaal, Lydenburg Distr., Jan. 1895 (fl), Williams 1383 (WU!).

Notes. Bilabrella schimperiana is rather easily distinguishable species by its spur shape and connection between lip and basal part of the gynostemium.

$B$. schimperiana and $B$. genuflexa differs from one another in the length of pedicel and ovary and length of anterior petal lobes. Szlachetko et al. (2010) followed Summerhayes (1968b) proposal to keep both species separately. Studying specimens of the above mentioned taxa from the wide range, we came to the conclusions that both are conspecific, as there are no discontinuum in the diagnostic features aforementioned and follow in this viewpoint of Gerrinck (1984).

Hildebrandt 175 at $\mathrm{K}$ is an abnormal form.

42. Bilabrella cataphysema (Rchb. f.) Szlach. \& KrasLap., Richardiana 3(3): 139. 2003. - Szlachetko et al., Orchid. of West-Central Africa 1: 221. 2010.

Habenaria cataphysema Rchb. f., Flora 50: 101. 1867.Rolfe, Fl. Trop. Afr. 7: 237. 1898. - Kraenzlin, Orchid. Gen. Sp.: 217. 1901. - Williamson, Orchid. S. Centr. Africa: 56. 1977. - Geerinck, Fl. Afr. Centr., Orchid. 1: 115. 1984. - la Croix \& Cribb, Fl. Zambes., Orchid. 11: 89. 1995.

Type (Szlachetko et al. 2010): Angola, Welwitsch 722 (lectotype, designated by Szlachetko et al. [2010: 221], BM!, K! - fragment) \& Angola, Welwitsch 743 (paratype, $\mathrm{BM}$ ! - fragment, $\mathrm{K}$ ! - fragment, $\mathrm{W} !)$.

Tubers 1-1.5 $\mathrm{cm}$ long, $1 \mathrm{~cm}$ in diameter, globose to ovoid. Stem 30-80(175) cm tall, erect, relatively delicate to rather stout, glabrous. Leaves $7-9,5.5-13.5 \mathrm{~cm}$ long, $0.4-0.8 \mathrm{~cm}$ wide, linear-lanceolate, acute, slightly deflexed from the stem, decreasing in size upwards. Inflorescence 11-23 cm long, many-flowered, rather 


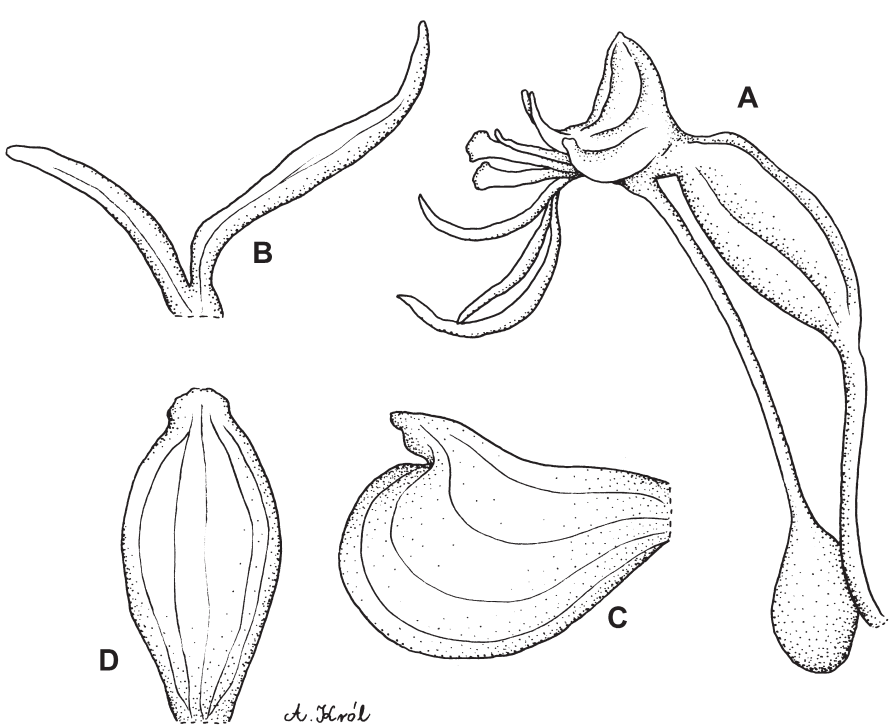

Fig. 126. Bilabrella cataphysema (Rchb. f.) Szlach. \& Kras-Lap. Explanations: A - pedicel, ovary, gynostemium, lip and spur; B - petal; C - lateral sepal; D - dorsal sepal (drawn by A. Król from lectotype of Habenaria cataphysema Rchb. f. - Welwitsch 722, K)

dense. Flowers pale green. Floral bracts 7-10 $\mathrm{mm}$ long, lanceolate, acuminate, glabrous. Pedicel and ovary 16$18 \mathrm{~mm}$ long, glabrous. Dorsal sepal 2.5-4 mm long, $2 \mathrm{~mm}$ wide, oblong obovate, obtuse, cochleate, deflexed. Petals bilobed almost to the base, shortly papillose; anterior lobe 5-7 $\mathrm{mm}$ long, $0.5-0.8 \mathrm{~mm}$ wide, filiform, acute; posterior lobe 3-4 $\mathrm{mm}$ long, 0.3-0.4 mm wide, filiform, obtuse. Lateral sepals 5-6 mm long, 3-4 mm wide, obliquely obovate, concave, glabrous, deflexed, laterally apiculate. Lip 3-lobed above the short basal part $1 \mathrm{~mm}$ long; the middle lobe $6-6.5 \mathrm{~mm}$ long, $0.5 \mathrm{~mm}$ wide, filiform; lateral lobes 3.3-4.2 mm long, 0.2-

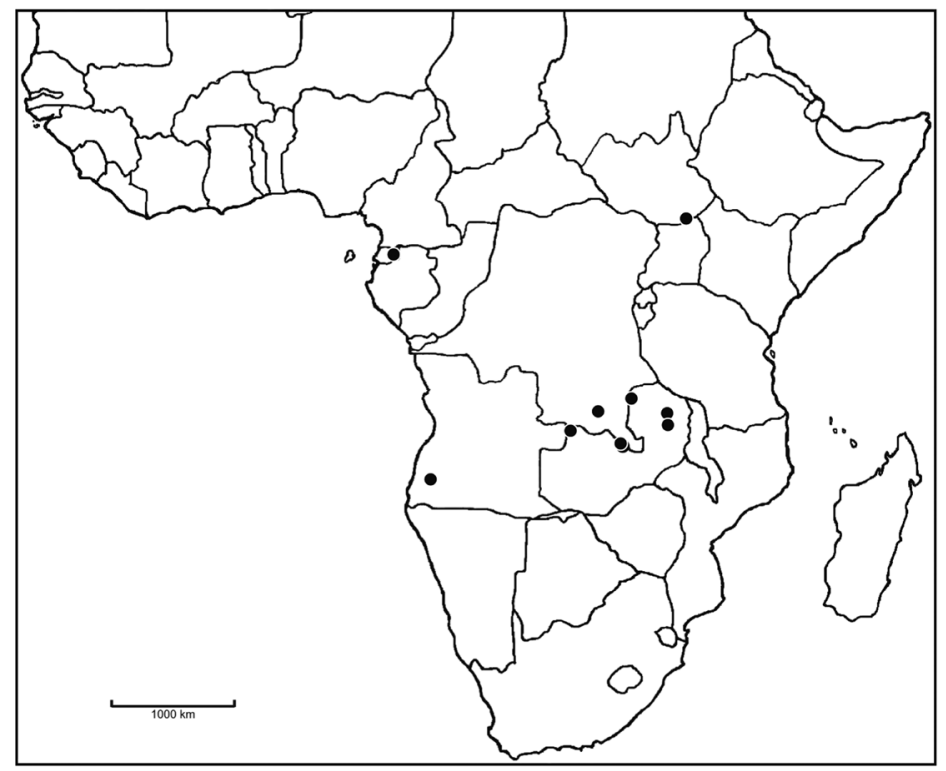

Fig. 127. Distribution of Bilabrella cataphysema (Rchb.f.) Szlach. \& Kras-Lap.
$0.4 \mathrm{~mm}$ wide, filiform, pendent. Spur 12-13 mm long, filiform, pendent, with apical third much swollen, globose, ampullaceous, 2-3 mm in diameter. Anther 2-3 mm tall; connective rounded; antherophores 2-3 $\mathrm{mm}$ long, slender, upcurved. Stigmaphores $4 \mathrm{~mm}$ long, straight, swollen and truncate apically. Rostellum middle lobe shorter than thecae. Auriculae oblong-ovoid, rather small (Fig. 126).

E c ology. Dambo, damp marshy places, perennially wet upland bogs, wet grassland. Flowering in December-June.

D is tribution. Sudan, Equatorial Guinea, Democratic Republic of the Congo (Zaire), Uganda, Angola, Zambia, Malawi - not seen (Williamson 1977), alt. 1200-1950 m (Fig. 127).

$\mathrm{S} p$ e c i men s ex a mined. Sudan. Eastern Equatoria: Imatongs Mts., in burned-over grassland at edge of flat rocks in mountain savannah, $1950 \mathrm{~m}, 26$ June 1947 (fl), MacLeay 74 (BM!); Imatong Mts., in burnedover grassland of mountain savannah, $1950 \mathrm{~m}$, 30 June 1947 (fl), MacLeay 122 (BM!). Equatorial Guinea. East part, 1893 (fl), Gregory s.n. (BM!). Democratic Republic of the Congo (Zaire). Haut-Katanga: Biano Plateau, savanne on front of Plateau, $1500 \mathrm{~m}, 10 \mathrm{Jan}$. 1981 (fl), Schaijes 1015 (BR!); Shaba Region, Biano Plateau, $40 \mathrm{~km}$ between N and NNE of Tenke, grassland, 1600 m, 29 Jan. 1984 (fl), Schaijes 2198 (K! \& spirit.). Uganda. Swamp, 1200 m, June 1917 (fl), Dummer 3213 (BM!). Angola. Huila: "Inter Lopollo et Humpata", Jan. 1860 (fl), Welwitsch 721 p.p. (BM!); "Inter Lopollo et Obay", 1250-1800 m, Feb. 1860 (fl), Welwitsch 722 (BM!, K! - fragment); Morro de Monino, 1250-1800 m, Apr. 1860 (fl), Welwitsch 743 (BM! - fragment, K! - fragment, W!). Zambia. Copperbelt: Chingola, moist, dambo, 18 Jan. 1956 (fl), Fanshawe 2740 (K!); Nkwa (Kitwe), in damp, marshy places, 14 Jan. 1936 (fl), Baron 13 (K!); Luapula: Kawambwa, Mbereshi River, wet grassland, 11 Jan. 1961 (fl), Holmes 0293 (AMES!, K!); Northern: Chinsali, Lake Young, Shiwa Ngandu, in damp grassland on lake shore, 1350 m, 17 Jan. 1959 (fl), Richards 10737 (AMES!, K!); Kasama, dambo, 7 Jan. 1961 (fl), Robinson 4239 (K!); Shiwa Ngandu, dambo, Feb. 1969 (fl), Williamson 1431 (K!); North-Western: Mwinilunga, Sinkabolo Dambo in boggy grassland, 21 Dec. 1937 (fl), Milne-Redhead 3757 (BM!, BR!, K!); Mwinilunga Distr., Matonchi, Sinkabolo dambo, 22 Dec. 1969 (fl), Williamson \& Simon 1811 (K!).

Notes. The species is easily distinguishable from other of the genus by very characteristic spur with globose apex.

43. Bilabrella weberana (Schltr.) Szlach. \& Kras-Lap., Richardiana 3(3): 142. 2003. - Szlachetko et al., Orchid. of West-Central Africa 1: 237. 2010. 


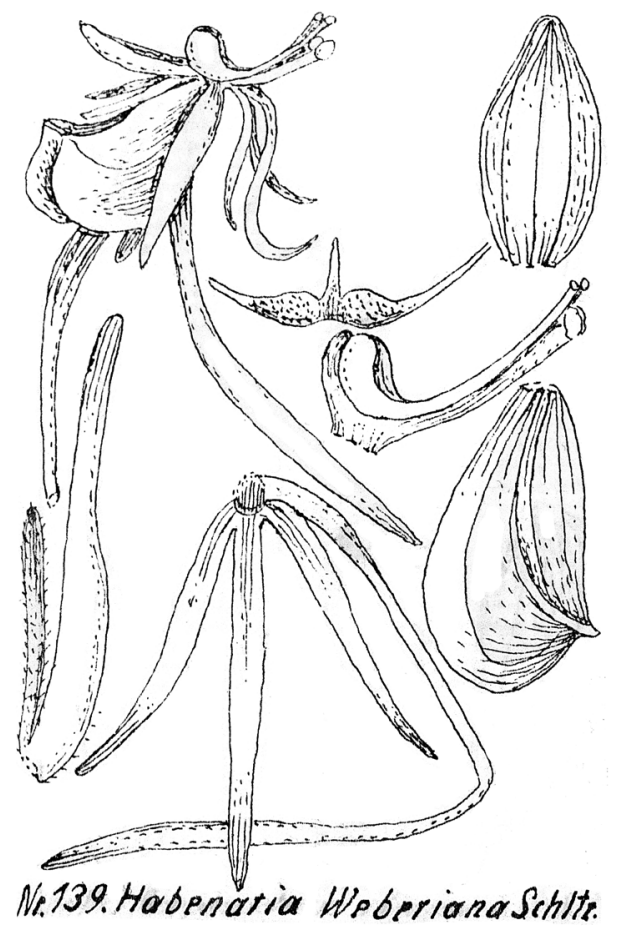

Fig. 128. Original Schlechter (1932) drawing of Habenaria weberana Schltr.

Habenaria weberana Schltr., Bot. Jahrb. Syst. 53: 516. 1915. - Schlechter, Repert. Bot. Sp. Nov Regni Veg. 68: tab. 35. 1932. - Summerhayes, FTEA, Orchid. 1: 94. 1968b. - Grosvenor, Excelsa 6: 83. 1976. - Williamson, Orchid. S. Centr. Afr.: 52. 1977. - Geerinck, Fl. Afr.

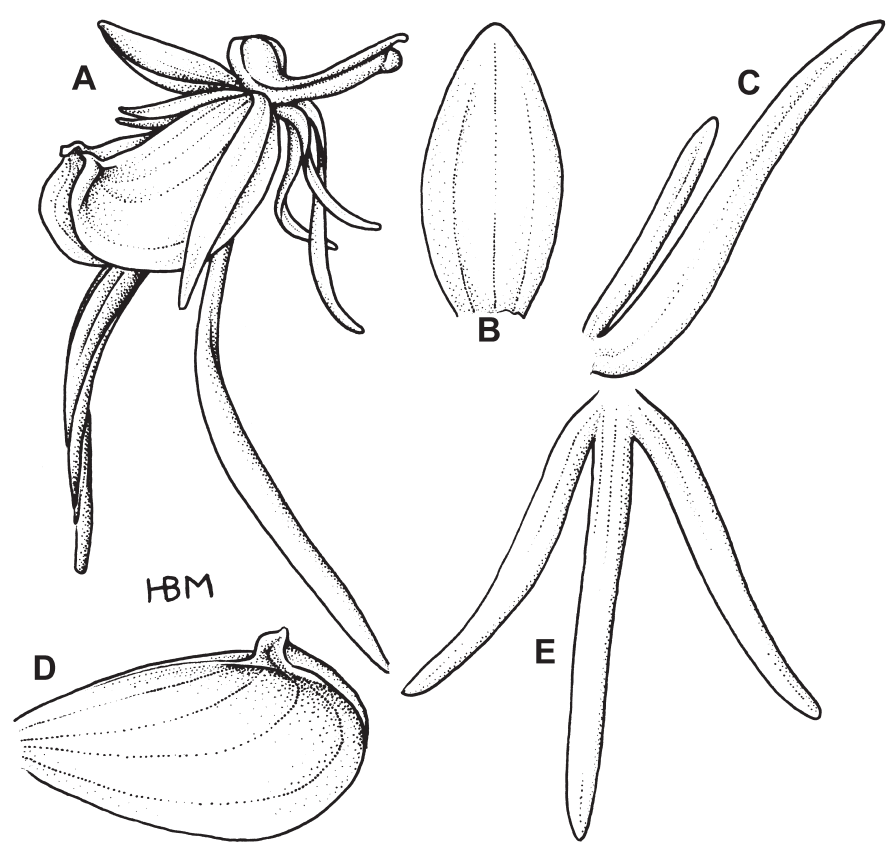

Fig. 129. Bilabrella weberana (Schltr.) Szlach. \& Kras-Lap. Explanations: A - flower; B - dorsal sepal; C - petal; D - lateral sepal; E - lip (drawn by H. B. Margońska from lectotype of Habenaria weberana Schltr. - Stolz 762, K)

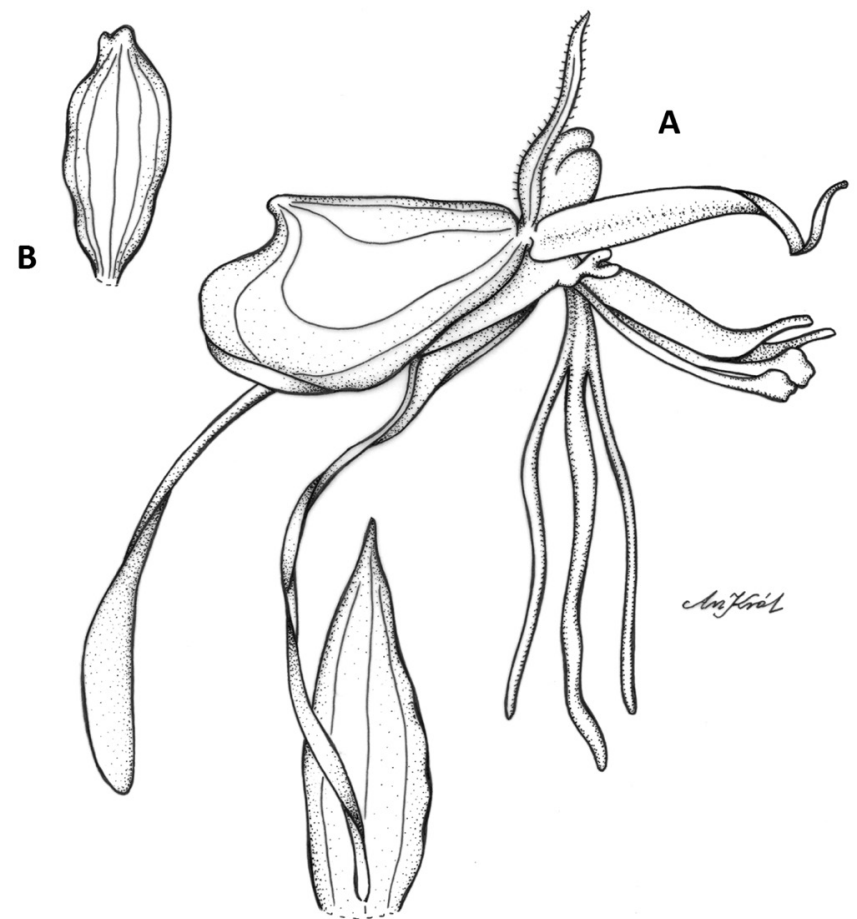

Fig. 130. Bilabrella weberana (Schltr.) Szlach. \& Kras-Lap. Explanations: A - flower; B - dorsal sepal (drawn by A. Król from Williamson 412, AMES)

Centr., Orchid. 1: 115. 1984. - la Croix et al., Orchid. Malawi: 80. 1991. - la Croix \& Cribb, Fl. Zambes., Orchid. 11: 111. 1995.

Type (Szlachetko et al. 2010): Tanzania, Stolz 672 (B+; lectotype, designated by Szlachetko et al. [2010: 237], K!; isolectotypes, C!, W-R!, S not seen).

Habenaria huillensis var. weberana (Schltr.) Geerinck, Bull. Jard. Bot. Nat. Belg. 52: 343. 1982.

Tubers 1-2 $\mathrm{cm}$ long, $0.5-1 \mathrm{~cm}$ in diameter, ellipsoid or globose. Stem $25-100 \mathrm{~cm}$ tall, erect, rather slender, glabrous. Leaves 7-13, 10-28 cm long, 0.6-2.5 cm wide, narrowly lanceolate, linear-lanceolate to linear, acute, erect or suberect, decreasing in size up the stem. Inflorescence 5-33 cm long, 5- to many-flowered, dense or lax. Flowers pale or yellow-green with white centre, with an unpleasant nocturnal smell. Floral bracts 7-27 mm long, ovate-lanceolate, acuminate. Pedicel and ovary 15-20 mm long, glabrous. Dorsal sepal 5-6.5 mm long, 2-3 mm wide, oblong-ovate, rounded, cochleate, glabrous. Petals bilobed nearly to the base, glabrous; anterior lobe $7-13 \mathrm{~mm}$ long, $2 \mathrm{~mm}$ wide, obliquely linear-lanceolate, obtuse; posterior lobe 4-6.5 mm long, $0.5 \mathrm{~mm}$ wide, linear-filiform, acute, ciliolate. Lateral sepals $6.5-8.5 \mathrm{~mm}$ long, $4.5-7 \mathrm{~mm}$ wide, obliquely obovate, cochleate at the apex, glabrous, laterally apiculate. Lip deflexed, 3-lobed above the basal 2-3 mm, glabrous; the middle lobe $10-12 \mathrm{~mm}$ long, $0.5 \mathrm{~mm}$ wide, linear, obtuse; lateral lobes 4.5-6.5 mm long, $0.4 \mathrm{~mm}$ wide, linear, subobtuse. Spur 15-20 mm long, narrowly 


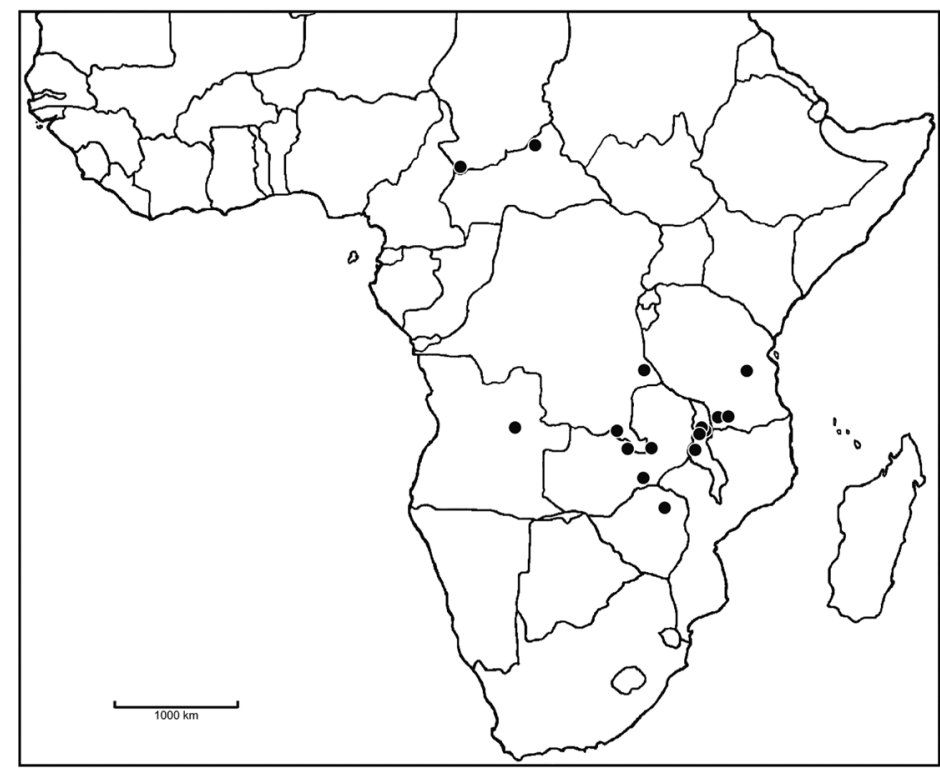

Fig. 131. Distribution of Bilabrella weberana (Schltr.) Szlach. \& Kras-Lap.

cylindrical, very slightly inflated in the apical half, acute, pendent. Anther 2-3 mm long; connective truncate; antherophores 4.5-6 mm long, slender, abruptly upcurved near the middle. Stigmaphores 3.5-6 mm long, narrowly cylindrical, truncate, straight. Rostellum middle lobe subulate, longer than connective. Auriculae 3-lobed, small (Figs 128-130).

E c o lo g y. Marshy and boggy grassland, in vlei, wet bog, loamy sand. Throughout the year.

Distribution. Chad, Central African Republic, Democratic Republic of the Congo (Zaire), Tanzania, Angola, Zambia, Malawi, Zimbabwe, alt. 580-1650 m (Fig. 131).

S pecimens examined. Chad. Logone Occidental: Bekas, 1 Oct. 1964 (fl), Avdru 1279 (K!). Central African Republic. Niao River $4 \mathrm{~km} \mathrm{~S}$ of Sakala Road embranchment at confluence of small E tributary, loamy sand, $8^{\circ} 32^{\prime} \mathrm{N}, 21^{\circ} 18^{\prime} \mathrm{E}, 600 \mathrm{~m}, 19$ Aug. 1985 (fl), Fay 7418 (K!). Democratic Republic of the Congo (Zaire). Haut-Katanga: Shaba Region, Kipopo, 14 km NW of Lubumbasbi, 1240 m, 7 Jan. 1983 (fl), Schaijes 1790 (K! \& spirit.); Shaba Region, Munuanga, 90,5 km nearly worth of Kolwezi, boggy grassland, 880 m, 7 Dec. 1983 (fl), Schaijes 2099 (K! \& spirit.); Nord-Kivu: Kitimba, 18 Jan. 1908 (fl), Kassner 2363 (HBG!). Tanzania. Kaskazini-Pemba: Nyassa Hochland, Mwaisumu Konde, 600 m, 8 June 1911 (fl), Stolz 762 (K!); Morogoro, Hannington s.n. (K!); Ruvuma: Songea Distr., about $6,5 \mathrm{~km} \mathrm{~W}$ of Songea, 990 m, 6 Feb. 1956 (fl), Milne-Redhead \& Taylor 8626 (AMES!, K! \& spirit.); About 1,5 km E of Songea in marshy ground among Dissotis sp. by stream, 1050 m, 13 Feb. 1956 (fl), Milne-Redhead \& Taylor 8681
(AMES!, K!); Ngare Nanyuki, Pittores Gorge, Arusha National Park, 1600 m, 1 Jan. 1971 (fl), Richards 26438 (AMES!, UGDA-DLSz! - fragment, drawing). Angola. Moxico: By River Mumbala, in boggy grassland, 8 Jan. 1938 (fl), Milne-Redhead 4002 (BR!, K!). Zambia. Central: Serenje Distr., by head of Kundalila Falls, near Karano, in vlei, 5 Feb. 1959 (fl), Ball 807 (K!); Serenje Distr., Kundalila falls (53 km ENE Serenje), dambo near camp-site, 1500 m, 4 Feb. 1973 (fl), Schelpe $2914 a$ (K!); Kundalila Falls, 15 km SE of Kanona, Feb. 1971 (fl), Williamson 2224 (K!); Copperbelt: Kitwe, Extension of Kent Avenue, 15 Feb. 1964 (fl), Morze 165 (K!); Ndola, in Plateau woodland, 10 Jan. 1954 (fl), Fanshawe 658 (K!); Lusaka: Chakwenga Headwaters, 100-129 km East of Lusaka, permanently wet dambo, 9 Feb. 1964 (fl), Robinson 6231 (K!); Shiwa Ngandu, Feb. 1968 (fl), Willimason 412 (AMES!, K!, UGDA-DLSz! - fragment, drawing). Malawi. Central: Kasungu National Park, in wet grass at edge of Dam, 1150 m, 9 Aug. 1987 (fl), Field (la Croix) 1046 (K!); Kasungu to Mzimba, 13 Feb. 1971 (fl), Westwood 612 (K!); Northern: Mzimba Distr., Lunyangwa River at Mzuzu Govt. Sec. Sch. Mzuzu, 1350 m, 24 Mar. 1971 (fl), Pawek 4517 (K!); Nkhata Bay Distr., Mzenga dambo, 580 m, 10 Feb. 1986 (fl), la Croix 801 (K!); Nkhata Bay Distr., Vipya Plateau, 5 miles SW of Mzuzu, elephant rock, in wet bog, 1650 m, 3 Feb. 1974 (fl), Pawek 8066 (K!); Viphya Plateau, Luckilemu dambo, 1300 m, 21 Feb. 1984 (fl), la Croix 557 (K!); S. Viphya, elephant rock dambo, 1550 m, 12 Jan. 1986 (fl), la Croix 771 (K!). Zimbabwe. Harare: Salisbury Distr., Chacoma, in vlei, 27 Jan. 1950 (fl), Greatrex 26744 (AMES!, K!); Salisbury Distr., W of Ehworth rock hilles, in vlei, 1350 m, 19 Feb. 1950 (fl), Greatrex 26898 (K!); Salisbury Distr., vlei in Umwinzidale, 1500 m, 12 Jan. 1963 (fl), Smith 505 (K!).

$\mathrm{N}$ ot es. This species is similar to Bilabrella linguiformis, but has prominently longer leaves and glabrous petals. Similar to B. galpinii and B. hebes. From both of them differs in the spur shape, and additionally, from $B$. hebes by the form of petals.

44. Bilabrella linguiformis (Summerh.) Szlach. \& Kras, Richardiana 9(4): 158. 2009.

Habenaria linguiformis Summerh., Kew Bull. 1932: 340. 1932. - Summerhayes, FWTA ed. 2, 3: 196. 1968a.

Type (hoc loco selectus): Nigeria, Lely 343 A (lectotype, $\mathrm{K}$ !).

Stem ca. $30 \mathrm{~cm}$ tall, erect, rather stout. Leaves 9-12, gathered in the lower part of the stem, 4-6 cm long, 0.9$1.2 \mathrm{~cm}$ wide, lanceolate, narrowly lanceolate to linearlanceolate, acute, recurved, decreasing in size upwards. Inflorescence to $10 \mathrm{~cm}$ long, laxly several-flowered. Flowers greenish-white. Floral bracts 9-15 mm long, 


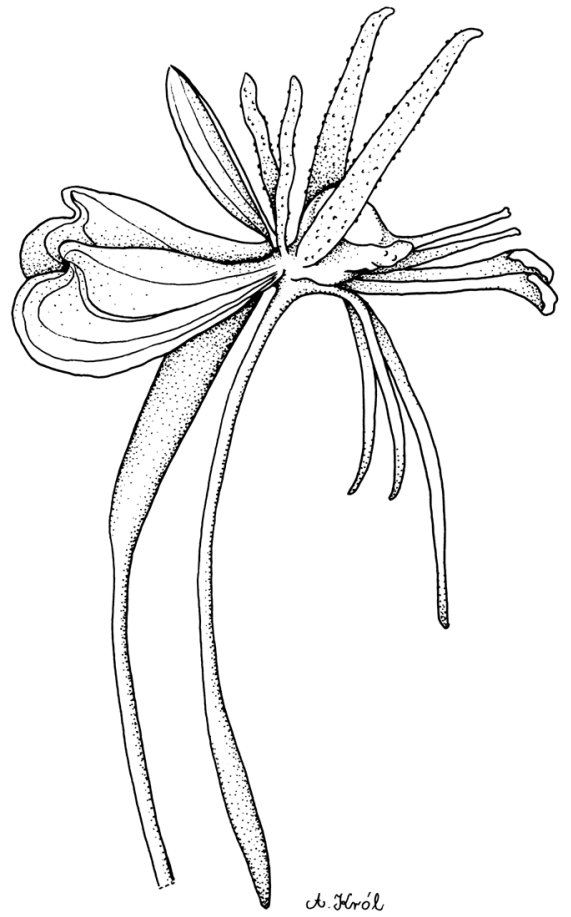

Fig. 132. Bilabrella linguiformis (Summerh.) Szlach. \& Kras flower (drawn by A. Król from lectotype of Habenaria linguiformis Summerh. - Lely $343 \mathrm{~A}, \mathrm{~K}$ !)

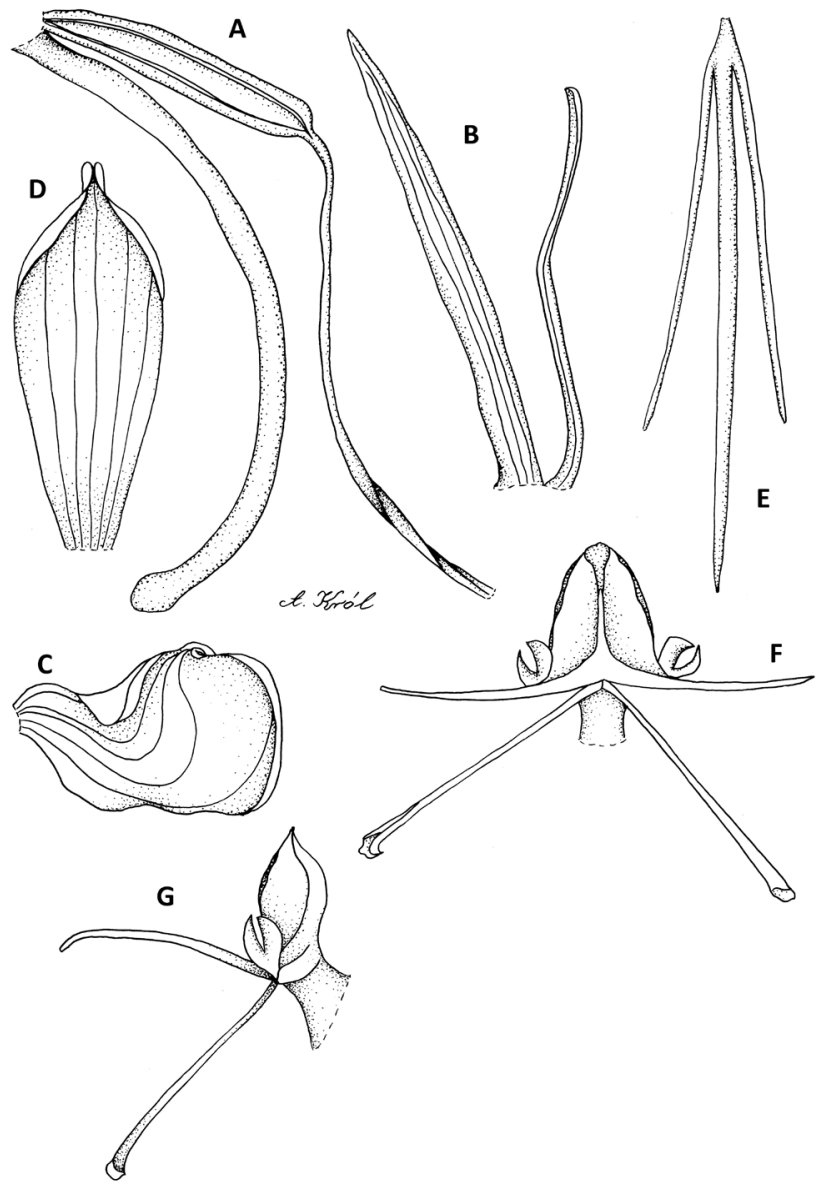

Fig. 133. Bilabrella linguiformis (Summerh.) Szlach. \& Kras Explanations: A - ovary, pedicel, spur; B - petal; C - lateral sepal; D - dorsal sepal; E - lip; F - gynostemium, front view; G - gynostemium, side view (drawn by A. Król from lectotype of Habenaria linguiformis Summerh. Lely $343 A, \mathrm{~K})$

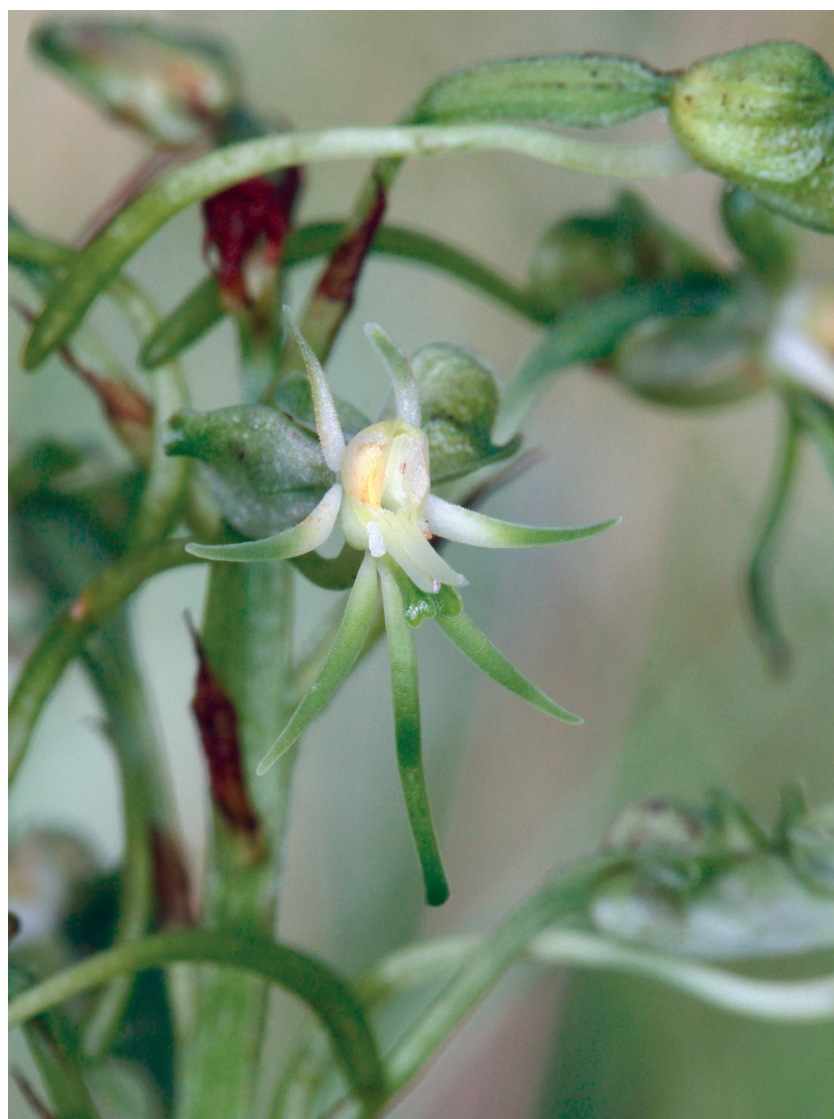

Fig. 134. Bilabrella linguiformis (Summerh.) Szlach. \& Kras flower close-up (phot. L. Grobler)

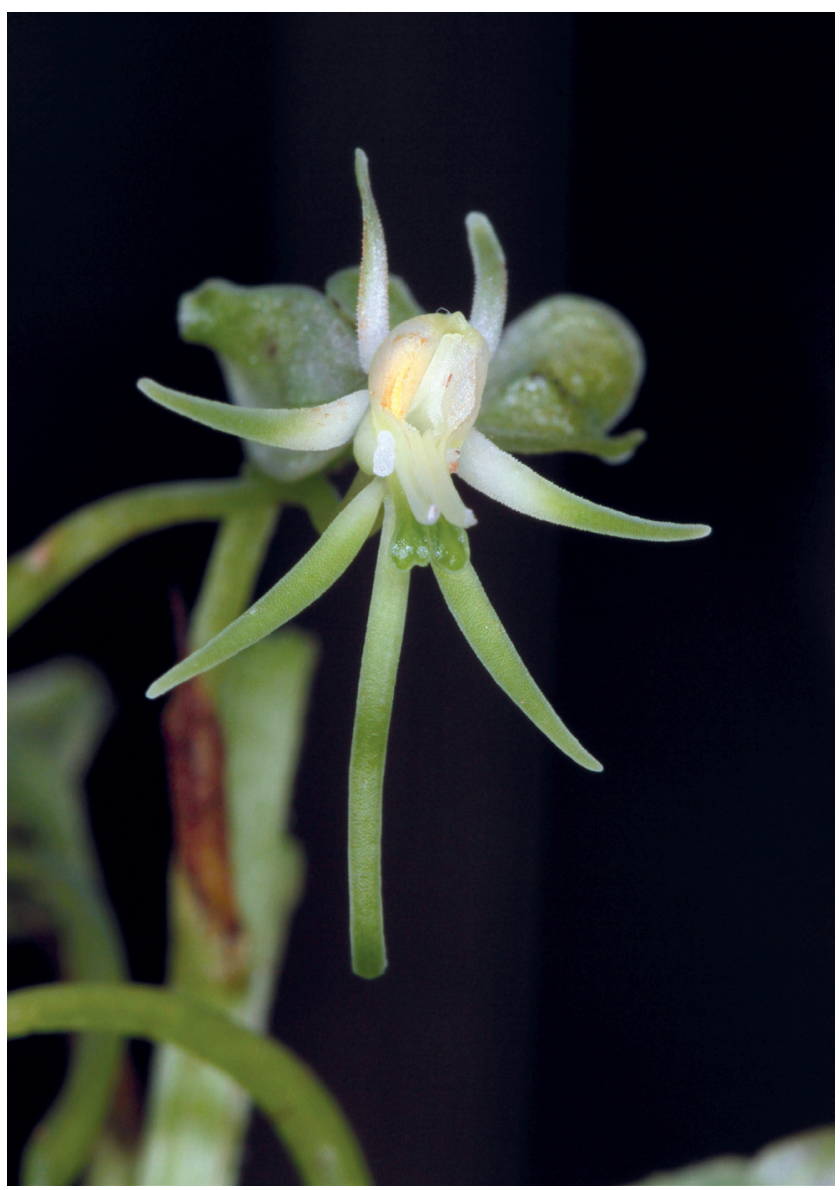

Fig. 135. Bilabrella linguiformis (Summerh.) Szlach. \& Kras flower close-up (phot. L. Grobler) 


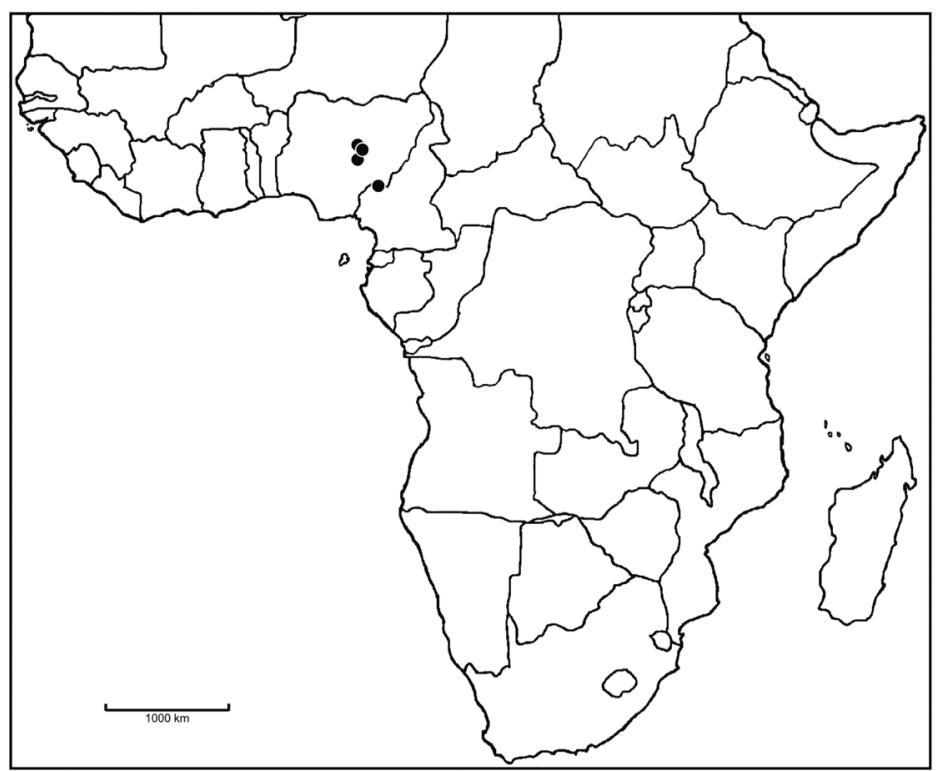

Fig. 136. Distribution of Bilabrella linguiformis (Summerh.) Szlach. \& Kras

narrowly lanceolate, acuminate. Pedicel and ovary to $20 \mathrm{~mm}$ long. Dorsal sepal $6 \mathrm{~mm}$ long, $2 \mathrm{~mm}$ wide, elliptic-obovate to oblong-obovate, obtuse, concave. Petals bilobed to the base; anterior lobe $8-8.5 \mathrm{~mm}$ long, 0.5-0.8 mm wide, linear-lanceolate, acute, papillose; posterior lobe $5 \mathrm{~mm}$ long, $0.2 \mathrm{~mm}$ wide, filiform, obtuse, papillose. Lateral sepals $7 \mathrm{~mm}$ long, 3-5 mm wide, obliquely oblong obovate, laterally apiculate. Lip 3-lobed; the middle lobe 6-10 $\mathrm{mm}$ long, $0.5 \mathrm{~mm}$ wide, linear-filiform, obtuse; lateral lobes 6-7 mm long, $0.5 \mathrm{~mm}$ wide, linear-filiform, acute, pendent. Spur 16$18 \mathrm{~mm}$ long, filiform, incurved, apically slightly swollen, attenuate towards mamillate, acute apex. Anther $2 \mathrm{~mm}$ tall; connective rounded; antherophores $4 \mathrm{~mm}$ long, slender, straight. Stigmaphores 5-6 mm long, slender, swollen apically, obliquely truncate. Auriculae $1.5 \mathrm{~mm}$ long, ligulate (Figs 132-135).

E c o log y. Rocky hillside. June-July.

D istribution. Nigeria, Cameroon, alt. 1100-1290 m (Fig. 136).

Specimens examined. Nigeria. Plateu: N'gall, 1290 m, June 1957 (fl), King 27 (K! \& spirit.); Plain between Bukuru and Hepham, 1290 m, July 1921 (fl), Lely $343 A$ (K!); Northern Region, hills between Kuru village and Plateau Provinicial, on steep rocky hillside, 1200 m, July 1958 (fl), Summerhayes 160 (K!). Cameroon. Northwest: Road between Essu and Munkep, Kunyang, 30 km N Wum, 1100-1200 m, 6 July 1975 (fl), Letouzey 13959 (BR!, K!, P!).

Notes. As mentioned above this species is similar to $B$. weberana. It differs from the latter, however, by short leaves and papillose petals. The flowers of this species resemble those of $B$. hebes, both species however, differ clearly in the vegetative parts - in B. linguiformis leaves are short and wide (ca. 5 times longer than wide), wheras in $B$. hebes - narrow and long (ca. 30 times longer than wide).

45. Bilabrella subaequalis (Summerh.) Szlach. \& KrasLap., Richardiana 3(3): 142. 2003.

Habenaria subaequalis Summerh., Kew Bull. 16: 297. 1962. - Grosvenor, Excelsa 6: 83. 1976. - la Croix \& Cribb, Fl. Zambes., Orchid. 11: 106. 1995.

Type: Zimbabwe, De Wild 4455 (holotype, K!; isotypes, K!, SRGH not seen).

Tubers 1.2-1.6 cm long, 0.4-0.7 cm wide, ovoid. Stem 15-35 cm tall, erect, slender, leafy. Leaves 7-10, 6-11 cm long, 0.4-1 cm wide, linear-lanceolate, acute to acuminate, the longest leaves gathered in the lower part of the stem, upper ones grading into the bracts. Inflorescence $4-9 \mathrm{~cm}$ long, fairly laxly to densely 5-30-flowered. Flowers white, occasionally described as green and white, scented. Floral bracts 6-8 mm long, lanceolate, acuminate. Pedicel and ovary $10-17 \mathrm{~mm}$ long, arcuate. Sepals reflexed. Dorsal sepal 5.4-6 mm long, 3-3.7 mm wide, elliptic to obovate-elliptic, obtuse, apiculate, concave, reflexed. Petals 2-lobed almost to
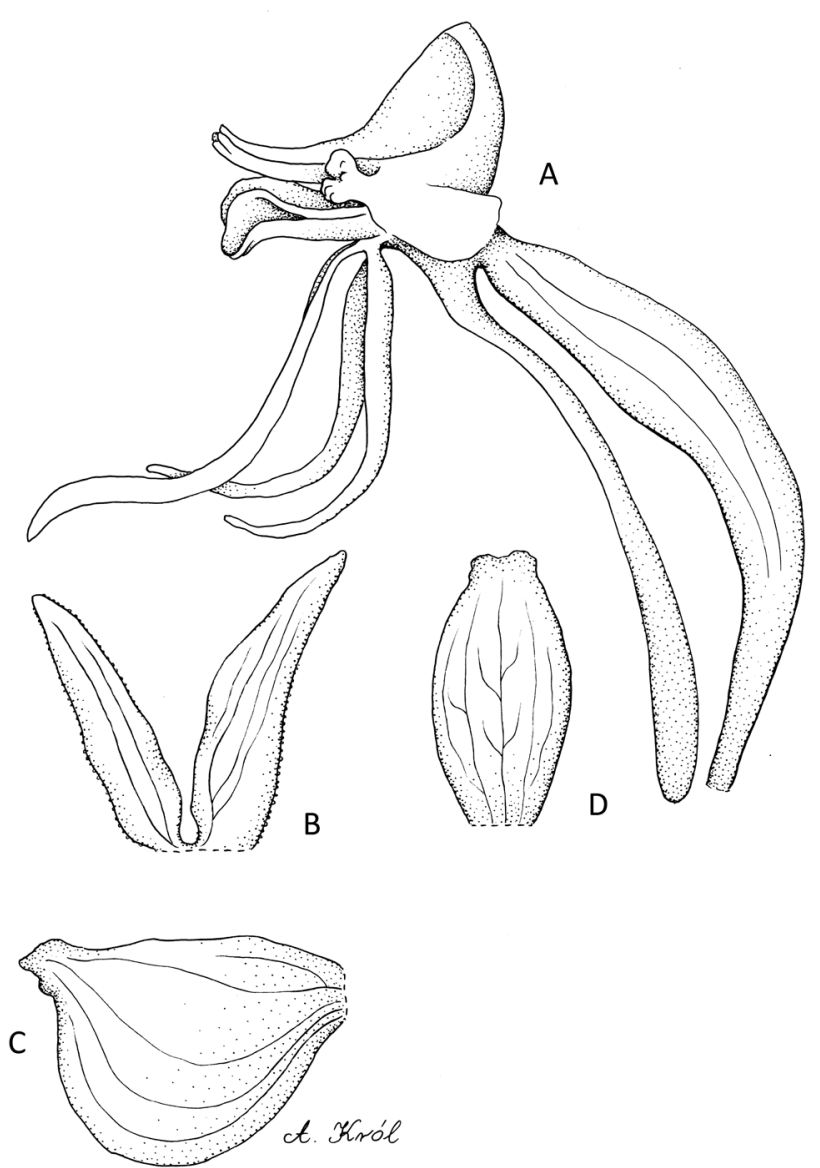

Fig. 137. Bilabrella subaequalis (Summerh.) Szlach. \& Kras-Lap. Explanations: A - pedicel, ovary, gynostemium, lip and spur; B - petal; C lateral sepal; D - dorsal sepal (drawn by A. Król from holotype of Habenaria subaequalis Summerh. - De Wild 4455, K) 
base, papillose-ciliolate; anterior lobe 5.6-7.5 $\mathrm{mm}$ long, 1.6-2 mm, oblong-lanceolate, acute or obtuse; posterior lobe 5.5-6 mm long, 1.2-1.6 mm wide, oblong-lanceolate to ligulate, acute to obtuse. Lateral sepals 6-7 mm long, 3.5-5 mm wide, obliquely obovate, deflexed, with a lateral apiculus, cochleate. Lip 3-lobed almost to the base; the middle lobe 9-10 mm long, 0.6-0.7 $\mathrm{mm}$ wide, linear-lanceolate, subacute; lateral lobes $6.5-7.8 \mathrm{~mm}$ long, $0.5-0.8 \mathrm{~mm}$ wide, linear to linear-lanceolate, subacute. Spur 10-13 mm long, filiform, much swollen in the apical third, blunt. Anther ca. $3.5 \mathrm{~mm}$ tall; connective truncate; antherophors $3.5 \mathrm{~mm}$ long, straight. Stigmaphores $4.5 \mathrm{~mm}$ long, narrowly cylindrical, truncate apically. Rostellum middle lobe as long as thecae. Auriculae large, irregular, occasionally bifid (Fig. 137). E c ology. Grassland, hillsides, granite and rocky soils, damp submontane or plateau grassland, usually in marshy ground, almost always amongst rocks. February-April.

D i s tribution. Democratic Republic of the Congo (Zaire), Zimbabwe, alt. 1380-1980 m (Fig. 138).

Specimens examined. Democratic Republic of the Congo (Zaire). Haut-Katanga: Plateau Kundelungu, env. $3 \mathrm{~km}$ of WNW from the spring of the Lutshipuka, 1680 m, 25 Mar. 1969 (fl), Lisowski 4006 (UGDA-DLSz!). Zimbabwe. Goromonzi: Rilly slope of Domboshawa "whole-back", 1500 m, 2 Mar. 1961 (fl), Greatrex 119792 (K!); Inyanga: Above Mtarazi Falls, 2 Mar. 1958 (fl), Beasley 67 (83549) (K!); In hollow with grass among granite boulders on Rodel Farm, 1890 m, 26 Feb. 1966 (fl), Chase 8382 (K!); On footpath to Mtarazi Falls amongest grass, $1380 \mathrm{~m}$, 7 Mar. 1969 (fl), Jacobsen 3780 (K!); Manicaland: Troutbeck. North Downs, in marshy ground among rocks, 1980 m, 8 Mar. 1981 (fl), Philcox, Leppard, Duri \& Urayai 8931 (K!); Wet hillside above Rhodes Hotel, 1800 m, 2 Mar. 1956 (fl), Whellan \& Davies 995 (K!); Vumba Mts., castle Beacon, 1880 m, 18 Mar. 1956 (fl), Chase $6015 A$ (K!); Vumba, Vorseland, in damp ground on granite slope, 1500 m, Mar. 1949 (fl), De Wild 2806 (22718) (K! \& spirit.); Melsseter: Chimanimani, grassland, 1500 m, 21 Feb. 1954 (fl), Ball 237 (46248) (K!); Chimanimani Mts., Mar. 1957 (fl), Goodier 190 (K!); Goodier 197 (K!); Chimanimani Mts., Bundi Velley, growing by side of path through the valley, $1500 \mathrm{~m}$, 5 Apr. 1969 (fl), Grosvenor 481 (K!); Chimanimani Mts., between The Hut and Digby's pool, in grassland, 1650 m, 10 Apr. 1967 (fl), Grosvenor 376 (K!); Chimanimani Mts., east of National Parks Office, $1600 \mathrm{~m}$, 20 Mar. 1981 (fl), Philcox, Leppard, Duri \& Urayai 9028 (K!); East-facing slope of Mt. Peni, in grazed montane grassland, 1560 m, 16 Mar. 1981 (fl), Philcox, Leppard, Duri \& Urayai 8989 (K!); East-facing slope of Mt. Peni, in grazed montane grassland, $1560 \mathrm{~m}, 16$ Mar. 1981 (fl), Philcox, Leppard, Duri \& Urayai 8989A (K!);

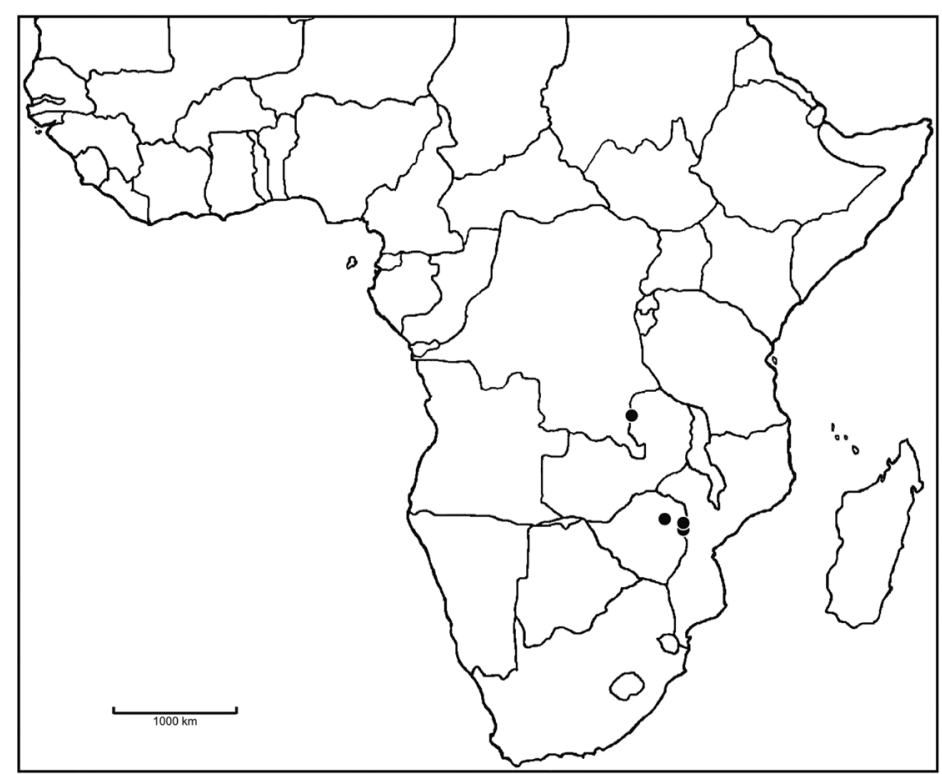

Fig. 138. Distribution of Bilabrella subaequalis (Summerh.) Szlach. \& Kras-Lap.

Mutare: Himalaya Range, Engwa, De Wild 4455 (K!). N ot es. Bilabrella subaequalis, like $B$. welwitschii, B. macrotidion and B. pauper, has the two petal segments nearly equal in length, but in this species they are also similar in shape and other characters. This species is unusual in Bilabrella in having white flowers (la Croix \& Cribb 1995).

46. Bilabrella hebes (la Croix \& P. J. Cribb) Szlach. \& Kras-Lap., Richardiana 3(3): 140. 2003.

Habenaria hebes la Croix \& P. J. Cribb, Kew Bull. 48(2): 357. 1993. - la Croix \& Cribb, Fl. Zambes., Orchid. 11: 94. 1995.

Type: Zambia, Robinson 6601 (holotype, K!).

Tubers $1.2 \mathrm{~cm}$ long, $1 \mathrm{~cm}$ in diameter, ovoid. Stem 40-45 cm tall, slender, leafy. Leaves 8-13, the lowest 1-2 sheathing with rather obscure reticulate veining; the next 2-5 suberect, to about $11 \mathrm{~cm}$ long and $0.3 \mathrm{~cm}$ wide, narrowly linear, acute; the upper 5-6 bract-like and appressed to the stem. Inflorescence $8-10 \mathrm{~cm}$ long, fairly laxly 6-9-flowered. Flowers green. Floral bracts to $15 \mathrm{~mm}$ long, oblong-lanceolate, acute to acuminate. Pedicel 20-22 mm long, ovary 10-13 mm long, straight or slightly arched. Dorsal sepal 4-5.5 mm long, 1.5$2 \mathrm{~mm}$ wide, elliptic-obovate to oblong obovate, obtuse, concave. Petals bilobed to the base; anterior lobe 10.5$14 \mathrm{~mm}$ long, 0.5-0.9 mm wide, deflexed, narrowly linear-lanceolate, subulate apically, slightly papillose, lose towards base; posterior lobe $4-5 \mathrm{~mm}$ long, $0.4 \mathrm{~mm}$ wide, linear-subulate, erect but curving back, papillose. Lateral sepals 7-8 $\mathrm{mm}$ long, 3-5 $\mathrm{mm}$ wide, obliquely oblong-obovate from a narrow base, with a lateral apiculus, cochleate. Lip trilobed almost to the base; the 


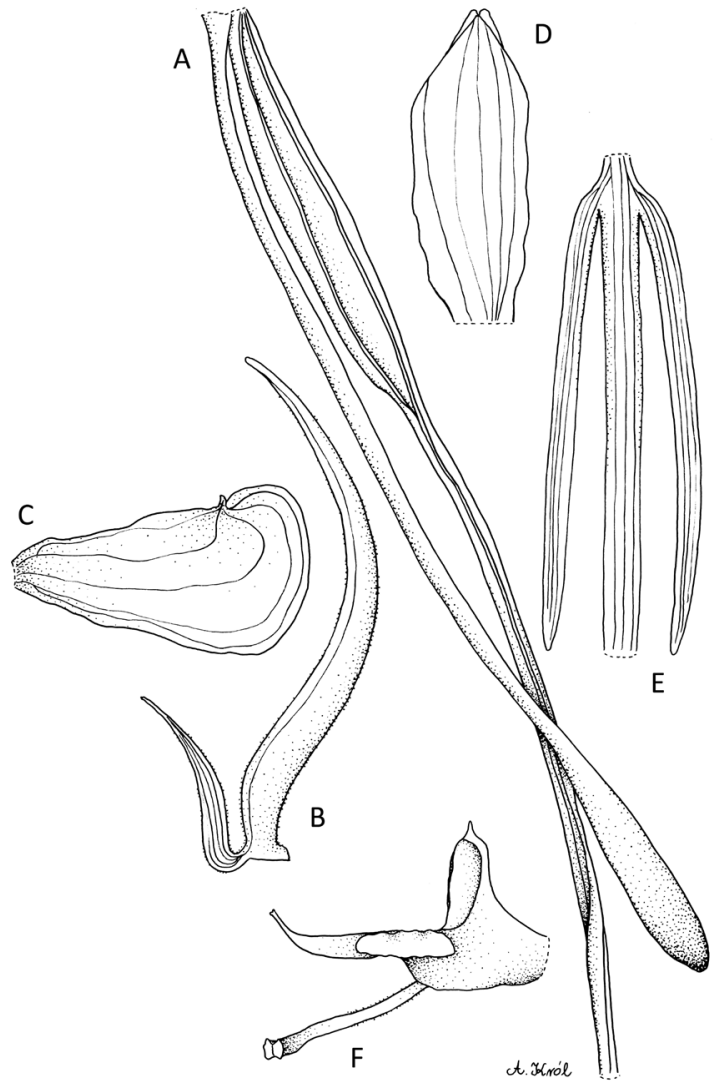

Fig. 139. Bilabrella hebes (la Croix \& P.J. Cribb) Szlach. \& KrasLap.

Explanations: A - ovary, pedicel, spur; B - petal; C - lateral sepal; D - dorsal sepal; E- lip; F- gynostemium, side view (drawn by A. Król from holotype of Habenaria hebes la Croix \& P. J. Cribb - Robinson 6601, K) middle lobe 11-16 mm long, 0.6-0.9 mm wide, linear or narrowly lanceolate, obtuse; lateral lobes $8-11 \mathrm{~mm}$ long, $0.5 \mathrm{~mm}$ wide, linear-lanceolate, subacute. Spur 26$28 \mathrm{~mm}$ long, straight, more or less parallel to the ovary and pedicel, very slender but swollen to c. $1.5 \mathrm{~mm}$ wide, in apical $6 \mathrm{~mm}$. Gynostemium shortly stalked. Anther $2 \mathrm{~mm}$ high; connective apiculate; antherophores $5 \mathrm{~mm}$ long, the apices gently upturned. Stigmaphores $5 \mathrm{~mm}$ long, cylindrical, truncate, straight, minutely papillate. Rostellum middle lobe shorter than thecae. Auriculae large, obliquely ellipsoid (Figs 139-140).

E c ology. Seasonally damp grassland and sandy plateau grassland. April-December.

Distribution. Malawi, Zambia, alt. 1400-2700 m (Fig. 141).

Specimens examined. Malawi. Nyassaland, Mlanje Mt, 2700 m, 16 Apr. 1938 (fl), Emson 04 (AMES!, UGDA-DLSz! - fragment, drawing). Zambia. North-Western: Mwinilunga Distr., Sinkabolo Dambo, in moist grassland around grey termite mounds, 21 Dec. 1937 (fl), Milne-Redhead 3769 (K!); 30 km E of Mwinilunga, Sandy Plateau, grassland, 1400 m, 17 Apr. 1960 (fl), Robinson 3666 (K!); Mwinilunga Distr., Ikelenge, seasonally damp grassland, 16 Apr. 1965 (fl), Robinson 6601 (K!).

Notes. Bilabrella hebes appears to be somewhat similar to B. ugandensis and B. macrostele which have a sterile stalk at the base of the gynostemium. It most
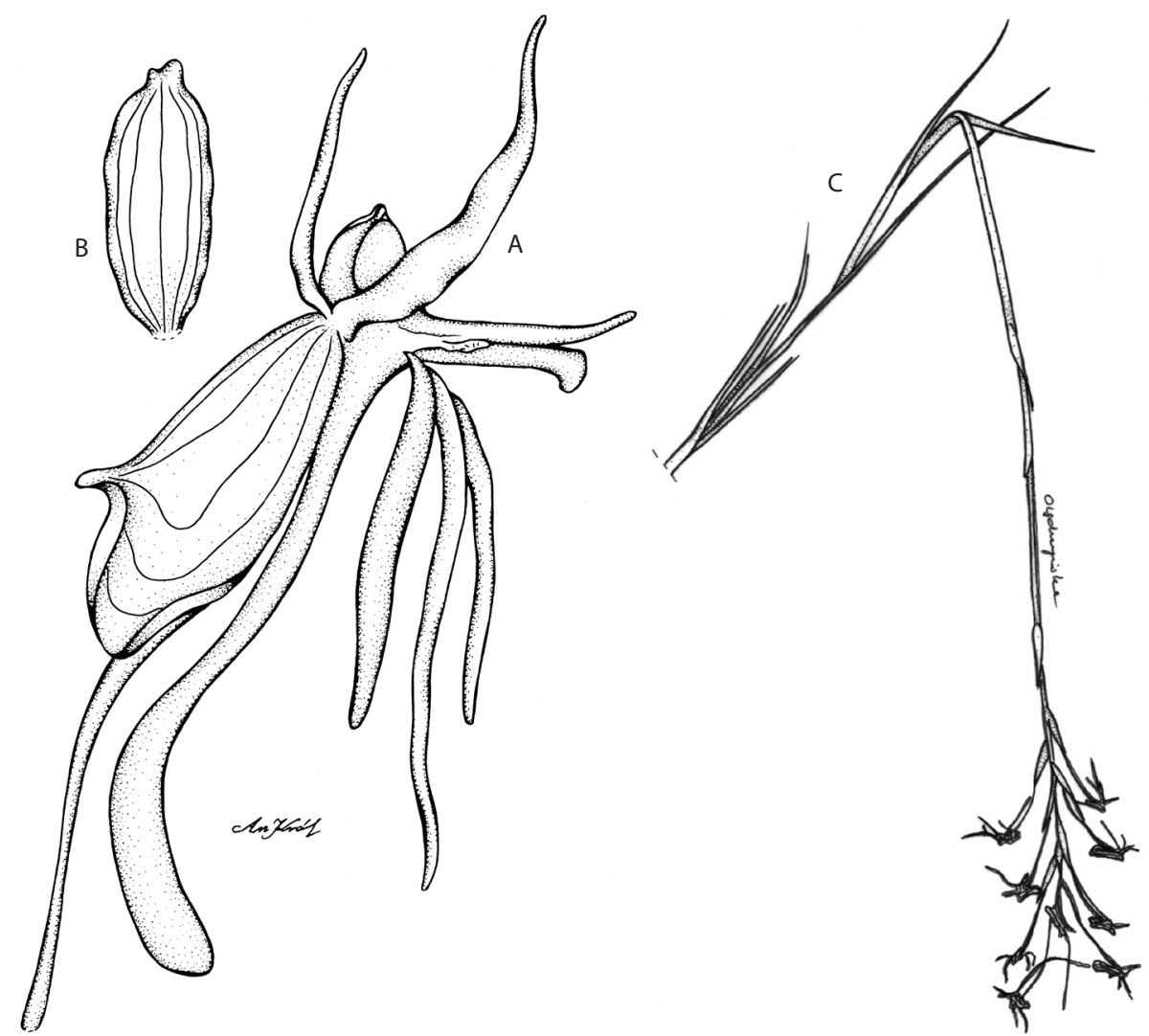

Fig. 140. Bilabrella hebes (la Croix \& P.J. Cribb) Szlach. \& Kras-Lap.

Explanations: A - flower; B - dorsal sepal (drawn by A. Król from Emson 04b, AMES); C - habit (drawn by N. Olędrzyńska from Robinson 6601, K) 


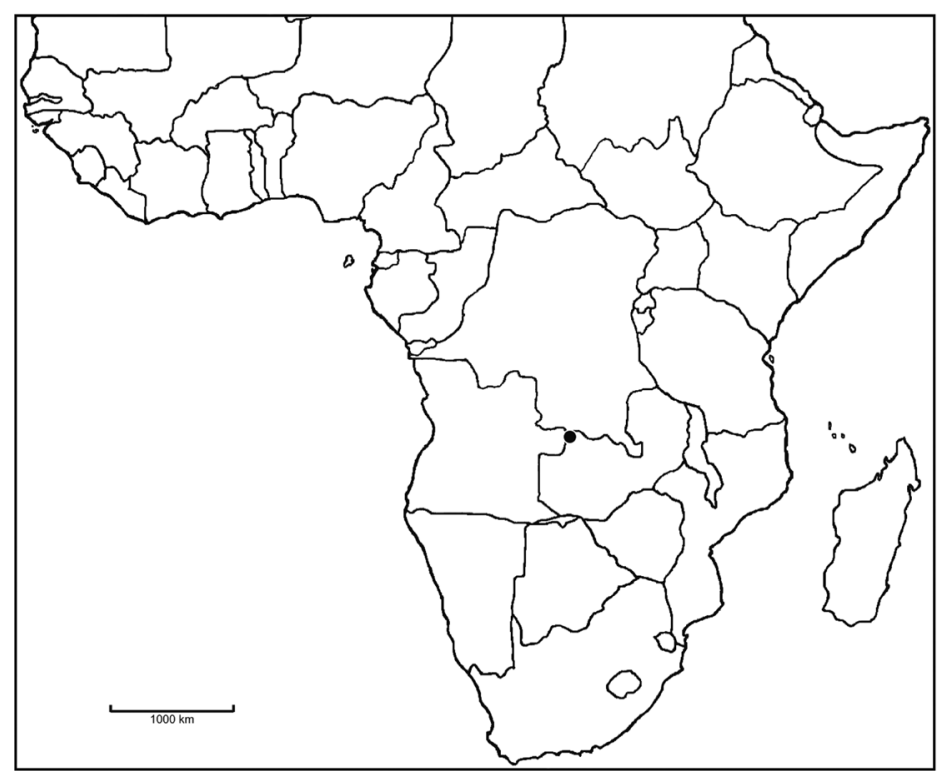

Fig. 141. Distribution of Bilabrella hebes (la Croix \& P.J.Cribb) Szlach. \& Kras-Lap.

closely resembles $B$. ugandensis but differ from it in the much narrower leaves, shorter and fewer-flowered inflorescence, longer pedicel and ovary and longer spur which is straight and not corved as in B. ugandensis and is more slender for most of its lenght but more inflated at the apex. It differs from similar B. subaequalis in having petal lobes of very different size and form.

47. Bilabrella orthocaulis (Schltr.) Szlach. \& Kras, Richardiana 6(4): 197. 2006.

Habenaria orthocaulis Schltr., Bot. Jahrb. Syst. 53: 519. 1915. - Schlechter, Repert. Sp. Nov Regni Veg. 68: tab. 31. 1932.

Type: Tanganyika, Stolz $1186(\mathrm{~B}+)$.

Stem $20-30 \mathrm{~cm}$ tall, erect, relatively delicate, glabrous. Leaves $6-7$, up to $10 \mathrm{~cm}$ long and $0.3(1) \mathrm{cm}$ wide, linear, acute, slightly deflexed from the stem, decreasing in size upwards. Inflorescence to $7 \mathrm{~cm}$ long, laxly 4-13-flowered. Floral bracts elliptic-lanceolate, acuminate, glabrous. Pedicel and ovary $15-22 \mathrm{~mm}$ long, glabrous. Dorsal sepal 5-10 mm long, 2-4 mm wide, obovate, obscurely 3-lobed just above the middle, acute, cochleate. Petals bilobed to the base, glabrous; anterior lobe 10-11 mm long, 0.3-0.7 mm wide, linearfalcate, acute; posterior lobe 7-8 $\mathrm{mm}$ long, $0.4-0.8 \mathrm{~mm}$ wide, linear-lanceolate, acute. Lateral sepals $6-13 \mathrm{~mm}$ long, 5-8 mm wide, obliquely elliptic-ovate, apiculate, concave, glabrous, deflexed. Lip 3-lobed to the base; the middle lobe 11-14 mm long, 0.7-1 mm wide, linear, subacute; lateral lobes 7-10 mm long, 0.6-0.8 $\mathrm{mm}$ wide, linear-lanceolate, acute, pendent. Spur 14-22 mm long, filiform, pendent, with apical quarter slightly swollen,

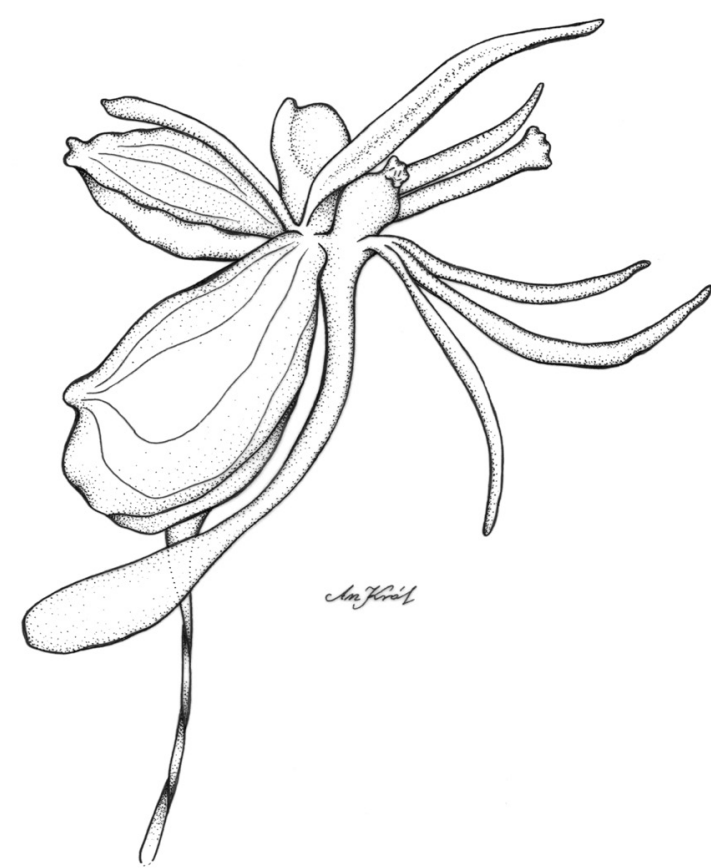

Fig. 142. Bilabrella orthocaulis (Schltr.) Szlach. \& Kras: flower (drawn by A. Król from Polhill \& Paulo 1569b, AMES)

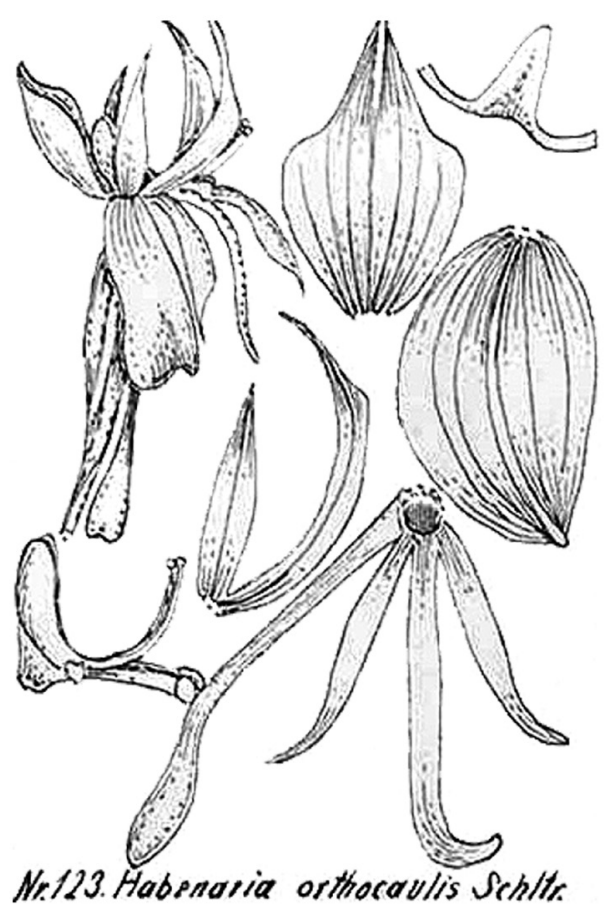

Fig. 143. Bilabrella orthocaulis (Schltr.) Szlach. \& Kras - original Schlechter (1932) drawing of Habenaria orthocaulis Schltr.

blunt. Anther 2-5 mm tall; connective triangular, obtuse; antherophores 3-9 mm long, slender, upcurved. Stigmaphores 4-8 mm long, cylindrical, straight. Rostellum middle lobe shorter than thecae. Auriculae subglobose to more or less notched apically (Figs 142-143).

E c ology. No records. February-April. D i stribution. Democratic Republic of the Congo (Zaire), Tanzania, alt. 1680-1800 m (Fig. 144). 


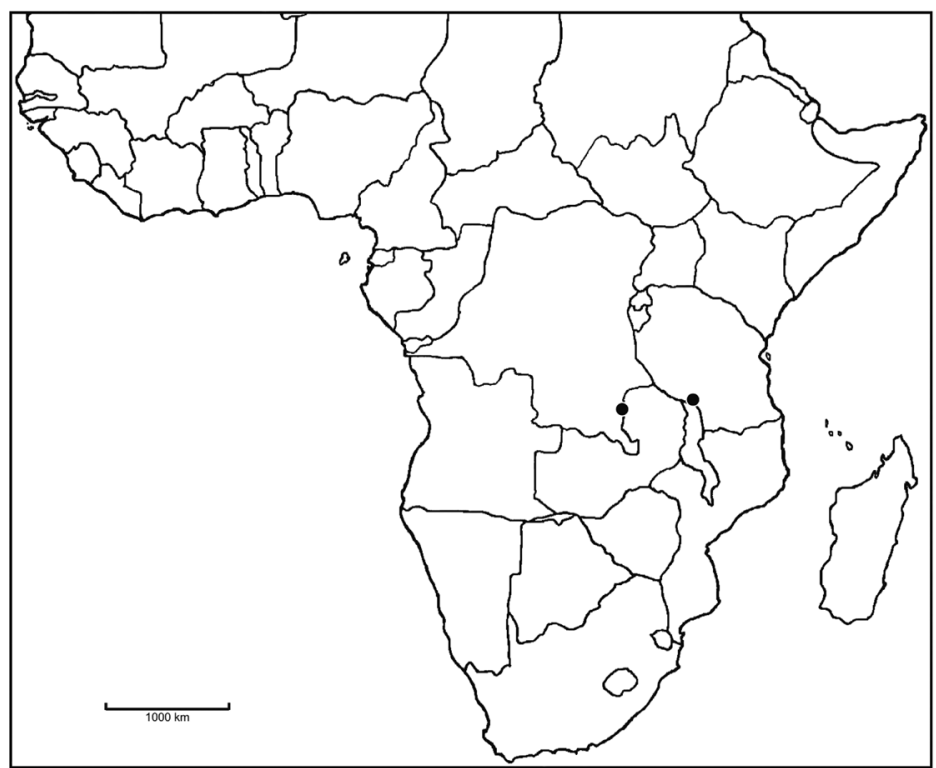

Fig. 144. Distribution of Bilabrella orthocaulis (Schltr.) Szlach. \& Kras

Specimens examined. Democratic Republic of the Congo (Zaire). Haut-Katanga: Haut-Shaba, Kundelungu Plateau, 18,4 km from the Lualala Porte, 10¹9'40"S, 27'52'40”'E, 1680 m, 21 Apr. 1971 (fl), Lisowski 66325 (UGDA-DLSz!). Tanzania. Mbeya: Rungwe Distr., Upper Mbaka, 1800 m, Apr. 1912 (fl), Stolz $1186(\mathrm{~B}+)$; Iringa Distr., 10 miles S of Dabaga, 22 Feb. 1962 (fl), Polhill \& Paulo 1569b (AMES!, UGDA-DLSz! - fragment, drawing).

Notes. The species is unique in the genus in having obscurely 3-lobed dorsal sepal.

\section{Bilabrella ugandensis (Summerh.) Szlach. \& Kras-} Lap., Richardiana 3(3): 143. 2003.

Habenaria ugandensis Summerh., Kew Bull. 16: 299. 1962. - Summerhayes, FTEA, Orchid. 1: 101. 1968b.

Type: Uganda, Lind 2464 (holotype, K!).

Tubers $2 \mathrm{~cm}$ long, $1 \mathrm{~cm}$ in diameter, ellipsoid, rather densely tomentose. Stem to $70 \mathrm{~cm}$ tall, erect, rather slender to stout, leafy throughout its length. Leaves 11-12, to $9 \mathrm{~cm}$ long and 2-5 cm wide, the lowermost 1-2 reduced to sheaths, the middle 6-7 suberect, ovate-lanceolate to lanceolate, acute, the upper ones lanceolate, smaller, similar to the bracts. Inflorescence to $30 \mathrm{~cm}$ long, rather loosely many-flowered. Flowers white with brown veins. Floral bracts 10-15 mm long, ovate-lanceolate, acuminate. Pedicel with ovary $20 \mathrm{~mm}$ long, curved. Dorsal sepal $6 \mathrm{~mm}$ long and $2.5 \mathrm{~mm}$ wide, narrowly elliptic to narrowly obovate, obtuse, reflexed, convex. Petals bilobed to the base; anterior lobe $10-12 \mathrm{~mm}$ long, $1 \mathrm{~mm}$ wide, spreading forwards, linear-filiform, acute; posterior lobe $6 \mathrm{~mm}$ long and $0.5 \mathrm{~mm}$ wide, reflexed, linear-filiform. Lateral sepals $8 \mathrm{~mm}$ long and $4 \mathrm{~mm}$

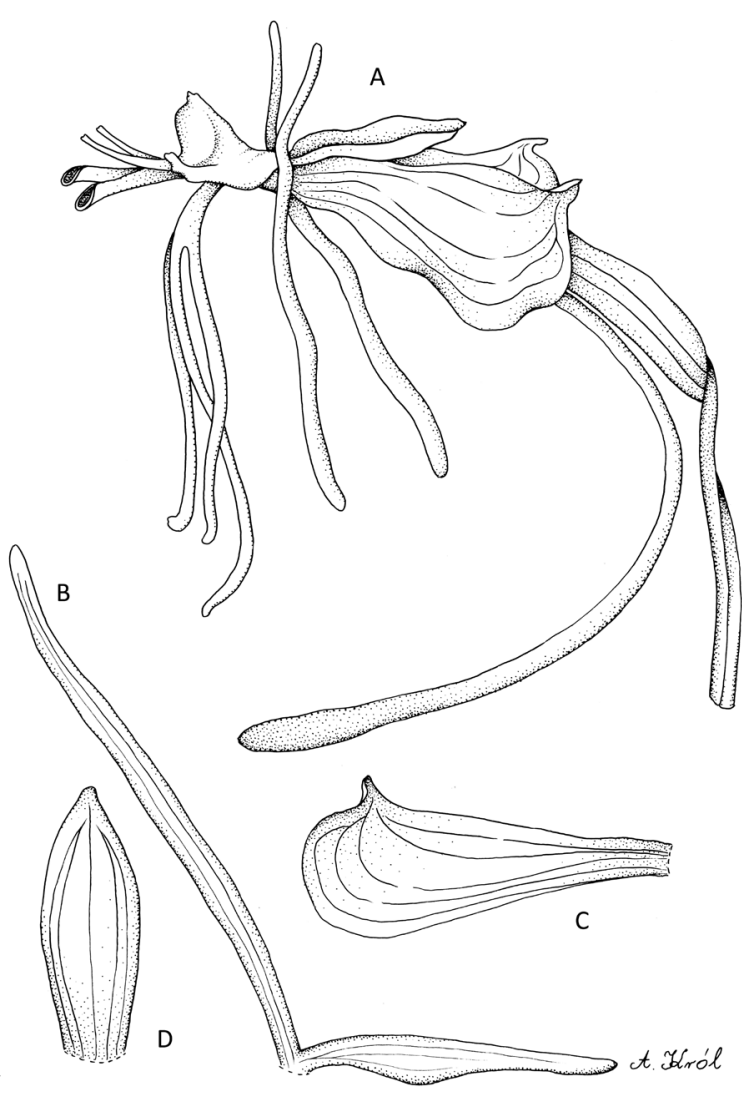

Fig. 145. Bilabrella ugandensis (Summerh.) Szlach. \& Kras-Lap. Explanations: A - flower; B - petal; C - lateral sepal; D - dorsal sepal (drawn by A. Król from holotype of Habenaria ugandensis Summerh. Lind 2464, K)

wide, deflexed, obliquely and narrowly obovate, apiculus lateral. Lip deflexed, 3-lobed from an undivided base 1-2 mm long; the middle lobe $10-12 \mathrm{~mm}$ long, $0.5 \mathrm{~mm}$ wide, linear, obtuse; lateral lobes $8 \mathrm{~mm}$ long, $0.5 \mathrm{~mm}$

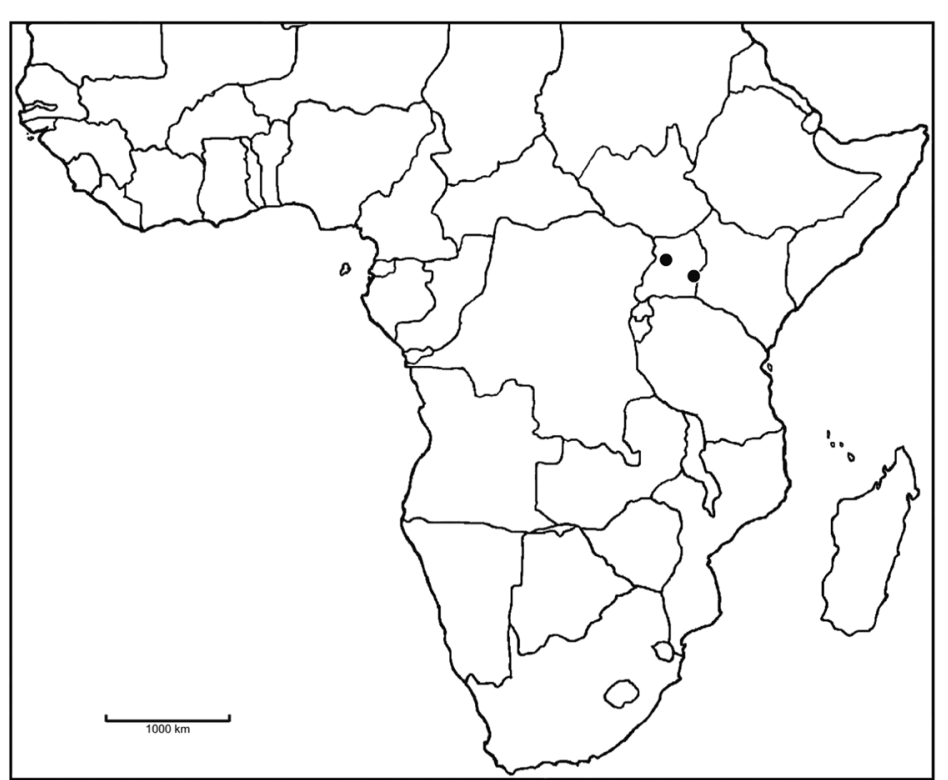

Fig. 146. Distribution of Bilabrella ugandensis (Summerh.) Szlach. \& Kras-Lap. 
wide, linear, acute. Spur $20 \mathrm{~mm}$ long, incurved, slightly swollen in the apical half, blunt. Gynostemium distinctly stalked, the stalk $2 \mathrm{~mm}$ long. Anther $3.5 \mathrm{~mm}$ tall; connective shortly apiculate; antherophores $4 \mathrm{~mm}$ long, slender, straight. Stigmaphores $5 \mathrm{~mm}$ long, narrowly cylindrical, obliquely truncate at the apex. Rostellum middle lobe shorter than thecae. Auriculae prominent, oblong-ovoid, irregular (Fig. 145).

E c o logy. Grassland, swamp, rocky granish hill. May.

D i s tribution. Endemic for Uganda? Alt. $1200 \mathrm{~m}$ (Fig. 146).

Specimens examined. Uganda. Embira: Oruchor Hill, Bulita, 6 miles N of Busia, Samia-Bugwe Co., an a rocky granish hill, 1200 m, 24 May 1951 (fl), Wood 228 (K!); $185 \mathrm{~km}$ on Masindi-Bombo road, Lind 2464 (K!)

$\mathrm{N}$ o t e $\mathrm{s}$. This species is characterised by the relatively small leaves, the rather long incurved spur and the distinct stalk of the gynostemium. Additionally, it differs from Bilabrella galpinii by longer inflorescence. It is similar to B. ndiana and B. macrostele in having stalked gynostemium, but despite both of them $B$. ugandensis has much longer antherophores and stigmaphores than fertile part of the anther.

49. Bilabrella galpinii (Bolus) Szlach.\& Kras-Lap., Richardiana 3(3): 140. 2003.

Habenaria galpinii Bolus, Icon. Orchid. Austro-Afr. 1(1): t. 17. 1893. - Kraenzlin, Orchid. Gen. Sp.: 218. 1901. - Rolfe, Fl. Cap. 5(3): 125. 1913. - Stewart et al., Wild Orchid. S. Afr.: 88. 1982. - la Croix \& Cribb, Fl. Zambes., Orchid. 11: 93. 1995. - Kurzweil \& Linder, Wild Orchids of Southern Africa: 128. 1999.

Type: Republic of South Africa, Galpin 392a (BOL not seen, K!, PRE not seen).

Stem 20-60 cm tall, erect or decumbent, slender, leafy. Leaves 4-8, 5-17 cm long, 0.5-1.7 cm wide, scattered, lanceolate to linear-laceolate, acuminate, the margins undulate, erect-spreading, the upper gradually smaller and passing into bracts. Inflorescence $11-15 \mathrm{~cm}$ long, loosely to densely many-flowered. Flowers green, green and white or yellow-green. Floral bracts 12$17 \mathrm{~mm}$ long, linear-lanceolate, acute, pubescent. Pedicel and ovary about 20-25 mm long, slender. Dorsal sepal 3-6 mm long, 1-2 mm wide, ovate to elliptic-ovate, obtuse, concave, externally scabrid. Petals bilobed to the base; anterior lobe 8-11 mm long, 0.8-1.2 mm wide, linear-lanceolate in the lower half, subulate above, acute, spreading; posterior lobe 5-6.5 $\mathrm{mm}$ long, $0.5 \mathrm{~mm}$ wide, linear, acute, erect. Lateral sepals 4.5-8 mm long, 3-4 mm wide, obliquely obovate, obtuse, obliquely apiculate. Lip 3-lobed, with basal claw c. $2 \mathrm{~mm}$ long, segments spreading; the middle lobe $10-13 \mathrm{~mm}$ long,

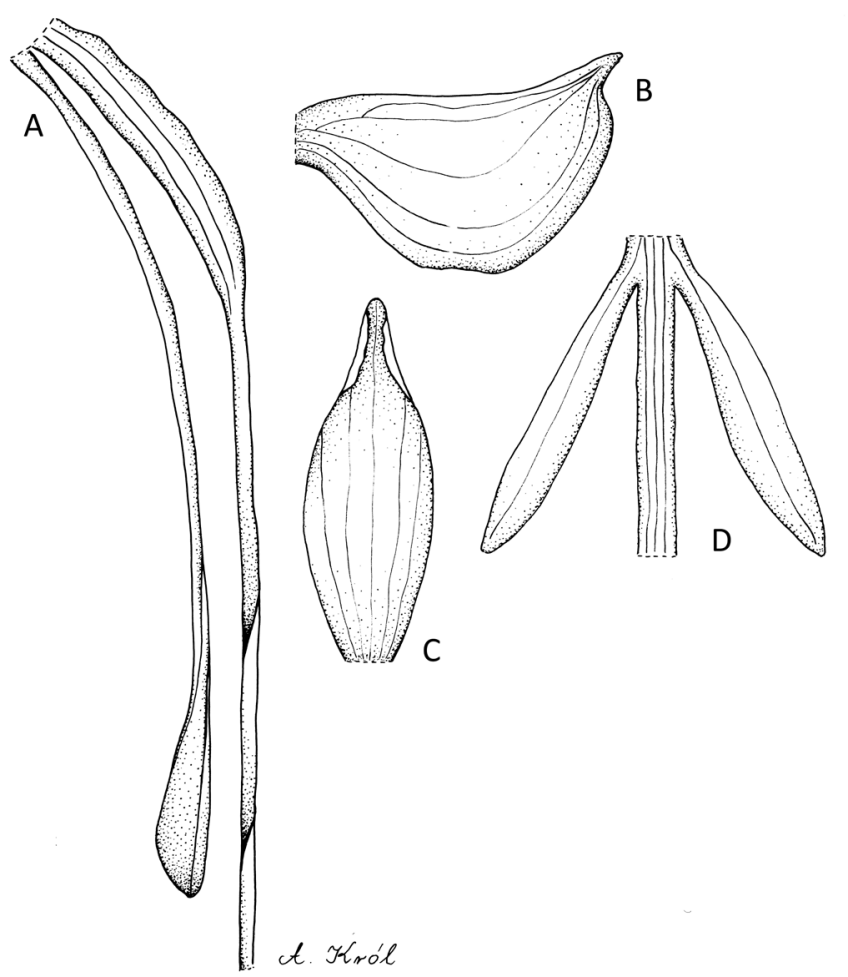

Fig. 147. Bilabrella galpinii (Bolus) Szlach.\& Kras-Lap. Explanations: A - ovary, pedicel, spur; B - lateral sepal; C - dorsal sepal; D - lip (drawn by A. Król from Habenaria galpinii Bolus - Hiliard \& Burtt 10263, E)

0.4-0.8 mm wide, linear, acute to acuminate; lateral lobes 7-8 $\mathrm{mm}$ long, $0.4 \mathrm{~mm}$ wide, linear-lanceolate, acuminate. Spur 13-23 mm long, pendulous, filiform, incurved, gradually inflated downward, blunt. Anther $3 \mathrm{~mm}$ tall, subglobose; connective shortly apiculate; antherophores 4.6-5.7 $\mathrm{mm}$ long, slender, straight. Stigmaphores $5.5 \mathrm{~mm}$ long, narrowly cylindrical, truncate. Rostellum middle lobe as long as thecae. Auriculae prominent, irregular (Fig. 147).

E c o log y. In open grassland, in vlei, in Eucalyptus plantation, rocky soil, along stream or on stony hillside. January-May.

Distribution. Zambia, Zimbabwe, Republic of South Africa, alt. 300-1800 m (Fig. 148).

S pecimens examined. Zambi. Southern: Matobo, Miller 4168 (K!). Zimbabwe. Harare: Salisbury, Matobo, Farm Besna Kobila, damp soil over rock, 1440 m, Mar. 1957 (fl), Miller 4161 (K!); Salisbury, Matobo, moist grassland on farm, $1440 \mathrm{~m}$, Mar. 1958 (fl), Miller 5127 (K!); Urungwe National Park, 276-286 km from Salisbury, on Chirundu road, rocky outcrops in open savannah woodland, 1000-1200 m, 21 Feb. 1981 (fl), Philox, Leppard, Dini 8800 (K! \& spirit.); Manicaland: Inyanga, Susurumba, 5 Feb. 1955 (fl), Ball 502 (SRGH 50243) (K! \& spirit.); Inyanga, in Eucalyptus plantation round orchard, $1800 \mathrm{~m}$, Mar. 1954 (fl), Payne 24 (SRGH 45946) (K! \& spirit.); Mashonaland West: Mwami, Apr. 1926 (fl), Band 


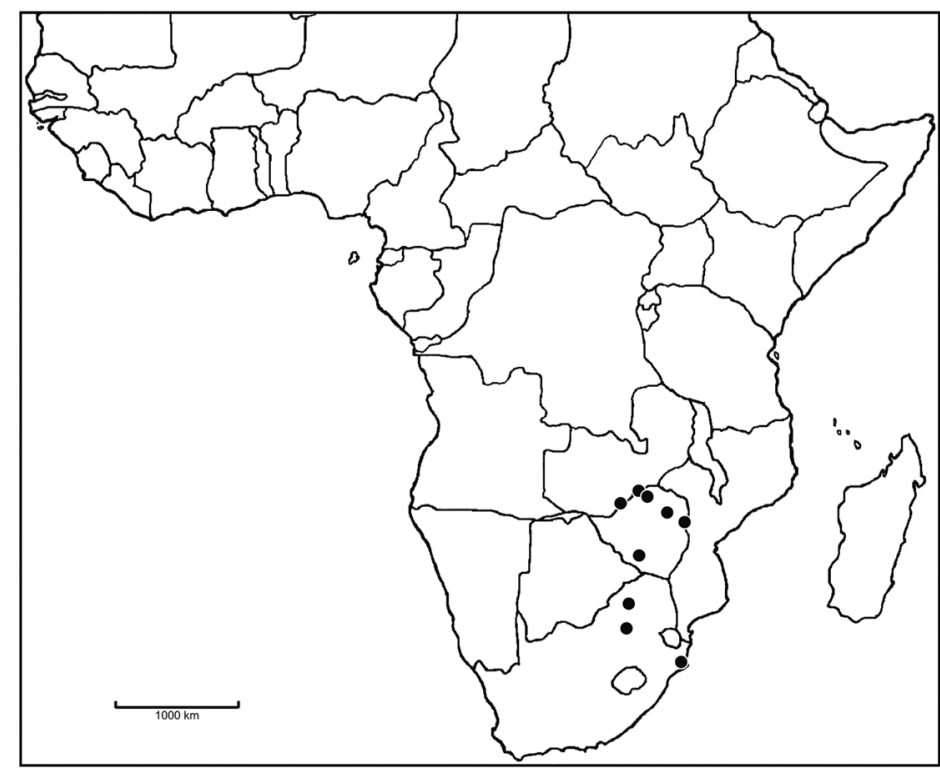

Fig. 148. Distribution of Bilabrella galpinii (Bolus) Szlach. \& Kras-Lap.

44 (BM!); Midlands: Belingwe Distr., Sikanajena hill, granite outcrop, W slope, sponge mat at wales edge of perennial stream, 1420 m, 4 May 1973 (fl), Pope, Biegel, Simon 1109 (K!). Republic of South Africa. Gauteng: Near Johannesburg, 1580 m, Galpin $392 a$ (K!); Pretoria, Iraskop Peak, on upper slopes, 500-1700 m, 31 Jan. 1937 (fl), Galpin 14441 (K!); Pretoria, Bakenkop, 9 miles E of Sabie, humus-rich sand among rocks, 900 m, 5 Mar. 1968 (fl), Leistner \& Mauve 3233 (K!); Kwazulu-Natal, Port Shepstone Distr., Hluhluwe Nature Reserve, 300 m, 26 Apr. 1977 (fl), Hiliard \& Burtt 10263 (E!); Limpopo: Transvaal, Palala area, Bamboeskloof, near source of Palala River, open grassveld, in vlei, grey sandy soil, 9 Mar. 1978 (fl), Venter 4220 (K!).

Notes. Bolus (1893) describing his new species, Habenaria galpinii, based on Galpin 392 collection from South Africa. In 1913 Rolfe proposed $H$. incurva based on the same collection. We had the opportunity to study type specimen of both species. Both differs from each other in some features - proportion in length between lateral and middle lip lobes and between anterior and posterior petal lobes. In our opinion these are key differences in this genus and both species should be maintained separately. Because relatively frequently Bilabrella species occur in mixed populations, it is obvious that Galpin collected two different species under the same number 392 . We proposed to segregate this collection into two different subcollection indicated by the letter $a$ and $b$.

B. galipinii can be distinguished from B. ugandensis by longer leaves, shorter inflorescence and sessile gynostemium. It is somewhat similar to $B$. weberana either, but has spur inflated apically, blunt and lip lobes acute.

\subsubsection{Section Biauriculatae Szlach. \& Kras, sect. nov.}

Type species: Bilabrella altior (Rendle) Szlach. \& KrasLap. [ $\equiv$ Habenaria altior Rendle]

Auricles bipartite, sessile or stalked.

\subsubsection{Subsection Decapterae Szlach. \& Kras, subsect. nov.}

Type species: Bilabrella decaptera (Rchb. f.) Szlach. \& Kras [三 Habenaria decaptera Rchb. f.].

Lip 3-lobed near the middle, both lateral lobes crossing each other below the middle lobe.

A monotypic subsection.

50. Bilabrella decaptera (Rchb. f.) Szlach. \& Kras, Richardiana 9(4): 158. 2009. - Szlachetko et al., Orchid. of West-Central Africa 1: 212. 2010.

Habenaria decaptera Rchb. f., Flora 65: 31. 1882.

Type: Angola, Mechow 438 (lectotype, designated by Szlachetko et al. [2010: 212] W-R!, K! - drawing).

Stem $45-60 \mathrm{~cm}$ tall, erect, rather stout, leafy. Leaves 9-15, up to $19 \mathrm{~cm}$ long and $1.7 \mathrm{~cm}$ wide, the longest near the middle of the stem, linear-lanceolate, acute, erect-spreading, decreasing in size up the stem. Inflores-

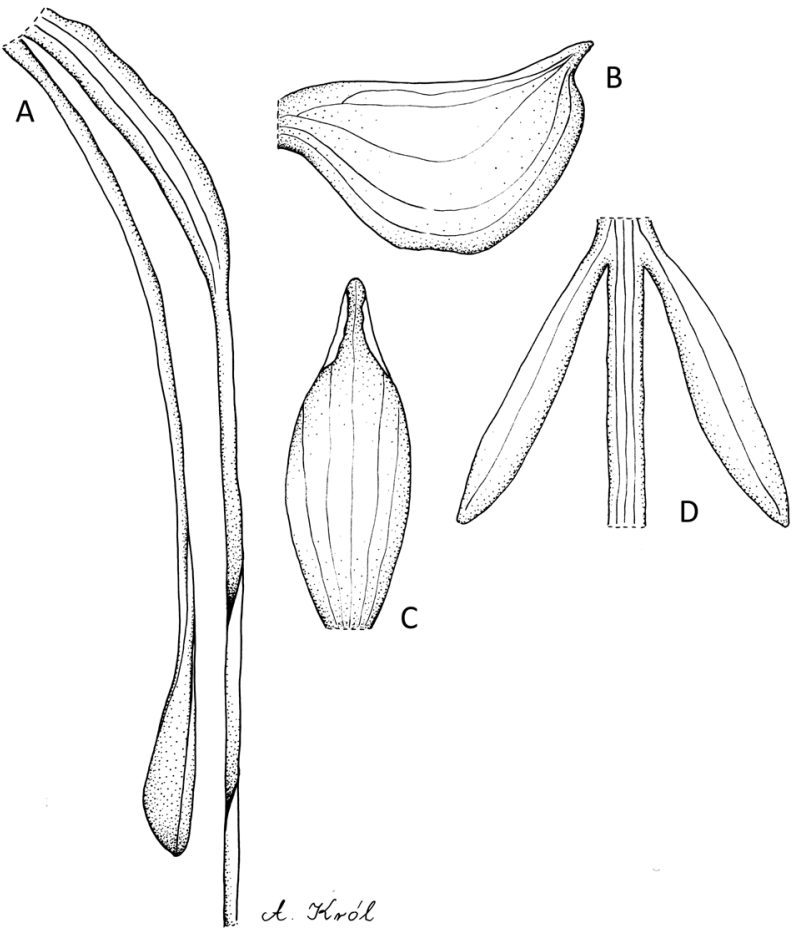

Fig. 149. Bilabrella decaptera (Rchb. f.) Szlach. \& Kras Explanations: A-flower; B-C - lip, various views (drawn by H.B. Margońska from lectotype of Habenaria decaptera Rchb. f. - Mechow 438, W-R) 


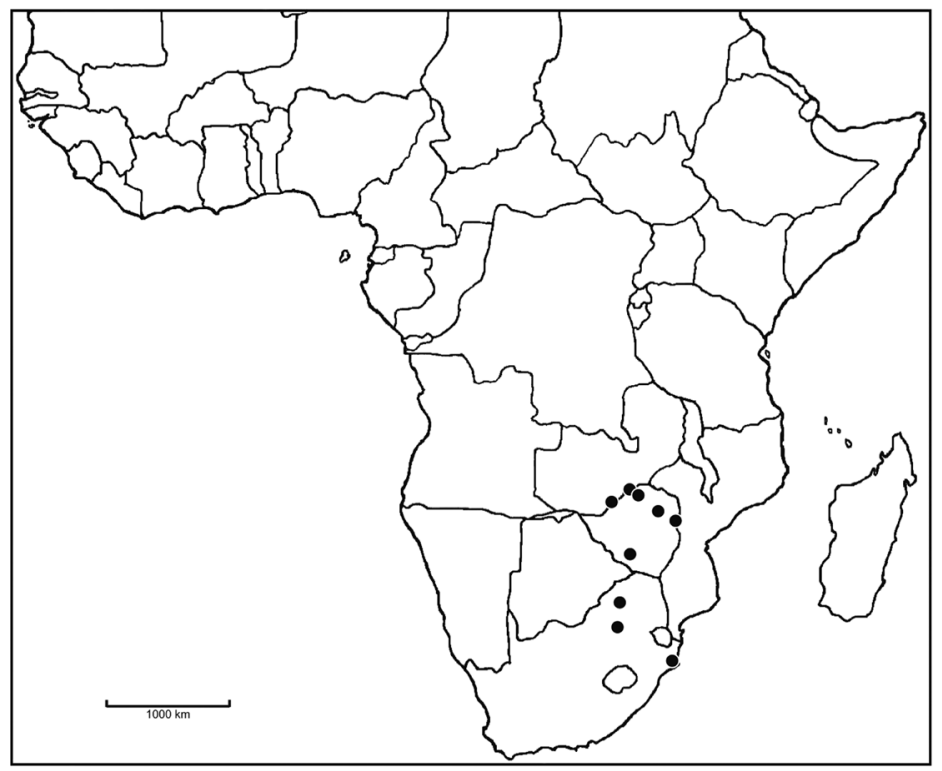

Fig. 150. Distribution of Bilabrella decaptera (Rchb.f.) Szlach. \& Kras

cence $8-11.5 \mathrm{~cm}$ long, densely multiflowered. Flowers white. Floral bracts $20 \mathrm{~mm}$ long, ovate-lanceolate, acuminate, densely ciliolate on margins, sparsely on outside. Pedicel and ovary to $27 \mathrm{~mm}$ long, slender. Dorsal sepal $7.5 \mathrm{~mm}$ long, $3.5 \mathrm{~mm}$ wide, oblongcuneate, concave, apiculate. Petals bilobed to the base, glabrous, thin; anterior lobe $9.5 \mathrm{~mm}$ long, $3 \mathrm{~mm}$ wide, oblong-oblanceolate, obtuse; posterior lobe $8.5 \mathrm{~mm}$ long, $2.5 \mathrm{~mm}$ wide; obliquely oblanceolate, subobtuse. Lateral sepals $8 \mathrm{~mm}$ long, $4 \mathrm{~mm}$ wide, obliquely cuneate to oblong-obovate, slightly concave in the centre, shortly apiculate. Lip 3-lobed near the middle, undivided basal part $5 \mathrm{~mm}$ long, triangular-ovate; the middle lobe $4.5 \mathrm{~mm}$ long, $3.5 \mathrm{~mm}$ wide, ovate, obtuse, with irregulary toothed margins; lateral lobes $4 \mathrm{~mm}$ long, $1.8 \mathrm{~mm}$ wide, ligulate-falcate, obtuse, crossed below the middle one. Spur $30 \mathrm{~mm}$ long, filiform, swollen towards subacute apex. Anther $2.5 \mathrm{~mm}$ tall; connective truncate; antherophores subequal to stigmaphores, $3 \mathrm{~mm}$ long. Rostellum middle lobe distinctly shorter than connective. Auriculae small, deeply and equally bilobed (Fig. 149).

E c o lo g y. Unknown. Flowering in January-February (Kraenzlin 1901).

Distribution. Endemic to Angola? (Fig. 150).

Specimens examined. Angola. Malange, Jan.-Feb. 1880 (fl), Mechow 438 (K! - drawing, W-R!). Notes. We have examined only type material 3 specimens - kept at W-R (35807) and drawing deposited at K. Very unusual feature of this species is lip 3-lobed near the middle, with both lateral lobes crossing together above the middle one. All other characters placed Habenaria decaptera in Bilabrella.

\subsubsection{Subsection Martiales Szlach. \& Kras, subsect. nov.}

Type species: Bilabrella martialis (Rchb. f.) Szlach. \& Kras-Lap. [三 Habenaria martialis Rchb. f.].

Both petal lobes lanceolate, oblong-lanceolate or oblanceolate, anterior lobe smaller than posterior one.

\section{Key to species}

1. Spur twisted. Petals divided distinctly above the base 51. B. peltates

1. Spur not twisted. Petals divided to the base

2. The upper part of posterior petal lobe clearly toothed, auriculae obscurely bilobed

52. B. martialis

2. The upper part of posterior petal lobe entire, glabrous, auriculae inconspicuous

53. B. kilimanjari

51. Bilabrella peltastes (Rchb. f.) Szlach. \& Kras-Lap., Richardiana 3(3): 142. 2003.

Habenaria peltastes Rchb. f., Otia Bot. Hamburg.: 99. 1881. - Rolfe, Fl. Trop. Afr. 7: 238. 1898. - Kraenzlin, Orchid. Gen. Sp.: 213. 1901.

Type (hoc loco selectus): Eritrea, Steudner 701 (lectotype, W-R!; isolectotype, K! - fragment).

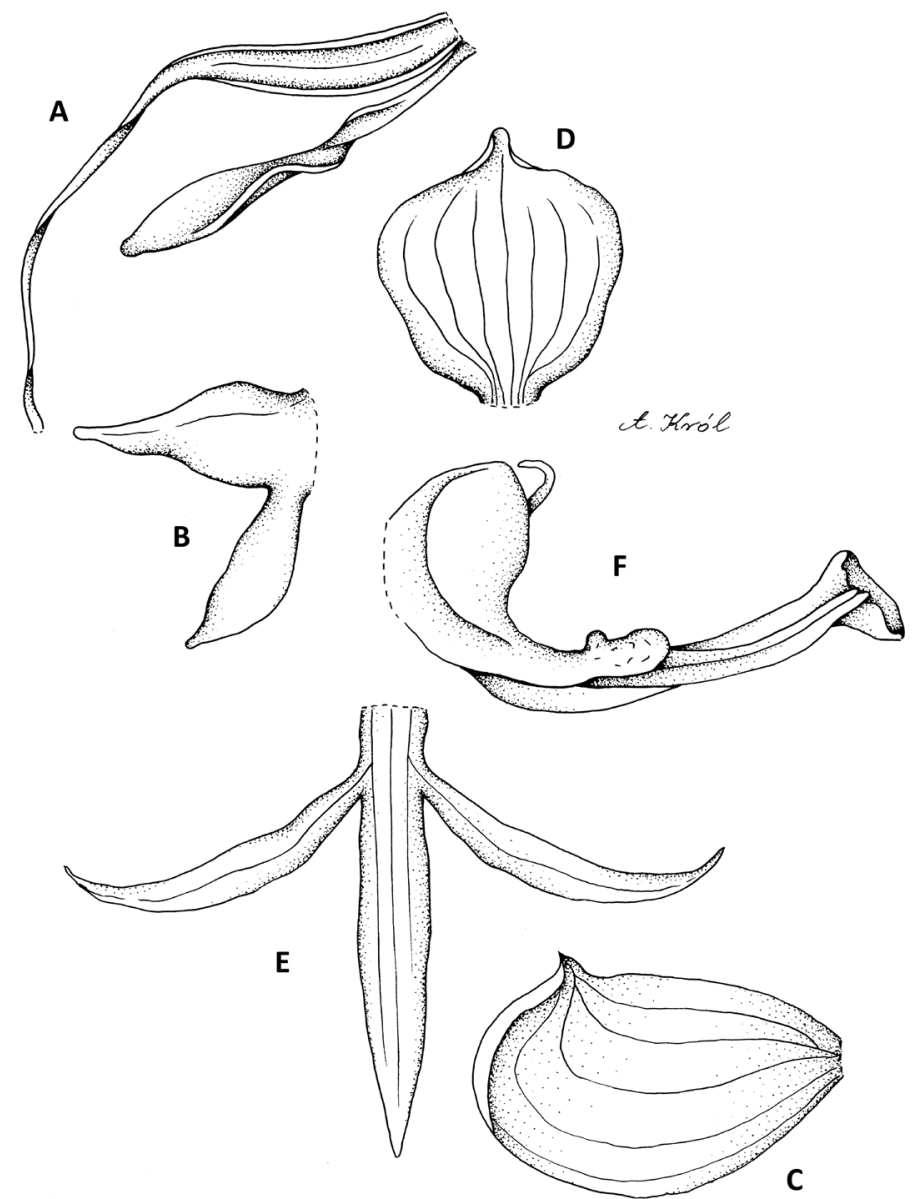

Fig. 151. Bilabrella peltastes (Rchb. f.) Szlach. \& Kras-Lap. Explanations: A - ovary, pedicel, spur; B - petal; C - lateral sepal; D - dorsal sepal; E - lip; F - gynostemium, side view (drawn by A. Król from lectotype of Habenaria petalstes Rchb. f. - Steudner 701, W-R) 
Stem to $100 \mathrm{~cm}$ tall, erect, with two sheaths at the base. Leaves 6-7 on the lower part of the stem, lanceolate, acuminate, nerved below. Inflorescence elongate, lax, many-flowered. Flowers completely glabrous. Flower bracts $12 \mathrm{~mm}$ long, lanceolate-elliptic, acuminate. Pedicellate ovary $24 \mathrm{~mm}$ long, slender. Dorsal sepal $5 \mathrm{~mm}$ long, $5 \mathrm{~mm}$ wide, suborbicular to orbicular-obovate, shortly apiculate, subobtuse, concave. Petals bilobed above the base; anterior lobe $2.5 \mathrm{~mm}$ long, $0.8 \mathrm{~mm}$ wide, oblong- or ligulate-oblanceolate, shortly apiculate, nerveless; posterior lobe $3.5 \mathrm{~mm}$ long, $1.2 \mathrm{~mm}$ wide, obliquely triangular-lanceolate, apiculate, 2-nerved. Lateral sepals $6 \mathrm{~mm}$ long, $4 \mathrm{~mm}$ wide, obliquely obovate, apiculate in the superior angle, concave. Lip 3-lobed above the basal $2 \mathrm{~mm}$ claw; the middle lobe $6 \mathrm{~mm}$ long, $1.8 \mathrm{~mm}$ wide, linear- or ligulatelanceolate, acuminate; lateral lobes $4 \mathrm{~mm}$ long, $0.5 \mathrm{~mm}$ wide, linear-lanceolate, acute, gently upcurved. Spur $15 \mathrm{~mm}$ long, narrowly cylindrical-clavate, subobtuse, twisted near the middle, parallel to the pedicel and ovary. Anther $2.8 \mathrm{~mm}$ long, antherophores and stigmaphores 4-4.5 mm long. Rostellum middle lobe longer than connective, deflexed apically. Auriculae large, shallowly and very unequally bilobed (Fig. 151).

Ec ology. No records. August.

Distribution. Eritrea only? (Fig. 152).

$\mathrm{S} p$ ecimens examined. Eritrea. Anseba: Lalamba near Keren, Aug. 1861 (fl), Steudner 701 (W$\mathrm{R}$ !, $\mathrm{K}$ ! - drawing.).

Notes. The vegetative parts are known from the original description only. Reichenbach (cf. Rolfe 1898) stated that the type specimen was kept at B where, however, Kraenzlin had been unable to find it. The

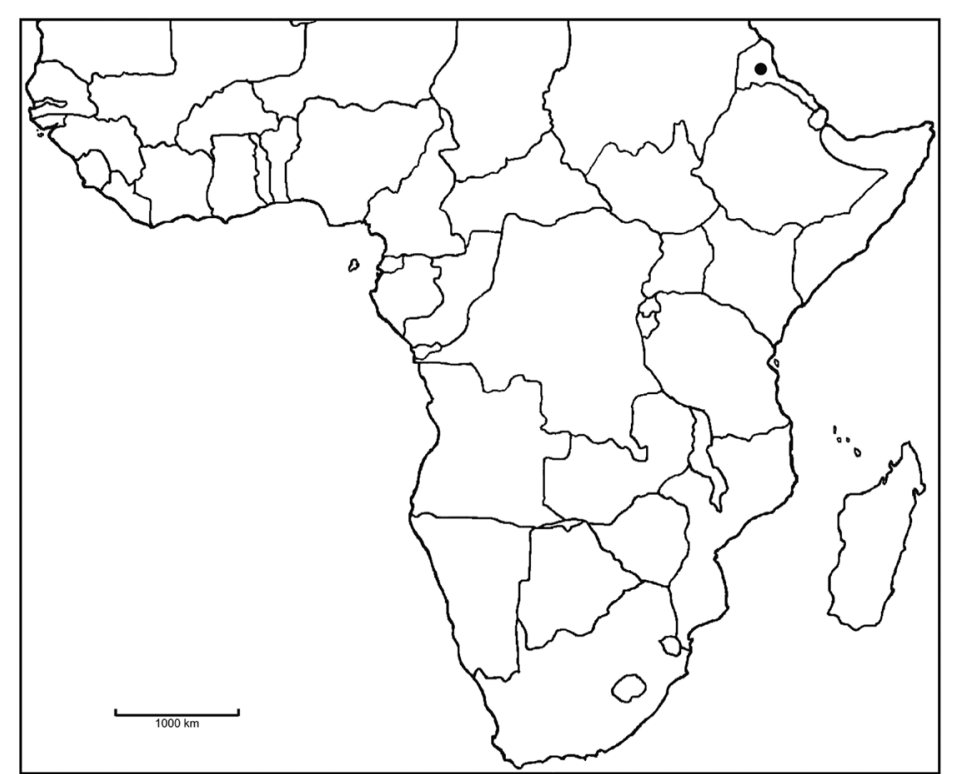

Fig. 152. Distribution of Bilabrella peltastes (Rchb.f.) Szlach. \& Kras-Lap. only materials we examined kept at W-R and probably represented part of the type specimen consist of tracing of the plant habit and some flowers in envelope. The specimen at $\mathrm{K}$ bears a single flower in envelope and Summerhayes drwing of it.

Bilabrella peltastes is probably related to $B$. martialis, differing from it however, in several features, as petal lobation, lip morphology, ratio between anther and stigma and anther projections and spur length and shape.

52. Bilabrella martialis (Rchb. f.) Szlach. \& Kras, Richardiana 9(4): 159. 2009.

Habenaria martialis Rchb. f., Otia Bot. Hamburg.: 99. 1881. - Rolfe, Fl. Trop. Afr. 7: 236. 1898.

Type: Mozambique, Kirk s.n. (lectotype, designated by Szlachetko et al. [2010: 227], K!).

Habenaria uhligii Kraenzl., Bot. Syst. Jahrb. 43: 396. 1909.

Type: Tanzania, Uhlig $1(\mathrm{~B}+$; lectotype, designated by Szlachetko et al. [2010: 227], K! - fragment) \& Uhlig 11 (B+; paratype, $\mathrm{K}$ ! - fragment).

Habenaria lutaria Schltr., Bot. Syst. Jahrb. 53: 515. 1915.

Type: Tanzania, Stolz 214 (B+; lectotype, designated by Szlachetko et al. [2010: 227], K!; isolectotypes, C!, $\mathrm{S}$ not seen).

Stem $25-40 \mathrm{~cm}$ tall, erect, slender, glabrous. Leaves 4-8, 8-11 cm long, 0.8-2.5 cm wide, linear-lanceolate, acute, erect to spread, decreasing in size up the stem. Inflorescence $9 \mathrm{~cm}$ long, 25 -flowered, lax. Floral bracts 8-20 mm long, elliptic-ovate, acuminate. Pedicel and ovary 13-20 mm long, glabrous. Dorsal sepal 5-7.5 mm long, 2-3 mm wide, oblong-elliptic, obtuse, glabrous. Petals bilobed to the base; anterior lobe 3.5-6 $\mathrm{mm}$ long, $1.2-2 \mathrm{~mm}$ wide, obliquely narrowly lanceolate to ovate, obtuse, glabrous; posterior lobe 5.5-7.5 mm long, 0.8$2 \mathrm{~mm}$ wide, obliquely lanceolate to oblanceolate, acute, the upper part clearly toothed, glabrous. Lateral sepals 6-8.5 mm long, 2-4.5 mm wide, obliquely ellipticovate, concave, laterally acute, glabrous. Lip 3-lobed to the base, glabrous; the middle lobe 5-11 mm long, 0.4-0.9 mm wide, linear-filiform, subobtuse; lateral lobes 3-7 mm long, 0.6-1.2 mm wide, obliquely lanceolate, spread, subacute. Spur 13-17 mm long, narrowly cylindrical in the upper part, inflated in the apical third, blunt, parallel to the ovary. Anther 2.5-3.5 mm tall; connective truncate, shortly apiculate; antherophores 3-4 mm long, slender, straight. Stigmaphores 2-3 mm long, narrowly clavate to cylindrical, relatively massive, truncate. Rostellum middle lobe subulate, shorter than thecae. Auriculae obscurely bilobed (Figs 153155).

E c o log y. No records. January-March. 

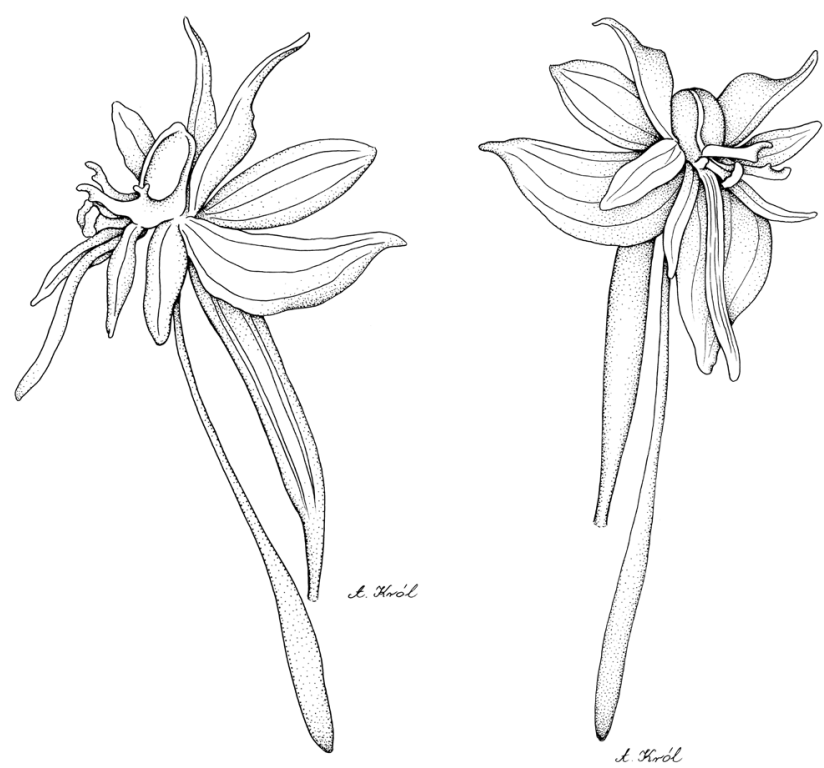

Fig. 153. Bilabrella martialis (Rchb. f.) Szlach. \& Kras - flower (drawn by A. Król from lectotype of Habenaria lutaria Schltr. Stolz 214, K)

Distribution. Tanzania, Mozambique, Angola, alt. 550-1550 m (Fig. 156).

Specimens examined. Tanzania. Mbeya: Near Nyasa Mountain, Station Kyimbila, 1550 m, 1909 (fl), Stolz 214bis (C!); Rungwe Distr., Mwaya (Muaja), 550 m, Mar. 1910 (fl), Stolz 214 (B+, C!, K!, S n.v.); Tanga: Masai Steppe, by R. Pangani, Uhlig $1 \& 11(\mathrm{~B}+)$. Mozambique. Mouth of Rovuma river, 29 Mar. 1861 (fl), Kirk s.n. (K!). Angola. Moxico: Ikula Hot Springs by River Zambezi, 17 Jan. 1938 (fl), Milne-Redhead 4189 (AMES!, BM!, BR!, K!, UGDA-DLSz! - fragment, drawing).

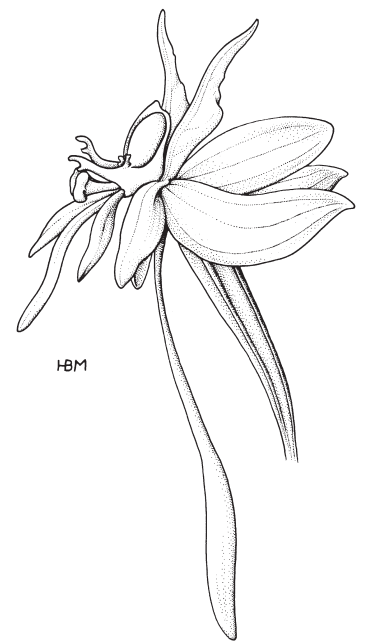

Fig. 154. Bilabrella martialis (Rchb. f.) Szlach. \& Kras - flower (drawn by H.B. Margońska from lectotype of Habenaria martialis Rchb. f. - Kirks.n., K)

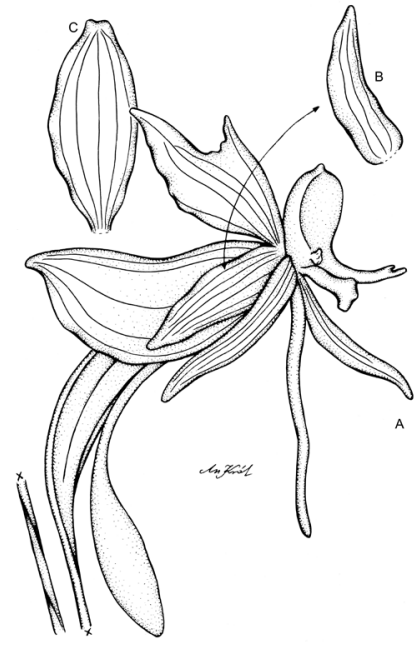

Fig. 155. Bilabrella martialis (Rchb. f.) Szlach. \& Kras Explanations: A - flower; B - dorsal sepal; $\mathrm{C}$ - anterior petal lobe (drawn by A. Król from Milne-Redhead 4189, AMES)

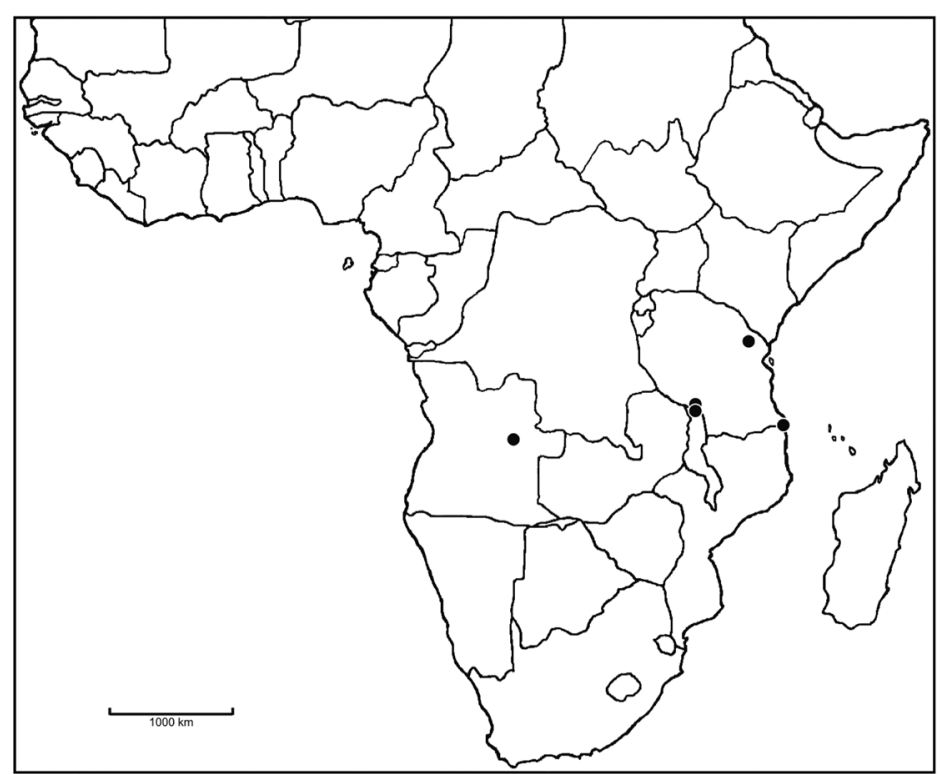

Fig. 156. Distribution of Bilabrella martialis (Rchb.f.) Szlach. \& Kras

Notes. Antherophores and stigmaphores of Bilabrella martialis are relatively short and massive. The species appears to be very similar - maybe conspecific - to B. kilimanjari. The features of B. martialis absent in B. kilimajari are: the upper part of the posterior petal lobe clearly toothed, the lip lateral lobes evidently lanceolate and obscurely bilobed auriculae. Examination of the type specimens of $H$. uhligii and H. lutaria leads us to the conclusion that they are conspecific with B. martialis.

53. Bilabrella kilimanjari (Rchb. f.) Szlach. \& KrasLap., Richardiana 3(3): 141. 2003. - Szlachetko et al., Orchid. of West-Central Africa 1: 227. 2010.

Habenaria kilimanjari Rchb. f., Otia Bot. Hamburg.: 119. 1881. - Rolfe, Fl. Trop. Afr. 7: 240. 1898. - Kraenzlin, Orchid. Gen. Sp.: 213. 1901. - Symmerhayes, Bull. Misc. Inform., Kew 1933: 249. 1933. - Summerhayes, Kew Bull. 16: 284. 1962. - Summerhayes, FTEA, Orchid. 1: 83. 1968b. - Geerinck \& Coutrez, Not. Taxon. Orchid. Afr. Centr., Habenaria: 9. 1977, (as H. kilimandjari). - Williamson, Orchid. S. Centr. Afr.: 51. 1977. - Geerinck, Fl. Afr. Centr., Orchid. 1: 110. 1984. - la Croix et al., Orchid. Malawi: 72. 1991. - la Croix \& Cribb, Fl. Zambes., Orchid. 11: 98. 1995. - Stewart \& Campbell, Orchids of Kenya: 136. 1996.

Type: Tanzania, New s.n. (lectotype, designated by Szlachetko et al. [2010: 227], K!).

Habenaria theodorii Kraenzl., Vierteljahrschr. Naturf. Ges. Zürich 74: 107. 1929.

Type: Zaire, Kassner 2486 (B+; lectotype, designated by Szlachetko et al. [2010: 227], K!, isolectotype, $\mathrm{BR}$ !). 


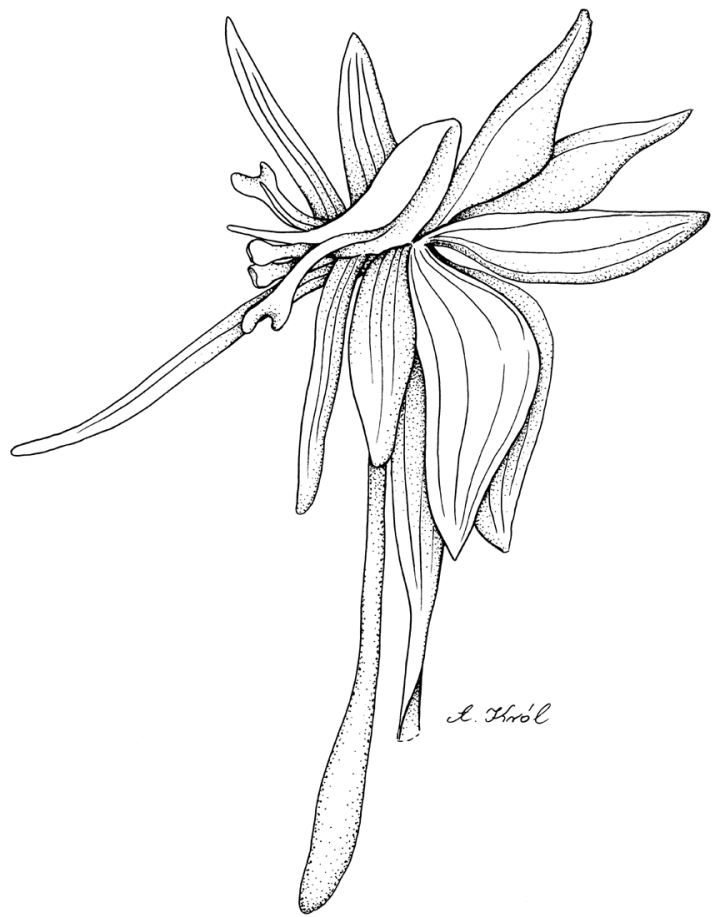

Fig. 157. Bilabrella kilimanjari (Rchb. f.) Szlach. \& Kras-Lap.flower (drawn by A. Król from lectotype of Habenaria theodorii Kraenzl. - Kassner 2486, K)

Tubers $1-2.5 \mathrm{~cm}$ long, $0.5-1.5 \mathrm{~cm}$ in diameter, ovoid or ellipsoid. Stem $15-90 \mathrm{~cm}$ tall, erect, rather slender to relatively stout, glabrous. Leaves 7-13, 8-23 cm long, $1-2.5 \mathrm{~cm}$ wide, linear-lanceolate to narrowly lanceolate, acute, erect or suberect, decreasing in size up the stem. Inflorescence $4-37 \mathrm{~cm}$ long, multiflowered, dense or lax. Flowers white with the apex of spur green. Floral bracts $8-30 \mathrm{~mm}$ long, elliptic-lanceolate to oblong-

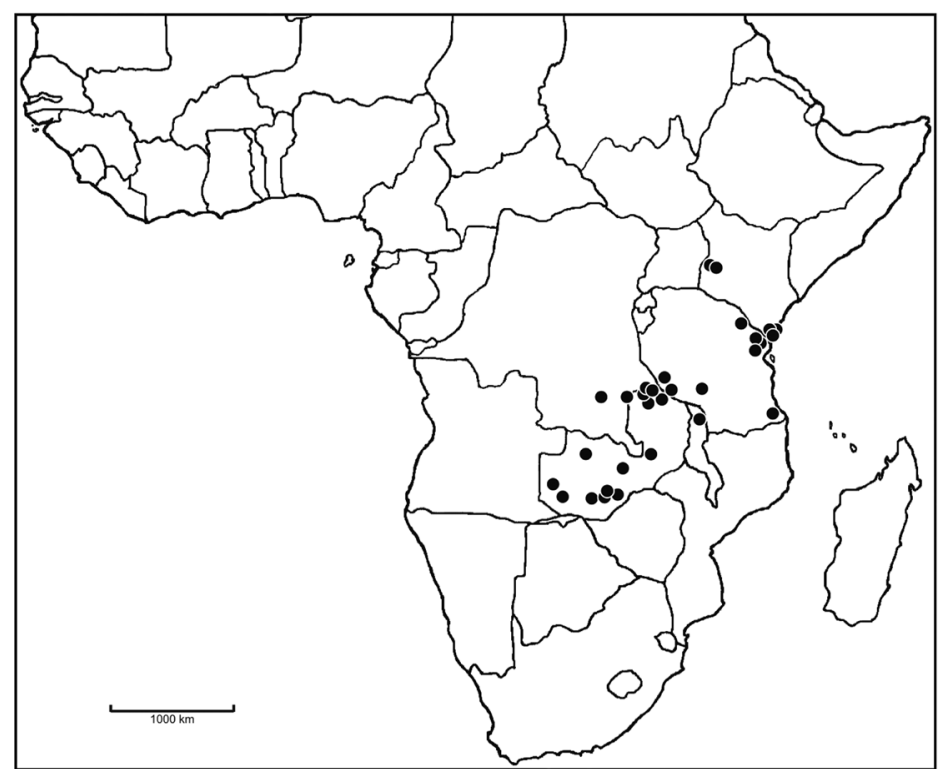

Fig. 158. Distribution of Bilabrella kilimanjari (Rchb.f.) Szlach. \& Kras-Lap. lanceolate, acuminate. Pedicel and ovary $15-25 \mathrm{~mm}$ long, glabrous. Dorsal sepal 5-7.5 mm long, 2-3 mm wide, oblong-elliptic, obtuse, cochleate, glabrous. Petals bilobed to the base; anterior lobe $3.5-5 \mathrm{~mm}$ long, 1.2-1.5 mm wide, narrowly lanceolate, obtuse, glabrous; posterior lobe 5-7.5 mm long, 1-2 mm wide, lanceolate to oblanceolate, acute, the upper part entire, glabrous. Lateral sepals 6-8.5 mm long, 2.5-4.5 mm wide, obliquely elliptic-ovate to semi-orbicular, slightly concave, glabrous. Lip 3-lobed nearly to the base, thin, glabrous; the middle lobe $5-11 \mathrm{~mm}$ long, $0.4 \mathrm{~mm}$ wide, filiform, subobtuse; lateral lobes 3-5 mm long, $0.6 \mathrm{~mm}$ wide, narrowly lanceolate, subacute. Spur 13-17 mm long, narrowly cylindrical, parallel to the ovary, inflated in the apical third, blunt. Anther $3 \mathrm{~mm}$ tall; connective rounded; antherophores $3.5 \mathrm{~mm}$ long, slender, slightly upcurved. Stigmaphores $2 \mathrm{~mm}$ long, rather massive. Rostellum middle lobe as long as connective. Auriculae inconspicuous, bifid (Fig. 157).

E c o logy. Periodically flooded grassland, often with scattered bush, dark clay loam soil in marsh. JanuaryDecember.

Distribution. Democratic Republic of the Congo (Zaire), Kenya, Tanzania, Zambia, Malawi, alt. 90$2400 \mathrm{~m}$ (Fig. 158).

Specimens examined. Democratic Republic of the Congo (Zaire). Haut-Katanga: Regions of Tchowa and Mweru Wantipa, 25 Aug. 1939 (fl), Breds 3686 (K!); Mweru Wantipa, 6 Jan. 1944 (fl), Breds 5904 (BR!); Luente, near swamp, 24 Feb. 1906 (fl), Kassner 2486 (BM!); Haut-Lomami: National Park Upemba, Mabwe sector, savannah, E side of Upemba lake, 600 m, 14 Jan. 1959 (fl), De Wilde 700 (BR!); National Park Upemba, De Witte 5205 (BR!); Prov. Bukama Terr., Mabwe, National Park Upemba, 15 Jan. 1949 (fl), De Witte 5254 (BR!, K!); Kasaï: Kapanga, 1932 (fl), Overlaet 532 (BR!). Kenya. Coast: Likoni, grassland scrub, Aug. 1933 (fl), Blake 5996 (K!); Kwale Distr., Digo country near Mwele in swamp, July 1936 (fl), Dale 3547 (K!); North Eastern: Kichongolia, Matovia on Gazi-Mwele Road, in somewhat swampy land, 90 m, Gragam 1596 (K!); Rift Valley: Trans Nzoia, prison farm near Kitale, in grass, wet savannah, 1800 m, July 1970 (fl), Griesback via Tweedie 3828 (K!); Uasin Gishu, Bwent forest, in grass, $2250 \mathrm{~m}$, Aug. 1970 (fl), Griesback via Tweedie 3830 (K!). Tanzania. Kilimanjaro: Kilimanjaro, New s.n. (K!); Lindi: Lindi Distr., Lutamba-Sea, $40 \mathrm{~km}$ W from Lindi, 200-250 m, 5 Mar. 1935 (fl), Schlieben 6085 (BM!, BR!); Mbeya: Usangu, Ruiwa, in swampy grassland, $1300 \mathrm{~m}, 11 \mathrm{Feb}$. 1979 (fl), Cribb, Grey-Wilson \& Mwasumbi 11399 (K!); Rukwa: Chikanda (Chimambwe), Rukwa Valley, dotted in Chloris and Hyparrhenia zones of flood plain, able to stand some flooding by brackish lake water, 8 Feb. 1938 (fl), Michelwe 1477 (K!); Rukwa 
Valley, open grassy plain, seasonally flooded, among short grass, 900 m, 8 Feb. 1947 (fl), Pielou 85 (AMES!, $\mathrm{K}$ !); Central Rukwa, Kafukola, flood plain grassland, 792 m, 12 Feb. 1954 (fl), Siame 325 (K!); North Rukwa, Nziga, Mimosa woodland by the plain, 780.6 m, 2 Feb. 1955 (fl), Siame 565 (K!); Ufipa, Nziga, open plain with Cynodon, Cyperus and small patches of Sokwa, 900 m, 15 Feb. 1950 (fl), Bullock 2453 (AMES!, BR!, $\mathrm{K}$ ! - spirit.); Ufipa Distr., Raft valley, in slightly bare patches chloris near bush of flood plain, $810 \mathrm{~m}, 10 \mathrm{Apr}$. 1936 (fl), Lea 21 (K!); Ufipa Distr., Soda Locust Camp. Rukwa Escarpment, in damp grassland in flood plain, 780 m, 8 Feb. 1962 (fl), Richards 16027 (K!); Tanga: Korogwe, Mkujani in extra wet patches of black soil, grass in large numbers but quickly over, $296 \mathrm{~m}, 27$ June 1963 (fl), Auchlsed 275 (K!); Korogwe Distr., Magemga Estate, among grass in law lying pand, in swampy during the rains, 300 m, 11 July 1952 (fl), Fauekm s.n. (K!); Korogwe Distr., Magemga Estate, 11 July 1952 (fl), Faulkner 974 (AMES!, BR!); Korogwe, west foot of W Usamabaras, locally common amongst grasses on a black cotton soil subject to periodical flooding, $330 \mathrm{~m}$, 6 July 1934 (fl), Greenway 4016 (K!); Korogwe, Nov. 1984 (fl), Mathew s.n. (K!); Lushoto Distr., LushotoMombo road, 4 miles SE Lushoto, Western Usambaras, grassland with scattered clumps of bushes, $1250 \mathrm{~m}$, 10 June 1953 (fl), Drummond \& Hemsley 2885 (K!); Flats by Lwengera River, 4 miles ENE of Korogwe, river valley flats, in grassy areas on cracking soils, $300 \mathrm{~m}$, 27 June 1953 (fl), Drummond \& Hemsley 3061 (AMES!, K!); T3 Korogwe Distr., Kisalaka, Near Mnyusi Rly Station, 20 June 1972 (fl), Semsei 4248(BR!, K!); Maurui, July 1894 (fl), Volkens 2371 (BM!). Zambia. Northern: Musosa, Mission antiecridienne, 1939 (fl), Bredo 3686 (BR!); Western: (Barotse Prov. of F.Z.). Songea Distr., $10 \mathrm{~km} \mathrm{~S}$ of Senanga, flats on E side of Zambezi River, clay soil, 31 Jan 1975 (fl), Brummitt, Chisumpa \& Polhill 14193 (BR!, K!); North-Western: east part of country, near Kasempa, Lufuu River, 1921-1922 (fl), Foster s.n. (BM!, K!); Northern: Mporokoso Distr., Chipilipili Plain, Mweru-Wantipa, in grassland, clay soil damp, 1000 m, 15 Dec. 1960 (fl), Foster 13712 (K!); Arbercon Distr., Mpulugu, marsh near N'mkole, flooded grassland, 780 m, 20 Feb. 1964 (fl), Foster 19054 (K!); Southern: Mazabuka, near bank of Miangama Stream, 4 miles W of Monze African Compound, open dambo, very wet, 23 Jan. 1963 (fl), Linley 313 (K!); Central: Broken Hill, dambo at Mukobeko Road, 24 Jan. 1961 (fl), Morze 54 (K! \& spirit.); Serenje, grass dambos, Jan. 1967 (fl), Odgers 248 (K!); Southern: Namwala Distr., 1935 (fl), Read 19 (AMES!, K!); Namwala, on the edge of the Mulela flood plain on dark clay loam soil, 15 Jan. 1964 (fl), Rensburg 2771 (K!); Northern: Mbala Distr., Mpulungu, 900 m, 12 Feb. 1957 (fl), Richards 8175 (AMES!, BR!, K!); Southern: Mbeza, N of Mapanza, in dambo, 990 m, 21 Jan. 1954 (fl), Robinson 476 (BR!, K!); Simasunda, Mapanza N Dambo, 1050 m, 2 Feb. 1957 (fl), Robinson 2126 (K!); Western: Mongu, damp flood plain, 20 Jan. 1966 (fl), Robinson 6808 (K!). Malawi. Northern: Nyika, Chelinda Camp, 21 Apr. 1982 (fl), la Croix \& Johnston-Stewart 318 (K!); Mwanemba, 2400 m, Feb. 1903 (fl), McCloune 57 (K!).

$\mathrm{N}$ otes. Bilabrella kilimanjari is closely related to B. martialis and differs mostly in the posterior petal lobes. In the former they have entire margins, wheras in the latter irregularly toothed. As stated previously, both species might be conspecific and were treated as such by Szlachetko et al. (2010).

\subsubsection{Subsection Disparilae Szlach. \& Kras, subsect. nov.}

Type species: Bilabrella disparilis (Summerh.) Szlach. \& Kras-Lap. [三 Habenaria disparilis Summerh.].

Anterior petal lobes bilobulate.

\section{Key to species}

1. Both anterior petal lobules oblong triangular, acute, glabrous 54. B. disparilis 1. Anterior petal lobe rounded, with laterally placed acute apex, ciliolate in the lower part

55. B. usambarae

54. Bilabrella disparilis (Summerh.) Szlach. \& Kras.-Lap., Richardiana 3(3): 140. 2003. - Szlachetko et al., Orchid. of West-Central Africa 1: 225. 2010.

Habenaria disparilis Summerh., Kew Bull. 16: 277. 1962. - Grosvenor, Excelsa 6: 83. 1976. - Geerinck \& Coutrez, Not. Taxon. Orchid. Afr. Centr., Habenaria: 8. 1977. - Williamson, Orchid. S. Centr. Afr.: 53. 1977. - Geerinck, Fl. Afr. Centr., Orchid. 1: 102. 1984. - la Croix et al., Orchid. Malawi: 70. 1991. - la Croix \& Cribb, Fl. Zambes., Orchid. 11: 90. 1995.

Type: Malawi, Robson 1345 (holotype, K! \& spirit.).

Tubers $2.5-3 \mathrm{~cm}$ long, 1-2 $\mathrm{cm}$ in diameter, ellipsoid. Stem $50-90 \mathrm{~cm}$ tall, erect, robust, glabrous. Leaves $7-13,10-20 \mathrm{~cm}$ long, $1.5-2.5 \mathrm{~cm}$ wide, linear-lanceolate, acute, decreasing in size up the stem; lower leaf sheaths reticulately veined. Inflorescence $10-30 \mathrm{~cm}$ long, fairly densely many-flowered, almost all flowers opening together. Flowers green, white in centre. Floral bracts $20 \mathrm{~mm}$ long, elliptic-lanceolate, acuminate, somewhat scarious. Pedicel and ovary 12-22 mm long, glabrous. Sepals all reflexed. Dorsal sepal 5-5.5 mm long, $2 \mathrm{~mm}$ wide, oblong elliptic-obovate, obtuse, concave. Petals bilobed to the base, glabrous; anterior lobe $10-13 \mathrm{~mm}$ long, 2.5-3 mm wide, elliptic- or ovate-lanceolate, bilobulate, both lobules oblong triangular, acute; posterior lobe 6-9 $\mathrm{mm}$ long, less than $1 \mathrm{~mm}$ wide, linear-filiform, 


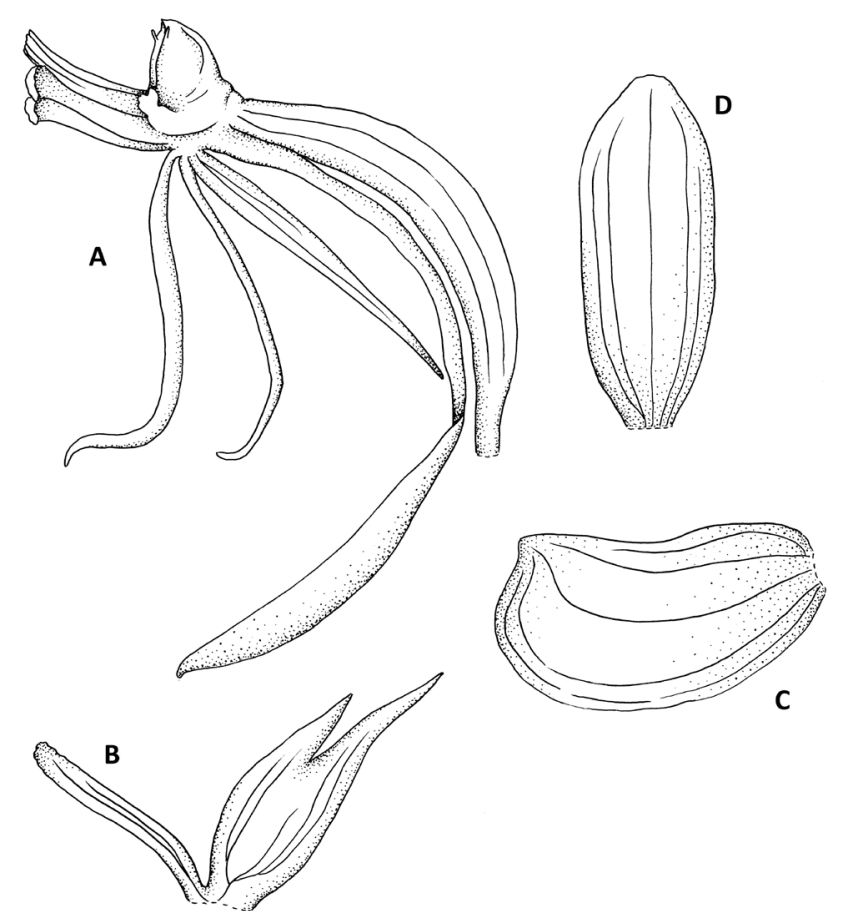

Fig. 159. Bilabrella disparilis (Summerh.) Szlach. \& Kras-Lap. Explanations: A - pedicel, ovary, gynostemium, lip and spur; B - petal; C lateral sepal; D - dorsal sepal (drawn by A. Król from holotype of Habenaria disparilis Summerh. - Robson 1345, K)

obtuse. Lateral sepals 7-8 mm long, 3-4.5 mm wide, obliquely elliptic-obovate, concave, laterally shortly apiculate. Lip 3-lobed to the base, deflexed; the middle lobe 10.5-16 mm long, 0.7-1 mm wide, linear, subacute; lateral lobes $10-11 \mathrm{~mm}$ long, $0.5 \mathrm{~mm}$ wide, linearfiliform, acute. Spur 20-30 mm long, curving forwards with 1 twist about the middle, inflated in apical third,

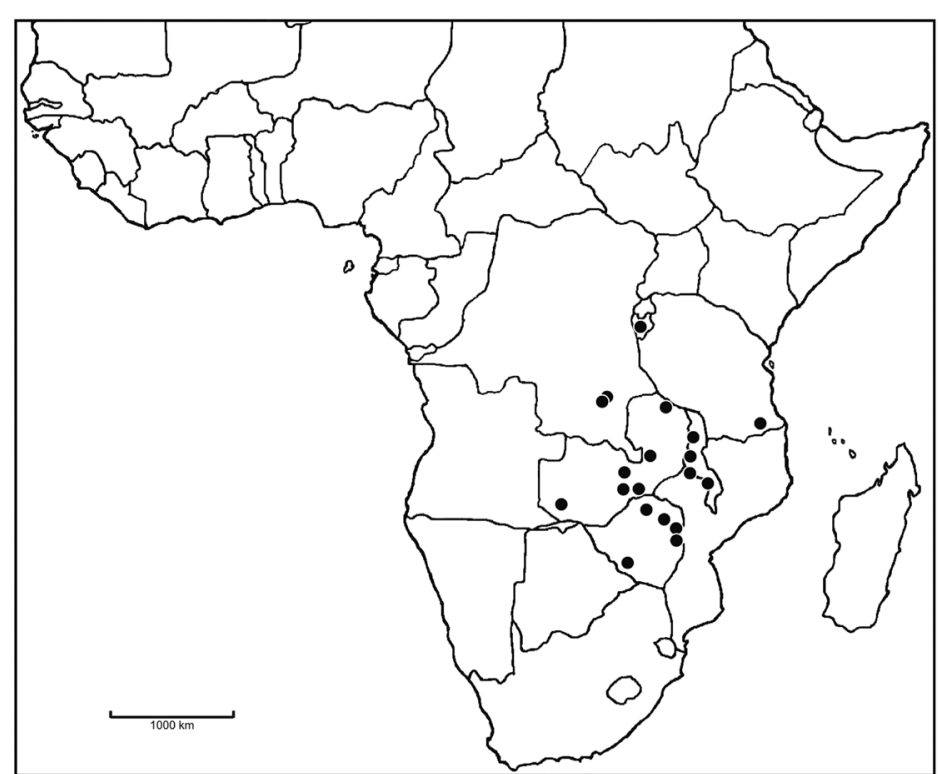

Fig. 161. Distribution of Bilabrella disparilis (Summerh.) Szlach. \& Kras-Lap.

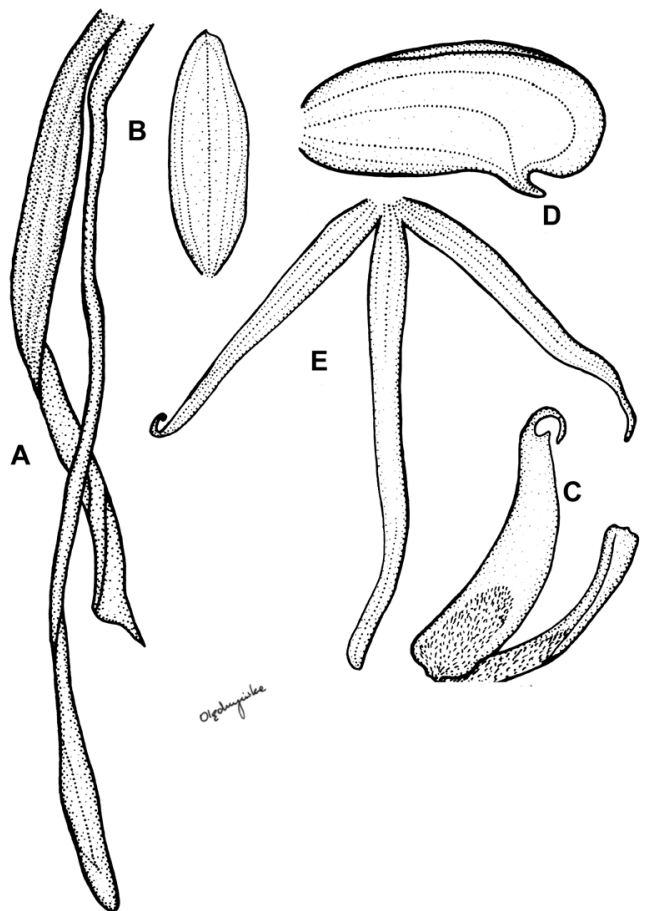

Fig. 160. Bilabrella disparilis (Summerh.) Szlach. \& Kras-Lap. Explanations: A - ovary, pedicel, spur; B - dorsal sepal; C - petal; D - lateral sepal; E - lip (drawn by N. Olędrzyńska from Kornaś 3246, KRA)

acute. Anther 2.5-3 mm long; connective rounded, shortly apiculate; antherophores 4.5-6 $\mathrm{mm}$ long, slender, straight or apically upturned. Stigmaphores $5-7 \mathrm{~mm}$ long, narrowly cylindrical, straight, glandular, diverging at an angle of $60^{\circ}$. Rostellum middle lobe shorter than thecae. Auriculae shortly stalked, bilobed (Figs 159$160)$.

Ec ology. In Brachystegia and open mixed woodland, grassland, also in road cuttings, red clay soil and granite. Flowering in January-December.

Distribution. Democratic Republic of the Congo (Zaire), Burundi, Tanzania, Zambia, Malawi, Zimbabwe, alt. 1050-2000 m (Fig. 161).

Specimens examined. Democratic Republic of the Congo (Zaire). Haut-Katanga: Shaba, Mitwaba-Mamono, km 20 (zone Mitwaba), 8²9'S, $27^{\circ} 20^{\prime} \mathrm{E}, 1450$ m, 1 Feb. 1986 (fl), Bamps \& Malaisse 8490 (BR!); Simama s/riv. Dikuluwe Terr., Mitwaba, 8 Jan. 1957 (fl), Brynaert 606 (BR!); Park National Upemba, Mukawa-Kabwe Kanono, 3 Feb. 1949 (fl), Brynaert s.n. (BR!); 11 Feb. 1948 (fl), De Witte 1347 (BR!, K!); 11 Feb. 1948 (fl), De Witte 3306 (BR!, K!); 1820 m, 3 Jan. 1949 (fl), De Witte 5079 (BR!, K!); Park National Upemba, Kaziba, 1140 m, 10 Feb. 1978 (fl), s.n. (BR!). Burundi. Kayanza: Isabu, environs the $\mathrm{Bu}-$ rarama, 2000 m, 10 Jan. 1993 (fl), Breyne 5916 (BR!). Tanzania. Mtwara: Marika, 8 Jan. 1971 (fl), Symoens, De Bilde \& Schweird 8 (BR!); Lake Tanganyika, Jan. 1895 (fl), Baum 59 (K!). Zambia. Central: Broken 
Hill, on sandy loams of mixed Julbernardia-Joberlinia woodland, 18 Jan. 1961 (fl), Morze 50 (AMES!, K!); Lusaka: Chakwenga Headwaters, 100-129 km East of Lusaka, Brachystegia woodland, 9 Feb. 1964 (fl), Robinson 6228 (K!); Chakwenga Headwaters, 100-129 km East of Lusaka, Brachystegia woodland, 14 Feb. 1965 (fl), Robinson 6383 (K!); 6 miles East of Lusaka, grassland, 1260 m, 20 Feb. 1956 (fl), King 322 (K!); 15 m west of Lusaka, Feb. 1969 (fl), Williamson 1379 (K!); Northern: Abercorn Distr., under trees in open Brachystegia woodland, 30 Dec. 1949 (fl), Bullock 2139 (BR!, K!); Abercorn Distr., Kawimbe, at side of gorge, also in lower grassland, 1680 m, 24 Jan. 1957 (fl), Richards 7961 (K! \& spirit.); Kale Dambo, in sandy peaty soil dry, among grass, with scattered trees, 5 Jan. 1955 (fl), Richards 3899 (AMES!, K!); Mbala, little Poland, woodland, sandy soil, $1590 \mathrm{~m}$, 21 Jan. 1970 (fl), Sanane 1000 (K!); Serewse Distr., Kundalila Falls, $15 \mathrm{~km}$ SE of Kanona, wet dambo above falls, $13^{\circ} 00^{\prime} \mathrm{S}, 30^{\circ} 30^{\prime} \mathrm{E}$, Jan. 1972 (fl), Williamson 2151 (K!). Malawi. Central: Dedza Distr., $12 \mathrm{~km}$ from Dedza road to Lilongwe, $1 / 2 \mathrm{~km}$ from highway on track to Rock Paintings National Movement, in wet on rocky slopes above quarry in Brachystegia woodland, 3 Feb. 1982 (fl), Gassner \& Cribb 209 (K!); Kasungu Distr., Chulu N.A. Kasungu-Lundazi, 1050 m, 15 Jan. 1959 (fl), Robson 1191 (BM!); Lilongwe Distr., Dzalanyama Forest Reserve, above Chiunjiza Road, $5 \mathrm{~km}$ south-east of Chaulongwe Falls, by stream on rocky hill-side in open woodland, 1260 m, 22 Mar. 1970 (fl), Brummitt 9299 (K!); Ntcheu, growing on steep road cuttings, 1150 m, 3 Jan. 1983 (fl), la Croix \& la Croix 384 (K!); Ntcheu Distr., Nkhande Hill, 1370 m, 29 Jan. 1959 (fl), Robson 1345 (BM!, K!); Northern: Viphya, on road side on Viphya, in scrubby Brachystegia woodland with red clay soil, 1450 m, 18 Apr. 1982 (fl), Johnston-Stewart 311 (K!); Namwra Distr., Likvciro Hill, in Brachystegia woodland, 1200 m, 21 Mar. 1980 (fl), Morris 842 (K!). Zimbabwe. Harare: $60 \mathrm{~km}$ N of Harare, Brachystegia woodland, below granite, 1200 m, 5 Feb. 1997 (fl), la Croix 1189 (K!); Kambanji Hill, in sparse grass on rocky hill under open mixed woodland, 1600 m, 22 Jan. 1997 (fl), la Croix 1180 (K!); Salisbury, Inyanga, $1500 \mathrm{~m}$, 31 Jan. 1958 (fl), Baasley 43 (82876) (K!); Salisbury, Inyanga, meadow, 1650 m, 5 Feb. 1955 (fl), Ball 490 (50246) (K!); Salisbury, in bush country, Jan. (fl), Eyles 6934 (K!); Saisbury Distr., generally distributed in "bush", 10 Jan. 1946 (fl), Greatrex 28 (K!); Salisbury Distr., red soil grassland, 1440 m, 8 Jan. 1958 (fl), Leach 4235 (K!); Salisbury, 8 July 1920 (fl), Norlindh \& Weimarck s.n. (K!); Salisbury Distr., Lochinvar, in Msasa bush, 1350 m, 30 Dec. 1945 (fl), Wild 593 (K!); Manicaland: Inyanga, Rhodes Inyanga Experiment Station, in open grassland, 4 Jan. 1975 (fl), Burrows 718 (K!); Inyanga, 1850 m, 30 Jan. 1931 (fl), Norlindh \&
Weimarck 4749 (K!); Umtali Distr., grassland, 1080 m, 4 Apr. 1954 (fl), Chase 5217 (K!); Mashonaland East: Ruwa, overgrown fireline, Rockall Farm, 1470 m, Jan. 1962 (fl), Miller 8144 (K!); Mashonalad West: Lomagundi, Alaska mine, on dolomite with Cuo ores, 3 Feb. 1965 (fl), Wild 6775 (K!); Matebeleland South: Matobo Distr., Besna Kobila Farm, 16 Apr. 1962 (fl), Miller 8241 (K!).

$\mathrm{N}$ o t e $\mathrm{s}$. This species is easily separated from any other representatives of the genus by very peculiar anterior petal lobe, which is bilobulate with both lobules being oblong triangular and acute, and diverging stigmaphores and rostellophores.

Robson 1191 collection at K appears to represent an intermediate form to $B$. leucoeras.

55. Bilabrella usambarae Kras \& Szlach., Polish. Bot. Journ. 53(2): 100. 2008.

Type: Tanzania, Meinkof 125 (holotype, HBG!).

Vegetative parts unknown. Pedicel and ovary $11.5 \mathrm{~mm}$ long, glabrous. Sepals all reflexed. Dorsal sepal $6 \mathrm{~mm}$ long, $1.8 \mathrm{~mm}$ wide, oblong elliptic-obovate, obtuse, concave. Petals bilobed to the base, ciliolate in the lower part; anterior lobe $5.1 \mathrm{~mm}$ long, $1.8 \mathrm{~mm}$ wide, 7-nerved, rounded, with laterally placed acute apex; posterior lobe $6 \mathrm{~mm}$ long, $0.3 \mathrm{~mm}$ wide, linear, acute. Lateral sepals $7.5 \mathrm{~mm}$ long, $4 \mathrm{~mm}$ wide, obliquely elliptic-obovate, concave, with apex long $1 \mathrm{~mm}$. Lip

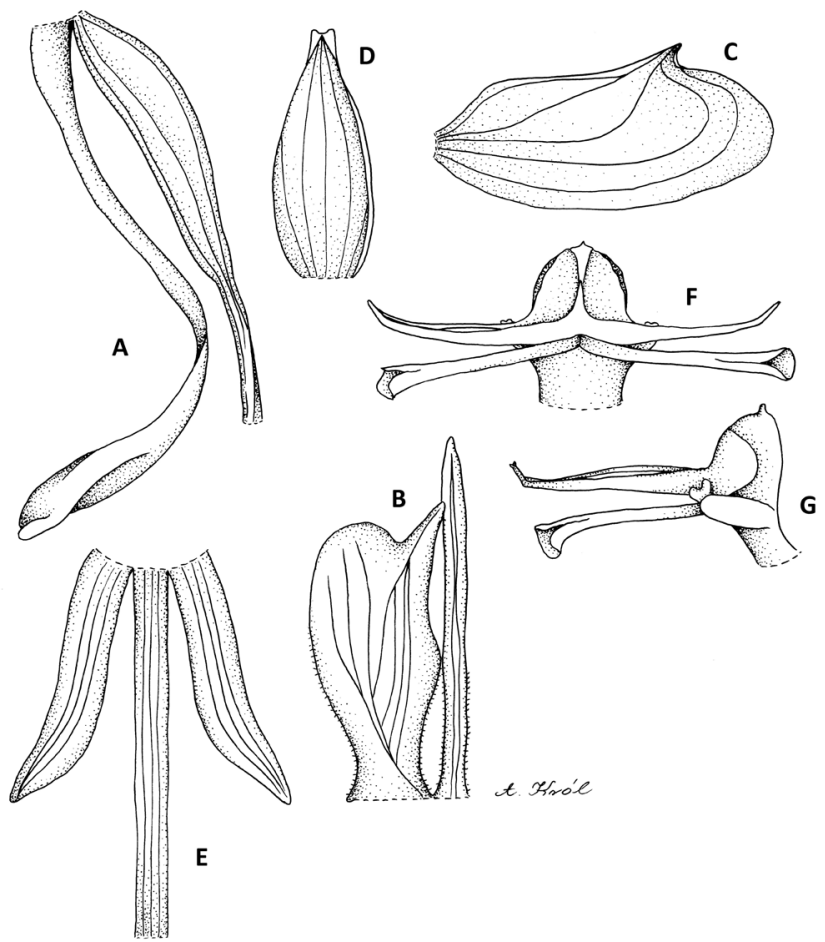

Fig. 162. Bilabrella usambarae Kras \& Szlach.

Explanations: A - ovary, pedicel, spur; B - petal; C - lateral sepal; D - dorsal sepal; E - lip; F - gynostemium, front view; $\mathrm{G}$ - gynostemium, side view (drawn by A. Król from holotype-Meinkof 125, HBG) 


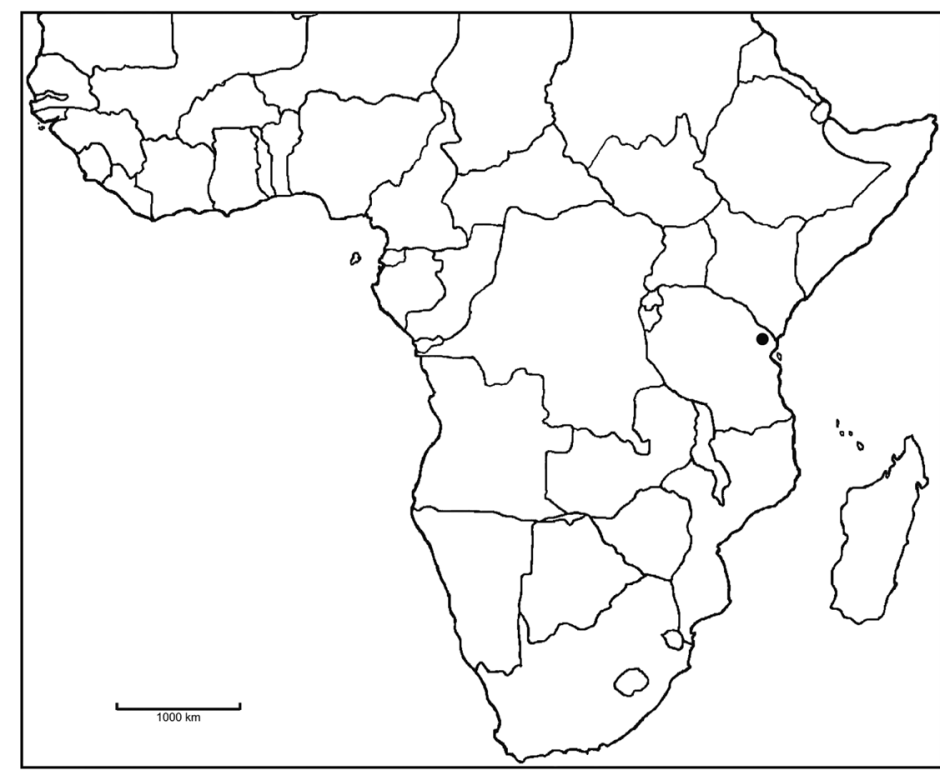

Fig. 163. Distribution of Bilabrella usambarae Kras \& Szlach.

3-lobed to the base; the middle lobe $0.5 \mathrm{~mm}$ wide, linear, obtuse; lateral lobes usually slightly shorter, $4.7 \mathrm{~mm}$ long, $1 \mathrm{~mm}$ wide, linear. Spur $16 \mathrm{~mm}$ long, curving forwards with 1 twist about the middle, inflated in apical third. Anther $2.5 \mathrm{~mm}$ long; connective rounded, shortly apiculate; antherophores $5.5 \mathrm{~mm}$ long, slender, slightly upcurved apically. Stigmaphores $4 \mathrm{~mm}$ long, narrowly cylindrical, truncate. Rostellum middle lobe shorther than connective. Auricules bilobed (Fig. 162).

Ecology. No records.

D i stribution. Tanzania only? (Fig. 163).

S pecimens examined. Tanzania. Tanga: Usambara, Meinkof 125 (HBG!).

N o te s. Similar to Bilabrella disparilis, but the anterior petal lobes are not so deeply bilobulate. One of the lobule is rounded and the other one is triangular, acute. In contrast to $B$. disparilis petals are ciliolate in the lower part. The another similar species is $B$. thomsonii. B. usambarae differs from $B$. thomsonii by lobation of petals and narrow spur, longer than pedicel and ovary.

\subsubsection{Subsection Dalzielae Szlach. \& Kras,} subsect. nov.

Type species: Bilabrella dalzielii (Summerh.) Szlach. \& Kras [三 Habenaria dalzielii Summerh.].

Petals bilobulate above basal 2-3 $\mathrm{mm}$.

\section{Key to species}

1. Leaves up to 10 times longer than wide. Petals more or less 3-lobulate

56. B. tetrapetaloides

1. Leaves ca. 15-20 times longer than wide. Petals bilobed
56. Bilabrella tetrapetaloides (Schltr.) Szlach. \& Kras, Richardiana 6(4): 197. 2006.

Habenaria tetrapetaloides Schltr., Bot. Jahrb. Syst. 20(50): 34. 1895. - Kraenzlin, Orchid. Gen. Sp.: 228. 1901. - Rolfe, Fl. Cap. 5(3): 129. 1913.

Type (hoc loco selectus): Capland, Schlechter 4464 (B+; lectotype, BM!; isolectotypes, BOL not seen, $\mathrm{K}$ !, WU!, Z not seen).

Stem about $30-50 \mathrm{~cm}$ tall, erect, stout, leafy. Leaves $8-15 \mathrm{~cm}$ long, to $1.5 \mathrm{~cm}$ wide, erect, linear-lanceolate, acute, somewhat coriaceous, decreasing upwards into imbricating sheaths. Inflorescence 7.5-12.5 cm long, cylindrical, dense, many-flowered. Flowers green. Floral bracts 12-15 mm long, lanceolate or ovate-lanceolate, acuminate. Pedicel and ovary 20-25 mm long, slender. Dorsal sepal $5 \mathrm{~mm}$ long, ovate-lanceolate to oblongovate, cucullate, subobtuse. Petals 2-lobed; anterior lobe 8.7-10 mm long, oblong, linear, obtuse; posterior lobe to $8 \mathrm{~mm}$ long, unequally 2-lobed in the upper half, the upper segment subulate, acute. Lateral sepals $7 \mathrm{~mm}$ long, obliquely obovate, apiculus laterally placed. Lip 3-lobed; the middle lobe 10-25 mm long, linear, acute; lateral lobes 8-20 mm long, linear, acute, diverging. Spur
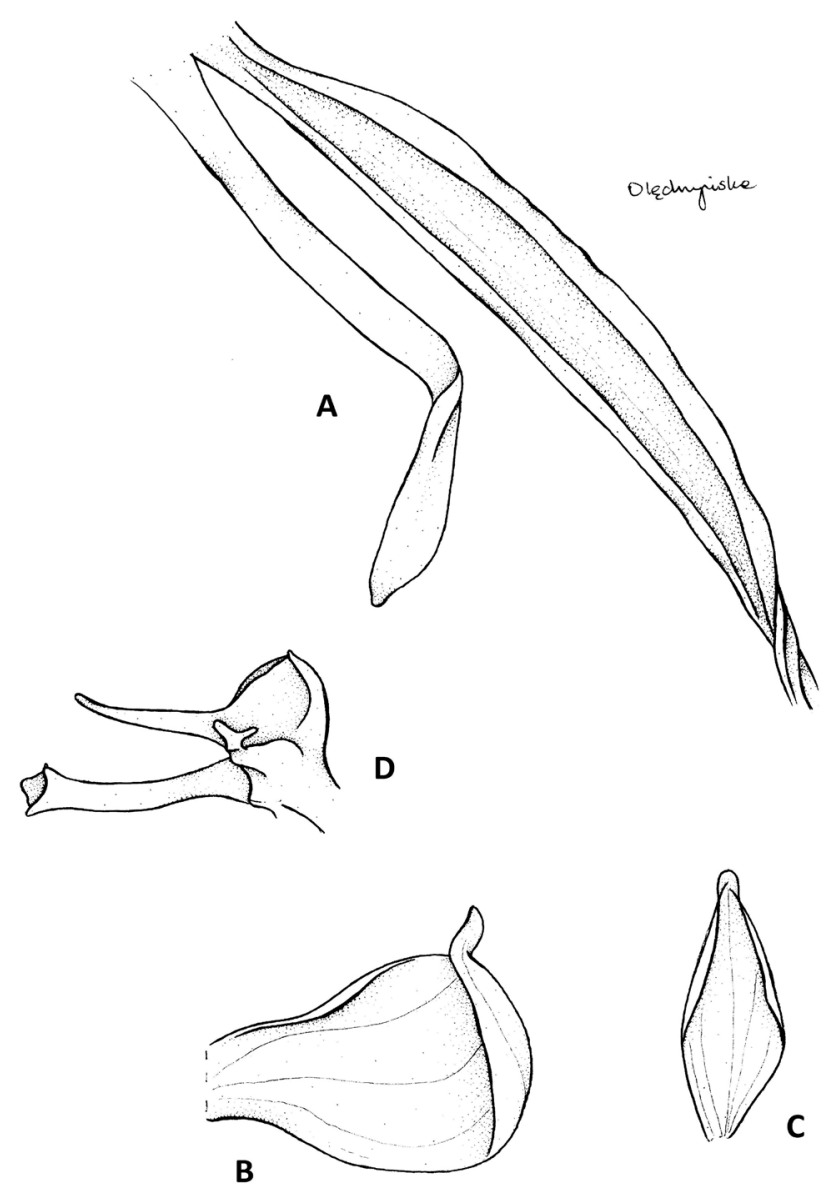

Fig. 164. Bilabrella tetrapetaloides (Schltr.) Szlach. \& Kras Explanations: A - ovary, pedicel and spur; B - dorsal sepal; C-lateral sepal; $\mathrm{D}$ - gynostemium (drawn by N. Olędrzyńska from isolectotype of Habenaria tetrapetaloides Schltr. - Schlechter 4464, K) 
$20 \mathrm{~mm}$ long, curved, cylindrical, somewhat twisted and clavate above. Anther $3 \mathrm{~mm}$ long; connective obtuse, shortly apiculate; antherophores $7 \mathrm{~mm}$ long, slender, straight. Stigmaphores about 6-9 mm long, narrowly cylindrical, truncate, straight. Rostellum middle lobe shorter than connective. Auriculae prominent, shortly stalked; unequally bilobed (Fig. 164).

E c o logy. In damp places. February.

Distribution. Republic of South Africa, alt. $2000 \mathrm{~m}$.

Specimens examined. Republic of South Africa. Transvaal, Houtbosh Prov., 2000 m, 11 Feb. 1894 (fl), Schlechter 4464 (BM!, K!, WU!).

$\mathrm{N}$ o t e $\mathrm{s}$. The species is very characteristic by its unique 3-lobed petals, probably not closely related to any other members of the genus.

57. Bilabrella dalzielii (Summerh.) Szlach. \& Kras, Richardiana 9(4): 158. 2009.

Habenaria dalzielii Summerh., Kew Bull. 1932: 339. 1932.

Type (hoc loco selectus): Nigeria, Dalziell 222 (lectotype, K!).

Tuber up to $1 \mathrm{~cm}$ long and wide, globose. Stem up to $90 \mathrm{~cm}$ long, erect, slender, glabrous. Leaves $8,5-27 \mathrm{~cm}$ long, $0.7-1.2 \mathrm{~cm}$ wide, linear to linear-lanceolate, acute, suberect, decreasing in size upwards. Inflorescence 13$16 \mathrm{~cm}$ long, laxly multiflowered. Flowers greenish. Floral bracts $7-13 \mathrm{~mm}$ long, lanceolate, acuminate. Pedicel and ovary 20-30 mm long. Dorsal sepal 6-7 mm long, 3-4 mm wide, obovate to olbong-obovate, obtuse. Petals bilobed above the basal 2-3 $\mathrm{mm}$; anterior lobe 5-6 $\mathrm{mm}$ long, $0.5 \mathrm{~mm}$ wide, linear to linear-filiform, obtuse; posterior lobe 4-5 $\mathrm{mm}$ long, 1-2 $\mathrm{mm}$ wide, obliquely linear-lanceolate, subacute to subobtuse. Lateral sepals 7-10 $\mathrm{mm}$ long, $4 \mathrm{~mm}$ wide, obliquely semi-obovate to

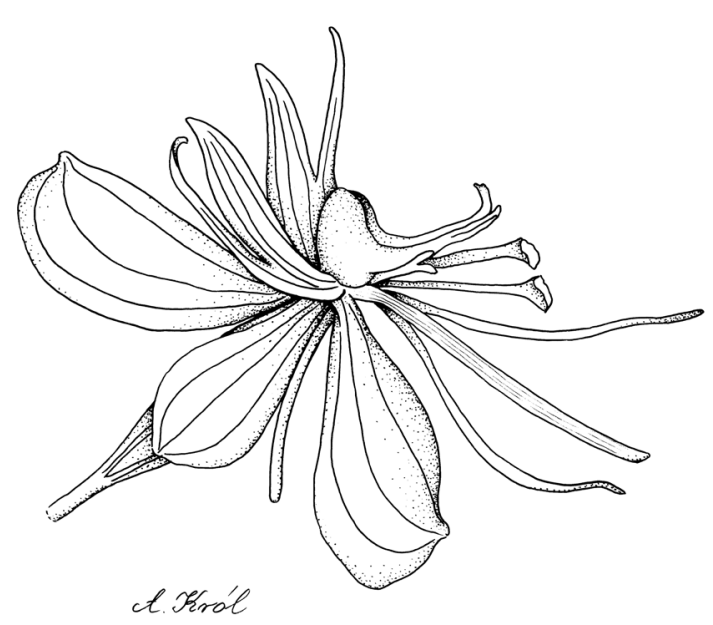

Fig. 165. Bilabrella dalzielii (Summerh.) Szlach. \& Kras - flower (drawn by A. Król from lectotype of Habenaria dalzielii Summerh. - Dalziel 222, K)

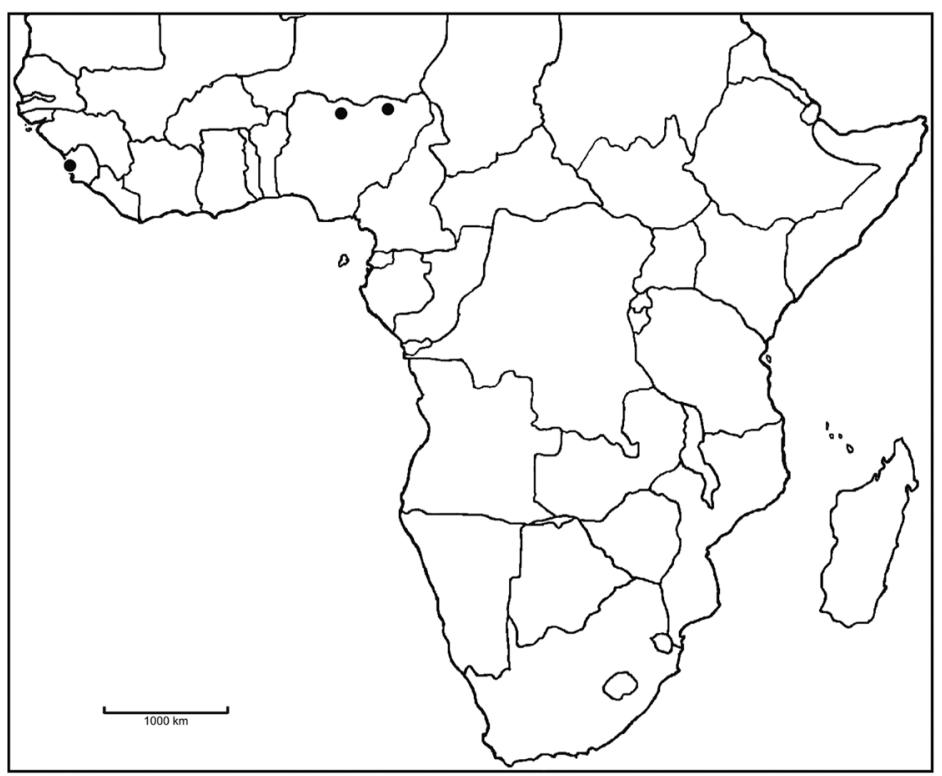

Fig. 166. Distribution of Bilabrella dalzielii (Summerh.) Szlach. \& Kras

oblong-obovate, laterally shortly apiculate. Lip 3-lobed above the basal $1-2 \mathrm{~mm}$; the middle lobe $8-10 \mathrm{~mm}$ long, 0.7-1 mm wide, linear, obtuse; lateral lobes $8.5-10.5 \mathrm{~mm}$ long, $0.5 \mathrm{~mm}$ wide, linear-filiform, subacute. Spur 5-7 mm long, cylindrical-filiform, apically slightly inflated, incurved. Anther $3 \mathrm{~mm}$ tall; connective rounded; antherophores 4-4.5 mm long, slender, upcurved apically. Stigmaphores 6-7 mm long, slender cylindricalfiliform, truncate and widened apically. Auriculae long stalked, deeply bilobed (Fig. 165).

E c o lo g y. Terrestrial in swamp. Flowering in JuneOctober.

D istribution. Sierra Leone, Nigeria (Fig. 166). Specimens examined. Sierra Leone. Western Area Urban: Hill Station, Oct. 1926 (fl), Deighton 2173 (pp) (K!); Hill Station, Deighton 2173a (K!). Nigeria. Plateau: Northern part of country, Gurum, June 1958 (fl), King 92 (K! \& spirit.); Yobe: Kilboa country, in a swamp, Aug. 1909 (fl), Dalziel 222 (K!).

$\mathrm{N}$ ot e s. A very characteristic member of Bilabrella easily distinguished by the form of petals, together with long stalked auriculae, the obovate dorsal sepal and almost equal lobes of the lip. The pedicels often have a peculiar sharp upward bend just below the ovary. Under his no. 2173 Deighton also collected a specimen of the abnormal form of $B$. anaphysema.

\subsubsection{Subsection Biauriculatae}

Both petal lobes different, anterior lobe larger than posterior one, lip 3-lobed at the base.

\section{Key to species}

1. Posterior petal lobe wider than anterior one .......... 2 
1. Posterior petal lobe narrower than or subequal to anterior one 3 2. Petals bilobed nearly to the base, anterior lobe $6-6.5 \times$ $1 \mathrm{~mm}$, papillate-puberulate, posterior lobe $5-5.5 \times 2-2.5$ $\mathrm{mm}$, ciliolate

58. $B$. angustissima

2. Petals bilobed distinctly above the base, glabrous, anterior lobe $4 \times 0.5 \mathrm{~mm}$, posterior lobe $3 \times 2.1 \mathrm{~mm} \ldots$

59. B. kraenzliniana

3. Spur twisted 4

3. Spur not twisted 19

4. Spur prominently swollen below the middle, swollen part at least twice wider than the basal one ... 5 4. Spur only slightly swollen towards apex ............. 11

5. Spur geniculate near the middle ......................... 6

5. Spur not geniculate ....................................... 8

6. Spur distinctly longer than lip middle lobe

60. B. altior

6. Spur subequal tot he lip middle lobe 7

7. Petals densely pubescent and ciliate, anterior lobe 9-11 $\times 1.2-2 \mathrm{~mm}$, posterior lobe 5.5-6 × 0.2-0.3 mm ...

61. B. kyimbilae

7. Petals puberulous or papillose with ciliolate margins, anterior lobe $8 \times 2-3 \mathrm{~mm}$, posterior lobe $6-7 \times 0.3-$ $0.5 \mathrm{~mm}$

62. B. thomsonii

8. Lip lateral lobes oblong-lanceolate, subobtuse, different than the middle one

63. B. welwitschii 8. Lip lateral lobes linear, similar tot he middle one .... 9 9. Anterior petal lobe glabrous, posterior one ciliolate 64. B. leucoceras

9. Both petal lobes more or less ciliolate 10 10. Leaves up to $1(1.5) \mathrm{cm}$ wide, anterior petal lobe $7-9 \times$ $2 \mathrm{~mm}$, posterior lobe $3-5 \times 0.3 \mathrm{~mm}$.. 65. B. kubangensis 10. Leaves $1-3 \mathrm{~cm}$ wide, anterior petal lobe $9-18 \times 1.5-$ $2.5 \mathrm{~mm}$, posterior lobe $5.5-7 \mathrm{~mm} \times 0.3 \mathrm{~mm}$

66. B. myodes

11. Spur usually shorter than pedicellate ovary ....... 12

11. Spur distinctly longer than pedicel and ovary ....... 15

12. Stigmaphotes ciliate 67. B. humbertii

12. Stigmaphores glabrous

68. B. burttii

13. Lip 3-lobed nearly to the base

13. Lip 3-lobed above distinct claw, 1.5-2 mm long ... 14

14. Both petal lobes completely papillose

69. B. maitlandii

14. Anterior petal lobe glabrous, posterior one ciliolate 70. B. involuta

15. Both petal lobes similar in width, filiform

\section{B. kornasiorum}

15. Both petal lobes of different width, only posterior one filiform 16

16. Leaves $0.2-0.8 \mathrm{~cm}$ wide 17

16. Leaves 1-3 cm wide 18

17. Spur ca twice longer than pedicellate ovary 72. B. tubifolia

17. Spur as long as pedicellate ovary
18. Floral bracts $12 \mathrm{~mm}$ long, ciliolate along margins, minutely glandular outside, stigmaphores $3 \mathrm{~mm}$ long, relatively massive, cylindrical, ciliate ... 74. B. bertauxiana 18. Floral bracts $10-25 \mathrm{~mm}$ long, glabrous, stigmaphores $4 \mathrm{~mm}$ long, slender cylindrical-clavate, minutely papillate 75. $B$. retinervis 19. Posterior petal lobe linear to ligulate, but never filiform ..................................................... 20 19. Posterior petal lobe filiform ........................... 22 20. Spur shorter than pedicellate ovary, both petal lobes papillose

76. B. furcipetala

20. Spur as long as or longer than pedicellate ovary, petals not as above. 21 21 . Floral bracts rather densely ciliolate along margins and outwards, spur filiform, equal in thickness nearly to apex, acute

77. B. renziana 21. Floral bracts glabrous, spur narrowly cylindrical, apically inflated, subacute . 78. B. rehmannii 22. Spur prominently shorter than pedicel and ovary ... 23

22. Spur usually as long as pedicel and ovary, occasionally somewhat longer or shorter .......................... 27 23. Spur cylindrical-filiform, not swollen, pendent ... 24 23. Spur cylindrical, somewhat swollen in the apical half, sigmoid 26 24 . Anterior petal lobe linear, ca $1 \mathrm{~mm}$ wide, ciliolate 79. B. linderi

24. Anterior petal lobe ovate-lanceolate to narrowly oblanceolate, 1.5-2.5 mm wide, glabrous ............... 25 25. Anterior petal lobe ovate-lanceolate, auriculae stalked, deeply bilobed, lobes elongate ....

80. B. macrotidion

25. Anterior petal lobe narrowly oblanceolate, auriculae sessile, massive, very unequally bilobed....

81. B. bosseriana

26. Petals glabrous; anterior lobe $13.5 \times 1.1 \mathrm{~mm}$, linearlanceolate, posterior lobe $6 \times 0.5 \mathrm{~mm}$, linear

82. B. luegiana

26. Anterior petal lobe $7.5-9 \times 3.5-4 \mathrm{~mm}$, obliquely ovate-lanceolate to semi-ovate, margins minutely crenate, minutely papillose-pubescent above, mainly towards the base, posterior lobe $6 \times 0.4-0.5 \mathrm{~mm}$, linear, papillose-pubescent above with ciliolate margins .......

83. B. riparia

27. Spur clavately swollen towards apex ............... 28

27. Spur filiform, almost not swollen .................... 32

28. Auriculae very large, massive .......................... 29

28. Auriculae small ............................................. 30

29. Pedicellate ovary up to $27 \mathrm{~mm}$ long, lip middle lobe 15-17 mm long, spur up to $22 \mathrm{~mm}$... 84. B. tangheana 29. Pedicellate ovary up to $13.5 \mathrm{~mm}$ long, lip middle lobe $9 \mathrm{~mm}$ long, spur $14 \mathrm{~mm}$ long ... 85. B. stanislawii 30. Lateral sepals oblong-obovate to elliptic-obovate, with short, erect apiculus placed near the middle of upper margin

86. B. cribbiana 
30. Laterl sepals obliquely oblong-obovate, apiculus subapically placed 31 31. Spur parallel to the ovary, pendent, anterior petal lobe glabrous, posterior lobe ciliolate .... 87. B. haareri 31. Spur horizontal or upcurved, petals densely pubescent 88. B. petraea 32. Ovary ciliate 89. B. incurva

32. Ovary glabrous 33

33. Spur $45-70 \mathrm{~mm}$ long, ca twice longer than pedicel and ovary 90. B. plectomaniaca 33. Spur much shorter, nearly as long as pedicellate ovary ..... 34 34. Anterior petal lobe glabrous

91. B. pseudokaessneriana

34. Antrior petal lobe papillose and/or ciliate 35 35. Lip 3-lobed just above the base ... 92. B. limnophila 35. Lip 3-lobed above the basal undivided part up to $1.5 \mathrm{~mm}$ long 93. B. chirensis

58. Bilabrella angustissima (Summerh.) Szlach. \& Kras, Richardiana 6(4): 196. 2006.

Habenaria angustissima Summerh., Kew Bull: 249. 1933. - Summerhayes, FWTA ed. 2, 3: 196. 1968a.

Type (hoc loco selectus): Guinea, Pobeguin 1680 (lectotype, K!; P not seen).

Tuber single, $2 \mathrm{~cm}$ long, $0.5-1 \mathrm{~cm}$ in diameter, ellipsoid. Stem 15-30 cm tall, erect, delicate, slender, glabrous. Leaves 3-6, 4-10 cm long, up to $1 \mathrm{~cm}$ wide, linear or narrowly lanceolate, acute, suberect, the upper ones much smaller, erect, adpressed to the stem, bract-like, the lower 1-2 stheath-like. Inflorescence

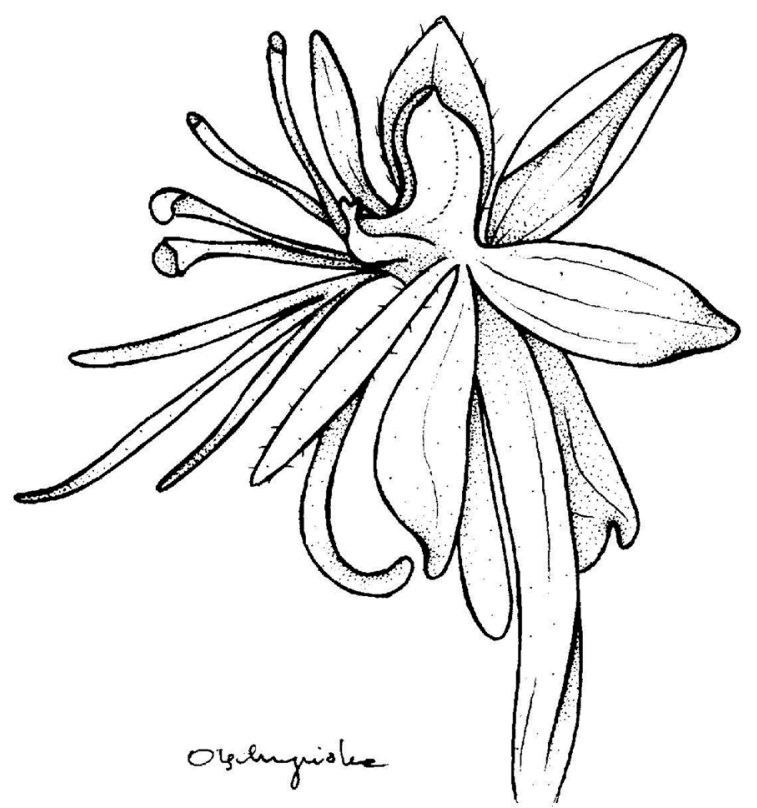

Fig. 167. Bilabrella angustissima (Summerh.) Szlach. \& Kras flower (drawn by N. Olędrzyńska from lectotype of Habenaria angustissima Summerh. - Pobeguin 1680, K)

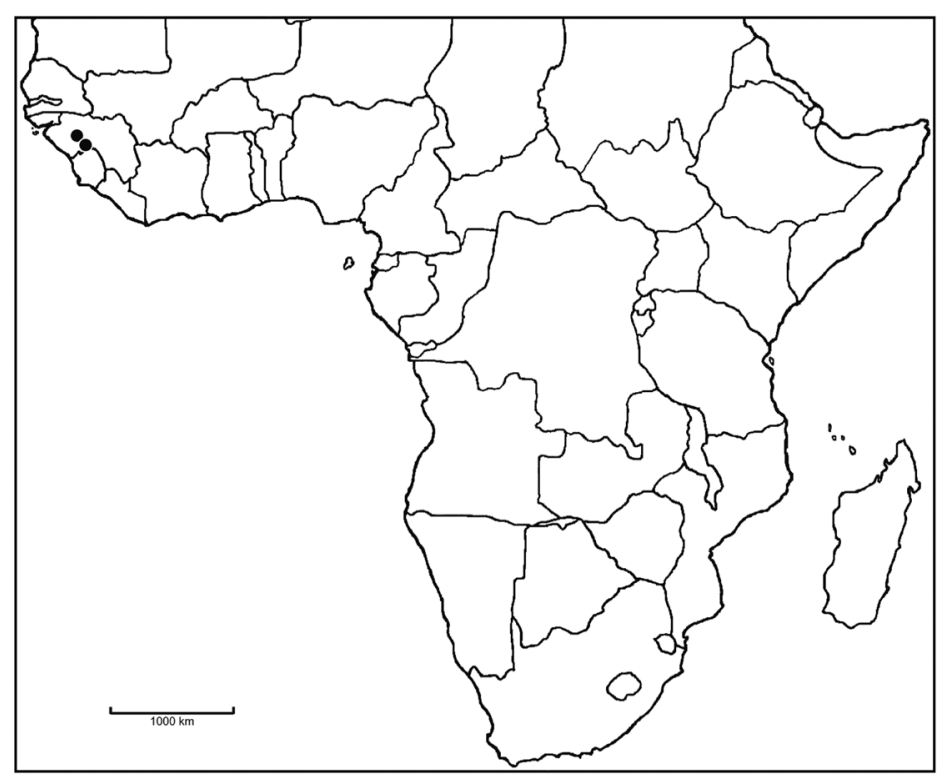

Fig. 168. Distribution of Bilabrella angustissima (Summerh.) Szlach. \& Kras

4-10 cm long, 3-12-flowered, lax. Flowers small, resupinate. Floral bracts up to $11 \mathrm{~mm}$ long, lanceolate, aristate-acuminate, herbaceous. Pedicel and ovary 10-15 mm long, slender. Dorsal sepal 5.5-6 mm long, 3-3.5 mm wide, narrowly elliptic or elliptic-ovate, concave, glabrous, reflexed. Petals bilobed nearly to the base; anterior lobe 6-6.5 $\mathrm{mm}$ long, up to $1 \mathrm{~mm}$ wide, oblanceolate-ligulate, acute, papillate-puberulate; posterior lobe 5-5.5 $\mathrm{mm}$ long, 2-2.5 $\mathrm{mm}$ wide, obliquely rhomboid-elliptic above the narrow base, acute, ciliolate. Lateral sepals 7-7.5 mm long, 3-3.5 mm wide, obliquely cuneate-obovate, cochleate in the apical half, with short, ca $0.5 \mathrm{~mm}$ long, lateral apiculus, glabrous, unbranched. Lip 3-lobed above an undivided basal part $1-1.5 \mathrm{~mm}$ long; the middle lobe $8-8.5 \mathrm{~mm}$ long, $0.5 \mathrm{~mm}$ wide, linear, acute or obtuse; lateral lobes $5.5 \mathrm{~mm}$ long, $0.5 \mathrm{~mm}$ wide, linear, acute, pendent. Spur 7-8 $\mathrm{mm}$ long, pendent, cylindrical-clavate, slightly swollen towards the blunt apex. Anther 3.5-4 mm long, ellipsoid; connective truncate; antherophores $5 \mathrm{~mm}$ long, filiform, upcurved. Stigmaphores $5 \mathrm{~mm}$ long, slender, narrowly clavate, truncate at the apex. Rostellum middle lobe shorter than connective. Auriculae rather small, bilobed (Fig. 167).

E c o log y. No record. Flowering July-August. Distribution. Appears to be endemic to Guinea (Fig. 168).

S pecimens examined. Guinea. Kindia: Bondakuri, SW of Pita, 5 July 1962 (fl), Adames 291 (AMES!, K!); Mamou, Aug. 1907 (fl), Pobeguin 1680 (K!, P!).

N o t e s. A small, rather slender species, very reminiscent of $B$. ichneumonea, but differing in quite a number 
of minor features and possibly more nearly related to some other species of Bilabrella. It shares with B. ichneumonea the extremely narrow leaves. Perhaps the most interesting feature is the wide posterior petal lobe, which is much wider than the anterior lobe (cf. Summerhayes 1933). The same character can be found also in B. kraenzliniana from Kenya. It seems, however, that they are not closely related to each other as differences between them concern many morphological characters.

59. Bilabrella kraenzliniana Kras \& Szlach., Polish. Bot. Journ. 53(2): 97. 2008.

Type: Kenya, Graham 3736 B (holotype, BR!).

Plant $36.2 \mathrm{~cm}$ tall, rather erect. Leaves $8.5 \mathrm{~cm}$ long, $0.7 \mathrm{~cm}$ wide, linear, acute, spread, the upper ones sheath-like, sparsely hairy. Inflorescence $6.2 \mathrm{~cm}$ long, 12-flowered, lax. Floral bracts $21 \mathrm{~mm}$ long, $4 \mathrm{~mm}$ wide, oblong-lanceolate, acuminate, hairy on external sides. Pedicel and ovary $11 \mathrm{~mm}$ long, slender. Dorsal sepal $7 \mathrm{~mm}$ long, 2.5-3 mm wide, oblong-elliptic to oblongobovate, shortly apiculate, blunt, concave, glabrous. Petals bilobed distinctly above the base, glabrous; anterior lobe $4 \mathrm{~mm}$ long, $0.5 \mathrm{~mm}$ wide, linear-filiform, acute; posterior lobe wide $3 \mathrm{~mm}$ long, $2.1 \mathrm{~mm}$ wide, obliquely triangular-ovate, acute. Lateral sepals $7.5 \mathrm{~mm}$ long, $5.5 \mathrm{~mm}$ wide, oblong-obovate, cochleate at the apex, with the apex $0.5 \mathrm{~mm}$ long, erect. Lip 3-lobed at

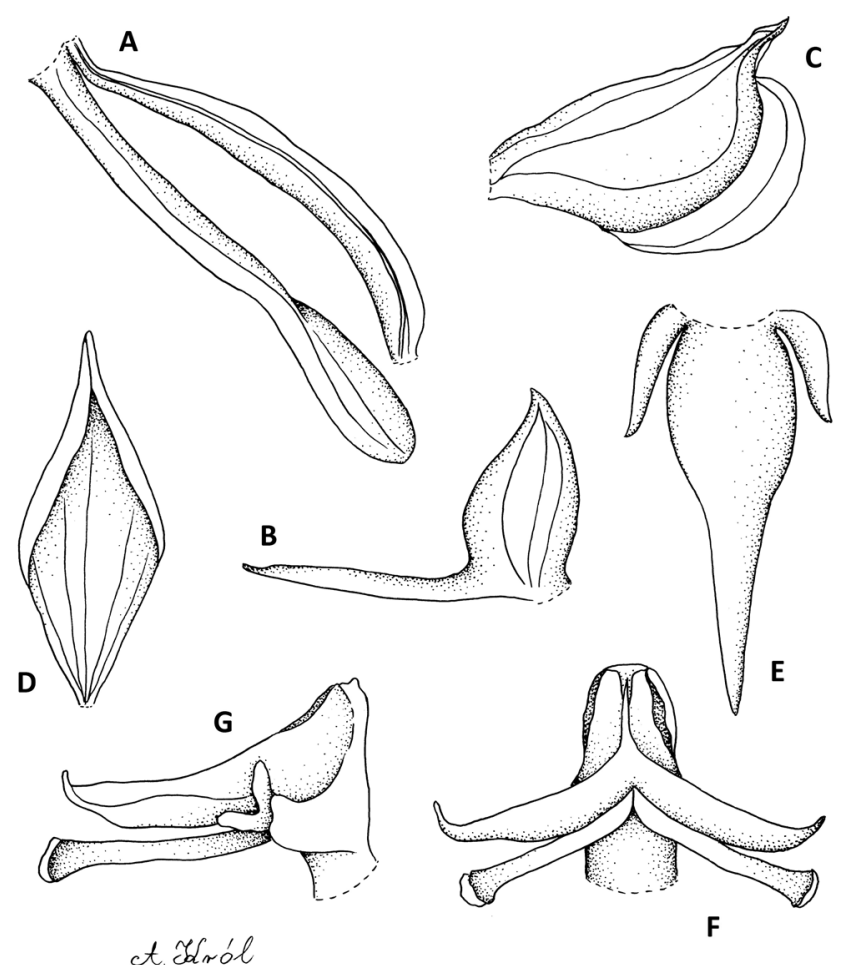

Fig. 169. Bilabrella kraenzliniana Kras \& Szlach.

Explanations: A - ovary and spur; B - petal; C - lateral sepal; D - dorsal sepal; E - lip; F - gynostemium, front view; G - gynostemium, side view (drawn by A. Król from holotype - Graham 3736B, BR)

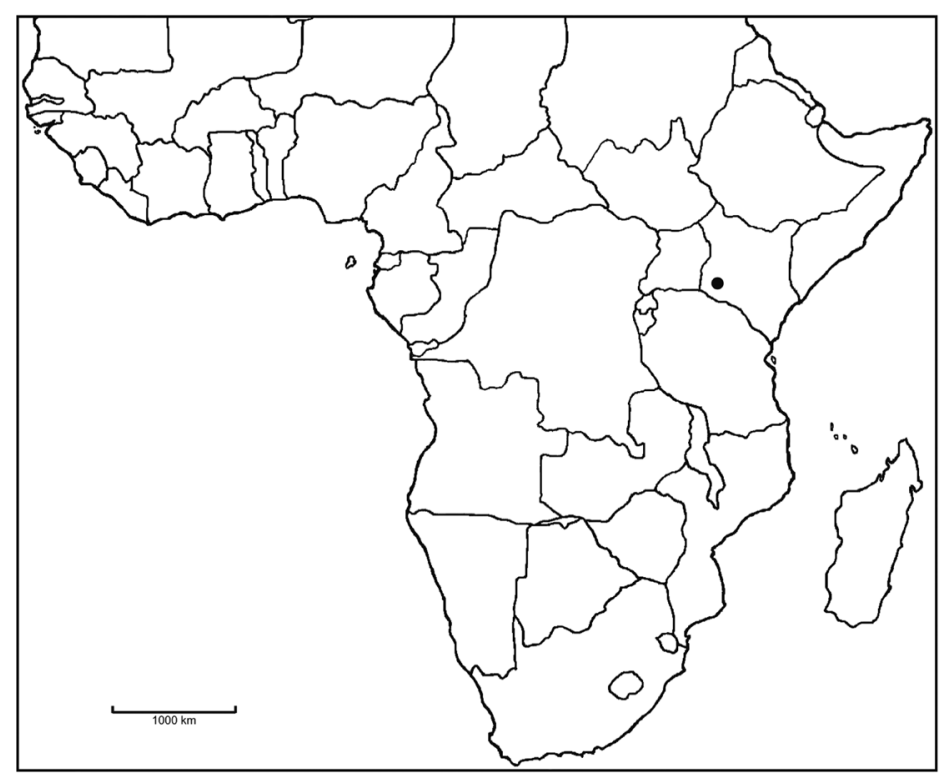

Fig. 170. Distribution of Bilabrella kraenzliniana Szlach. \& Kras

the base, glabrous; the middle lobe $6 \mathrm{~mm}$ long, 1-2 mm wide, obovate in the basal half, thickened, lanceolate above, thiner, acute; lateral lobes $2 \mathrm{~mm}$ long, $0.4 \mathrm{~mm}$ wide, obliquely narrowly lanceolate at the base, filiform above, acute. Spur $13 \mathrm{~mm}$ long, $1.6 \mathrm{~mm}$ in diameter at the apex, cylindrical, slightly twisted near the middle, distinctly swollen in the apical third, blunt. Anther $3.1 \mathrm{~mm}$ long; connective truncate; antherophores $5 \mathrm{~mm}$ long, rather massive. Stigmaphores $4 \mathrm{~mm}$ long, narrowly cylindrical, truncate. Rostellum middle lobe $1.9 \mathrm{~mm}$ long, $0.2 \mathrm{~mm}$ wide, shorter than connective, linear-triangular, acute. Auriculae bilobed, rather massive (Fig. 169).

E c o logy. No records. Flowers in November.

D i s t r i bu ti o n. Known from Kenya only, alt. 2550 m (Fig. 170).

Specimens examined. Kenya. Rift Valley: Sitotan, near Lumbwa, 2550 m, Nov. 1936 (fl), Graham $3736 B$ (BR!, K!).

$\mathrm{N}$ o te s. Unique in the genus by its peculiar lip morphology, especially by obovate lower part of the middle lobe. Similar somewhat to $B$. welwitschii from which it may be distinguished by the shape of petals - the anterior petal lobe is linear and the posterior lobe is obliquly ovate, acute. As mentioned above, this species shares with $B$. angustissima posterior petal lobe much wider than anterior one, but the form of this lobe, as well as lip form clearly separate both species.

60. Bilabrella altior (Rendle) Szlach. \& Kras-Lap., Richardiana 3(3): 139. 2003. - Szlachetko et al., Orchid. of West-Central Africa 1: 238. 2010.

Habenaria altior Rendle, J. Linn. Soc., Bot. 30: 394. 1895. - Rolfe, Fl. Trop. Afr. 7: 245. 1898. - Summerhayes, Kew Bull. 16: 273. 1962. - Summerhayes, 
FTEA, Orchid. 1: 86. 1968b. - Piers, Orchid. E. Afr.: 32. 1968. - Stewart \& Campbell, Orchid. Kenya: 130. 1996.

Type: Tanzania, W.E.Taylor s.n. (lectotype, designated by Szlachetko et al. [2010: 238], BM!; isolectotype, $\mathrm{BM}$ !).

Habenaria lykipiensis Rolfe, Fl. Trop. Afr. 7: 243. 1898. - Piers, Orchid. E. Afr.: 32. 1968.

Type: Kenya, Thomson s.n. (lectotype, designated by Szlachetko et al. [2010: 238], K!).

Habenaria ranicolorata Rolfe, Fl. Trop. Afr. 7: 244. 1898.

Type: Tanzania, H.H. Johnston 159 (lectotype, designated by Szlachetko et al. [2010: 238], K!).

Tubers $1.5-2.5 \mathrm{~cm}$ long, $1 \mathrm{~cm}$ in diameter, ovoid or ellipsoid. Stem $20-95 \mathrm{~cm}$ tall, erect, stout, glabrous. Leaves 9-13, 7-22 cm long, 1-2 cm wide, lanceolate, narrowly lanceolate to linear-lanceolate, acute, erect or suberect, decreasing in size up the stem. Inflorescence 4-25 cm long, 7- to many-flowered, rather dense. Flowers green and white. Floral bracts $10-30 \mathrm{~mm}$ long, lanceolate-ovate, acuminate. Pedicel and ovary 20-30 mm long, glabrous. Dorsal sepal 6-9 mm long, 2-4.5 mm wide, oblong-elliptic or oblong-obovate, with truncate, thickened apex, cochleate, glabrous. Petals bilobed nearly to the base, ciliate pubescent in the lower part or throughout; anterior lobe 8-13 mm long, 1.1$2.5 \mathrm{~mm}$ wide, obliquely linear-lanceolate, acuminate; posterior lobe $5-7.5 \mathrm{~mm}$ long, $0.7 \mathrm{~mm}$ wide, linear, acute. Lateral sepals $8.5-11 \mathrm{~mm}$ long, 4.5-7 mm wide, obliquely elliptic, semi-orbicular to obovate, cochleate at the apex, apiculus laterally placed, glabrous. Lip deflexed, 3-lobed above the basal $1 \mathrm{~mm}$, glabrous; the middle lobe 10-17 $\mathrm{mm}$ long, 0.5-1 mm wide, linear,

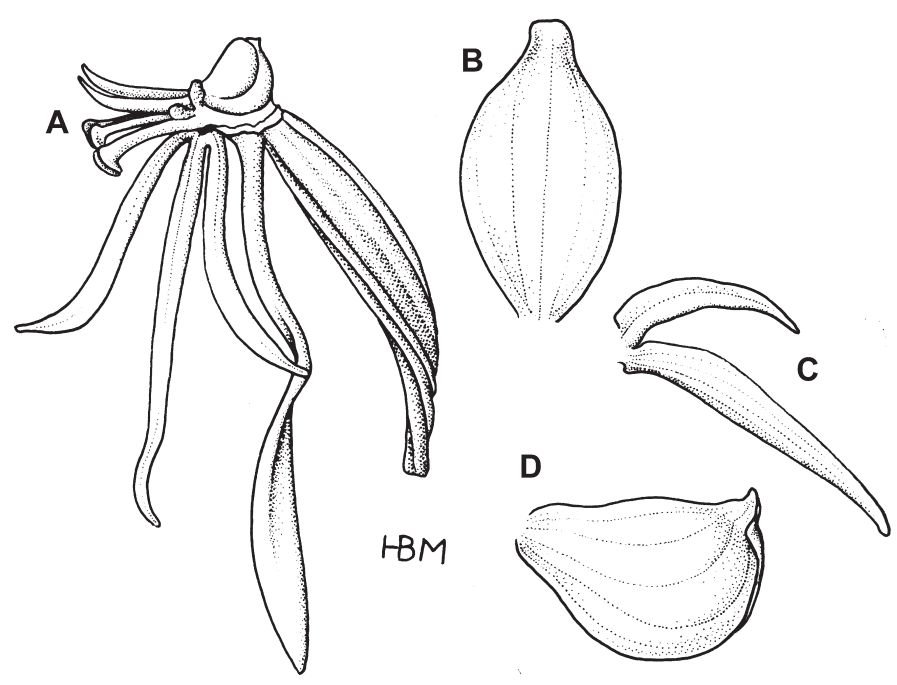

Fig. 171. Bilabrella altior (Rendle) Szlach. \& Kras-Lap. Explanations: A - pedicel, ovary, gynostemium, lip and spur; B - dorsal sepal; C - petal; D - lateral sepal (redrawn by H. B. Margońska from lectotype material - W.E. Taylor s.n., K)

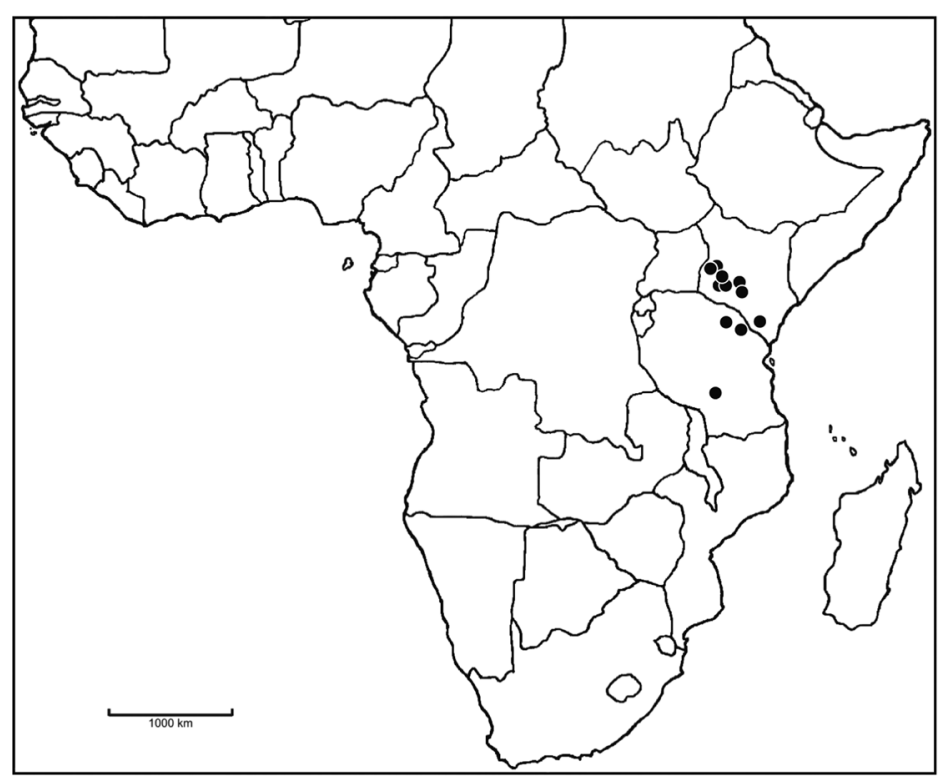

Fig. 172. Distribution of Bilabrella altior (Rendle) Szlach. \& Kras-Lap.

acute to subobtuse; lateral lobes $6.5-13 \mathrm{~mm}$ long, $1.1 \mathrm{~mm}$ wide, linear-lanceolate to linear, acute. Spur 15-22 mm long, narrowly cylindrical, swollen in the apical half, blunt, pendent, more or less geniculate near the middle. Anther 2-4 mm long; connective truncate to shallowly notched; antherophores 4-7 mm long, slender, gradually upcurved in the apex. Stigmaphores 4.5-7.5 mm long, narrowly cylindrical, straight, slightly widened and truncate at the apex. Rostellum middle lobe distinctly shorter than thecae. Auriculae medium-sized, bilobed in the upper half (Fig. 171).

E c o logy. Grassland, among rocks. Flowering in January-December.

D i s tributio n. Kenya, Tanzania, alt. 1450-3300 m (Fig. 172).

Specimens examined. Kenya. Coast: Taita Distr. \& Hills, Ngangao Forest, upper edge among rocks, 19 Nov. 1969 (fl), Bally 13638 (K!) (as H. thomsonii); Nyanza: Kericho, Stone River, Oct. 1943 (fl), Copley 121 (K!) (as H. lykipensis); Rift Valley: Cherangani, in grassland, 2550 m, Sep. 1935 (fl), Dale 3447 (K!); Laikipia, Thomson s.n. (K!); Forest Station, in camp, $2300 \mathrm{~m}$, Fries 306 (K!); Coll Station near Lumbwa, 2550 m, Nov. 1936 (fl), Graham 3736 (K!); Naivasha Distr., Gilgil, Okolands, Sep. 1953 (fl), Piers s.n. (K!); Naivasha Distr., Gilgil, Simba River, 2250 m, 12 Oct. 1953 (fl), s.n. (K!); Ravine Distr., in grass, 2700 m, Tweedie 3829 (K! \& spirit.); Eldama Ravine, Whyte s.n. (K!); Tinderet, 2100 m, Nov. 1933 (fl), s.n. (K!); Western: Elgon, Oct. 1940 (fl), Tweedie 427 (K! \& spirit.); E Elgon, Terriet Hill, grass slades in woodland, 3300 m, Dec. 1943 (fl), Tweedie 617 (K!); Sine loc., 2700 m, Nov. 1938 (fl), Mainwaring 6000 (K!); Sine loc., 2490 m, 16 Dec. 1931 (fl), Ramsdew 1539 
(K!). Tanzania. Arusha: Ngorongoro rest camp, grassland, 1800 m, 3 Apr. 1941 (fl), Bally 2244 (K!); Lava Fields, between Burunga \& Kibati, E of Ngorongonro Volcano, short grass on smooth lavas, 5 Jan. 1931 (fl), Burtt 3209 (K!); Iringa: Mufindi Distr., Lulando Forest, closed canopy forest, $08^{\circ} 30^{\prime} \mathrm{S}, 35^{\circ} 40^{\prime} \mathrm{E}, 1450 \mathrm{~m}, 19 \mathrm{Apr}$. 1987 (fl), Lovett \& Congdon 2018 (K!); Kilimanjaro: Kilimanjaro East, Useri, 2400 m, June 1927 (fl), Haarer 549 (K!); Kilimanjaro, Marangu, grassland, Dec. 1957 (fl), Ivens 1066 (K!); Kilimanjaro, Johnston 159 (K!); Kilimanjaro Mt., higher slopes above Marangu, 3000 m, Taylor 1888 (K!); Higher slopes of Kilimanjaro above Marangu, 3000 m, 1886 (fl), s.n. (BM!).

Note s. According to Rendle (1895) it is very near the Ethiopian Habenaria schimperana, but has not the characteristically much twisted spur, while the flower-spike is short and dense, and the bracts are larger, wider and ciliolate. B. altior appears to be related to $B$. weberana as well. Unlike the latter however, its petals lobes are ciliate-pubescent, spur is incurved, and slightly swollen in the apical half. In $B$. weberana anterior petals lobe is glabrous, posterior one ciliolate, and spur is pendent, filiform. It appears that both $\mathrm{Ha}$ benaria lykipensis Rolfe and $H$. ranicolorata $\mathrm{Rchb}$. f. are conspecific with $B$. altior.

There is a group of 3 species with similarly formed spur, which is geniculate and twisted near the middle and prominently swollen below. They are B. altior, $B$. kyimbilae and $B$. thomsonii. From the species mentioned, $B$. altior has the longest spur, distinctly longer than lip middle lobe. In both other taxa spur and lip middle lobe have similar length.

61. Bilabrella kyimbilae (Schltr.) Szlach. \& Kras-Lap., Richardiana 3(3): 141. 2003. - Szlachetko et al., Orchid. of West-Central Africa 1: 229. 2010.

Habenaria kyimbilae Schltr., Bot. Syst. Jahrb. 53: 515. 1915. - Schlechter, Repert. Sp. Nov Regni Veg. 68: tab. 28. 1932. - Summerhayes, Kew Bull. 16: 284. 1962. Summerhayes, FTEA, Orchid. 1: 87. 1968b. - Geerinck $\&$ Coutrez, Not. Taxon. Orchid. Afr. Centr., Habenaria: 9. 1977. - Williamson, Orchid. S. Central Afr.: 51. 1977. - Geerinck, Fl. Afr. Centr., Orchid. 1: 111. 1984. - la Croix \& Cribb, Fl. Zambes., Orchid. 11: 98. 1995.

Type: Tanzania, Stolz 785 (B+; lectotype, designated by Szlachetko et al. [2010: 229], K!; isolectotypes, C!, $\mathrm{S}$ not seen, W-R!).

Tubers 1-2.5 cm long, 0.8-1.4 cm in diameter, almost globose to ellipsoid. Stem $20-90 \mathrm{~cm}$ tall, erect, rather slender, glabrous. Leaves 6-11, 8-29 cm long, 0.6-2 cm wide, linear-lanceolate, acute, adpressed to the stem, decreasing in size upwards. Inflorescence $5-25 \mathrm{~cm}$ long, densely 10- to many-flowered. Flowers green, yellowish-green to whitish-green. Floral bracts 10-
$25 \mathrm{~mm}$ long, ovate-lanceolate, acuminate. Pedicel and ovary 15-26 mm long, glabrous. Dorsal sepal 5-6 mm long, 2-3 mm wide, narrowly obovate to elliptic, with ligulate obtuse apex, concave, glabrous. Petals bilobed nearly to the base, densely pubescent and ciliate; anterior lobe 9-11 mm long, 1.2-2 mm wide, lanceolate, linear-lanceolate to narrowly lanceolate, acute, falcate; posterior lobe 5.5-6 $\mathrm{mm}$ long, 0.2-0.3 $\mathrm{mm}$ wide, linear to filiform, subobtuse to subacute. Lateral sepals 6.5$8.5 \mathrm{~mm}$ long, 5-6 $\mathrm{mm}$ wide, oblong obovate to ellipticobovate, cochleate, strongly deflexed, apiculus ca. $0.5 \mathrm{~mm}$ long, laterally placed. Lip 3-lobed above the basal $1 \mathrm{~mm}$ claw, glabrous; the middle lobe $10-12 \mathrm{~mm}$ long, 0.5-0.8 mm wide, linear, obtuse, incurved; lateral lobes $6.5-8.5 \mathrm{~mm}$ long, $0.8-1 \mathrm{~mm}$ wide, linear-lanceolate to oblong lanceolate, acute. Spur 10-13.5 mm long, cylindrical, geniculate in the middle, swollen in the distal half, blunt. Anther $3 \mathrm{~mm}$ tall; connective truncate with small apiculus; antherophores 3.5-5 mm long, massive with delicate upturned apices. Stigmaphores 3-4 mm long, cylindrical, truncate, straight. Rostellum middle lobe as long as connective. Auricles very unequally bifid (Figs 173-174).

Ecology. Grassland, among rocks, damp places, Brachystegia woodland. February-November.

D i stribution. Democratic Republic of the Congo (Zaire), Tanzania, Malawi, alt. 600-2700 m (Fig. 175). S pecimens examined. Democratic Republic of the Congo (Zaire). Haut-Katanga: Near Elisabethville,

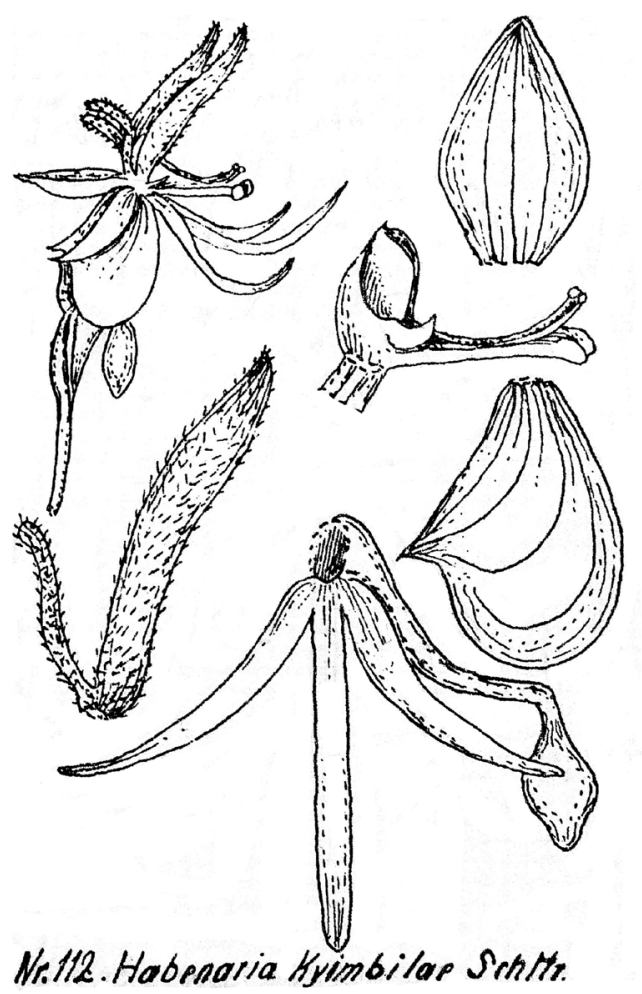

Fig. 173. Original Schlechter (1932) drawing of Habenaria kyimbilae Schltr. 
in very shady undergrowth near a stream, $1250 \mathrm{~m}$, May 1946 (fl), Qnane 8016 (K!); Marungu, Kipizi, common in savannah, 2200 m, Apr. 1945 (fl), Dubois 1415 (K!); Sud-Kivu: Ituri, Haut Haya, near Kahanda, 1360 m, 4 Aug. 1975 (fl), Lisowski 41103 (UGDA-DLSz!). Tanzania. Iringa: Luponde Estate, on grass vergts at the edge ao the tea and maize fields, 2200 m, 10 May 1989 (fl), Spurrier 81 (K!); Makete Distr., Kitulo Plateau, high altitude grassland between Kitulo and Matamba, 0905'S, 335ㅇ'E, 2600 m, 30 Mar. 1987 (fl), Lovett \& Congdon 1858 (K!); Makete Distr., Matamba village, grassy roadside, $08^{\circ} 55^{\prime} \mathrm{S}, 33^{\circ} 50^{\prime} \mathrm{E}, 2000 \mathrm{~m}, 30$ Mar. 1987 (fl), Lovett \& Congdon 1876 (K!); Milo, open scrub, 2040 m, 4 May 1963 (fl), Mdbbs 12A (K!); Mufindi, 08³0'S, 35²0’E, 1830 m, Nov. 1987 (fl), Keeley s.n. (K!); Mwakite, highland grassland, edge of cultivated area, 2000 m, 3 Feb. 1989 (fl), Adam 238 (K!); T7, Njombe Distr., Poroto Mts., Kitulo Plateau Ndumbi Valley, montane grassland along small stream, rich dark brown volcanic soils, $9^{\circ} 03^{\prime} \mathrm{S}, 34^{\circ} 00^{\prime} \mathrm{E}, 2500 \mathrm{~m}$, 24 Mar. 1991 (fl), Bidgood, Congdon, Vollesen 2137 (K!); Southern Prov., Njombe Distr., Mbeya, Kitulo Sheep Project, grassland, 2700 m, 1 Mar. 1970 (fl), Fuller 109 (K!); Southern Highlands Prov., Njombe Distr., Njombe, in clumps of vegetation on rocks in river at top of Ruhidji falls, May 1951 (fl), Eggeling 6108 (K!); In grassland, 1800 m, 2 Sep. 1987 (fl), Leysen 156 (K!), Njombe, Dansland, in marsh, in 1-2" water, short grass, 2160 m, 15 Mar. 1989 (fl), Spurrier

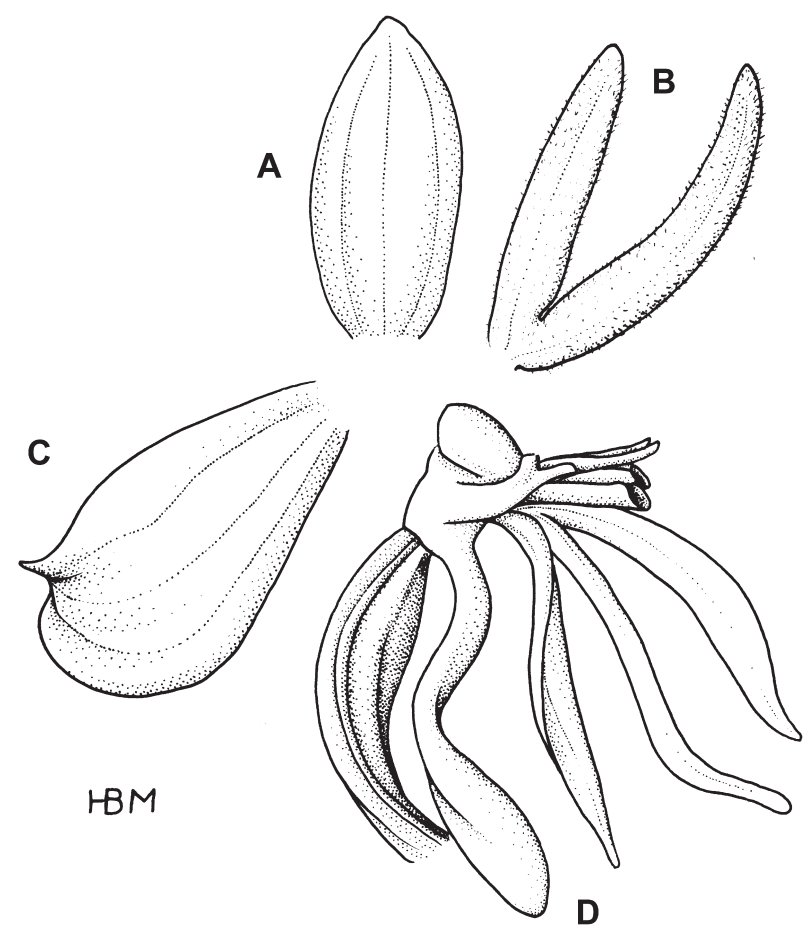

Fig. 174. Bilabrella kyimbilae (Schltr.) Szlach. \& Kras-Lap. Explanations: A - dorsal sepal; B - petal; C - lateral sepal; D - lip, spur, pedicel and gynostemium, side view (redrawn by H. B. Margońska from lectotype of Habenaria kyimbilae Schltr. - Stolz 785, K).

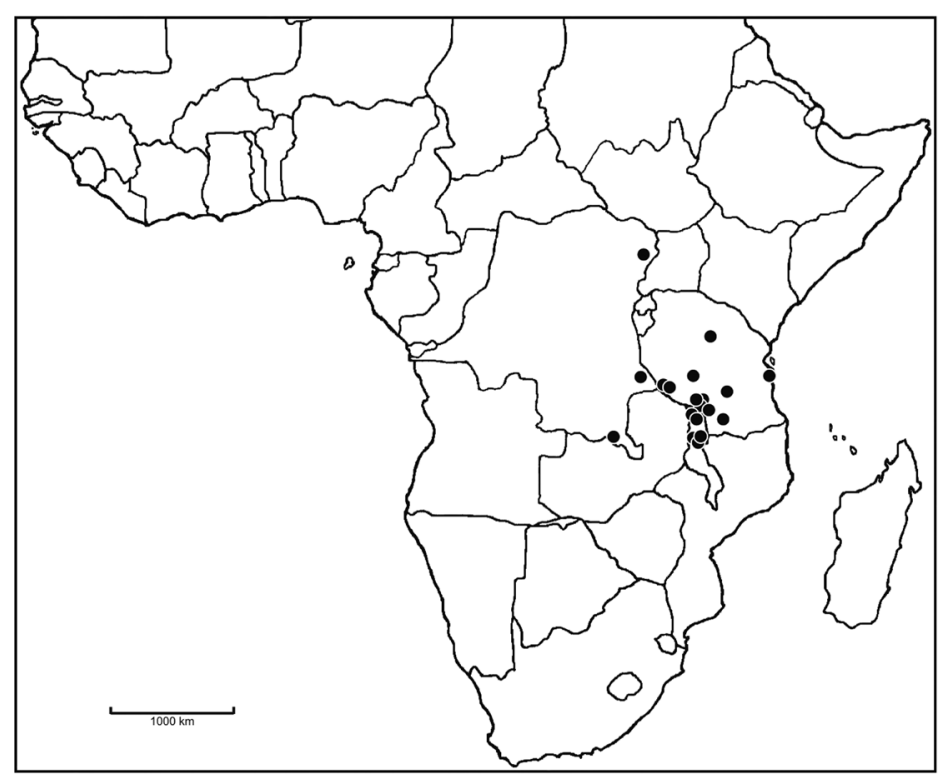

Fig. 175. Distribution of Bilabrella kyimbilae (Schltr.) Szlach. \& Kras-Lap.

64 (K!); Njombe, Luponde Estate, grassland, black soil or red-quite a wide range, from fertile cultivated land to rocky, 2200 m, 13 Apr. 1989 (fl), Spurrier 70 (K!); Mbeya, Kyimbila Distr., north of lake Nyasa, 14 Mar. 1914 (fl), Stolz 2581 (BM!, K!); Stolz 2581bis (BM!); Mbeya Distr., Ntumbi area, Usafwa, on road to Kiwira Forest Camp, grassland, $9^{\circ} 05^{\prime} \mathrm{S}, 33^{\circ} 40$ 'E, $2100-2200 \mathrm{~m}$, Pettersson, Hedren \& Kibuwa 202 (K!); Rungwe Distr., Kyimbila, Stolz 785 (K!); Mt. Rungwe, N slopes, in ecotone between forest and grassland, 2130-2220 m, 14 Mar. 1937 (fl), Thompson 905 (AMES!, K!); Rukwa: Ufipa Distr., Malonje (eastern summit), among rocks on level ground at the summit, 2400 m, 1 Apr. 1950 (fl), Bullock 2767 (K!); Ruvuma, Southern Prov., Kitulo Sheep Project, grassland, 2700 m, 1 Mar. 1970 (fl), Fuller 113 (K!); Songea Distr., Matongo Hills, Mpapa in secondary bushland, 1530-1680 m, 24 May 1956 (fl), Milne-Redhead \& Taylor 10426(K! \& spirit.) \& $10426 a$ (AMES!, UGDA-DLSz! - fragment, drawing); Tanga: Ukonga, Lumakali River, in meadow, 2000 m, 4 Feb. 1914 (fl), Stolz 2490 A (K!). Malawi. Central: Kasungu Distr., Msuka Hills, 10 May 1947 (fl), Benson 1261 (K!); Northern: Chitipa Distr., Misuku Hills, 1350 m, 10 May 1947 (fl), Benson 1238 (K!); 9 May 1947 (fl), Benson 1239 (K!); Benson 1245 (K!); Chitipa Distr., Misuku Hills, Mughesse rain forest, 1650 m, 6 July 1973 (fl), Pawek 7007 (K!); Karonga Distr., Mussissi Mt., in often grassland on quarty soil among rocks, 1950 m, 2 May 1947 (fl), Benson 1246 (K!); Kondowe, 600-1800 m, July 1896 (fl), Whyte s.n. (K!); Mzimba Distr., South Vipya Plateau, $30 \mathrm{~km}$ south of Chikangawa on link road to Luwawa,Ggassland, 1670 m, 8 May 1970 (fl), Brummitt 10471 (K!); Mzimba, Apr. 1954 
(fl), Jackson 1307 (K!); Mzimba Distr., Mzuzu, Lunyangwa River at waterworks, edge dambo, in grassland, 1350 m, 27 June 1973 (fl), Pawek 6951 (C!); Mzuzu, Lunyangwa, in grass in woodland, near edge of riverside forest, 1330 m, 23 May 1986 (fl), la Croix 841 (K!); Mzimba Distr., Mzuzu, Marymount, dambo (wet meadow), gray-sandy soil, 1350 m, 28 June 1969 (fl), Pawek 2505 (K!); Mzimba Distr., Mzuzu, Marymount, Brachystegia woodland, 1350 m, 23 June 1975 (fl), Pawek 9801 (AMES!, K!); The same locality, frequent in Brachystegia woodland, 1350 m, 31 July 1975 (fl), Pawek 9913 (AMES!, K!); Mzimba Distr., 10 m N Chikangawa, open grassland, 1650 m, 29 Mar. 1978 (fl), Phillips 3345 (K!); 5 miles west of Mzuzu, edge of rain forest, $1260 \mathrm{~m}, 8$ June 1976 (fl), Phillips 1874 (K!); Mzuzu, in garden, overgrass, 1290 m, 1 July 1983 (fl), s.n. (K!); Nyika National Park, frequent in grassland around Chilinda, montane grassland, $2250 \mathrm{~m}, 12$ Mar. 1983 (fl), Elias 15 (K!); The same locality, 14 Mar. 1983 (fl), Elias 18 (K!); Nyika National Park, montane grassland beside Chelinda-Kasaramba road, $2220 \mathrm{~m}$, 7 Apr. 1984 (fl), la Croix 596 (K!); Nyika National Park, Chisanga Falls, just part footpath to Falls, top of bank where it is cut away by road, 24 Apr. 1982 (fl), la Croix \& Johnston-Stewart 327 (K!); Rumphi Distr., Uzumara forest, occasional in grassland and in dirt road, 1980 m, 25 May 1975 (fl), Pawek 9640 (K!). Viphya, Chikangawa, in montane grassland, $1750 \mathrm{~m}, 10$ May 1987 (fl), Cornelius (la Croix) 1037 (K!). Viphya, montane grassland olong top of cutting beside road, 18 Apr. 1982 (fl), la Croix \& Johnston-Stewart 312 (K!); Viphya, Nkhata Bay Distr., 2 miles east on Parker Road, grassland, 1650 m, 25 May 1969 (fl), Pawek 2422 (K!); Nkhata Bay Distr., Vipya Link Rd, 70 miles SW of Mzuzu, grassland, 1740 m, 16 May 1971 (fl), Pawek 4825 (K!); Northern Viphya, Uzumara F.R., grassland at edge of forest, on beside path, $1900 \mathrm{~m}, 26$ Apr. 1987 (fl), la Croix 1035 (K!); South Viphya, Wozi Hill, near Chikangawa, in grassland, on rocky hill, 1800 m, 5 Apr. 1983 (fl), Dowsett-Lemaire 713 (K!); South Viphya, Kawandama, very common in montane grassland, 1800-1850 m, 14 May 1983 (fl), Dowsett-Lemaire 714 (K!); South Viphya, small rocky hill between Elephant Rock and Chikangawa, in grass among rocks, $1750 \mathrm{~m}$, 10 Apr. 1986 (fl), la Croix 831 (K!).

Notes. This species is characterized by peculiar geniculate spur near the middle, which distal part is swollen and form a kind of blunt ampoulle. Bilabrella kyimbilae can be easily separated from similar B. thomsonii by petals. They are densely pubescent and ciliate in the former and puberulous or papillose with ciliolate margins in the latter.

The type specimen of Habenaria furcipetala is evidently a slightly abnormal form in which the size of the anterior petal lobe has been reduced. Schlech- ter (1915) mentions that he was able to find only one "normal"specimen among the 60 or so plants in the gathering of Stolz 1189. The others had the petals more or less converted into stamens.

62. Bilabrella thomsonii (Rchb. f.) Szlach. \& KrasLap., Richardiana 3(3): 143. 2003.

Habenaria thomsonii Rchb. f. apud Oliv., J. Linn. Soc., Bot. 21: 404. 1885. - Summerhayes, FTEA, Orchid. 1: 85. 1968b. - Robyns \& Tournay, Fl. Spermat. Parc Natl. Albert 3: 432. 1955. - Rolfe, Fl. Trop. Afr. 7: 242. 1984. - Stewart \& Campbell, Orchids of Kenya: 141. 1996.

Type: Kenya, Thomson s.n. (lectotype, designated by Summerhayes [1968b: 85], K!; isolectotype, BR!). Habenaria humilior var. brevicalcarata Rendle, J. Bot. 33: 280. 1895.

Type: Kenya, Jackson s.n. (BM not seen).

Stem $35-50 \mathrm{~cm}$ tall, erect, slender to rather stout, leafy throughout its length. Leaves 7-10, 9-12 cm long and $1.5-2.5 \mathrm{~cm}$ wide, the lowermost sheath-like, the next 3 or 4 suberect, lanceolate or widely lanceolate, acute, the remainder smaller, lanceolate, resembling the bracts. Inflorescence 6-12 cm long, densely 12-35-flowered.
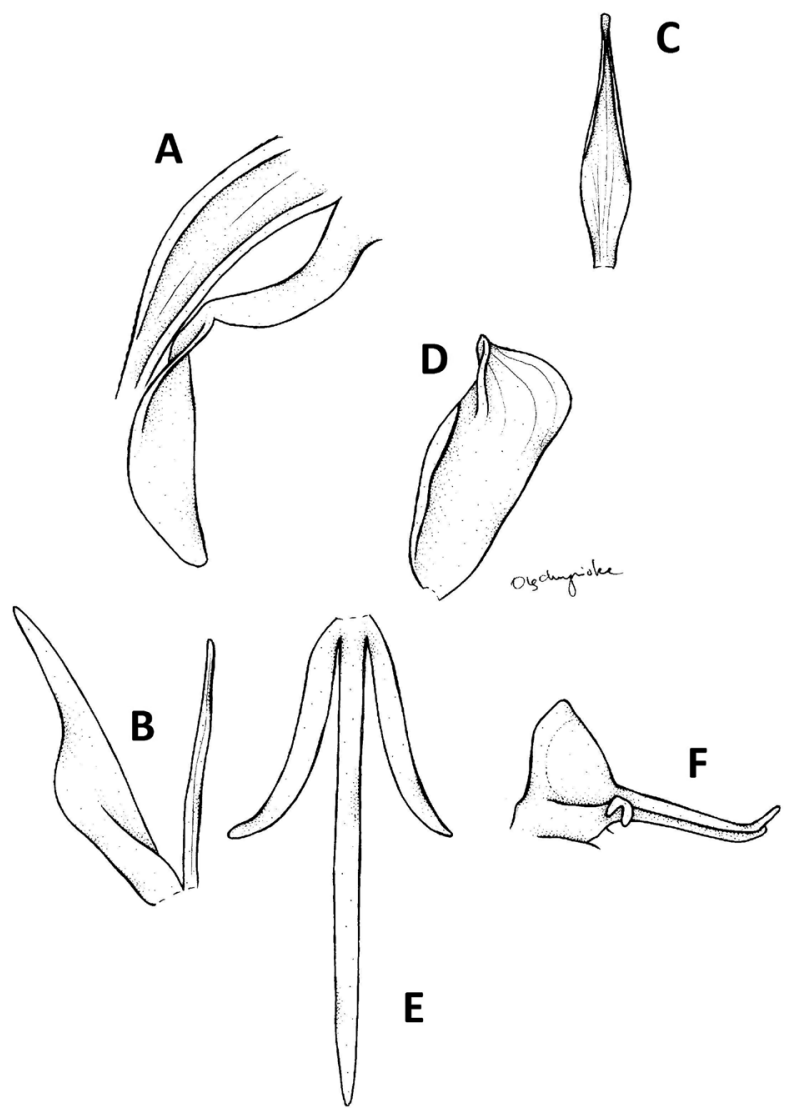

Fig. 176. Bilabrella thomsonii (Rchb. f.) Szlach. \& Kras-Lap. Explanations: A - ovary and spur; B - petal; C - dorsal sepal; D - lateral sepal; E-lip; F - gynostemium (drawn by N. Olędrzyńska from isolectotype of Habenaria thomsonii Rchb. F. - Thomson s.n., BR) 


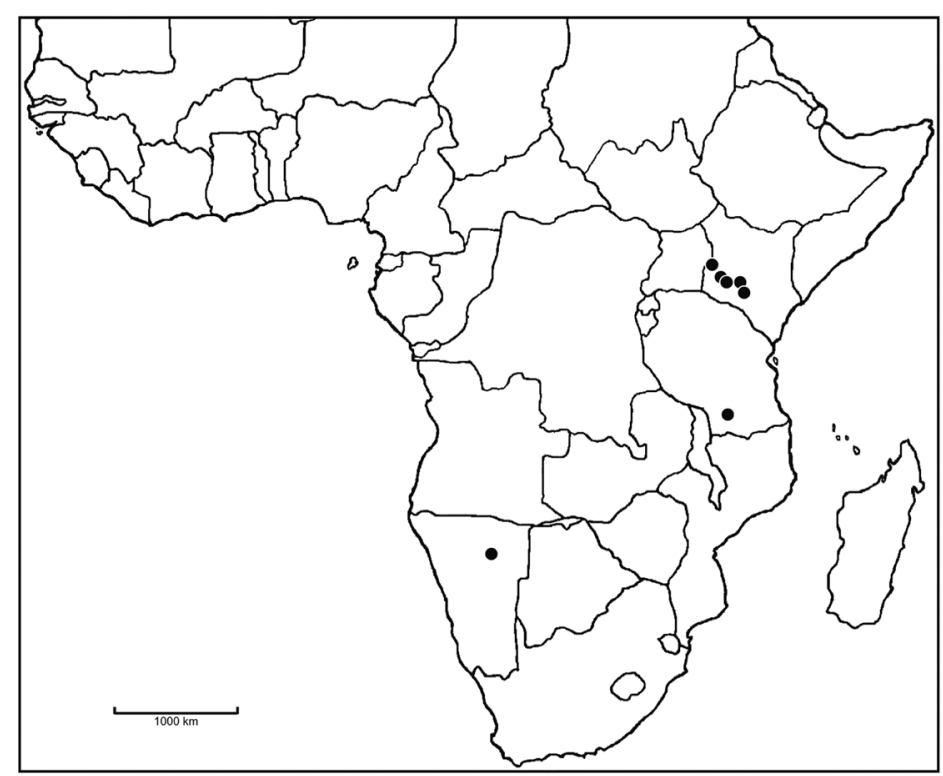

Fig. 177. Distribution of Bilabrella thomsonii (Rchb.f.) Szlach. \& Kras-Lap.

Flowers green with white centre. Floral bracts 10 $18 \mathrm{~mm}$ long, lanceolate or widely lanceolate, acuminate, often equalling or longer than the pedicel with ovary. Pedicel with ovary 14-17 mm long, curved. Dorsal sepal 6-7 mm long, $3 \mathrm{~mm}$ wide, elliptic or oblong-elliptic, apiculate, obtuse, convex, reflexed. Petals bilobed to the base, both lobes puberulous or papillose with ciliolate margins; anterior lobe $8 \mathrm{~mm}$ long, 2-3 $\mathrm{mm}$ wide, spreading forwards, obliquely oblong-obovate in the lower half, linear-lanceolate above, acute; posterior lobe 6-7 mm long, 0.3-0.5 $\mathrm{mm}$ wide, reflexed, linear-filiform. Lateral sepals 6.5-8.5 mm long, 3-6 mm wide, deflexed, very obliquely oblong obovate with a lateral apiculus, cochleate. Lip decurved, 3-lobed from a very short undivided base; the middle lobe 11-12 $\mathrm{mm}$ long, 0.5 $0.9 \mathrm{~mm}$ wide, linear, acute; lateral lobes 6-7.5 $\mathrm{mm}$ long, 0.5-0.9 mm wide, curved forwards, linear-lanceolate. Spur 10-12 mm long, parallel to the ovary, twisted and geniculate near the middle, swollen in the distal half, blunt. Anther nearly $3 \mathrm{~mm}$ tall; connective rounded; antherophores over $4 \mathrm{~mm}$ long, the apex abruptly upturned. Stigmaphores $4 \mathrm{~mm}$ long, slender. Rostellum middle lobe shorter than connective. Auriculae mediumsized, deeply bifid (Fig. 176).

E c ology. Damp or swampy grassland. MarchNovember.

Distribution. Kenya, Tanzania, alt. 2400-3000 m (Fig. 177).

Specimens examined. Kenya. Rift Valley: Ainabkoi, 2400 m, 11 Nov. 1949 (fl), Bickford 7764 (K!); Sitoton, near Lumbwa, 2550 m, Nov. 1936 (fl), Graham 3736 (K!); Mau Forest, Sep. 1895 (fl), Jackson 1889 (BM!). Naivasha Prov., Aberdare Mts.
Kinagop, 2550 m, Oct. 1934 (fl), Synge S. 1119 (BM!); Naivasha Prov., Naivasha Distr., Aberdare Mts. Sussex Farm, Kinagop, 2550 m, 1 Nov. 1934 (fl), Taylor 1578 (BM!); Laikipia Plateau, 2000-2700 m, Thomson s.n. (K!); Western: NE Elgon, open grass slade at upper edge of pools forest, $3000 \mathrm{~m}$, Oct. 1948 (fl), Tweedie 727 (K!). Tanzania. Iringa: Makeje Distr., Kitulo Plateau, open grassland, along river stream, $09^{\circ} 05^{\prime} \mathrm{S}, 33^{\circ} 58^{\prime} \mathrm{E}$, 2400 m, 1 Mar. 1991 (fl), Suleiman \& Fundi 54 (K!). Notes. By spur and petals forms it appears to be similar to Habenaria kyimbilae, from which it differs by puberulous or papillose petals with ciliolate margins.

63. Bilabrella welwitschii (Rchb. f.) Szlach. \& KrasLap., Richardiana 3(3): 142. 2003. - Szlachetko et al., Orchid. of West-Central Africa 1: 224. 2010.

Habenaria welwitschii Rchb. f., Flora 48: 179. 1865. - Rolfe, Fl. Trop. Afr. 7: 235. 1898. - Rendle, Cat. Welw. Afr. Pl. 2: 12. 1899. - Robyns \& Tournay, Fl. Spermat. Parc Nat. Albert 3: 434. 1955. - Summerhayes, FTEA, Orchid. 1: 83. 1968b. - Geerinck \& Coutrez, Not. Taxon. Orchid. Afr. Centr., Habenaria: 12. 1977. - Cribb \& Leedal, Mount. Fl. S. Tanzania: 215. 1982. - Geerinck, Fl. Afr. Centr., Orchid. 1: 108. 1984. - la Croix et al., Orchid. Malawi: 80. 1991. - la Croix \& Cribb, Fl. Zambes., Orchid. 11: 111. 1995.

Type: Angola, Welwitsch 721 (lectotype, designated by Szlachetko et al. [2010: 224], BM!; isolectotypes, $\mathrm{K}$ !, P!, W-R!).

Habenaria leptostigma Schltr., Bot. Syst. Jahrb. 38: 146. 1906.

Type: Mozambique, Schlechter $6995(\mathrm{~B}+)$.

Habenaria keiliana Kraenzl., Bot. Syst. Jahrb. 43: 393. 1909.

Type: Rwanda, Keil 225 (B+).

Tubers $1.5 \mathrm{~cm}$ long, $0.9 \mathrm{~cm}$ in diameter, almost globose. Stem 20-62 cm tall, erect, very delicate, glabrous. Leaves 7-12, 5-26 cm long, 1-1.5 cm wide, linear to linear-lanceolate, acute, gathered in the lower part of the stem, suberect, decreasing in size up the stem. Inflorescence 6-18 cm long, 10-30-flowered, rather lax. Flowers white or greenish-white. Floral bracts $8-20 \mathrm{~mm}$ long, ovate-lanceolate, acuminate, glabrous. Pedicel and ovary 15-25 mm long, glabrous. Dorsal sepal 5-6.5 mm long, 2-2.5 mm wide, elliptic, oblong-ovate, obtuse, cochleate, glabrous, deflexed. Petals bilobed almost to the base, rather fleshy; anterior lobe 4-5.5 $\mathrm{mm}$ long, $1.5-3 \mathrm{~mm}$ wide, obliquely ovate, obovate or deltoid, acute, spreading forwards and sideways, glabrous; posterior lobe 4-6 mm long, 0.4-0.5 mm wide, linearsubulate to filiform, acute, shortly ciliolate. Lateral sepals 7-9 mm long, 4-6 mm wide, obliquely oblong elliptic to obovate, cochleate, apiculus laterally placed, 


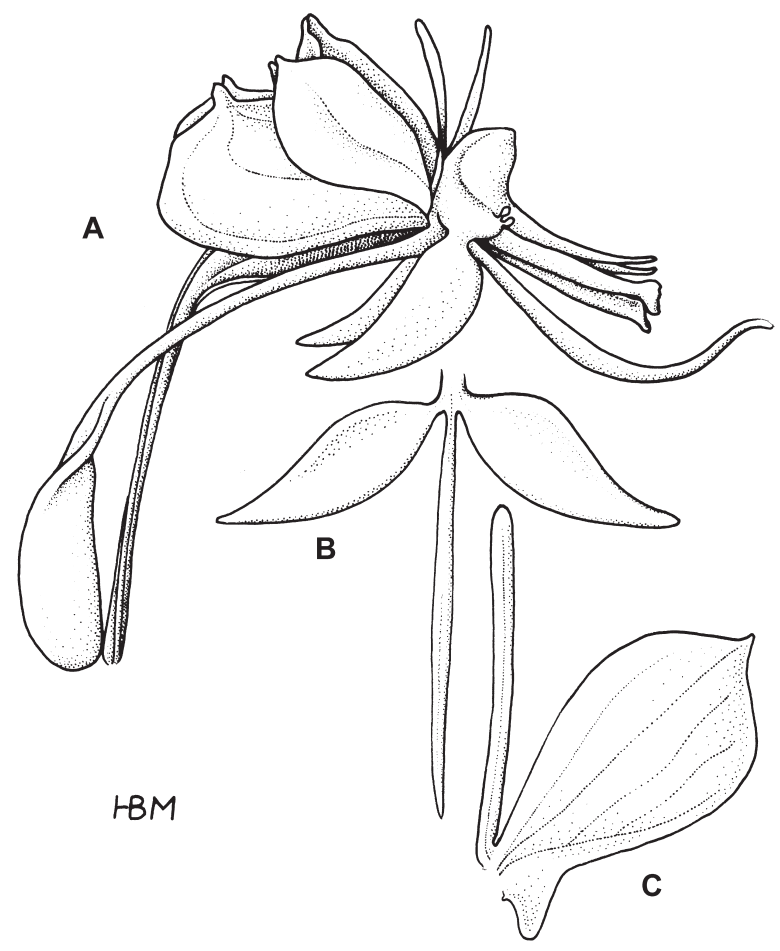

Fig. 178. Bilabrella welwitschii (Rchb. f.) Szlach. \& Kras-Lap. Explanations: A - flower; B - lip; C - petal (drawn by H. B. Margońska from isolectotype of Habenaria welwitschii Rchb. f. - Welwitsch 721, W-R)

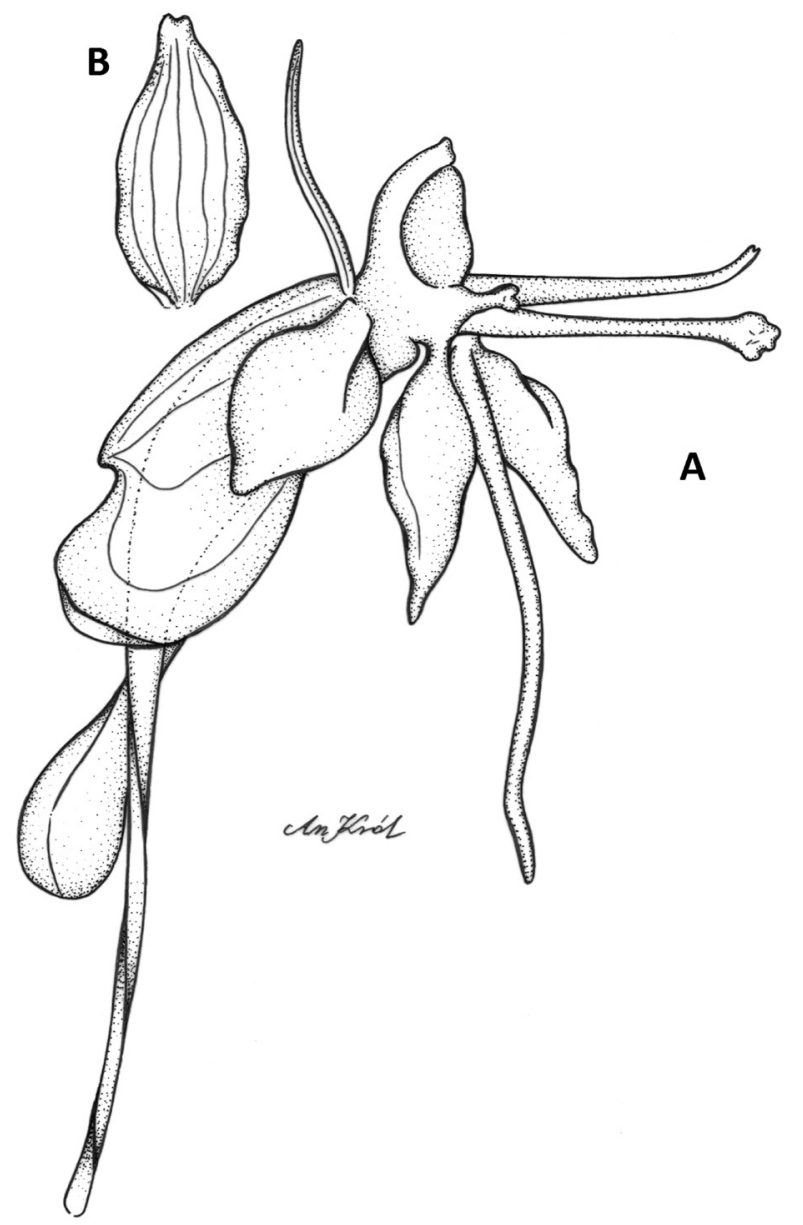

Fig. 179. Bilabrella welwitschii (Rchb. f.) Szlach. \& Kras-Lap. Explantions: A - flower; B - dorsal sepal (drawn by A. Król from Polhill \& Paulo 1569, AMES) glabrous. Lip 3-lobed above the basal undivided part to $1.5 \mathrm{~mm}$ long, delicate, glabrous; the middle lobe 8-12 mm long, 0.5-0.6 mm wide, linear, obtuse to acute; lateral lobes 4-6.5 mm long, 1-1.9 mm wide, oblonglanceolate, subobtuse. Spur 10-16 mm long, narrowly cylindrical to filiform, twisted and much swollen in the apical half, $2 \mathrm{~mm}$ in diameter, blunt, parallel to the ovary or upcurved. Anther $2.5 \mathrm{~mm}$ long; connective rounded; antherophores 5-6 mm long, slender, slightly curved apically. Stigmaphores 5-7 mm long, narrowly cylindrical, straight, truncate. Rostellum middle lobe shorter than thecae. Auriculae small, deeply bifid (Figs 178-179).

E c ology. Marshy places, rock crevices, marsh. January-December.

Distribution. Democratic Republic of the Congo (Zaire), Uganda, Burundi, Tanzania, Angola, Malawi, alt. up to $2700 \mathrm{~m}$ (Fig. 180).

Specimens examined.emocratic Republic of the Congo (Zaire). Haut-Katanga: Shaba Prov. (Katanga), Kundelungu Plateau, in very wet parts of marsh at Katshupa, 1700 m, 5 Mar. 1975 (fl), Hooper \& Townsend 541 (K!); Haut-Shaba, Plateau Kundelungu, $2 \mathrm{~km}$ of $2 \mathrm{E}$ from Lutshipuka, $1600 \mathrm{~m}, 8$ Jan. 1971 (fl), Lisowski 65979 (UGDA-DLSz!); Shaba Region, Biano Plateau of $22 \mathrm{~km}$ NE of Tenke, $1575 \mathrm{~m}, 19$ Dec. 1982 (fl), Schaijes 1654 (K! \& spirit.); Haut-Lomami: Upemba Natural Park, land of Mubale spring, savannah, 1880 m, 16 Jan. 1948 (fl), Witte 3237 (K!); Nord-Kivu: Sabinio to Vissoke, Mashiga swamp, in short Cyperus and grass growth, $2250 \mathrm{~m}, 14$ Dec. 1930 (fl), Burtt 2995 (K!); Sabinio to Vissoke, South Mashiga swamp, 2250 m, 14 Dec. 1930 (fl), Taylor 3001 (K!). Uganda. Northern: Moyo Distr., Muhabura Mt., in wet grass overlying rock, 2500 m, 29 Dec. 1970 (fl), Hunford s.n. (K!); Western: Kigezi Distr., Virunga Mts., foot of pass between Mgahinga and Sabinio, 2100 m, 25 Dec. 1934 (fl), Taylor 2046 (BM!); Kisoro Distr., below Sabinio Volcano in short grassed Cyperus marsh on lava plain, on marsh to Bunagana Camp, 2100 m, 13 Dec. 1930 (fl), Burtt 2990 (K!). Burundi. Bubanza: Teza, 313'S, 29'33'E, 2550 m, 26 Feb. 1980 (fl), Reekmans 8645 (K!). Tanzania. Iringa: Dabaga Highlands, 10 miles S of Dabaga on the Kibengu road, streamside marsh in upland grassland, 1965 m, 22 Feb. 1962 (fl), Polhill \& Paulo 1569 (AMES!, K!); Njombe, Dansland Dambo, dambo margin - damp but out standing water in short grass, 2160 m, 29 Jan. 1989 (fl), Spurrier 47 (K!); 90 km past Iringa on road to Mbeya, common in wet dambo, 2700 m, 4 Feb. 1976 (fl), Cribb \& Grey-Wilson 10547 (K!); Njombe Distr., Dansland, Uwemba-Ludewa road past Luponde Tea Estate, swampy grassland, growing in water, 9'34,66'S, 34'39,03'E, 2157 m, 18 Feb. 2003 (fl), Bytebier \& Sakwa 2540 (K!); Njombe, Vwembi, Hakafilo River, 2200 m, 10 Jan. 1978 (fl), Leedal 4799 


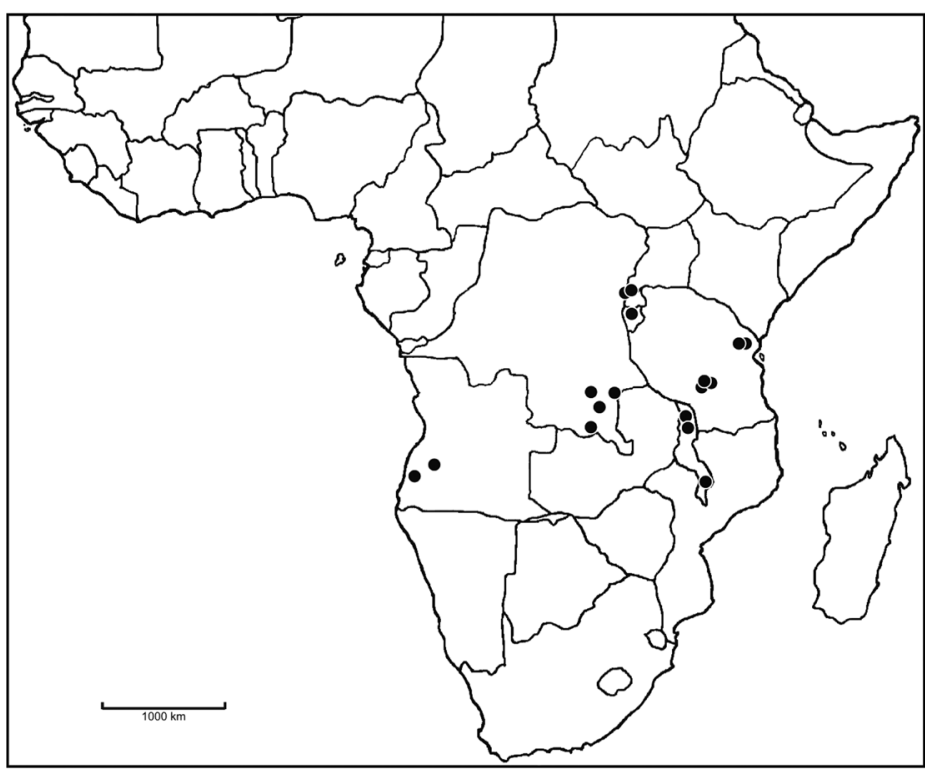

Fig. 180. Distribution of Bilabrella welwitschii (Rchb.f.) Szlach. \& Kras-Lap.

(K!); Main road between Sao Hill and Lugoda turn off, in marsh, 20 Mar. 1986 (fl), Bidgood \& Keeley 356 (K!); Sao Hill, meadow land \& swamp margins in dense grass, $1860 \mathrm{~m}$, Feb. 1959 (fl), Watermeyer 20 (K!); Sao Hill, damp meadow, 1860 m, Watermeyer 256 (K!); Tanga: Lutindi Peak, in gneiss rock crevices in association with Aeolanthus usambarensis, Bidens, Streotocarpus saxorum and Cyperus on a rocky mountain peak, 1350 m, 28 Oct. 1935 (fl), Greenway 4142 (K!); West Usambara Mts., Mazumbai Forest Reserve, 1650 m, 30 Oct. 1980 (fl), Tanner 237 (K!). Angola. Huambo: Huila and Mocamedes, Chipipa, 18 Feb. 1960 (fl), Stopp BO207 (K!); Huíla: Between Lopollo and Humpata, Jan. 1860 (fl), Welwitsch 721 (BM!, K!, P!, W-R!). Malawi. Northern: Muhuju, Chinzama Plateau, on seepage slopes, 2200 m, 28 Mar. 1983 (fl), la Croix \& Johnston-Stewart 477 (K!); Mzimba Distr., Mzuzu, Marymount, hard soil, grassy flat, 1350 m, 4 July 1975 (fl), Pawek 9807 (K!); Southern: Mulanje Mt., by Nandalanda path from Chinzania to Thuchila, under a large rock in montane grassland, 2000 m, 13 Apr. 1985 (fl), Jankins 4 (K!).

$\mathrm{N}$ ote $\mathrm{s}$. This species is easily distinguishable from other of the genus by peculiar petals (anterior lobe obliquely ovate, obovate or deltoid, acute) and lip shape (lateral lobes are oblong-lanceolate, subobtuse, different in form from the middle one). It shares similar spur form, i.e. narrowly cylindrical to filiform in the basal part, twisted and much swollen in the apical half, occasionally forming an ampoule), with $B$. leucoceras, $B$. kubangensis and B. myodes, but in all of those species lip lobes are similar in form, i.e. linear-filiform.
64. Bilabrella leucoceras (Schltr.) Szlach. \& Kras-Lap., Richardiana 3(3): 141. 2003.

Habenaria leucoceras Schltr., Bot. Jahrb. Syst. 53: 518. 1915. - Summerhayes, FTEA, Orchid. 1: 90. 1968b. Williamson, Orchid. S. Central Afr.: 53. 1977. - la Croix et al., Orchid. Malawi: 73. 1991. - la Croix \& Cribb, F1. Zambes., Orchid. 11: 99. 1995.

Type (as holotype): Tanzania, Stolz 2490 (B+; lectotype, designated by Summerhayes [1968b: 90], BM!, isolectotype, $\mathrm{K}$ !).

Tubers $1.5-2 \mathrm{~cm}$ long, $0.5-1.5 \mathrm{~cm}$ in diameter, ellipsoid or ovoid, sparsely tomentose. Stem 30-60 cm tall, glabrous, erect, rather stout, leafy throughout its length. Leaves 7-11, 7-20 cm long, $0.5-1.5 \mathrm{~cm}$ wide, the lowest 1 or 2 reduced to sheaths, together with the sheath of the lowermost foliage leaf whitish with purplish-green reticulated veins, the middle leaves erect or suberect, rather stiff, linear or very narrowly lanceolate, acute, the upper leaves adpressed to the stem, imbricate, much smaller, lanceolate, acuminate, resembling the lower bracts but more herbaceous. Inflorescence $7-15 \mathrm{~cm}$ long, rather loosely 6-25-flowered. Flowers green or whitish-green. Floral bracts 15-30 mm long, thin and chaffy, lanceolate, acuminate, often longer than the pedicel with ovary. Pedicel with ovary 16-25 mm long. Dorsal sepal 6-8 $\mathrm{mm}$ long, 2-3 $\mathrm{mm}$ wide, narrowly elliptic, obtuse, convex, reflexed. Petals bilobed to the base; anterior lobe 12.5-16.5(19) $\mathrm{mm}$ long, 2-3.5 mm wide, spreading, versatile, oblong to linear-lanceolate,

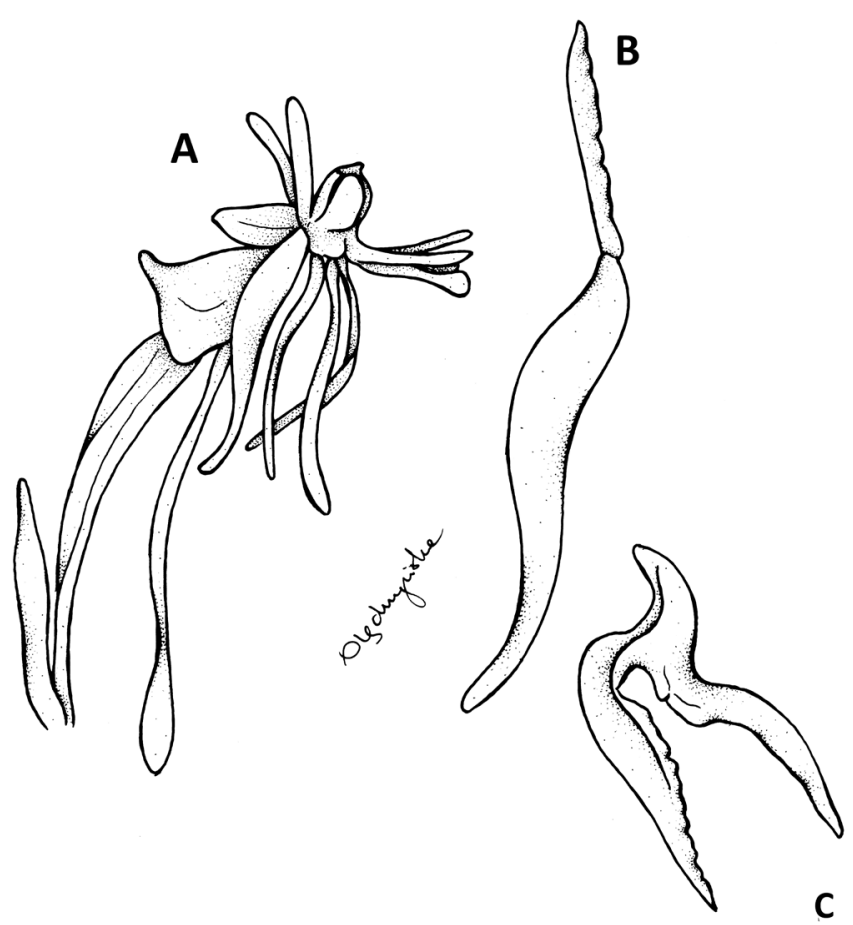

Fig. 181. Bilabrella leucoceras (Schltr.) Szlach. \& Kras-Lap. Explantions: A - flower; B - petal; C - rostellum (redrawn by N. Olędrzyńska from Williamson 1977) 


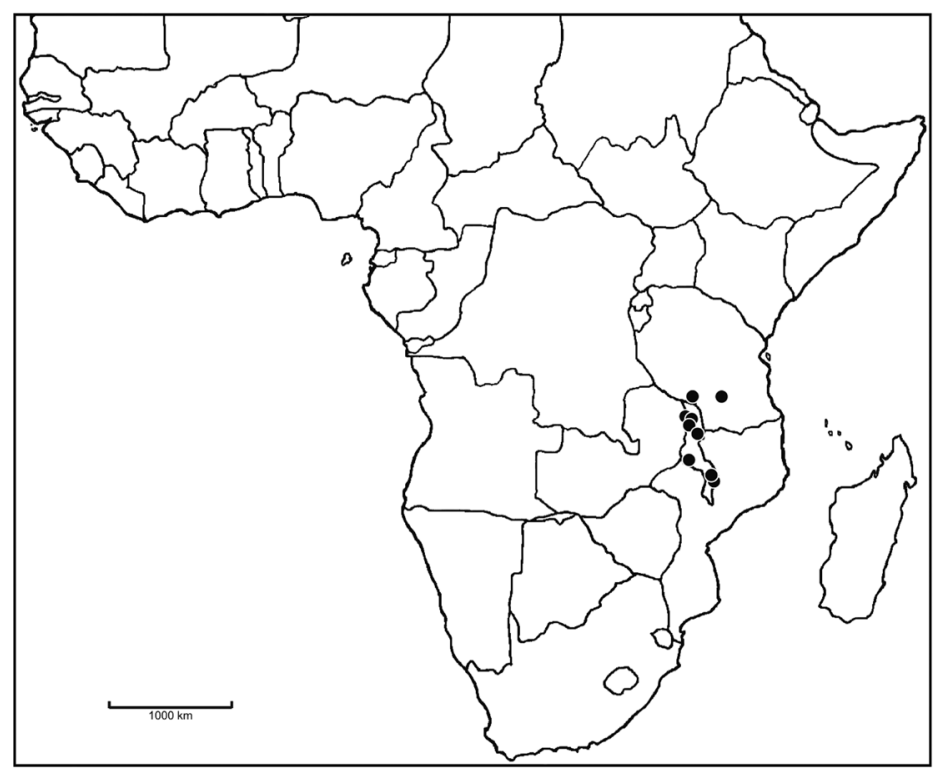

Fig. 182. Distribution of Bilabrella leucoceras (Schltr.) Szlach. \& Kras-Lap.

curved, acute, glabrous; posterior lobe 7-9.5 mm long, to $1 \mathrm{~mm}$ wide, reflexed, linear or linear-filiform, ciliolate. Lateral sepals 8-11 mm long, 4.5-7 mm wide, obliquely obovate or elliptical with the lateral apiculus, deflexed. Lip deflexed, 3-lobed nearly to the base; the middle lobe 12.5-16(19) mm long, 0.5-1 mm wide, linear, acute, incurved; lateral lobes 8.5-12.5 mm long, 0.5-0.9 mm wide, linear. Spur 15-18.5 mm long, incurved, twisted once or twice in the middle, apical third or quarter much swollen, blunt. Anther 2-4 mm tall; connective obtuse; antherophores 3-5 mm long, slender, straight. Stigmaphores 3.5-5 mm long, cylindrical-clavate, truncate. Rostellum middle lobe shorter than connective. Auriculae medium-sized, bilobed (Fig. 181).

E c o log y. Grassland, Brachystegia woodland, brown soil. January-May, October.

D istribution. Tanzania, Zambia, Malawi, alt. 1500-2550 m (Fig. 182).

S p e c im en s e x a m ined. Tanzania. Iringa: Mufindi Distr., in grassland of edge of guarry, $1800 \mathrm{~m}$, 30 Apr. 1989 (fl), Leyser 250 (K!); Njombe, Dansland, wide spraed in grassland, laterite soil, $2000 \mathrm{~m}$, May 1991 (fl), Spurrier 123 (K!); Iringa-Kisolanza Farm, Njombe-Jakoby, grassland - poor peat and sand soil seasonally wet, 1500 m, Apr. 1992 (fl), Spurrier 147 (K!); Mbeya: Mbeya Mt., in upland grassland on dark brown soil, 2550 m, 13 May 1956 (fl), Milne-Redhead \& Taylor 10313 (AMES!, K!); Mbeya Distr., Southern Highlands Prov., Mbeya Range, locally common in mountain grassland, 2400 m, Apr. 1959 (fl), Procter 1191 (K!); Rungwe Distr., Rumakali R, Stolz 2490 (K!). Zambia. Eastern: Lundazi Distr., Nyika Plateau, near Rest House, in grassland, Mar. 1967 (fl), Williamson 274 (K!); Lundazi Distr., Nyika Plateau, grassland,
Oct. 1967 (fl), Williamson \& Simon 943 (K!); Nyika, grassland near Rest House, Mar. 1967 (fl), Williamson \& Odgers 274 (K!); Nyika National Park, 0,5 km SW of Zambian Govt. Rest House, 17 Apr. 1986 (fl), Philcox, Pope \& Chisumpa 9976 (K!). Malawi. Central: Nsipo, Nchefu-Dedza road, 19 Jan. 1972 (fl), Westwood 586 (K!); Northern: Rumphi Distr., Malawi National Park Nyika Plateau, near junction Junip. For. Kasar. Road, grassland, 2340 m, 11 Apr. 1969 (fl), Pawek 2118 (K!); Rumphi Distr., Nyika Plateau, brought down from Nyika, grassland, 2250 m, 1 May 1974 (fl), Pawek 8566 (K!); Rumphi Distr., Dembo Road, Nyika Plateau, grassland, $10,6^{\circ} \mathrm{S}, 11,9^{\circ} \mathrm{E}, 2300 \mathrm{~m}, 17$ Apr. 1975 (fl), Pawek 9296 (K!); Nyika Plateau, 1 km N of Zambian Govt. Rest House, damp area at base of rock outcrop, 2260 m, 17 Apr. 1986 (fl), Philcox, Pope \& Chisumpa 9954 (K!); Nyika National Park, grassland near Dam 3, 2100 m, 7 Apr. 1984 (fl), la Croix 594 (K!); Nyika, not infrequent in montane grassland, 21 Apr. 1982 (fl), la Croix \& Johnston-Stewart 317 (K!); Viphya Plateau, 2 Mar. 1972 (fl), Westwood 663 (K!); Southern: Mlanje Distr., slopes of Mlanje Mountain, on Tuchila "skyline" path, mountside with scattered Brachystegia, $1650 \mathrm{~m}$, 10 Apr. 1970 (fl), Brummitt 9788 (K!); Malosa Nat., in secondary montane grassland at edge of Brachystegia woodland, 1500 m, 3 Apr. 1983 (fl), la Croix \& Johnston-Stewart 481 (K!); Zomba Plateau, rare in open grassland, 1800 m, 25 Apr. 1949 (fl), Wiehe N/84 (K!). Notes. This species is similar to Bilabrella kubangensis and $B$. myodes in many respects, but differs form both of them by having glabrous anterior petal lobes.

65. Bilabrella kubangensis (Schltr.) Szlach. \& KrasLap., Richardiana 3(3): 141. 2003. - Szlachetko et al., Orchid. of West-Central Africa 1: 237. 2010.

Habenaria kubangensis Schltr. in Warb., Kuene-Sambesi-Exped. Baum: 205. 1903. - Schlechter, Repert. Sp. Nov Regni Veg. 68: tab. 28. 1932.

Type: Angola, Baum 425a (B+; lectotype, designated by Szlachetko et al. [2010: 237], K!; isolectotype, W-R!).

Tubers 1-1.5 cm long, 0.5-1 cm in diameter, ellipsoid. Stem 35-45 cm tall, erect, slender, glabrous. Leaves 5-9, up to $18 \mathrm{~cm}$ long, up to $1(1.5) \mathrm{cm}$ wide, narrowly lanceolate to linear-lanceolate, acute, gathered in the lower part of the stem, erect or suberect, decreasing in size up the stem. Inflorescence $5-11 \mathrm{~cm}$ long, 15- to many-flowered, dense. Floral bracts 10 $\mathrm{mm}$ long, glabrous. Pedicel and ovary 12-17 mm long, glabrous. Dorsal sepal 5-5.5 mm long, 2-3 mm wide, oblong-elliptic to ovate, truncate, cochleate, glabrous. Petals bilobed to the base; anterior lobe 7-9 $\mathrm{mm}$ long, $2 \mathrm{~mm}$ wide, obliquely oblong-lanceolate, acuminate, thick, fleshy, ciliolate at the base; posterior lobe 3-5 mm 


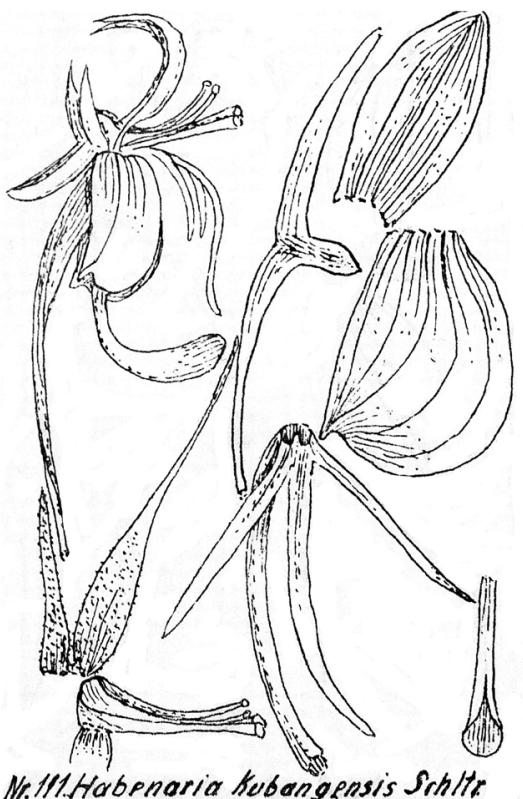

Fig. 183. Original Schlechter's (1932) drawing of Habenaria kubangensis Schltr.

long, $0.3 \mathrm{~mm}$ wide, filiform, acute, thin, ciliolate. Lateral sepals $7.5 \mathrm{~mm}$ long, $4.5-5.5 \mathrm{~mm}$ wide, obliquely obovate to elliptic-obovate, cochleate at the apex, glabrous, apiculus laterally placed. Lip deflexed, 3-lobed above the basal 2-3 mm, glabrous; the middle lobe $9-11 \mathrm{~mm}$ long, $0.5-0.8 \mathrm{~mm}$ wide, linear, acute; lateral lobes $6 \mathrm{~mm}$ long, $0.5-0.8 \mathrm{~mm}$ wide, linear, acute, apices slightly upcurved. Spur $12 \mathrm{~mm}$ long, narrowly cylindrical, inflated in the apical third, subacute, pendent, twisted. Anther

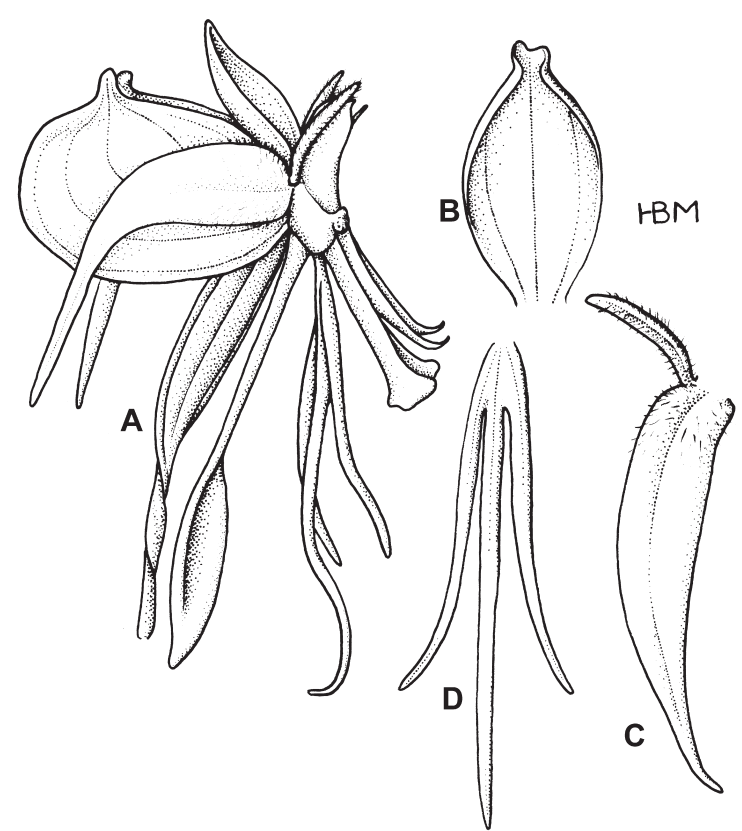

Fig. 184. Bilabrella kubangensis (Schltr.) Szlach. \& Kras-Lap. Explantions: A - flower; B - dorsal sepal; C - petal; D - lip (drawn by H. B. Margońska from isolectotype of Habenaria kubangensis Schltr. - Baum $425 a$, W-R)

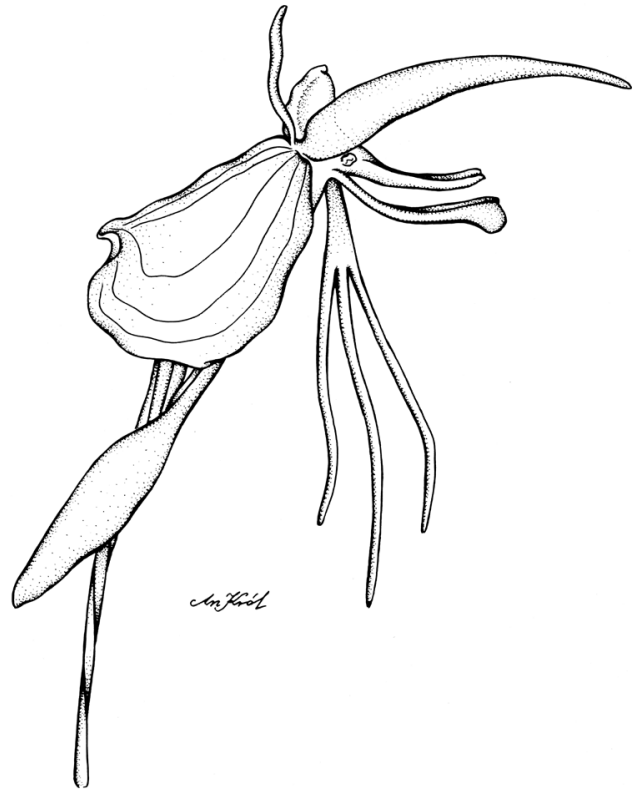

Fig. 185. Bilabrella kubangensis (Schltr.) Szlach. \& Kras-Lap. flower (drawn by A. Król from Holmes 0118, AMES)

$2 \mathrm{~mm}$ long; oblong-ellipsoid; connective truncate; antherophores $5 \mathrm{~mm}$ long, slender, gently upcurved in the apical half. Stigmaphores $5 \mathrm{~mm}$ long, narrowly cylindrical, gently upcurved, with ligulate apex. Rostellum middle lobe distinctly shorter than thecae. Auriculae small, deeply bilobed (Figs 183-185).

E c o logy. No records. May-December.

Distribution. Democratic Republic of the Congo (Zaire), Angola, Zambia, alt. 1100-1500 m (Fig. 186). Specimens examined. Democratic Republic of the Congo (Zaire). Haut-Katanga: Shaba Region,

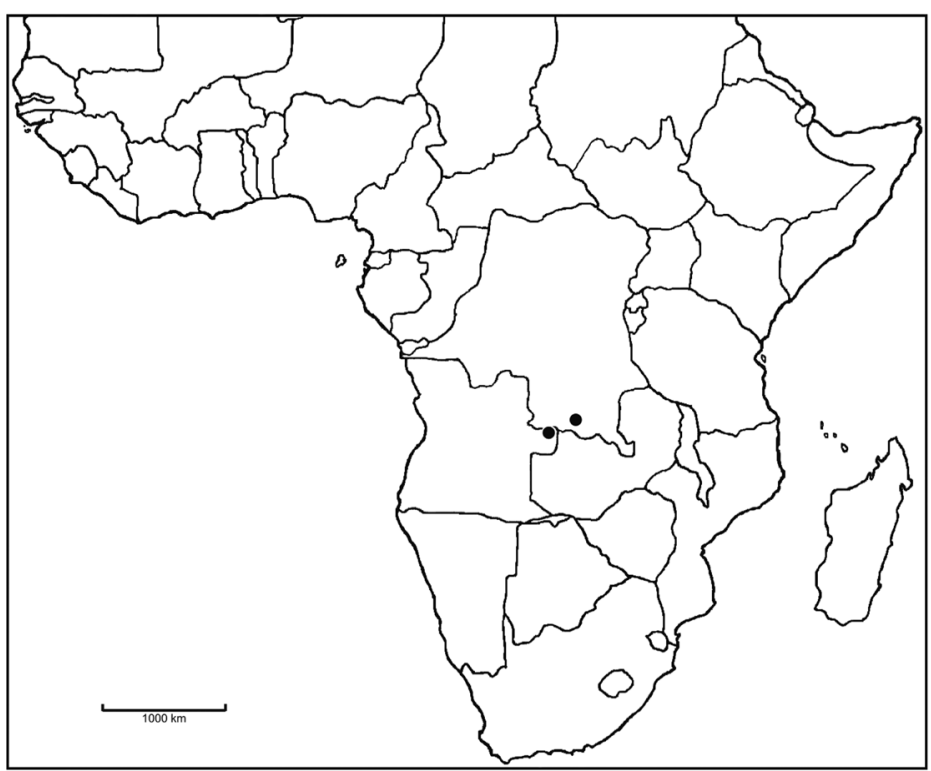

Fig. 186. Distribution of Bilabrella kubangensis (Schltr.) Szlach. \& Kras-Lap. 
5,5 km of S from Kolwenzi, Waniba, $1500 \mathrm{~m}, 25$ May 1986 (fl), Schaijes 2870 (K! \& spirit.). Angola. Western: Kubango River, between Kalolo and Kavungu, 1100 m, Nov. 1899 (fl), Baum 425a (BM!, HBG!, $\mathrm{K}$ !, W!). Zambia. Mwinilunga, edge of stream, 6 Dec. 1958 (fl), Holmes 0118 (AMES!, K!, UGDA-DLSz! fragment, drawing).

N o te s. In many respects this species is very similar to Bilabrella myodes, but usually has narrower leaves, shorter both petal lobes and differently positioned gynostemium. All projections are pendent, in contrast to $B$. myodes where they are straight forward. In some respects $B$. kubangensis is similar to $B$. weberana but has ciliolate posterior and basal part of anterior petal lobes, and by oblong-lanceolate anterior petal lobe.

66. Bilabrella myodes (Summerh.) Szlach. \& KrasLap., Richardiana 3(3): 141. 2003.

Habenaria myodes Summerh., Kew Bull. 16: 287. 1962. - Summerhayes, FTEA, Orchid. 1: 91. 1968b. Williamson, Orchid. S. Central Afr.: 52. 1977. - la Croix \& Cribb, Fl. Zambes., Orchid. 11: 100. 1995. - Miller \& Morris, Plants of Dhofar: 220. 1988.

Type: Zimbabwe, Greatrex in GHS 27347 (holotype, K!; isotypes, AMES!, BR!, K!, UGDA-DLSz! - fragment, drawing, SRGH not seen).

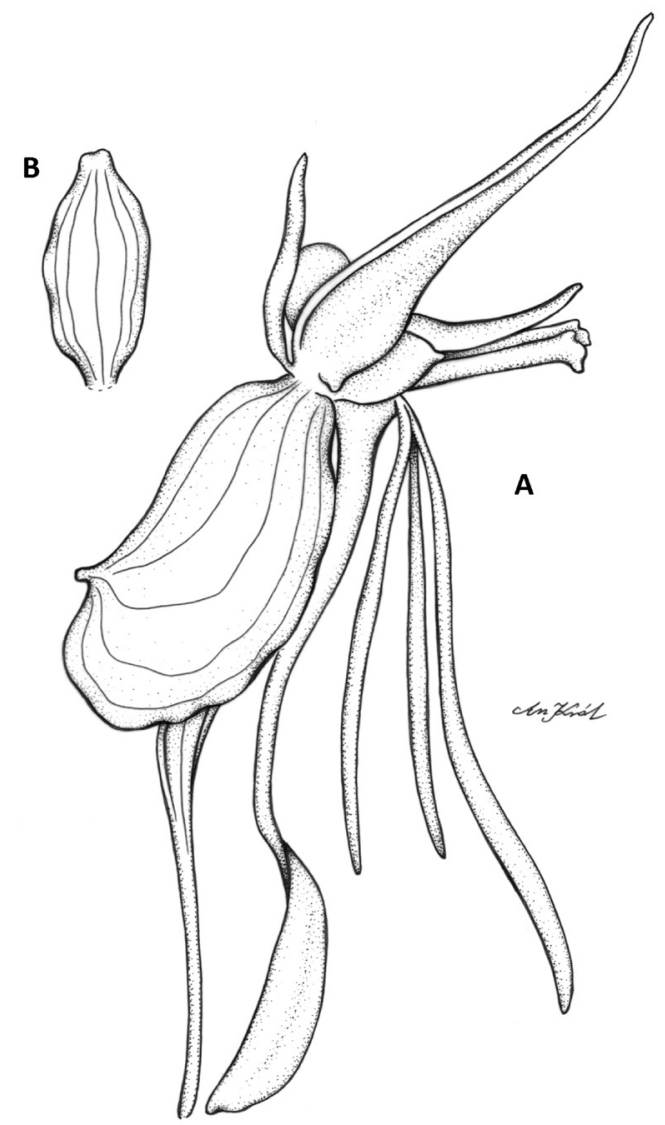

Fig. 187. Bilabrella myodes (Summerh.) Szlach. \& Kras-Lap. Explanations: A - flower; B - dorsal sepal (drawn by A. Król from isotype Greatrex 27347, AMES)
Tubers 1-2 $\mathrm{cm}$ long and in diameter, globose to ovoid, densely tomentose. Stem $20-75 \mathrm{~cm}$ tall, erect, slender to rather stout, leafy throughout its length. Leaves 7-14, 7-22 cm long, 1-3 cm wide, the lowermost rarely reduced to a sheath, the middle ones bunched in the lower part of the stem, suberect or spreading, narrowly lanceolate to oblong-lanceolate, acute, the upper 3-6 much smaller, adpressed to the stem, lanceolate, acuminate. Inflorescence 6-27 cm long, rather loosely 5 - to many-flowered. Flowers green with white centre, fragrant at night. Flower bracts 10-25 mm long, lanceolate, acuminate, usually distinctly shorter than the pedicel with ovary. Pedicel with ovary $18-25 \mathrm{~mm}$ long, curved. Dorsal sepal 5-7.5 mm long, 3-4 mm wide, narrowly elliptical to ovate, obtuse, convex, reflexed. Petals bilobed nearly to the base, ciliolate; anterior lobe 9-18 mm long, 1.5-2.5 mm wide, spreading forwards, linear or oblong lanceolate, apiculate, curved; posterior lobe 5.5-7 $\mathrm{mm}$ long, $0.3 \mathrm{~mm}$ wide, reflexed, linear to filiform, subacute. Lateral sepals $7.5-10 \mathrm{~mm}$ long, $5-6.5 \mathrm{~mm}$ wide, obliquely obovate, oblong elliptic or semi-orbicular, apiculus somewhat lateral, deflexed. Lip deflexed, 3-lobed from an undivided base just over $1 \mathrm{~mm}$ long; the middle lobe $10-17 \mathrm{~mm}$ long, $0.8-1 \mathrm{~mm}$ wide, linear, attenuate towards the apex, obtuse; lateral lobes 6.5-12 mm long, 0.3-0.5 mm wide, linear-lanceolate, acute, pendent. Spur 15-20 mm long, parallel to the ovary or \pm incurved, twisted in the middle, swollen in the apical half, blunt to subobtuse. Anther $3 \mathrm{~mm}$ tall, ovoid-conical; connective truncate to slightly notched; antherophores 4-6 mm long, slightly upcurved. Stigmaphores 4-6 mm long, narrowly cylindrical, truncate. Rostellum middle lobe shorter than thecae. Auriculae small, bifid in the upper part (Figs 187-190).

E c o lo gy. Grassland, savannah, rocky soil. JanuaryMay, November-December.

D i stribution. Uganda, Rwanda, Tanzania, Malawi, Zimbabwe, alt. 900-2400 m (Fig. 191).

S pecimens ex a mined. Uganda. Lake Mutanda, 1 Feb. 1939 (fl), Loveridge 462 (AMES!, K!, UGDADLSz! - fragment, drawing); Grassland, 1800 m, Jan. 1947 (fl), Parsegler 2313 (K!). Rwanda. East: Ikirungu, Terr. Biumba, in savannah, an clay, Dec. 1932 (fl), Baequet $176(\mathrm{~K}$ !). Tanzania. Kilimanjaro: Mt. Kilimanjaro, above Marungu on River Ona, shade of Eucalyptus plantation, 1800 m, Nov. 1965 (fl), Beesley 170 (K!); Ruvuma: Songea Distr., Matongo Mts., at edge of plantation, 1470 m, 15 Apr. 1960 (fl), Hay 89 (AMES!, K!); Songea Distr., Matongo Hills, just S of Songea, 15 Apr. 1960 (fl), Hay s.n. (K!); Songea Distr., rock outcrop on R. Luhira near shangano fish ponds in about $8 \mathrm{~cm}$ of stagnant water in pool at rock surface, $1060 \mathrm{~m}, 25 \mathrm{Apr}$. 1956 (fl), Milne-Redhead \& Taylor 9913 (K!); Songea Distr., rock outcrop on R. Luhira near shangano fish ponds in about $8 \mathrm{~cm}$ of stagnant water in pool at rock 


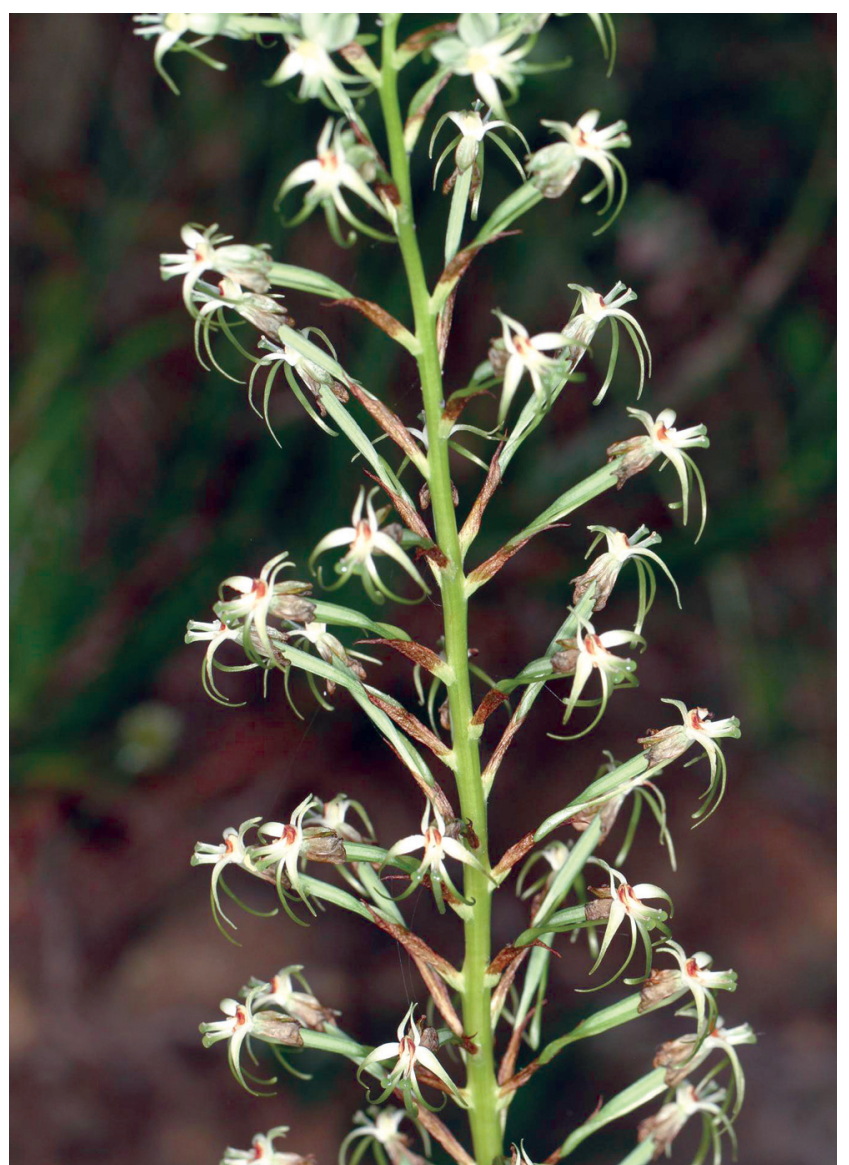

Fig. 188. Bilabrella myodes (Summerh.) Szlach. \& Kras-Lap. inflorescence (phot. L. Grobler)

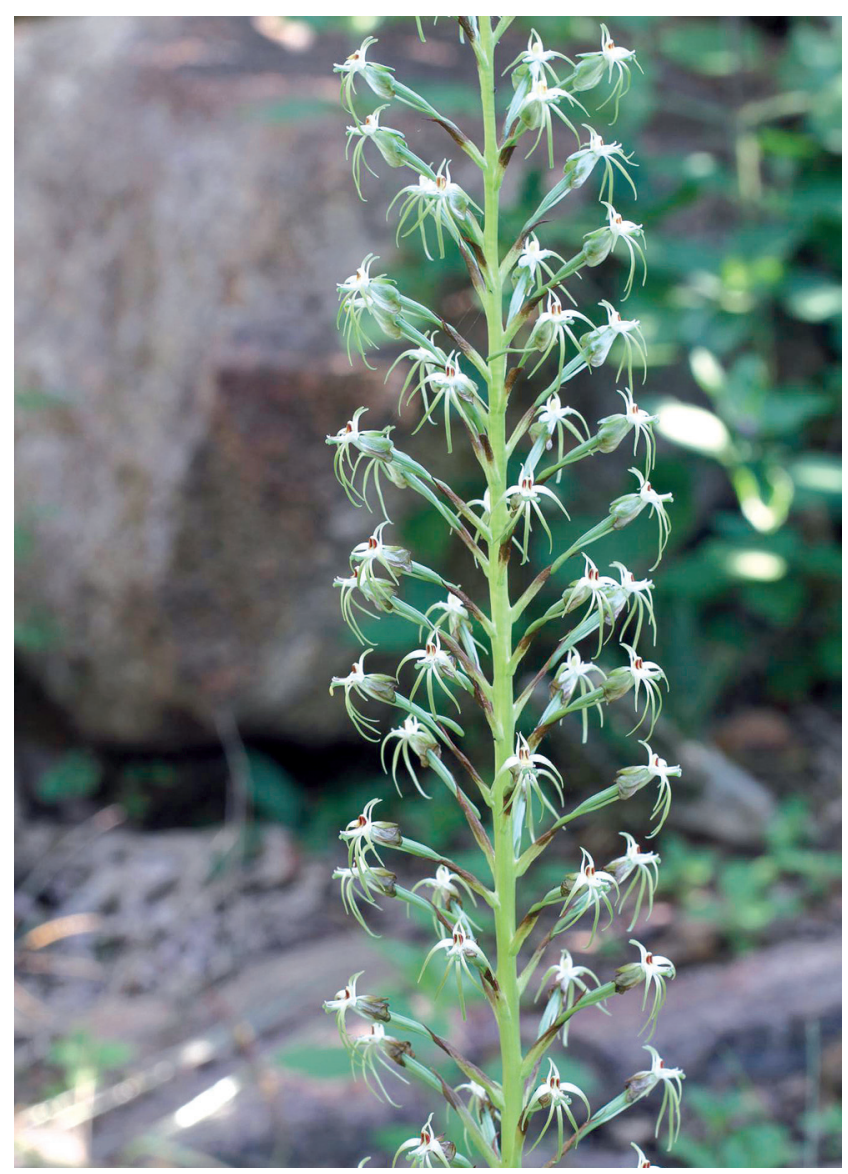

Fig. 189. Bilbarella myodes (Summerh.) Szlach. \& Kras-Lap. inflorescence close-up (phot. L. Grobler)

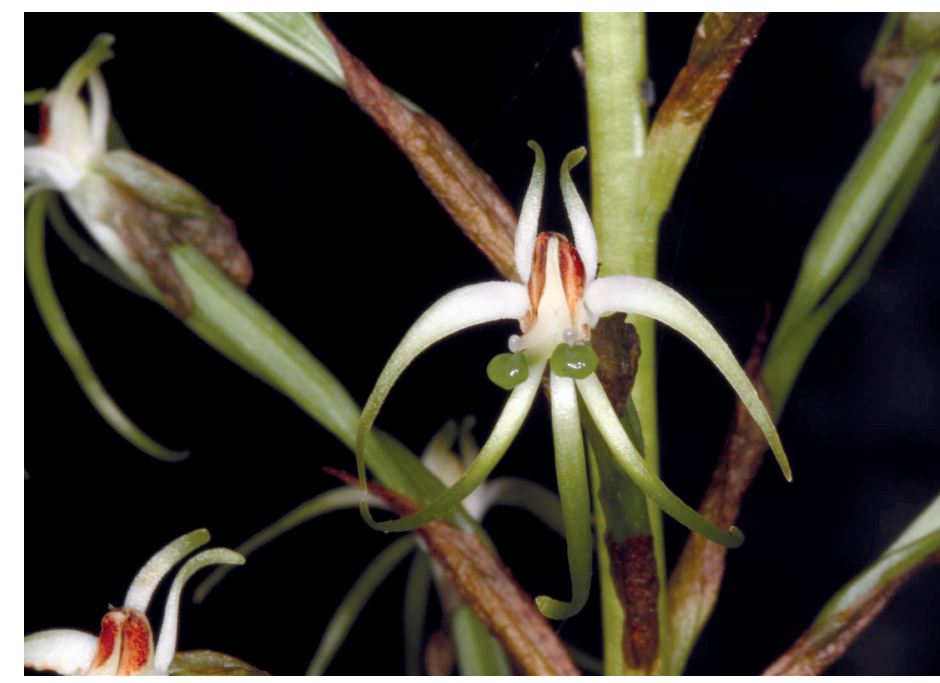

Fig. 190. Bilabrella myodes (Summerh.) Szlach. \& Kras-Lap. flower close-up (phot. L. Grobler)

surface, 1060 m, 25 Apr. 1956 (fl), Milne-Redhead \& Taylor 9913 A (AMES!, K!); Songea Distr., top of ridge on Litenga Hill in small pocket of soil on exposed rock surface in open, dominant over a all area but mostly now over, 1200 m, 19 Apr. 1956 (fl), Milne-Redhead \& Taylor 9770 (K!); Kasaï: Ulunga Distr., Maberge, in grass in savannah, very frequent, $1000 \mathrm{~m}, 30$ Apr. 1932 (fl), Schlieben 2159 A (K!). Malawi. Northern: Karonga Distr., Misuku, at edge of evergreen forest, among grass, 1500 m, 9 May 1947 (fl), Benson 1242 (K!); Southern: Blantyre, S Lunzu Road, in bud, large colony in open patch of long grass, in light Brachystegia woodland, 900 m, 1 Apr. 1982 (fl), la Croix 305 (K!); Chiradzulu Distr., Chiradzulu Mt., south-west side, grassy steep

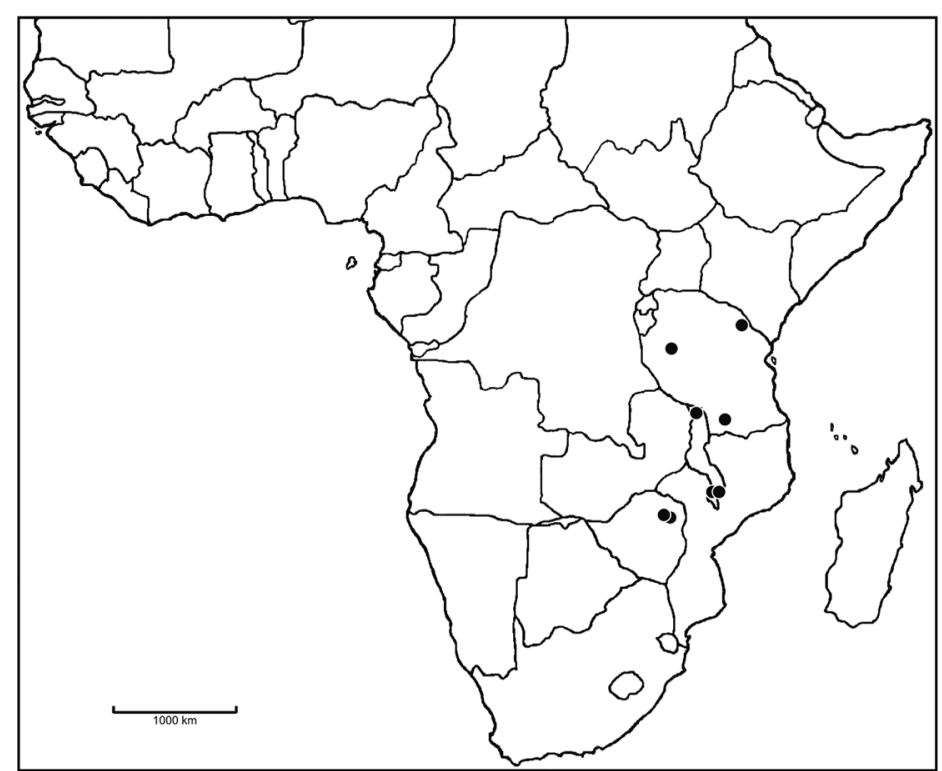

Fig. 191. Distribution of Bilabrella myodes (Summerh.) Szlach. \& Kras-Lap. 
rocky hillside, 1350-1500 m, 14 Apr. 1970 (fl), Brummitt \& Banda 9832 (K!); Mlanje Mt., open grassland, 2400 m, 4 Apr. 1938 (fl), Benson 4 (K!); Zomba Mt., on road, on top, sladed grassy bank, 8 Apr. (fl), Ash 706 (K!). Zimbabwe. Harare: 11 miles N of Salisbury, Graterex $91 A$ (K! - drawing); Damboshawa Distr., on granite whole black in hillside, 17 Mar. 1950 (fl), Graterex 27241 (K!); Goromonzi Distr., Chinaimora Tribal Trust Land on Damboshawa Hill, in grassy places over granite, 3 Apr. 1977 (fl), Grosvenor \& Renz 1305 (K!); Salisbury, Cleveland, in humus by rocks, 2 Apr. 1950 (fl), Graterex 27347 (AMES!, K!); Salisbury, Botanic Garden Extension, Christon Bank, in grassland with rocks, 12 Mar. 1969 (fl), Grosvenor 476 (K!); Scattered over areas NE of Salisbury in shady situations usually near rocks, 10 Apr. 1945 (fl), Graterex 91 (K!); "Himalayas" Butea south, open woodland, 20 Mar. 1955 (fl), Ball 536 (50845) (K!).

Notes. Bilabrella myodes can be confused with $B$. kubangensis and $B$. leucoceras. In contrast to the latter, $B$. myodes has both petal lobes more or less ciliolate. It differs from $B$. kubangensis by having wider leaves, larger petal lobes and position of the gynostemium projections.

67. Bilabrella humberti (Szlach. \& Olsz.) Szlach. \& Kras-Lap., Richardiana 3(3): 140. 2003. - Szlachetko et al., Orchid. of West-Central Africa 1: 232. 2010.

Habenaria humberti Szlach. \& Olsz., Fl. Cam., Orchid. 34(1): 166. 1998.

Type: Zaire, Humbert s.n. (holotype, P!).

Stem ca. $85 \mathrm{~cm}$ tall, erect, robust, glabrous. Leaves ca. 13, the middle ones more or less petiolate, spread; petiole up to $1.5 \mathrm{~cm}$ long, wide; blade up to $22 \mathrm{~cm}$ long and $2.5 \mathrm{~cm}$ wide, lanceolate, acute, the upper ones ca. $5 \mathrm{~cm}$ long, erect, loosely adpressed to the stem. Inflorescence $20 \mathrm{~cm}$ long, ca. 30-flowered, dense, cylindrical. Floral bracts $15 \mathrm{~mm}$ long, ovate-lanceolate, acuminate, glabrous, with ciliate margins. Pedicel and ovary up to $25 \mathrm{~mm}$ long; pedicel glabrous; ovary papillate. Dorsal sepal 6-7 mm long, 3-4 mm wide, oblong-obovate, concave, obtuse with reflexed apex, glabrous, deflexed. Petals bilobed to the base, rather fleshy, minutely ciliolate; anterior lobe $13 \mathrm{~mm}$ long, 1.2-1.5 mm wide, linear-lanceolate, acute, falcate, pendent; posterior lobe $6 \mathrm{~mm}$ long, $0.6 \mathrm{~mm}$ wide, linear, slightly falcate, acute, margins somewhat involute, upcurved. Lateral sepals $8 \mathrm{~mm}$ long, $5.5 \mathrm{~mm}$ wide, broadly obovate, cochleate near the apex with involute apical margin, apiculus laterally placed, ca. $1 \mathrm{~mm}$ long, glabrous. Lip 3-lobed to the base, slightly thickened above the base, glabrous; the middle lobe $15 \mathrm{~mm}$ long, up to $1 \mathrm{~mm}$ wide, linearfiliform, acute; lateral lobes $11 \mathrm{~mm}$ long, $0.5 \mathrm{~mm}$ wide, filiform, acute, pendent. Spur $18 \mathrm{~mm}$ long, $0.7 \mathrm{~mm}$ in

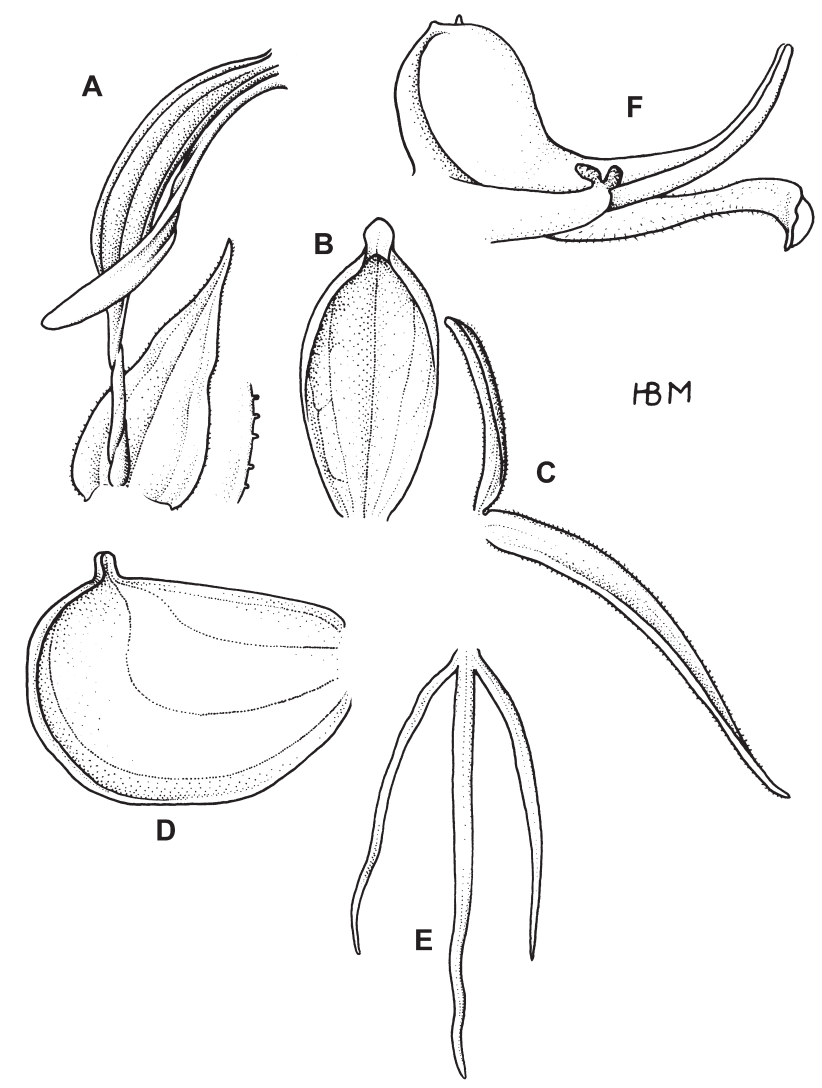

Fig. 192. Bilabrella humberti (Szlach. \& Olsz.) Szlach. \& Kras-Lap. Explanations: A - ovary, pedicel, spur and bract; B - dorsal sepal; C petal; D - lateral sepal; E - lip; F - gynostemium, side view (drawn by H. B. Margońska from holotype of Habenaria humberti Szlach. \& Olsz. - Humbert s.n., P)

diameter near the middle, $2 \mathrm{~mm}$ in diameter at the apex, narrowly cylindrical and parallel to the ovary up to the middle, then twisted once, swollen above and slightly upcurved, blunt. Anther $3 \mathrm{~mm}$ long, ovoid; connective

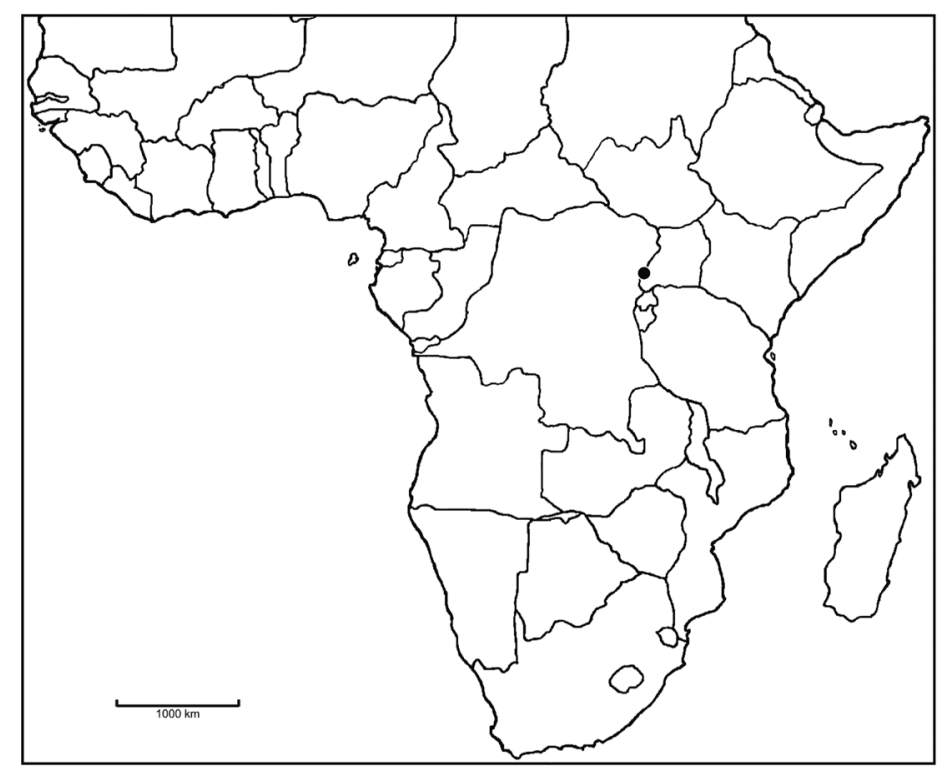

Fig. 193. Distribution of Bilabrella humberti (Szlach. \& Olsz.) Szlach. \& Kras-Lap. 
truncate; antherophores $5 \mathrm{~mm}$ long, gently upcurved. Stigmaphores narrowly cylindrical, the apex, bent down, truncate, ciliate. Rostellum middle lobe as long as connective. Auriculae small, deeply bilobed (Fig. 192).

Ec ology. No records. May.

D i s tribution. Known so far from the type collection only (Fig. 193).

Specimens examined. Democratic Republic of the Congo (Zaire). Nord-Kivu: SW Ruwenzori, Lume valley, May 1929 (fl), Humbert s.n. (P!).

Notes. The unique character of $B$. humbertii - not found elsewhere in species having twisted and obscurely swollen spur - are ciliolate stigmaphores.

68. Bilabrella burttii (Summerh.) Szlach. \& Kras-Lap., Richardiana 3(3): 139. 2003.

Habenaria burttii Summerh., Kew Bull. 16: 275. 1962.

- Summerhayes, FTEA, Orchid 1: 99. 1968b.

Type: Tanzania, B.D. Burtt 4624 (holotype, K!).

Stem 40-55 cm tall, erect, slender to rather stout, leafy throughout its length, almost entirely glabrous. Leaves 5-6, the middle 2-3 erect or suberect, linear, the

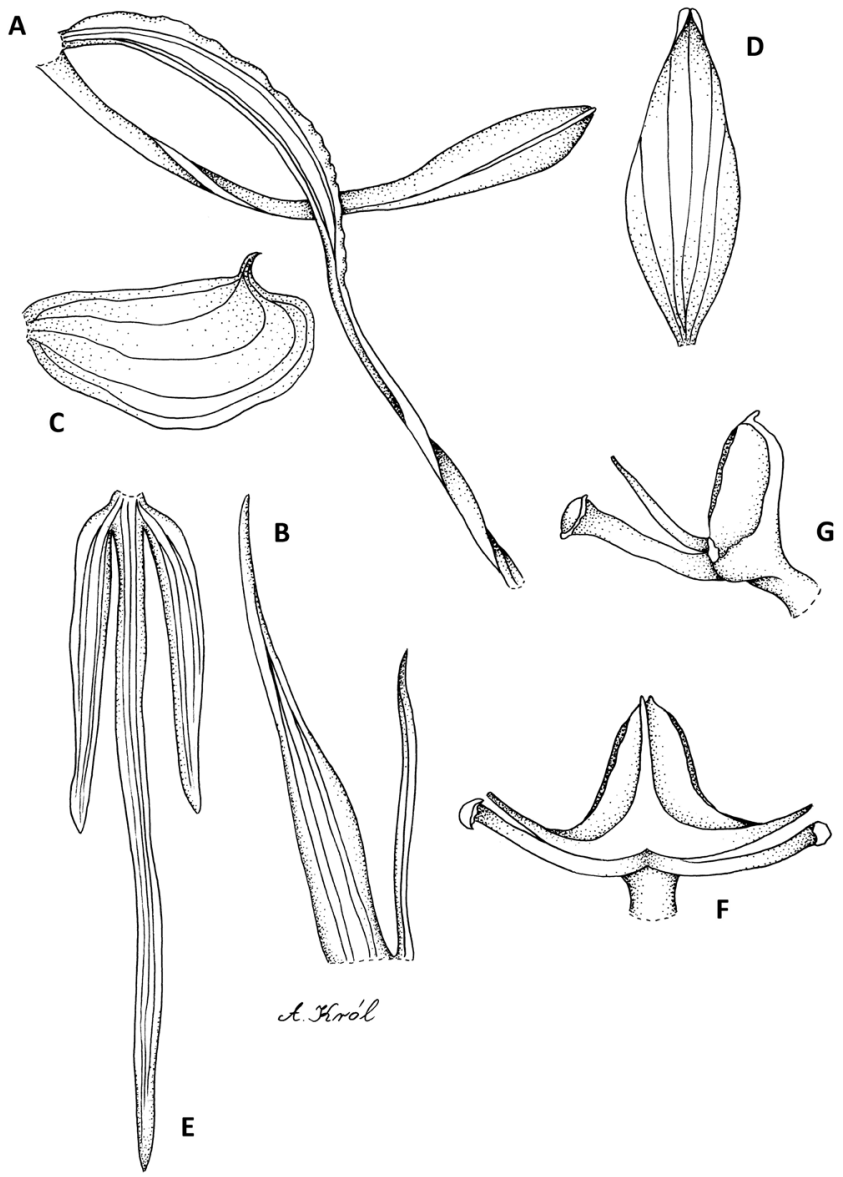

Fig. 194. Bilabrella burtti (Summerh.) Szlach. \& Kras-Lap. Explanations: A - ovary, pedicel, spur; B - petal; C - lateral sepal; D - dorsal sepal; E - lip; F - gynostemium, front view; $\mathrm{G}$ - gynostemium, side view (drawn by A. Król from holotype of Habenaria burttii Summerh. - Burtt $4624, \mathrm{~K})$

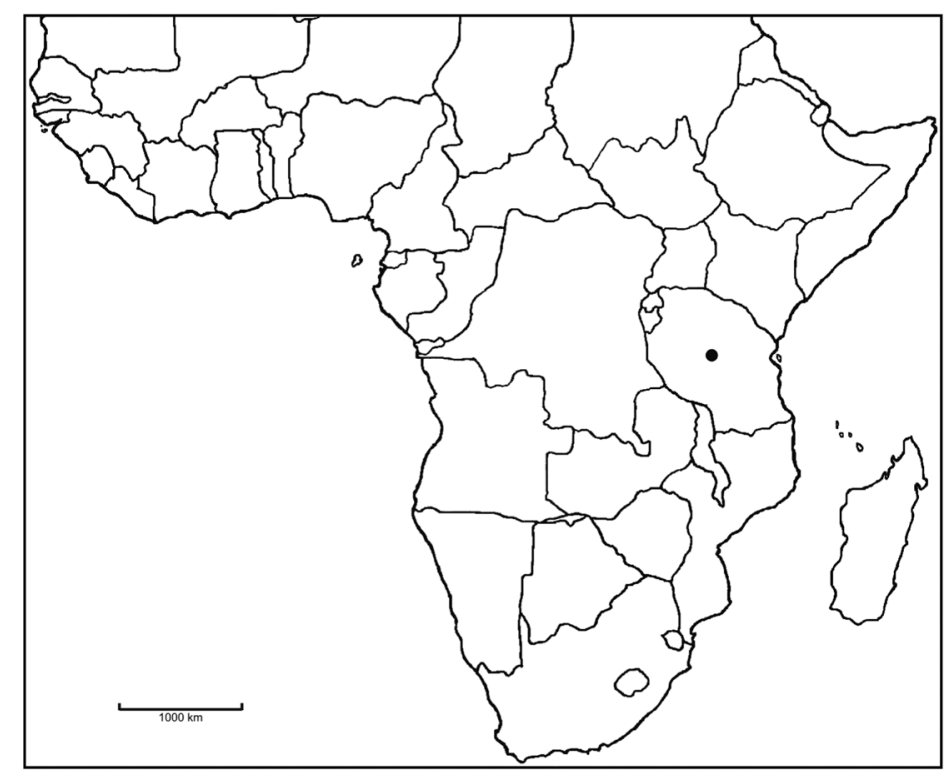

Fig. 195. Distribution of Bilabrella burttii (Summerh.) Szlach. \& Kras-Lap.

largest $14-16 \mathrm{~cm}$ long and $1-1.5 \mathrm{~cm}$ wide, the upper ones smaller, lanceolate, acuminate. Inflorescence $10-30 \mathrm{~cm}$ long, rather laxly to subdensely many-flowered. Flowers green with white petals. Floral bracts 10-20 mm long, lanceolate, acuminate. Pedicel and ovary $15-20 \mathrm{~mm}$ long, much curved. Dorsal sepal 4-5 mm long, 1.5$2 \mathrm{~mm}$ wide, narrowly elliptical to oblong-ovate, obtuse, reflexed. Petals bilobed nearly to the base; anterior lobe 8-10 mm long, 1-2 $\mathrm{mm}$ wide at base, spreading forwards, obliquely lanceolate, subulate in the upper part, acute; posterior lobe nearly $5 \mathrm{~mm}$ long, 0.2 $0.5 \mathrm{~mm}$ wide, filiform, acute. Lateral sepals $6.5-7 \mathrm{~mm}$ long, $4 \mathrm{~mm}$ wide, obliquely obovate to elliptic-ovate, with lateral apiculus, deflexed. Lip deflexed, 3-lobed nearly to the base; the middle lobe 11-12 mm long, 0.4$0.8 \mathrm{~mm}$ wide, incurved, linear, subobtuse to subacute; lateral lobes 5-9 mm long, 0.6-1 mm wide, oblanceolatelinear, acute. Spur 10-18 mm long, strongly upcurved, somewhat swollen in the apical third, subacute. Anther 2-5 mm tall, ellipsoid-conical; connective rounded; antherophores $3 \mathrm{~mm}$ long, very slender, upcurved. Stigmaphores $3 \mathrm{~mm}$ long, narrowly cylindrical, truncate, upcurved. Rostellum middle lobe slightly longer than connective. Auriculae tiny, unequally bilobed (Fig. 194).

E c ology. Terrestrial in seasonally wet bog. Flowers in January.

Distribution. Not known outside Tanzania, alt. $1260 \mathrm{~m}$ (Fig. 195).

Specimens examined. Tanzania. Dodoma: Kazikazi, 1260 m, Jan. 1933 (fl), Burtt 4624 (K!).

$\mathrm{N}$ otes. This species is chiefly characterised by the upcurved spur which is very slender in the lower part and widened towards the apex to form a club-shaped 
swelling. The very spreading flowers with markedly arcuate ovaries and pedicels provide another striking feature of the species. It is clearly allied to Bilabrella humbertii, but despite the latter its stigmaphores are glabrous. The other similar species is $B$. maitlandii from Cameroon, but it has lip 3-lobed above distinct claw, 1.5-2 mm long. The lip of B. burttii is 3-lobed to the base.

69. Bilabrella maitlandii (Summerh.) Szlach. \& KrasLap., Richardiana 3(3): 141. 2003. - Szlachetko et al., Orchid. of West-Central Africa 1: 226. 2010.

Habenaria maitlandii Summerh., Kew Bull. 1932: 341. 1932. - Summerhayes, FWTA, ed. 1, 2: 412. 1936. - Summerhayes, FWTA, ed. 2, 3: 196. 1968a. - Szlachetko \& Olszewski, Fl. Cam., Orchid. 34(1): 172. 1998.

Type (as holotype): Cameroon, Maitland 1386 (lectotype, designated by Szlachetko \& Olszewski [1998: 172], B!; isolectotype, K!).

Stem 30-32 cm tall, erect. Leaves 3-7, 7-10 cm long, $1-1.4 \mathrm{~cm}$ wide, narrowly lanceolate, acute, suberect, decreasing in size up the stem. Inflorescence $7-10 \mathrm{~cm}$ long, 9-16-flowered, rather lax. Flowers white or green. Floral bracts 14-15 mm long, ovate-lanceolate, acuminate. Pedicel and ovary 16-21 mm long, glabrous. Dorsal sepal 4.5-5.5 $\mathrm{mm}$ long, 2-3 $\mathrm{mm}$ wide, obovate to oblong obovate, apiculate, obtuse, cochleate, glabrous. Petals bilobed to the base, both parts completely papillose; anterior lobe 6.8-8.5 $\mathrm{mm}$ long, $1-1.5 \mathrm{~mm}$ wide, lanceolate or lanceolate-elliptic to oblong-lanceolate, acute to acuminate; posterior lobe $4.5-5.5 \mathrm{~mm}$ long, $0.2-0.5 \mathrm{~mm}$

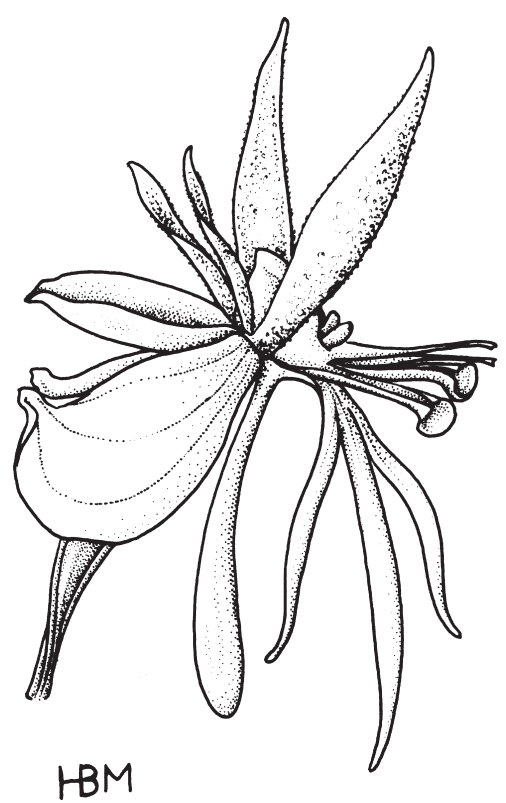

Fig. 196. Bilabrella maitlandii (Summerh.) Szlach. \& Kras-Lap. flower (drawn by H.B. Margońska from isolectotype of Habenaria maitlandii Summerh. - Maitland 1386, K)

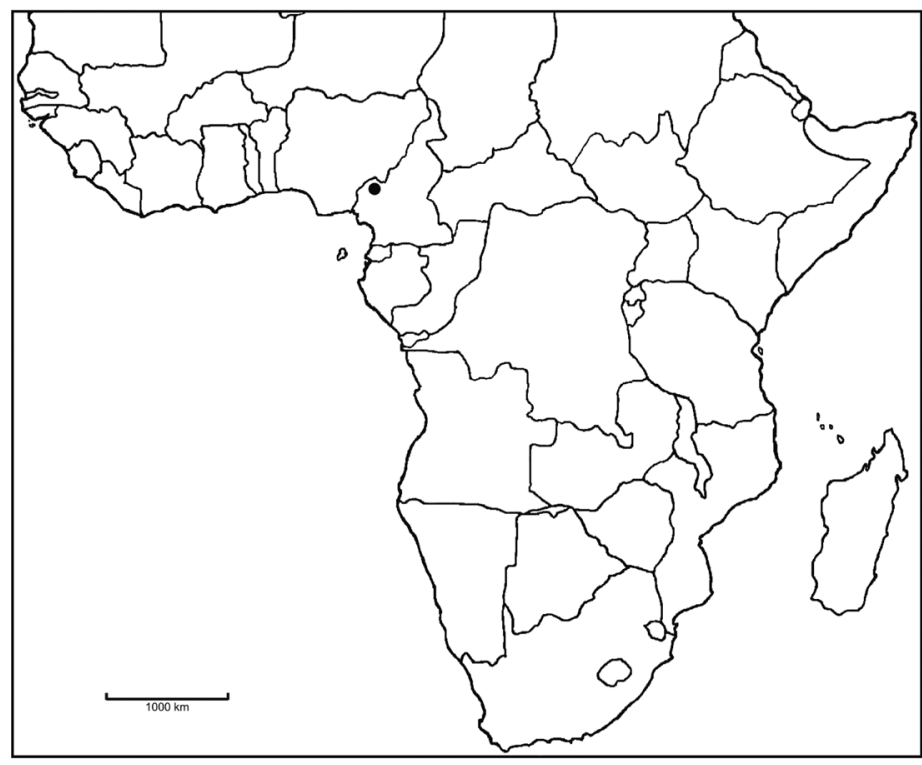

Fig. 197. Distribution of Bilabrella maitlandii (Summerh.) Szlach. \& Kras-Lap.

wide, linear-filiform, acute. Lateral sepals $6.5-7.5 \mathrm{~mm}$ long, 4-5 mm wide, obliquely obovate, concave, with laterally placed apiculus. Lip 3-lobed above the basal 1.5-2 mm long, glabrous; the middle lobe $9-10 \mathrm{~mm}$ long, 0.5-0.8 mm wide, linear, acute; lateral lobes $6-8 \mathrm{~mm}$ long, $0.5 \mathrm{~mm}$ wide, linear, subacute. Spur $8.5-11 \mathrm{~mm}$ long, narrowly cylindrical, twisted and swollen in the apical half, blunt, pendent. Anther $2 \mathrm{~mm}$ long; connective obtuse; antherophores $4 \mathrm{~mm}$ long, delicate, almost filiform, straight. Stigmaphores $4 \mathrm{~mm}$ long, narrowly cylindrical, straight, truncate. Rostellum middle lobe as long as connective. Auriculae shortly stalked, deeply bilobed (Fig. 196).

E c ology. No records. June.

D i s trib u ti o n. Known so far from Cameroon only, alt. $1680 \mathrm{~m}$ (Fig. 197).

Specimens examined. Cameroon. Northwestern: Uchan near Bamenda, 1680 m, June 1931 (fl), Maitland 1386 (B!, K!).

N o t e s. Similar to Bilabrella involuta from Republic of South Africa, but both petal lobes are completely papillose (vs anterior petal lobe glabrous, posterior one ciliolate). Some collections from Democratic Republic of the Congo (Lisowski 65753, 65759) and Ethiopia (Westphol \& Westphol-Stevels 1679) share some intermediate characters with $B$. leucoceras, as for example larger leaves, slightly longer spur and lip middle lobe.

70. Bilabrella involuta (Bolus) Szlach. \& Kras-Lap., Richardiana 6(4): 197. 2006.

Habenaria involuta Bolus, J. Linn. Soc., Bot. 25: 165. 1889. - Kraenzlin, Orchid. Gen. Sp.: 229. 1901.

Type: Republic of South Africa, Sanderson 833 (BOL not seen, K!). 


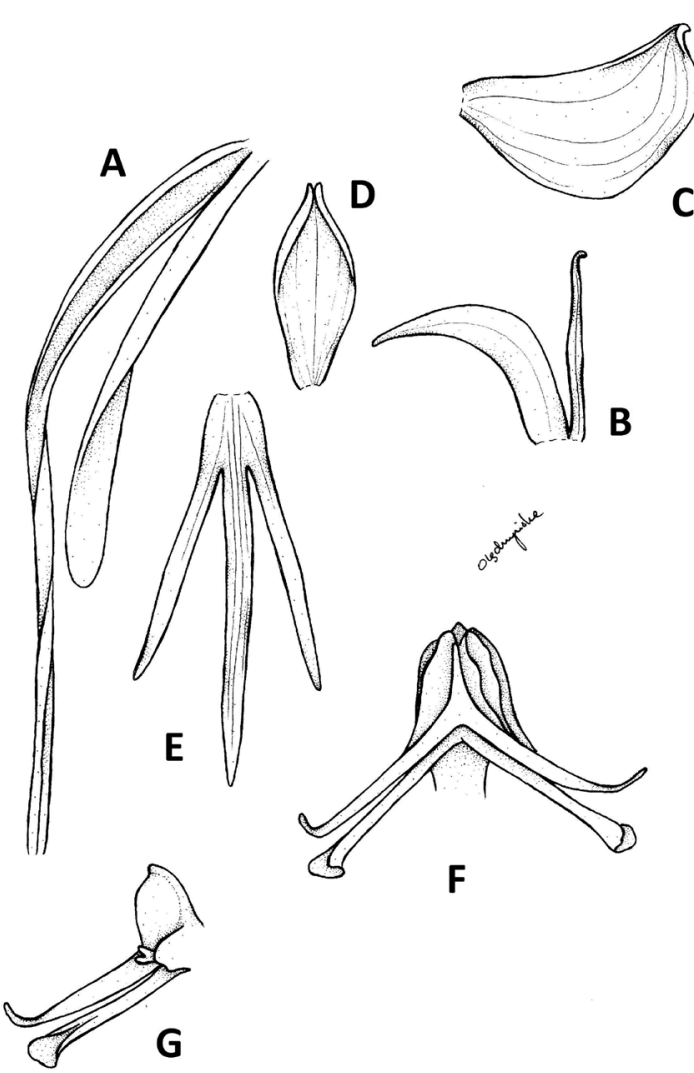

Fig. 198. Bilabrella involuta (Bolus) Szlach. \& Kras-Lap. Explanations: A - pedicel, ovary and spur; B - petal; C - lateral sepal; $\mathrm{D}$ - dorsal sepal; E - lip; F-G - gynostemium, various views (drawn by N. Olędrzyńska from type material of Habenaria involuta Bolus - Sanderson $833, \mathrm{~K})$

Stem $60 \mathrm{~cm}$ tall, erect, glabrous. Leaves 3-4, to $22 \mathrm{~cm}$ long and $1.5 \mathrm{~cm}$ wide, linear-lanceolate, acute, decreasing in size up the stem. Inflorescence $14 \mathrm{~cm}$ long, 10-18-flowered. Floral bracts 15-20 mm long, oblong lanceolate, acuminate. Pedicel and ovary $15-20 \mathrm{~mm}$ long, slender. Dorsal sepal $5 \mathrm{~mm}$ long, $2 \mathrm{~mm}$ wide, ovate-lanceolate to oblong-obovate, shortly apiculate, obtuse, concave, erect. Petals bilobed to the base; anterior lobe $7 \mathrm{~mm}$ long, $1.1 \mathrm{~mm}$ wide, oblong-lanceolate to linear-lanceolate, falcate, acuminate, glabrous; posterior lobe $5 \mathrm{~mm}$ long, $0.3 \mathrm{~mm}$ wide, filiform, obtuse, ciliolate. Lateral sepals 5-6 mm long, $4 \mathrm{~mm}$ wide, semi-orbicular, obovate-cuneate, laterally apiculate, concave, reflexed. Lip 3-lobed above the undivided basal $2 \mathrm{~mm}$, to $9 \mathrm{~mm}$ long in total; the middle lobe linear, twice longer; the middle lobe $8.5 \mathrm{~mm}$ long, $0.5 \mathrm{~mm}$ wide, linear with lanceolate, acute, revolute apex; lateral lobes $6 \mathrm{~mm}$ long, $0.5 \mathrm{~mm}$ wide, linear, acute. Spur 10-20 mm long, narrowly cylindrical, clavate in the apical half, obtuse at the apex. Anther $2 \mathrm{~mm}$ long; connective shortly apiculate; antherophores $5 \mathrm{~mm}$ long, slender, abruptly upcurved at the apex. Stigmaphores 4-4.5 mm long, narrowly cylindrical, truncate. Rostellum middle lobe subulate, slightly shorter than thecae. Auriculae obscure, bilobed (Fig. 198).

E c o log y. Sandy soil near the river. February.

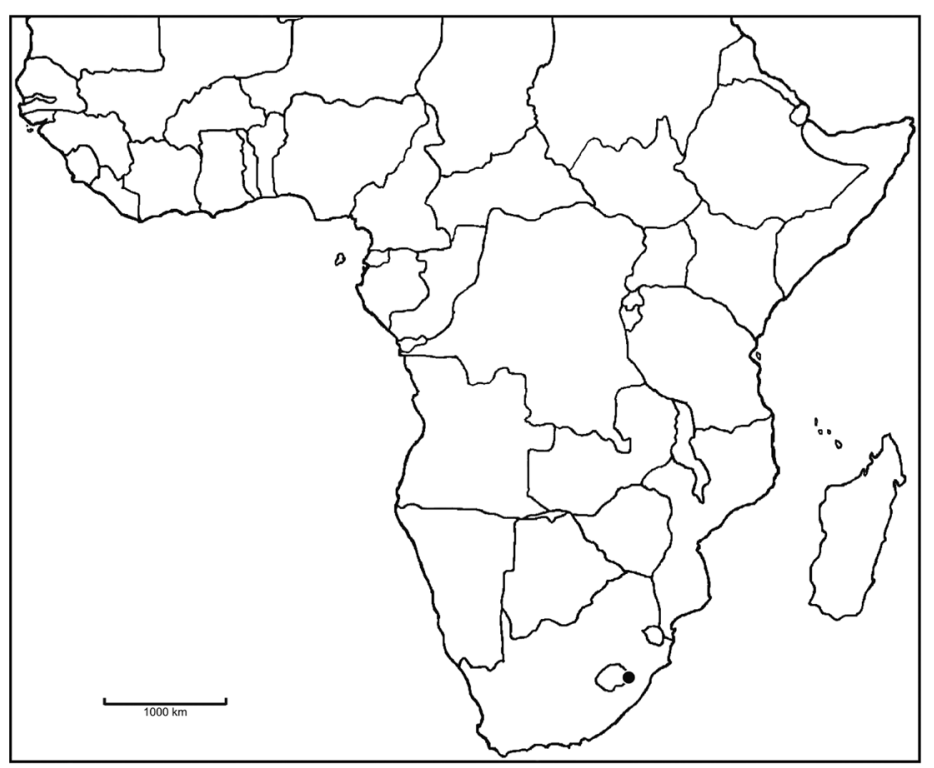

Fig. 199. Distribution of Bilabrella involuta (Bolus) Szlach. \& Kras-Lap.

Distribution. Republic of South Africa, alt. $1650 \mathrm{~m}$ (Fig. 199).

Specimens examined. Republic of South Africa. Kwazulu-Natal, Sanderson 833 (K!); Loteni Nature Reserve, sandy soil in boulden bed of river, 1650 m, 11 Feb. 1981 (fl), Stewart 2212 (K!).

N o te s. Bilabrella involuta remids in many characters Cameroonian B. maintlandii. Both species can be separated by details of petals (anterior petal lobe glabrous, posterior one ciliolate in $B$. involuta vs both petal lobes completely papillose in $B$. maintlandii). The species has habit and appearance of $B$. dives and of $B$. kilimanjari, but the flowers are different from either (Bolus 1889).

71. Bilabrella kornasiorum (Szlach. \& Olsz.) Szlach. \& Kras-Lap., Richardiana 3(3): 141. 2003. - Szlachetko et al., Orchid. of West-Central Africa 1: 220. 2010.

Habenaria kornasiorum Szlach. \& Olsz., Fl. Cam., Orchid. 34(1): 180. 1998.

Type: Central African Republic, Tisserant s.n. (holotype, $\mathrm{P} !)$.

Tubers unknown. Stem $70 \mathrm{~cm}$ tall, erect, rather stout, glabrous. Leaves 8 , up to $11 \mathrm{~cm}$ long and $2 \mathrm{~cm}$ wide, lanceolate, acute, slightly deflexed from the stem, decreasing in size up the stem. Inflorescence $21 \mathrm{~cm}$ long, ca. 40-flowered, rather dense. Floral bracts $15 \mathrm{~mm}$ long, glabrous. Pedicel and ovary $20 \mathrm{~mm}$ long, glabrous, erect. Dorsal sepal 5.5-6 mm long, 1.8-2 mm wide, oblong obovate, obtuse with short apiculus, cochleate, glabrous. Petals bilobed to the base, rather fleshy, ciliolate; anterior lobe $12 \mathrm{~mm}$ long, $0.6 \mathrm{~mm}$ wide, filiform, slightly twisted; posterior lobe $5.5 \mathrm{~mm}$ long, $0.3 \mathrm{~mm}$ wide, filiform, with recurved apex. Lateral sepals 


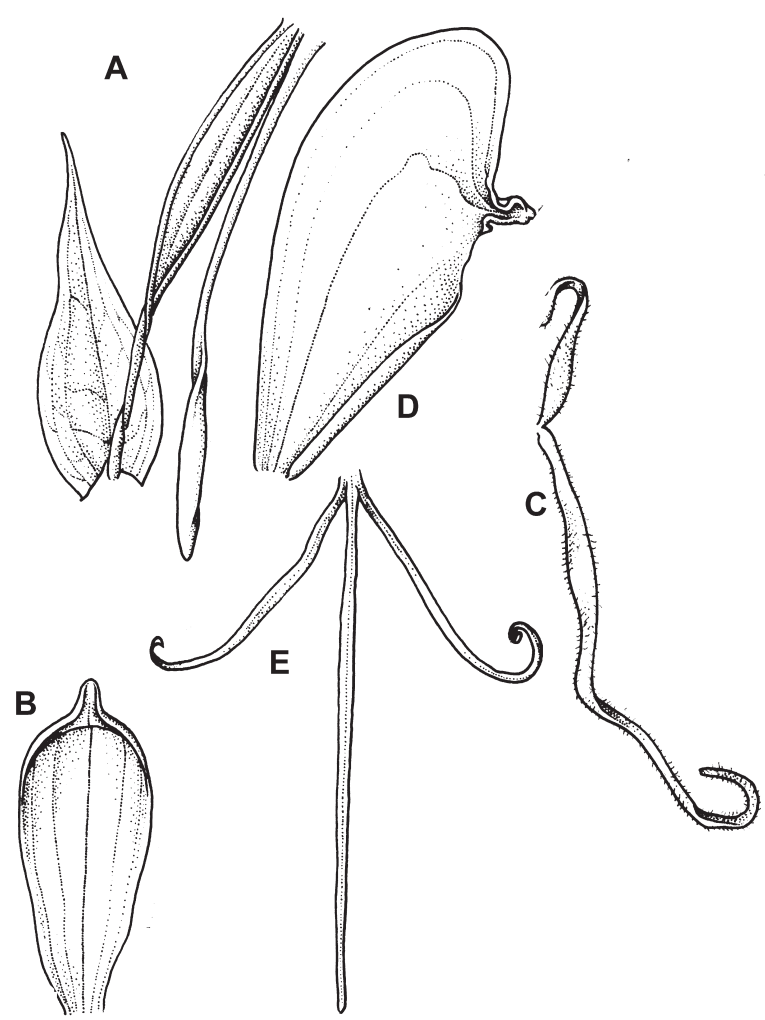

Fig. 200. Bilabrella kornasiorum (Szlach. \& Olsz.) Szlach. \& Kras-Lap.

Explanations: A - ovary, pedicel, spur and bract; $\mathrm{B}$ - dorsal sepal; C - petal; D - lateral sepal; E - lip (drawn by D. L. Szlachetko from holotype of $\mathrm{Ha}$ benaria kornasiorum Szlach. \& Olsz. - Tisserant s.n., P)

9-10 $\mathrm{mm}$ long, $5 \mathrm{~mm}$ wide, oblong-obovate, concave, with laterally placed apiculus $1 \mathrm{~mm}$ long, glabrous. Lip 3-lobed above the short basal part $1.3 \mathrm{~mm}$ long, rather fleshy, glabrous; the middle lobe $14-15 \mathrm{~mm}$ long, $0.3 \mathrm{~mm}$ wide, filiform; lateral lobes $9 \mathrm{~mm}$ long, $0.3 \mathrm{~mm}$

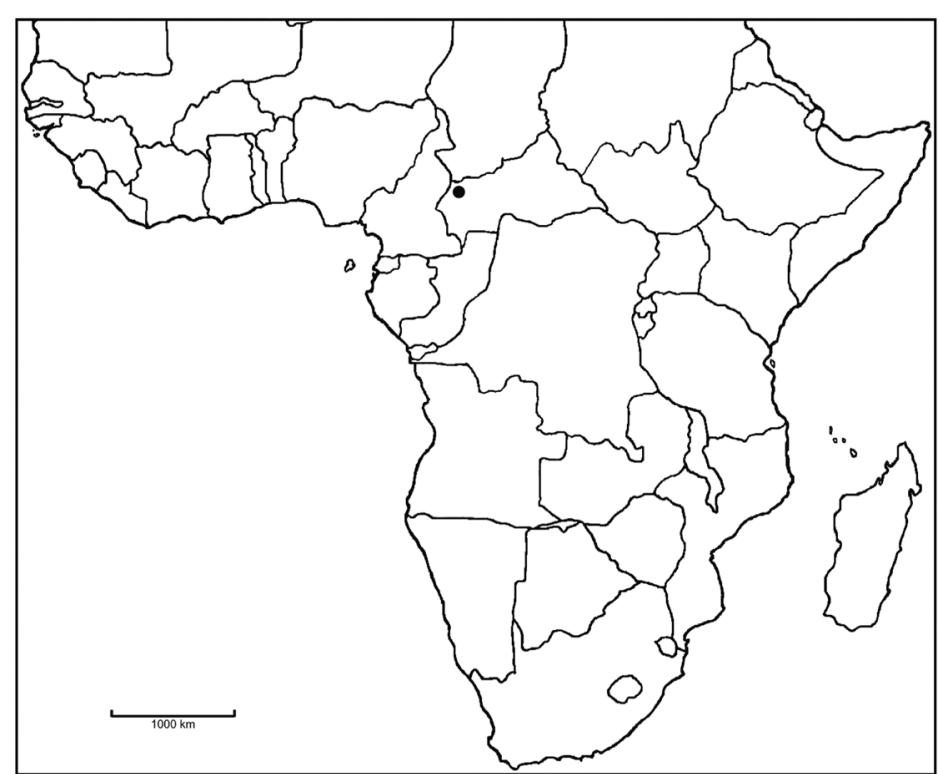

Fig. 201. Distribution of Bilabrella kornasiorum (Szlach. \& Olsz.) Szlach. \& Kras-Lap. wide, filiform with upcurved apices. Spur 20-22 mm long, $0.5 \mathrm{~mm}$ in diameter at the base, $1 \mathrm{~mm}$ in diameter at the apex, narrowly cylindrical, in the apical third once twisted and slightly swollen, subacute, pendent. Anther $1.5 \mathrm{~mm}$ tall; connective apiculate; antherophores $7.5 \mathrm{~mm}$ long, filiform, slightly upcurved. Stigmaphores $7.5 \mathrm{~mm}$ long, very slender, almost filiform, with ligulate apices. Rostellum middle lobe shorter than thecae. Auriculae stalked, shallowly bilobed (Fig. 200).

E c ology. Savannah. September.

D is tribution. Known so far from type collection only (Fig. 201).

Specimens examined. Central African Republic. Lobaye: Station the Boukoko, Bozoum, 11 Sep. 1032 (fl), Tisserant s.n. (P!).

$\mathrm{N}$ ot e s. The unique character of this species are filiform both petal lobes.

Bilabrella kornasiorum can be misidentified with $B$. altior, but has narrower, ciliolate petal lobes, slightly twisted spur in the lower part, shortly stalked gynostemium with slender stigmaphores abruptly curved down near the apex (Szlachetko et al. 2010).

72. Bilabrella tubifolia (la Croix \& P. J. Cribb) Szlach. \& Kras-Lap., Richardiana 3(3): 143. 2003.

Habenaria tubifolia la Croix \& P. J. Cribb, Kew Bull. 48: 359. 1993. - la Croix \& Cribb, Fl. Zambes., Orchid. 11: 109. 1995.

Type: Zambia, Richards 5063 (holotype, K!).

Stem $18-20 \mathrm{~cm}$ tall, erect, slender, leafy. Leaves numerous, the lower 10-12 clustered near stem base, up to $8 \mathrm{~cm}$ long and $0.2-0.8 \mathrm{~cm}$ wide, tubular with a sheathing base, the bases overlapping; the ca. 6 uppermost leaves bract-like, appressed to stem, lanceolate or ovate, acuminate. Inflorescence 5-7 cm long, fairly laxly 6-11-flowered. Flowers pale green. Floral bracts 12-13 $\mathrm{mm}$ long, lanceolate, acute, the margins erose. Pedicel and ovary $15 \mathrm{~mm}$ long, arched. Sepals reflexed. Dorsal sepal $5 \mathrm{~mm}$ long, 1.5-2 $\mathrm{mm}$ wide, oblong to bolongelliptic, obtuse. Petals 2-lobed almost to the base; anterior lobe $12-13 \mathrm{~mm}$ long, $2.5-3 \mathrm{~mm}$ wide, spreading, curved lanceolate to bolong-lanceolate, long acute, slightly papillose at the base; posterior lobe $5 \mathrm{~mm}$ long, $0.2 \mathrm{~mm}$ wide, almost filiform, acute, erect. Lateral sepals 8-9 mm long, 3-4 mm wide, obliquely elliptic-obovate, with a lateral apiculus. Lip reflexed, lying \pm parallel to ovary, 3-lobed to ca. $1 \mathrm{~mm}$ from base, all lobes fleshy, linear, somewhat papillose; the middle lobe $10-11 \mathrm{~mm}$ long, 0.4-0.5 mm wide, linear, obtuse; lateral lobes 8-8.5 $\mathrm{mm}$ long, $0.4 \mathrm{~mm}$ wide, linear, acute. Spur 25-28 mm long, parallel to pedicel and ovary, slender, the apical $5 \mathrm{~mm}$ thickened and slightly bent up, blunt. Anther $1.5 \mathrm{~mm}$ tall; connective truncate; antherophores $4 \mathrm{~mm}$ long. Stigmaphores $3 \mathrm{~mm}$ long, slender, cylindrical, 


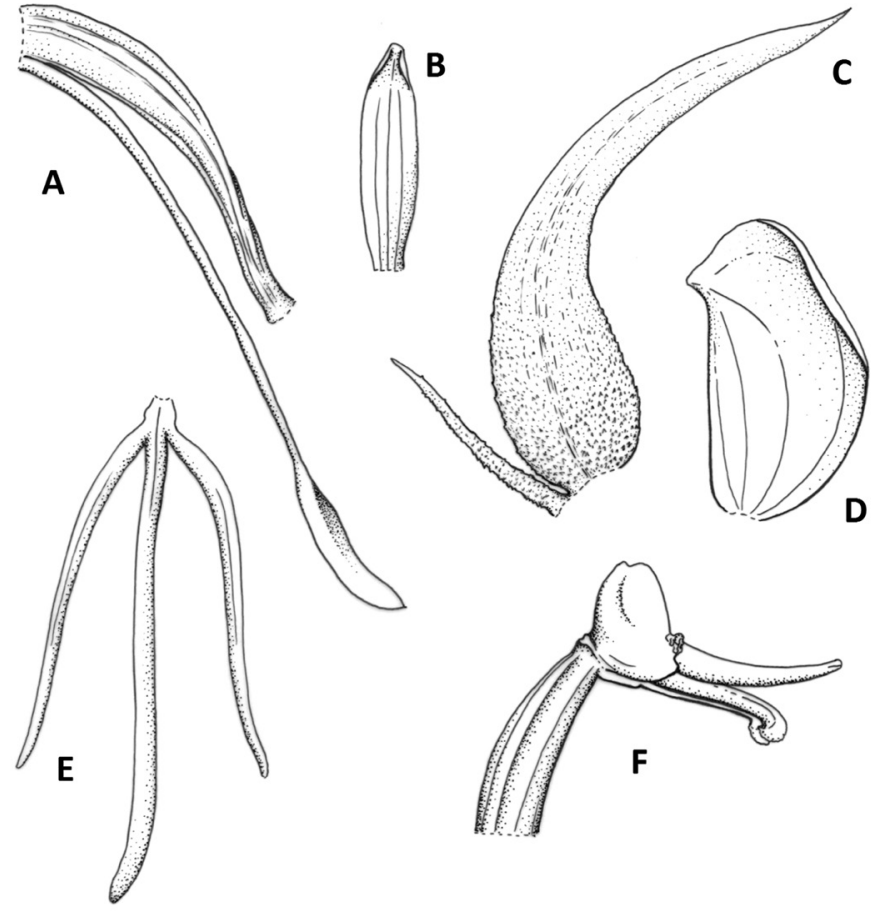

Fig. 202. Bilabrella tubifolia (la Croix \& P.J. Cribb) Szlach. \& Kras-Lap.

Explanations: A - ovary, pedicel, spur; B - dorsal sepal; C - petal; D - lateral sepal; E - lip; F - gynostemium, side view (redrawn by A. Król from la Croix \& P.J. Cribb 1993)

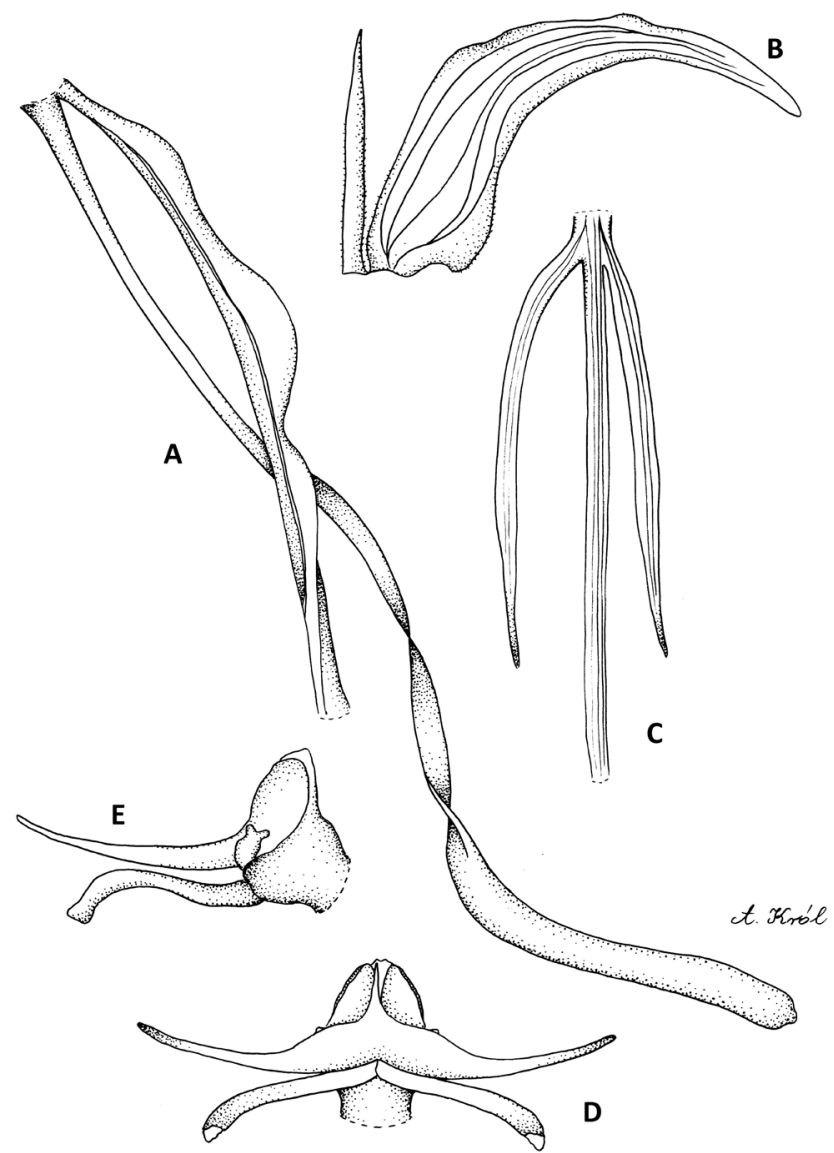

Fig. 203. Bilabrella tubifolia (la Croix \& P. J. Cribb) Szlach. \& Kras-Lap.

Explanations: A - ovary, pedicel, spur; B - petal; C - lip; D - gynostemium, front view; E-gynostemium, side view (drawn by A. Król from holotype of Habenaria tubifolia la Croix \& P.J. Cribb - Richards 5063, K) with ligulate apex. Rostellum middle lobe shorter than thecae. Auriculae small, bifid (Figs 202-203).

E c o logy. No records. March.

D istribution. Apparently endemic, known only from type collection from Zambia.

Specimens examined. Zambia: Mauser's Farm, 28 Mar. 1955 (fl), Richards 5063 (K!).

$\mathrm{N}$ otes. It bears some resemblance to Bilabrella strangulans but the crowded, tubular leaves are distinctive. Also, the inflorescence is fewer-flowered and the spur is longer and not wrapped round the pedicel as in B. strangulans.

73. Bilabrella strangulans (Summerh.) Szlach. \& KrasLap., Richardiana 3(3): 142. 2003. - Szlachetko et al., Orchid. of West-Central Africa 1: 233. 2010.

Habenaria strangulans Summerh., Kew Bull. 16: 295. 1962. - Grosvenor, Excelsa 6: 83. 1976. - Williamson, Orchid. S. Central Afr.: 55. 1977. - la Croix, Orchid. Malawi: 79. 1991. - la Croix \& Cribb, Fl. Zambes., Orchid. 11: 106. 1995.

Type: Zimbabwe, Pollitt 42189 (holotype, K!).

Tubers $2 \mathrm{~cm}$ long, $1 \mathrm{~cm}$ in diameter, ovoid. Stem $15-60 \mathrm{~cm}$ tall, erect, slender, glabrous. Leaves up to 14 , 6-16 cm long, 0.4-0.7 cm wide, linear, acute, loosely adpressed to the stem, decreasing in size upwards. Inflorescence $8-20 \mathrm{~cm}$ long, loosely 10-30-flowered. Flowers green. Floral bracts 12-15 mm long, oblong-lanceolate, glabrous. Pedicel and ovary 20-23 mm long. Dorsal sepal 5-6 $\mathrm{mm}$ long, 2-3 $\mathrm{mm}$ wide, oblong-obovate to oblong-elliptic, concave, obtuse, glabrous, deflexed.
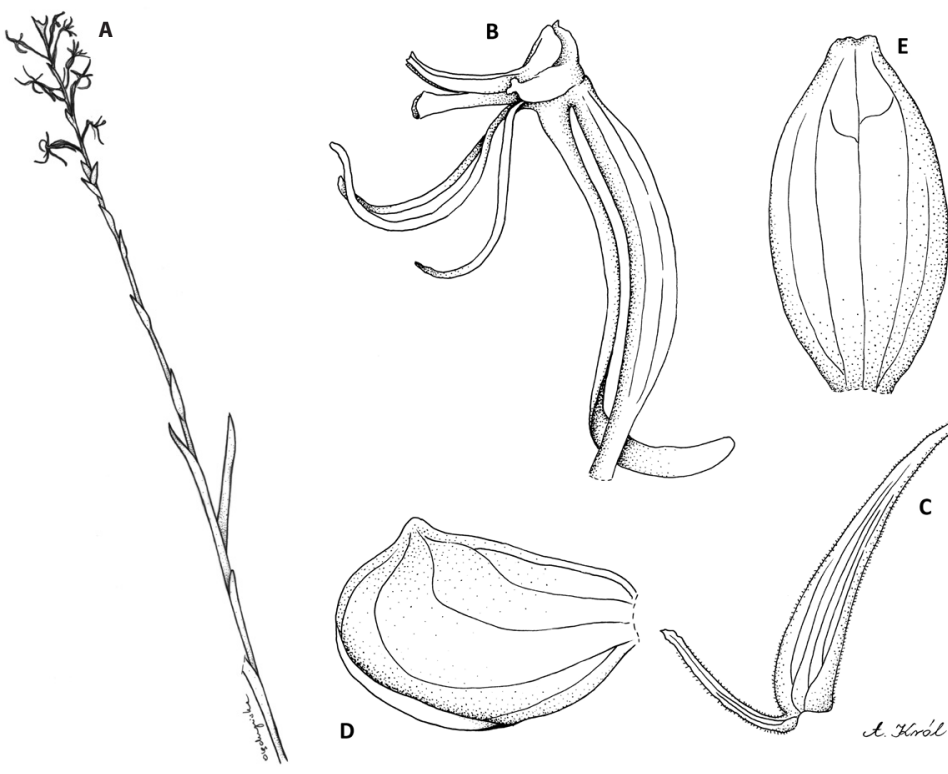

Fig. 204. Bilabrella strangulans (Summerh.) Szlach. \& Kras-Lap. Explanations: A - habit; B - pedicel, ovary, gynostemium, lip and spur; C petal; D - lateral sepal; E - dorsal sepal (drawn by A. Król from holotype of Habenaria strangulans Summerh. - Pollitt 42189, K) 


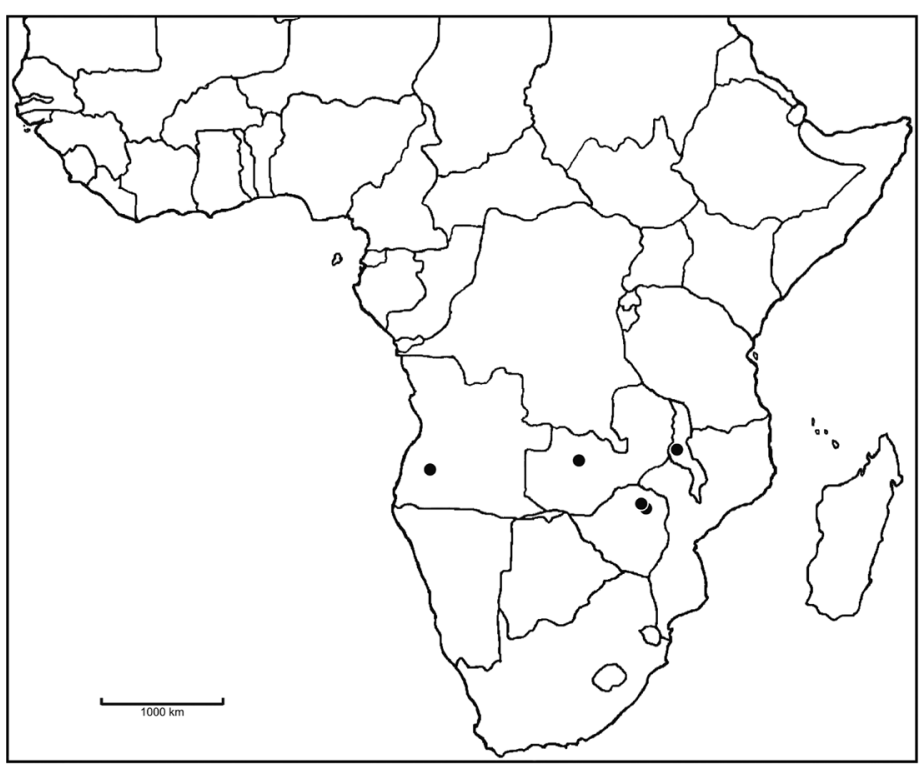

Fig. 205. Distribution of Bilabrella strangulans (Summerh.) Szlach. \& Kras-Lap.

Petals bilobed to the base; anterior lobe $10-12.5 \mathrm{~mm}$ long, $2 \mathrm{~mm}$ wide, obliquely lanceolate to obliquely oblong-lanceolate, with acute, subulate apex, glabrous or minutely ciliolate in the lower half; posterior lobe 4.5-6 mm long, 0.6-0.7 mm wide, filiform-linear, erect, acute, minutely ciliolate. Lateral sepals 7-8 $\mathrm{mm}$ long, 5-5.5 mm wide, obliquely obovate, cochleate near the apex with involute apical margin, apiculus laterally placed, glabrous. Lip 3-lobed to the base, glabrous, all lobes pendent with slightly incurved apices; the middle lobe $11.5-12.5 \mathrm{~mm}$ long, $0.5 \mathrm{~mm}$ wide, linear-filiform, acute; lateral lobes $8.5-9.5 \mathrm{~mm}$ long, 0.3-0.5 $\mathrm{mm}$ wide, linear-filiform, acute. Spur ca 22-24 mm long, narrowly cylindrical and parallel to the ovary up to the middle, then slightly swollen and wrapped round the pedicel, blunt. Anther 2-2.5 mm long, conical-ovoid; connective obtuse; antherophores 4-5 mm long, very slender, slightly upcurved. Stigmaphores 4-5 mm long, narrowly cylindrical, truncate, straight. Rostellum middle lobe longer than connective. Auriculae very unequally bilobed (Fig. 204).

E c o log y. Open woodland but also in shade. MarchApril.

Distribution. Angola, Zambia, Malawi, Zimbabwe, alt. 1200-1300 m (Fig. 205).

S pecimens examined. Angola. Huíla, 1884 (fl), Antunes 14 (K! - fragment.). Zambia. Williamson 263 (K! \& spirit.); Kafue Game, in woodland. Mar. 1967 (fl), Williamson 303 (K!). Malawi. Central: Kasungu Distr., Mi, 30 miles N of Kasungu, sandy, Brachystegia woodland, 1250 m, 7 Apr. 1978 (fl), Pawek 14347 (K!). Zimbabwe. Harare: Umtali Distr., "Rhodes View", Circular Drive, commonage, 11 miles NE of Salisbury, 1200 m, 15 Mar. 1960 (fl), Chase 7293 (K!); "Rhodes
View", Circular Drive, commonage, 11 miles NE of Salisbury, 1200 m, 15 Mar. 1960 (fl), Chase 7293 A (K!); Salisbury Distr., few specimens on NE border, in open bushland, Mar. (fl), Geatrex 94 (K!); Mashonaland Central: Umvukwes, Aranbira Estates, 1300 m, Mar. 1953 (fl), Pollitt 42189 (K!).

Notes. An easily distinguishable species by the spur wrapping round the pedicel in the distal half. By its grass-like leaves it resembles Bilabrella tubifolia. Despite the latter, however, it has completely different spur and usually much wider anterior petal lobe.

This species resembles somewhat also B. anaphysema in general appearance, both having rather narrow leaves in the lower part of the stem, but may be distinguished by the very different spur. In $B$. anaphysema this is considerably shorter than the ovary and pedicel and is incurved sharply just below the base of the swollen apical part. In B. strangulans, on the other hand, the spur equals the ovary and pedicel in length while the apical part is curved more or less closely round the pedicel; from this feature the specific epithet is derived.

The sheet of Antunes 14 in the Coimbra Herbarium contains two specimens of $B$. huillensis (Rchb. f.) Szlach. \& Kras-Lap. and the upper part of a large specimen of B. strangulans, consisting of the inflorescence and a small part of the stem below it. Other gatherings of this number which Summerhayes (cf. 1962) examined consist of B. huillensis only.

74. Bilabrella bertauxiana (Szlach. \& Olsz.) Szlach. \& Kras-Lap., Richardiana 3(3): 139. 2003. - Szlachetko et al., Orchid. of West-Central Africa 1: 236. 2010.

Habenaria bertauxiana Szlach. \& Olsz., Fl. Cam., Orchid. 34(1): 178. 1998.

Type: Zaire, Kassner 2363a (holotype, P!).

Stem $65 \mathrm{~cm}$ tall, erect, rather stout, glabrous. Leaves 8 , $10-15 \mathrm{~cm}$ long, $1.5 \mathrm{~cm}$ wide, lanceolate, acute, erect or suberect, decreasing in size up the stem. Inflorescence $18 \mathrm{~cm}$ long, ca. 20-flowered, rather lax. Floral bracts $12 \mathrm{~mm}$ long, ciliolate along margins, minutely glandular outside. Pedicel and ovary $22 \mathrm{~mm}$ long, glabrous. Dorsal sepal $6 \mathrm{~mm}$ long, 1.8-2 mm wide, oblong-elliptic, with truncate, thickened apex, cochleate, glabrous. Petals bilobed nearly to the base, rather thick; anterior lobe $8 \mathrm{~mm}$ long, $1.5 \mathrm{~mm}$ wide, lanceolate, acuminate, glabrous; posterior lobe $5.5 \mathrm{~mm}$ long, $0.7 \mathrm{~mm}$ wide, linear, obtuse, densely ciliolate. Lateral sepals $8 \mathrm{~mm}$ long, $5 \mathrm{~mm}$ wide, obliquely elliptic-orbicular, deeply cochleate at the apex, apiculus $0.7 \mathrm{~mm}$ long, laterally placed, glabrous. Lip 3-lobed to the base, thick, fleshy, glabrous; the middle lobe $10 \mathrm{~mm}$ long, $0.9 \mathrm{~mm}$ wide, linear, acute; lateral lobes $8 \mathrm{~mm}$ long, $1.1 \mathrm{~mm}$ wide, lanceolate, acuminate, with apices curved upwards. Spur $30 \mathrm{~mm}$ long, $1.5 \mathrm{~mm}$ in diameter, filiform, twisted 


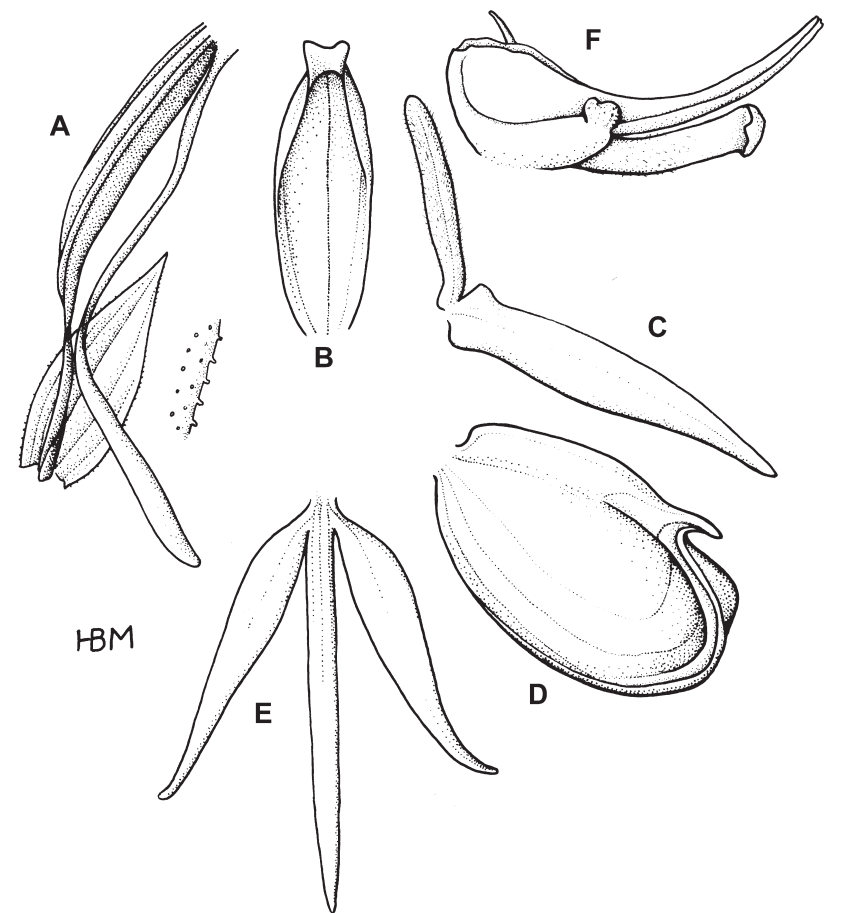

Fig. 206. Bilabrella bertauxiana (Szlach. \& Olsz.) Szlach. \& Kras-Lap.

Explanations: A - ovary, pedicel, spur and bract; $\mathrm{B}$ - dorsal sepal; $\mathrm{C}$ - petal; D - lateral sepal; E - lip; F - gynostemium, side view (drawn by H. B. Margońska from holotype of Habenaria bertauxiana Szlach. \& Olsz. Kassner 2363a, P)

once near the middle and below slightly thickened, subacute, more or less incurved. Anther $2.5 \mathrm{~mm}$ long, bent back; connective rounded; antherophores $6 \mathrm{~mm}$ long, slender, gently upcurved. Stigmaphores $3 \mathrm{~mm}$ long, relatively massive, cylindrical, ciliate outwards. Rostellum middle lobe as long as or slightly longer than connective. Auriculae more or less bilobed (Fig. 206).

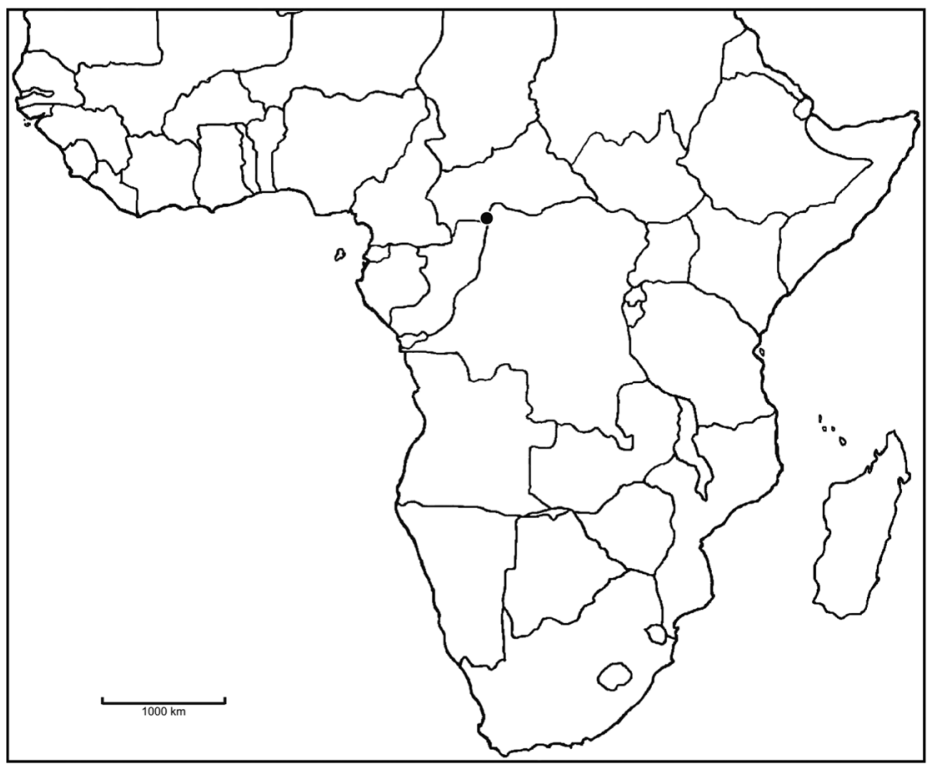

Fig. 207. Distribution of Bilabrella bertauxiana (Szlach. \& Olsz.) Szlach. \& Kras-Lap.
E c o logy. Terrestrial in forest. Flowers in January. D i s tribution. Known so far from the type collection only (Fig. 207).

Specimens examined. Democratic Republic of the Congo (Zaire). Équateur: Kitambo, 15 Jan. 1908 (fl), Kassner 2363a (P!).

N o t e s. Bilabrella bertauxiana appears to be similar to $B$. retinervis from which it differs in length of floral bracts (12 $\mathrm{mm}$ vs $10-25 \mathrm{~mm}$ ), which are ciliolate along margins, minutely glandular outside (vs glabrous) and relatively massive stigmaphores which are ciliate (vs slender, minutely papillate). According to Szlachetko et al. (2010) B. bertauxiana can be related to $B$. haareri from which it differs in the form of anterior petal lobe, in having spur longer than pedicellate ovary, and antherophores longer than stigmaphores.

75. Bilabrella retinervis (Summerh.) Szlach. \& KrasLap., Richardiana 3(3): 142. 2003. - Szlachetko et al., Orchid. of West-Central Africa 1: 222. 2010.

Habenaria retinervis Summerh., Kew Bull. 16: 291. 1962. - Summerhayes, FTEA, Orchid. 1: 98. 1968b. Grosvenor, Excelsa 6: 83. 1976. - Geerinck \& Coutrez, Not. Taxon. Orchid. Afr. Centr., Habenaria: 11. 1977. - Williamson, Orchid. S. Central Afr.: 54. 1977. Geerinck, Fl. Afr. Centr., Orchid. 1: 117. 1984. - la Croix et al., Orchid. Malawi: 77. 1991. - la Croix \& Cribb, Fl. Zambes., Orchid. 11: 103. 1995.

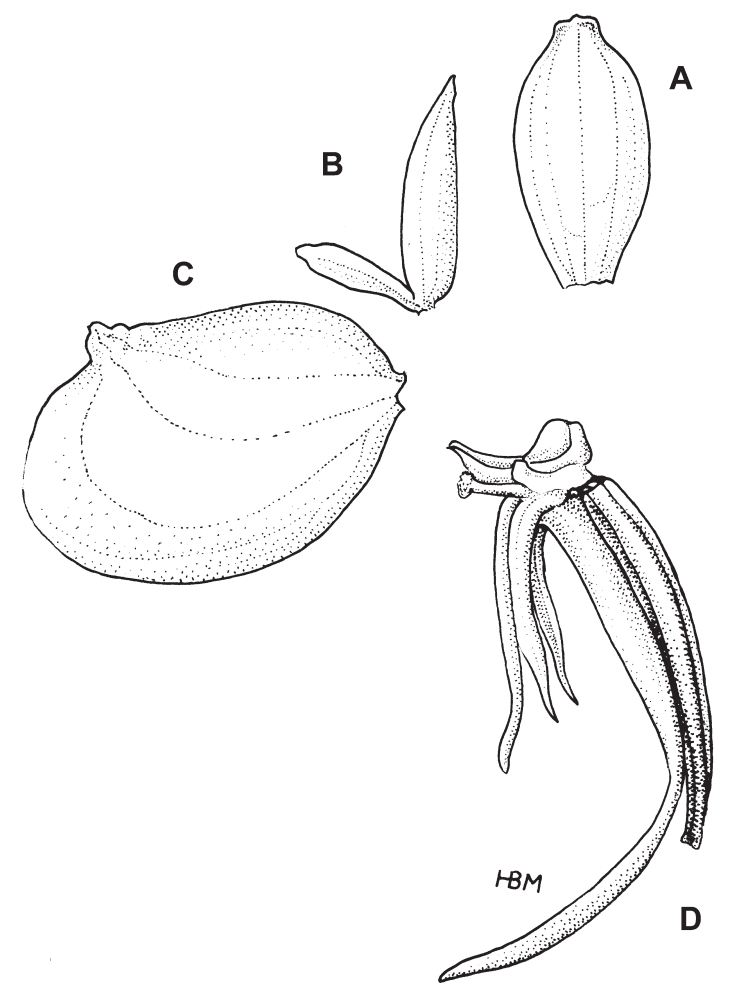

Fig. 208. Bilabrella retinervis (Summerh.) Szlach. \& Kras-Lap. Explanations: A - dorsal sepal; B - petal; C - lateral sepal; D - ovary, pedicel, spur and gynostemium, side view (redrawn by H. B. Mragońska from holotype of Habenaria retinervis Summerh. - Milne-Redhead 3805, K) 


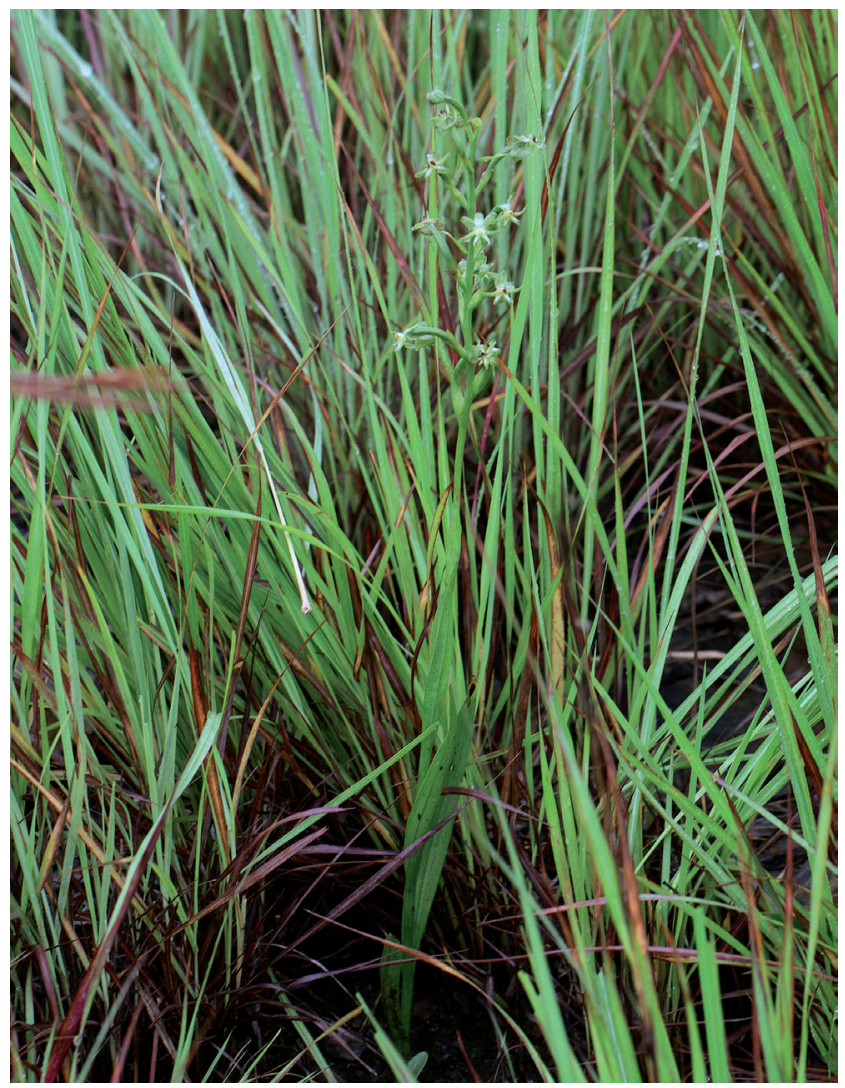

Fig. 209. Bilabrella retinervis (Summerh.) Szlach. \& Kras-Lap. habit (phot. L. Grobler)

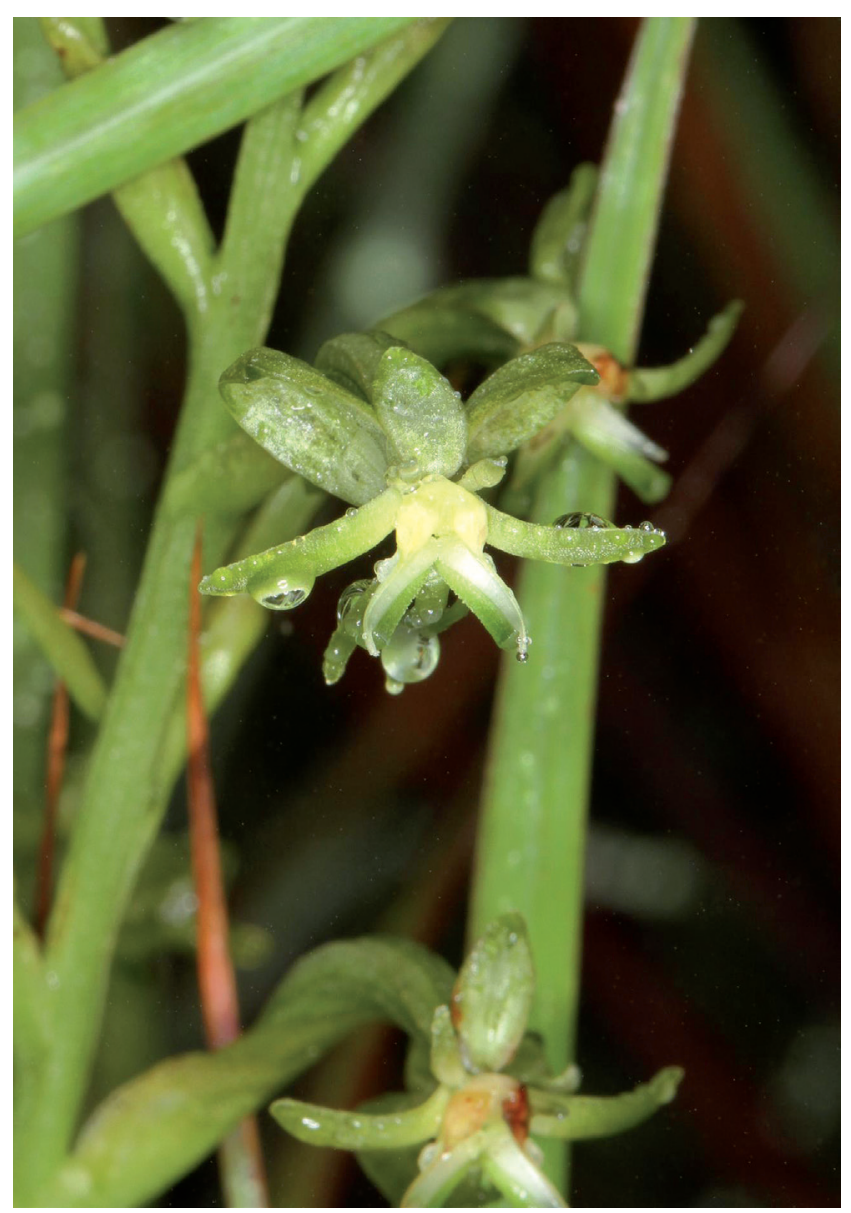

Fig. 211. Bilabrella retinervis (Summerh.) Szlach. \& Kras-Lap. inflorescence close-up (phot. L. Grobler)

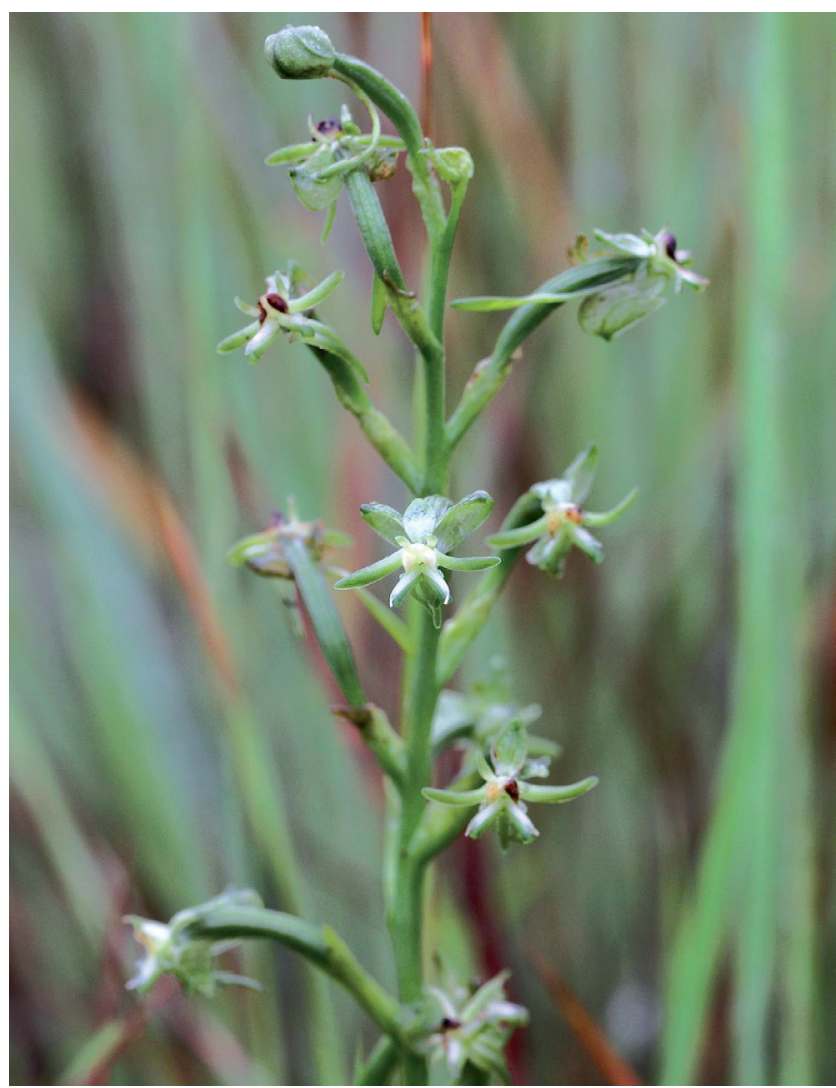

Fig. 210. Bilabrella retinervis (Summerh.) Szlach. \& Kras-Lap.inflorescence (phot. L. Grobler)

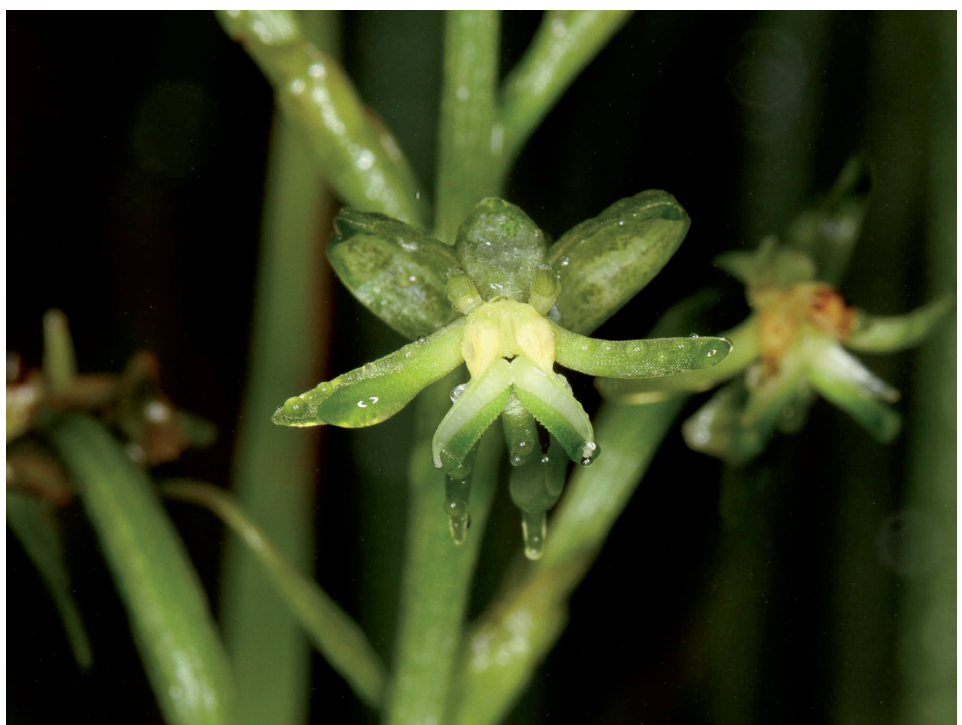

Fig. 212. Bilabrella retinervis (Summerh.) Szlach. \& Kras-Lap. flower close-up (phot. L. Grobler)

Type: Zambia, Milne-Redhead 3805 (holotype, K! \& spirit.).

Tubers 1-3 cm long, 1-1.5 $\mathrm{cm}$ in diameter, ovoid to ellipsoid. Stem $35-85 \mathrm{~cm}$ tall, erect, slender. Leaves 7-11, 5-14 cm long, 1-3 cm wide, lanceolate to ellipticlanceolate, acute, erect to suberect, decreasing in size 
up the stem. Inflorescence 10-27 cm long, fairly laxly 8-28-flowered. Flowers green with whitish lip and petals. Floral bracts $10-25 \mathrm{~mm}$ long, ovate-lanceolate, acuminate. Pedicel and ovary 20-30 mm long. Dorsal sepal 5-6 mm long, 1.5-3 mm wide, oblong-elliptic to elliptic, concave, obtuse, reflexed. Petals bilobed almost to the base; anterior lobes 9.5-11.5 mm long, 1-2.5 mm wide, lanceolate, acute, projecting forwards, glabrous; posterior lobe 5-6.5 $\mathrm{mm}$ long, $0.5-1 \mathrm{~mm}$ wide, ligulate to linear-ligulate, denticulate towards the apex, erect, ciliolate on margins. Lateral sepals 7-9.5 mm long, 4.5-6.5 mm wide, obliquely obovate to elliptic, shortly apiculate, concave, reflexed. Lip deflexed, 3-lobed to the base; the middle lobe 11-13.5 mm long, 0.5-1 mm wide, linear, obtuse, incurved; lateral lobes $7.5-11 \mathrm{~mm}$ long, 1-1.2 mm wide, narrowly lanceolate, pendent, acute. Spur 25-32 mm long, pendent, slender, narrowly cylindrical, slightly swollen towards the apex. Anther 2-3 mm long, rather massive; connective truncate; antherophores $4 \mathrm{~mm}$ long, slender, almost straight. Stigmaphores $4 \mathrm{~mm}$ long, slender cylindrical-clavate, minutely papillate. Rostellum middle lobe longer than connective. Auriculae bilobed, lobes subglobose (Figs 208-212).

E c ology. Brachystegia woodland among grass, forest margins. March-December.

Distribution. Ethiopia, Democratic Republic of the Congo (Zaire), Tanzania, Angola, Zambia, Malawi, alt. 70-2000 m (Fig. 213).

S pecimens ex a mined. Ethiopia. Oromiya: In Teclea-Acokanthera woodland, Stand 325, 69 road km W of Shashamane, 1750 m, 1 Aug. 1963 (fl), Beals 372 (K!). Democratic Republic of the Congo (Zaire). HautKatanga: Kanavia, in savannah, following a Dambo, 1907 (fl), Qunne s.n. (K!); Kolwezi, Dikuluwe, 21 Jan. 1960 (fl), Duvigneaud 51550 (BRLU!); 44 km northnorth-west of Kolwezi, woodland, 1070 m, 24 Jan. 1983 (fl), Schaijes 1828 (K!); Kasaï-Oriental: Kundelungu, waterside of river the Kalunda, 1590 m, 9 Jan. 1971 (fl), Lisowski 12636 (UGDA-DLSz!); Env. 25 km au NW de Lubumbashi, Mount Tumbwe, 1470 m, 18 Jan. 1970 (fl), Lisowski 65768 (UGDA-DLSz!); Shaba Region, Kipopo, 14 km north-west of Lubumbashi, seasonally flooded grassland, $1240 \mathrm{~m}, 8$ Jan. 1983 (fl), Schaijes 1790B (K!). Tanzania. Ruvuma: Songea Distr., Matagoro Hill just S of Songea among grass in Brachystrgia woodland, 1350 m, 27 Feb. 1956 (fl), Milne-Redhead \& Taylor 8890 (K!). Angola. Huambo: Sa da Bandeira, Vila Arriaga km 20, 144'서. $13^{\circ} 29^{\prime} \mathrm{E}$, 1950-2000 m, 19 Apr. 1973 (fl), Bamps, Martins \& Matos 4596 (BR!). Zambia. Central: Broken Hill, in the mixed Brachystegia-Uapaca woodland with lateritic out-crops, 22 Jan. 1961 (fl), Morze 53B (K!); Road to Kasula, in Uapaca and Protea bush, open bush, grass, 1 Jan. 1952 (fl), Richards 229 (K!); Northern: Aber-

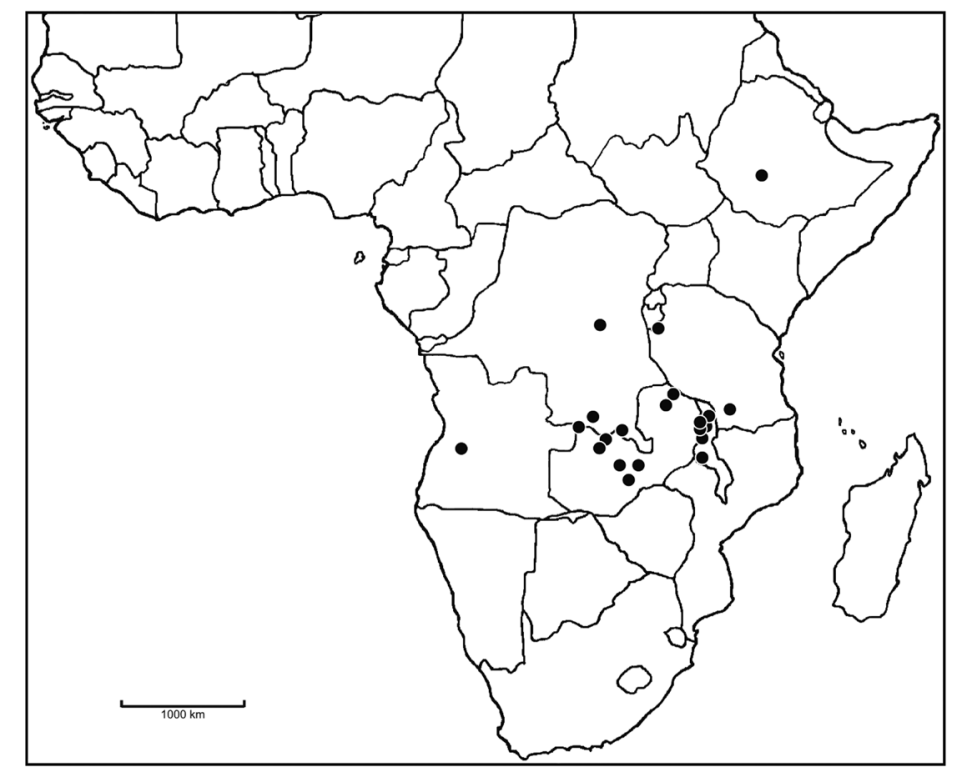

Fig. 213. Distribution of Bilabrella retinervis (Summerh.) Szlach. \& Kras-Lap.

corn Distr., Chenda Farm, grassland near the Dam, 1740 m, 13 Jan. 1965 (fl), Richards 19558 (K!). The same locality, Richards 19562 (K!); Abercon Distr., road to Uningi Pans, on open grassland and marshy land, 1500 m, 12 Feb. 1967 (fl), Richards $22080 b$ (K!); Kasama Distr., woodland, 31 Jan. 1961 (fl), Robinson 4327 (K!); Mbala Distr., Chila Dambo, Mbala, woodland, by an ant hill, sandy loam, 1590 m, 18 Jan. 1968 (fl), Richards 22925 (K!); North-western: Mwinilunga Distr., $52 \mathrm{~km}$ S of Mwinilunga on road to Kabompo, degraded Brachystegia spiciformis, B. boehmii woodland on gravel ridge, 1300 m, 25 Jan. 1975 (fl), Brummitt, Polhill \& Chisumpa 14102 (K!); Mwinilunga Distr., just E of Matonchi Farm, in Brachystegia woodland, 5 Jan. 1938 (fl), Milne-Redhead 3805B (K! \& spirit.); Mwinilunga Distr., Matonchi River, gallery forest woodland, 18 Feb. 1975 (fl), Willimason \& Gassner 2426 (K!); Mwinilunga Distr., Matonchi, below old homestead, Brachystegia woodland, 21 Dec. 1969 (fl), Williamson \& Simon 1796 (K!); Solwezi Distr., 18 km W of Muheba R. $59 \mathrm{~km} \mathrm{~W}$ of Mutanda, mixed miombo woodland on Kalahari sand, $12^{\circ} 20^{\prime} \mathrm{S}, 25^{\circ} 50^{\prime} \mathrm{E}, 1400 \mathrm{~m}$, 19 Jan. 1975 (fl), Brummitt, Polhill \& Chisumpa 13857 (K!); Solwezi Distr., $55 \mathrm{~km}$ NE of Kasempa, $6 \mathrm{~km}$ E of Kayimbwe on road to Kitwe, mixed miombo, 26 Jan. 1975 (fl), Brummitt, Polhill \& Chisumpa 14149 (K!); Solwezi Distr., in woodland, 16 Jan. 1960 (fl), Holmes 0192 (K!); 6 miles of Lusaka, red soil under Brachystegia-Isoberlinia woodland, 1260 m, 2 Feb. 1958 (fl), King 412 (K!). Malawi. Central: Nchisi Forest Reserve, among sparse herbs on the forest edge and in woodland, 1300-1450 m, 31 Jan. 1983 (fl), Dowsett-Lamaire 607 (K!); Lilongwe Distr., Chickewa, Brachystegia woodland, 27 Jan. 1953 (fl), Jackson 1036 (K!); Northern: 
Lunyangwa Forest Reserve, near Mzuzu in grassy charing, like dry dambo, in woodland, $1320 \mathrm{~m}, 25 \mathrm{Jan}$. 1986 (fl), la Croix 786 (K!); Mzimba Distr., Mzuzu, Marymount, Brachystegia woodland, 1350 m, 6 Feb. 1974 (fl), Pawek 8068 (K!); Mzimba Distr., 4 miles $\mathrm{N}$ of Champira, roadside in Brachystegia woodland, 1290 m, 8 Apr. 1978 (fl), Pawek 14353 (K!); Mzimba, Road W, Lower dam, in savannah, 14 Feb. (fl), Westwod 607 (K!); 10 miles $\mathrm{N}$ of Mzimba, Upga, frequent all over Plateau, Feb. 1968 (fl), Williamson 798 (K!); 5 mi. NW of Mzuzu, in Brachywoodland, on grassy meadow, 1350 m, 7 Apr. 1974 (fl), Pawek 8306 (K!); 6 miles of Mzuzu toward Lupaso, edge grassy flat, mostly along roadside, 7 Mar. 1976 (fl), Pawek 10886 (K!); Nyika Plateau, 1 mile N of Mi, 1470 m, 2 Feb. 1978 (fl), Pawek 13710 (K!); Rumphi Distr., Nyika approach, $3 \mathrm{~m}$ after burn off from Mi, Brachystegia woodland, a stony slope, 1500 m, 17 Jan. 1987 (fl), la Croix 939 (K!); Rumphi Distr., 33 miles NW of Rumphi, 1/2 mile on Bowe road, Brachystegia woodland, 1450 m, 3 Mar. 1977 (fl), Pawek 12389 (K!); Rumphi, Nyika foothill, Brachystegia woodland, Feb. 1968 (fl), Williamson, Simon \& Ball 798 (K!); N Viphya, S 8 to Uzumala, SW of Mphompaka, Brachystegia woodland, 1360 m, 6 Jan. 1987 (fl), la Croix 925 (K!).

N o t e s. This species is characterised by the relatively few-flowered rather lax inflorescences, the relatively short leaves spaced out at intervals along the stem and the beautifully veined sheaths at the base of the stem These are white with a green reticulation and from this feature the specific epithet is derived. The species differs from Habenaria sochensis by the characters given in the diagnosis (cf. Summerhayes 1962). It is also similar to Bilabrella bertauxiana, what we discussed above.

Szlachetko et al. (2010) compared B. retinervis to $B$. compta and stated that both species can be separated based on posterior petal lobe, stigmaphores and rostellophores, which are prominently longer in the former species.

76. Bilabrella furcipetala (Schltr.) Szlach. \& Kras, Richardiana 6(4): 197. 2006.

Habenaria furcipetala Schltr., Bot. Jahrb. Syst. 53: 514. 1915. - Schlechter, Repert. Spec. Nov Regni Veg. Beih. 68: tab. 26. 1932.

Type: Tanganyika, Stolz 1189 (lectotype, designated by Szlachetko et al. [2010: 229], B!; isolectotype, K!). Habenaria furcipetala var. andromaniaca Schltr., Bot. Jahrb. Syst. 53: 514. 1915.

Type: Nyassa, Schlechter $1189(\mathrm{~B}+)$ - abnormal form.

Stem up to $60 \mathrm{~cm}$ tall, erect, rather stout, glabrous. Leaves 5-10, up to $18 \mathrm{~cm}$ long and $0.8 \mathrm{~cm}$ wide, linear to linear-lanceolate, acute, erect to suberect, decreasing in

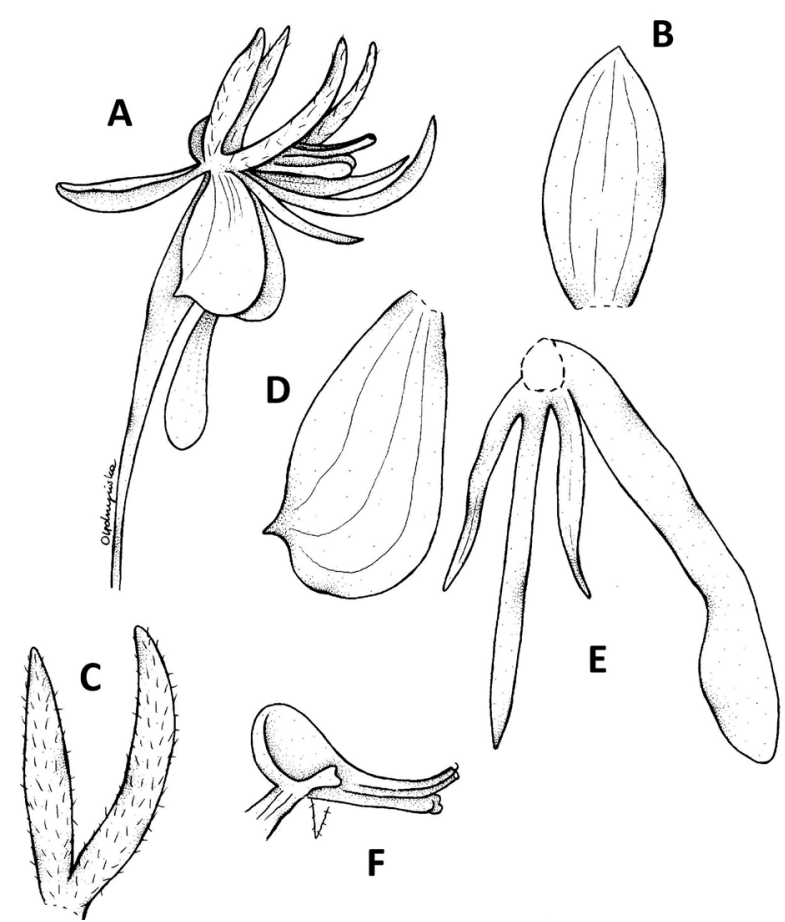

Fig. 214. Bilabrella furcipetala (Schltr.) Szlach. \& Kras Explanations: A - flower; B - dorsal sepal; C - petal; D - lateral sepal; E - lip and spur; F - gynostemium (redrawn by N. Olędrzyńska from Schlechter 1932)

size upwards. Inflorescence up to $12 \mathrm{~cm}$ long, sublaxly 12-20-flowered. Floral bracts $13 \mathrm{~mm}$ long, ellipticlanceolate, acuminate. Pedicellate ovary ca $15 \mathrm{~mm}$ long, glabrous. Dorsal sepal $5.5 \mathrm{~mm}$ long, elliptic, obtuse, glabrous, deflexed. Petals bilobed or furculate above the base, minutely papillose; anterior lobe $6.5 \mathrm{~mm}$ long, $0.8 \mathrm{~mm}$ wide, oblong-lanceolate, falcate, subobtuse; posterior lobe $5 \mathrm{~mm}$ long, $0.8-0.9 \mathrm{~mm}$ wide, obliquely linear-lanceolate to oblong lanceolate, subacute. Lateral sepals $6.5 \mathrm{~mm}$ long, $3 \mathrm{~mm}$ wide, obliquely obovate, concave, laterally apiculate, deflexed. Lip 3-lobed almost to the base; the middle lobe $8 \mathrm{~mm}$ long, $0.7 \mathrm{~mm}$ wide, linear, acute; lateral lobes $5 \mathrm{~mm}$ long, $0.7 \mathrm{~mm}$ wide, linear to linear-lanceolate, acute, divergent. Spur $10 \mathrm{~mm}$ long, subcurved near the middle, subclavate at the apex. Anther $3 \mathrm{~mm}$ tall; connective rounded; antherophores $5 \mathrm{~mm}$ long, slender, gently upturned apically. Stigmaphores $4.5 \mathrm{~mm}$ long, narrowly cylindrical-clavate, truncate. Rostellum middle lobe shorter than connective. Auriculae large, rather massive, apically bilobed, hence appearing cordate (Fig. 214).

E c o logy. No records. May.

Distribution. Tanzania, alt. $1800 \mathrm{~m}$ (Fig. 215).

Specimens examined. Tanzania. Mbeya: Rungwe Distr., upper slopes of Mbaka, 1800 m, 1 May 1912 (fl), Stolz 1189 (B!, K!).

$\mathrm{N}$ ot es. Along with Bilabrella renziana and B. rehmannii it form a group of species with linear to ligulate posterior petal lobe. In these species this lobe is never 


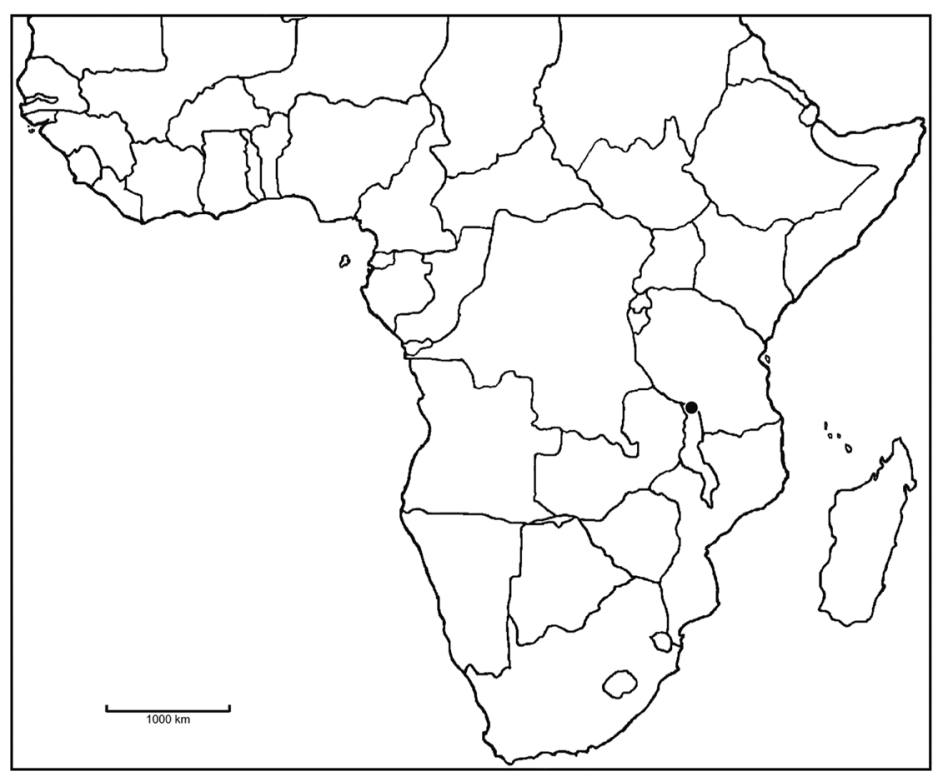

Fig. 215. Distribution of Bilabrella furcipetala (Schltr.) Szlach. \& Kras

filiform. B. furcipetala can be easily distinguished from aforementioned species by having spur shorter than pedicellate ovary and papillose both petal lobes.

77. Bilabrella renziana (Szlach. \& Olsz.) Szlach. \& Kras-Lap., Richardiana 3(3): 142. 2003. - Szlachetko et al., Orchid. of West-Central Africa 1: 228. 2010.

Habenaria renziana Szlach. \& Olsz., Fl. Cam., Orchid. 34(1): 182. 1998.

Type: Zaire, Kassner $2457 a$ (holotype, P!).

Tuber $1.5 \mathrm{~cm}$ long, $1 \mathrm{~cm}$ in diameter, globose. Stem $47 \mathrm{~cm}$ tall, erect, delicate, glabrous. Leaves 8 , up to $9 \mathrm{~cm}$ long, and $0.7 \mathrm{~cm}$ wide, erect, linear-lanceolate, acute, adpressed to the stem, decreasing in size up the stem. Inflorescence $9 \mathrm{~cm}$ long, loosely 5 -flowered. Floral bracts $13 \mathrm{~mm}$ long, rather densely ciliolate along margins and outwards. Pedicel and ovary ca. $25 \mathrm{~mm}$ long, erect, glabrous. Dorsal sepal $6 \mathrm{~mm}$ long, $2 \mathrm{~mm}$ wide, narrowly elliptic, with ligulate obtuse apex, concave, glabrous. Petals bilobed nearly to the base, rather thin; anterior lobe $5.5 \mathrm{~mm}$ long, $1.2 \mathrm{~mm}$ wide, slightly obliquely linear-lanceolate, acute, glabrous; posterior lobe $4 \mathrm{~mm}$ long, $0.8 \mathrm{~mm}$ wide, linear, acute, densely ciliolate. Lateral sepals $7 \mathrm{~mm}$ long, $4 \mathrm{~mm}$ wide, almost orbicular or widely obovate, strongly cochleate at the apex, apiculus ca. $1 \mathrm{~mm}$ long, laterally placed, strongly deflexed. Lip 3-lobed nearly to the base, glabrous; the middle lobe $12 \mathrm{~mm}$ long, $1 \mathrm{~mm}$ wide, linear, obtuse; lateral lobes $8-8.5 \mathrm{~mm}$ long, $1.2 \mathrm{~mm}$ wide, lanceolate with subulate, slightly upcurved apex. Spur $27 \mathrm{~mm}$ long, $1 \mathrm{~mm}$ in diameter, filiform, equal in thickness nearly to apex, acute. Gynostemium similar to H. huillensis (Fig. 216).

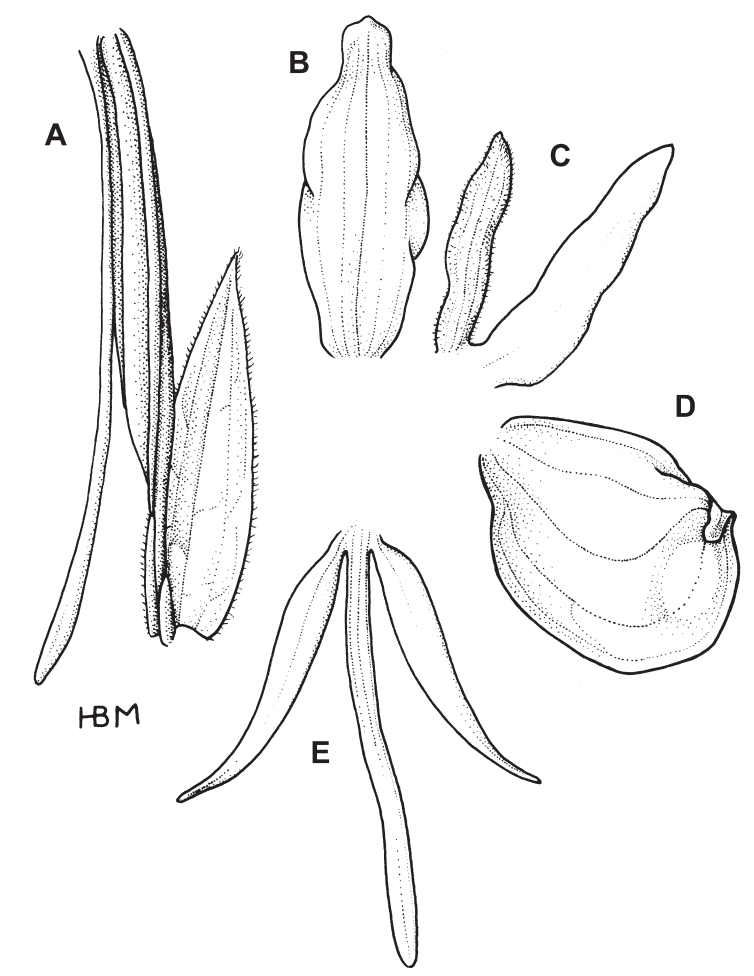

Fig. 216. Bilabrella renziana (Szlach. \& Olsz.) Szlach. \& Kras-Lap. Explanations: A - ovary, pedicel, spur and floral bract; B - dorsal sepal; C - petal; D - lateral sepal; E - lip (drawn by H. B. Margońska from holotype of Habenaria renziana Szlach. \& Olsz. - Kassner 2457a, P)

E c ology. No records. February.

D i s tribution. Known so far from the type collection only (Fig. 217).

Specimens examined. Democratic Republic of the Congo (Zaire). Kasaï Oriental: Lupaka Riv., 6 Feb. 1908 (fl), Kassner 2457a (P!).

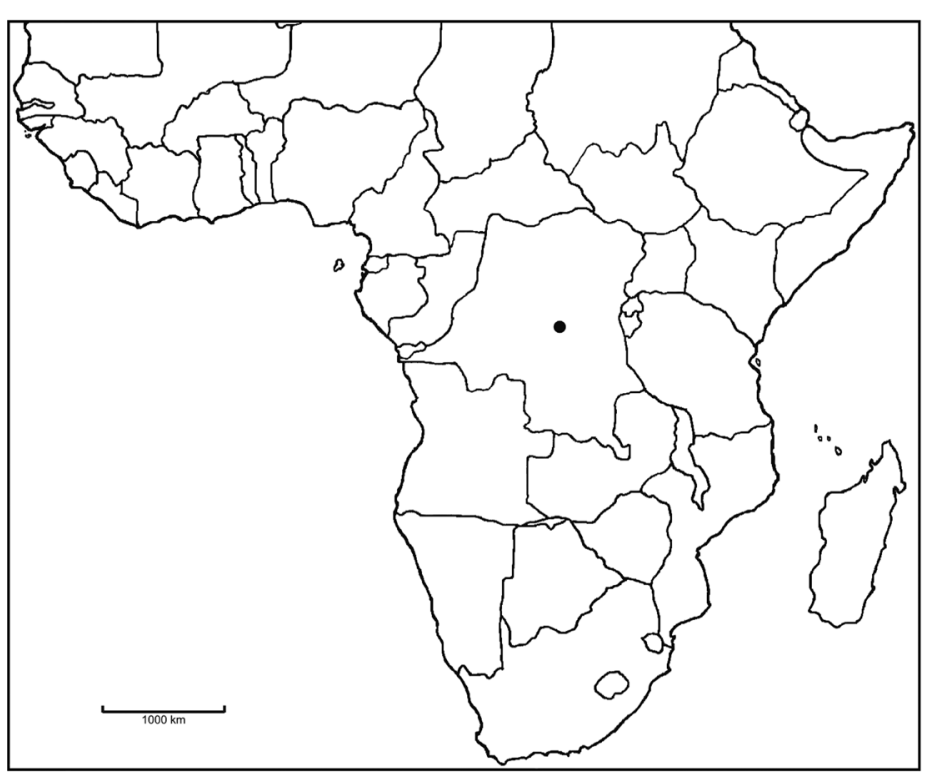

Fig. 217. Distribution of Bilabrella renziana (Szlach. \& Olsz.) Szlach. \& Kras-Lap. 
Note s. The most similar species to Bilabrella renziana is B. rehmannii. They can be separated by the following characters: floral bracts rather densely ciliolate along margins and outwards (glabrous in the latter), spur filiform (vs narrowly cylindrical), equal in thickness nearly to apex (vs apically inflated), acute (subacute). Szlachetko et al. (2010) regarded B. renziana to $B$. subequalis and $B$. kilimanjari. It differs, however, from the former by having non-swollen spur, longer than pedicellate ovary, and somewhat different architecture of the gynostemium. Unlike B. kilimanjari, posterior petal lobe of $B$. renziana is linear, acute, shorter than anterior one.

78. Bilabrella rehmannii (Bolus) Szlach. \& Kras, Richardiana 6(4): 197. 2006.

Habenaria rehmannii Bolus, J. Linn. Soc., Bot. 25: 169. 1889. - Kraenzlin, Orchid. Gen. Sp.: 224. 1901.

Type: Republic of South Africa, Rehmann 5780 (holotype, $\mathrm{K}$ !; isotype, $\mathrm{Z}$ not seen).

Stem to $45 \mathrm{~cm}$ long, erect, rather stout, glabrous. Leaves 4-9, 3-23 cm long, 0.7-1.3 cm wide, linearlanceolate, acuminate, erect or spreading, gradually

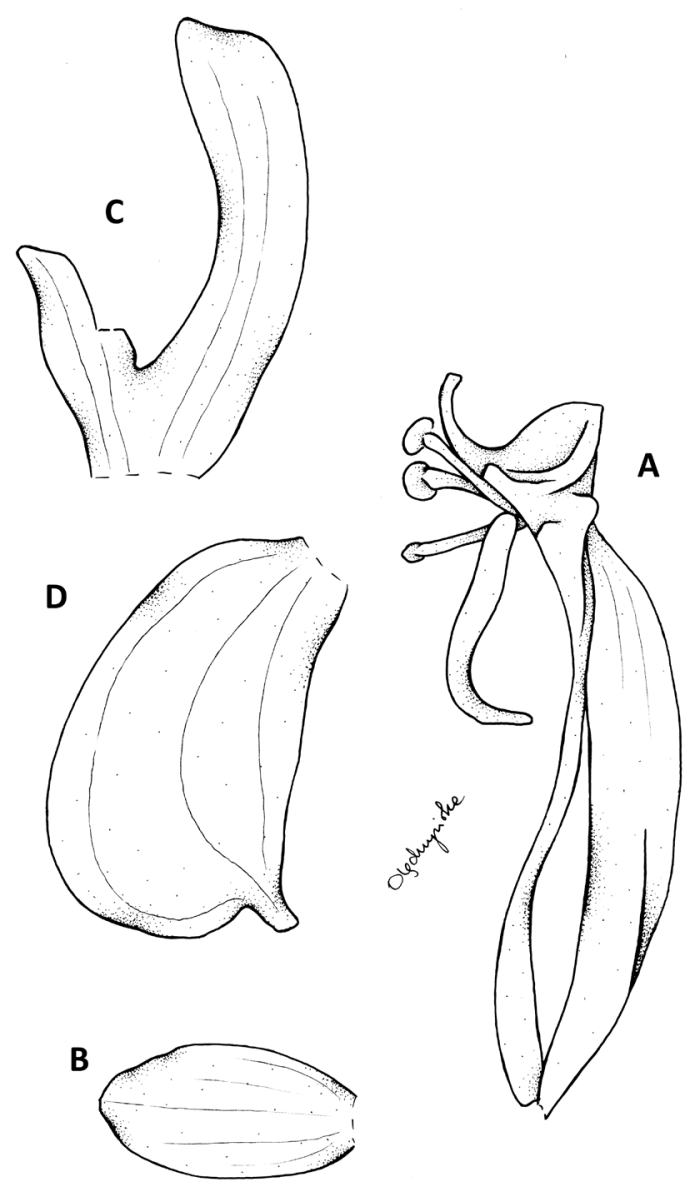

Fig. 218. Bilabrella rehmannii (Bolus) Szlach. \& Kras Explanations: A - ovary, gynostemium, lip, spur; B - dorsal sepal; C - petal; D - lateral sepal (redrawn from N. Olędrzyńska from holotype of Habenaria rehmannii Bolus - Rehmann 5780, K)

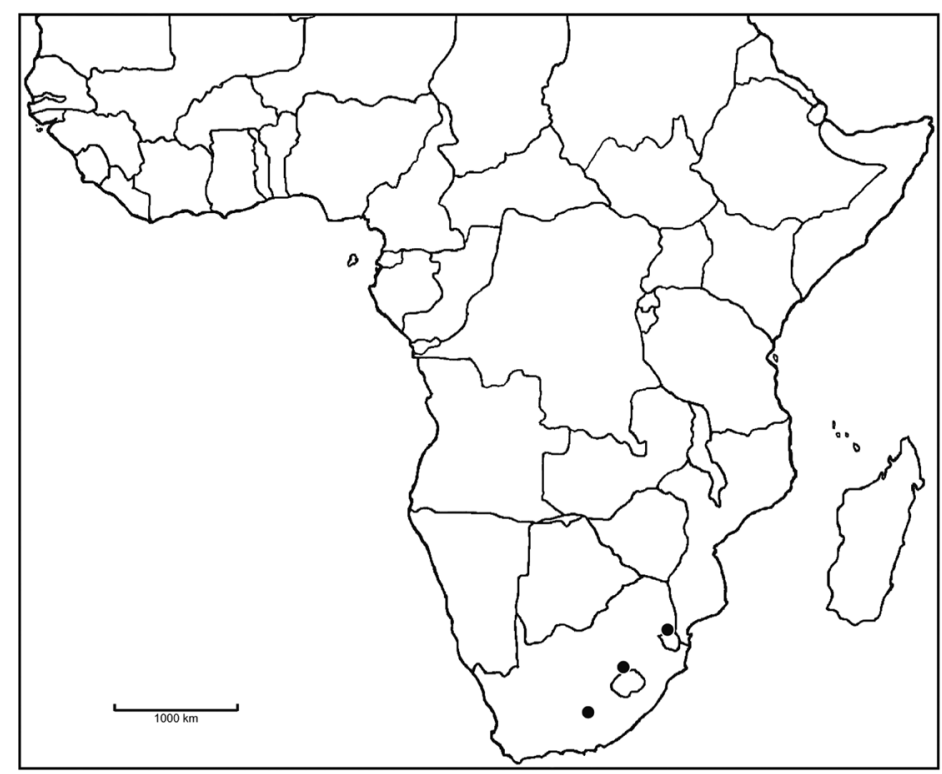

Fig. 219. Distribution of Bilabrella rehmannii (Bolus) Szlach. \& Kras

decreasing in size upwards. Inflorescence $6.5-14 \mathrm{~cm}$ long, laxly 9-24-flowered. Floral bracts 9-13 mm long, lanceolate, acuminate. Pedicellate ovary to $25 \mathrm{~mm}$ long, slender. Dorsal sepal 4-5 mm long, 2-2.5 mm wide, ovate to elliptic, apiculate to obtuse, concave, erect or reflexed. Petals bilobed to the base; anterior lobe 3.5$8 \mathrm{~mm}$ long, $0.5-1 \mathrm{~mm}$ wide, linear-lanceolate to ligulate, falcate, dentate at the apex, 2-nerved; posterior lobe 3-5 mm long, 0.5-0.9 mm wide, linear, obtuse, erect. Lateral sepals 6-8 $\mathrm{mm}$ long, 4-4.5 mm wide, obliquely obovate, cuneate, laterally apiculate, concave at the apex. Lip 3-lobed, deflexed; the middle lobe 10-11 mm long, $0.6-0.8 \mathrm{~mm}$ wide, linear to linear-lanceolate, acute; lateral lobes $8 \mathrm{~mm}$ long, $0.4-0.6 \mathrm{~mm}$ wide, linear, acute, incurved. Spur 15-25 mm long, pending, narrowly cylindrical, apically inflated, subacute. Anther 2-3 mm long; connective broadly triangular, subobtuse; antherophores 3-3.5 mm long, narrowly cylindrical, straight. Stigmaphores $4 \mathrm{~mm}$ long, narrowly cylindrical-clavate, slightly pendent at the apex. Rostellum middle lobe distinctly shorter than thecae. Auricles prominent, very unequally bilobed (Fig. 218).

E c o logy. On damp rocks. March-December.

D i stribution. Republic of South Africa, Swaziland, alt. $1320 \mathrm{~m}$ (Fig. 219).

Specimens examined. Republic of South Africa. Mpumalanga: Barberton, 1890 (fl), Culver 74 (K!); Transvaal, Middelburg Distr., Farm "Welvesolier" (W. Mayer), 20 Mar. 1981 (fl), Hilliard \& Burtt 14428 (E!, K!); Transvaal, 2530 B.B. Mac Mac Pods and vicinity, on damp rocks, 16 Mar. 1981 (fl), Hilliard \& Burtt 14387 (E!); Transvaal, Houtbosh, 1875-1880 (fl), Rehmann 5780 (BM!). Swaziland: 1320 m, Dec. 1905 (fl), Bolus 12309 (K!). 
$\mathrm{N}$ o t e s. Bilabrella rehmannii is similar to B. renziana from Democratic Republic of the Congo, what we discussed above.

79. Bilabrella linderi (Summerh.) Szlach. \& Kras-Lap., Richardiana 3(3): 141. 2003.

Habenaria linderi Summerh., Kew Bull. 1931: 381. 1931. - Summerhayes, FTEA, Orchid. 1: 85. 1968 b. - Stewart \& Campbell, Orchids of Kenya: 137. 1996.

Type: Uganda, Linder 2586 (holotype: AMES not seen; drawing, $\mathrm{K}$ !).

Habenaria microsaccus Kraenzl., Bot. Jahrb. Syst. 43: 393. 1909.

Type: Burundi, Keil 63 (B+, K!) \& Uganda, Linder $2586(\mathrm{~B}+)$.

Tubers 1-5 cm long, $1 \mathrm{~cm}$ in diameter, ellipsoid, sparsely tomentose. Stem $35-50 \mathrm{~cm}$ tall, erect, slender or rather stout, leafy throughout its length. Leaves $7-13,6-25 \mathrm{~cm}$ long, 1-1.5 $\mathrm{cm}$ wide, the lowermost sometimes sheath-like, the next 4-6 suberect, lanceolate or lanceolate-linear, acute, the largest adpressed to the stem, much smaller, lanceolate, similar to the bracts. Inflorescence $10-20 \mathrm{~cm}$ long, rather densely to laxly 10-35-flowered. Flowers green. Floral bracts 10-30 mm long, lanceolate, acuminate. Pedicel with ovary $15-$ $30 \mathrm{~mm}$ long, somewhat curved. Dorsal sepal $8 \mathrm{~mm}$ long, $3 \mathrm{~mm}$ wide, oblong elliptic, obtuse, convex, reflexed. Petals 2-lobed almost to the base; anterior lobe 5-9 $\mathrm{mm}$ long, $1 \mathrm{~mm}$ wide, linear, obtuse, ciliolate; posterior lobe 5-9 mm long, $1 \mathrm{~mm}$ wide, filiform, ciliolate. Lateral sepals 9-10 mm long, 4-6 mm wide, obliquely obovate or semi-orbicular, subacute to subobtuse, deflexed. Lip deflexed, 3-lobed from a short undivided base; the middle lobe 8-12 mm long, $1 \mathrm{~mm}$ wide, linear, obtuse; lateral lobes $7-10 \mathrm{~mm}$ long, $0.9 \mathrm{~mm}$ wide, linearlanceolate, obtuse. Spur 10-14 mm long, parallel to the ovary, cylindrical-filiform, scarcely swollen towards apex, blunt. Anther $4 \mathrm{~mm}$ tall, broadly ovoid; connective rounded; antherophores 4-5 mm long, slender, filiform, almost straight. Stigmaphores 4.5-7 mm long, relatively massive, narrowly cylindrical in the lower part, swollen and truncate at the apex. Rostellum middle lobe shorter than connective. Auriculae large, bilobed (Fig. 220). E c ology. Grassland, swamp. September-August. D i stributio n. Uganda, Kenya, alt. 2100-2700 m (Fig. 221).

S pecimens examined. Uganda. Central: Mpigi Distr., Kitala, 1800 m, Aug. 1931 (fl), Blake 1418 (K!); Mpigi Distr., Kigezi, N Muko, roadside grass, 2100 m, July 1962 (fl), Leakey OR 4 (K!); Mpigi Distr., Kigezi, Bufundi, Apr. (fl), Linder 2586 (K!); Mpigi Distr., 22 miles Kisoro Road, Kigezi, rocky grassland, 2100 m, Mar. 1948 (fl), Purseglove 2608 (K!); Kachwekano Farm, Kigezi, Permenent pasture, 2190 m, Apr. 1950 (fl), Purseglove 3361 (K!); Eastern: Iganga Distr., Butandiga, Bguishu, moist grass, $2100 \mathrm{~m}$, 2 Sep. 1932 (fl), Thomas 480 (K!); Kapchorwa Distr., Mt. Elgon, 2550 m, 1 Dec. 1930 (fl), Lugard 270 (K!); Kapchorwa Distr., NE Elgon, open grass slade in forest, 2700 m, Oct. 1948 (fl), Tweedie 728 (K!); Kapchorwa Distr., NE Elgon, Kisano swamp, grassy glade between swamp and forest, 2400 m, Sep. 1958 (fl), Tweedie 1725 (K!); Jemakikwa, common grassland, 13 Jan. 1912 (fl),
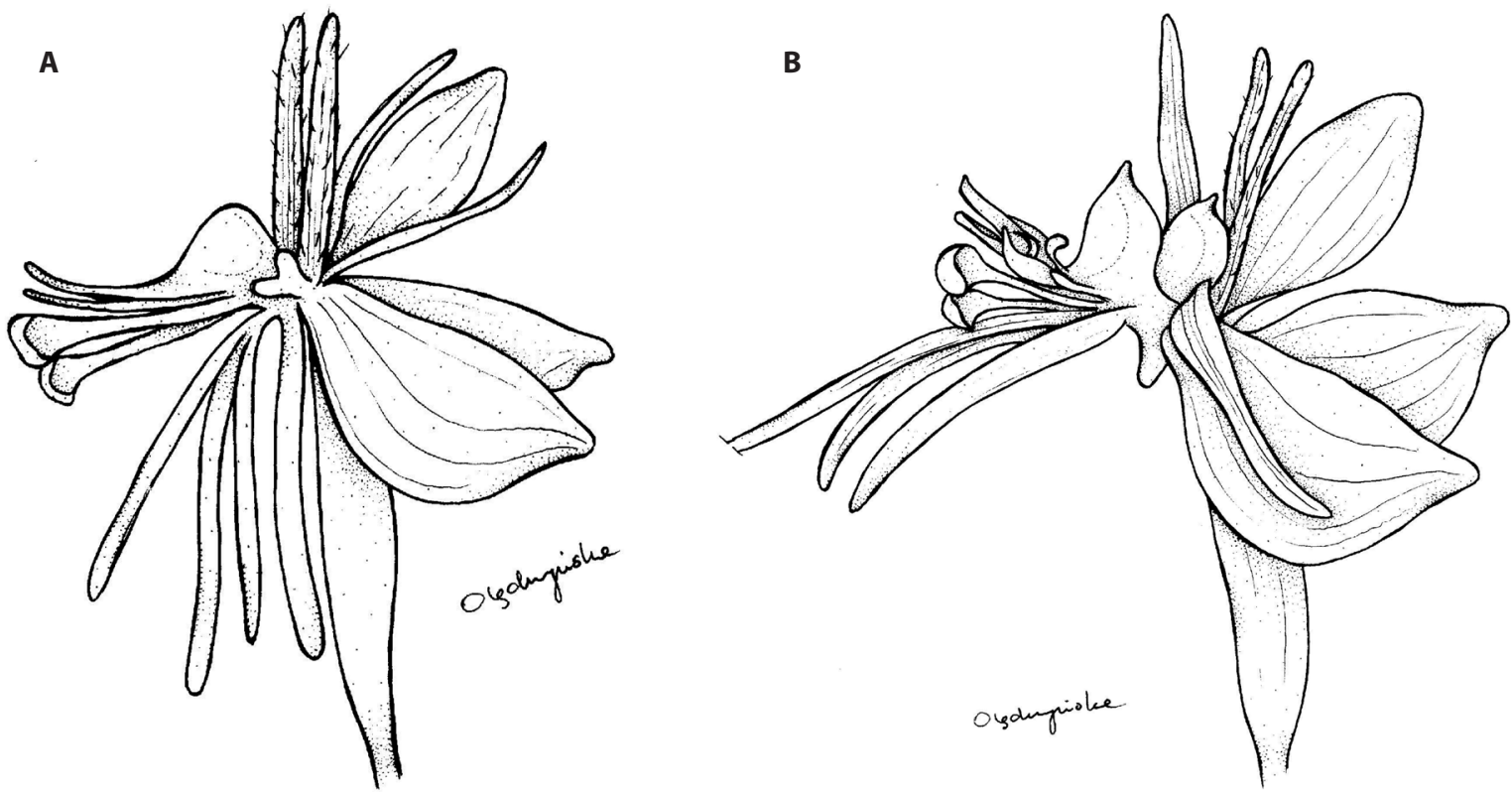

Fig. 220. Bilabrella linderi (Summerh.) Szlach. \& Kras-Lap.

Explanations: A - flower of the type material of Habenaria linderi Summerh. - Linder 3586, K; B - flower of the type of Habenaria microsaccus Kraenzl. - Keil 63, K (redrawn by N. Olędrzyńska) 


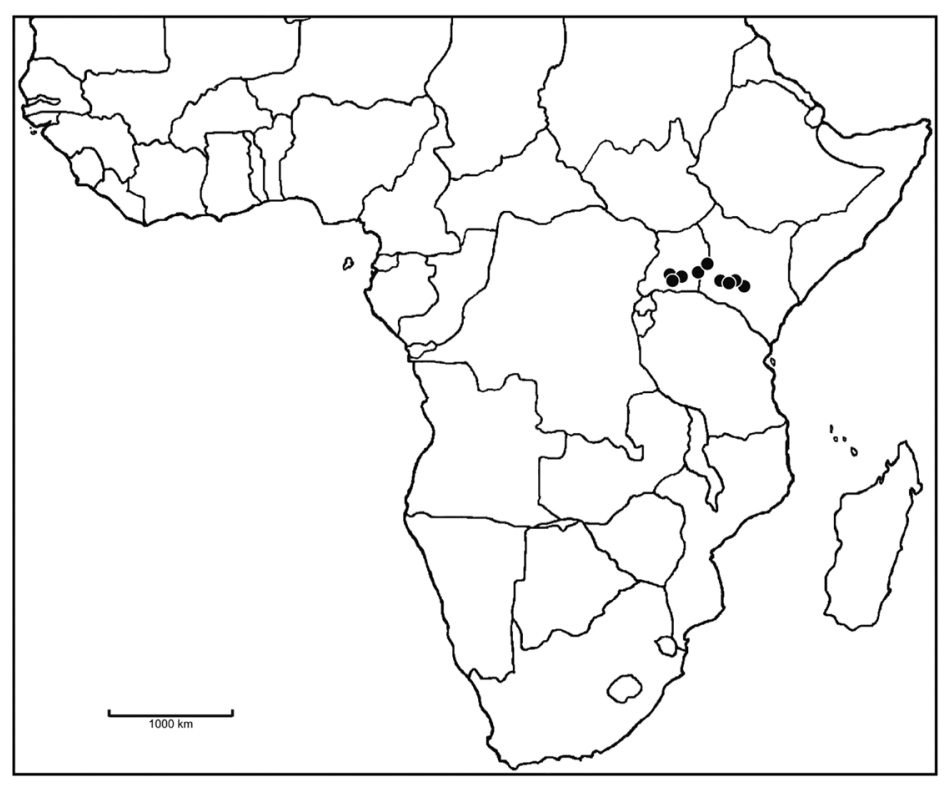

Fig. 221. Distribution of Bilabrella linderi (Summerh.) Szlach. \& Kras-Lap.

Bakes 33 (K!). Kenya. Central: Aberdare Mts., James s.n. (K!). K 3-4, Nyanduma Distr., E end Sasumua Dam, share of dam, 2430 m, 13 Oct. 1970 (fl), Dyson 610 (K!); K3, Nauziha Distr., Sasumua Dam, exposed subsoil on stram, 0'46'S, 642'E, 2170 m, 5 Dec. 1970 (fl), Dyson 617 (K!); K4, Nyanduma Distr., Sasumua Dam, near South Kinangop (Njiame), old quarry site just below dam, bottom flat and kept water logged by spring flowing from top of quarry face, colonised by Typha in deeper areas, sedges, Xyris, etc. in shallow areas, $0^{\circ} 46^{\prime} \mathrm{N}, 36^{\circ} 42^{\prime}$ E, 2400 m, 22 Oct. 1977 (fl), Gilbert 4873 (K!); Rift Valley: Londiani, rainfall 43", in open grassland, generally badly drained and boggy in rains, rainy season, $2250 \mathrm{~m}$, 19 July 1951 (fl), Davis 9 (K!); Sitotan, near Lumbwa, 2550 m, Nov. 1936 (fl), Graham 3736C (K!); Western: NE Elgon, Oct. 1948 (fl), Tweedie 428 (K! \& spirit.); Molo, grass, $2700 \mathrm{~m}$, Oct. 1950 (fl), Tweedie 872 (AMES!, K! \& spirit.); Mt. Elgon, Kisawo swamp, Sep. 1958 (fl), Tweedie 1425 (K! \& spirit.).

N o t e s. Describing H. microsaccus, Kraenzlin (1909) mentioned that it was characterised by two special features, namely, the very short spur, from which the trivial name was derived and the "sonderbaren flügelartigen Anhängsel der Anthere". An examination of the drawings of the type specimen shows that these so-called appendages, which, incidentally, are practically free from the anther to their base, are two additional anthers. Each one is provided with 2 properly constructed thecae and anther canals, though the latter, on account of the absence of supporting rostellumarms, tend to curl up towards to the anther. There are also the usual staminodes on the outside of each lateral anther. No pollinia were seen. This occurrence of two incomplete and ineffective additional anthers must be considered as an abnormal or teratological phenomenon, as in general habit and floral structure Keil 63, the type of $H$. microsaccus, is a typical example of Bilabrella. In most respects, i.e., habit, sepals, petals, lip (except the spur), gynostemium and rostellum it agrees with the type of $H$. linderi. Both possess petals which, instead of being bilobed almost to the base, as is usual in genus Bilabrella, have a united basal portion which is at least $1 \mathrm{~mm}$ long. It is evident from a comparison with both normal and obviously teratological specimens of other species that the type of $H$. microsaccus Kraenzl. is a monstrosity and the name must be rejected under Art. 65 of the International Rules of Nomenclature. It is probable that the very short spur is also an abnormality since in abnormal specimens of other species belonging to Bilabrella a somewhat similar reduction in the length of the spur is a frequent occurrence.

H. microsaccus was described from near Usumbura at the north end of Lake Tanganyika. B. linderi was first discovered at Bufundi in the extreme SW of Uganda; it has since been found on Mt. Elgon (both Uganda and Kenya sides) and on the Mau Plateau in Kenya. The name $B$. linderi must be accepted as the correct name for this species.

The unique character of this species, not found elsewhere, are linear anterior petal lobes, ca $1 \mathrm{~mm}$ wide, ciliolate.

80. Bilabrella macrotidion (Summerh.) Szlach. \& KrasLap., Richardiana 3(3): 141. 2003. - Szlachetko et al., Orchid. of West-Central Africa 1: 226. 2010.

Habenaria macrotidion Summerh., Kew Bull. 16: 285. 1962. - Williamson, Orchid. S. Central Afr.: 53. 1977. - la Croix \& Cribb, Fl. Zambes., Orchid. 11: 100. 1995. Type: Zambia, Richards 12318 (holotype, K!).

Tubers $2 \mathrm{~cm}$ long, $0.8 \mathrm{~cm}$ in diameter, ellipsoid. Stem $40-55 \mathrm{~cm}$ tall, erect to slightly flexuose, slender to delicate, glabrous. Leaves 6-8, up to $16 \mathrm{~cm}$ long and $1 \mathrm{~cm}$ wide, linear-lanceolate, acute, erect, decreasing in size up the stem. Inflorescence 10-16 cm long, laxly manyflowered. Flowers pale green. Floral bracts 7-10 mm long, oblong-lanceolate, acuminate. Pedicel and ovary 17-23 mm long, glabrous. Dorsal sepal 5-6 mm long, $3 \mathrm{~mm}$ wide, oblong elliptic-obovate, obtuse to shallowly truncate, concave. Petals bilobed nearly to the base; anterior lobe 6-7.5 mm long, 2-2.5 mm wide, ovate-lanceolate, subobtuse, glabrous; posterior lobe 5-6 mm long, $0.5 \mathrm{~mm}$ wide, linear-filiform, obtuse, densely papillose-ciliolate. Lateral sepals 7-8.5 mm long, $5 \mathrm{~mm}$ wide, obliquely obovate, concave, shortly apiculate. Lip 3-lobed above the basal 1.5-2 mm claw, deflexed; the middle lobe $9-10 \mathrm{~mm}$ long, $0.5 \mathrm{~mm}$ wide, 


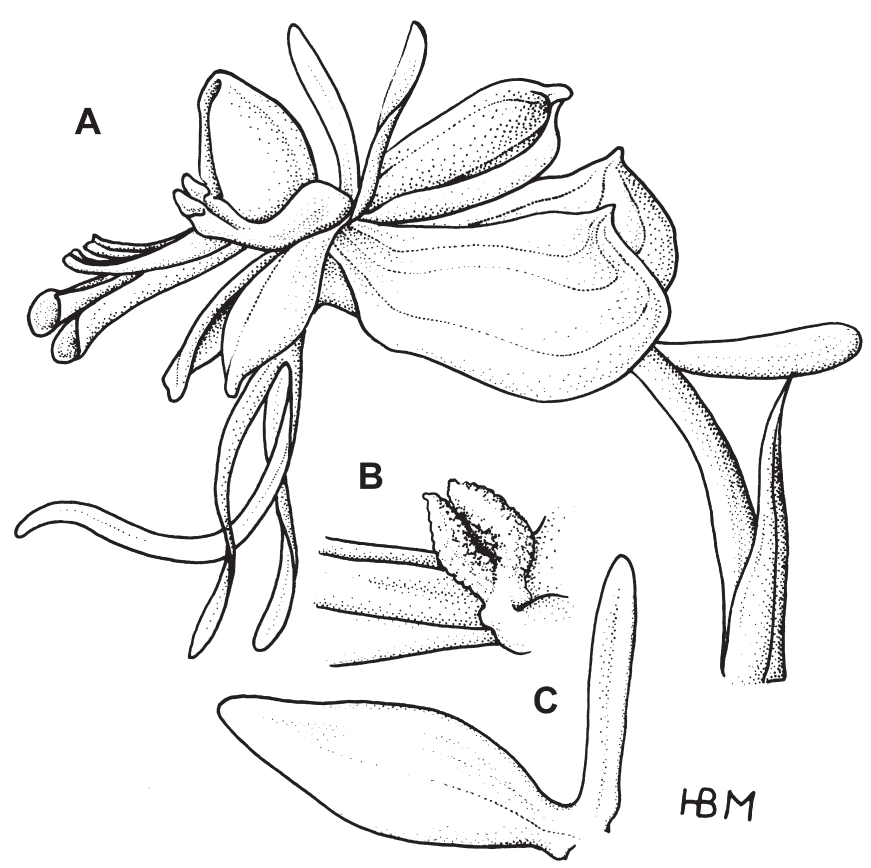

Fig. 222. Bilabrella macrotidion (Summerh.) Szlach. \& Kras-Lap. Explanations: A - flower; B - auriculae, C - petal (redrawn by H.B. Margońska from Summerhayes 1962)

linear, obtuse; lateral lobes 5-6 mm long, $0.3 \mathrm{~mm}$ wide, linear, subobtuse. Spur 8-10 mm long, cylindrical-filiform, blunt. Anther 3-4 mm long; connective truncate; antherophores $4 \mathrm{~mm}$ long, slender, gently upturned in the apical half. Sigmaphores 4-4.5 mm long, narrowly cylindrical, truncate apically. Rostellum middle lobe shorter than connective. Auriculae stalked, deeply bilobed, lobes elongate (Fig. 222).

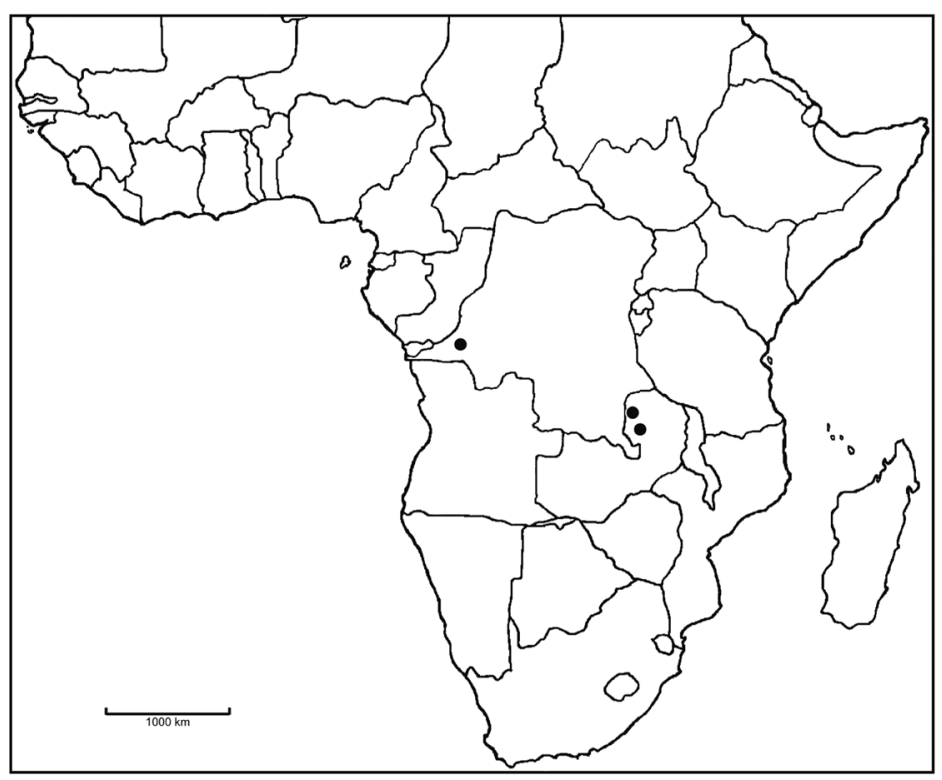

Fig. 223. Distribution of Bilabrella macrotidion (Summerh.) Szlach. \& Kras-Lap.
E c o logy. Swampy ground. January.

Distribution. Democratic Republic of the Congo (Zaire), Zambia, alt. 1050-1235 m (Fig. 223).

Specimens examined. Democratic Republic of the Congo (Zaire). Haut-Katanga: Shaba Region, $7 \mathrm{~km}$ north west of Lubumbashi, Kiboko valley, boggy gassland, 1235 m, 9 Jan. 1983 (fl), Schaijes 1795 (K!). Zambia. Luapula: Kawambwa Distr., Mbereshi, W of Kawambwa, 1050 m, 11 Jan. 1960 (fl), Richards 12318 (K!); Northern: Lake Bangweulu, in spongy ground near southern shore, 13 Jan. 1959 (fl), Watmough 12 (BR!, K!).

Notes. This characteristic member of Bilabrella is especially notable for the long auricules of the gynostemium. These are deeply bifid, the two narrow lobules being parallel in position. The species is allied to $B$. welwitschii in general structure, particularly the wide short anterior petal lobe, but differs also in the structure of the lip. B. pauper is also allied to the present species, but has smaller flowers with distinctly shorter anther canals, and shorter pedicels. Both of these species have shortly bifid and much wider auriculae (cf. Summerhayes 1962).

81. Bilabrella bosseriana (Szlach. \& Olsz.) Szlach. \& Kras-Lap., Richardiana 3(3): 139. 2003. - Szlachetko et al., Orchid. of West-Central Africa 1: 227. 2010.

Habenaria bosseriana Szlach. \& Olsz., Fl. Cam., Orchid. 34(1): 170. 1998.

Type: Cameroon, Leeuwenberg 10199 (holotype, P!; isotypes, $\mathrm{C}$ !, $\mathrm{K}$ !, MO!, WAG!).

Tuber $3 \mathrm{~cm}$ long, $1.5 \mathrm{~cm}$ in diameter, oblong. Stem $95 \mathrm{~cm}$ tall, erect, rather stout, glabrous. Leaves 11, up to $35 \mathrm{~cm}$ long and $1 \mathrm{~cm}$ wide, linear-lanceolate, acute, erect or suberect, decreasing in size up the stem. Inflorescence $19 \mathrm{~cm}$ long, multiflowered, rather dense. Flowers green with white centre. Floral bracts ca. $10 \mathrm{~mm}$ long, widely ovate-lanceolate, acute, ciliolate along margins. Pedicel and ovary up to $20 \mathrm{~mm}$ long, glabrous. Dorsal sepal ca. $6 \mathrm{~mm}$ long, ca. $2.5 \mathrm{~mm}$ wide, oblong, obtuse, cochleate, glabrous. Petals bilobed to the base, rather thin; anterior lobe $8 \mathrm{~mm}$ long, $1.5-2 \mathrm{~mm}$ wide, narrowly oblanceolate, acute, slightly falcate, glabrous; posterior lobe 5.5-6 mm long, $0.4 \mathrm{~mm}$ wide, linear or subulate, acute, densely ciliate. Lateral sepals 7-7.5 mm long, $5 \mathrm{~mm}$ wide, obliquely obovate, with laterally placed apiculus, slightly concave, apical margin involute, glabrous. Lip 3 -lobed above the basal undivided $2 \mathrm{~mm}$, rather thin, glabrous; the middle lobe $10 \mathrm{~mm}$ long, $0.4 \mathrm{~mm}$ wide, filiform, subacute; lateral lobes $6 \mathrm{~mm}$ long, $0.3 \mathrm{~mm}$ wide, filiform, acute, slightly curved upwards. Spur $10 \mathrm{~mm}$ long, narrowly cylindrical-filiform, straight, parallel to the ovary, blunt. Anther $2.5 \mathrm{~mm}$ tall; connective shortly apiculate; antherophores $4 \mathrm{~mm}$ long, 

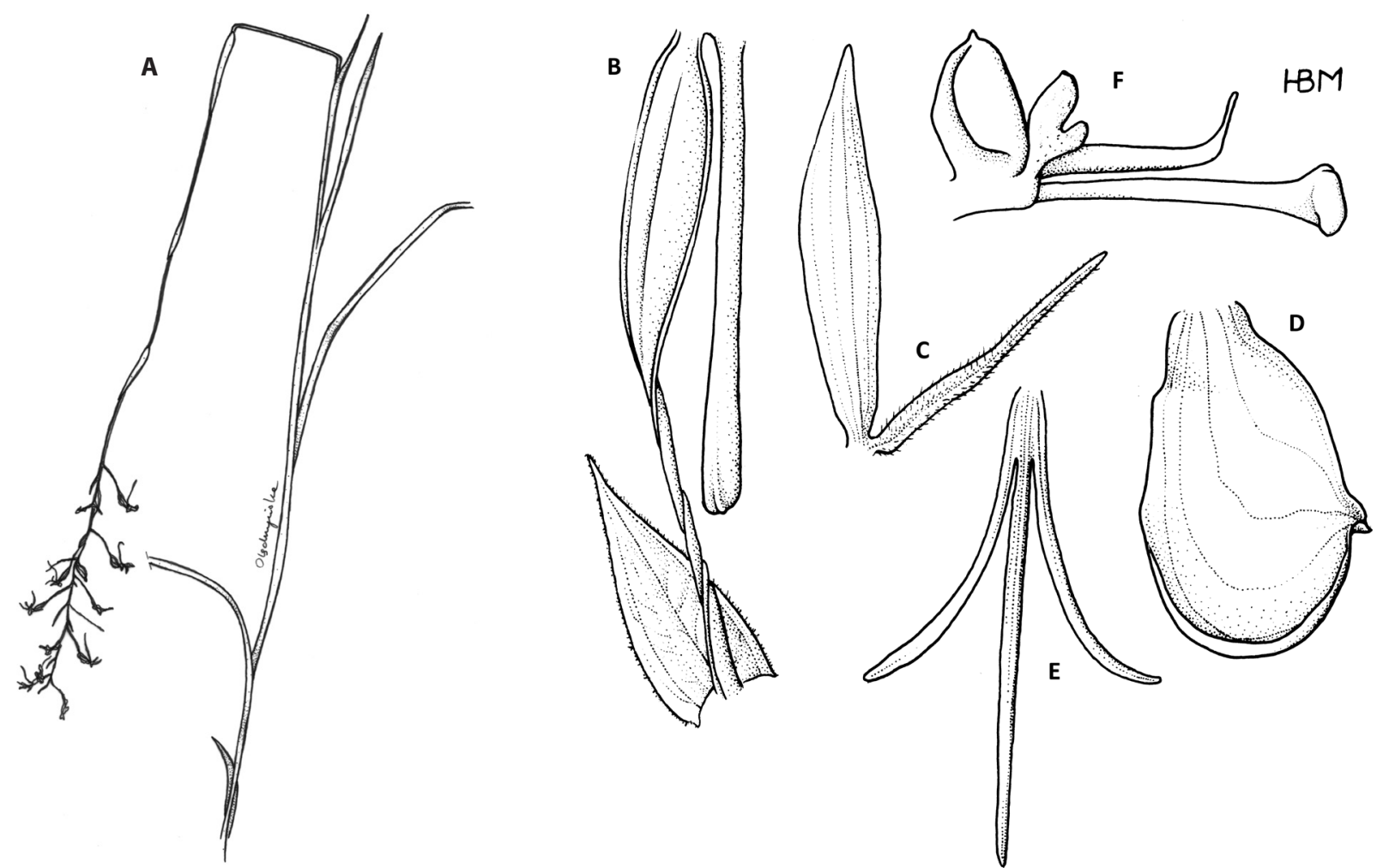

Fig. 224. Bilabrella bosseriana (Szlach. \& Olsz.) Szlach. \& Kras-Lap.

Explanations: A - habit; B - ovary, pedicel, spur and bract; C - petal; D - lateral sepal; E - lip; F - gynostemium, side view (drawn by H. B. Margońska form holotype of Habenaria bosseriana Szlach. \& Olsz. - Leeuwenberg 10199, P)

slender, abruptly upturned at the apex. Stigmaphores $5.5 \mathrm{~mm}$ long, narrowly cylindrical-clavate, truncate. Rostellum middle lobe as long as thecae. Auriculae sessile, massive, very unequally bilobed (Fig. 224).

E c ology. Dambo. Flowers in January-July.

D is tribution. Cameroon, Democratic Republic of the Congo (Zaire), alt. 1160-1200 m (Fig. 225).

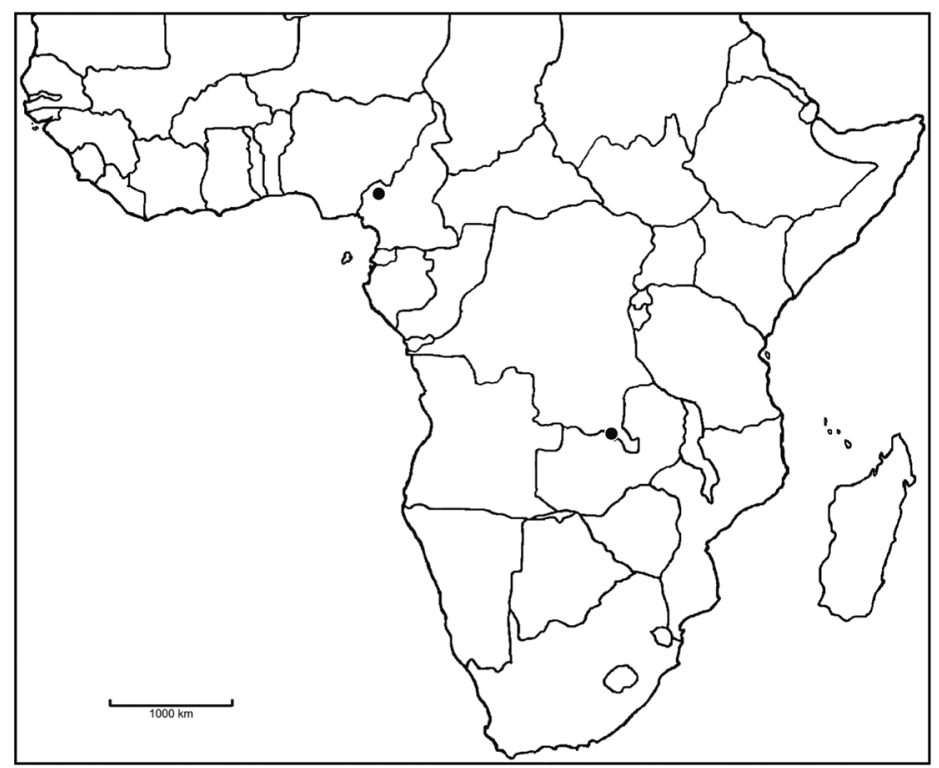

Fig. 225. Distribution of Bilabrella bosseriana (Szlach. \& Olsz.) Szlach. \& Kras-Lap.
S pecimens ex a mined. Cameroon. West: Lake Monoum, NNW de Foumbot, 5'36’ N, 10³3'E, $1200 \mathrm{~m}$, 11 July 1972 (fl), Leeuwenberg 10199 (C!, K!, MO!, P!, WAG!). Democratic Republic of the Congo (Zaire). Haut-Katanga: Lubumbashi-Kasenga, 43 km Dembo Lwamisamba, Zone Kipushi, the awash dambo, 11'26'S, $27^{\circ} 43^{\prime} \mathrm{E}, 1160$ m, 23 Jan. 1986 (fl), Bamps \& Malaisse 8264 (MO!).

$\mathrm{N}$ ot es. In some respects it is similar to Bilabrella macrotidion, but has shorter anther (2.6 mm long), longer stigmaphores (5.5 $\mathrm{mm}$ long), and auriculae bilobed in the apical half only. Szlachetko et al. (2010) compared B. bosseriana to B. chirensis and stated that the former species has ciliate posterior petal lobe, glabrous anterior lobe, shorter rostellum middle lobe, straight stigmaphores longer than upcurved antherophores and different spur form.

82. Bilabrella luegiana Kras \& Szlach., Polish. Bot. Journ. 53(2): 98. 2008.

Type: Republic of South Africa, Rudatis 240 (holotype, E!, isotypes,: AMES!, UGDA-DLSz! - fragment, drawing).

Stem $61 \mathrm{~cm}$ tall, erect, slender, glabrous. Leaves 8 , 4.5-18 cm long, $1-1.2 \mathrm{~cm}$ wide, linear-lanceolate, acute, spredaing or adpressed to the stem, decreasing in size upwards. Inflorescence $18 \mathrm{~cm}$ long, laxly 25 -flowered. 


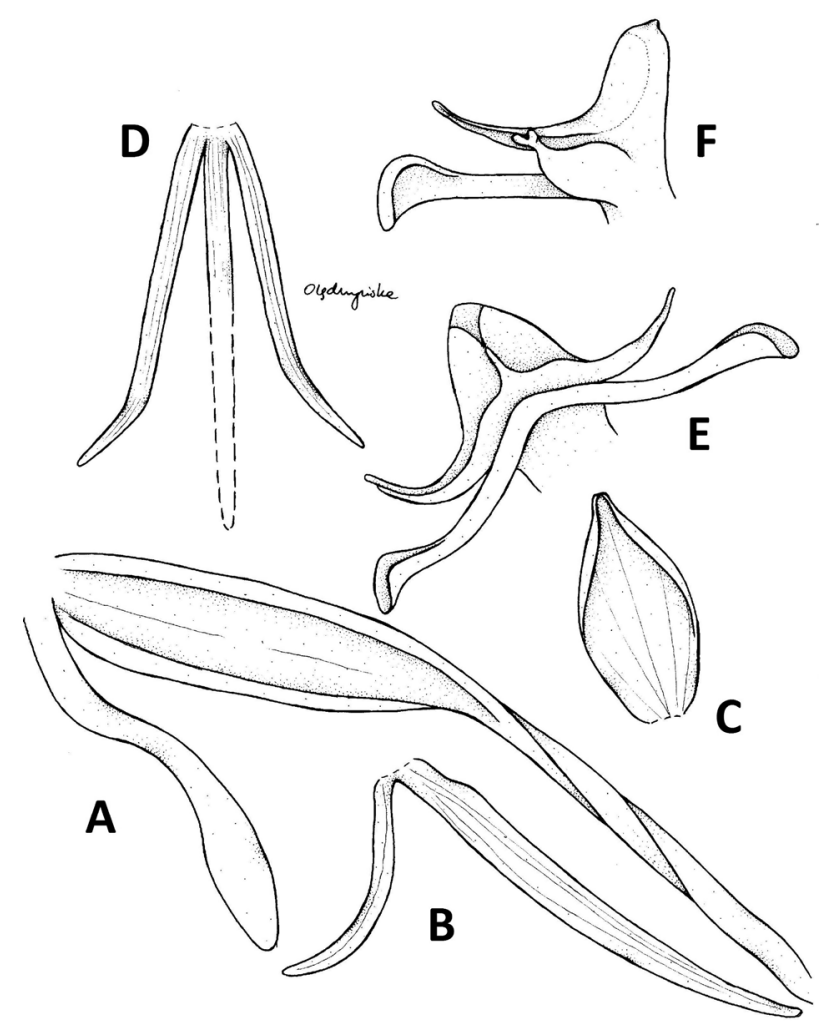

Fig. 226. Bilabrella luegiana Kras \& Szlach.

Explanations: A - ovary, pedicel and spur; B - petal, C - dorsal sepal; D lip; E - gynostemium, front view; F - gynostemium, side view (drawn by N. Olędrzyńska from holotype - Rudatis 240, E)

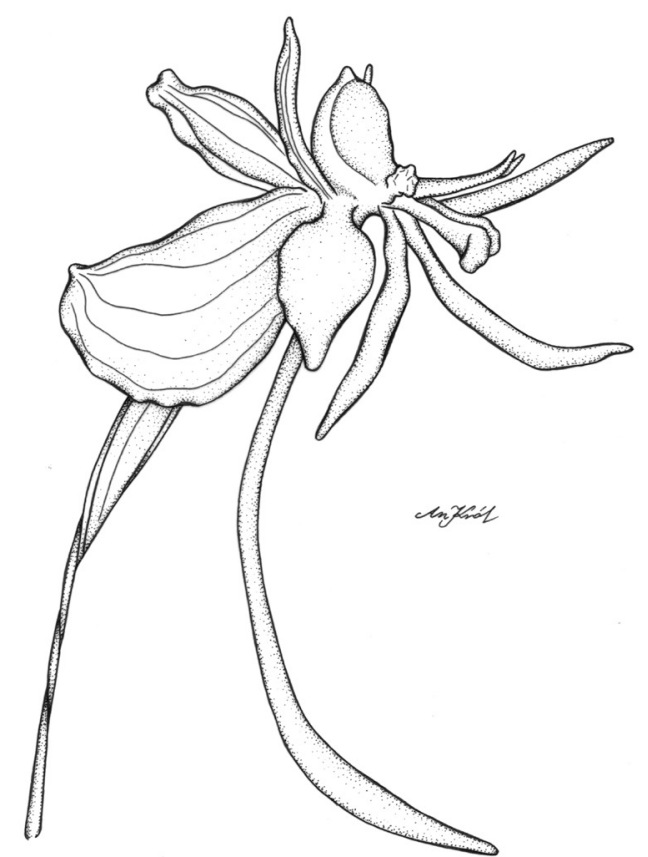

Fig. 227. Bilabrella luegiana Kras \& Szlach. - flower (drawn by A. Król from Rudatis 246, AMES)

Floral bracts 13-20 mm long, ovate-lanceolate, acuminate. Pedicel and ovary $23 \mathrm{~mm}$ long, slender, glabrous. Dorsal sepal $6 \mathrm{~mm}$ long, $2 \mathrm{~mm}$ wide, elliptic, apiculate, obtuse, concave, glabrous. Petals bilobed to the base, glabrous; anterior lobe $13.5 \mathrm{~mm}$ long, $1.1 \mathrm{~mm}$ wide,

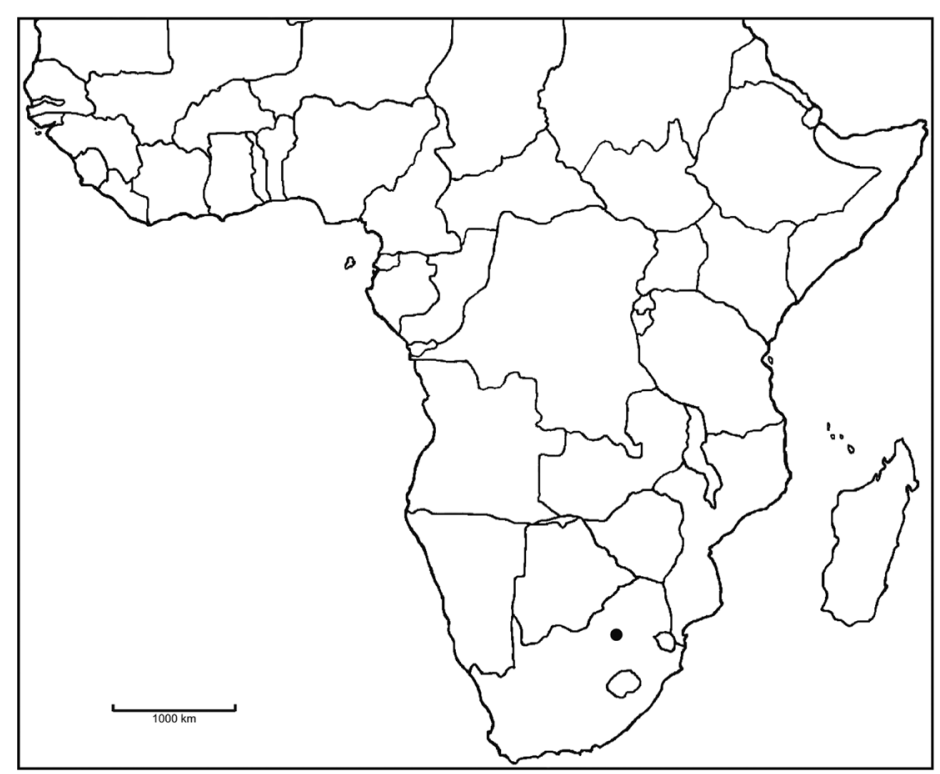

Fig. 228. Distribution of Bilabrella luegiana Szlach. \& Kras

linear-lanceolate, subacute to acute; posterior lobe $6 \mathrm{~mm}$ long, $0.5 \mathrm{~mm}$ wide, linear, obtuse, falcate. Lateral sepals $7.5 \mathrm{~mm}$ long, $4.1 \mathrm{~mm}$ wide, elliptic-obovate, cochleate, strongly deflexed, apiculus ca. $0.5 \mathrm{~mm}$ long, laterally placed. Lip 3-lobed nearly the base, glabrous; the middle lobe $10 \mathrm{~mm}$ long, $0.5 \mathrm{~mm}$ wide, linear; lateral lobes $9 \mathrm{~mm}$ long, $0.6 \mathrm{~mm}$ wide, linear, acute, slightly upcurved. Spur $12 \mathrm{~mm}$ long, narrowly cylindrical, sigmoid in the middle, slightly swollen in apical third, blunt. Anther $3 \mathrm{~mm}$ tall, ovoid-ellipsoid; connective rounded; antherophores $5 \mathrm{~mm}$ long, gently upcurved. Stigmaphores $5.5 \mathrm{~mm}$ long, clavate with rather massive truncate apices. Rostellum middle lobe half as long as thecae. Aurticles small, bifid (Figs 226227).

E c o logy. No records. February.

Distribution. Republic of South Africa, alt. $400 \mathrm{~m}$ (Fig. 228).

Specimens examined. Republic of South Africa. Kwazulu-Natal: Alexandra Distr., Station Dumisa, 400 m, 2 Feb. 1908 (fl), Rudatis 240 (E!, $\mathrm{K}$ !).

$\mathrm{N}$ o t e s. This species diffres from Bilabrella involuta and $B$. riparia in anterior petal lobes which are twice longer than posterior lobes, linear-filiform. B. luegiana is similar to $B$. kyimbilae but differs by pendent, not geniculate spur.

83. Bilabrella riparia (Renz \& Grosvenor) Szlach. \& Kras-Lap., Richardiana 3(3): 142. 2003. Habenaria riparia Renz \& Grosvenor, Candollea 34: 362. 1979. la Croix et al., Orchid. Malawi: 77. 1991. - la Croix \& Cribb, Fl. Zambes., Orchid. 11: 103. 1995.

Type: Malawi, Renz \& Grosvenor 11801 (holotype, SRGH not seen; isotype, $\mathrm{K}$ !). 

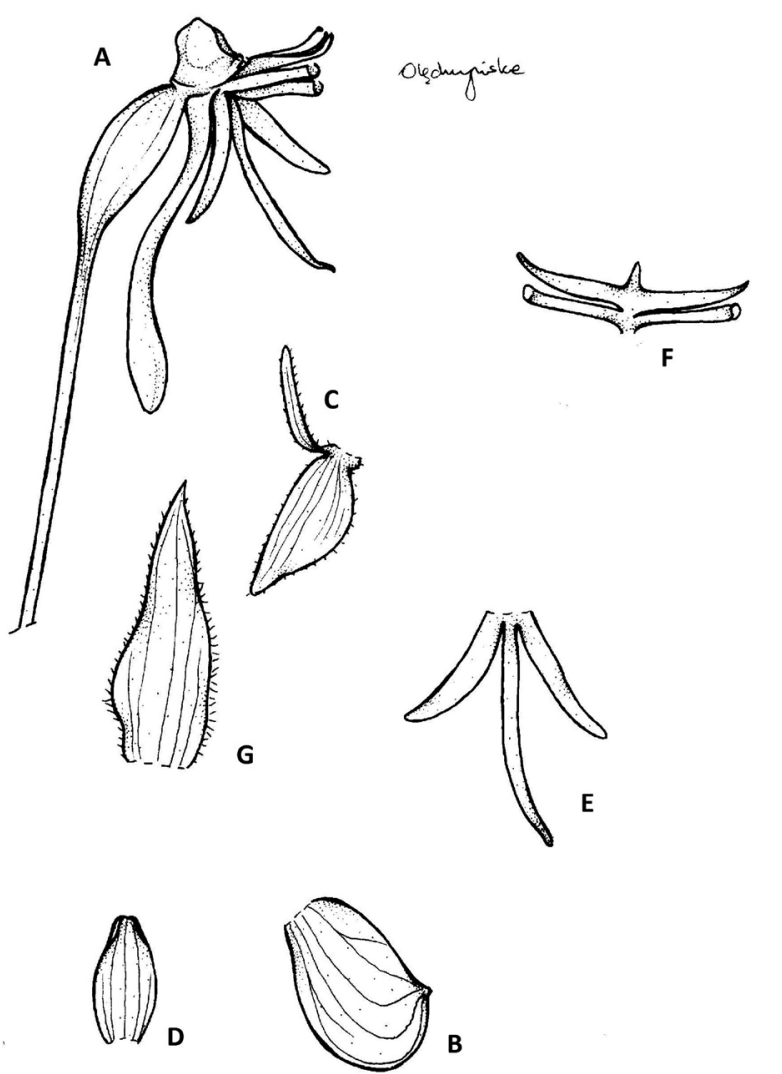

Fig. 229. Bilabrella riparia (Renz \& Grosvenor) Szlach. \& KrasLap.

Explanations: A - pedicel, ovary, gynostemium, lip and spur; B - lateral sepal; C - petal; D - dorsal sepal; E - lip; F - rostellum and stigmaphores, spread; G - floral bract (redrawn by N. Olędrzyńska from Renz \& Grosvenor 1979

Tubers small, ellipsoid. Stem up to $80 \mathrm{~cm}$ tall, slender, erect, leafy, glabrous. Leaves 7-10, up to $20 \mathrm{~cm}$ long, 1-2 cm wide, the lowermost reduced to a sheath, succeeded by two erect, well developed linear or nar- rowly lanceolate, acute, long-sheathed leaves, those above decreasing immediately in size and becoming bract-like. Inflorescence $8-16 \mathrm{~cm}$ long, cylindric, fairly laxly or fairly densely several- to many-flowered. Flowers green, becoming whitish towards the centre. Flower bracts to $20 \mathrm{~mm}$ long, green, ovate-lanceolate or lanceolate, acuminate, margins ciliate. Pedicel 20$25 \mathrm{~mm}$ long. Ovary 7-9 mm long, papillose, slighty curved outwards. Dorsal sepal about $7 \mathrm{~mm}$ long and $3 \mathrm{~mm}$ wide, ovate-elliptic, obtuse, concave, apex somewhat hooded, reflexed. Petals bilobed nearly to the base; anterior lobe 7.5-9 $\mathrm{mm}$ long, 3.5-4 $\mathrm{mm}$ wide, spreading, obliquely ovate-lanceolate to semi-ovate, slightly narrowed at base, margins minutely crenate, minutely papillose-pubescent above, mainly towards the base; posterior lobe $6 \mathrm{~mm}$ long, 0.4-0.5 $\mathrm{mm}$ wide, erect or recurved, linear, papillose-pubescent above with ciliolate margins. Lateral sepals $10 \mathrm{~mm}$ long and $6 \mathrm{~mm}$ wide, obliquely obovate to elliptic-obovate, deflexed, with a lateral apiculus. Lip 3-lobed to the base; the middle lobe 12-14 mm long, $1 \mathrm{~mm}$ wide, linear, acute, often curved; lateral lobes 6-7 mm long, 1.5-2 mm wide, spreading forwards, sickle-shaped, lanceolate-linear, subacute. Spur 16-19 mm long, cylindrical, sigmoid, hanging down, swollen in apical half. Anther $3 \mathrm{~mm}$ tall; connective obtuse; antherophores $7 \mathrm{~mm}$ long, slender, apically abruptly upcurved. Stigmaphores $6 \mathrm{~mm}$ long, narrowly cylindrical, truncate, straight. Rostellum middle lobe shorter than thecae. Auriculae bilobed (Figs 229-230).

E c ology. Wet grass near stream, grassland. February-March.

D istribution. Malawi, alt. 2150-2250 m (Fig. 231).
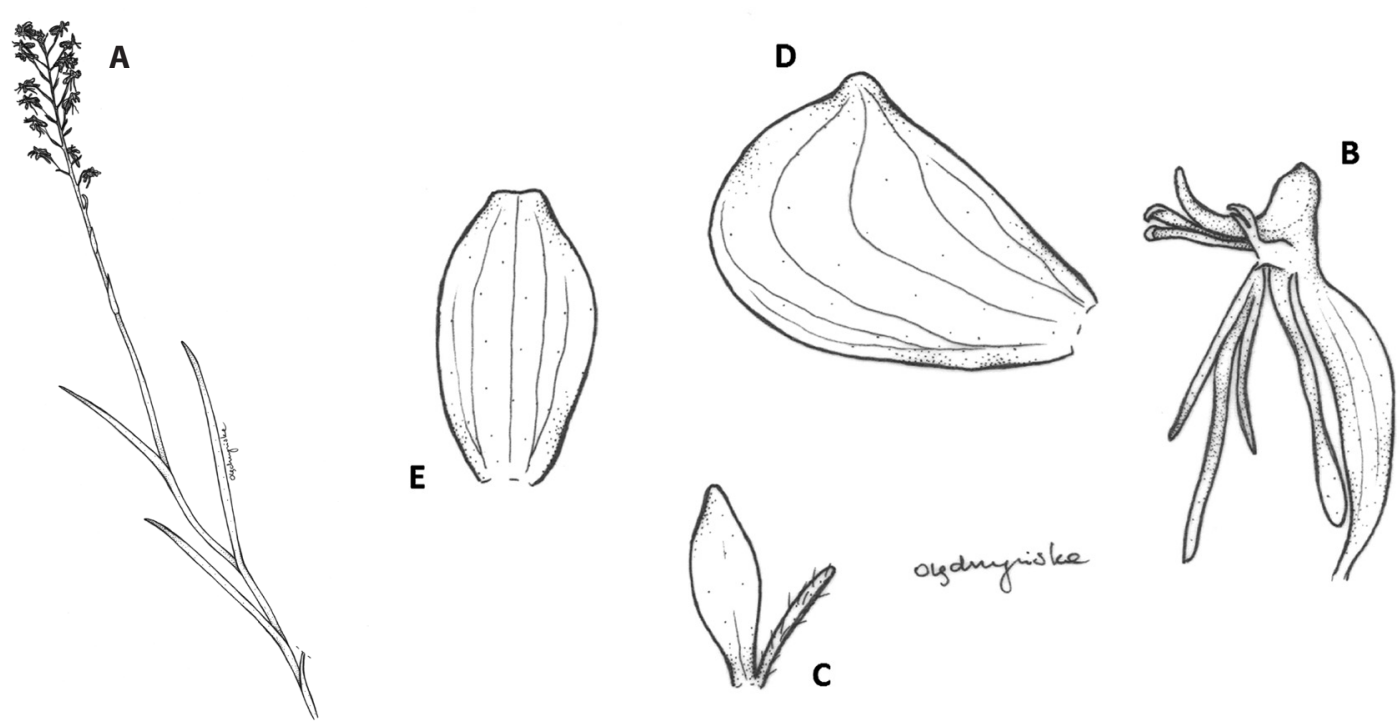

Fig. 230. Bilabrella riparia (Renz \& Grosvenor) Szlach. \& Kras-Lap.

Explanations: A - habit; B - pedicel, ovary, gynostemium, spur and lip; C - petal; D - lateral sepal; E - dorsal sepal (drawn by N. Olędrzyńska from Richards $12318, \mathrm{~K})$ 


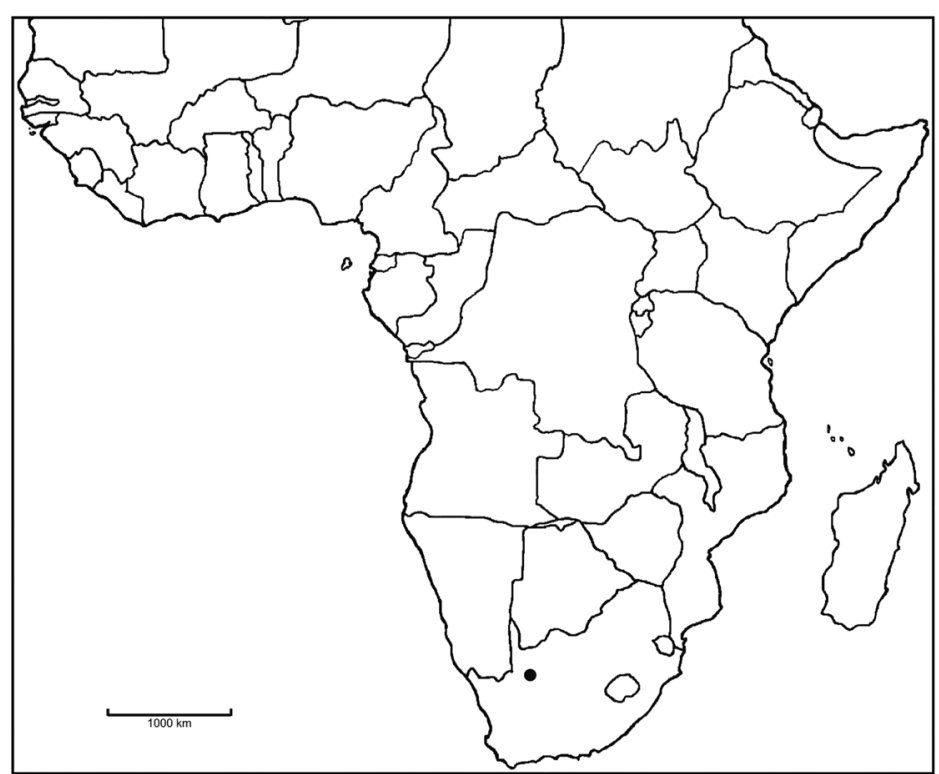

Fig. 231. Distribution of Bilabrella riparia (Renz \& Grosvenor) Szlach. \& Kras-Lap.

Specimens examined. Malawi. Northern: Nyika N Plateau, Dambo Bridge, in grassland stream, 2250 m, 13 Feb. 1987 (fl), la Croix 965 (K!); Rumphi Distr., Nyika-Plateau, Dembo Bridge, $6 \mathrm{~km}$ E of Chelinda Camp, in swampy grassland near stream, $2150 \mathrm{~m}$, Mar. 1977 (fl), Grosvenor \& Renz 11801 (K!).

Note s. The species is similar to Bilabrella welwitschii, from which it differs by the larger flowers and the lateral lobes of the lip, which are falcately lanceolate-linear and not obliquely obovate. Also the petals - especially the anterior lobe - are $+/$ - papillose-pubescent, whereas the petals of $B$. welwitschii are glabrous. B. riparia is also allied in habit to $B$. weberana, however the latter has a lip with a distinct undivided base, which is $2-3 \mathrm{~mm}$ long, and narrowly curved-lanceolate anterior petal lobes, which are about twice as long as the posterior lobes (Renz \& Grosvenor 1979).

Close to the East African B. thomsonii, but differing in the longer spur, longer pedicel and ovary, and slightly narrower leaves (la Croix \& Cribb 1995).

84. Bilabrella tangheana (Geerinck \& Schaijes) Szlach. \& Kras, Richardiana 9(4): 159. 2009.

Habenaria tangheana Geerinck \& Schaijes, Bull. Jard. Bot. Belg. 57(34): 482. 1987.

Type: Congo, Schaijes 1879 (holotype, BR!).

Stem $40-50 \mathrm{~cm}$ tall, erect, slender, leafy. Leaves 8 , $7-10 \mathrm{~cm}$ long, $0.15-0.8 \mathrm{~cm}$ wide, narrowly linear, long acuminate, black glandular, decreasing in size upwards. Inflorescence 14-15 cm long, laxly 15-17-flowered. Flowers green. Floral bracts $20 \mathrm{~mm}$ long, oblonglanceolate, acuminate. Pedicel up to $17 \mathrm{~mm}$ long. Ovary up to $10 \mathrm{~mm}$ long. Dorsal sepal 5-6 $\mathrm{mm}$ long, $1.5-2 \mathrm{~mm}$

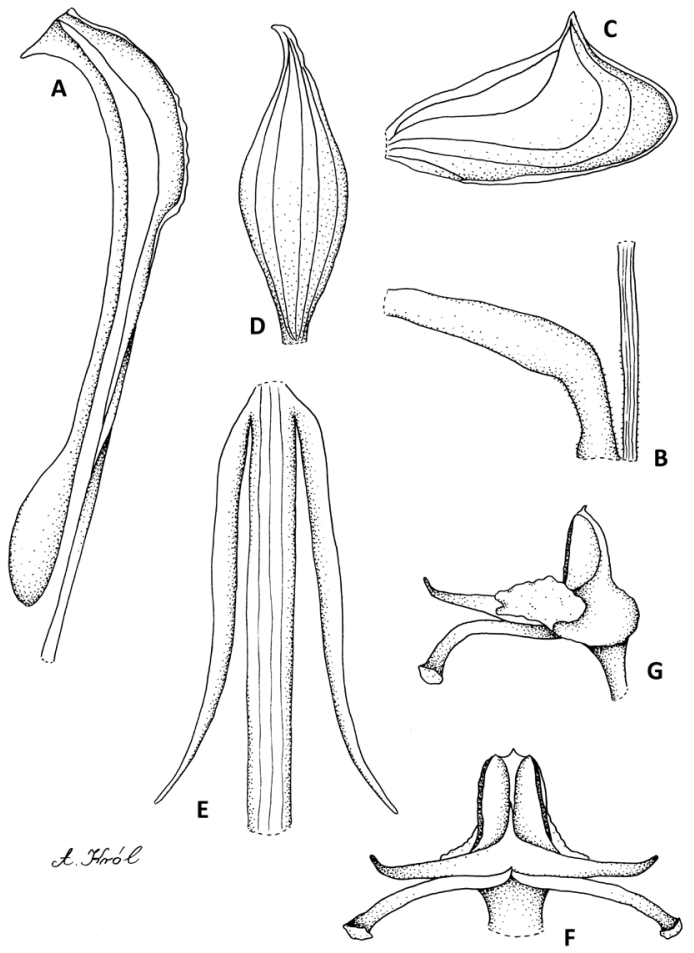

Fig. 232. Bilabrella tangheana (Geerinck \& Schaijes) Szlach. \& Kras

Explanations: A - ovary, pedicel, spur; B - petal; C - lateral sepal; D - dorsal sepal; E - lip; F - gynostemium, front view; $\mathrm{G}$ - gynostemium, side view (drawn by A. Król from holotype of Habenaria tangheana Geerinck \& Schaijes - Schaijes 1879, BR)

wide, elliptic to ovate-elliptic, obtuse to subacute, subcarinate. Petals bilobed to the base; anterior lobe $12 \mathrm{~mm}$ long, $1 \mathrm{~mm}$ wide, linear-lanceolate, acute, minutely papillose, especially in the lower part; posterior lobe $5 \mathrm{~mm}$ long, 0.3-0.5 $\mathrm{mm}$ wide, filiform, acute, glabrous. Lateral sepals 7-8 mm long, 4-5 mm wide, obliquely

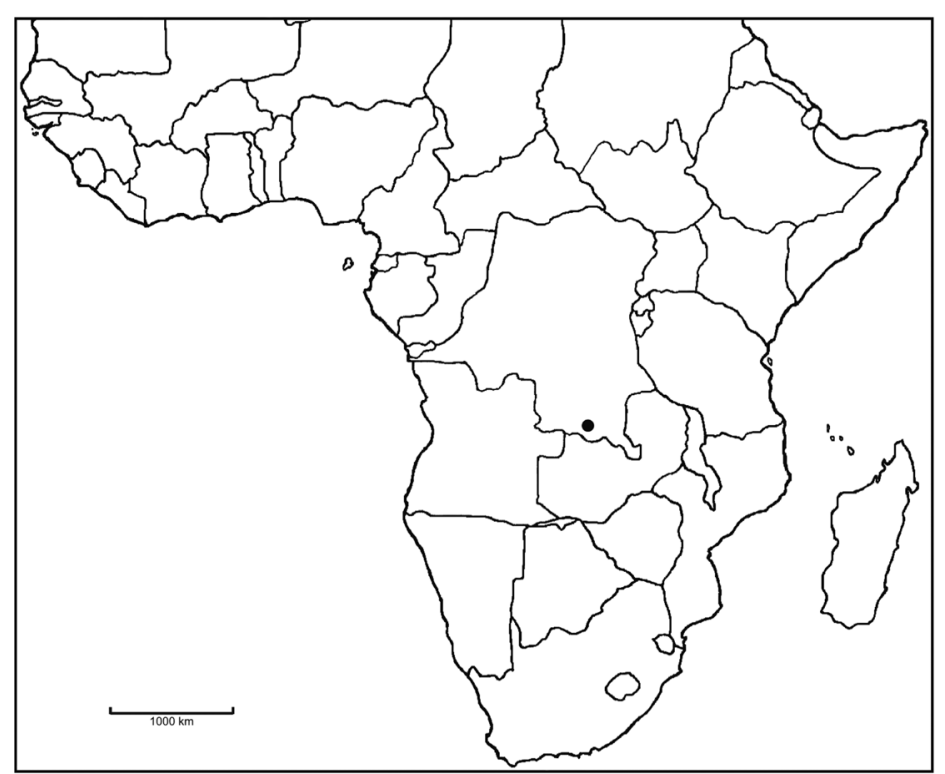

Fig. 233. Distribution of Bilabrella tangheana (Geerinck \& Schaijes) Szlach. \& Kras 
subelliptic to oblong-obovate, laterally apiculate. Lip $20 \mathrm{~mm}$ long, 3-lobed almost to the base; the middle lobe $15-17 \mathrm{~mm}$ long, $1 \mathrm{~mm}$ wide, linear; lateral lobes 9-10 mm long, $0.5 \mathrm{~mm}$ wide, linear-lanceolate, attenuate towards the apex, upcurved apically. Spur up to $22 \mathrm{~mm}$ long, narrowly cylindrical, swollen in the apical quarter, blunt, parallel to the ovary. Anther $2 \mathrm{~mm}$ tall, ellipsoid; connective truncate, shortly apiculate; antherophores $3.5 \mathrm{~mm}$ long, slender, straight, apically slightly upturned. Stigmaphores 3.5-4 mm long, narrowly cylindrical, truncate, bent down. Rostellum middle lobe half as long as thecae. Auriculae huge, bifid apically (Fig. 232).

Ec o logy. Grassy savannah. March.

D i stribution. Democratic Republic of the Congo (Zaire), alt. $1400 \mathrm{~m}$ (Fig. 233).

Specimens examined. Democratic Republic of the Congo (Zaire). Haut-Katanga: $42 \mathrm{~km}$ WSW de Kolwezi, savannah steppe, 1400 m, Mar. 1983 (fl), Schaijes 1879 (BR!).

Notes. Bilabrella tangheana is very unique in the genus by its huge auriculae, bifid apically. In this respect it reminds somewhat $B$. stanislawii, but has longer pedicel and ovary ( $27 \mathrm{~mm}$ vs $13.5 \mathrm{~mm}$ ), lip middle lobe (15-17 $\mathrm{mm}$ vs $9 \mathrm{~mm}$ ) and spur (22 $\mathrm{mm}$ vs $14 \mathrm{~mm})$.

85. Bilabrella stanislawii Kras \& Szlach., Polish. Bot. Journ. 53(2): 99. 2008.

Type: Democratic Republic of the Congo (Zaire), Lisowski 11147 (holotype, UGDA-DLSz!).

Stem $21 \mathrm{~cm}$ tall, glabrous, erect, slender, leafy throughout its length. Leaves 8 , the 4 lowermost reduced to a sheath, lanceolate-linear, acute, the largest $7.9 \mathrm{~cm}$ long and $0.4 \mathrm{~cm}$ wide, the upper leaves much smaller, adpressed to the stem, lanceolate, acuminate, similar to the lower bracts. Inflorescence $6.8 \mathrm{~cm}$ long, laxly 12-flowered. Floral bracts 8-9 mm long, lanceolate, acuminate. Pedicel with ovary $13.5 \mathrm{~mm}$ long. Dorsal sepal $3.9 \mathrm{~mm}$ long and $1.2 \mathrm{~mm}$ wide, oblong-elliptic, shortly apiculate, truncate, concave. Petals bilobed nearly to the base; anterior lobe 7-9 $\mathrm{mm}$ long, $0.7 \mathrm{~mm}$ wide, linearto oblong-lanceolate, glabrous; posterior lobe $4 \mathrm{~mm}$ long, $0.4 \mathrm{~mm}$ wide, reflexed, linear-filiform, ciliolate. Lateral sepals $6 \mathrm{~mm}$ long and $4 \mathrm{~mm}$ wide, obliquely oblong-obovate, concave, with laterally placed apiculus $0.5 \mathrm{~mm}$ long, deflexed. Lip 3-lobed nearly to the base, the middle lobe shorter than laterals; the middle lobe $9 \mathrm{~mm}$ long, $0.5 \mathrm{~mm}$ wide, linear, obtuse; lateral lobes $10.5 \mathrm{~mm}$ long, $0.5 \mathrm{~mm}$ wide, linear, subacute. Spur $14 \mathrm{~mm}$ long, cylindrical, slightly swollen at the base and in the apical third, subacute, pendent. Anther just over $3 \mathrm{~mm}$ tall, ellipsoid-ovoid; connective rounded, shortly apiculate; antherophores $4.5 \mathrm{~mm}$ long, rather slender, abruptly upcurved apically. Stigmaphores $5 \mathrm{~mm}$ long,
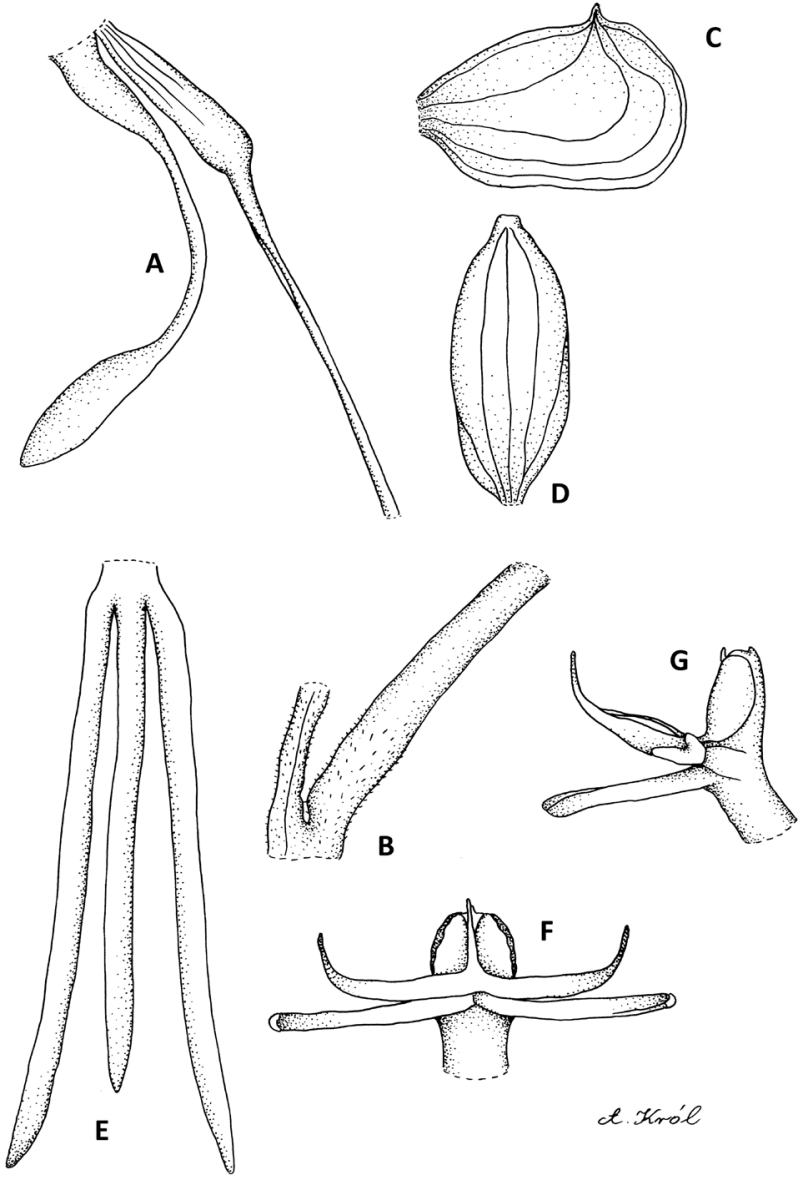

Fig. 234. Bilabrella stanislawii Kras \& Szlach. Explanations: A - ovary, pedicel, spur; B - petal; C - lateral sepal; D - dorsal sepal; E - lip; F - gynostemium, front view; $\mathrm{G}$ - gynostemium, side view (drawn by A. Król from holotype - Lisowski 11147, UGDA-DLSz)

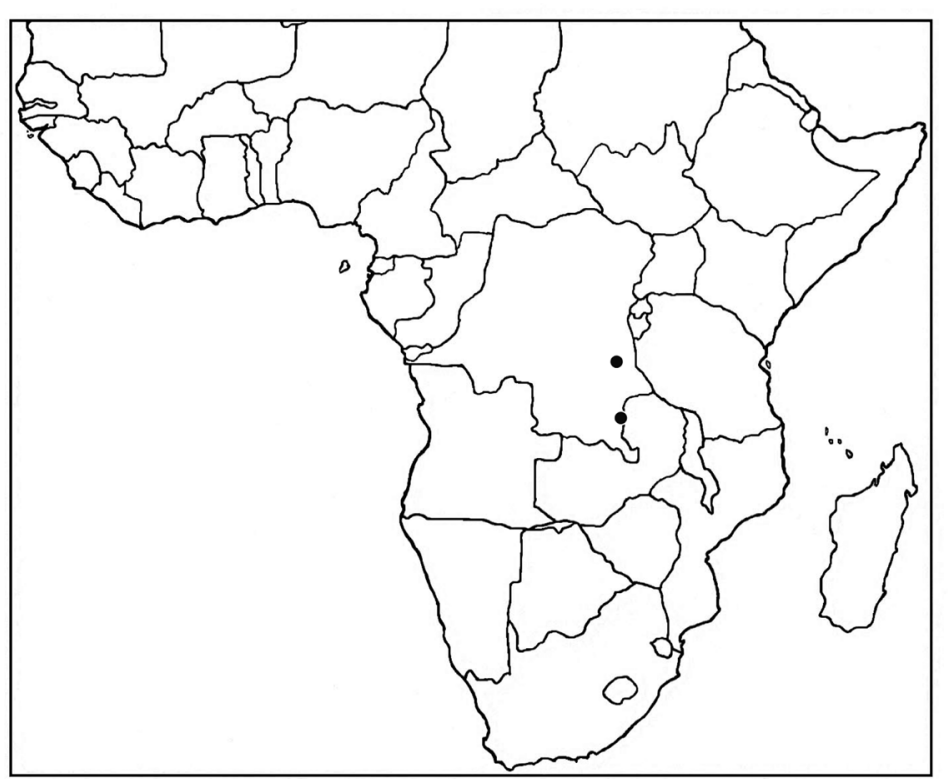

Fig. 235. Distribution of Bilabrella stanislawii Szlach. \& Kras

narrowly cylindrical, straight. Rostellum middle lobe longer than connective, subulate. Auriculea unequally bilobed, rather massive (Fig. 234).

E c o lo gy. Damp grassland. January-April. 
D is tribution. Democratic Republic of the Congo (Zaire), alt. $1650 \mathrm{~m}$ (Fig. 235).

Specimens examined. Democratic Republic of the Congo (Zaire). Haut-Katanga: Shaba Highland, the Kundelungu Plateau, 10,9 km of NNW of Lualala Poste, near the Munva stream, $1650 \mathrm{~m}$, 21 Apr. 1971 (fl), Lisowski 11147 (UGDA-DLSz!); The Kundelungu Plateau, source of the Lofoi, dump grassland, 1650 m, 10 Jan. 1971 (fl), Lisowski 65758 (UGDA-DLSz!).

N o te s. Bilabrella stanislawii differs from B. tanghea$n a$ by series of morphological characters, as lip, spur and pedicellate ovary length.

86. Bilabrella cribbiana (Szlach. \& Olsz.) Szlach. \& Kras-Lap., Richardiana 3(3): 140. 2003. - Szlachetko et al., Orchid. of West-Central Africa 1: 231. 2010.

Habenaria cribbiana Szlach. \& Olsz., Fl. Cam., Orchid. 34(1): 184. 1998.

Type: Congo, Lissouba 21 (holotype, P!).

Plant $32 \mathrm{~cm}$ tall, rather erect, delicate, glabrous. Leaves 7, up to $10 \mathrm{~cm}$ long, $0.6 \mathrm{~cm}$ wide, linear, acute, spread, the upper one sheath-like, second the longest, the upper decreasing in size up the stem. Inflorescence $4 \mathrm{~cm}$ long, 6-flowered. Floral bracts $10 \mathrm{~mm}$ long, hairy on both sides. Pedicel and ovary up to $20 \mathrm{~mm}$ long, glabrous. Dorsal sepal $6 \mathrm{~mm}$ long, $2 \mathrm{~mm}$ wide, oblongelliptic, obtuse, concave, glabrous. Petals bilobed to the base, glabrous, thicker than sepals; anterior lobe $7.5 \mathrm{~mm}$ long, $1.7 \mathrm{~mm}$ wide, obliquely lanceolate, subulate at the apex; posterior lobe $6 \mathrm{~mm}$ long, $0.4 \mathrm{~mm}$ wide, filiform, obtuse. Lateral sepals $8.5 \mathrm{~mm}$ long, $4 \mathrm{~mm}$ wide, oblong-

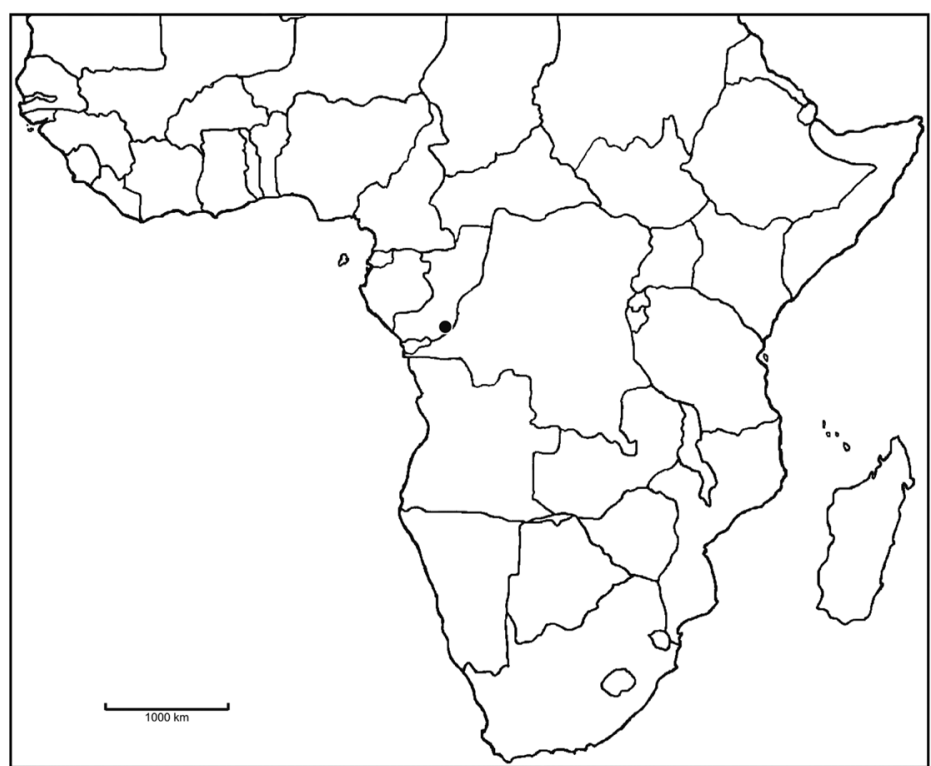

Fig. 237. Distribution of Bilabrella cribbiana (Szlach. \& Olsz.) Szlach. \& Kras-Lap.

obovate to elliptic-obovate, cochleate at the apex, with short, erect apiculus placed near near the middle of upper margin. Lip 3-lobed nearly to the base, glabrous; the middle lobe $11-12 \mathrm{~mm}$ long, $0.6 \mathrm{~mm}$ wide, linear or filiform, acute; lateral lobes $8.5 \mathrm{~mm}$ long, $0.5 \mathrm{~mm}$ wide, narrowly lanceolate, at the base, filiform above, acute, upcurved. Spur 17-18 mm long, $2 \mathrm{~mm}$ in diameter at the apex, narrowly cylindrical, distinctly swollen in the apical quarter or fifth, blunt. Anther $2.5 \mathrm{~mm}$ long; connective rounded; antherophores $7 \mathrm{~mm}$ long, slender, apically upcurved. Stigmaphores 5.5-6 mm long, very slender, slightly upcurved. Rostellum middle lobe

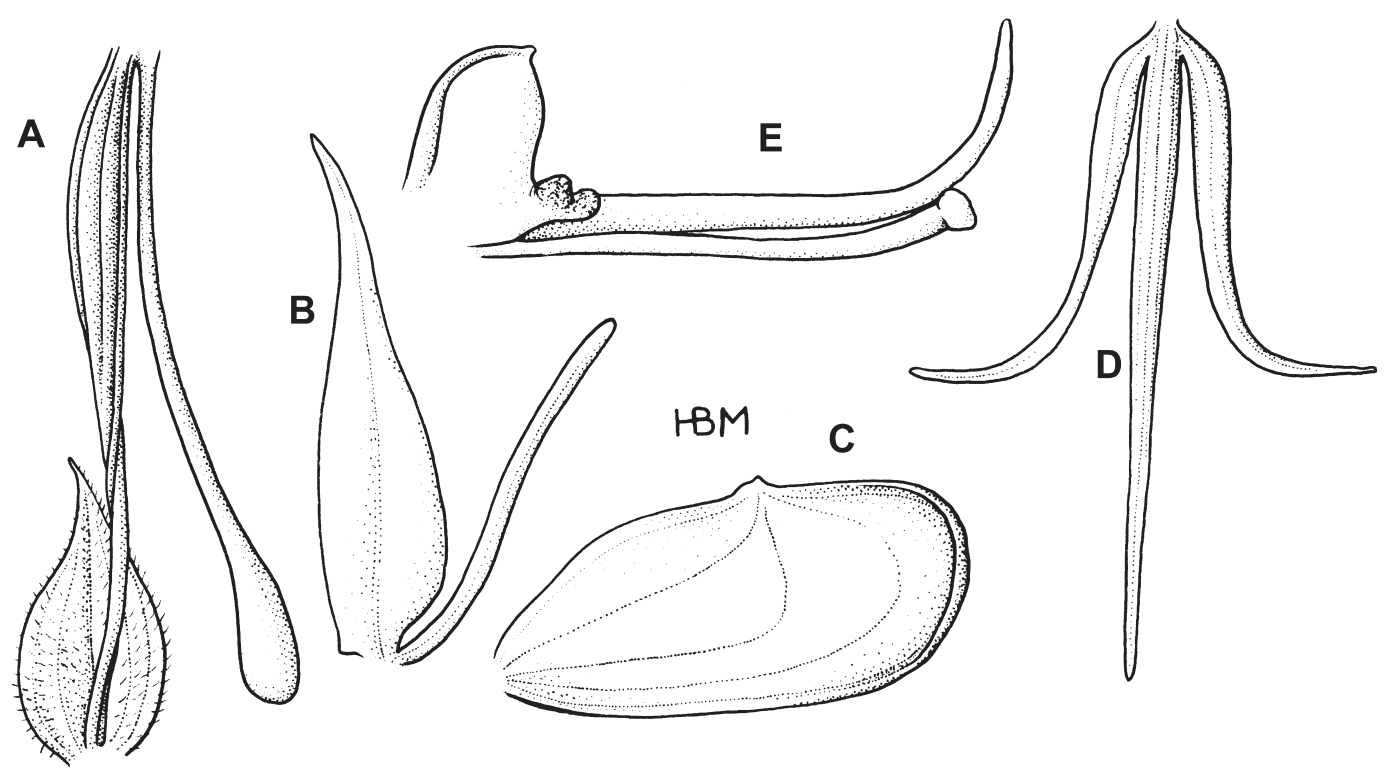

Fig. 236. Bilabrella cribbiana (Szlach. \& Olsz.) Szlach. \& Kras-Lap.

Explanations: A - ovary, pedicel, spur and bract; B - petal; C - lateral sepal; D - lip; E - gynostemium, side view (drawn by H. B. Margońska from holotype of Habenaria cribbiana Szlach. \& Olsz. - Lissouba 21, P) 
linear-triangular, acute, shorter than thecae. Auriculae shallowly bilobed, rather massive (Fig. 236).

E c o log y. On floatted vegetation. Flowers in August. D i s tributio n. Known from Congo only (Fig. 237). Specimens examined. Congo. Pool: Lifoula, Gamakala pond, 12 Aug. 1966 (fl), Lissouba 21 (P!). Notes. At the first glance it is similar to Bilabrella huillensis, but the spur of $B$. cribbiana is swollen near the apex, floral bract equals in length to pedicel and anterior petal lobe is lanceolate, ca. 4 times longer than wide. The other similar species appears to be $B$. cornutella. B. cribbiana has spur as long as pedicel and ovary, anterior petals lobe 4 times longer than wide, and auriculae bifid in the apical half, whereas $B$. cornutella - spur shorter than pedicel and ovary, anterior petals lobe 8 times longer than wide, and globose auriculae.

87. Bilabrella haareri (Summerh.) Szlach. \& KrasLap., Richardiana 3(3): 140. 2003.

Habenaria haareri Summerh., Kew Bull. 16: 281.1962.

- Summerhayes, FTEA, Orchid. 1: 93. 1968b.

Type: Tanganyika, Haarer 233 (holotype, K!; isotype, EA not seen).

Habenaria pedicellaris Kraenzl., Engl. Pfl. Ost-Afr. C.: 152. 1895, non Rchb. f., 1881.

Type: Tanzania, Schimper 1369c (B+).

Tubers $1 \mathrm{~cm}$ in diameter, globose, densely tomentose. Stem $30-50 \mathrm{~cm}$ tall, glabrous, erect, rather slender, leafy throughout its length. Leaves 8 , the lowermost sometimes reduced to a sheath, the lower 4 or 5 suberect or almost erect, lanceolate-linear, acute, the largest 10-14 $\mathrm{cm}$ long and $1 \mathrm{~cm}$ wide, the upper leaves much smaller, adpressed to the stem, lanceolate, acuminate, similar to the lower bracts. Inflorescence $9-16 \mathrm{~cm}$ long, laxly to rather densely 12-24-flowered. Floral bracts $15-25 \mathrm{~mm}$ long, lanceolate, acuminate, thin in texture, the lower ones longer than the pedicel with ovary. Pedicel with ovary $20 \mathrm{~mm}$ long. Dorsal sepal 6-7 mm long, $2.5 \mathrm{~mm}$ wide, narrowly elliptic, obtuse, convex, reflexed. Petals bilobed nearly to the base; anterior lobe 9-10 mm long, just over $1 \mathrm{~mm}$ wide, spreading forwards, oblong lanceolate to linear-lanceolate, curved, acute, glabrous; posterior lobe 5-6 mm long, $0.5 \mathrm{~mm}$ wide, reflexed, linear, ciliolate. Lateral sepals $8 \mathrm{~mm}$ long and 4-5 mm wide, deflexed, obliquely oblong-obovate, apiculus subapically placed. Lip deflexed, 3-lobed nearly to the base; the middle lobe $10-12 \mathrm{~mm}$ long, $0.5 \mathrm{~mm}$ wide, linear, obtuse; lateral lobes $6.5-8.5 \mathrm{~mm}$ long, $0.5 \mathrm{~mm}$ wide, lanceolate-linear, acute. Spur $20 \mathrm{~mm}$ long, parallel to the ovary, pendent, swollen in the apical third, subobtuse. Anther $2.5 \mathrm{~mm}$ tall, conical-ovoid; connective obtuse, shortly apiculate; antherophores $3 \mathrm{~mm}$ long, slender, gently upcurved. Stigmaphores $4.5 \mathrm{~mm}$ long, rather massive, cylindrical, truncate at

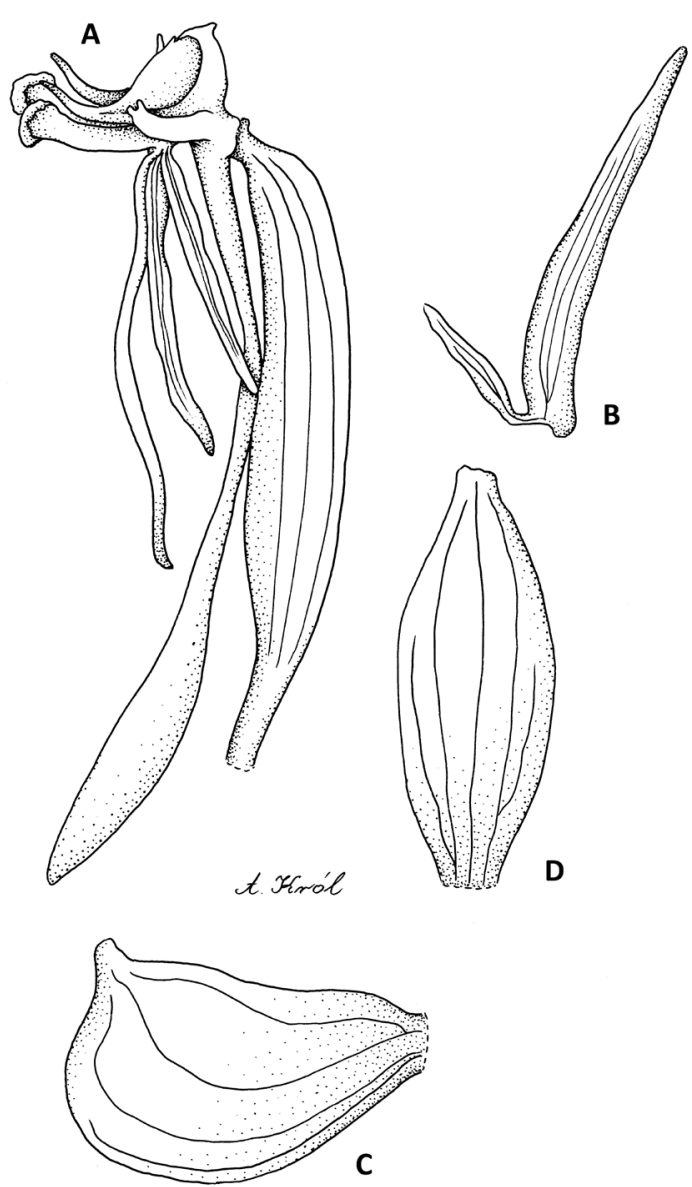

Fig. 238. Bilabrella haareri (Summerh.) Szlach. \& Kras-Lap. Explanations: A - pedicel, ovary, gynostemium, lip and spur; B - petal; C lateral sepal; D - dorsal sepal (drawn by A. Król from holotype of Habenaria haareri Summerh. - Haarer 233, K)

the apex, glandular. Rostellum middle lobe as long as connective. Auriculae small, unequally bilobed (Fig. 238).

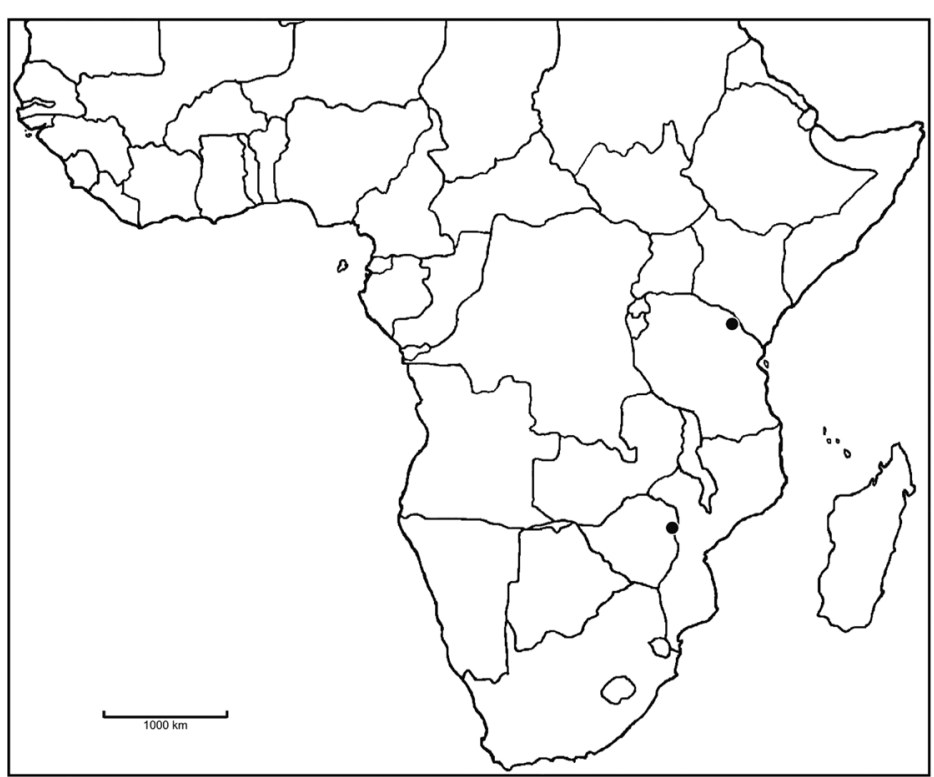

Fig. 239. Distribution of Bilabrella haareri (Summerh.) Szlach. \& Kras-Lap. 
E c o logy. In vlei. February-June.

D is tribution. Tanzania, Zimbabwe, alt. $1200 \mathrm{~m}$ (Fig. 239).

Specimens examined. Tanzania: Kilimanjaro: Kilimanjaro, E side, Mashati, Haarer 233 (K!); East Kilimanjaro, Keni, 1200 m, June 1927 (fl), Haarer $551 B$ (K!). Zimbabwe. Manicaland: Makonis Distr., Mkovi Garge Farm, near vlei, 16 Feb. 1976 (fl), Lancaster in Best 1281 (K!).

N o te s. Bilabrella haareri is similar in gynostemium morphology to B. leucoceras. In the former however, the spur is pendent, not twisted, whereas in the latter it is evidently twisted in the apical quarter. Also anterior petal lobe is different in both of the species. It can be distinguished from $B$. petraea by having pendent spur (vs horizontal) and glabrous anterior petal lobe and ciliolate posterior one (vs densely pubescent both petal lobes). Summerhayes (1962) describing Habenaria haareri compared it to $H$. isoantha, despite clear differences in the gynostemium structure, lateral sepals morphology and ciliation of petal lobes.

88. Bilabrella petraea (Renz \& Grosvenor) Szlach. \& Kras-Lap., Richardiana 3(3): 142. 2003.

Habenaria petraea Renz \& Grosvenor, Candollea 34(2): 358. 1979. - la Croix et al., Orchid. Malawi: 77. 1991. - la Croix \& Cribb, Fl. Zambes., Orchid. 11: 102. 1995.

Type: Malawi, Grosvenor \& Renz 1104 (holotype, SRGH not seen; isotype, $\mathrm{K}$ !).

Tubers small, ovoid. Stem $20-70 \mathrm{~cm}$ tall, erect, delicate. Leaves 5-9, 6-14 cm long, 0.5-2 cm wide, the lowermost 1-2 sheathing, spotted with black, the next few leaves semi-spreading, linear-lanceolate, folded, the uppermost grading into the bracts. Inflorescence $5-26 \mathrm{~cm}$ long, laxly or fairly densely several- to manyflowered. Flowers pale green, green, or green with the anterior petal lobes white. Flower bracts 10-22 mm long, lanceolate, acuminate, finely pubescent or glandular. Pedicel 16-20 mm long, semi-erect, ovary $8-10 \mathrm{~mm}$ long, horizontal, all becoming straight and semi-erect in fruit. Dorsal sepal 5.5-8 mm long, 2-3 mm wide, oblong elliptic to elliptic-obovate, obtuse, somewhat fleshy at apex, concave, reflexed. Petals densely pubescent, 2-lobed almost to the base; anterior lobes $9-13 \mathrm{~mm}$ long, $1.5-2 \mathrm{~mm}$ wide, linear-lanceolate to oblongoblanceolate, acute to subobtuse, spreading, hinged; posterior lobe 5-7 $\mathrm{mm}$ long, 0.6-0.8 $\mathrm{mm}$ wide, linear, acute to subobtuse, erect or recurved. Lateral sepals 8.3-10 mm long, 4-6 $\mathrm{mm}$ wide, obliquely obovate or oblong obovate, with subapical apiculus, deflexed. Lip deflexed, 3-lobed almost to the base; the middle lobe 12-15 mm long, 0.4-0.6 mm wide, flexuous, linear, obtuse; lateral lobes 8-10 mm long, 0.6-0.8 mm wide, linear-lanceolate, acute, slighty upcurved. Spur (12.5)

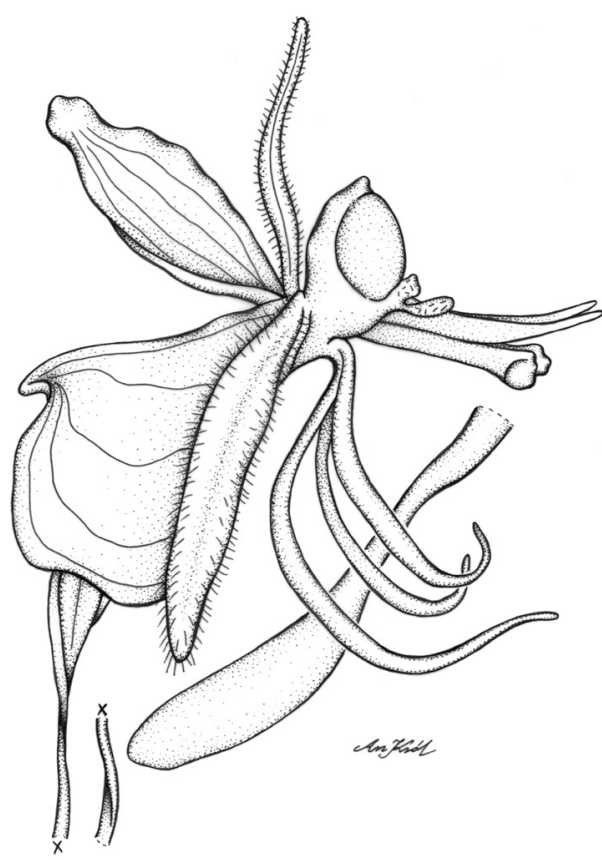

Fig. 240. Bilabrella petraea (Renz \& Grosvenor) Szlach. \& KrasLap. - flower (drawn by A. Król from Milne-Redhaed \& Taylor 10426a, AMES)

15-20 mm long, swollen towards apex, twisted in the middle, horizontal or upcurved so that the top is higher than the ovary, blunt. Anther 3.5-4 mm tall; connective rounded; antherophores 4-5 mm long, slender, apically upturned. Stigmatophores 3.6-5 mm long, (usually slightly shorter than anther canals), narrowly cylindrical, truncate. Rostellum middle lobe shorter than thecae. Auriculae unequally bilobed, rather large (Fig. 240). E c o logy. Grassland, margins of evergreen forest, granite. February-July.

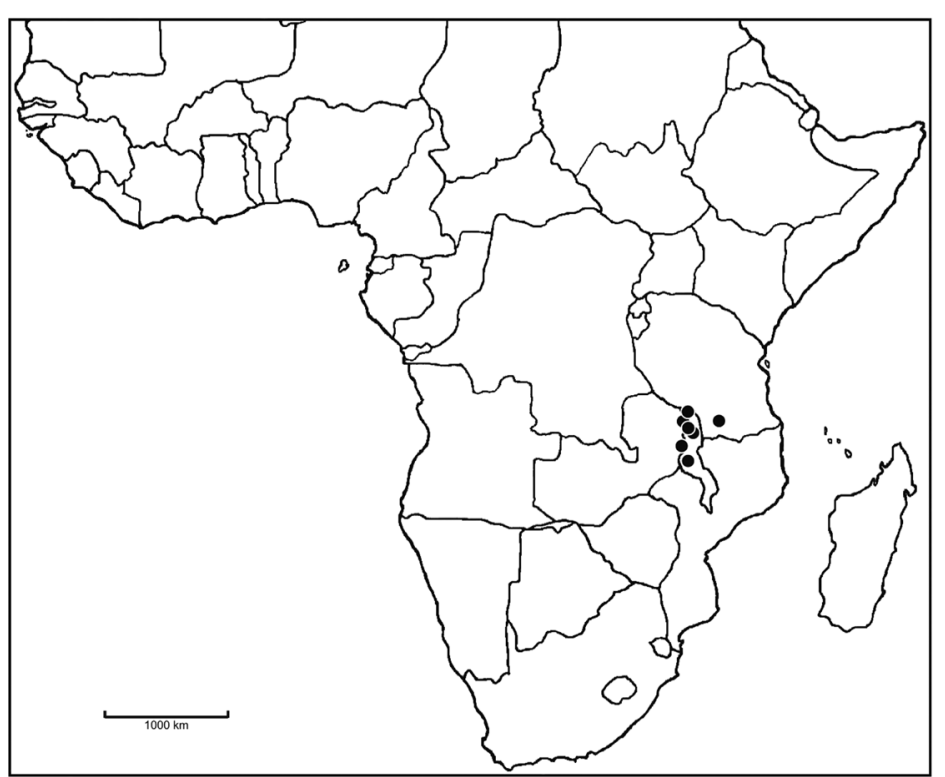

Fig. 241. Distribution of Bilabrella petraea (Renz \& Grosvenor) Szlach. \& Kras-Lap. 
D istribution. Tanzania, Zambia, Malawi, alt. 750-2400 m (Fig. 241).

Specimens examined. Tanzania. Ruvuma: Songea Distr., Matengo Hills, Luwiri Kitesa, in upland grassland above the forest, 1890 m, 24 May 1956 (fl), Milne-Redhead \& Taylor 10426A (AMES!, K!). Zambia. Eastern: Lundazi Distr., Nyika Plateau, Apr. 1969 (fl), Williamson 946 (K!); Northern: Nyika N Plateau, Chowo forest margin, monte grassland, 1950-2100 m, 5 Apr. 1983 (fl), Elias 20 (K!); Nyika, on Chowo rock, Mar. 1967 (fl), Williamson \& Odgers 273 (K!). Malawi. Central: Around Chilindira, Dam 1, monte grassland, 2250 m, 7 Mar. 1983 (fl), Elias 13 (K!); Northern: Chitipa Distr., Misuku Hills, Mugesse Forest, margins of evergreen (Entandrophragma etc.) forest, 1800 m, 14 July 1970 (fl), Brummitt 1210 (K!); Chilinda Plantation, grassland under pines, $2250 \mathrm{~m}$, 12 Mar. 1983 (fl), Elias 17 (K!); Mzuzu, path from Tech. Tolunyanga Research Stn., in grass near edge of forest, 1300 m, 9 May 1986 (fl), la Croix 839 (K!); Nyika, granite outcrops on Nyika, Feb. 1968 (fl), Williamson 832 (K!); Nyika N Plateau, dambo bridge, rock turf, 19 Feb. 1983 (fl), Elias 8 (K!); Dam 2, Nyika National Park, 750 m, 12 Mar. 1983 (fl), Elias 16 (K!); Nyika Plateau, Mt. Chosi, Grosvenor \& Renz 1104 (K!, SRGH not seen); Nyika Plateau, in peat-grit tussocks or humus pocket on granite, $2100 \mathrm{~m}, 11 \mathrm{Feb} .1960$ (fl), Holmes 214 (K!); Nyika National Park, in short grass beside Dam 3, 2100 m, 7 Apr. 1984 (fl), la Croix 595 (K!); Nyika National Park, Chilinda, in grass beside Dam, 2250 m, 7 Apr. 1984 (fl), la Croix 598 (K!); Nyika, damp grassland near Dembo Bridge, 2150 m, 13 Feb. 1987 (fl), la Croix 967 (K!); Nyika Plateau, Chilinda Valley, just below house, grassland, 2250 m, 5 Mar. 1976 (fl), Phillips 1361A (K!); Nyika Plateau, Dam 1-Dam 11, W side of road, grassland, 2265 m, 15 Mar. 1976 (fl), Phillips 1371 (K!); Nyika Plateau, Chilinda Valley $\mathrm{N}$ airfield turn, grassland, $2250 \mathrm{~m}, 16$ Mar. 1976 (fl), Phillips 1461 (K!); Nyika Plateau, near junction Dembo road, grassland, 20 Mar. 1976 (fl), Phillips 1505 (K!); Nyika Plateau, Dam 1, grassland, 2250 m, 3 Apr. 1976 (fl), Phillips 1624 (K!); Rumphi Distr., Nyika Plateau, Kasaramba view, grassland, 2400 m, 17 Apr. 1975 (fl), Pawek 9327 (K!); Rumpi Distr., Nyika, Lake Kaulime, grassland, 2280 m, 27 Mar. 1970 (fl), Pawek 23375A (K!); Rumphi Distr., Nyika Plateau, Chowa Rock, Mar. 1967 (fl), Williamson 273 (K!).

Notes. This species is related to the East African Habenaria altior, differing only in the spur position (la Croix \& Cribb 1995).

The species is also similar to B. kyimbilae, also having densely pubescent petals. It can however easely be distinguished by the narrow leaves, the lax, shorter and wideer inflorescence, the elongated pedicel, the longer, horizontally directed, sligtly sigmoid spur, which is only moderately inflated in the apical part, and the longer stigmatic arms and anther canals. B. kyimbilae has a wide range of distribution in tropical Africa, having been recorded from Zambia, Malawi, Tanzania and Zaire (Summerhayes 1968b). B. petraea is so far only known from the Nyika-Plateau, a high mountain area, which extends from the northeast of Zambia into Malawi as far as to an abrupt escarpment on the western border of Lake Malawi (formerly Lake Nyasa), and to the Mulanje mountains in the south of Malawi.

89. Bilabrella incurva (Rolfe) Szlach. \& Kras, Richardiana 9(4): 158. 2009.

Habenaria incurva Rolfe, Fl. Cap. 5(3): 133. 1912.

Type (hoc loco selectus): Republic of South Africa, Galpin $392 b$ (lectotype, K!; isolectotypes, BOL not seen, PRE not seen).

Stem $15 \mathrm{~cm}$ tall, erect, slender, leafy. Leaves 9 , $5-8 \mathrm{~cm}$ long, to $0.9 \mathrm{~cm}$ wide, gathered at the base of the stem, narrowly lanceolate to linear-lanceolate, long acuminate, decreasing in size upwards. Inflorescence $7 \mathrm{~cm}$ long, laxly 10-flowered. Floral bracts $9 \mathrm{~mm}$ long, oblong-lanceolate, acuminate. Pedicel and ovary $17 \mathrm{~mm}$ long, slender, ovary ciliate. Dorsal sepal $5 \mathrm{~mm}$ long, $2.3 \mathrm{~mm}$ wide, elliptic-obovate, obtuse, concave. Petals bilobed to the base; anterior lobe $5.3 \mathrm{~mm}$ long, $0.6 \mathrm{~mm}$ wide, linear, subobtuse, minutely papillose; posterior lobe $5 \mathrm{~mm}$ long, $0.4 \mathrm{~mm}$ wide, linear-filiform, obtuse,

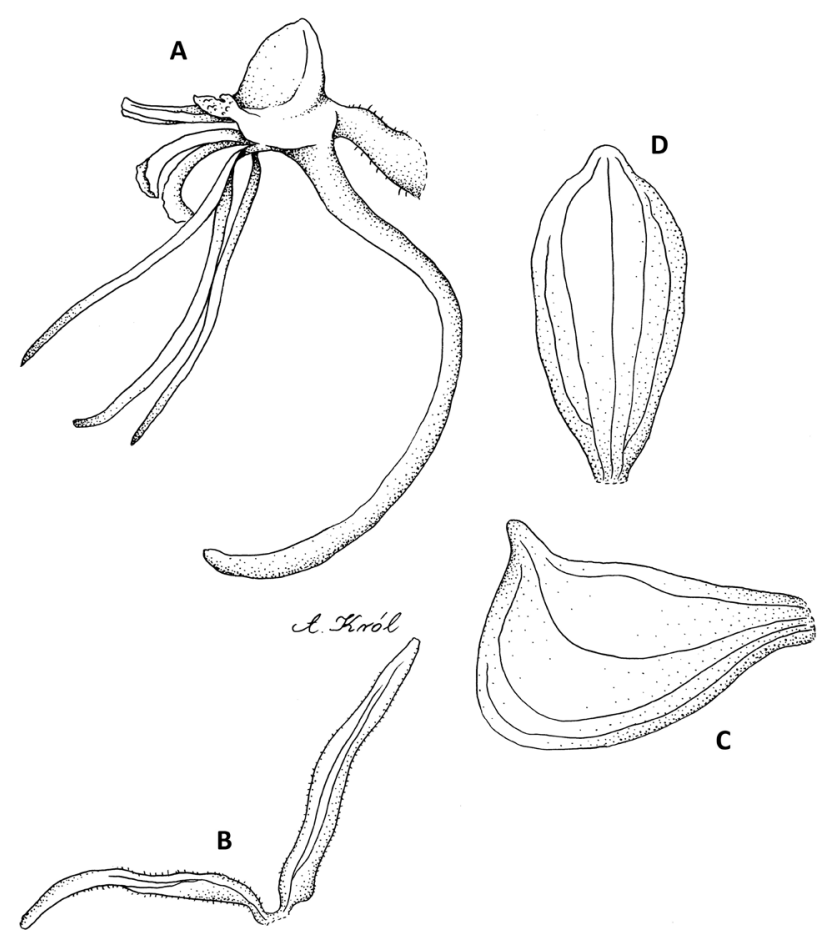

Fig. 242. Bilabrella incurva (Rolfe) Szlach. \& Kras Explanations: A - pedicel, ovary, gynostemium, lip and spur; B - petal; $\mathrm{C}$ - lateral sepal; D - dorsal sepal (drawn by A. Król from lectotype of Habenaria incurva Rolfe - Galpin 392b, K) 


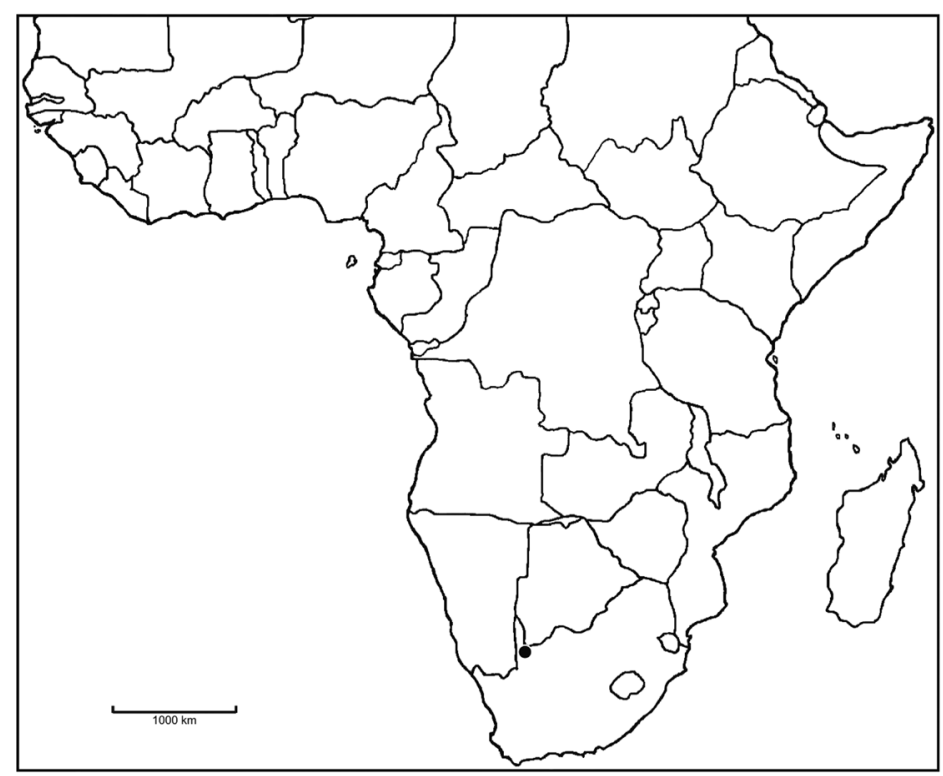

Fig. 243. Distribution of Bilabrella incurva (Rolfe) Szlach. \& Kras

ciliolate. Lateral sepals $5 \mathrm{~mm}$ long, $2.5 \mathrm{~mm}$ wide, obliquely oblong-obovate, laterally apiculate, obtuse, concave. Lip 3-lobed almost to the base; the middle lobe $7.5 \mathrm{~mm}$ long, $0.3 \mathrm{~mm}$ wide, linear-filiform, obtuse; lateral lobes $7.6 \mathrm{~mm}$ long, $0.2 \mathrm{~mm}$ wide, filiform, acute, pendent. Spur 13-14 mm long, narrowly cylindrical, not swollen, blunt, gently incurved. Anther $2 \mathrm{~mm}$ tall, conical-ovoid; connective rounded; antherophores $3 \mathrm{~mm}$ long, slender, straight. Stigmaphores slightly longer, narrowly cylindrical, ligulate apically. Rostellum middle lobes shorter than connective. Auriculae shortly stalked, unequally bifid apically (Fig. 242).

E c o lo gy. Hillsides, among rocks.

Distribution. Republic of South Africa, alt. $1800 \mathrm{~m}$ (Fig. 243).

Specimens examined. Republic of South Africa. Northern Cape: Kalahari Region, Transvaal, hillsides among rocks, $1800 \mathrm{~m}$, Galpin $392 b$ (K!).

Notes. Bilabrella incurva differs from similar $B$. plectomaniaca and B. pseudokaessneriana by having ciliate ovary (vs ovary glabrous).

90. Bilabrella plectomaniaca (Rchb. f. \& S. Moore) Szlach. \& Kras-Lap., Richardiana 3(3): 142. 2003.

Habenaria plectomaniaca Rchb. f. \& S. Moore, J. Bot. 16: 136. 1878. - Rolfe, Fl. Trop. Afr. 7: 245. 1898. Kraenzlin, Orchid. Gen. Sp.: 227. 1901. - Robyns \& Tournay, Fl. Spermat. Parc Natl. Albert 3: 434. 1955. Piers, Orchid. E. Afr.: 23. 1959. - Summerhayes, FTEA, Orchid. 1: 96. 1968b. - Stewart \& Campbell, Orchids of Kenya: 139. 1996.

Type (as holotype): Kenya, Wakefield s.n. (lectotype, designated by Summerhayes [1968b: 96], K!; isolectotype, W-R!).
Stem 50-100 cm tall, erect, stout, leafy throughout its length. Leaves 10-15, 13-32 cm long, 0.5-2 cm wide, the lowermost 1-2 reduced to sheaths, the middle 5-7 suberect, linear or lanceolate-linear, acute, the upper leaves adpressed to the stem, much smaller, lanceolate, acuminate, similar to the lower bracts. Inflorescence 10-30 cm long, loosely to rather densely 7 - to manyflowered. Flowers spreading, white or greenish-white. Flower bracts 10-30 mm long, lanceolate, acuminate, thin and scarious, shorter than the pedicel with ovary. Pedicel with ovary $25-35 \mathrm{~mm}$ long, slightly curved or straight. Dorsal sepal 8-9 mm long, $3 \mathrm{~mm}$ wide, reflexed, narrowly elliptical, obtuse, convex. Petals bilobed nearly to the base; anterior lobe $12-15 \mathrm{~mm}$ long, $2.5-4.5 \mathrm{~mm}$ wide, spreading forwards, lanceolate, acute; posterior lobe $8 \mathrm{~mm}$ long and $1 \mathrm{~mm}$ wide, upright or reflexed, linear, obtuse. Lateral sepals 11-13 mm long, 6-8 mm wide, deflexed, obliquely obovate with lateral apiculus. Lip projecting forwards, 3-lobed from an undivided base $1 \mathrm{~mm}$ long; the middle lobe $16-20 \mathrm{~mm}$ long, $1 \mathrm{~mm}$ wide, linear, obtuse; lateral lobes 11-17 mm long, lanceolatelinear, subacute, wideer than the middle lobe. Spur 45$70 \mathrm{~mm}$ long, incurved, somewhat swollen in the apical third or quarter. Anther 3-4.5 mm tall, ellipsoid-ovoid; connective truncate; antherophores 7-8 $\mathrm{mm}$ long, very slender, almost filiform, straight. Stigmaphores 8-9 mm long, narrowly cylindrical, truncate and slightly swollen at the apex, straight. Rostellum middle lobe slightly longer than connective. Auriculae stalked, very small, bilobed (Figs 244-245).
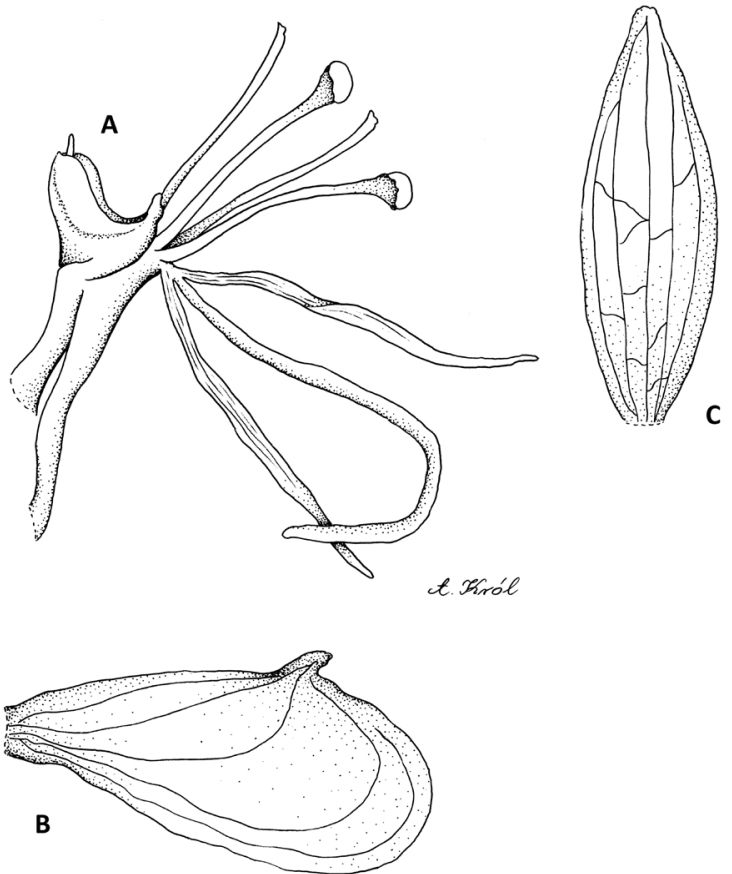

Fig. 244. Bilabrella plectomaniaca (Rchb. f. \& S. Moore) Szlach. \& Kras-Lap.

Explanations: A - gynostemium, lip and spur basal part; B - lateral sepal; $\mathrm{C}$ - dorsal sepal (drawn by A. Król from lectotype of Habenaria plectomaniaca Rchb. f. \& S. Moore - Wakefield s.n., K) 


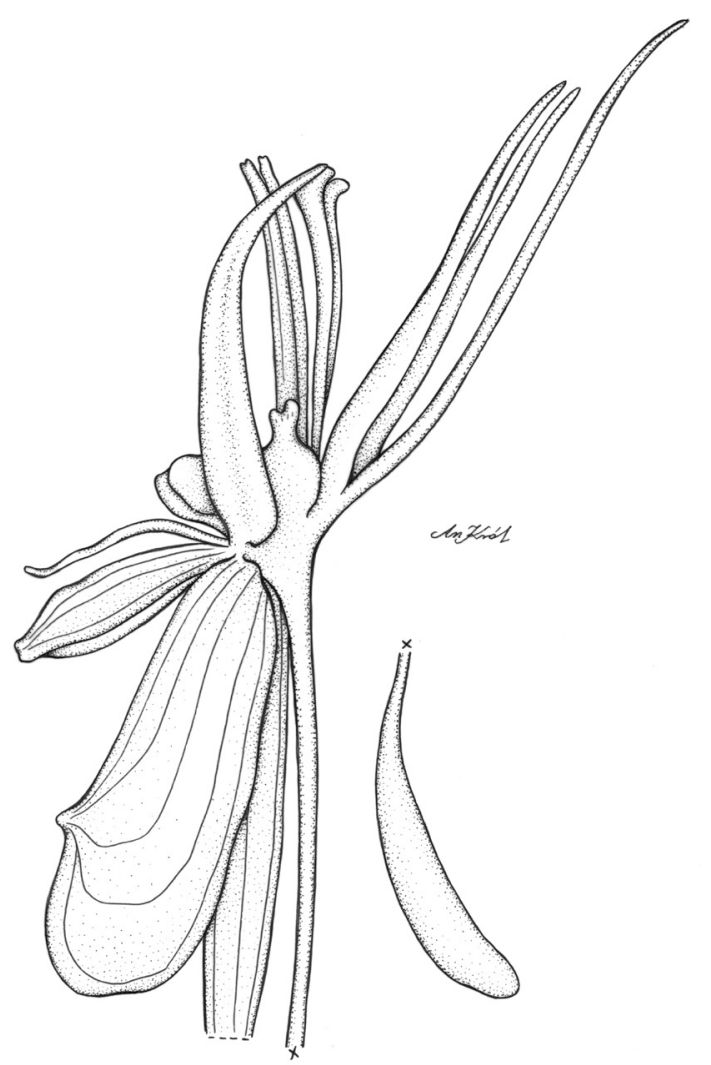

Fig. 245. Bilabrella plectomaniaca (Rchb. f. \& S. Moore) Szlach. \& Kras-Lap. - flower (drawn by A. Król from Vaughan 2822, AMES)

E c o logy. Grassland, sandy soil. June-October. Distribution. Eritrea, Kenya, Tanzania, alt. to $750 \mathrm{~m}$ (Fig. 246).

Specimens examined. Eritrea. Northern

Red Sea: Ambori, Oct. 1893 (fl), Holst $2878 a$ (K!). Kenya. K7. Coast: Kilifi Distr., near Bomani, Mtivapa

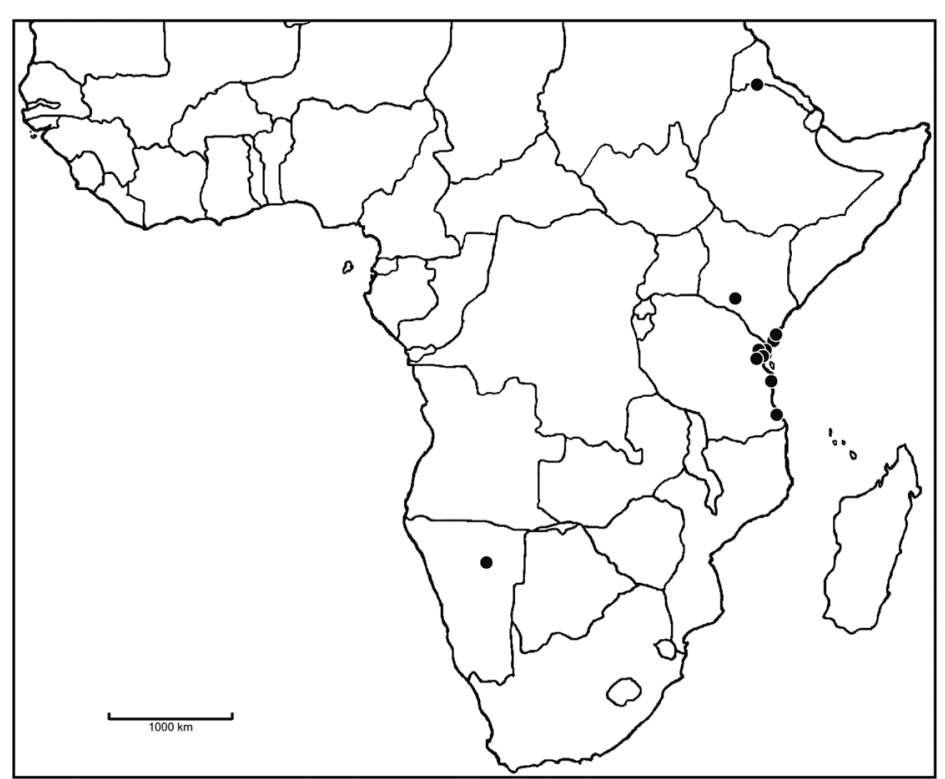

Fig. 246. Distribution of Bilabrella plectomaniaca (Rchb.f. \& S. Moore) Szlach. \& Kras-Lap. settlement Scheme, 75 m, 27 July 1970 (fl), Adams 33 (BR!, K!); The same locality, Adams 34 (BR!, K!); Kwale Distr., Mwele Mdogo Forest, Shimba Hills, 12 miles SW of Kwale, open grassland areas on hillside, 400 m, 28 Aug. 1953 (fl), Drummond \& Hemsley 3968 (K!); Kwale, Mnazi-wa-Chorzi (Digo), grassland, Graham A.532 (1934) (K!); Kwale Distr., Shimba Hills, in grassland, 420 m, Graham 1593 (K!); Kwale Distr., Shimba Hills, Sheldrick Falls area, grassland, 04 ${ }^{\circ} 16^{\prime}$ S, 39'25'E, 150 m, 29 Sep. 1992 (fl), Luke 3317 (K!); Rabai Hills, Mombaz, July-Sep. 1885 (fl), Taylor s.n. (BM!); Nyika country near Mombasa, Wakefield s.n. (K!, W-R!). Tanzania. Dar-es-Salaam: Mile 5. Pugu Road, in grass by railway, Aug. 1936 (fl), Voughan 2400 (K!); Lindi: June 1927 (fl), Agrcenltnal 1 (K!); Morogoro: Nyingwa, near Handeni border, Tuliani, $750 \mathrm{~m}$, 18 June 1924 (fl), Dao 22/17/5 (K! - icono.); Tanga: Korogwe, in grass, 296 m, 31 July 1963 (fl), Archbold 285 (K!); Handeni Distr., Kwa Mkono, in grass, 8 July 1983 (fl), Archbold 3005 (K!); Korogwe Distr., Ruvuland Estate, damp, swamp ground: among long grass, 300 m, 28 July 1952 (fl), Faulkner 1009 (K!); Grass steppe, 50 cm, 27 July 1932 (fl), Geilinger 1154 (K!); Kwa Mkono, well up the mountain in long grass, $510 \mathrm{~m}$, 30 June 1966 (fl), Cuchlsald 815 (K!); Pangani Distr., Msebugwe Forest, Mwera Chieldom, on well drained damp sandy soil, 150 m, 12 July 1955 (fl), Tanner 1991 (BR!, K!); Pugu Road, miles 8, marshy ground, $30 \mathrm{~m}$, 22 June 1939 (fl), Voughan 2822 (AMES!, BR!, K!, UGDA-DLSz! - fragment, drawing).

N o t e s. Similar species to Bilabrella plectomaniaca are $B$. cataphysema, $B$. anaphysema and $B$. ichnemonea. Unlike all aforementioned species, $B$. plectomaniaca has very slender anther and stigma projections. It has also very long spur, ca twice longer than pedicel and ovary.

91. Bilabrella pseudokaessneriana Szlach. \& Kras, Richardiana 9(4): 159. 2009, nom nov pro Habenaria kaessneriana Kraenzl., Viertelj. Nat. Ges. Zurich 74: 104. 1929.

Type (hoc loco selectus): Congo, Kassner 2413 (B+; lectotype, HBG!; isolectotypes, BM!, K!), non Kraenzl., 1912.

Stem 30-110 cm tall, erect, stout or realtively delicate, glabrous. Leaves 4-6, 8-35 cm long, up to 1.5 (occasionally 2) cm wide, linear-lanceolate, acute, suberect, decreasing in size up the stem. Inflorescence $6-25 \mathrm{~cm}$ long, 15-17-flowered, lax. Floral bracts 10-25 mm long, oblong-lanceolate, acuminate, glabrous. Pedicel and ovary $17-25 \mathrm{~mm}$ long, glabrous. Dorsal sepal $5.2 \mathrm{~mm}$ long, $2.75 \mathrm{~mm}$ wide, oblong-elliptic, obtuse, glabrous. Petals bilobed to the base; anterior lobe $6.3 \mathrm{~mm}$ long, $1.3 \mathrm{~mm}$ wide, obliquely lanceolate or linear-lanceolate, 


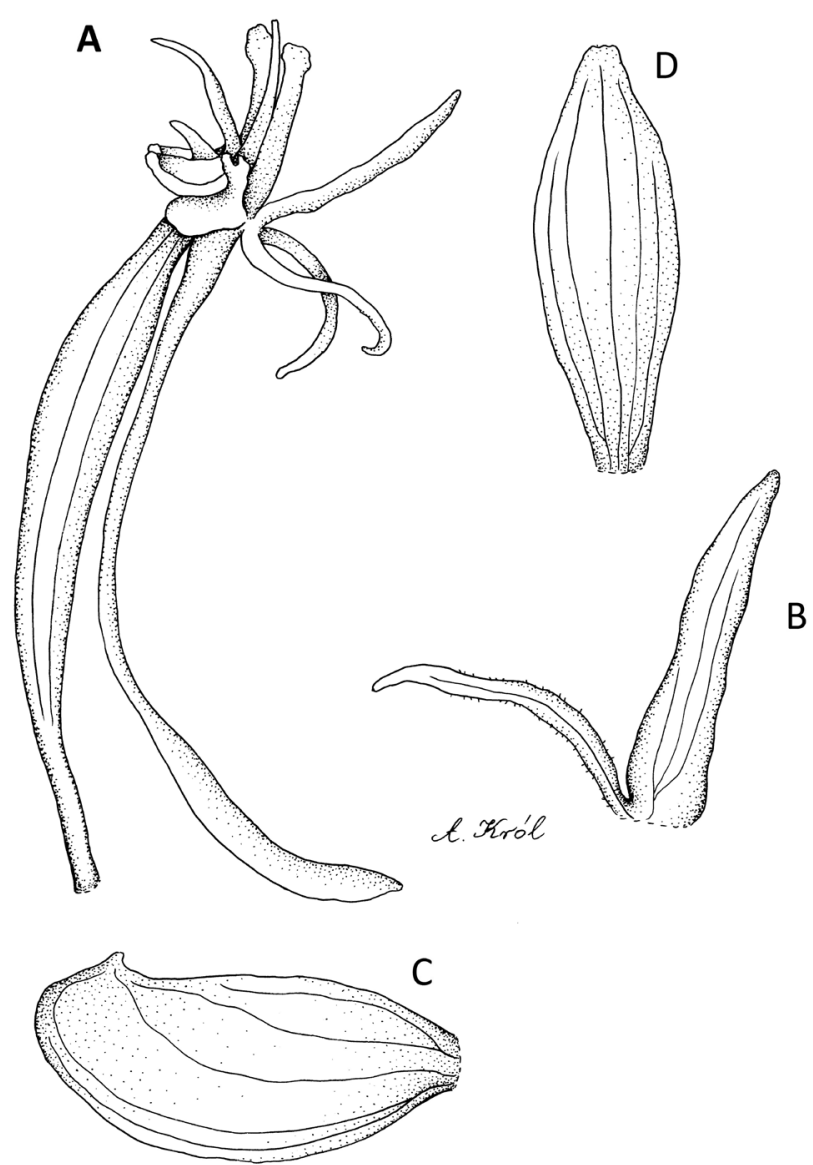

Fig. 247. Bilabrella pseudokaessneriana Szlach. \& Kras

Explanations: A - pedicel, ovary, gynostemium, lip and spur; B - petal; C - lateral sepal; D - dorsal sepal (drawn by A. Król from isolectotype of Habenaria kaessneriana Kraenzl. - Kassner 2413, K)

apiculate, rather thick, glabrous; posterior lobe $4.7 \mathrm{~mm}$ long, $0.5 \mathrm{~mm}$ wide, linear or filiform, thin, ciliolate, sometimes glabrous. Lateral sepals $7.3 \mathrm{~mm}$ long, $3 \mathrm{~mm}$ wide, obliquely oblong-elliptic, concave, with apicaly placed short, erect apiculus, glabrous. Lip 3-lobed at the base, glabrous; the middle lobe $6 \mathrm{~mm}$ long, $0.6 \mathrm{~mm}$ wide, linear-lanceolate, acute; lateral lobes $5-5.5 \mathrm{~mm}$ long, $0.3 \mathrm{~mm}$ wide, linear-lanceolate, acuminate. Spur $21 \mathrm{~mm}$ long, narrowly cylindrical, moderately swollen in the apical third, subacute, pendent. Anther $1.2 \mathrm{~mm}$ long; connective rounded; antherophores $3 \mathrm{~mm}$ long, slender, straight. Stigmaphores $4 \mathrm{~mm}$ long, narrowly cylindrical, truncate, straight. Rostellum middle lobe as long as connective. Auriculae medium-sized, bifid (Fig. 247).

E c o log y. In vlei. February.

D istribution. Democratic Republic of the Congo (Zaire) (Fig. 248).

Specimens examined. Democratic Republic of the Congo (Zaire). Haut-Katanga: Near Kimiwa, 27 Feb. 1907 (fl), Kassner 190 (K!).

N o tes. Bilabrella pseudokaessneriana along with B. limnophila and B. chirensis have slender, non-twisted

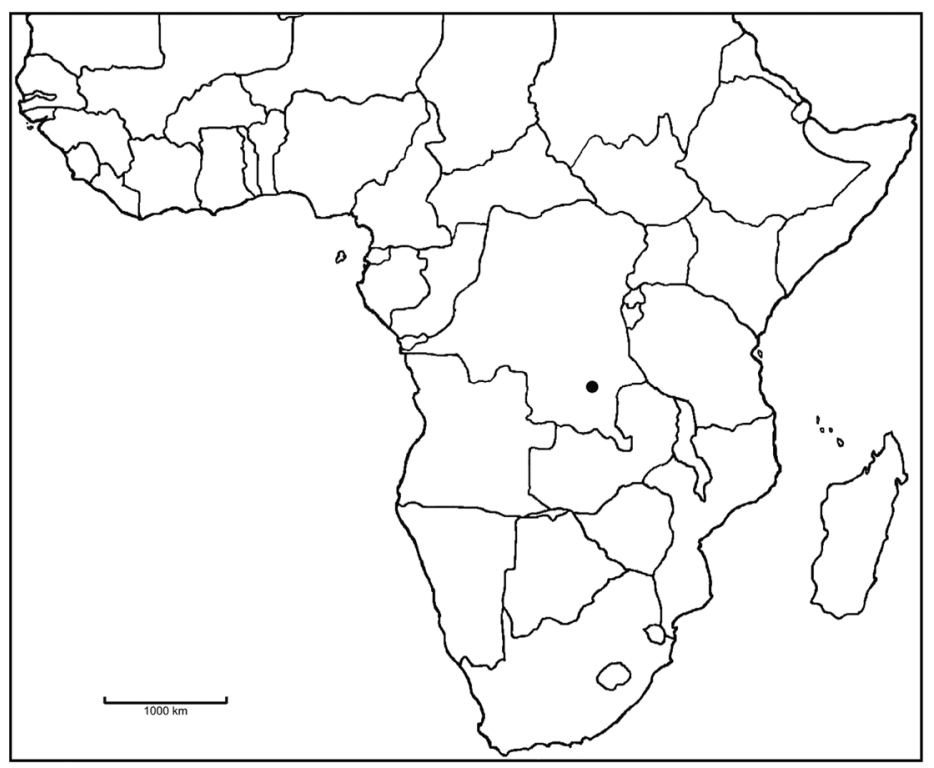

Fig. 248. Distribution of Bilabrella pseudokaessneriana (Kraenzl.) Szlach. \& Kras

spur, nearly as long as pedicellate ovary. Despite both of these species its anterior petal lobes are glabrous. This species is similar also to $B$. huillensis but the posterior petal lobe is linear and the lip lateral lobes are linearlanceolate.

92. Bilabrella limnophila (Summerh.) Szlach. \& Kras, Richardiana 9(4): 158. 2009.

Habenaria limnophila Summerh., Kew Bull. 1931: 381.1931.

Type (as holotype): Tanganyika, Haarer 1477 (lectotype, designated by Summerhayes [1968b], K!; isolectotype, EA not seen).

Stem $45-75 \mathrm{~cm}$ tall, erect, glabrous, leafy. Leaves $6-10$, to $33 \mathrm{~cm}$ long and $1.6 \mathrm{~cm}$ wide, suberect, linear, acute, gradually transforming upwards into bracts. Inflorescence 10-20 cm long, subdensely multiflowered. Flowers greenish, greenish-white or white. Floral bracts lanceolate, acuminate. Pedicellate ovary 13-20 mm long, slender. Dorsal sepal 4.5-6 mm long, $1.5-2 \mathrm{~mm}$ wide, elliptic-obovate, shortly apiculate, obtuse, concave. Petals bilobed to the base; anterior lobe 7-8.5 mm long, 0.7-0.8 mm wide, linear-lanceolate, acute, papillose at the base or entire; posterior lobe 4.5-6 mm long, $0.1 \mathrm{~mm}$ wide, filiform, acute, papillose. Lateral sepals 6-8 mm long, 3.5-4.5 mm wide, obliquely obovate, laterally apiculate, cochleate. Lip 3-lobed just above the base, glabrous; the middle lobe $12-13 \mathrm{~mm}$ long, $0.7 \mathrm{~mm}$ wide, linear, subacute; lateral lobes $8-8.5 \mathrm{~mm}$ long, $0.2 \mathrm{~mm}$ wide, linear-filiform, acute, pendent. Spur 18-19 mm long, narrowly cylindrical, falcate, slightly ampulaceous at the apex, blunt. 


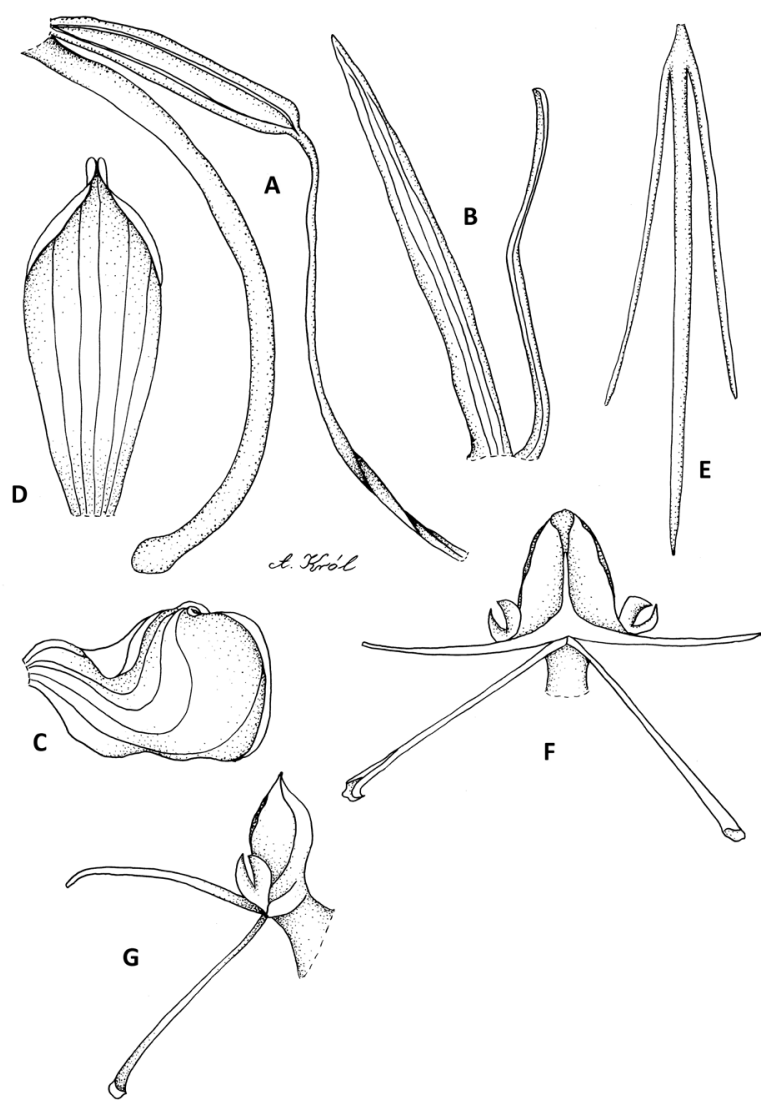

Fig. 249. Bilabrella limnophila (Summerh.) Szlach. \& Kras Explanations: A - ovary, pedicel, spur; B - petal; C - lateral sepal; D - dorsal sepal; E - lip; F - gynostemium, front view; G - gynostemium, side view (drawn by A. Król from lectotype of Habenaria limnophila Summerh. Haarer $1477, \mathrm{~K}$ )

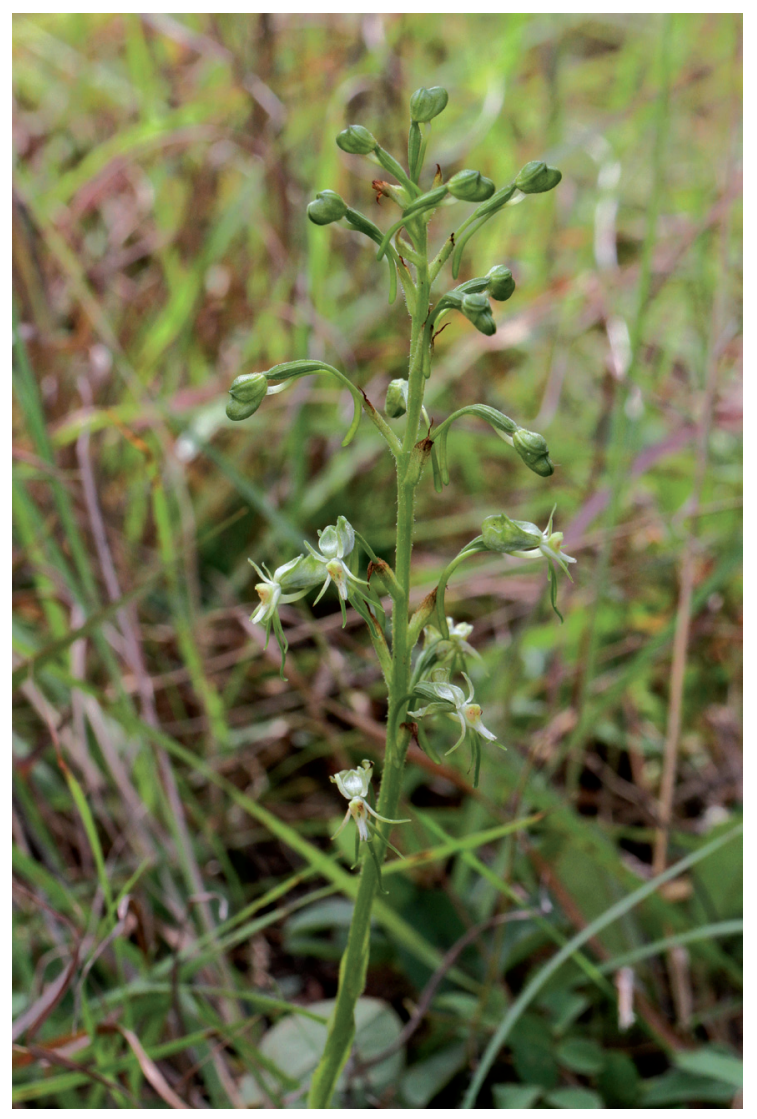

Fig. 251. Bilabrella limnophila (Summerh.) Szlach. \& Kras inflorescence (phot. L. Grobler)

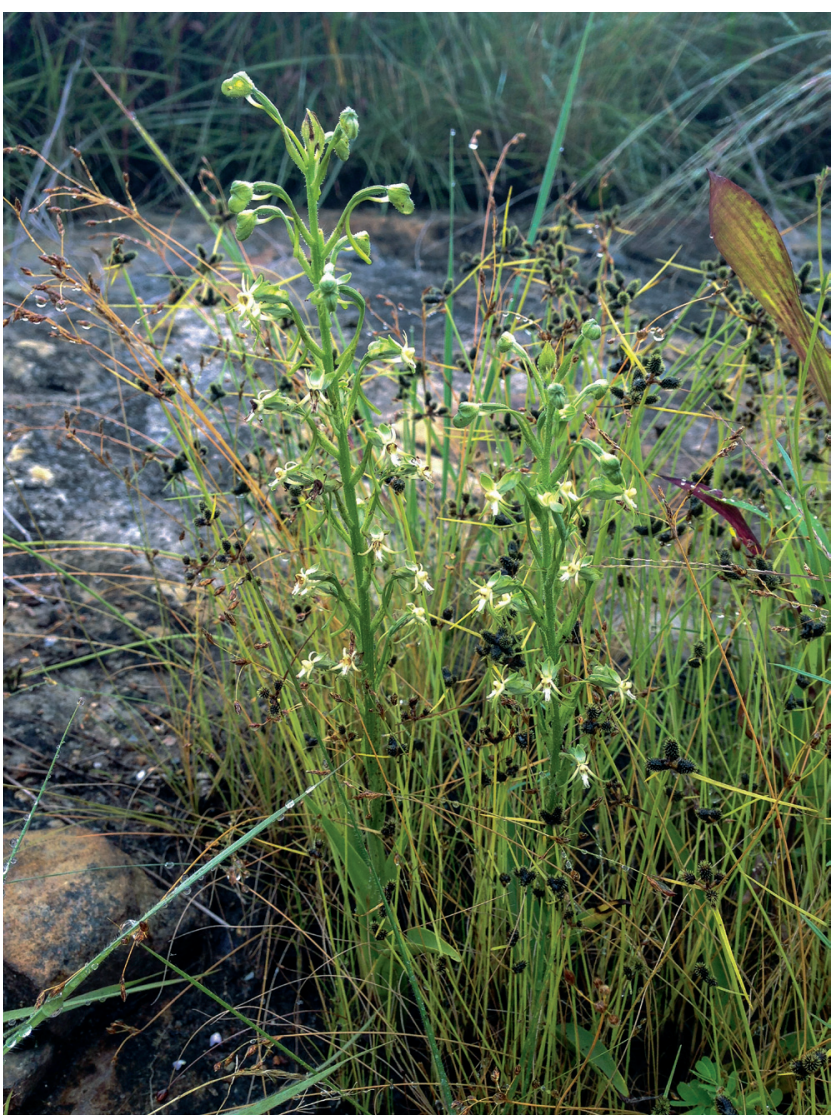

Fig. 250. Bilabrella limnophila (Summerh.) Szlach. \& Kras - habit (phot. L. Grobler)

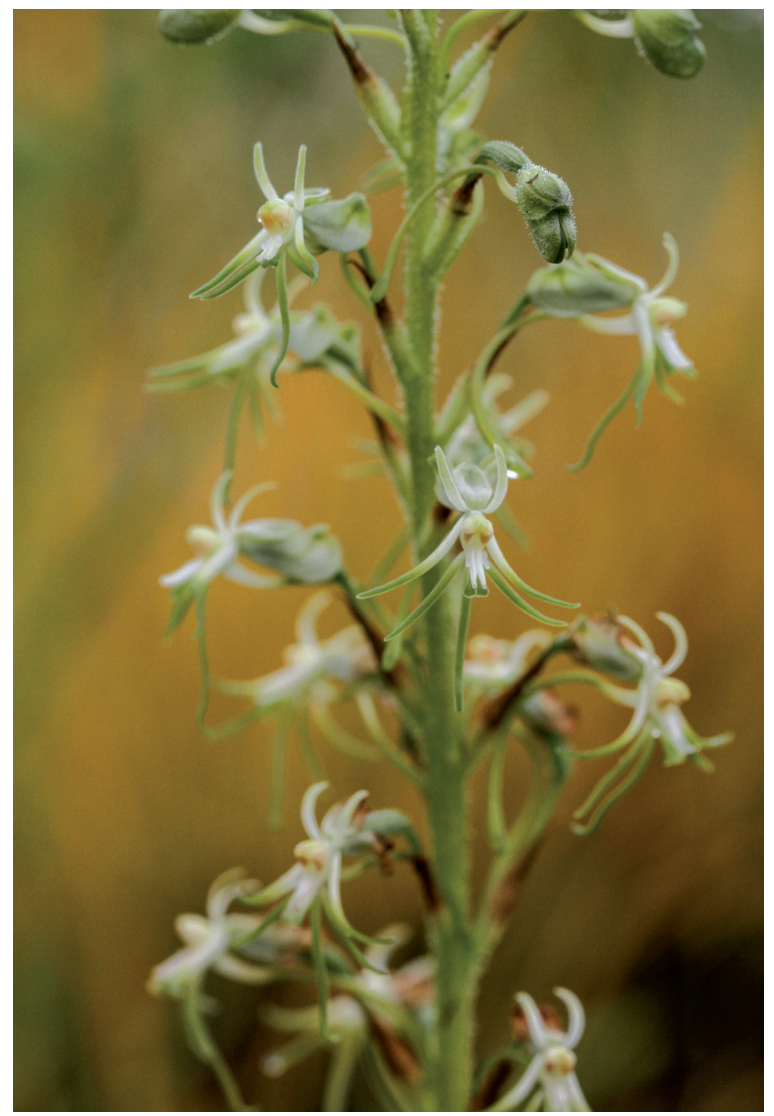

Fig. 252. Bilabrella limnophila (Summerh.) Szlach. \& Kras - flower close-up (phot. L. Grobler) 


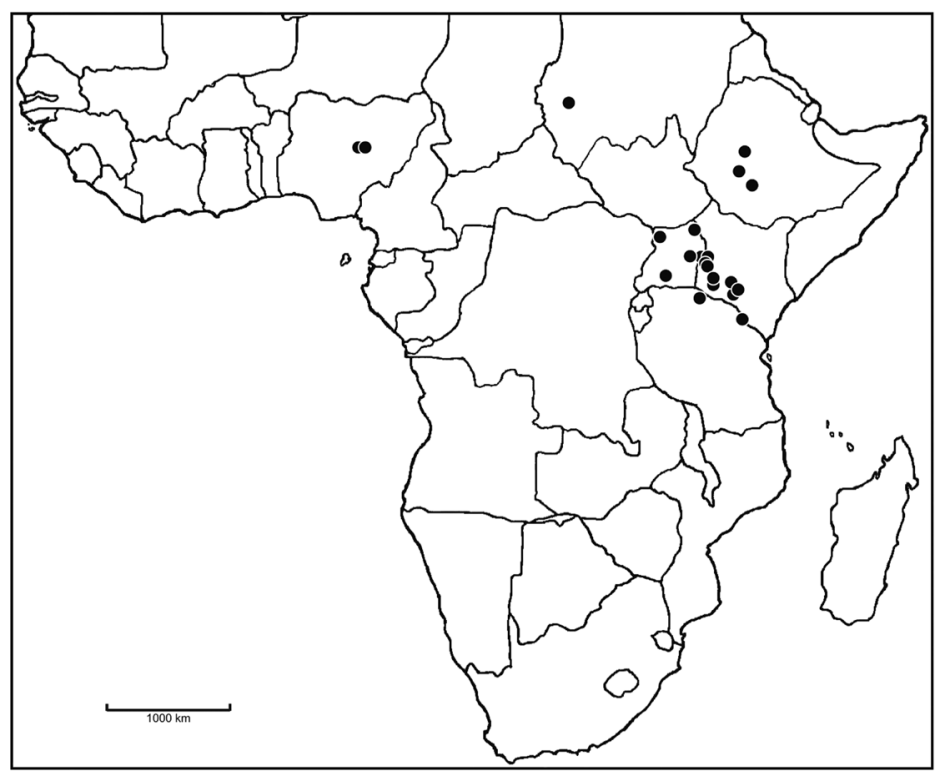

Fig. 253. Distribution of Bilabrella limnophila (Summerh.) Szlach. \& Kras

Anther $3 \mathrm{~mm}$ tall; connective rounded; antherophores 4.5-5 mm long, filiform. Stigmaphores 5-6 mm long, filiform, slightly widened apically, truncate. Rostellum middle lobe distinctly shorter than connective. Auriculae $1.5-2 \mathrm{~mm}$ long, relatively large, stipitate, bifid at the apex (Figs 249-252).

E c o lo g y. Grassland, wet savannah, swampy ground. September-February, May-August.

D i s tr i bu ti o n. Sudan, Nigeria, Ethiopia, Uganda, Kenya, Tanzania, alt. 1110-2600 m (Fig. 253).

S p e c i men s ex a mined. Sudan. North Darfur: Jebel Marra, 1110 m, 20 Aug. 1964 (fl), Wickens 2171 (K!). Nigeria. Plateau: State Distr., along the motor road, in a swampy place very near Ropp, 12 July 1970 (fl), Daramola \& Ekwuno FHI 62970 (K!); Plain between Bukuru and Hepham, 1290 m, 4 July 1921 (fl), Lely 343 (K!); Bauchi Plateau, June 1930 (fl), Lely P. 379 (K!); Northern region, in grassland, June 1962 (fl), King 28 (K!); Northern Region, Kuru Station, 1 July 1958 (fl), Summerhayes 148 (K!); N'gell, widely spread in Plateau Prov., in sandy ground, 1290 m, June 1956 \& 1958 (fl), King 89 (K!); Pankshin Distr., Kurra, Gashish, 22 June 1963 (fl), Okafor FHI 59233 (K!); Rafin Bauna (N) F. R., savannah woodland in grassy areas, 23 July 1962 (fl), Lawlor \& Hall FHI 45582 (K!); Savannah wet places, 19 July 1980 (fl), Sharland 1359 (K!); Savannah Mar VII, 25 July 1987 (fl), Sharland 2055 (K!). Ethiopia. Oromiya: Keffa Prov., Jimma, at airport, square $81 . \mathrm{B}$, very common in area of marshy ground behind airport buildings, in damp marshy area, 1676 m, 8 Aug. 1971 (fl), Ash 1105 (K!); Shola Region, $8 \mathrm{~km} \mathrm{~W}$ of Ghedo, Wetter areas of rough grassland, $9^{\circ} 02^{\prime} \mathrm{N}, 37^{\circ} 25^{\prime} \mathrm{E}, 2300$ m, 20 Sep. 1975 (fl), Gilbert \& Thulin 917 (K!); Kaffa Prov., Jimma-Agaro road, marshland, 1620 m, 7 Sep. 1962 (fl), Thomas 27 (K!); Arussi Prov., $35 \mathrm{~km}$ ESE of Shashamane, alnog road to Dodollo, undulating country devoid of any treegrowth, grasses and herbs covering the gentle slopes, small creek with marshy borderlands on bottom of valley, $7^{\circ} 03^{\prime} \mathrm{N}$, $38^{\circ} 49^{\prime} \mathrm{E}, 2600$ m, 26 July 1970 (fl), De Wilde 6847 (K!). Uganda. Eastern: Kapchorwa Distr., N Kapchorwa, Sebei, grassland, 1950 m, 28 June 1962 (fl), Leqakcy 3 (K!); Kapchorwa Distr., M. Elgon, common in wet grassland near camp, 2100 m, 7 Sep. 1954 (fl), Lind 248 (K!); Kapchorwa Distr., Mt. Elgon, grassland above camp, 1950 m, Lind 279 (K!); Kapchorwa Distr., Sipi, Bugishu, swampy ground, 1800 m, 31 Aug. 1932 (fl), Thomas 447 (K!); Soroti Distr., Serere, at Rapai, in swamp, 1110 m, July 1926 (fl), Maitland 1308B (K!); Wet places, 1200 m, Aug. 1931 (fl), Staff 2253 (K!); North-western part of country, West Nile Distr., valley first own the Congo border due West of Deiba, marshy ground by a small stream, 1440 m, 2 Aug. 1953 (fl), Chaneella 94 (K!); North-eastern part of country, near Morunyanai, SW Karamoja, tall grass on black clay plains, grazed primarily during dry season, $1200 \mathrm{~m}$, 3 July 1957 (fl), Dyson-Hudson 274 (K!); Swampy land, 30 May 1910 (fl), Fryffe 256 (K!). Kenya. Central: Kabete, W Nairobi, in damp, 1860 m, 1930 (fl), Mettam 168b (K!); Karura forest, 1740 m, May 1937 (fl), Someren 7308 (K!); Kiambu, 1710 m, Mainwaring s.n. (K!); Nairobi: Kirichwa Ndogo valley, on black cotton soil, occasionally waterlogged, 1650 m, 10 July 1947 (fl), Bally 5134 (K!); Murang'a Distr., Thika, N side of Thika River, E of the Nairobi - Fort Hall Road, level area opposite road to Horticultural Research Station, marsh with some common or characteristic species, $1^{\circ} 02^{\prime}$ 'S, 37 $37^{\circ} 05^{\prime} \mathrm{E}, 1520 \mathrm{~m}, 11$ July 1971 (fl), Faden \& Kabuye 71/566 (K!); Nairobi, 1650 m, 22 Sep. 1916 (fl), Dowson 376 (K!); Thika Road House, grassland, locally scarce in Themeda, Setaria trinervia, Aristida, Eragrostis and Fuirena assoc, 3 July 1951 (fl), Verdcourt 533 (K!); Nairobi, Aug. 1903 (fl), Whyte s.n. (K!); Themeda grassland with Acacia drepanolobium on shallow black cotton soil over volcanic rock at edge of forest, 1755 m, 16 July 1967 (fl), Wood 852 (K!); Rift Valley: Kipkarren Distr., rock pools, Hill 26 (136) (K!); Sotik, in grassland, 1800 m, 13 June 1950 (fl), Bally B7842 (K!); Kitale, in swamp, 19 July 1952 (fl), Bogdon 10192 (K!); Kitale, grassland, 1950 m, Feb. 1935 (fl), Thorold 2779 (K!); Kitale, marshland amongst tall grass, $1860 \mathrm{~m}$, Wiltshire 32 (K!); Western: Broderick Falls, swampy ground in wet savannah, $1500 \mathrm{~m}$, June 1964 (fl), Tweedie 2836 (K!); Mt. Elgon, Fack 302 (K!); Elgon, 2100 m, June 1932 (fl), Fack 303 (3055) (K!); Mt. Elgon, in swamp, 1950 m, 20 July 1931 (fl), Lugard 673 (K!); SW Elgon, 1980 m, 12 July 1958 (fl), Symes 395 (K!); Elgon, on shallow wet soil over rock, $2010 \mathrm{~m}$, June 1931 (fl), Tweedie 15 (K!); E Kakamega, Nandi, 
neer Kapsabet, in swamp, 1950 m, Aug. 1933 (fl), Dale 3170 (K!). Tanzania. Kagera: Lake Prov., North Mara Distr., Shirati to Utegi road, on grassy patches between patches of bush, 1200 m, 23 May 1957 (fl), Gane 119 (K!); Kilimanjaro: Moshi Distr., Mpololo, $1050 \mathrm{~m}$, Aug. 1928 (fl), Haarer 1477 (K!).

$\mathrm{N}$ o t e s. Bilabrella limnophila is similar to B. chirensis. Both share similar form and length of the spur and and gynostemium morphology, but can be separated by the lip, which is 3-lobed just above the base. In B. chirensis the lip is 3-lobed above the basal undivided part up to $1.5 \mathrm{~mm}$ long.

This species, which, judging from the number of specimens seen, must be fairly common in the highlands between Mts. Elgon, Kenya and Kilimanjaro, is characterised chiefly by the slighty falcate spur which ends abruptly in a very slight swelling but is not at all clavate as in so many other allied species. B. limnophila is apparently an inhabitant of swampy land, occuring especially in the plains in the neighbourhood of Nairobi (Summerhayes 1931).

93. Bilabrella chirensis (Rchb. f.) Szlach. \& Kras-Lap., Richardiana 3(3): 139. 2003.

Habenaria chirensis Rchb. f., Otia Bot. Hamburg.: 99. 1881. - Rolfe, Fl. Trop. Afr. 7: 238. 1898. - Summerhayes, FWTA, ed. 1, 2: 412. 1936. - Piers, Orchid. E. Afr.: 15. 1959. - Copley, Tweedie \& Carroll, Journ. E. Afr. Nat. Hist. Soc. 24(4): 22. 1964. - Summerhayes, FWTA, ed. 2, 3: 196. 1968a. - Piers, Orchid. E. Afr.: 22. 1968. - Summerhayes, FWTA, ed. 2, 3: 196. 1968. Summerhayes, FTEA, Orchid. 1: 88. 1968b. - Geerinck \& Coutrez, Not. Taxon. Orchid. Afr. Centr., Habenaria: 8. 1977. - Geerinck, Fl. Afr. Centr., Orchid. 1: 107. 1984. - Stewart \& Campbell, Orchids of Kenya: 131. 1996. - Edwards, Demissew, Hedberg, Flora of Ethiopia and Eritrea 6: 219. 1997. - Szlachetko \& Olszewski, Fl. Cam., Orchid. 34(1): 170. 1998. - Demissew, Cribb, Rasmusen, Field guide to Ethiopian Orchids: 93. 2004.

Type: Ethiopia, Quartin Dillon s.n. (lectotype, designated by Szlachetko et al. [2010: 219], W-R!, K! - drawing).

Tubers 1-4 cm long, $0.5-1.3 \mathrm{~cm}$ in diameter, globose, ovoid to oblong ellipsoid. Stem $20-100 \mathrm{~cm}$ tall, erect, delicate to stout, glabrous. Leaves 7-13, 8-30 cm long, $0.5-1.5 \mathrm{~cm}$ wide, erect or spread, linear-lanceolate, acute, decreasing in size up the stem. Inflorescence 6-30 cm long, loosely or densely 12-50-flowered. Flowers white, greenish-white or green except for the white processes and auriculae, with an unpleasent smell. Floral bracts 8-19 mm long, oblong to ovate-lanceolate, acuminate, sparsely glandular along margins. Pedicel and ovary $20 \mathrm{~mm}$ long, glabrous. Dorsal sepal 4.5$6 \mathrm{~mm}$ long, $2 \mathrm{~mm}$ wide, oblong ovate, narrowly elliptic,

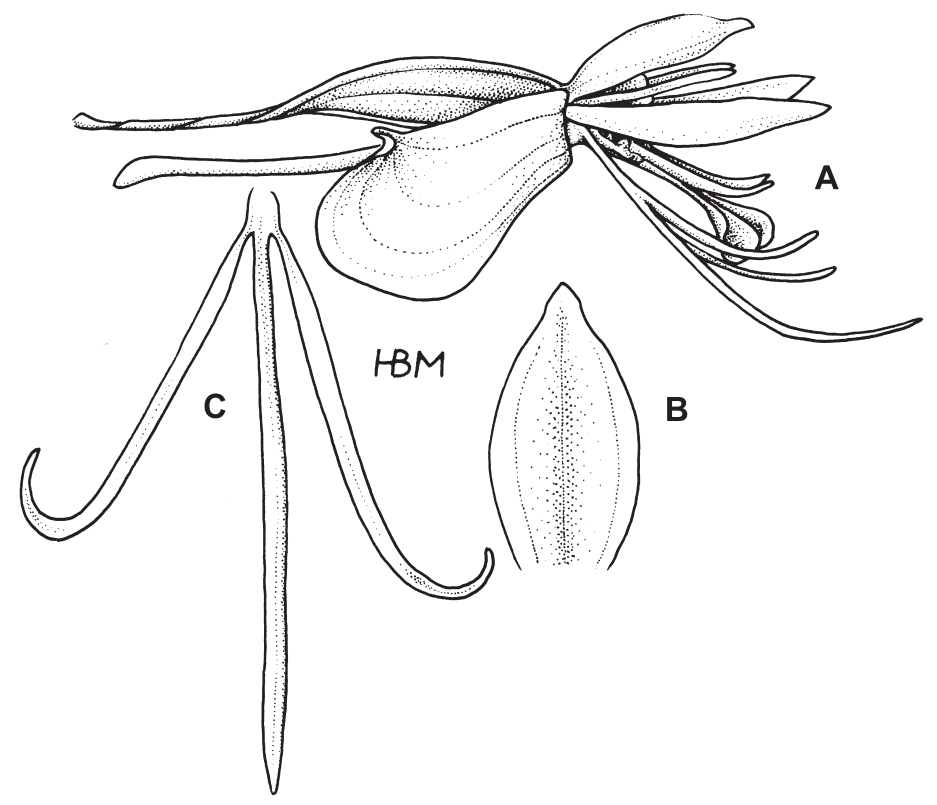

Fig. 254. Bilabrella chirensis (Rchb. f.) Szlach. \& Kras-Lap. Explanations: A - flower; B - dorsal sepal; C - lip (drawn by H. B. Margońska from lectotype of Habenaria chirensis Rchb. f. - Quartin-Dillon s.n., W-R)

obtuse to shortly apiculate, cochleate, glabrous. Petals bilobed nearly to the base; anterior lobe 5-9 $\mathrm{mm}$ long, 0.5-1.2 mm wide, linear-oblanceolate, acute, rather thick, fleshy, papillose and ciliate in the lower part or glabrous; posterior lobe 4-6 mm long, 0.2-0.5 mm wide, filiform, acute, thin, delicate, papillose and ciliate. Lateral sepals 6-9 mm long, 3-5 mm wide, obliquely obovate, with involute apical margin, apiculus ca. $0.5 \mathrm{~mm}$ long, laterally placed, glabrous. Lip 3-lobed above the basal undivided part up to $1.5 \mathrm{~mm}$ long, rather

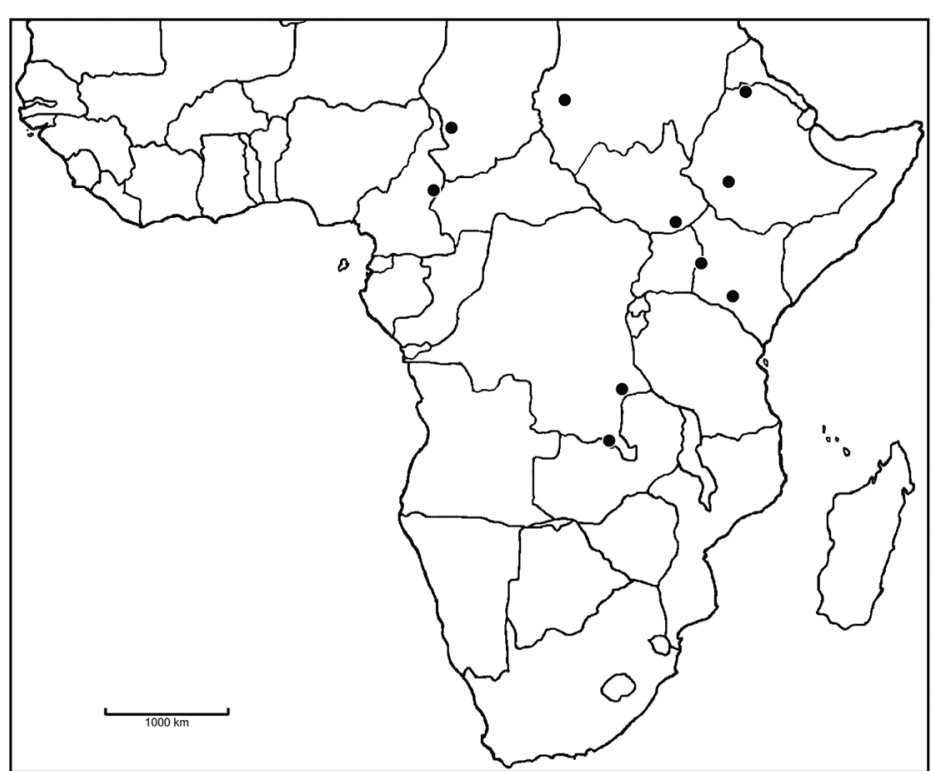

Fig. 255. Distribution of Bilabrella chirensis (Rchb.f.) Szlach. \& Kras-Lap. 
thick; the middle lobe 9-12 $\mathrm{mm}$ long, $0.5-1 \mathrm{~mm}$ wide, linear or filiform, acute; lateral lobes 6-8 mm long, 0.2-0.6 mm wide, filiform, slightly curved upwards. Spur 10-20 mm long, $0.8-1 \mathrm{~mm}$ in diameter, narrowly cylindrical, sometimes with slightly swollen blunt apex, parallel to the ovary or incurved. Anther 2-3 mm tall; connective rounded; antherophores $5 \mathrm{~mm}$ long, slender, slightly upcurved at the apex. Stigmaphores ca. 4-6 mm long, slender, narrowly cylindrical, truncate apically. Rostellum middle lobe shorter than thecae. Auriculae large, shortly stalked, unequally or subequally bilobed (Fig. 254).

E c o log y. Terrestrial in burned-over grassland, sandy slopes, damp grassland, swamp, wet places among rocks, savannah. Flowering in June-July, February, December.

D i st ribution. Chad, Sudan, Ethiopia, Cameroon, Democratic Republic of the Congo (Zaire), Uganda, Kenya, alt. 1200-1950 m (Fig. 255).

Specimens ex a mined. Chad. Chari-Baguirmi: Lake Chad, Baguirmi S. Bahr Erguig region, between Boulbai and Djomour, Chevalier 9484 (P!). Sudan. North Darfur: Jebel Marra, 1200 m, Wickens 2215 (K!); Equatoria: Gilo, Imatong Mts., in burned-over grassland at edge of flat rocks in mountain savannah, 1950 m, 26 June 1947 (fl), MacLeay 73 A (BM!). Ethiopia. Central: Steppes of the Ihipa-Thika, TanaYunci basin, sandy slopes with Acacia, Camp. 34, 18 July 1893 (fl), Gregory s.n. (BM!); Oromiya: Along road from Bonga to Jimma, 26 July 1975 (fl), Jansen 2343 (WAG!); Kaffa Prov., 26 km along road Jimma to Addis Ababa, open, sloping country, grassvegetation with patches of cultivation (Zea mais, etc.) some scattered trees and shrubs, $7^{\circ} 42^{\prime} \mathrm{N}, 37^{\circ} 0^{\prime} \mathrm{E}$, 1800 m, 10 July 1969 (fl), De Wilde 5428 (WAG!); About $5 \mathrm{~km}$ WSW of Jimma, along road to Bonga, De Wilde 7584 (BR!, C!, P!, WAG!); Tigray: Chire, Quartin-Dillon s.n. (W-R!). Cameroon. Adamaoua: Mdere and Meiganga, June 1939 (fl), Jacques-Felix 4126 (K!, P!). Democratic Republic of the Congo (Zaire). Haut-Katanga: Haut-Shaba, Luapula valley, near the Kinioma, dambo, 25 Dec. 1968 (fl), Lisowski 65555 (UGDA-DLSz!); Lubumbashi, 2 Feb. 1970 (fl), Lisowski 66421 (UGDA-DLSz!). Uganda. Eastern: Kapchorwa Distr., Sebei, N'Kapchorwa, grassland, 1950 m, 28 June 1962 (fl), Leakey 3 (BR!). Kenya. Nairobi: St. Austeris Mission, June 1951 (fl), Piers P.52 (K! - icono \& spirit.).

N o te s. This species can be confused with Bilabrella limnophila as both share many common morphological characters. At the best of our knowledge both can be distinguished by lip lobation - it is 3-lobed either at the base (B. limnophila) or above the basal claw $1.5 \mathrm{~mm}$ long (B. chirensis). It seems to be discriminative character.
B. chirensis appears to be similar also to B. bertauxiana, from which differs easily by its shorter, blunt spur, usually partly ciliate and papillose anterior petal lobes, and form of the lip lateral lobes. The length of the spur and width of the leaves distinguished it easily from B. strangulans.

\section{Incertae sedis}

The following species have been included to the section Replicatae by Kraenzlin (1901). Unfortunately, we had no access to any material representing these species, neither herbarium specimens, nor illustrations. In result, we were not able to create our own opinion on their taxonomic position. We decided to gather them in this chapter.

1. Habenaria barberae Schltr., Bot. Jahrb. Syst. 20(50): 7. 1895

Type: Eastern Cape, Barber s.n. (holotype: $\mathrm{B}+$ ).

2. Habenaria johannae Kraenzl., Bot. Jahrb. Syst. 16: 77. 1893. - Kraenzlin, Orchid. Gen. Sp.: 219. 1901. Perrier de la Bathie, Fl. Madagasc. 1: 65. 1939. - Puy, Cribb et al., Orchids of Madagascar: 153. 1999.

Type: Johanna Island, Hildebrandt 1883 (holotype?: $\mathrm{B}+$ ).

3. Habenaria pristichila Kraenzl., Bot. Jahrb. Syst. 51: 372. 1914.

Type: Basin of Kongo, Kassner 2931.

4. Habenaria petromedusa Webb. in Hook., Niger Fl. 180. 1849. - Kraenzlin, Orchid. Gen. Sp.: 213. 1901.

Type: Capverdische Island. Exam. spec. Museum Paris.

5. Habenaria sigillum Thouars, Hist. Orchid. 20. 1822. - Lindley, Gen. Sp. Orchid. Pl.: 326. 1835. - Kraenzlin, Orchid. Gen. Sp.: 218. 1901.

Type: Madagascar, Thouars s.n. (iconotype, W-R!).

\section{Undereminated materials}

In many cases preservation of the herbarium materials made the correct determination impossible. Below we listed such collections:

Guinea. July 1900 (fl), Pobeguin 362 (P!). Sierra Leone. Koinadugu: Loma Mts., high altitude, grassland, 25 Sep. 1945 (fl), Paul Jaeger I FAV (K!); Loma Mts., high altitutude, grassland, 25 Dec. 1945 (fl), Paul Jaeger 1984 (K!). Nigeria. Plataeu: Northern Part Oho, 1230 m, 9 Oct. 1961 (fl), King 144 (K!). Ethiopia. 
Oromiya: $170 \mathrm{~km}$ from Mojo, road to Shashemene, fields, Acacia woodland, grass vegetation and some other shrubs, black loamy soil, +/- 1850 m, 6 Sep. 1967 (fl), Westphal and Westphal-Stevels 1679 (C!). Central African Republic. Haute-Kotto: Kotto Mt. (OubanguiChari-A.E.F.), 23 Apr. 1921 (fl), Bequeathed 1967 (BM!); Ouaka: Swamp near Riv. Bayedon, 30 km S Ippy, May 1929 (fl), Bequeathed s.n. (BM!); The area around the Waka (Oubangui-Chari A.E.F.), plateau near River Gumburu, 60 km N Bambari, 1924 (fl), Tisserant 1.975 (BM!); Nana-Grébizi: Oubangui, Yagbanga River, $40 \mathrm{~km} \mathrm{~N}$ Besson, blooming through all year, 1914 (fl), Tisserant 199 (P!); Ouham-Pendé: At Bozoum Oubangui Chari (A.E.F.) region, 8 Oct. 1931 (fl), Tisserant 2.935 (BM!). Gabon. Ngounié: Around Haute-Ngounye, 1924 (fl), Bequeathed s.n. (BM!); Woleu-Ntem: Bengo, 1933 (fl), Bequeathed 9.120 (BM!); Bengo, 1933 (fl), Bequeathed s.n. (BM!). Democratic Republic of the Congo (Zaire). Kasaï Oriental: Lake Kivu beetwen Mont Ngoma and Kisenji, 1410 m, 21 Jan. 1931 (fl), Burtt 3238 (K!); Haut-Katanga: Env. 8 km of W from Lubumbashi, 25 Dec. 1968 (fl), Lisowski 65753 (UGDA-DLSz!); Env. $8 \mathrm{~km}$ of W from campus of univeristy in Hasapa, 12 Jan. 1968 (fl), Lisowski 65759 (UGDA-DLSz!); $12 \mathrm{~km} \mathrm{~W}$ from L'shi, near Tasamakale village, 25 Jan. 1970 (fl), Lisowski 66396 (UGDA-DLSz!). Kenya. Western: Mt. Elgon, 1920 (fl), Benham s.n. (BM!). Tanzania. Area of the Ruhudje river, landscape of the Lupembe, north waterside of the river, May 1931 (fl), Schlieben 884 (BM!). Angola. Lunda Sul: Saurimo, 24 Oct. 1932 (fl), Young 1151 (BM!); Moxico, Rao d'Oaxaca, May 1842 (fl), Ghiesbreght 271 (P!); Southern: Kirk Range, grassland, 17 Mar. 1955 (fl), Excell, Mendonca \& Wild 998 (BM!); 19651966, Martins s.n. (P!). Zambia. Luapula: Kashiba, 27 Jan. 1908 (fl), Kassner 2412 (BM!); North-Western: Mwinilunga Distr., Sinkabolo Dambo in boggy grassland, Oct. 1937-Feb. 1938 (fl), Milne-Redhead 3457 (BM!). Malawi. Southern: Nyasaland, Limbe, open forest on hillside facing north west, $1200 \mathrm{~m}, 29$ Feb. 1948 (fl), Goodwin 78 (BM!). Mozambique. Sofala: Near Gonda, 4 Mar. 1882 (fl), Christenson 148 (W!); Maputo: Gazaland, 900 m, 1907 (fl), Swynnerto 744 (BM!); Kiki Highlands, s.n. 302 (W!) (cf. H. genuflexa). Zimbabwe. Harare: Salisbury Distr., Umtali, 1080 m, 21 Mar. 1955 (fl), Chase 5505 (K!); Salisbury Distr., Umtali, grassland South of Chase arenal, $1410 \mathrm{~m}, 9$ Nov. 1961 (fl), Chase 5847 (K!); Salisbury Distr., Umtali, 21 Mar. 1955 (fl), Clare 5505 (K!); Salisbury, 2 Feb. 1943 (fl), Greatrex 9556 (K!); Manicaland: Inyanga \& Harare, Salisbury, 21 Jan. 1942 (fl), Hopkins 8606 (K!); Mashonaland West: Inselberg nahe bankot, ZW 15, Gwangwaza, 17'29'40"S, 30²8'41'”E, 1390 m, 5 Mar. 1994 (fl), Sejne 1143 (K!); Matabeleland North:
Victoria, 1909 (fl), Monro 874 (BM!); Victoria, 1909-12 (fl), Monro 1723A (BM!); George Prov., 3 Mar. 1893 (fl), Schlechter 2325 (BM!). Republic of South Africa. Kwazulu-Natal: „In planitie prope fl. Umlaas”, 18381840 (fl), Krauss 101 (BM!); Mpumalanga: Transvaal, Lydenburg Distr., near city Lydenburg, Jan. 1895 (fl), Wilms 1383 (E!). Spec. indent. Sine loc., Guinzius (?) 108 (W!); Sine loc., 9 Mar. 1962 (fl), Kirk s.n. (W!); Kalccunda River, 9 Jan. 1971 (fl), Lisowski 12626 (UGDA-DLSz!); Sine loc., Tweedie 406 (K!); Sine loc., Tweedie 2191 (K!, W-R 43221!); Sine loc., Wallich s.n. (WU!).

\section{Conclusions}

1. The genus Bilabrella is easy to distinguish from all other taxa of this rank among the Habenariinae based on the specific features combination: slender stigmaphores, rostellophores and antherophores, short stamen, deeply divided, 2-lobed petals and 3-lobed labellum, heavily asymmetrical lateral sepals and the strongly retrorse backwards dorsal sepal.

2. Species boundaries within the genus involve subtle features related mostly to gynostemium morphology, petals, labellum and spur. The actual importance of these features to the taxonomy of the genus can only be ascertained by field research.

3. The majority of Bilabrella Lindl. species are found in Tanzania, mostly in savannas. Research on herbarium materials would seems to indicate that this region may be considered the centre for genus differentiation.

4. Wide distribution and abundance of many species, often collected intermediate forms between species clearly indicate that Bilabrella is relatively young genus fast evolving and remembering somewhat the Holarctis genus Dactylorhiza Neck. ex Nevski.

Acknowledgments. We would like to express our special thanks to the former Curator of K, Dr. Phillip Cribb, former Curator of W, Dr. Ernst Vitek and former Director of Museum National d'Histoire Naturelle in Paris, Prof. Philippe Morat, as well as Curators of AMES, BM, BR, BRLU, C, K, W and WU for their hospitality during our personal visits. We would like to thank also, the Curators of E, F, HBG, LBV, LISU, MA, MO, P and WAG for loan valuable materials. We are grateful to Dr. Hanna B. Margońska (HBM) and Anna Król M.Sc.(AK) for permission to using their drawings in this work and Mr. Lourens Grobler and Mr. R. Wendt for their kind permission to use colour photographs of various species of Bilabrella. Dr. G. Chiron is thanked for the Latinization of the diagnoses. These study were supported by the Polish Ministry of Education and Science (KBN grant no. 2P04C 05627 ) and the COBICE and SYNTHESYS AT-TAF-1703 projects. 


\section{References}

Bamps P. 1982. Flore D'Afrique Centrale. Repertoire des lieux de recolte. Jardin botanique national de Belgique, Bruxelles.

Bateman R. M., Hollingsworth P. M., Preston J., Yi-Bo L., Pridgeon A. M. \& Chase M. W. 2003. Molecular phylogenetics and evolution of Orchidinae and selected Habenariinae (Orchidaceae). Bot J Linn Soc 142: $1-40$.

Batista J. A. N., Borges K. S., de Faria M. W. F., Proite K., Ramalho A. J., Salazar G. A. \& van den Berg C. 2013. Molecular phylogenetics of the species-rich genus Habenaria (Orchidaceae) in the new world based on nuclear and plastid DNA sequences. Mol Phylogenet Evol 67: 95-109.

Bolus H. 1889. Contributions to South-African Botany. IV. J Linn Soc Bot 25: 156-210.

Bolus H. 1893. Icones Orchidearum Austro-Africanarum Extra-Tropicarum, W.Wesley \& Son, London 1(1): t. 17.

Bolus H. 1913. Orchids of South Africa. 3: tab. 22-24. W. Wesley \& Son, London.

Brummit R. K. 2001 World Geographical Scheme for Recording Plant Distribution. 2: 1-152. Edition Carnegic Mellon University, Pittsburgh.

la Croix I. \& Cribi P. J. 1995. Orchidaceae. 11(1): 59-148. In: G. V. Pope (ed.). Flora Zambesiaca. Whitstable Litho Printers Ltd, Whitstable.

VAN DER Cingel N. A. 2001. An atlas of Orchid pollination America, Africa, Asia and Australia. A. A. Baklema, Rotterdam, 134.

CRIBB P. J. 1989. Flora of Tropical East Africa. A.A. Baklema, Rotterdam 3: 413-651.

Du Puy D., Cribb P. J., Bosser J., Hermans J. \& Hermnas C. 1999. The Orchids of Madagascar. 150-155 pp. Kew Publishing, London.

GeErinck D. 1984. Flore d'Afrique Centrale (Zaire-RwandaBurundi). 52-142 pp. Jardin botanique national de Belgique, Meise.

Greuter W., Burdet H. M., Chaloner G., Demoulin V., Grolle R., Hawksworth D. L., Nicolson D. H., Silva P. c., Stafleu F. A., Voss E. G. \& McNeill J. 1987. International Code of Botanical Nomenclature adopted by the Fourteenth International Botanical Congress, Berlin, July-August 1987. Article 64.1. Regnum Veg. 328. 1988.

Holmgren P. K., Holmgren N. H. \& Barnett L. C. 1990. Index Herbariorum. 693 pp. New York Botanical Garden, New York.

Inda L. A., Pimentel M. \& Chase M. W. 2010. Contribution of mitochondrial cox 1 intron sequences to the phylogenetics of tribe Orchideae (Orchidaceae): do the distribution and sequence of this intron tell us something about its evolution? Taxon 59: 1053-1064.

Inda L. A., Pimentel M. \& Chase M. W. 2012. Phylogenetics of tribe Orchideae (Orchidaceae: Orchidoideae) based on combined DNA matrices: inferences regarding timing of diversification and evolution of pollination syndromes. Ann Bot London 110: 71-90.
Jin W. T., Jin X. H., Schuiteman A., Li D. Z., Xiang X. G., Huang W. C., Li J. W. \& Huang L. Q. 2014. Molecular systematics of subtribe Orchidinae and Asian taxa of Habenariinae (Orchideae, Orchidaceae) based on plastid matK, rbcL and nuclear ITS. Mol Phylogenet Evol 77: 41-53.

Kraenzlin F. 1891. Beiträge zu einer Monographie der Gattung Habenaria Willd. (Allgemeiner Teil.). Botanische Jahrbücher für Systematik, Pflanzengeschichte und Pflanzengeographie 16: 5-41.

KraenZlin F. 1901. Orchidacearum Genera et Species. 174469 pp. Mayer and Meyer, Berlin.

Kraenzlin F. 1909. Orchidaceae africanae. X. Bot. Jahrb. Syst. 43: 393 Mayer and Meyer, Berlin, 399.

Kras M. \& SzlachetKo D. L. 2008. Four new species of Bilabrella (Orchidaceae, Habenariinae) from Africa. Polish Botanical Journal 53(2): 97-101.

Kras M. \& SzlachetKo D. L. 2009. Materials to the revision of Habenariinae (Orcidaceae, Orchidoideae).9. Bilabrella. Richardiana 9(4): 157-160.

Kras M. \& SzlachetKo D. L. 2013. The genus Bilabrella Lindl. (Orchidaceae, Habenariinae): general characteristic and research history of the genus. Biodiv. Res. Conserv. 32: 9-24.

Linder H. P. \& Kurzweil H. 1999. Flora of Southern Africa. A. A. Balkema 124-132.

LiNDLEY J. 1834. Orchis foliosa. Edwards's Botanical Register 20: sub. t. 1701 .

PodBIELKowski Z. 1987. Fitogeografia części świata - Europa, Azja, Afryka. 262-338 pp. PWN, Warszawa.

Reichenbach H. G. f. 1849. Orchidiographische Beiträge. Linnaea 22: 859.

RendLe A. B. 1895. Flora of Eastern Tropical Africa. Journal of the Linnean Society. Botany 30: 393.

Rolfe R. A. 1898. Flora of Tropical Africa. L.Reeve \& Co., London, 7: 206-249.

Rolfe R. A. 1913. Flora Capensis. 3-313 pp. L. Reeve \& Co., London.

Schlechter R. 1906. Orchidaceae africanae. IV. Bot. Jahrb. Syst. 38: 146-150.

Schlechter R. 1915. Beiträge zur Flora von Afrika. XLV. Bot. Jahrb. Syst. 53: 512-519.

Stewart J. \& Campbell B. 1996. Orchids of Kenya. 127-143 pp. Timber Press Inc, Portland Oregon.

Summerhayes V. S. 1942. African Orchids. XII. Botanical Museum Leaflets [Harvard University] 10(9): 257-280.

Summerhayes V. S. 1960. African Orchids. XXVII. Kew Bulletin 14(1): 126-157.

Summerhayes V. S. 1962. African Orchids. XXVIII. Kew Bulletin 16(2): 253-314.

Summerhayes V. S. 1966. African Orchids. XXX. Kew Bulletin 20(2): 165-199.

Summerhayes V. S. 1968a. Flora of West Tropical Africa. The Whitefriars Press Ltd., London 3(1): 180-276.

Summerhayes V. S. 1968b. Flora of Tropical East Africa. 1-235 pp. Minister for Overseas Development by the Crown Agents for Oversea Governments and Administrations, London. 
SzlachetKo D. L. 1995. Systema Orchidalium. 32-35 pp. W. Szafer Institute of Botany, Polish Academy of Sciences, Kraków.

Szlachetko D. L. 2003a. Habenariinee (Orchidaceae, Orchidoideae) - Contribution to the Revision of the Subtribe. 3. Die Orchidee 54(2): 82-84.

Szlachetko D. L. 2003b. Habenariinee (Orchidaceae, Orchidoideae) - Contribution to the Revision of the Subtribe. 3. Die Orchidee 54(2): 85-87.

SzlachetKo D. L. 2003c. Habenariinee (Orchidaceae, Orchidoideae) - Contribution to the Revision of the Subtribe. 6. Die Orchidee 54(3): 70-72.

Szlachetko D. L. 2003d. Materiaux pour la revision de Habenaria (Orchidaceae, Orchidoideae) - 2. Richardiana 3(4): 153-157.

SzlachetKo D. L. 2004a. Habenariinae (Orchidaceae, Orchidoideae)-Contribution to the revision of the Subtribe. 7. Die Orchidee 55(1): 57-59.

SzlachetKo D. L. 2004b. Habenariinae (Orchidaceae, Orchidoideae)-Contribution to the revision of the Subtribe. 9. Die Orchidee 55(3): 50-51.

SzlachetKo D. L. 2004c. Habenariinae (Orchidaceae, Orchidoideae) - Contribution to the revision of the Subtribe. 10. Die Orchidee 55(4): 91-93.

SzlachetKo D. L. 2004d. Habenariinae (Orchidaceae, Orchidoideae) - Contribution to the revision of the Subtribe. 11. Die Orchidee 55(4): 93-96.

SzlachetKo D. L. 2004e. Materiaux pour la revision des Habenariinae (Orchidaceae, Orchidoideae) -4. Richardiana 4(2): 52-65.

SzlachetKo D. L. 2004f. Materiaux pour la revion des Habenariinae (Orchidaceae, Orchidoideae) - 5. Richardiana 4(3): 103-108.

SzlachetKo D. L. 2005a. Habenariinae (Orchidaceae, Orchidoideae) - Contribution to the Revision of the Subtribe. 13. Die Orchidee 56(2): 73-75.

SzlachetKo D. L. 2005b. Habenariinae (Orchidaceae, Orchidoideae) - Contribution to the Revision of the Subtribe. 14. Die Orchidee 56(3): 65-66.

SzlachetKo D. L., GóRniak M. \& Tukalıo P. 2003. Materiaux pour la revision de Habenaria (Orchidaceae, Orchidoideae) - 3. Richardiana 3(4): 158-162.

Szlachetko D. L. \& Kowalkowska A. 2007. Contributions to the orchid flora of Guinea, West Africa. Pol. Bot. Stud. 25: 1-259.

SzlachetKo D. L. \& Kras M. 2006a. Notes sur le genre Plantaginorchis (Orchidaceae, Orchidoidae, Habenariinae). Richardiana 6(1): 31-32.

Szlachetko D. L. \& Kras M. 2006b. Notes sur le genre Habenella. Richardiana 6(1): 33-39.
SzlachetKo D. L. \& Kras M. 2006c. Materiaux pour la revision taxonomique de Habenaria Willdenow (Orchidaceae, Orchidoideae). Richardiana 6(1): 40-43.

SzlachetKo D. L. \& Kras M. 2006d. Materiaux pour la revision des Habenariinae (Orchidaceae, Orchidoideae) -6. Richardiana 6(3): 139-146.

SzlachetKo D. L. \& Kras M. 2006. Materiaux pour la revision des Habenariinae (Orchidaceae, Orchidoideae) - 7. Richardiana 6(4): 178-179.

SzlachetKo D. L. \& Kras M. 2006f. Materiaux pour la revision taxonomique de Habenaria (Orchidaceae, Orchidoideae) - 8. Richardiana 6(4): 196-197.

Szlachetko D. L. \& Kras-Łapińska M. 2003. Habenariinee (Orchidaceae, Orchidoideae) - Contribution to the revision of the Subtribe. 1. Die Orchidee 54(1): 84-87.

SzlachetKo D. L. \& MargońsKa H. B. 2004. Habenariinae (Orchidaceae, Orchidoideae) - Contribution to the revision the Subtribe. 8. Die Orchidee 55(2): 40-41.

SzlachetKo D. L., Mytnik-Ejsmont J., Kras M., RutKowsKi P., Baranow P. \& GórniaK M. 2010. Orchidaceae of West-Central Africa. Vol. 1, 363 pp. Wyd. Uniw. Gdańskiego, Gdańsk.

Szlachetko D. L. \& Olszewski T. S. 1998a. Flore du Cameroun. Muséum National d'Historie Naturelle, Paris, 34(1): 102-186.

Szlachetko D. L. \& Olszewski T. S. 1998b. Renzorchis (Orchidaceae, Habenariinae), a new genus from Gabon. Adansonia 3: 323-326.

Szlachetko D. L. \& Rutkowski P. 2000. Gynostemia Orchidalium I. Acta Bot. Fennica 169: 162-165.

SzlachetKo D. L. \& SAwicka M. 2003a. Habenariinee (Orchidaceae, Orchidoideae) - Contribution to the revision of the Subtribe. 2. Die Orchidee 54(1): 88-90.

SzlachetKo D. L. \& Sawicka M. 2003b. Habenariinee (Orchidaceae, Orchidoideae) - Contribution to the revision of the Subtribe. 5. Die Orchidee 54(3): 67-69.

SzlachetKo D. L., SAwicka M. \& Kras-ŁapińSKa M. 2004. Flora du Gabon I. Museum National d'Historie Naturelle, Paris, 36: 28-46.

Thomas S. \& CribB P. J. 1996. New species of Habenaria (Orchidaceae) from Ethiopia. Kew Bull. 3(1): 145154.

www.ipni.org

http://www.tropicos.org

Willdenow K. L. 1805. Species Plantarum. Berolini, 4(1): 44.

Williamson G. 1977. Orchids of South Central Africa. 39-68 pp. J. M. Dent \& Sons Ltd., London.

Williamson G. 1980. Contribution to the Orchid Flora of South Central Africa. Pl Syst Evol 134: 53-77. 


\section{Index to scientific names}

Page numbers in boldface indicate description of species; page numbers marked with asteriks $(*)$ indicate drawings.

Acacia spp. .15

Ala Szlach. ...8

Alinorchis Szlach

Arachnaria Szlach. ............................................................

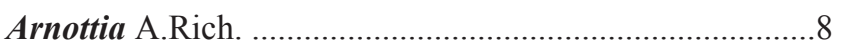

Ate Lindl. ............................................................... 8

Bertauxia Szlach. ............................................................. 8

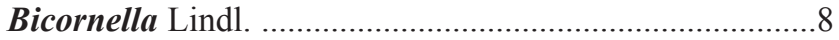

Bilabrella Lindl. .................................................................. 8

Section Antenniferae Szlach. \& Kras ................19, 28, 37

Section Biauriculatae Szlach. \& Kras .....................45, 98

Section Bilabrella ................................................45, 46

Section Bilabrellae Lindl. .......................................... 7, 9

Section Diselloidae Szlach. \& Kras .........................19, 23

Section Papillabiae Szlach. \& Kras ...........................19, 22

Section Replicatae Lindl. .......................7, 19, 45, 153, 161

Subgenus Bilabrella ...............................................19, 45

Subgenus Brevicolumna Szlach. \& Kras .....................19

Subsection Arachnoideae .....................................45, 78

Subsection Biauriculatae Szlach. \& Kras ..............46, 107

Subsection Bilabrella ...........................................45, 47

Subsection Dalzielae Szlach. \& Kras ....................46, 106

Subsection Decapterae Szlach. \& Kras ..............45, 98

Subsection Disparilae Szlach. \& Kras ................46, 103

Subsection Inaequilobae Szlach. \& Kras ...............45, 46

Subsection Martiales Szlach. \& Kras .....................45, 99

Bilabrella woodii(Schltr.) Szlach.\& Kras ...16, 28,38,39, 163

Bilabrella altior (Rendle) Szlach. \& Kras-Lap. $6,14,18,98,108,110,111,112,126,146,161$

Bilabrella anaphysema (Rchb. f.) Szlach. \& Kras-Lap. ...... $8,12,12,15,17,47,49,128,148,161$

Bilabrella angustissima (Summerh.) Szlach. \& Kras ......... $18,108,109,161$

Bilabrella antennifera (A.Rich.) Szlach. \& Kras $16,28,43,161$

Bilabrella arianae (Geerinck) Szlach. \& Kras $13,16,19,22,45,161,163$ Bilabrella bertauxiana (Szlach. \& Olsz.) Szlach. \& KrasLap......................................14, 18, 108, 128, 132, 153, 161 Bilabrella bosseriana (Szlach. \& Olsz.) Szlach. \& KrasLap $18,108,137,161$

Bilabrella burttii (Summerh.) Szlach. \& Kras-Lap. $13,18,108,12$

Bilabrella caffra (Schltr.) Szlach. \& Kras $17,47,66,67,70,71,161,163$

Bilabrella cataphysema (Rchb. f.) Szlach. \& Kras-Lap. .... $12,13,17,78,87,148,161$

Bilabrella chirensis (Rchb. f.) Szlach. \& Kras-Lap. ........... $11,13,15,19,109,138,149, \mathbf{1 5 2}, 161$

Bilabrella compta (Summerh.) Szlach. \& Kras.-Lap. ....... $16,28,33,35,36,132,161$

Bilabrella confusa (Rolfe) Szlach. \& Kras ....17, 47, 51, 161 Bilabrella cornutella (Summerh.) Szlach. \& Kras-Lap. .... 17, 47, 58, 61, 144, 161
Bilabrella cribbiana (Szlach. \& Olsz.) Szlach. \& Kras-Lap. $11,18,58,108,143,161$ Bilabrella culcifera (Rolfe) Szlach. \& Kras ...17, 47, 77, 161 Bilabrella dalzielii (Summerh.) Szlach. \& Kras

$17,106, \mathbf{1 0 7}, 161$

Bilabrelladecaptera(Rchb.f.)Szlach.\&Kras...16,45,98, 161 Bilabrella diselloides (Schltr.) Szlach. \& Kras-Lap. $16,23,24,28,161$

Bilabrella disparilis (Summerh.) Szlach. \& Kras-Lap. ......... $11,17, \mathbf{1 0 3}, 161$

Bilabrella dives (Rchb. f.) Szlach. \& Kras $11,13,16,23,25,25,26,43,125,161$

Bilabrella falcata (G.Will.) Szlach. \& Kras-Lap. $13,17,47,66,161$ Bilabrella falcicornis Lindl.

$. .6,11,17,19,47,54,66,68,161,162,163$ Bilabrella furcipetala (Schltr.) Szlach. \& Kras $15,18,108,132,161$

Bilabrella galpinii (Bolus) Szlach.\& Kras-Lap. $12,15,17,79,90,97,1$

Bilabrella haareri (Summerh.) Szlach. \& Kras-Lap. 19, 109, 129, 144, 161, 162 Bilabrella hebes (la Croix \& P.J. Cribb) Szlach. \& Kras-Lap. $.11,13,17,79,90,92,93,161$ Bilabrella hirsutitrunci (G. Will.) Szlach. \& Kras-Lap. .... $.17,78,79,161,163$

Bilabrella hochstetteriana (Kraenzl. \& Schltr.) Szlach. \& Kras 11, 17, 47, 61, 161

Bilabrella huillensis (Rchb. f.) Szlach. \& Kras-Lap. .......... $12,17,47,63,128,144,149,161$ Bilabrella humberti (Szlach. \& Olsz.) Szlach. \& KrasLap. ..........................................12, 14, 18, 108, 122, 161 Bilabrella humblotii (Rchb. f.) Szlach. \& Kras $17,47,71,76,161$

Bilabrella humilior (Rchb. f.) Szlach. \& Kras-Lap. $12,17,47,62,71,76,161,162$ Bilabrella ichneumonea (Sw.) Szlach. \& Kras-Lap. 14, 15, 17, 47, 51, 55, 60, 109, 110, 162, 163 Bilabrella inaequiloba (Schltr.) Szlach. \& Kras-Lap. ......... $16,45,46,53,162$ Bilabrella incarnata (Lyall. ex Lindl.) Szlach. \& Kras-Lap. $.13,17,47,161,162,163$ Bilabrella incurva (Rolfe) Szlach. \& Kras .. 19, 109, 146, 162 Bilabrella involuta (Bolus) Szlach. \& Kras-Lap. 13, 18, 108, 124, 139, 162

Bilabrella isoantha (Schltr.) Szlach. \& Kras-Lap. $16,28,29,32,44,162$ Bilabrella kilimanjari (Rchb. f.) Szlach. \& Kras-Lap. .... $. .12,17,99,101,125,134,162,163$ Bilabrella kornasiorum (Szlach. \& Olsz.) Szlach. \& KrasLap. $18,108, \mathbf{1 2 5}, 162$ Bilabrella kraenzliniana Kras \& Szlach. ...........18, 108, 110 Bilabrella kubangensis (Schltr.) Szlach. \& Kras-Lap. ...... 18, 108, 117, 118, 122, 162 
Bilabrella kyimbilae (Schltr.) Szlach. \& Kras-Lap. $11,12,18,40,108, \mathbf{1 1 2}, 139,146,162$ Bilabrella leucoceras (Schltr.) Szlach. \& Kras-Lap. $.18,29,40,108,117,122,124,145,162$ Bilabrella limnophila (Summerh.) Szlach. \& Kras 19, 62, 109, 149, 153, 162 Bilabrella linderi (Summerh.) Szlach. \& Kras-Lap. .18, 108, 135, 162 Bilabrella linguiformis (Summerh.) Szlach. \& Kras ........... $17,79,90,162$ Bilabrella lisowskii (Szlach.) Szlach. \& Kras-Lap. $17,47,60,162$

Bilabrella luegiana Kras \& Szlach. .18, 108, 138 Bilabrella macrostele (Summerh.) Szlach. \& Kras-Lap. 12, 13, 15, 16, 19, 67, 94, 97, 161, 162, 163 Bilabrella macrotidion (Summerh.) Szlach. \& Kras-Lap. ... 11, 18, 93, 108, 136, 162 Bilabrella maitlandii (Summerh.) Szlach. \& Kras-Lap. ..... .18, 108, 124, 162

Bilabrella martialis (Rchb. f.) Szlach. \& Kras $17,99,100,162,163$

Bilabrella marxiana (Schltr.) Szlach. \& Kras 12, 13, 16, 28, 31, 33, 34, 162 Bilabrella myodes (Summerh.) Szlach. \& Kras-Lap. $. .8,12,13,15,18,108,117,118, \mathbf{1 2 0}, 162$ Bilabrella ndiana (Rendle) Szlach. \& Kras-Lap. 16, 28, 30, 31, 97, 162, 163 Bilabrella nyikana (Rchb. f.) Szlach. \& Kras-Lap. 17, 47, 76, 78, 161, 162

Bilabrella orangana (Rchb. f.) Szlach. \& Kras $13,16,28,42,162$

Bilabrella orthocaulis (Schltr.) Szlach. \& Kras $17,79,95,162$

Bilabrella pauper (Summerh.) Szlach. \& Kras-Lap. $17,47, \mathbf{5 1}, 54,93,137,162$

Bilabrella pedicellaris (Rchb. f.) Szlach \& Kras $.12,17,47,54,162$ Bilabrella peltastes (Rchb. f.) Szlach. \& Kras-Lap. ..99, 162 Bilabrella petraea (Renz \& Grosvenor) Szlach. \& Kras-Lap. .19, 109, 145, 162

Bilabrella plectomaniaca (Rchb. f. \& S. Moore) Szlach. \& Kras-Lap. ....................................14, 19, 109, 147, 162 Bilabrella pseudokaessneriana (Kraenzl.) Szlach. \& Kras .19, 109, 147, 148, 161, 162

Bilabrella rehmannii (Bolus) Szlach. \& Kras

18, 108, 132, 134, 162

Bilabrella renziana (Szlach. \& Olsz.) Szlach. \& Kras-Lap. 14, 18, 108, 132, 133, 135, 162

Bilabrella replicata (Hochst. ex A.Rich.) Szlach. \& Kras ... $.17,47,62,163$

Bilabrella retinervis (Summerh.) Szlach. \& Kras-Lap. ... 14, 18, 29, 40, 42, 108, 129, 163

Bilabrella riparia (Renz \& Grosvenor) Szlach. \& KrasLap. 18, 108, 139, 163

Bilabrella robusta (Welw.) Szlach. \& Kras-Lap.

$13,16,23,163$

Bilabrella schimperiana (Hochst. ex A.Rich.) Szlach. \& Kras-Lap. ......11, 12, 13, 15, 17, 78, 80, 161, 162, 163 Bilabrella sochensis (Rchb. f.) Szlach. \& Kras-Lap. ........ $11,15,16,28,31,39, \mathbf{4 0}, 163$
Bilabrella stanislawii Kras \& Szlach. .....14, 18, 108, 142 Bilabrella stenophylla (Summerh.) Szlach. \& Kras .......... $. .16,28,39,42,163$

Bilabrella stolzii (Schltr.) Szlach. \& Kras-Lap. 16, 28, 29, 36, 38, 163 Bilabrella strangulans (Summerh.) Szlach. \& Kras-Lap. .. $.15,18,108,127,153,163$ Bilabrella subaequalis (Summerh.) Szlach. \& Kras-Lap. $.11,17,79,92,95,163$ Bilabrella tangheana (Geerinck \& Schaijes) Szlach. \& Kras ......................................14, 18, 108, 141, 143, 163 Bilabrella tetrapetaloides (Schltr.) Szlach. \& Kras $13,17, \mathbf{1 0 6}, 163$

Bilabrella thomsonii (Rchb. f.) Szlach. \& Kras-Lap. ......... $15,18,106,108,112,114,141,162,163$ Bilabrella tisseranti (Szlach. \& Olsz.) Szlach. \& KrasLap. 16, 28, 37, 163

Bilabrella tortilis (P.J. Cribb) Szlach. \& Kras-Lap. $15,16,28,54,163$ Bilabrella tubifolia (la Croix \& P.J. Cribb) Szlach. \& KrasLap. ..........................12, 13, 15, 18, 108, 126, 128, 163 Bilabrella ugandensis (Summerh.) Szlach. \& Kras-Lap. .. $. .17,79,94,95,96,98,163$

Bilabrella urundiensis (Summerh.) Szlach. \& Kras .......... .15, 17, 29, 47, 53, 163

Bilabrella usambarae Kras \& Szlach. .........17, 103, 105 Bilabrella vollesenii (Thomas \& P.J. Cribb) Szlach. \& Kras-Lap. ...................................15, 16, 28, 32, 34, 163 Bilabrella weberana (Schltr.) Szlach. \& Kras-Lap. $.12,17,78, \mathbf{8 8}, 98,112,120,141,161,163$ Bilabrella welwitschii (Rchb. f.) Szlach. \& Kras-Lap. $. .15,18,93,108,110,115,141,162,163$ Bilabrella williamsoni (P.J. Cribb) Szlach. \& Kras .......... $.13,16,23,28,35,44,163$

Bonatea Willd. ..8, 9

Bonatea bilabrella Lindl. $.69,161$

Bonatea incarnata Lyall. ex Lindl.

47,161

Bonatea tetrapetala Lindl. $68,69,161$

Brachystegia spp. .......15, 23, 25, 30, 41, 42, 54, 63, 64, $66,75,77,104,105,112,114,118,121,128,131,132$ Centrostigma Schltr. ..................................................... 8 Ceratopetalae Kraenzl. .................................................... 9 Ceratopetalorchis Szlach., Górn., Tukałło ............8, 9, 10 Ceratopetalorchis sanfordiana (Szlach. \& Olszewski) Szlach., Górniak \& Tukałło

Coeloglossum Lindl. . .10

Cynorkis Thouars

Dactylorhiza Neck. ..............................................6, 154

Diplomeris D.Don. .................................................... 8

Eucalyptus L.Hér. .................15, 41, 42, 77, 84, 97, 120

Fimbrorchis Szlach.

Gymnadenia R.Br.

Habenaria Willd. $6,7,8$

Habenaria altior Rendle ..............6, 98, 110, 146, 161 Habenaria anaphysema Rchb. f. .................8, 49, 161 Habenaria anaphysema Robyns \& Tournay ........128, 161 Habenaria anaphysema Robyns \& Tournay $80,107,148,161$

Habenaria angustissima Summerh. 109,161

Habenaria antennifera A.Rich. $7,28,43,161$ 
Habenaria arianae Geerinck in Geerinck \& Coutrez ......... 22,161

Habenaria bertauxiana Szlach. \& Olsz. 128,161

Habenaria bilabrella (Lindl.) Kraenzl. 69,161

Habenaria bosseriana Szlach. \& Olsz. 137,161

Habenaria brachyceratitis Willd.

....6

Habenaria burttii Summerh. 123,161

Habenaria caffra Schltr. $67,70,161$

Habenaria cataphysema Rchb. f. $.87,161$

Habenaria chirensis Rchb. f.

152,161

Habenaria compta Summerh.

33, 161

Habenaria confusa Rolfe

$.51,161$

Habenaria cornutella Summerh.

58,161

Habenaria cribbiana Szlach. \& Olsz.

143,161

Habenaria culcifera Rolfe

$.77,161$

Habenaria dactylostigma Kraenzl.

Habenaria dalzielii Summerh.

$106,107,161$

Habenaria decaptera Rchb. f.

$.98,161$

Habenaria diptera Schltr.

.47, 161

Habenaria diselloides Schltr.

$23,24,37,161$

Habenaria disparilis Summerh.

103,161

Habenaria dives Rchb. f.

$26,39,161$

Habenaria elatior Schltr.

76,161

Habenaria falcata G.Will.

66,161

Habenaria falcicornis (Lindl.) Bolus ........68, 70, 76, 161

Habenaria furcipetala Schltr. .....................114, 132, 161

Habenaria furcipetala var. andromaniaca Schltr. ..132, 161

Habenaria galpinii Bolus

97, 161

Habenaria genuflexa Rendle .........7, 50, 80, 85, 154, 161

Habenaria haareri Summerh. ..............................144, 161

Habenaria hebes la Croix \& P.J. Cribb .............93, 161

Habenaria hirsutitrunci G. Will. ......................79, 161

Habenaria hochstetteriana Kraenzl. \& Schltr. .....61, 161

Habenaria huillensis Rchb. f. .....................63, 133, 161

Habenaria huillensis Schltr. var. weberana (Schltr.)

Geerinck

89,161

Habenaria huillensis sensu Geerinck \& Coutrez ...19, 161

Habenaria humberti Szlach. \& Olsz. ..................122, 161

Habenaria humblotii Rchb. f. ..............................71, 161

Habenaria humilior Rchb. f. ...........32, 33, 71, 76, 161

Habenaria humilior Rchb. f. var. brevicalcarata Rendle ...

114,162

Habenaria humilior sensu Geerinck

$.72,162$

Habenaria ichneumonea (Sw.) Lindl.

$.55,162$

Habenaria ichneumonea Kraenzl.

80,162

Habenaria inaequiloba Schltr.

$7,46,162$

Habenaria incarnata (Lyall. ex Lindl.) Rchb. f.

$.8,32,47,162$

Habenaria incurva Rolfe

$98,146,162$

Habenaria ingrata Rendle

31,162

.124, 162

Habenaria involuta Bolus

$29,49,145,162$

Habenaria johannae Kraenzl. .................................153

Habenaria kaessneriana Kraenzl. .......................148, 162

Habenaria keiliana Kraenzl. ............................115, 162

Habenaria kilimanjari Rchb. f. .......................19, 101, 162

Habenaria kornasiorum Szlach. \& Olsz. ............125, 162

Habenaria kubangensis Schltr. .....................34, 118, 162

Habenaria kyimbilae Schltr.

$112,115,162$

Habenaria leptobrachiata Ridley

$.43,162$
Habenaria leptostigma Schltr.

115,162

Habenaria leucoceras Schltr.

117,162

Habenaria limnophila Summerh.

$7,149,162$

Habenaria linderi Summerh.

135,162

Habenaria linguiformis Summerh. .......................90, 162

Habenaria lisowskii Szlach. .................................60, 162

Habenaria lutaria Schltr. ...................................100, 162

Habenaria lykipiensis Rolfe ................................111, 112

Habenaria macroceratitis Willd. ..................................6

Habenaria macrostele Summerh. ............19, 20, 22, 162

Habenaria macrotidion Summerh. ......................136, 162

Habenaria maitlandii Summerh. ........................124, 162

Habenaria martialis Rchb. f. ......................99, 100, 162

Habenaria marxiana Schltr. ..................................34, 162

Habenaria microsaccus Kraenzl. ........................135, 162

Habenaria mundtii Kraenzl. .............................69, 162

Habenaria myodes Summerh. .........................120, 162

Habenaria ndiana Rendle ..............................7, 31, 162

Habenaria nyikana Rchb. f. ..............................76, 162

Habenaria orangana Rchb. f. ..........................42, 162

Habenaria orthocaulis Schltr. ............................95, 162

Habenaria pantothrix Kraenzl. ex Engl. ..............43, 162

Habenaria pauper Summerh. .........................7, 52, 162

Habenaria pedicellaris Kraenzl. .................32, 144, 162

Habenaria pedicellaris Rchb. f. ...........................54, 162

Habenaria pedicellaris Rolfe ..............................54, 162

Habenaria peltastes Rchb. f. .......................47, 99, 162

Habenaria petraea Renz \& Grosvenor ............145, 162

Habenaria petromedusa Webb. in Hook. .................153

Habenaria plectomaniaca Rchb. f. \& S. Moore ...147, 162

Habenaria pristichila Kraenzl. ...................................153

Habenaria ranicolorata Rolfe ...........................111, 162

Habenaria rehmannii Bolus .........................76, 134, 162

Habenaria renziana Szlach. \& Olsz. .................133, 162

Habenaria replicata Hochst. ...............................62, 163

Habenaria retinervis Summerh. ...................45, 129, 163

Habenaria riparia Renz \& Grosvenor ...............139, 163

Habenaria robusta Welw. ex Rchb. f. ...............23, 163

Habenaria rutenbergiana Kraenzl. .......................47, 163

Habenaria schimperiana Hochst. ex A.Rich.

..7, 50,78,80, 163

Habenaria sigillum Thouars ........................................153

Habenaria simense Rchb. f. ........................7, 43, 163

Habenaria similis Schltr. ................................31, 163

Habenaria sochensis Rchb. f. .....35, 40, 48, 132, 163

Habenaria $s p$. Williamson .................................22, 163

Habenaria sp. Williamson ..................................79, 163

Habenaria stenoloba Schltr. ..........................80, 84, 163

Habenaria stenophylla Summerh. ......................39, 163

Habenaria stolzii Schltr. ...........................26, 36, 163

Habenaria strangulans Summerh. .....................127, 163

Habenaria subaequalis Summerh. .......................92, 163

Habenaria tangheana Geerinck \& Schaijes ....141, 163

Habenaria tetrapetala (Lindl.) Rchb. f. ..............69, 163

Habenaria tetrapetala Kraenzl. ex Rolfe .......67, 68, 163

Habenaria tetrapetaloides Schltr. .................76, 106, 163

Habenaria theodorii Kraenzl. ...........................101, 163

Habenaria thomsonii Rchb. f. apud Oliv. ....111, 114, 163

Habenaria tisseranti Szlach. \& Olsz. ................37, 163

Habenaria tortilis P.J. Cribb .............................28, 163 
Habenaria tournayana Geerinck

19,163

Habenaria tubifolia la Croix \& P.J. Cribb

126,163

Habenaria ugandensis Summerh.

96,163

Habenaria uhligii Kraenzl.

100,163

Habenaria urundiensis Summerh.

$.53,163$

Habenaria vollesenii Thomas \& P.J. Cribb

32,163

Habenaria weberana Schltr.

89,163

Habenaria welwitschii Rchb. f.

$53,115,163$

Habenaria williamsonii P.J. Cribb

44,163

Habenaria woodii Schltr.

38,163

Habenariinae Benth $5,8,9,11,13,15$

Habenella Small ..8

Kraenzlinorchis Szlach.

Kryptostoma (Summerh.) Geerinck

...8

Kusibabella Szlach.

Lowiorchis Szlach.

Macrura Szlach. \& Sawicka

Medusorchis Szlach.

Megalorchis H.Perr.

Mirandorchis Szlach. \& Kras-Lap.

Monadeniorchis Szlach. \& Kras

Montolivaea Rchb. f.

Ochyrorchis Szlach.
Orchidaceae Juss. $.7,8$

Orchidales Bromhaed ..........................................6, 8

Orchidoideae ....................................................... 8

Orchis L. ......................................................................6

Orchis ichneumonea Sw. ...................................56, 163

Physoceras Schltr. .......................................................... 8

Piperia Rydb. ..........................................................6

Plantaginorchis Szlach. ................................................ 8

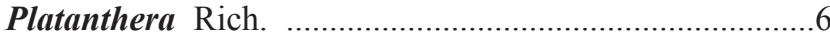

Platycoryne Rchb. f. ...................................... 8, 11, 37

Platycorynoides Szlach. .................................8, 10, 11

Podandriella Szlach. .................................................... 8

Pseudocoeloglossum Szlach. ........................................ 8

Pseudohemipilia Szlach. .......................................... 8

Renzorchis Szlach. \& Olszewski ................8, 9, 10, 45

Roeperocharis Rchb. f. ............................................. 8

Schlechterorchis Szlach. .............................................. 8

Senghasiella Szlach. .....................................................8

Smithanthe Szlach. \& Marg. .........................................8

Trachypetalum Szlach. \& Sawicka ..........................8

Tsaiorchis Tang. \& Wang. .........................................8

Uapaca spp. ..................................15, 41, 54, 75, 131

Veyretella Szlach. \& Olszewski ......................................8 
Index of synonyms

Bonatea bilabrella Lindl. = Bilabrella falcicornis Lindl.

Bonatea incarnata Lyall. ex Lindl. = Bilabrella incarnata (Lyall. ex Lindl.) Szlach. \& Kras-Lap.

Bonatea tetrapetala Lindl. = Bilabrella falcicornis Lindl.

Habenaria Willd. sect. Replicatae Kraenzl. = Bilabrella Lindl.

Habenaria altior Rendle $=$ Bilabrella altior $($ Rendle $)$ Szlach. \& Kras-Lap.

Habenaria anaphysema Rchb. f. = Bilabrella anaphysema (Rchb. f.) Szlach. \& Kras-Lap.

Habenaria anaphysema Robyns \& Tournay = Bilabrella schimperiana (Hochst. ex A. Rich.) Szlach. \& Kras-Lap.

Habenaria angustissima Summerh. = Bilabrella angustissima (Summerh.) Szlach. \& Kras

Habenaria antennifera A. Rich. = Bilabrella antennifera (A. Rich.) Szlach. \& Kras

Habenaria arianae Geerinck = Bilabrella arianae (Geerinck) Szlach. \& Kras

Habenaria bertauxiana Szlach. \& Olsz. = Bilabrella bertauxiana (Szlach. \& Olsz.) Szlach. \& Kras-Lap.

Habenaria bilabrella (Lindl.) Kraenzl. = Bilabrella falcicornis Lindl.

Habenaria bosseriana Szlach. \& Olsz. = Bilabrella bosseriana (Szlach. \& Olsz.) Szlach. \& Kras-Lap.

Habenaria burttii Summerh. = Bilabrella burttii (Summerh.) Szlach. \& Kras-Lap.

Habenaria caffra Schltr. = Bilabrella caffra (Schltr.) Szlach. \& Kras

Habenaria cataphysema Rchb. f. = Bilabrella cataphysema (Rchb. f.) Szlach. \& Kras-Lap.

Habenaria chirensis Rchb. f. = Bilabrella chirensis (Rchb. f.) Szlach. \& Kras-Lap.

Habenaria compta Summerh. = Bilabrella compta (Summerh.) Szlach. \& Kras.-Lap.

Habenaria confusa Rolfe = Bilabrella confusa (Rolfe) Szlach. \& Kras

Habenaria cornutella Summerh. = Bilabrella cornutella (Summerh.) Szlach. \& Kras-Lap.

Habenaria cribbiana Szlach. \& Olsz. = Bilabrella cribbiana (Szlach. \& Olsz.) Szlach. \& Kras-Lap.

Habenaria culcifera Rolfe = Bilabrella culcifera (Rolfe) Szlach. \& Kras

Habenaria dalzielii Summerh. = Bilabrella dalzielii (Summerh.) Szlach. \& Kras
Habenaria decaptera Rchb. f. = Bilabrella decaptera $($ Rchb. f.) Szlach. \& Kras

Habenaria diptera Schltr. = Bilabrella incarnata $($ Lyall. ex Lindl.) Szlach. \& Kras-Lap.

Habenaria diselloides Schltr. $=$ Bilabrella diselloides $($ Schltr.) Szlach. \& Kras-Lap.

Habenaria disparilis Summerh. = Bilabrella disparilis (Summerh.) Szlach. \& Kras-Lap.

Habenaria dives Rchb. f. $=$ Bilabrella dives (Rchb. f.) Szlach. \& Kras

Habenaria elatior Schltr. = Bilabrella nyikana (Rchb. f.) Szlach. \& Kras-Lap.

Habenaria falcata G.Will. = Bilabrella falcata (G.Will.) Szlach. \& Kras-Lap.

Habenaria falcicornis (Lindl.) Bolus = Bilabrella falcicornis Lindl.

Habenaria furcipetala Schltr. = Bilabrella furcipetala (Schltr.) Szlach. \& Kras

Habenaria furcipetala var. andromaniaca Schltr. = Bilabrella furcipetala (Schltr.) Szlach. \& Kras

Habenaria galpinii Bolus = Bilabrella galpinii (Bolus) Szlach.\& Kras-Lap.

Habenaria genuflexa Rendle = Bilabrella schimperiana (Hochst. ex A. Rich.) Szlach. \& Kras-Lap.

Habenaria haareri Summerh. = Bilabrella haareri (Summerh.) Szlach. \& Kras-Lap.

Habenaria hebes la Croix \& P. J. Cribb = Bilabrella hebes (la Croix \& P. J. Cribb) Szlach. \& Kras-Lap.

Habenaria hirsutitrunci G. Will. = Bilabrella hirsutitrunci (G. Will.) Szlach. \& Kras-Lap.

Habenaria hochstetteriana Kraenzl. \& Schltr. = Bilabrella hochstetteriana (Kraenzl. \& Schltr.) Szlach. \& Kras

Habenaria huillensis Rchb. f. = Bilabrella huillensis (Rchb. f.) Szlach. \& Kras-Lap.

Habenaria huillensis Schltr. var. weberana (Schltr.) Geerinck = Bilabrella weberana (Schltr.) Szlach. \& Kras-Lap.

Habenaria huillensis sensu Geerinck \& Coutrez = Bilabrella macrostele (Summerh.) Szlach. \& Kras-Lap.

Habenaria humberti Szlach. \& Olsz. = Bilabrella humberti (Szlach. \& Olsz.) Szlach. \& Kras-Lap.

Habenaria humblotii Rchb. f. = Bilabrella humblotii (Rchb. f.) Szlach. \& Kras

Habenaria humilior Rchb. f. = Bilabrella humilior (Rchb. f.) Szlach. \& Kras-Lap.

Habenaria humilior Rchb. f. var. brevicalcarata Rendle = Bilabrella thomsonii (Rchb. f.) Szlach. \& Kras-Lap. 
Habenaria humilior sensu Geerinck = Bilabrella humilior (Rchb. f.) Szlach. \& Kras-Lap.

Habenaria ichneumonea $($ Sw.) Lindl. = Bilabrella ichneumonea (Sw.) Szlach. \& Kras-Lap.

Habenaria ichneumonea Kraenzl. = Bilabrella schimperiana (Hochst. ex A. Rich.) Szlach. \& Kras-Lap.

Habenaria inaequiloba Schltr. = Bilabrella inaequiloba (Schltr.) Szlach. \& Kras-Lap.

Habenaria incarnata (Lyall. ex Lindl.) Rchb. f. = Bilabrella incarnata (Lyall. ex Lindl.) Szlach. \& Kras-Lap.

Habenaria incurva Rolfe = Bilabrella incurva (Rolfe) Szlach. \& Kras

Habenaria ingrata Rendle $=$ Bilabrella ndiana $($ Rendle $)$ Szlach. \& Kras-Lap.

Habenaria involuta Bolus = Bilabrella involuta (Bolus) Szlach. \& Kras-Lap.

Habenaria isoantha Schltr. = Bilabrella isoantha (Schltr.) Szlach. \& Kras-Lap.

Habenaria kaessneriana Kraenzl. = Bilabrella pseudokaessneriana (Kraenzl.) Szlach. \& Kras

Habenaria keiliana Kraenzl. = Bilabrella welwitschii $($ Rchb.

f.) Szlach. \& Kras-Lap.

Habenaria kilimanjari Rchb. f. = Bilabrella kilimanjari (Rchb. f.) Szlach. \& Kras-Lap.

Habenaria kornasiorum Szlach. \& Olsz. = Bilabrella kornasiorum (Szlach. \& Olsz.) Szlach. \& Kras-Lap.

Habenaria kubangensis Schltr. = Bilabrella kubangensis (Schltr.) Szlach. \& Kras-Lap.

Habenaria kyimbilae Schltr. = Bilabrella kyimbilae (Schltr.) Szlach. \& Kras-Lap.

Habenaria leptobrachiata Ridley = Bilabrella antennifera (A. Rich.) Szlach. \& Kras

Habenaria leptostigma Schltr. = Bilabrella welwitschii (Rchb. f.) Szlach. \& Kras-Lap.

Habenaria leucoceras Schltr. = Bilabrella leucoceras (Schltr.) Szlach. \& Kras-Lap.

Habenaria limnophila Summerh.= Bilabrella limnophila (Summerh.) Szlach. \& Kras

Habenaria linderi Summerh. = Bilabrella linderi (Summerh.) Szlach. \& Kras-Lap.

Habenaria linguiformis Summerh. = Bilabrella linguiformis (Summerh.) Szlach. \& Kras

Habenaria lisowskii Szlach. = Bilabrella lisowskii (Szlach.) Szlach. \& Kras-Lap.

Habenaria lutaria Schltr. = Bilabrella martialis (Rchb. f.) Szlach. \& Kras

Habenaria lykipiensis Rolfe = Bilabrella altior (Rendle) Szlach. \& Kras-Lap.
Habenaria macrostele Summerh. = Bilabrella macrostele (Summerh.) Szlach. \& Kras-Lap.

Habenaria macrotidion Summerh. = Bilabrella macrotidion (Summerh.) Szlach. \& Kras-Lap.

Habenaria maitlandii Summerh. = Bilabrella maitlandii (Summerh.) Szlach. \& Kras-Lap.

Habenaria martialis Rchb. f. = Bilabrella martialis $($ Rchb. f.) Szlach. \& Kras

Habenaria marxiana Schltr. = Bilabrella marxiana (Schltr.) Szlach. \& Kras

Habenaria microsaccus Kraenzl. = Bilabrella linderi (Summerh.) Szlach. \& Kras-Lap.

Habenaria mundtii Kraenzl. = Bilabrella falcicornis Lindl.

Habenaria myodes Summerh. = Bilabrella myodes (Summerh.) Szlach. \& Kras-Lap.

Habenaria ndiana Rendle = Bilabrella ndiana (Rendle) Szlach. \& Kras-Lap.

Habenaria nyikana Rchb. f. = Bilabrella nyikana (Rchb. f.) Szlach. \& Kras-Lap.

Habenaria orangana Rchb. f. $=$ Bilabrella orangana $($ Rchb. f.) Szlach. \& Kras

Habenaria orthocaulis Schltr. = Bilabrella orthocaulis (Schltr.) Szlach. \& Kras

Habenaria pantothrix Kraenzl. ex Engl. = Bilabrella antennifera (A. Rich.) Szlach. \& Kras

Habenaria pauper Summerh. = Bilabrella pauper (Summerh.) Szlach. \& Kras-Lap.

Habenaria pedicellaris Kraenzl. = Bilabrella haareri (Summerh.) Szlach. \& Kras-Lap.

Habenaria pedicellaris Rchb. f. = Bilabrella pedicellaris (Rchb. f.) Szlach \& Kras

Habenaria pedicellaris Rolfe $=$ Bilabrella pedicellaris $(\mathrm{Rchb}$. f.) Szlach \& Kras

Habenaria peltastes Rchb. f. = Bilabrella peltastes $($ Rchb. f.) Szlach. \& Kras-Lap.

Habenaria petraea Renz \& Grosvenor = Bilabrella petraea (Renz \& Grosvenor) Szlach. \& Kras-Lap.

Habenaria plectomaniaca Rchb. f. \& S. Moore= Bilabrella plectomaniaca (Rchb. f. \& S. Moore) Szlach. \& Kras-Lap.

Habenaria ranicolorata Rolfe $=$ Bilabrella altior $($ Rendle $)$ Szlach. \& Kras-Lap.

Habenaria rehmannii Bolus $=$ Bilabrella rehmannii $($ Bolus $)$ Szlach. \& Kras

Habenaria renziana Szlach. \& Olsz. = Bilabrella renziana (Szlach. \& Olsz.) Szlach. \& Kras-Lap.

Habenaria replicata Hochst. ex A. Rich. = Bilabrella replicata (Hochst. ex A. Rich.) Szlach. \& Kras

Habenaria retinervis Summerh. = Bilabrella retinervis (Summerh.) Szlach. \& Kras-Lap. 
Habenaria riparia Renz \& Grosvenor = Bilabrella riparia (Renz \& Grosvenor) Szlach. \& Kras-Lap.

Habenaria robusta Welw. ex Rchb. f. = Bilabrella robusta (Welw.) Szlach. \& Kras-Lap.

Habenaria rutenbergiana Kraenzl. = Bilabrella incarnata (Lyall. ex Lindl.) Szlach. \& Kras-Lap.

Habenaria schimperiana Hochst. ex A. Rich. = Bilabrella schimperiana (Hochst. ex A. Rich.) Szlach. \& Kras-Lap.

Habenaria simense Rchb. f. = Bilabrella antennifera (A. Rich.) Szlach. \& Kras

Habenaria similis Schltr. = Bilabrella ndiana (Rendle) Szlach. \& Kras-Lap.

Habenaria sochensis Rchb. f. = Bilabrella sochensis $($ Rchb. f.) Szlach. \& Kras-Lap.

Habenaria sp. Williamson = Bilabrella arianae $($ Geerinck $)$ Szlach. \& Kras

Habenaria sp. Williamson = Bilabrella hirsutitrunci (G. Will.) Szlach. \& Kras-Lap.

Habenaria stenoloba Schltr.= Bilabrella schimperiana (Hochst. ex A. Rich.) Szlach. \& Kras-Lap.

Habenaria stenophylla Summerh. = Bilabrella stenophylla (Summerh.) Szlach. \& Kras

Habenaria stolzii Schltr. = Bilabrella stolzii (Schltr.) Szlach. \& Kras-Lap.

Habenaria strangulans Summerh. = Bilabrella strangulans (Summerh.) Szlach. \& Kras-Lap.

Habenaria subaequalis Summerh.= Bilabrella subaequalis (Summerh.) Szlach. \& Kras-Lap.

Habenaria tangheana Geerinck \& Schaijes = Bilabrella tangheana (Geerinck \& Schaijes) Szlach. \& Kras

Habenaria tetrapetala (Lindl.) Rchb. f. = Bilabrella falcicornis Lindl.

Habenaria tetrapetala Kraenzl. ex Rolfe = Bilabrella caffra (Schltr.) Szlach. \& Kras
Habenaria tetrapetaloides Schltr. = Bilabrella tetrapetaloides (Schltr.) Szlach. \& Kras

Habenaria theodorii Kraenzl. = Bilabrella kilimanjari (Rchb. f.) Szlach. \& Kras-Lap.

Habenaria thomsonii Rchb. f. apud Oliv. = Bilabrella thomsonii (Rchb. f.) Szlach. \& Kras-Lap.

Habenaria tisseranti Szlach. \& Olsz. = Bilabrella tisseranti (Szlach. \& Olsz.) Szlach. \& Kras-Lap.

Habenaria tortilis P. J. Cribb = Bilabrella tortilis (P. J. Cribb) Szlach. \& Kras-Lap.

Habenaria tournayana Geerinck = Bilabrella macrostele (Summerh.) Szlach. \& Kras-Lap.

Habenaria tubifolia la Croix \& P. J. Cribb =Bilabrella tubifolia (la Croix \& P. J. Cribb) Szlach. \& Kras-Lap.

Habenaria ugandensis Summerh. = Bilabrella ugandensis (Summerh.) Szlach. \& Kras-Lap.

Habenaria uhligii Kraenzl. = Bilabrella martialis (Rchb. f.) Szlach. \& Kras

Habenaria urundiensis Summerh. = Bilabrella urundiensis (Summerh.) Szlach. \& Kras

Habenaria vollesenii Thomas \& P. J. Cribb $=$ Bilabrella vollesenii (Thomas \& P. J. Cribb) Szlach. \& Kras-Lap.

Habenaria weberana Schltr. = Bilabrella weberana (Schltr.) Szlach. \& Kras-Lap.

Habenaria welwitschii Rchb. f. = Bilabrella welwitschii (Rchb. f.) Szlach. \& Kras-Lap.

Habenaria williamsoni $\mathrm{P}$. J. Cribb = Bilabrella williamsoni (P. J. Cribb) Szlach. \& Kras

Habenaria woodii Schltr. = Bilabrella woodii (Schltr.) Szlach. \& Kras

Orchis ichneumonea Sw. = Bilabrella ichneumonea (Sw.) Szlach. \& Kras-Lap. 


\section{Index to numbered collections}

The number is refer to the corresponding species in the text and in the numerical list of species presented above

Acocks 13361 (dives).

Adam 238 (kyimbilae); 302 (ichneumonea); 12745 (schimperiana); 14579 (pauper); 14745 (ichneumonea); 15230 (schimperiana); 24343 (schimperiana); 24677 (schimperiana); 29966 (schimperiana); 29971 (schimperiana).

Adames 82 (schimperiana); 252 (schimperiana); 291 (angustissima); 326 (schimperiana).

Adams 33 (plectomaniaca); 34 (plectomaniaca).

Adamson 437 (humilior); 449 (humilior).

Afzelius s.n. (ichneumonea).

Agrcenltnal 1 (plectomaniaca).

Ake Assi 2630 (ichneumonea).

Allen 489 (schimperiana); 532 (humilior).

Allison 14 (orangana).

Andrews 1838 (humilior).

Antunes 14 - fragment (huillensis); 14-fragment (strangulans).

Arbonnier 99 (macrostele); 152 (pauper); 192 (arianae); 222 (macrostele); 317 (ndiana); 321 (macrostele); 331 (macrostele); 377 (huillensis); 378 (pauper); 383 (huillensis); 791 (ndiana).

Archbold 285 (plectomaniaca); 3005 (plectomaniaca).

Ash 514 (macrostele); 706 (myodes); 1105 (limnophila); 2600 (schimperiana); 3129 (schimperiana); 3654 (schimperiana).

Astle 1560 (schimperiana).

Attims 20 (schimperiana); 75 (cornutella).

Auchlsed 275 (kilimanjari).

Avdru 1279 (weberana).

Baasley 43 (disparilis).

Baequet 176 (myodes).

Bagshawe 461 (humilior); s.n. (hochstetteriana); s.n. (humilior).

Bakes 33 (linderi).

Baldwin 9434 (schimperiana); 9802 (ichenumonea).

Ball 188 (anaphysema); 214 (macrostele); 236 (46247) (falcicornis); 237 (46248) (subaequalis); 252 (humilior); 490 (disparilis); 502 (SRGH 50243) (galpinii); 536 (50845) (myodes); 807 (weberana); 46249 (humilior).

Bally \& Hardy 10761 (37).

Bally 2244 (altior); 2430 (ndiana); 5134 (limnophila); B7842 (limnophila); 7870 (ndiana); 7871 (ndiana); 13638 (altior); 131478 (humilior).
Bamps \& Malaisse 8264 (bosseriana); 8381 (arianae); 8490 (disparilis).

Bamps 7149 (dives).

Bamps, Martins \& Matos 4596 (retinervis).

Band 44 (galpinii).

Baron 13 (cataphysema); 213 (incarnata); 2253 (incarnata); 6092 (incarnata).

Bates 1439 (cornutella); s.n. (schimperiana).

Batten-Poole 183 (huillensis); 309 (schimperiana).

Baum 59 (disparilis); 425 (compta); 425a (kubangensis); $425 \mathrm{~b}$ (ichneumonea).

Baur 632 (dives); 816 (falcicornis).

Bax 408 (humilior).

Beals 372 (retinervis).

Beasley 67 (subaequalis).

Beesley 170 (myodes).

Benson 4 (myodes); 11 (humilior); 59 (anaphysema); 60 (anaphysema); 106 (schimperiana); 1238 (kyimbilae); 1239 (kyimbilae); 1242 (myodes); 1245 (kyimblae); 1246 (kyimbilae); 1261 (kyimbilae).

Bequaert 6196 (humilior).

Bequeathed 311 (78); 1967 (ichneumonea).

Bester 2170 (falcicornis); 2177 (dives); 2182 (dives); 2195 (falcicornis).

Beuson 232 (sochensis).

Bickford 7764 (thomsonii).

Bidgood \& Congdon 2207 (humilior).

Bidgood \& Keeley 356 (welwitschii); 385 (humilior).

Bidgood, Congdon, Vollesen 2137 (kyimbilae).

Bidgood, Mbago \& Vollesen 2582 (schimperiana).

Bingham \& Peters 12132 (schimperiana).

Bingham \& Beel 12050 (anaphysema).

Bjornstad 1510 (schimperiana).

Blacmore, Brummitt \& Banda 424 (macrostele).

Blake 1418 (linderi); 2577 (schimperiana); 5996 (kilimanjari).

Boardman 338 (caffra).

Bogdon 10192 (limnophila).

Bolus 477 (huillensis); 12309 (rehmannii); s.n. (falcicornis).

Bos 2264 (schimperiana).

Bossche s.n. (cornutella). 
Bowhen s.n. (falcicornis).

Bredo 3686 (kilimanjari).

Breds 3686 (kilimanjari); 5904 (kilimanjari).

Breyne 5916 (disparilis).

Brockman 194 (replicata).

Brodhurst Hill 27 (humilior).

Broun 330 (cornutella).

Brown 142 (schimperiana); 18101 (caffra).

Brummitt, Seyani \& Patel 14949 (schimperiana).

Brummitt \& Banda 9832 (myodes).

Brummitt \& Drummond 15855 (huillensis).

Brummitt 1210 (petraea); 9140 (macrostele); 9183 (sochensis); 9299 (disparilis); 9644 (humilior); 9788 (leucoceras); 10471 (kyimbilae).

Brummitt, Chisumpa \& Polhill 14193 (kilimanjari).

Brummitt, Polhill \& Benda 16051 (macrostele); 16079 (humilior); 16369 (humilior).

Brummitt, Polhill \& Chisumpa 13857 (retinervis); 14102 (retinervis); 14149 (retinervis).

Brummitt, Polhill \& Patel 15939 (macrostele).

Brummitt, Seyani \& Patel 14949 (schimperiana).

Bryant s.n. (huillensis).

Brynaert 606 (disparilis); s.n. (disparilis).

Buchanan 14 (dives); 302 (schimperiana); 306 (macrostele); 910 (macrostele); 1315 (dives); 1413 (dives); 14455 (dives).

Bullock 2139 (disparilis); 2453 (kilimanjari); 2669 (schimperiana); 2767 (kyimbilae); 3686 (sochensis).

Bunting 70 (anaphysema).

Burchell 5178 (falcicornis).

Burger 510 (schimperiana).

Burgess 15 (ndiana).

Burrows 718 (disparilis).

Burtt 2990 (welwitschii); 2995 (welwitschii); 3209 (altior); 4624 (burttii); 4625 (schimperiana).

Bytebier \& Sakwa 2540 (welwitschii).

Bytebier 2223 (dives).

Caille 2 (pauper); 8 (ichneumonea); 14966 (schimperiana).

Callens 3811 (anaphysema).

Chaessens 638 (humilior).

Chancellor 16 (pauper); 95 (huillensis).

Chaneella 94 (limnophila).

Chapman 1 (pauper); 18 (FHI 70818) (schimperiana); 1821 (schimperiana).
Chase 5217 (disparilis); 6015A (subaequalis); 6050 (macrostele); 6374 (sochensis); 7078 (schimperiana); 7271a (macrostele); $7271 \mathrm{~b}$ (schimperiana); 7293 (strangulans); 7293A (strangulans); 7908 (nyikana); 7958 (nyikana); 7966 (nyikana); 8382 (subaequalis); 50818 (huillensis).

Cheesman s.n. (humilior); s.n. (schimperiana).

Chevalier 2555 (schimperiana); 5375 (huillensis); 6052 (huillensis); 9484 (chirensis); 18802 (schimperiana); 34172 (schimperiana); s.n. (ichneumonea).

CNAD 1909 (schimperiana); 1969 (schimperiana).

Compton 26595 (caffra).

Cooper 1093 (orangana); 1096 (orangana); 1098 (orangana); 1099 (dives); s.n. (falcicornis).

Copley 121 (altior); B599 (humilior).

Corby 759 (35973) (anaphysema).

Corly 355 (schimperiana).

Cremers 1148 (schimperiana).

Cribb \& Grey-Wilson 10547 (welwitschii); 10799 (tortilis).

Cribb, Grey-Wilson \& Mwasumbi 11399 (kilimanjari); 11409 (ichneumonea); 11423 (humilior).

Crook 445 (humilior); 447 (41632) (macrostele).

Cuchlsald 815 (plectomaniaca).

Culver 28 (caffra); 74 (rehmannii).

Curle \& Schelpe 12 (schimperiana); 13 (humilior); 57 (humilior).

Cuthbert 28 (diselloides).

Dale 10 (84998) (nyikana); 2846 (schimperiana); 3170 (limnophila); 3547 (kilimanjari).

Dalziell 222 (dalzielii).

Dandon 112 (schimperiana).

Dandus 355 (huillensis).

Daneloj 372 (anaphysema).

Dao 22/17/5 (plectomaniaca).

Daramola \& Ekwuno FHI 62970 (limnophila).

Davies s.n. (schimperiana).

Davis 9 (linderi); 10 (humilior); 11 (schimperiana).

De Wild 2806 (22718) (subaequalis); 4455 (subaequalis).

De Wilde \& Gilbert 272 (schimperiana).

De Wilde 700 (kilimanjari); 5419 (humilior); 5428 (chirensis); 6847 (burttii); 6998 (schimperiana); 7092 (schimperiana); 7167 (schimperiana); 7502 (humilior); 7503 (humilior); 7504 (schimperiana); 7552 (schimperiana); 7584 (chirensis); 7829 (humilior); s.n. (schimperiana).

De Wilde, Arends \& de Bruijn 9160 (schimperiana); s.n. (schimperiana). 
De Witte 1347 (disparilis); 3306 (disparilis); 5079 (disparilis); 5205 (kilimanjari); 5254 (kilimanjari).

Decary 6165 (incarnata).

Deighton 2064 (ichneumonea); 2173 (pp) (dalzielii); 2173a (dalzielii); 3044 (schimperiana); 3766 (schimperiana).

Delarge 71 (arianae); 102 (arianae).

Deman 45 (schimperiana).

Descamps s.n. (schimperiana).

Descoings 5911 (cornutella).

Detilleux 389 (cornutella).

Deveyre 500 (schimperiana).

Dillewijn 100 (schimperiana).

Dinklagle 1768 (schimperiana); 2984 (schimperiana).

Dordge 5573 (dives).

Dovigneaud 5254 (huillensis); 30270 (huillensis).

Dowsett-Lemaire 195 (anaphysema); 233 (anaphysema); 276 (diselloides); 338 (hirsutitrunci); 355 (hirsutitrunci); 356 (sochensis); 359 (diselloides); 607 (retinervis); 632 (sochensis); 713 (kyimbilae); 714 (kyimbilae).

Dowson 376 (limnophila).

Drege 64 (falcicornis); 4566 (falcicornis).

Drewe s.n. (57833) (falcicornis).

Drummond \& Hemsley 2885 (kilimanjari); 2941 (ndiana); 3061 (kilimanjari); 3968 (plectomaniaca); 4647 (cornutella).

Drummond S109 (macrostele).

Dubois 1415 (kyimbilae).

Dummer 16 (pedicellaris); 2686 (cornutella); 3213 (cataphysema).

Duparquet s.n. (schimperiana).

Dusen s.n. (schimperiana).

Duvigneaud 51550 (retinervis).

Dyson 374 (schimperiana); 610 (linderi); 617 (linderi).

Dyson-Hudson 274 (limnophila).

Edwards \& Potgieter 1898 (falcicornis).

Edwards s.n. (humilior).

Eggeling 571 (cornutella); 3054 (schimperiana); 3407 (huillensis); 4230 (humilior); 5997 (huillensis); 6107 (humilior); 6108 (kyimbilae).

Elias 2 (diselloides); 8 (petraea); 12 (hirsutitrunci); 13 (petraea); 14 (hirsutitrunci); 15 (kyimbilae); 16 (petraea); 17 (petraea); 18 (kyimbilae); 20 (petraea).

Emson 04 (hebes).

Eood 737 (dives).

Evrard 2.444 (pauper).
Exell \& Mendonca 1984 (anaphysema).

Exell, Mendonca \& Wild 241 (macrostele); 717 (macrostele); 801 (sochensis); 1079 (macrostele).

Eyles 1037 (nyikana); 1477 (schimperiana); 6930 (schimperiana); 6934 (disparilis); 7288 (sochensis).

Fack 302 (limnophila); 303 (3055) (limnophila).

Faden \& Kabuye 71/566 (limnophila).

Falter \& Tisserant 1525 (schimperiana); 2935 (schimperiana).

Fanlkner A431 (ichneumonea).

Fanshawe 658 (weberana); 2740 (cataphysema); 11.163 (sochensis).

Faulkner 974 (kilimanjari); 1009 (plectomaniaca); 1491 (ndiana); s.n. (kilimanjari).

Fay 5639 (ichneumonea); 5829 (huillensis); 6604 (schimperiana); 6629 (schimperiana); 6690 (schimperiana); 7418 (weberana); 7419 (ichneumonea).

Festo \& Francis 1033 (schimperiana).

Field (la Croix) 1046 (weberana).

Foster 13712 (kilimanjari); 19054 (kilimanjari); s.n. (kilimanjari).

Fotius 2204 (schimperiana); 2530 (ichneumonea).

Fourcade 1135 (falcicornis).

Fox 42 (incarnata).

Fries 306 (altior).

Friis, Tadesse \& Vollesen 3267 (humilior); 3283 (schimperiana).

Fryffe 256 (limnophila).

Fuller 109 (kyimbilae); 113 (kyimbilae).

Galpin 392a (galpinii); 392b (incurva); 1400 (falcicornis); 9478 (dives); 11703 (orangana); 14441 (galpinii).

Gamwell 130 (pedicellaris).

Gane 119 (limnophila).

Gassner \& Cribb 209 (disparilis).

Geerling \& Bokdam 901 (schimperiana); 915 (schimperiana).

Geilinger 1154 (plectomaniaca).

Generd 583 (falcicornis).

Gerrard 383 (dives); 1480 (ichneumonea).

Germain 6038 (urundiensis); 6531 (urundiensis); 6531A (schimperiana).

Gilbert \& Getachew 3044 (schimperiana).

Gilbert \& Sebsebe 8550U (humilior); 8602 (vollesenii).

Gilbert \& Tadessa 6813 (humilior) 
Gilbert \& Thulin 783 (humilior); 917 (limnophila).

Gilbert 4873 (linderi).

Gilbert, Sebsebe \& Vollesen 80620 (vollesenii).

Gillett 17443 (ichneumonea).

Gilliand 1539 (humilior).

Goodier 190 (subaequalis); 197 (subaequalis); 198 (macrostele).

Gossweiler 560 (schimperiana); 2344 (ichneumonea); 3307 (anaphysema); 3910 (anaphysema).

Gragam 1596 (kilimanjari).

Graham 1593 (plectomaniaca); 2820 (901) (schimperiana); 3736 (altior); 3736A (thomsonii); 3736B (kraenzliniana); 3736C (linderi); A.532 (plectomaniaca).

Graterex 25 (schimperiana); 26 (ichneumonea); 28 (disparilis); 86 (ichneumonea); 90b (sochensis); 91 (myodes); 91A (myodes); 94 (strangulans); 7287 (schimperiana); 26744 (weberana); 26898 (weberana); 27210 (sochensis); 27241 (myodes); 27347 (myodes); GHS 35789 (robusta); 91703 (ichneumonea); 119792 (subaequalis).

Greenway \& Doughty 8527 (ndiana).

Greenway \& Kanui 13942 (humilior).

Greenway 1724 (ndiana); 4016 (kilimanjari); 4142 (welwitschii); 5498 (ichneumonea); 7771 (humilior); 8513 (humilior).

Gregory s.n. (cataphysema); s.n. (chirensis); s.n. (ndiana).

Greinying 45 (dives).

Griesback via Tweedie 3828 (kilimanjari); 3830 (kilimanjari).

Grosvenor \& Renz 973 (macrostele); 1018 (macrostele); 1104 (petraea); 1194 (diselloides); 1213 (williamsoni); 1298 (nyikana); 1305 (myodes); 11801 (riparia).

Grosvenor 304 (schimperiana); 376 (subaequalis); 476 (myodes); 481 (subaequalis); 482 (macrostele); 502 (schimperiana).

Gutz 63 (ichneumonea).

Haarer B2 (humilior); 233 (haareri); 549 (altior); 551B (haareri); 1477 (limnophila); 2370 (humilior); 2430 (humilior); 2502 (schimperiana).

Hall 219 (anaphysema); 2350 (schimperiana); 37048 (schimperiana); 43107 (schimperiana).

Hale B4077 (humilior).

Halleck s.n. (falcicornis).

Hanham s.n. (ichneumonea).

Hannington s.n. (weberana).

Hansfort S.797 (hochstetteriana).

Hardy \& Bally 10761B (huillensis).

Harvey 56 (ichneumonea); 155 (schimperiana); 1366 (schimperiana).
Hauman 489 (schimperiana).

Hay 89 (myodes); s.n. (myodes).

Hazel 668 (huillensis).

Heed 197 (macrostele).

Heischberg 163 (ichneumonea); 176 (schimperiana).

Hendricks 4975bis (macrostele).

Hepper, Field \& Mhoro 5250 (humilior).

Hericourt s.n. (schimperiana).

Hildbrandt 175 (schimperiana).

Hiliard \& Burtt 6255 (macrostele); 6302 (huillensis); 7748 (dives); 10263 (galpinii); 13937 (dives); 14387 (rehmannii); 14428 (rehmannii); 17730 (caffra); 17731 (dives); 18109 (dives).

Hill 26 (136) (limnophila).

Hillier 905 (schimperiana).

Hirschberg 187 (huillensis).

Hislop 34 (schimperiana).

Holmes 0118 (kubangensis); 0192 (retinervis); 0269 (schimperiana); 0293 (cataphysema); 0312 (anaphysema); 214 (petraea).

Holst 2878a (plectomaniaca); 8958 (ndiana).

Hooper \& Townsend 541 (welwitschii).

Hooper, Townsend \& Mwasumbi 1917 (humilior).

Humbert 7789 (macrostele); s.n. (humberti).

Humblot 426 (humblotii).

Hunford s.n. (welwitschii).

Hutton s.n. (falcicornis).

Ivens 1066 (altior).

Jackson 1036 (retinervis); 1094 (humilior); 1307 (kyimbilae); 1889 (thomsonii); 2034 (ichneumonea); 3846 (schimperiana); 4046 (huillensis); 4074 (humilior); s.n. (renziana).

Jacobsen 3724 (macrostele); 3780 (subaequalis).

Jacques-Felix 1802 (tisseranti); 1820 (schimperiana); 1888 (schimperiana); 1951 (schimperiana); 4126 (chirensis); 4583 (pauper); 7038 (ichneumonea); 7096 (schimperiana); 8073 (schimperiana); 8211 (huillensis); 8212 (schimperiana); 8221 (huillensis).

Jaeger 1552 (schimperiana); 1663 (anaphysema); 5239 (schimperiana).

James s.n. (linderi)

Jangoua 680 (confusa).

Jankins 4 (welwitschii); 119 (humilior).

Jansen 2343 (chirensis). 
Johnston 159 (altior); 856 (dives).

Johnston-Stewart 296 (humilior); 311 (disparilis).

Jordan 539 (schimperiana); 607 (schimperiana); 935 (schimperiana).

Joung s.n. (schimperiana).

Kassner 190 (pseudokaessneriana); 2363 (weberana); 2363a (bertauxiana); 2413 (pseudokaessneriana); 2414 (huillensis); 2457a (renziana); 2458 (huillensis); 2486 (kilimanjari); 2363 (weberana); 3564 (huillensis).

Keay 28 (schimperiana); s.n. (FHI 20175) (schimperiana); s.n. (FHI 22440) (huillensis).

Keeley 64 (humilior); 98 (schimperiana); PK37 (ichneumonea); s.n. (kyimbilae).

Keil 63 (linderi); 225 (welwitschii).

Kerr s.n. (dives).

Killak 42 (schimperiana).

Killick 1353 (orangana).

King 27 (linguiformis); 28 (limnophila); 32 (huillensis); 33 (huillensis); 33B (huillensis); 47 (schimperiana); 89 (limnophila); 92 (dalzielii); 175 (anaphysema); 189 (schimperiana); 322 (disparilis); 358 (sochensis); 412 (retinervis); 463 (schimperiana).

Kirk 10/83 (nyikana); s.n. (martialis); s.n. (nyikana); s.n. (woodii).

Kiss 35 (schimperiana).

Klaine 2057 (schimperiana); 2058 (schimperiana).

Klein 12b (schimperiana); 13 (schimperiana).

Klug Hamburg BFH 689 (antennifera).

Koechlin 4715 (schimperiana).

Koning 7207 (schimperiana).

Koning \& Hiemstra 9049 (nyikana).

Kornaś 3033 (ichneumonea).

Krook 109 (dives); 139 (dives).

Kwatha \& Patel 22 (schimperiana).

la Croix \& Johnston-Stewart 312 (kyimbilae); 317 (leucoceras); 318 (kilimanjari); 327 (kyimbilae); 477 (welwitschii); 481 (leucoceras).

la Croix \& Killick 455 (macrostele).

la Croix \& la Croix 384 (disparilis).

la Croix 105 (macrostele); 109 (schimperiana); 112 (nyikana); 113 (nyikana); 120 (sochensis); 128 (macrostele); 303 (sochensis); 304 (nyikana); 305 (myodes); 306 (sochensis); 341 (macrostele); 467 (ndiana); 471 (sochensis); 557 (weberana); 573 (schimperiana); 577 (sochensis); 586 (sochensis); 594 (leucoceras); 595 (petraea); 596 (kyimbilae); 598 (petraea);
603 (diselloides); 606 (hirsutitrunci); 632 (schimperiana); 668 (macrostele); 675 (nyikana); 771 (weberana); 786 (retinervis); 788 (schimperiana); 796 (ichneumonea); 801 (weberana); 823 (sochensis); 831 (kyimbilae); 839 (petraea); 841 (kyimbilae); 925 (retinervis); 927 (anaphysema); 939 (retinervis); 965 (riparia); 967 (petraea); 986 (diselloides); 994 (schimperiana); 999 (diselloides); 1001 (williamsoni); 1004 (sochensis); 1035 (kyimbilae); 1037 (kyimbilae); 1180 (disparilis); 1189 (disparilis) .

Labat, Haevermans, Randrianjohany \& Rabenantoandro 2972 (incarnata).

Lancaster in Best 1281 (haareri).

Lawlor \& Hall 29 (schimperiana); 470 (schimperiana); 660 (schimperiana); FHI 45582 (limnophila).

Le Testu 311 (nyikana); 911 (nyikana); 1508 (anaphysema); 1524 (schimperiana); 1524a (schimperiana); 1575 (anaphysema); 1924 (ichneumoena); 3126 (anaphysema); 3923 (schimperiana); 4029 (cornutella); 5061 (schimperiana); 9120 (schimperiana); 1.908 (anaphysema); 1.975 (anaphysema); 1.9756 (anaphysema); 2.935 (anaphysema); 3.296 (ichneumonea); 3.923 (ichneumonea); 5.061 (anaphysema); 9.120 (anaphysema); s.n. (anaphysema).

Lea 21 (kilimanjari).

Leach 4235 (disparilis); 82891 (schimperiana).

Leakey 3 (chirensis); OR 4 (linderi).

Lebrun 3254 (cornutella).

Leedal 74 (schimperiana); 441 (sochensis); 450 (stolzii); 4799 (welwitschii).

Leeuwenberg 8377 (schimperiana); 10199 (bosseriana).

Leistner \& Mauve 3233 (galpinii).

Lejoly 82/1015 (schimperiana).

Lely 343 (limnophila); 343A (linguiformis); P.379 (limnophila); 508 (schimperiana); P732 (huillensis); 1512 (schimperiana).

Leqakcy 3 (limnophila).

Leroy 30 (schimperiana).

Letouzey 1359 (schimperiana); 5500 (cornutella); 5662 (schimperiana); 6041 (schimperiana); 8060 (schimperiana); 8505 (pauper); 13959 (linguiformis); 14177 (schimperiana).

Lewalle 485 (arianae); 449 (arianae); 574 (arianae); 1397 (huillensis); 1555 (arianae); 4230 (pauper).

Lewis 1393 (schimperiana).

Leyn 152 (humilior).

Leysen 156 (kyimbilae).

Leyser 120 (isoantha); 250 (leucoceras).

Lind 248 (limnophila); 279 (limnophila); 2464 (ugandensis).

Linder 1506 (schimperiana); 1943 (caffra); 2016 (schimperiana); 2586 (linderi).

Linley 313 (kilimanjari); 314 (schimperiana); 610 (humilior). 
Lisowski 4006 (subaequalis); 10288 (schimperiana); 10319 (schimperiana); 11147 (stanislawii); 12636 (retinervis); 40660 (schimperiana); 41103 (kyimbilae); 41300 (schimperiana); 56051 (schimperiana); 56856 (schimperiana); 65555 (chirensis); 65560 (huillensis); 65746 (huillensis); 65758 (stanislawii); 65760 (huillensis); 65765 (huillensis); 65767 (huillensis); 65768 (retinervis); 65979 (welwitschii); 66294 (ichneumonea); 66325 (orthocaulis); 66326 (ichneumonea); 66388 (arianae); 66421 (chirensis); C-239 (lisowskii).

Lisowski, Malaisse \& Symoens 869 (arianae); 13.112 (anaphysema).

Lissouba 21 (cribbiana).

Litchford 366 (schimperiana).

Lock s.n. (cornutella).

Long 536 (falcicornis).

Longfield 85 (hochstetteriana).

Louis 4447 (cornutella); 4449 (cornutella).

Loveridge 462 (myodes).

Lovett \& Congdon 1858 (kyimbilae); 1858A (stenophylla); 1876 (kyimbilae); 2018 (altior).

Lovett \& Kayombo 4243 (schimperiana).

Lovett 622 (humilior); 1188 (schimperiana).

Lovett, Duncan \& Thomas 2817 (schimperiana).

Lugard 110 (ndiana); 270 (linderi); 673 (limnophila).

Luke 3317 (plectomaniaca).

Lusaha National History blub 237 (sochensis).

Luwiika, Harder, Schmidt \& Zimba 624 (humilior).

Luwiika, Harder, Zimba \& Nawa 76 (schimperiana).

Lyall 179 (incarnata).

Maas Geesteranus 5854 (schimperiana).

Mabberley \& McCall 3 (schimperiana); 59 (humilior).

Mabberley \& Tweedie 1120 (schimperiana).

Mabberley 1188 (humilior).

Macaulay 1173 (sochensis); 1173bis (sochensis).

MacInnes 162 (schimperiana).

Maclaud 94 (schimperiana); 229 (ichneumonea); 232 (pauper); 241 (schimperiana); 270 (pauper).

MacLeay 73A (chirensis); 74 (cataphysema); 103 (schimperiana); 122 (cataphysema).

MacLoughlin 60 (ichneumonea); 112 (macrostele).

Mac Owan s.n. (falcicornis).

Mainwaring 6000 (altior); s.n. (limnophila).

Maitland 1308A (ichneumonea); 1308B (limnophila); 1386 (maitlandii); 1562 (pauper); 1640 (schimperiana); s.n. (schimperiana).
Makany 1383 (schimperiana); 1383(!) (schimperiana).

Marais 1462 (dives).

Mathew s.n. (kilimanjari).

Mavi 942 (schimperiana).

McCloune 57 (kilimanjari).

Mdbbs 12A (kyimbilae).

Mechow 437 (huillensis); 438 (decaptera).

Medley Wood 437 (dives); 1005 (dives); 1015 (dives); 1534 (dives); 8171 (dives); s.n. (dives).

Meelise 9247 (falcicornis).

Meinkof 125 (usambarae).

Melville \& Hooker 278 (schimperiana); 280 (ichneumonea); 445 (schimperiana).

Mesfin \& Vollesen 4139 (vollesenii).

Mettam 168a (humilior); 168b (limnophila).

Meyers 7761 (huillensis).

Michel 3178 (cornutella).

Michel, Reed 901 (culcifera).

Michelwe 1477 (kilimanjari).

Midelwre 1481 (nyikana).

Mihinton 1969 (schimperiana); 2006 (schimperiana).

Miller 2193 (schimperiana); 4105 (schimperiana); 4161 (galpinii); 4168 (galpinii); 4189 (martialis); 5127 (galpinii); 8144 (disparilis); 8241 (disparilis).

Milne-Redhead \& Taylor 7977 (anaphysema); 8626 (weberana); 8681 (weberana); 8890 (retinervis); 9583 (sochensis); 9727 (sochensis); 9770 (myodes); 9913 (myodes); 9913A (myodes); 9927 (ichneumoena); 10313 (leucoceras); 10384 (stenophylla); 10426 (kyimbilae); 10426a (bertauxiana).

Milne-Redhead 3570 (ichneumonea); 3570A (ichneumonea); 3757 (cataphysema); 3769 (hebes); 3805 (retinervis); 3805B (retinervis); 3907 (schimperiana); 4002 (weberana); 4189 (martialis).

Milton s.n. (schimperiana).

Moll 612 (dives); 3462 (dives).

Monro 230 (schimperiana); 865 (schimperiana); 904 (schimperiana); 956 (schimperiana); 1753 (schimperiana); 1786 (ichneumonea).

Mooney 6153 (antennifera); 8051 (schimperiana); 9169 (schimperiana); 9265 (schimperiana).

Moos 16497 (falcicornis); 19242 (dives); 19243 (falcicornis). Moreau 211 (ndiana).

Moreaus 289 (sochensis).

Moriarty 160 (macrostele).

Morris 719 (macrostele); 736 (macrostele); 842 (disparilis); 871 (macrostele); 887 (macrostele); 926 (humilior). 
Morze 50 (disparilis); 53A (humilior); 53B (retinervis); 54 (kilimanjari); 62 (sochensis); 87 (robusta); 165 (weberana).

Moss 7565 (falcicornis); 16497 (falcicornis); 16628 (nyikana); 19242 (falcicornis).

Moussavou 1909 (schimperiana); 1969 (schimperiana).

Moyer \& Sunane 190 (schimperiana); 211 (schimperiana).

Munch 161 (schimperiana).

Mundt \& Maire s.n. (falcicornis).

Mutimushi 2461 (sochensis).

Mwasumbi \& Mdemu (humilior).

Napier 1144 (huillensis).

Ncube 74 (schimperiana).

Nelsh 707 (macrostele).

Nelson 16 (schimperiana).

New s.n. (kilimanjari).

Niangadouma \& Stone 409 (schimperiana).

Nicholson 149 (humilior); 238 (sochensis).

Noirfalise 217 (ichneumonea); 663 (schimperiana).

Nomann 288 (humilior).

Norlindh \& Weimarck 4488 (huillensis); 4989 (macrostele); 4749 (disparilis); 4835 (humilior); 4933 (schimperiana); 4989 (macrostele); s.n. (disparilis).

Nozeran s.n. (ichneumonea).

Odgers 187 (schimperiana); 248 (kilimanjari).

Okafor FHI 59233 (limnophila).

Onochie s.n. (FHI 33476) (schimperiana).

Ouren 20791 (humilior).

Overlaet 532 (kilimanjari); 1269 (ichneumonea).

Pappi 641 (schimperiana).

Parmentier \& Esomo 692 (schimperiana); 3636 (schimperiana).

Parmentier \& Nguema 548 (schimperiana).

Parsegler 2313 (myodes).

Parsons EA12365 (humilior).

Pauwals 1229 (cornutella).

Pawek 2118 (leucoceras); 2422 (kyimbilae); 2505 (kyimbilae); 4517 (weberana); 4825 (kyimbilae); 6951 (kyimbilae); 7007 (kyimbilae); 8066 (weberana); 8068 (retinervis); 8306 (retinervis); 8549 (diselloides); 8566 (leucoceras); 9296 (leucoceras); 9327 (petraea); 9640 (kyimbilae); 9801 (kyimbilae); 9807 (welwitschii); 9913 (kyimbilae); 10736 (schimperiana); 10886 (retinervis); 11491 (ichneumonea); 12389 (retinervis); 12525 (nyikana); 13710 (retinervis); 13917 (schimperiana); 14007 (schimperiana); 14025 (sochensis); 14166 (macrostele); 14347 (strangulans); 14353 (retinervis); 23375A (petraea).

Payne 24 (SRGH 45946) (galpinii).

Pegler 347 (falcicornis).

Penther 71 (falcicornis); 109 (dives); 136 (falcicornis); 139 (dives); 161 (falcicornis); 172 (falcicornis); 342 (falcicornis).

Perez -Vera 673 (schimperiana); 700 (schimperiana); 826 (schimperiana).

Perez Viso 2817bis (confusa).

Perrier de la Bathie 34 (incarnata).

Pettersson, Hedren \& Kibuwa 16 (schimperiana); 17 (isoantha); 202 (kyimbilae); 654 (humilior); 714 (humilior).

Pettersson \& Nilsson 209 (incarnata); 2676 (incarnata).

Phelan 252 (dives); 286 (huillensis).

Phillips 1130 (schimperiana); 1361A (petraea); 1371 (petraea); 1461 (petraea); 1505 (petraea); 1622 (schimperiana); 1624 (petraea); 1625 (diselloides); 1874 (kyimbilae); 3345 (kyimbilae); 4645 (schimperiana).

Philox, Leppard \& Dini 8797 (schimperiana); 8800 (galpinii).

Philox, Leppard, Duri \& Urayai 8896 (macrostele); 8920 (macrostele); 8931 (subaequalis); 8989 (subaequalis); 8989A (subaequalis); 8994 (macrostele); 9028 (subaequalis).

Philox, Pope \& Chisumpa 9954 (leucoceras); 9976 (leucoceras).

Phipps 369 (anaphysema).

Pielou 85 (kilimanjari).

Piers P.52 (chirensis); s.n. (altior).

Plowden s.n. (schimperiana).

Pobeguin 755 (ichneumonea); 759 (tisseranti); 1680 (angustissima); 2171 (tisseranti).

Polhill \& Paulo 1535 (schimperiana); 1569 (welwitschii); 1598 (urundiensis); 15696 (orthocaulis).

Pollitt 42189 (strangulans).

Polock 4 (sochensis).

Pooley 2205 (caffra).

Pope, Biegel, Simon 1109 (galpinii).

Poynton 5 (dives).

Prior s.n. (falcicornis).

Procter 1191 (leucoceras).

Purseglove 2224 (schimperiana); 2608 (linderi); 3361 (linderi).

Pyne 4 (schimperiana).

Qnane 8016 (kyimbilae). 
Quartin Dillon s.n. (antennifera); s.n. (chirensis); s.n. (humilior).

Quartin-Dillon \& Petit 106 (schimperiana); s.n. (humilior); s.n. (schimperiana).

Qunne s.n. (retinervis).

Ramsdew 1539 (altior).

Rand 269 (culcifera).

Ranger 267 (falcicornis).

Read 19 (kilimanjari).

Reck 6861 (falcicornis).

Reekmans 2380 (macrostele); 4710 (huillensis); 5902 (arianae); 8645 (welwitschii); 8698 (anaphysema); 8841 (williamsoni); 8842 (macrostele); 9818 (schimperiana).

Rehmann 5780 (rehmannii).

Reichenbach Herb. Orchid. Nr. 35855 s.n. (falcicornis); 38994 s.n. (falcicornis).

Reitsma J.M. \& B. 2758 (schimperiana).

Rensburg 2771 (kilimanjari).

Renvoize 2080 (humilior).

Richards 229 (retinervis); 462 (schimperiana); 783 (schimperiana); 888 (schimperiana); 1801 (humilior); 2136 (schimperiana); 3899 (disparilis); 4166 (schimperiana); 4274 (humilior); 4355 (schimperiana); 4401 (schimperiana); 4490 (humilior); 5063 (tubifolia); 5799 (humilior); 5867 (compta); 7848 (anaphysema); 7961 (disparilis); 8175 (kilimanjari); 8417 (tortilis); 8739 (schimperiana); 9942 (humilior); 10737 (cataphysema); 10737A (anaphysema); 10765 (humilior); 11133 (schimperiana); 12318 (macrotidion); 12461 (schimperiana); 12473 (hirsutitrunci); 15852 (schimperiana); 15978 (ichneumonea); 16027 (kilimanjari); 16195 (ichneumonea); 19558 (retinervis); 19562 (retinervis); 19729 (hirsutitrunci); 22080a (huillensis); 22080b (retinervis); 22107 (humilior); 22925 (retinervis); 22939 (schimperiana); 23314 (hochstetteriana); 26438 (weberana).

Richardson 69 (caffra).

Robertson 626 (huillensis).

Robinson 476 (kilimanjari); 550 (robusta); 2114 (humilior); 2126 (kilimanjari); 2139 (robusta); 3666 (hebes); 4153 (anaphysema); 4239 (cataphysema); 4327 (retinervis); 4967 (ichneumonea); 5637 (ichneumonea); 6228 (disparilis); 6231 (weberana); 6383 (disparilis); 6601 (hebes); 6808 (kilimanjari).

Robson 1191 (disparilis); 1345 (disparilis); 1512 (schimperiana); 2646 (anaphysema).

Rogers 30257 (falcicornis).

Rose-Innes 31331 (huillensis).

Rounee D2 (pauper); D3 (ichneumonea).

Rudatis 240 (luegiana); 548 (dives); 1302 (falcicornis); 1304 (falcicornis).
Rueppel N4 (antennifera).

Russell 72 (schimperiana).

Rutenberg s.n. (incarnata).

s.n. 14 (huillensis); 73 (huillensis); 152 (schimperiana); 224 (huillensis); 1391 (schimperiana); 5239 (schimperiana); 6153 (huillensis); 6494 (schimperiana); s.n. (altior); s.n. (disparilis); s.n. (dives); s.n. (falcicornis); s.n. (humilior); s.n. (kyimbilae); s.n. (linderi).

Saeger 001183 (pauper).

Sanane 730 (humilior); 1000 (disparilis); 1311 (ichneumonea).

Sanders s.n. (dives).

Sanderson 500 (caffra); 501 (dives); 560 (falcicornis); 833 (involuta).

Sanford 5109 (schimperiana).

Sankey 263 (orangana).

Savory 191 (schimperiana); 267 (schimperiana).

Schaijes 1015 (cataphysema); 1654 (welwitschii); 1681 (falcata); 1724 (ichneumonea); 1790 (weberana); 1790B (retinervis); 1791 (stenophylla); 1795 (macrotidion); 1827 (macrostele); 1828 (retinervis); 1879 (tangheana); 2099 (weberana); 2198 (cataphysema); 2870 (kubangensis).

Schelpe 2914 (humilior); 2914a (weberana); 4533 (dives); 5085 (dives); 5186 (falcicornis); 5257 (dives); s.n. (compta).

Schimper 596 (schimperiana); 607 (antennifera); III no. 703 (schimperiana); 1210 (schimperiana); 1324 (antennifera); 1324 (in part) (antennifera); 1364 (ichneumonea); 1369 (ichneumonea); 1369a (humilior); 1369b (pedicellaris); 1369c (haareri); 1369 (pedicellaris); 1373 (humilior); 1378 (schimperiana); 1645a (replicata); III. 1645b (hochstetteriana); 2703 (schimperiana).

Schinz 54 (dives).

Schlechter 1189 (furcipetala); 2325 (falcicornis); 4464 (tetrapetaloides); 4779 (nyikana); 6658 (dives); 6995 (welwitschii); 12824 (schimperiana); s.n. (caffra); s.n. (dives); s.n. (falcicornis).

Schlieben 797 (marxiana); 884 (marxiana); 1958 (pedicellaris); 2159 (marxiana); 2159A (myodes); 3758 (pedicellaris); 6085 (kilimanjari).

Schnell 1854 (schimperiana); 3027 (ichneumonea); 3727 (schimperiana); 6364 (schimperiana); 6816 (schimperiana); 7072 (schimperiana); 7461 (schimperiana).

Schuz A1 (schimperiana).

Schyff 2640 (nyikana).

Scott Elliot 276 (ndiana); 2576 (incarnata); 7198 in part (ndiana); 7854 (culcifera); 7922 (schimperiana); s.n. (falcicornis).

Segerback 1551 (schimperiana).

Segerbade 1170 (schimperiana). 
Semsei 2478 (ichneumonea); 4248 (kilimanjari).

Serand s.n. (schimperiana).

Seret 656 (schimperiana).

Sharland 1359 (limnophila); 2055 (limnophila).

Siame 325 (kilimanjari); 565 (kilimanjari).

Sidey 1971 (dives).

Sita \& Moutsambote s.n. (anaphysema); s.n. (ichneumonea).

Smith 505 (weberana); 675 (ichneumonea); 2798 (ichneumonea).

Snowden 91 (schimperiana); 915 (pedicellaris); 924 (humilior); 1275 (schimperiana); 1649 (anaphysema).

Sogerback 1291 (schimperiana).

Someren 7308 (limnophila).

Soyaux 211 (ichneumonea); 346 (schimperiana).

Spurrier 30 (schimperiana); 47 (welwitschii); 48 (anaphysema); 58 (humilior); 64 (kyimbilae); 66 (antennifera); 67 (macrostele); 69 (macrostele); 70 (kyimbilae); 81 (kyimbilae); 90 (stenophylla); 91 (humilior); 114 (diselloides); 120 (macrostele); 121 (stenophylla); 123 (leucoceras); 124 (sochensis); 147 (leucoceras); 30 (schimperiana); V.29 (huillensis); V.30 (schimperiana).

Staff 2253 (limnophila).

Steudner 701 (peltastes).

Stewart 1665 (dives); 2212 (involuta).

Stidolph 230,201 (schimperiana).

Stolz 2 (stolzii); 140 p.p (stolzii); 214 (martialis); 214bis (martialis); 713 (inaequiloba); 762 (weberana); 785 (kyimbilae); 1148 (isoantha); 1149 (marxiana); 1186 (orthocaulis); 1189 (furcipetala); 2490 (leucoceras); 2490A (limnophila); 2490B part (macrostele); 2490B part (stenophylla); 2581 (kyimbilae); 2581bis (kyimbilae); 2613 (diselloides); K59 (stolzii).

Stone 33 (schimperiana).

Stopp BO158 (huillensis); BO207 (welwitschii).

Strey 4116 (falcicornis); 8189 (woodii).

Suleiman \& Fundi 54 (thomsonii).

Summerhayes 21 (schimperiana); 29 (huillensis); 148 (limnophila); 160 (linguiformis); s.n. (FHI 22442) (huillensis).

Swant G17 (ndiana).

Swynnerton 744 (pedicellaris).

Symes 164 (humilior); 395 (limnophila).

Symoens, De Bilde \& Schweird 8 (disparilis).

Synge S. 1119 (thomsonii); S.1119a (hochstetteriana); S.1951 (cornutella).

Tanner 237 (welwitschii); 1991 (plectomaniaca); 4798 (schimperiana).
Taton 594 (schimperiana).

Taylor 14 (schimperiana); 1578 (thomsomii); 1888 (altior); 2046 (welwitschii); 3001 (welwitschii); s.n. (linderi); s.n. (plectomaniaca).

Teague 341 (huillensis).

Thollon 12 (schimperiana); 195 (schimperiana); 4069 (schimperiana); 4070 (schimperiana); s.n. (schimperiana).

Thomas 27 (limnophila); 86 (ichneumonea); 92 (schimperiana); 447 (limnophila); 480 (linderi); 2894 (schimperiana); 2978 (schimperiana); 3058 (schimperiana); 3136 (schimperiana); 3216 (schimperiana); 3650 (humilior); 3771 (schimperiana).

Thompson 905 (kyimbilae).

Thomson s.n. (altior); s.n. (thomdonii).

Thorold 2738 (humilior); 2779 (limnophila).

Tisserant 348 (schimperiana); 403 (schimperiana); 636 (schimperiana); 920 (schimperiana); 1508 (schimperiana); 1525 (schimperiana); 1579 (schimperiana); 1724 (schimperiana); 1908 (schimperiana); 2774 (tisseranti); 2935 (schimperiana); s.n. (kornasiorum); s.n. (welwitschii).

Troupin 1367 (pauper); 1568 (pauper); 1778 (pauper); 14.515 (macrostele); 15938 (humilior).

Tuller 112 (macrostele).

Tweedie 13 (humilior); 15 (limnophila); 207 (ndiana); 336 (ndiana); 404 (ndiana); 427 (altior); 428 (linderi); 483 (ndiana); 617 (altior); 628 (huillensis); 727 (thomsonii); 728 (linderi); 872 (linderi); 1349 (schimperiana); 1425 (linderi); 1725 (linderi); 2836 (limnophila); 3829 (altior).

Tyson 1071 (caffra); 1672 (caffra); 3080 (caffra).

Uhlig 1 (martialis); 11 (martialis).

van Nek 518 (schimperiana).

Vapier 3095 (humilior).

Vapin 2101 (humilior).

Venter 03010 (04010) (falcicornis); 4220 (galpinii); 5259 (dives).

Verdcourt 533 (limnophila).

Vigne 4785 (anaphysema).

Vogne 4785 (schimperiana).

Volkens 272 (pedicellaris); 2321 (pedicellaris); 2371 (kilimanjari).

Voughan 2400 (plectomaniaca); 2822 (plectomaniaca).

Wakefield s.n. (plectomaniaca).

Ward 29 (humilior).

Watermeyer 20 (welwitschii). 
Watmough 12 (macrotidion).

Welsh 192 (schimperiana); 652 (schimperiana).

Welwitsch 70 (anaphysema); 681 (confusa); 695 (robusta); 721 (welwitschii); 721 p.p. (cataphysema); 722 (cataphysema); 724 (huillensis); 724b (huillensis); 724c (huillensis); 743 (cataphysema); 744 (anaphysema); 1513 (dives).

Werdermann \& Oberdieck 2094 (orangana).

West 4740 (schimperiana).

Westphal \& Westhpal-Stevels 1679 (humilior).

Westwood 27 (huillensis); 27A (huillensis); 151 (schimperiana); 254 (schimperiana); 586 (leucoceras); 605 (schimperiana); 607 (retinervis); 612 (weberana); 625 (macrostele); 630 (macrostele); 657 (schimperiana); 663 (leucoceras); 665 (macrostele); 683 (macrostele).

Whellan \& Davies 995 (subaequalis).

White 6594 (humilior).

Whyte s.n. (altior); s.n. (kyimbilae); s.n. (limnophila).

Wickens 2171 (limnophila); 2215 (chirensis); 2459 (antennifera); 2600 (humilior); 2647 (antennifera); 2690 (antennifera).

Widebent 93 (ichneumonea).

Wiehe N/84 (leucoceras).

Wild 593 (disparilis); 950 (macrostele); 1868 (16211) (schimperiana); 3260 (27363) (nyikana); 4447 (macrostele); 46228 (macrostele); 6775 (disparilis).

Williams 74/44 (ndiana); 1383 (schimperiana); 11082 (schimperiana).

Williamson \& Drummond 1973 (hirsutitrunci).

Williamson \& Gassner 2294 (ichneumonea); 2295 (ichneumonea); 2376 (schimperiana); 2426 (retinervis); 2465 (ichneumonea).

Williamson \& Odgers 273 (petraea); 274 (hirsutitrunci).

Williamson \& Simon 943 (leucoceras); 1766 (arianae); 1775 (falcata); 1796 (retinervis); 1811 (cataphysema).

Williamson 99 (schimperiana); 143 (humilior); 257 (schimperiana); 263 (strangulans); 273 (petraea); 274 (leucoceras); 283 (williamsoni); 303 (strangulans); 387 (diselloides); 412 (weberana); 645 (anaphysema); 686 (schimperiana); 798 (retinervis); 832 (petraea); 946 (petraea); 1379 (disparilis); 1394 (humilior); 1431 (cataphysema); 1853 (schimperiana); 2055 (schimperiana); 2151 (disparilis); 2154 (humilior); 2167 (diselloides); 2224 (orthocaulis); 2227 (humilior).

Williamson, Simon \& Ball 798 (retinervis).

Wilson 976 (humilior).

Wiltshire 5 (schimperiana); 32 (limnophila).

Witte 3237 (welwitschii).

Wood 228 (ugandensis); 696 (falcicornis); 852 (limnophila); 1718 (antennifera); 3088 (falcicornis); 9374 (humblotii).
Wylie s.n. (dives).

Wylie sub Medley Wood 7601 (no. 9468 in Herb. Schltr.) (woodii).

Young s.n. (ichneumonea); s.n. (schimperiana).

\section{Incertae sedis:}

Barber s.n. (barberae). Hildebrandt 1883 (johannae).

Kassner 2931 (pristichila).

Thouars s.n. (sigillum).

\section{Undereminated materials:}

Benham s.n.

Bequethed 1967; 9.120; s.n.

Burtt 3238 .

Chase 5505; 5847.

Christenson 148.

Clare 5505.

Excell, Mendonca \& Wild 998.

Ghniesbreght 271.

Goodwin 78.

Greatrex 9556.

Guinzius (?) 108.

Hopkins 8606.

Kassner 2412.

King 144.

Kirk s.n.

Krauss 101.

Lisowski 12626; 65753; 65759; 66396.

Martins s.n.

Milne-Redhead 3457.

Monro 874; 1723A.

Paul Jaeger 1984; I FAV.

Pobeguin 362.

s.n. 302.

Schlechter 2325.

Schlieben 884 .

Sejne 1143.

Swynnerto 774.

Tisserant 199; 1.975; 2.935.

Tweedie 406; 2191.

Wallich s.n.

Wilms 1383.

Young 1151. 